\title{
DISEÑO DE ESTRATEGIAS DE SINCRONIZACIÓN Y ESTIMACIÓN DE CANAL PARA LA MEJORA DE COMUNICACIONES EN REDES INTELIGENTES DE ENERGÍA
}

Tesis de Doctorado

\section{MARTÍN ALEJANDRO COLOMBO}

Presentada ante la Facultad de Ingeniería de la Universidad Nacional de La Plata como requisito para la obtención del grado académico de

\section{DOCTOR EN INGENIERÍA}

Dirección de tesis:

Director: Dr. Miguel Ángel Mayosky

Codirector: Dr. Jesús Ureña Ureña

Subdirector: Dr. Álvaro Hernández Alonso

Jurado de tesis:

Dr. Juan Edmundo Cousseau

Dr. Fernando Cruz Roldán

Dra. Cecilia Galarza

Fecha de la defensa oral y pública: 10/09/2021 
Universidad

草 de Alcalá

UNIVERSIDAD DE ALCALÁ

UNIVERSIDAD NACIONAL DE LA PLATA

ESCUELA POLITÉCNICA SUPERIOR

FACULTAD DE INGENIERÍA

ELECRÓNICA: Sistemas

Doctorado en Ingeniería

Electrónicos Avanzados, Sistemas

Inteligentes

\title{
Diseño de Estrategias de Sincronización y Estimación de Canal para la Mejora de Comunicaciones en Redes Inteligentes de Energía
}

\author{
Autor: \\ Martín Alejandro Colombo \\ Directores: \\ Dr. Jesús Ureña Ureña y Dr. Álvaro Hernández Alonso \\ Dr. Miguel Mayosky
}

2021

TESIS DOCTORAL 



\section{RESUMEN}

En las últimas décadas se ha despertado un creciente interés en los sistemas de comunicaciones por la red eléctrica (Power-Line Communications - PLC) debido a su importante rol en el desarrollo de las redes eléctricas inteligentes. La red de distribución eléctrica se encuentra presente en zonas residenciales, comerciales e industriales, por lo que se convierte en un medio que presenta un bajo costo asociado al despliegue del sistema de comunicaciones digitales, en pos de satisfacer las necesidades de automatización y control. Sin embargo, los canales PLC presentan una marcada selectividad de frecuencia debido al efecto multicamino y diversas fuentes de ruido, lo que conforma un escenario desafiante para el desarrollo de sistemas de comunicaciones confiables. La multiplexación por división de frecuencias ortogonales (Orthogonal Frequency Division Multiplexing - OFDM) se ha propuesto como una solución eficiente para los sistemas PLC debido a su robustez frente a los canales de desvanecimiento selectivo en frecuencia. Sin embargo, los sistemas OFDM requieren de una sincronización adecuada, en conjunto con la estimación y ecualización del canal PLC para un desempeño apropiado.

La presente tesis contribuye en el desarrollo de estrategias eficientes de sincronización y estimación de canal para sistemas OFDM-PLC, que representen una disminución en la complejidad asociada respecto a variantes existentes en la literatura, y a su vez mantengan un desempeño competitivo. En primer lugar, se realiza el diseño de un símbolo piloto novedoso construido a partir de pares de secuencias complementarias, bajo el criterio de presentar adecuadas propiedades de correlación, un procesamiento de reducida complejidad, y la flexibilidad necesaria para poder aplicar técnicas de sincronización gruesa basadas en autocorrelación (AC) y correlación cruzada (CC), y efectuar una sincronización fina y estimación de canal a partir de esta última.

Con respecto a la sincronización gruesa, se realiza una comparación exhaustiva entre las técnicas basadas en AC y CC en las condiciones particulares del canal PLC. Luego, se desarrolla un algoritmo de sincronización fina a partir de la CC eficiente del símbolo piloto propuesto, en el que los cómputos realizados pueden reutilizarse posteriormente para obtener una estimación de la respuesta al impulso del canal, reduciendo la complejidad del conjunto respecto a un enfoque individual de cada etapa. Se realiza un análisis estadístico de las métricas planteadas en la etapa de sincronización gruesa así 
como aquellas planteadas en la etapa de sincronización fina, y se derivan umbrales de detección apropiados para los algoritmos de sincronización en cada caso.

Finalmente, se evalúa la propuesta de sincronización y estimación de canal conjunta en un escenario de transmisión en el que se limita el número de subportadoras activas a partir de una máscara espectral de compatibilidad electromagnética. Se evalúa la degradación de las propiedades del símbolo piloto propuesto, y a partir de ello se redefinen los umbrales de detección utilizados en las etapas de sincronización gruesa y fina. Se plantea la modificación de la correlación cruzada en el receptor para completar las subportadoras inactivas de manera tal de contrarrestar el efecto nocivo de la máscara espectral en la estrategia de estimación de canal.

A lo largo del trabajo se lleva a cabo un análisis de la complejidad asociada al cómputo de los algoritmos propuestos en los distintos escenarios planteados, y se verifica que la propuesta representa una opción de reducida complejidad gracias al diseño del símbolo piloto propuesto y su arquitectura de correlación eficiente. 


\begin{abstract}
In the last decades, there has been a growing interest in Power-Line Communications (PLC) systems due to their key role in the smart grids development. The power distribution network is present in residential, commercial and industrial areas, so it becomes a transmission medium that presents a low-cost deployment of digital communications system, in order to meet the needs of automation and control. However, PLC channels present high frequency selectivity due to the multipath effect and various noise sources, which make the development of reliable communications systems challenging. Orthogonal Frequency Division Multiplexing (OFDM) has been proposed as an efficient solution for PLC systems due to its robustness against frequency selective fading channels. However, OFDM systems require adequate synchronization and channel estimation and equalization for proper performance.
\end{abstract}

This thesis contributes to the development of efficient synchronization and channel estimation strategies for OFDM-PLC systems, which represent a decrease in the associated complexity with respect to existing strategies in the literature, while maintaining competitive performance. Firstly, we design a novel pilot symbol generated from complementary pair of sequences, pursuing adequate correlation properties, reduced complexity signal processing, and the necessary flexibility to apply coarse synchronization techniques based on auto-correlation (AC) and cross-correlation (CC), and to perform fine synchronization and channel estimation based on CC.

With respect to coarse synchronization, a comprehensive comparison is made between $\mathrm{AC}$ and $\mathrm{CC}$ based techniques under the particular conditions of the PLC channel. Then, a fine synchronization algorithm is developed from the efficient $\mathrm{CC}$ of the proposed pilot symbol, where the computations performed can be reused to obtain an estimate of the channel impulse response, reducing the complexity of the ensemble with respect to an individual approach for each stage. A statistical analysis of the metrics proposed in the coarse synchronization stage as well as those of the fine synchronization stage is performed, and appropriate detection thresholds are derived for the synchronization algorithms in each case.

Finally, the proposed joint synchronization and channel estimation is evaluated under a transmission scenario in which the number of active subcarriers is limited by means of an electromagnetic compatibility spectral mask. The degradation of the correlation properties of the proposed pilot symbol is evaluated, and therefore the detection 
thresholds used in the coarse and fine synchronization stages are redefined. A modification of the $\mathrm{CC}$ in the receiver is proposed, filling the inactive subcarriers in order to counteract the harmful effect of the spectral mask in the channel estimation strategy.

Throughout the work, an analysis of the complexity associated with the computation of the proposed algorithms in the different scenarios is carried out, and it is verified that the proposal represents an option of reduced complexity thanks to the design of the proposed pilot symbol and its efficient correlation architecture. 


\section{AGRADECIMIENTOS}

En primer lugar quiero agradecerles a mis directores, que sin su acompañamiento esta tesis no hubiera sido posible. Por el "lado español", a Jesús y Álvaro, por su compromiso, paciencia y dedicación en cada una de las reuniones semanales de los últimos años, pero principalmente por la calidez humana que me demostraron permanentemente, tanto en mis distintas estancias en Alcalá como en los encuentros virtuales. Por el "lado argentino", a Carlos y Miguel, que apostaron por mí para dar comienzo al largo recorrido que culmina con esta tesis, que siempre estuvieron para ayudarme y aconsejarme, y me dieron esa cuota de libertad para llevar adelante mi propio camino. Tuve la suerte de haber tenido directores académicamente excelentes, y aún mejores en el trato personal.

A todos mis compañeros del Departamento de Electrónica de la UAH, por los buenos momentos dentro y fuera del laboratorio, en especial con los que compartí durante mis estancias el fondo del pasillo 31.

A mis amigos, porque me acompañaron, cada uno a su manera, con asados y picadas de por medio. En especial a Fede, que siempre está. También a mis amigos/colegas Ripa, Santi y Nico C., que también "la sufrieron" y de algún modo nos acompañamos; a Rómulo que siempre está presente para dar una mano en lo que haga falta; y a Claudio y Gera, que me bancaron mucho en PByMN.

En el plano familiar, a mis viejos y hermanos, que me apoyan en todo lo que me propongo desde que tengo uso de razón. A la abu Zida, que es un ejemplo de fortaleza y vida. Mis mayores agradecimientos son para los amores de mi vida: a Lara, por su amor y apoyo permanente, y por complementarme en todo; a Emi, mi bebé, por ser un terremoto de amor; y a la pequeña vida que se está gestando actualmente, por traer aún más felicidad a la mía.

Finalmente, agradezco a la educación pública y gratuita argentina, en particular a la Universidad Nacional de La Plata y a todo el Departamento de Electrónica de la Universidad Nacional de la Patagonia San Juan Bosco. También al Consejo Nacional de Investigaciones Científicas y Técnicas por su apoyo, y a la Universidad de Alcalá por abrirme sus puertas. 



\section{ÍNDICE}

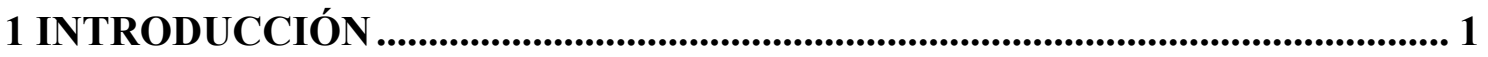

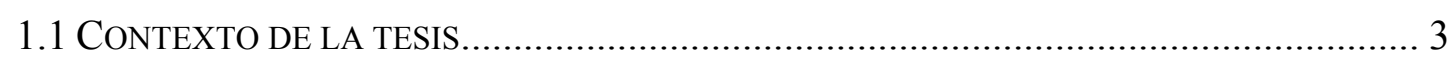

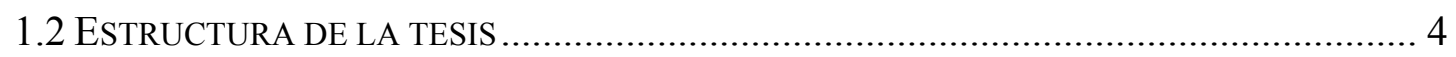

2 ESTADO DEL ARTE Y OBJETIVOS PLANTEADOS ......................................... 7

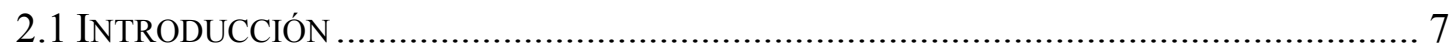

2.2 PLC COMO SOLUCIÓN PARA REDES ELÉCTRICAS INTELIGENTES................................ 8

2.3 MODULACIONES MULTIPORTADORA PARA PLC ...................................................... 9

2.4 CODIFICACIÓN PARA LA SINCRONIZACIÓN Y ESTIMACIÓN DE CANAL EN SISTEMAS

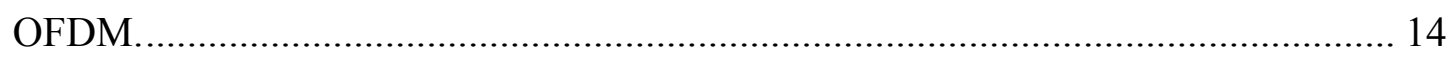

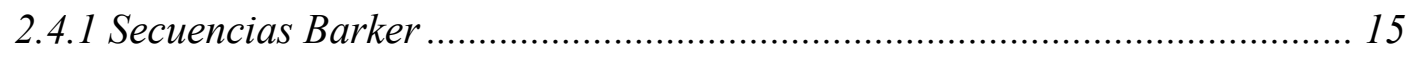

2.4.2 Secuencias pseudoaleatorias................................................................ 15

2.4.3 Pares y conjuntos de secuencias complementarias .................................. 17

2.4.4 Secuencias CAZAC ....................................................................................... 20

2.5 SINCRONIZACIÓN EN SISTEMAS OFDM.............................................................. 22

2.5.1 Sincronización basada en autocorrelación ................................................... 22

2.5.2 Sincronización basada en correlación cruzada ............................................ 28

2.5.3 Sincronización basada en CC incluyendo sufijo cíclico .............................. 32

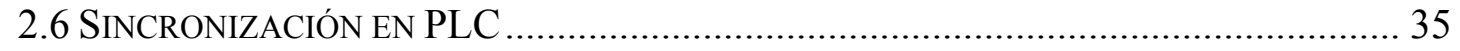

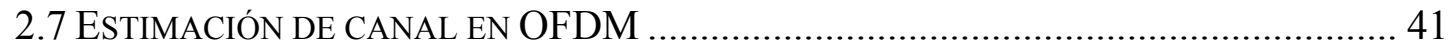

2.7.1 Estimación por mínimos cuadrados ........................................................... 42

2.7.2 Estimación por minimización del error cuadrático medio............................ 42

2.7.3 Estimación de canal por transformada discreta de Fourier ......................... 43

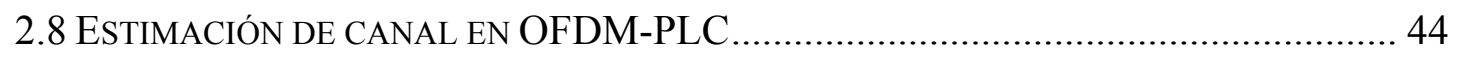

2.9 FORMULACIÓN DEL PROBLEMA Y OBJETIVOS PLANTEADOS .................................. 47

3 SINCRONIZACIÓN Y ESTIMACIÓN DE CANAL EN SISTEMAS OFDM-

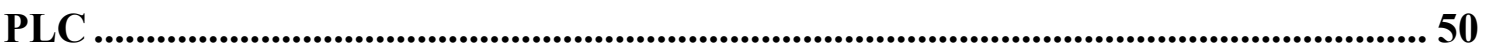

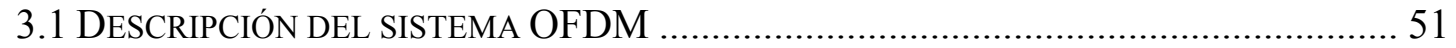

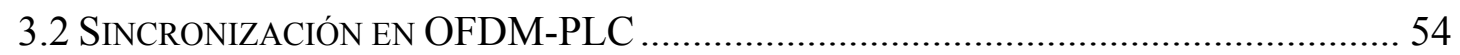

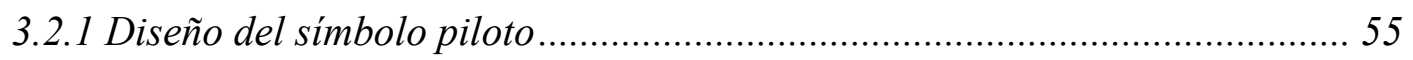

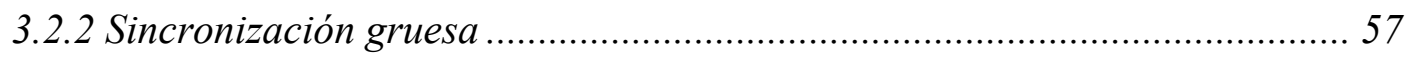

3.2.3 Correlación cruzada normalizada ........................................................... 59 


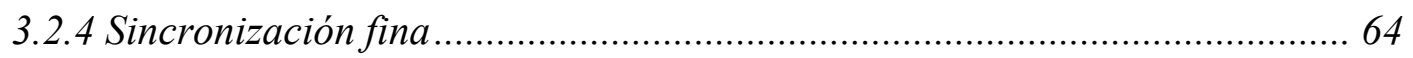

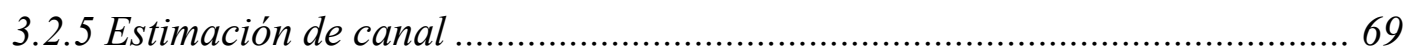

3.2.6 Complejidad de la propuesta............................................................. 71

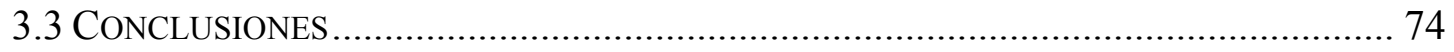

4 SINCRONIZACIÓN GRUESA EN SISTEMAS PLC-DMT ................................. 75

4.1 MODELO DE CANAL PLC PARA TRANSMISIONES BANDA BASE ................................ 76

4.2 DESCRIPCIÓN DEL SISTEMA Y DISEÑO DEL SÍMBOLO PILOTO ................................... 80

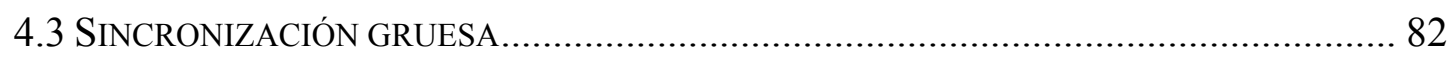

4.3.1 Métrica basada en autocorrelación bajo ruido de fondo blanco gaussiano. 85

4.3.2 Métrica basada en autocorrelación bajo ruido de fondo coloreado ............. 89

4.3.3 Métrica basada en correlación cruzada bajo ruido blanco gaussiano......... 96

4.3.4 Métrica basada en correlación cruzada bajo ruido de fondo coloreado... 104

4.3.5 Estimación de SNR a partir de las métricas de sincronización gruesa ...... 110

4.3.6 Métricas de sincronización gruesa bajo ruido impulsivo .......................... 114

4.3.7 Efecto del desvío en la frecuencia de muestreo en la sincronización gruesa 123

4.3.8 Resumen de desempeño de la etapa de sincronización gruesa .................. 125

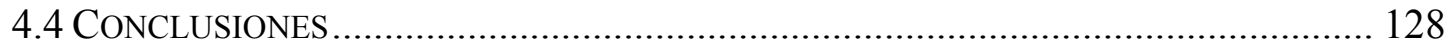

5 SINCRONIZACIÓN FINA Y ESTIMACIÓN DE CANAL EN SISTEMAS

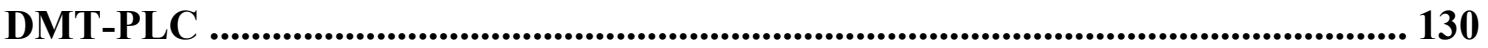

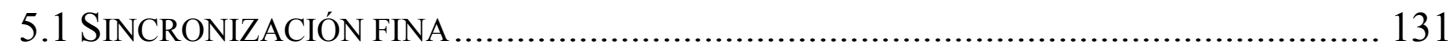

5.1.1 Análisis del canal PLC para la sincronización fina ................................ 131

5.1.2 Sincronización fina a partir de la correlación cruzada normalizada........ 135

5.1.3 Sincronización fina a partir de una correlación cruzada normalizada

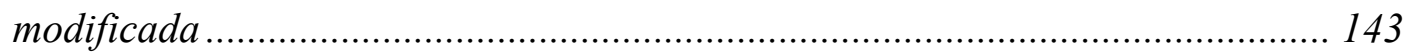

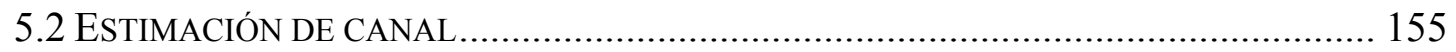

5.2.1 Estimación de canal bajo ruido blanco gaussiano..................................... 157

5.2.2 Estimación de canal bajo ruido de fondo coloreado................................ 157

5.2.3 Efecto del ruido impulsivo en la estimación de canal................................ 160

5.3 COMPLEJIDAD DE LA PROPUESTA DE SINCRONIZACIÓN Y ESTIMACIÓN DE CANAL 161

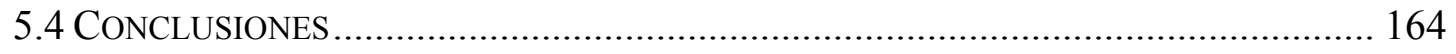

6 SINCRONIZACIÓN Y ESTIMACIÓN DE CANAL EN SISTEMAS DMT-PLC

BAJO UNA MÁSCARA ESPECTRAL DE TRANSMISIÓN ................................ 166 
6.1 DEFINICIÓN DE LA MÁSCARA ESPECTRAL DE TRANSMISIÓN Y SU EFECTO EN LAS PROPIEDADES DEL SÍMBOLO PILOTO PROPUESTO ..................................................... 168

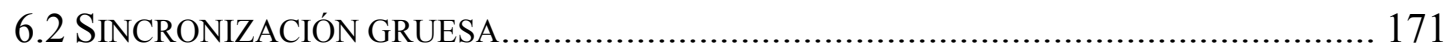

6.2.1 Inclusión de filtro notch en recepción .................................................... 174

6.2.2 Efecto del ruido impulsivo en la etapa de sincronización gruesa.............. 184

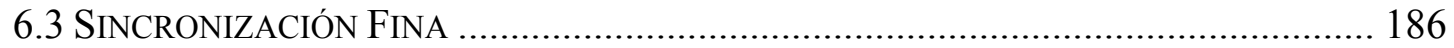

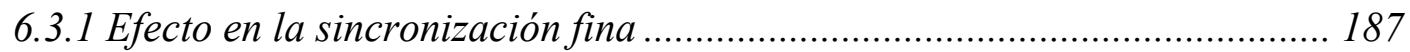

6.3.2 Propuesta de ajuste de umbral para sincronización fina ........................... 191

6.3.3 Efecto del ruido impulsivo en la sincronización fina ................................ 197

6.4 ESTIMACIÓN DE CANAL BAJO UNA MÁSCARA ESPECTRAL DE TRANSMISIÓN .......... 199

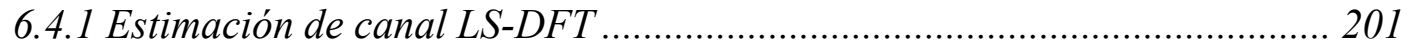

6.4.2 Estimación de canal DFT con interpolación.............................................. 204

6.4.3 Estimación de canal CPS-CC con interpolación ......................................... 209

6.4.4 Desempeño de la estimación de canal........................................................ 215

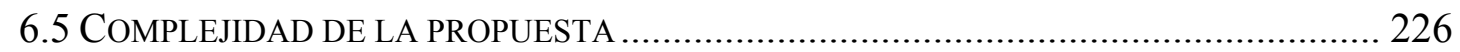

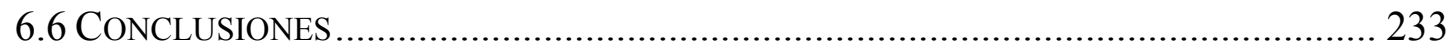

7 CONCLUSIONES Y TRABAJOS FUTUROS....................................................... 236

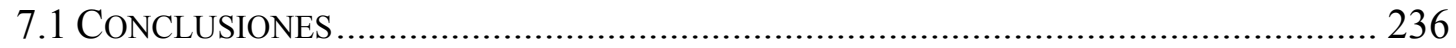

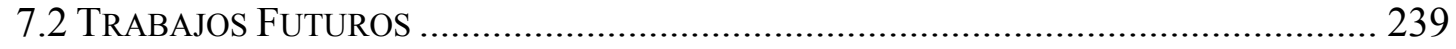

7.3 PUBLICACIONES DERIVADAS DE LA TESIS......................................................... 240

ANEXO A

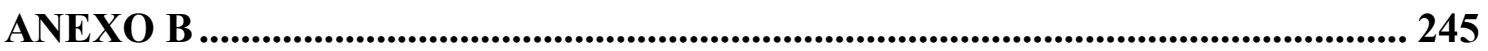

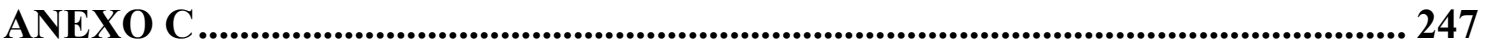

REFERENCIAS ............................................................................................................... 250 



\section{LISTA DE TABLAS}

Tabla 3.1 Perfil de ganancia promedio de los coeficientes para dos tipos de modelo de canal.

Tabla 3.2 Valores de diferentes umbrales para la etapa sincronización fina, para los canales $A$ y $B$ y distintas condiciones de SNR.

Tabla 3.3 Carga computacional asociada al cómputo de la correlación cruzada para distintos símbolos piloto.

Tabla 4.1 Parámetros característicos para la generación de dos variantes del modelo de canal PLC....78

Tabla 4.2 Parámetros de ajuste Log-normal para la ganancia del canal A, y de ajuste Log-normal y Gamma para la ganancia del canal B.

Tabla 5.1 Umbrales de sincronización fina y probabilidades de sincronización perfecta teóricas para los canales PLC tipo A y B bajo ruido blanco gaussiano. 142

Tabla 5.2 Umbrales de sincronización fina y probabilidades de sincronización perfecta teóricas para los canales PLC tipo A y B bajo ruido blanco gaussiano. 147

Tabla 5.3 Umbrales de sincronización fina y probabilidades de sincronización perfecta teóricas para los canales PLC tipo A y B bajo ruido de fondo coloreado.

Tabla 6.1 Detalle de portadoras activas al aplicar la máscara espectral de transmisión 169

Tabla 6.2 Valores de umbrales de sincronización fina para los canales PLC tipo A y B bajo ruido de fondo coloreado.

Tabla 6.3 Ganancia de error de las técnicas de estimación de canal respecto a LS, para distintos valores de SNR.

Tabla 6.4 Resumen preliminar de recursos demandados por la propuesta de sincronización y estimación de canal conjunta bajo una transmisión con y sin máscara espectral.....

Tabla A.1 Coeficientes más significativos del filtro equivalente utilizado para colorear el proceso de ruido blanco gaussiano.

Tabla C.1 Coeficientes más significativos del filtro notch utilizado para filtrar el ruido en las bandas inactivas en función de las bandas definidas por la máscara espectral de transmisión. 248 


\section{LISTA DE FIGURAS}

Fig. 2.1 Inserción del prefijo cíclico en símbolos OFDM.

Fig. 2.2 Diagrama en bloques del transmisor y receptor del sistema OFDM-PLC............................13

Fig. 2.3 Diagrama en bloques de un sistema DMT básico...................................................................... 14

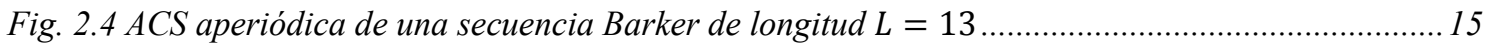

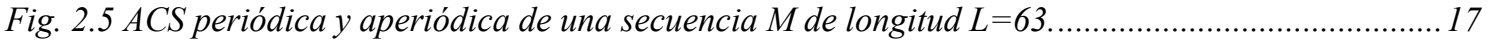

Fig. 2.6 Suma de ACS aperiódicas de un CPS de longitud $L=32$..................................................... 18

Fig. 2.7 Arquitecturas eficientes para la generación (arriba) y correlación (abajo) de un CPS..............19

Fig. 2.8 Descomposición de los elementos de una secuencia Zadoff-Chu de longitud L=64. .................221

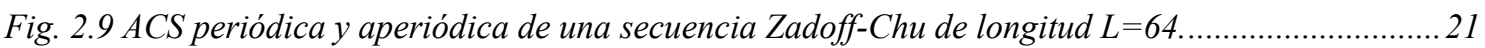

Fig. 2.10 Métricas de sincronización basadas en AC bajo un canal multicamino, sin el agregado de ruido......

Fig. 2.11 Correlación cruzada de distintos símbolos piloto extendidos con prefijo y sufijo cíclico con su correspondiente versión sin extender, bajo un canal multicamino sin el agregado de ruido............36

Fig. 3.1 Diagrama en bloques del transmisor y receptor del sistema OFDM-PLC................................52

Fig. 3.2 Función de correlación cruzada entre el símbolo extendido y el filtro acoplado del símbolo sin extender.

Fig. 3.3 CDF teórica y empírica de la correlación cruzada normalizada en las muestras previas al instante de sincronismo óptimo.

Fig. 3.4 CDF teórica y empírica de la correlación cruzada normalizada en el instante de sincronismo óptimo.

Fig. 3.5 Probabilidad de sincronización perfecta para la etapa de sincronización fina bajo distintos umbrales y condiciones de SNR para los canales $A$ y $B$.

Fig. 3.6 MSE de la sincronización fina para diferentes símbolos piloto basados en secuencias en los canales A (línea punteada) y B (línea continua).

Fig. 3.7 MSE de la estimación de canal con sincronización perfecta y luego de la sincronización fina para diferentes símbolos piloto basados en secuencias en los canales A (línea punteada) y B (línea

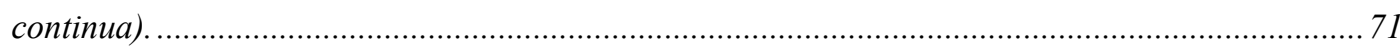

Fig. 3.8 Estructura equivalente para el cómputo de la métrica de sincronización gruesa...................... 72

Fig. 3.9 Diagrama en bloques del procesamiento de señal requerido para la propuesta de sincronización fina y estimación de canal conjunta.

Fig. 4.1 Respuestas al impulso correspondientes a una realización de los canales $A$ y $B$. 78

Fig. 4.2 CDFs empíricas obtenidas de la ganancia del canal A y su ajuste Log-normal, y del canal B y su ajuste Log-normal y Gamma. 79

Fig. 4.3 Diagrama en bloques de un sistema DMT básico.

Fig. 4.4 Función de correlación cruzada entre el símbolo extendido y el filtro acoplado del símbolo sin extender.

Fig. 4.5 Métricas para sincronización gruesa bajo una realización de canal A, sin agregado de ruido (izquierda) y con ruido de fondo coloreado y una SNR de $3 \mathrm{~dB}$ (derecha). 
Fig. 4.6 PDFs del valor de las métricas de sincronización gruesa en el instante de sincronización óptima $y$ anteriores, obtenidas por simulaciones, bajo un canal PLC tipo A normalizado con ruido blanco Gaussiano y distintos valores de SNR.

Fig. 4.7 Densidad espectral de potencia del ruido de fondo coloreado. 90

Fig. 4.8 Filtro equivalente aplicado a un proceso de ruido blanco Gaussiano para generar ruido de fondo coloreado.

Fig. 4.9 Respuesta al impulso del filtro obtenido en el dominio del tiempo a partir de la PSD del ruido de fondo coloreado.

Fig. 4.10 Secuencias de autocorrelación del símbolo piloto $\boldsymbol{x}$, el filtro que colorea el ruido $\boldsymbol{g}$ y la combinación de ambos.

Fig. 4.11 PDFs del valor de las métricas de sincronización gruesa en el instante de sincronización óptima y anteriores, obtenidas por simulaciones, bajo un canal PLC tipo A normalizado con ruido de fondo coloreado y distintos valores de SNR.

Fig. 4.12 Métrica basada en correlación cruzada del símbolo piloto propuesto, sin agregado de ruido bajo un canal PLC tipo A.

Fig. 4.13 Diagrama en bloques del cómputo equivalente de las variables aleatorias intervinientes en la métrica de sincronización bajo ruido blanco gaussiano.

Fig. 4.14 PDFs empírica y teórica del valor de la métrica de sincronización gruesa m3, $n$ en las muestras previas a la llegada del símbolo piloto con ruido de fondo blanco Gaussiano......

Fig. 4.15 PDFs del valor de la métrica de sincronización gruesa m3, $n$ en los instantes de sincronización óptimo y anteriores, obtenidos por simulaciones bajo un canal PLC tipo A normalizado con ruido de fondo blanco Gaussiano y distintos valores de SNR.

Fig. 4.16 Diagrama en bloques del cómputo equivalente de las variables aleatorias intervinientes en la métrica de sincronización bajo ruido de fondo coloreado.

Fig. 4.17 PDFs empírica y teórica del valor de la métrica de sincronización gruesa m3, $n$ en las muestras previas a la llegada del símbolo piloto con ruido de fondo coloreado. 108

Fig. 4.18 PDFs del valor de la métrica de sincronización gruesa $m 3, n$ en los instantes de sincronización óptimo y anteriores, obtenidos por simulaciones bajo un canal PLC tipo A normalizado con ruido de fondo coloreado y distintos valores de SNR. 109

Fig. 4.19 Probabilidad de sincronización en términos de la detección del símbolo piloto para las métricas basadas en autocorrelación y correlación cruzada bajo ruido de fondo coloreado........ 110

Fig. 4.20 PDFs del estimador de SNR obtenido a partir de las métricas basadas en autocorrelación. .. 112

Fig. 4.21 PDFs del estimador de SNR obtenido a partir de la métrica basada en correlación cruzada. 115

Fig. 4.22 Descomposición del modelo de ruido del canal PLC en ruido de fondo y diversas fuentes de ruido impulsivo.

Fig. 4.23 PDF empírica de las métricas de sincronización gruesa en el instante óptimo de sincronismo, con y sin eventos de ruido impulsivo con probabilidad de ocurrencia del $8 \%$ y una $S N R=0 \mathrm{~dB} \ldots 117$

Fig. 4.24 PDFs empíricas de las métricas de sincronización gruesa en los instantes previos a la llegada del símbolo piloto, con y sin eventos de ruido impulsivo con probabilidad de ocurrencia del 100\%. 
Fig. 4.25 Probabilidad de detección del símbolo piloto a partir de las métricas de sincronización gruesa, con y sin eventos de ruido impulsivo con probabilidad de ocurrencia de 8 y $100 \%$.

Fig. 4.26 Muestras de la señal recibida antes y después de aplicar el procesamiento no lineal de blanqueo, con SNR de $6 \mathrm{~dB}$. 120

Fig. 4.27 Resultado del uso de la etapa de AGC como medio para mitigar el efecto del ruido impulsivo. 121

Fig. 4.28 Efecto de las técnicas de blanqueo y AGC en las PDFs de la métrica m2, $n$ bajo eventos de ruido impulsivo con probabilidad de ocurrencia de $100 \%$.

Fig. 4.29 Efecto de las técnicas de blanqueo y AGC en las PDFs de la métrica m3, n bajo eventos de ruido impulsivo con probabilidad de ocurrencia de $100 \%$. 124

Fig. 4.30 Probabilidad de detección del símbolo piloto con y sin mitigación de eventos de ruido impulsivo con probabilidad de ocurrencia de $100 \%$.

Fig. 4.31 Probabilidad de detección del símbolo piloto con eventos de ruido impulsivo con probabilidad de ocurrencia de $8 \%$ y $100 \%$.

Fig. 4.32 PDFs empíricas de las métricas de sincronización gruesa en el instante óptimo de sincronismo, con y sin SFO, bajo de ruido de fondo coloreado y una SNR de $6 \mathrm{~dB}$.

Fig. 4.33 Probabilidad de detección del símbolo piloto con y sin SFO bajo ruido de fondo coloreado.. 126

Fig. 4.34 Probabilidad de detección del símbolo bajo ruido de fondo coloreado con y sin ruido impulsivo y $S F O$.

Fig. 4.35 Desempeño de las técnicas de sincronización propuestas en términos del RMSE, para distintas condiciones de SNR. 128

Fig. 5.1 CDFs empíricas de gCH(t1) a partir del generador de canal (izquierda) y de la aproximación considerando sólo el efecto del primer camino (derecha), para el canal A. 134

Fig. 5.2 CDFs empíricas de gCH(t1) a partir del generador de canal (izquierda) y de la aproximación considerando sólo el efecto del primer camino (derecha), para el canal A.

Fig. 5.3 Algoritmo de sincronización fina basado en la correlación cruzada normalizada, sin ruido agregado.

Fig. 5.4 PDFs empíricas de la raíz de la correlación cruzada normalizada en el instante óptimo de sincronismo para el canal A (izquierda) y B (derecha), bajo ruido de fondo coloreado y diferentes condiciones de SNR.

Fig. 5.5 PDFs empíricas de la raíz de la correlación cruzada normalizada en la zona de correlación nula para el canal A (izquierda) y B (derecha), bajo ruido blanco gaussiano y diferentes condiciones de SNR.

Fig. 5.6 Resultados de simulación de la probabilidad de sincronización perfecta bajo los canales PLC tipo A (izquierda) y B (derecha) con ruido blanco Gaussiano.

Fig. 5.7 PDFs empíricas de la raíz de la correlación cruzada normalizada modificada en el instante óptimo de sincronismo para el canal A (izquierda) y B (derecha), bajo ruido de fondo coloreado y diferentes condiciones de SNR.

Fig. 5.8 PDFs empíricas de la raíz de la correlación cruzada normalizada modificada en la zona de correlación nula para el canal A (izquierda) y B (derecha), bajo ruido blanco gaussiano $y$ diferentes condiciones de SNR. . 
Fig. 5.9 Resultados de simulación de la probabilidad de sincronización perfecta para la correlación cruzada normalizada modificada, bajo los canales PLC tipo A (izquierda) y B (derecha) con ruido blanco gaussiano.

Fig. 5.10 Desempeño de la etapa de sincronización fina propuesta en términos del RMSE, bajo los canales PLC tipo A (izquierda) y B (derecha) con ruido blanco gaussiano.

Fig. 5.11 PDFs empíricas de la raíz de la correlación cruzada normalizada en zona de correlación nula para el canal $A$ (izquierda) y $B$ (derecha), bajo ruido de fondo coloreado y diferentes condiciones de SNR.

Fig. 5.12 Resultados de simulación de la probabilidad de sincronización perfecta para la correlación cruzada normalizada modificada, bajo los canales PLC tipo A (izquierda) y B (derecha) con ruido de fondo coloreado.

Fig. 5.13 PDFs empíricas de la raíz de la correlación cruzada normalizada modificada en el instante óptimo de sincronismo para el canal A (izquierda) y $B$ (derecha), bajo ruido de fondo coloreado $e$ impulsivo, y una condición de $S N R=0 d B$.......

Fig. 5.14 PDFs empíricas de la raíz de la correlación cruzada normalizada en la zona de correlación nula para el canal A (izquierda) y $B$ (derecha), bajo ruido de fondo coloreado e impulsivo, y una condición de $S N R=0 d B$.

Fig. 5.15 Resultados de simulación de la probabilidad de sincronización perfecta para la correlación cruzada normalizada modificada, bajo los canales PLC tipo A (izquierda) y B (derecha) con ruido de fondo coloreado e impulsivo.

Fig. 5.16 Desempeño de la etapa de sincronización fina propuesta en términos del RMSE, bajo los canales PLC tipo A (izquierda) y B(derecha) con ruido de fondo coloreado e impulsivo. 155

Fig. 5.17 MSE de la estimación del canal PLC bajo ruido blanco gaussiano. 158

Fig. 5.18 MSE de la estimación del canal PLC bajo ruido de fondo coloreado.

Fig. 5.19 MSE de la estimación del canal PLC tipo A (izquierda) y B (derecha), bajo ruido de fondo coloreado e impulsivo.

Fig. 5.20 Estructura equivalente para el cómputo del numerador de la métrica de sincronización gruesa. 161

Fig. 5.21 Estructura equivalente para el cómputo del denominador de la métrica de sincronización gruesa. 161

Fig. 5.22 Diagrama en bloques del procesamiento de señal requerido para la propuesta de sincronización y estimación de canal conjunta.

Fig. 5.23 Diagrama en bloques del procesamiento no lineal para la mitigación del ruido impulsivo. ... 164

Fig. 6.1 Máscara espectral de compatibilidad electromagnética para transmisiones PLC en el estándar IEEE P1901 [IEEE 2010]. 169

Fig. 6.2 Transmisor del sistema DMT modificado incluyendo la máscara espectral de emisión. 170

Fig. 6.3 Función de correlación cruzada entre el símbolo piloto extendido con y sin máscara aplicada y el símbolo ideal mediante el filtro acoplado...... 171

Fig. 6.4 PDFs del valor de la métrica de sincronización gruesa basada en AC, en el instante de sincronización óptimo y anteriores, aplicando la máscara espectral de transmisión 
Fig. 6.5 PDFs del valor de la métrica de sincronización gruesa basada en CC, en el instante de sincronización óptimo y anteriores, aplicando la máscara espectral de transmisión. 173

Fig. 6.6 Probabilidad de sincronización en términos de la detección del símbolo piloto para las métricas basadas en AC y CC bajo ruido de fondo coloreado y máscara espectral de transmisión. 174

Fig. 6.7 Máscara espectral de transmisión y PSD normalizada del ruido de fondo coloreado.............. 175

Fig. 6.8 Respuesta en frecuencia del filtro notch de orden 2048........................................................ 176

Fig. 6.9 Receptor del sistema DMT modificado incluyendo el filtro notch ........................................ 176

Fig. 6.10 PDFs del valor de la métrica de sincronización gruesa basada en AC, en el instante de sincronización óptimo y anteriores, aplicando la máscara espectral de transmisión y el filtro notch en recepción para distintos valores de SNR. 177

Fig. 6.11 Secuencias de autocorrelación del filtro que colorea el ruido $\mathbf{g}$, el filtro notch $\boldsymbol{c}$ y la combinación de ambos. 178

Fig. 6.12 Diagrama equivalente del filtrado de muestras de ruido coloreado a partir del filtro notch en recepción. 178

Fig. 6.13 PDFs empírica y teórica del valor de la métrica de sincronización gruesa basada en AC en las muestras previas a la llegada del símbolo piloto con máscara espectral de transmisión y filtro notch en recepción.

Fig. 6.14 PDFs del valor de la métrica de sincronización gruesa basada en CC, en el instante de sincronización óptimo y anteriores, aplicando la máscara espectral de transmisión y el filtro notch en recepción para distintos valores de SNR.

Fig. 6.15 Secuencias de autocorrelación del filtro $\boldsymbol{f} \mathbf{1}$, el filtro acoplado del símbolo piloto $\boldsymbol{x}^{\prime} y$ la combinación de ambos.

Fig. 6.16 PDFs empírica y teórica del valor de la métrica de sincronización gruesa basada en CC en las muestras previas a la llegada del símbolo piloto con máscara espectral de transmisión y filtro notch en recepción.

Fig. 6.17 Probabilidad de sincronización en términos de la detección del símbolo piloto para las métricas basadas en AC y CC bajo ruido de fondo coloreado, máscara espectral de transmisión y filtro notch en recepción para distintos valores de SNR.

Fig. 6.18 Probabilidad de detección del símbolo piloto con y sin mitigación de eventos de ruido impulsivo con probabilidad de ocurrencia de $100 \%$.

Fig. 6.19 Probabilidad de detección del símbolo piloto con y sin mitigación de eventos de ruido impulsivo con probabilidad de ocurrencia de $8 \%$......

Fig. 6.20 Salida del filtro acoplado con y sin máscara espectral de transmisión, para una realización del canal $A$ sin el agregado de ruido. 188

Fig. 6.21 Probabilidad de sincronización fina perfecta considerando los umbrales $\alpha 1$ para las dos variantes de CCN, la ubicación del máximo de cada una, y el resultado de la etapa de sincronización gruesa, para los canales tipo A (izquierda y B (derecha).

Fig. 6.22 RMSE de la sincronización fina considerando los umbrales $\alpha 1$ para las dos variantes de CCN, la ubicación del máximo de cada una, y el resultado de la etapa de sincronización gruesa, para los canales tipo A (izquierda y $B$ (derecha). 
Fig. 6.23 Muestras de la correlación cruzada del símbolo piloto propuesto, luego de aplicar la máscara espectral de transmisión.

Fig. 6.24 Probabilidad de sincronización fina perfecta considerando los umbrales ak para las dos variantes de CCN, los umbrales $\alpha 1$ y la ubicación del máximo, para los canales tipo A (izquierda y $B$ (derecha). 193

Fig. 6.25 RMSE de la sincronización fina considerando los umbrales $\alpha k$ para las dos variantes de CCN, los umbrales $\alpha 1$ y la ubicación del máximo, para los canales tipo A (izquierda y B (derecha)..... 193

Fig. 6.26 Probabilidad de sincronización fina perfecta bajo distintas estrategias de umbralización para los canales tipo A (izquierda y $B$ (derecha).

Fig. 6.27 RMSE de la sincronización fina bajo distintas estrategias de umbralización para los canales tipo A (izquierda y $B$ (derecha).

Fig. 6.28 Desempeño de la etapa de sincronización fina en términos del RMSE, para los canales tipo A (izquierda y $B$ (derecha), con ruido de fondo coloreado e impulsivo. 198

Fig. 6.29 MSE de la estimación de canal en las subportadoras activas, para el sistema perfectamente sincronizado, con y sin máscara espectral de transmisión aplicada. 200

Fig. 6.30 Autovalores de la matriz del sistema lineal $\boldsymbol{F}_{\text {vir }}$ 203

Fig. 6.31 Subportadoras inactivas pertenecientes al sistema de ecuaciones completo y reducción propuesta. 204

Fig. 6.32 MSE de la estimación de canal al aplicar la técnica LS-DFT. 205

Fig. 6.33 Interpolación en el dominio de la frecuencia en las subportadoras inactivas intermedias para realización del canal tipo $A$ y $S N R=30 \mathrm{~dB}$. 206

Fig. 6.34 Resultado de extrapolar las muestras obtenidas a partir de los tres métodos de interpolación utilizados para completar las subportadoras inactivas fuera de la banda de transmisión....... 206

Fig. 6.35 Estimación de la respuesta al impulso luego del proceso de interpolación. 207

Fig. 6.36 MSE de la estimación de canal al aplicar las distintas técnicas INT-DFT para el canal PLC tipo A... 208

Fig. 6.37 Subportadoras necesarias para efectuar la interpolación y extrapolación lineal propuesta. ..210

Fig. 6.38 Filtro equivalente para el cómputo de la SDFT. 211

Fig. 6.39 Representación gráfica de la interpolación lineal propuesta para completar la parte real de cada banda intermedia inactiva.

Fig. 6.40 Proceso de modificación de la correlación cruzada a partir de la interpolación y extrapolación en las subportadoras inactivas, para una realización del canal PLC tipo A y $S N R=20 d B$.

Fig. 6.41 Diagrama del proceso de estimación de la CFR a partir del vector de estimación de la CIR. 216

Fig. 6.42 MSE de la estimación del canal PLC tipo A bajo sincronización perfecta para distintas técnicas de estimación de canal y la propuesta CPS-CC con interpolación.

Fig. 6.43 Respuesta en frecuencia del canal PLC sin el agregado de ruido, canal equivalente al incluir el filtro notch y la estimación de canal propuesta. 218

Fig. 6.44 MSE de la estimación del canal bajo sincronización perfecta al incorporar eventos de ruido impulsivo y la técnica de mitigación de blanqueo, para los canales tipo A (izquierda y B (derecha). 
Fig. 6.45 Proceso de modificación de la correlación cruzada, para una realización del canal PLC tipo A, un error de sincronismo de +80 muestras, y $S N R=30 \mathrm{~dB}$.

Fig. 6.46 MSE de la estimación del canal con sincronización conjunta, respecto al canal PLC y su versión desplazada por el error de sincronismo, para los canales tipo A (izquierda y B (derecha).

Fig. 6.47 MSE de la estimación del canal con sincronización conjunta, al incorporar eventos de ruido impulsivo y la técnica de mitigación de blanqueo, para los canales tipo A (izquierda y B (derecha)..

Fig. 6.48 Estructura equivalente para el cómputo del numerador de la métrica de sincronización gruesa.

Fig. 6.49 Estructura equivalente para el cómputo del denominador de la métrica de sincronización gruesa.

Fig. 6.50 Filtro equivalente para el cómputo de la SDFT. ............................................................228

Fig. 6.51 Diagrama en bloques para computar la estimación de la respuesta al impulso del canal.......221

Fig. 6.52 Diagrama en bloques del procesamiento de señal requerido para la propuesta de sincronización y estimación de canal conjunta.

Fig. A.1 Respuesta al impulso del filtro equivalente que colorea el ruido de fondo y coeficientes más significativos incluidos en la Tabla A.1.

Fig. C.1 Respuesta al impulso del filtro notch y coeficientes más significativos incluídos en la Tabla C.1. 



\section{LISTA DE ACRÓNIMOS}

$\mathrm{AC}$

ACS

ADC

AFE

AGC

AMR

ASCET

AWGN

$\mathrm{BB}$

BPSK

CAZAC

$\mathrm{CC}$

CCD

$\mathrm{CCN}$

CCS

CDF

CENELEC

CFO

CFR

CIR

$\mathrm{CP}$

CPS

CSS

DAC

DC

DFT

DMT

DSL

EMC

FBMC

FCC

FDE

FFT

FIR

GPC
Autocorrelación

Autocorrelation Sequence

Analog to Digital Converter

Analog Front-End

Automatic Gain Control

Advanced Metering Infrastructure

Adaptive Sine-modulated/Cosine-modulated filter bank Equalizer for Transmultiplexers

Additive White Gaussian Noise

Broadband

Binary Phase Shift Keying

Constant Amplitude Zero Auto-Correlation

Correlación Cruzada

Correlación Cruzada Diferencial

Correlación Cruzada Normalizada

Cross-Correlation Sequence

Cumulative Distribution Function

Comité Européen de Normalisation Électrotechnique

Carrier Frequency Offset

Channel Frequency Response

Channel Impulse Response

Cyclic Prefix

Complementary Pair of Sequences

Complementary Set of Sequences

Digital to Analog Converter

Direct Current

Discrete Fourier Transform

Discrete Multi-Tone

Digital Subscriber Line

Electromagnetic Compatibility

Filter Bank Multi-Carrier

Federal Communications Commission

Frequency Domain Equalizer

Fast Fourier Transform

Finite Impulse Response

Generalize Pairwise Complementary 


\begin{tabular}{|c|c|}
\hline HAN & Home Area Network \\
\hline $\mathrm{ICDF}$ & Inverse Cumulative Distribution Function \\
\hline ICI & Inter-Carrier Interference \\
\hline IDFT & Inverse Discrete Fourier Transform \\
\hline IEEE & Institute of Electrical and Electronics Engineers \\
\hline IFFT & Inverse Fast Fourier Transform \\
\hline IIR & Infinite Impulse Response \\
\hline ISI & Inter-Symbol Interference \\
\hline ITU & International Telecommunication Union \\
\hline LFSR & Linear Feedback Shift Register \\
\hline LMMSE & Linear Minimum Mean Square Error \\
\hline LS & Least Squares \\
\hline LSI & Linear Shift-Invariant \\
\hline LTE & Long Term Evolution \\
\hline LTI & Linear Time-Invariant \\
\hline MIMO & Multiple-Input Multiple-Output \\
\hline ML & Maximum Likelihood \\
\hline MMSE & Minimum Mean Square Error \\
\hline MOS & Modulatable Orthogonal Squence \\
\hline MSE & Mean Square Error \\
\hline NAN & Neighborhood Area Network \\
\hline NB & Narrowband \\
\hline NBI & Narrowband Interference \\
\hline OFDM & Orthogonal Frequency Division Multiplexing \\
\hline OQAM & Offset Quadrature Amplitude Modulation \\
\hline PAPR & Peak-to-Average Power Ratio \\
\hline PDF & Probability Density Function \\
\hline PDP & Power Delay Profile \\
\hline PLC & Power-Line Communications \\
\hline $\mathrm{PN}$ & Pseudo-Noise \\
\hline PRIME & Power-Line Intelligent Metering Evolution \\
\hline PSD & Power Spectral Density \\
\hline PSK & Phase Shift Keying \\
\hline PSP & Perfect Synchronization Probability \\
\hline QAM & Quadrature Amplitude Modulation \\
\hline QPSK & Quadrature Phase Shift Keying \\
\hline RI & Ruido Impulsivo \\
\hline RMSE & Root Mean Square Error \\
\hline
\end{tabular}


SCADA

SDFT

SFO

SG

SNR

TCL

TDS

VLC

WSS

$\mathrm{ZC}$

$\mathrm{ZCC}$

$\mathrm{ZCZ}$

$\mathrm{ZF}$
Supervisory Control and Data Acquisition

Sliding Discrete Fourier Transform

Sampling Frequency Offset

Smart Grids

Signal-to-Noise Ratio

Teorema Central del Límite

Time-Domain Synchronous

Visible Light Communication

Wide-Sense Stationary

Zadoff-Chu

Zero Correlation Code

Zero Correlation Zone

Zero Forcing 



\section{INTRODUCCIÓN}

El concepto de las Redes Eléctricas Inteligentes (Smart Grids - SG) [Bamberger 2006] [Val Beitia 2011] se encuentra en pleno auge en todo el mundo como una respuesta a los problemas de generación y distribución de energía eléctrica debido al incremento de la demanda y la obsolescencia de la red eléctrica [Jingfang 2012]. Adicionalmente, el crecimiento de las energías renovables en el panorama energético a nivel mundial ha cambiado notablemente los flujos de energía en la red eléctrica; ahora los usuarios no sólo consumen sino que también pueden producir energía eléctrica a través de la misma red, haciendo que el flujo energético pueda ser bidireccional [Top 2012]. De esta manera, las SG deben ser capaces de equilibrar la oferta y la demanda entre productores y consumidores.

Las SG deben proporcionar monitoreo, protección y optimización de forma automática a la operación de los diferentes elementos interconectados a la red eléctrica, así como aumentar la fiabilidad y la eficiencia con una fácil integración de fuentes de energía renovables y alternativas, a través de técnicas avanzadas de control, automatización y comunicación. También deben permitir controlar dispositivos inteligentes en hogares o edificios a fin de ahorrar energía de los consumidores. Para ello es necesario realizar comunicaciones bidireccionales [Ye 2013] para mejorar el control, la eficiencia, la fiabilidad y la seguridad.

Por lo tanto, una de las claves fundamentales de las redes eléctricas inteligentes es disponer de esquemas de comunicación eficientes para tareas de monitorización y control; o para ofrecer información en tiempo real del consumo y permitir el equilibrio de la oferta y gestión de la demanda [Gao 2012]. Muchas de las tecnologías de 
Diseño de Estrategias de Sincronización y Estimación de Canal para la Mejora de Comunicaciones en Redes Inteligentes de Energía

comunicación que deben adoptar las redes eléctricas inteligentes ya han sido utilizadas en aplicaciones industriales, tales como redes de sensores para la industria manufacturera y redes inalámbricas en telecomunicaciones, y están siendo adaptadas para su uso en este nuevo paradigma de inteligencia e interconexión [Ye 2013].

Dentro de las tecnologías de comunicaciones, una de las que está cobrando cada vez más importancia es la transmisión de datos por la red eléctrica de alimentación (PowerLine Communications - PLC) [Galli 2011]. Dado que la red de distribución eléctrica se encuentra presente en zonas residenciales, comerciales e industriales, se convierte en un medio atractivo para la comunicación de datos digitales en pos de satisfacer las necesidades de comunicación, automatización y control.

El canal eléctrico presenta múltiples fuentes de ruido impulsivo, ruido de fondo coloreado [Cortés 2010], y una marcada selectividad en frecuencia, por lo que el desarrollo de sistemas de comunicaciones debe ser robusto para lidiar con el ambiente hostil que representa el canal PLC [Tonello 2014]. Las técnicas de modulación mutiportadora resultan adecuadas para hacer frente a la selectividad en frecuencia del canal, siendo la multiplexación por división de frecuencias ortogonales (Orthogonal Frequency Division Multiplexing - OFDM) la técnica de modulación mayoritariamente empleada en sistemas PLC de banda ancha [Maneerung 2011] [IEEE 2010]. Sin embargo, los sistemas OFDM requieren de una sincronización adecuada, en conjunto con la estimación y ecualización del canal PLC para un desempeño apropiado. Debido a su importancia en el sistema de comunicaciones, el desarrollo de algoritmos de sincronización y estimación de canal para sistemas OFDM es motivo de investigación constante, y las particularidades del canal PLC demandan del desarrollo de estrategias específicas [Godoy 2012] [Bogdanović 2014]. Adicionalmente, la marcada selectividad en frecuencia del canal PLC requiere usualmente de un sistema OFDM con un número elevado de subportadoras [IEEE 2010], lo que incrementa considerablemente la complejidad de los algoritmos desarrollados para sistemas inalámbricos.

La presente tesis contribuye en el desarrollo de estrategias eficientes de sincronización y estimación de canal para sistemas OFDM-PLC, que representen una disminución en la complejidad asociada respecto a variantes existentes en la literatura, y a su vez mantengan un desempeño competitivo. 


\subsection{Contexto de la tesis}

La presente tesis se ha llevado a cabo en el marco de un convenio de cotutela internacional iniciado en el año 2016 entre la Universidad Nacional de La Plata (La Plata, Argentina), y la Universidad de Alcalá (Alcalá de Henares, España). Adicionalmente, parte de las actividades se han desarrollado como docente/investigador en la Universidad Nacional de la Patagonia San Juan Bosco (Comodoro Rivadavia, Argentina).

La tesis se ha desarrollado bajo los proyectos de investigación: "Arquitecturas eficientes en SoCs para tecnologías de capa física fiables en comunicaciones sobre redes eléctricas" (SOC-PLC) (ref. TEC2015-64835-C3-2-R) financiado por el Ministerio de Economía y Competitividad de España; "Filtrado eficiente en canales de propagación desfavorables empleando conjuntos de secuencias complementarias multinivel" (FI048/2014) y "Algoritmos de sincronización para esquemas de comunicación multiportadora en redes eléctricas”(PI1471/2018), financiados por la Universidad Nacional de la Patagonia San Juan Bosco.

Particularmente, esta tesis hace frente a las etapas de sincronización y estimación de canal en los sistemas de comunicaciones multiportadora por la red eléctrica. El principal objetivo es el desarrollo de algoritmos de complejidad reducida -y desempeño competitivo- de manera tal que puedan implementarse a partir de arquitecturas eficientes, cuestión de particular interés en los proyectos mencionados.

Adicionalmente, se ha participado de manera secundaria en los proyectos: "Mejorando y fomentando la vida activa y bienestar de las personas con demencia y deterioro cognitivo leve mediante el uso de técnicas de localización" (POM), (ref. PID2019105470RA-C33), financiado por el Ministerio de Ciencia e Innovación de España; y. "Mejora y robustecimiento de sistemas de localizacion en interiores para aplicaciones en robótica y asistencia a personas" (TARSIUS), (Ref. TIN2015-71564-C4) con continuación en "Sistemas de Posicionamiento Local: Enfoque Holístico desde las Tecnologías Base hasta las Aplicaciones” (MICROCEBUS - ref. RTI2018-095168-BC51, ambos financiado por el Ministerio de Economía y Competitividad de España. El aporte en estos proyectos se produjo a partir de tareas relacionadas con el uso de técnicas avanzadas de codificación para la sincronización de sistemas de localización.

Esta tesis ha sido además apoyada económicamente por la Universidad Nacional de la Patagonia San Juan Bosco; por una beca doctoral del Centro Nacional de 
Diseño de Estrategias de Sincronización y Estimación de Canal para la Mejora de Comunicaciones en Redes Inteligentes de Energía

Investigaciones Científicas y Técnicas (CONICET, Argentina), en el Centro de Investigación y Transferencia Golfo San Jorge; y una beca del programa Bec.AR financiada por la Jefatura de Gabinetes de la Nación Argentina que permitió realizar una primera estancia en el Grupo de Ingeniería Electrónica Aplicada a Espacios Inteligentes y Transporte (GEINTRA), de la Universidad de Alcalá, entre Mayo y Septiembre del 2016. Además, se realizó una segunda estancia de investigación en el mismo grupo entre los meses de Enero y Mayo del 2018.

\subsection{Estructura de la tesis}

La presente tesis se encuentra organizada en los siguientes capítulos, además del presente:

- Capítulo 2: "Estado del arte y objetivos planteados". En este capítulo se realiza una revisión de las comunicaciones por la red eléctrica y su importancia en el desarrollo de las redes eléctricas inteligentes. Luego, se recopilan las distintas técnicas de sincronización y estimación de canal para sistemas OFDM existentes en la literatura, con particular énfasis en el ámbito de PLC de banda ancha. A partir de ello, se plantea la problemática asociada a la sincronización y estimación de canal en OFDM-PLC, y los objetivos de la presente tesis.

- Capítulo 3: "Sincronización y estimación de canal en sistemas OFDM-PLC”. En este capítulo se plantea un esquema de transmisión OFDM paso banda, y se realiza el diseño de un símbolo piloto con adecuadas propiedades de correlación a partir de pares de secuencias complementarias. Luego, se plantea una métrica de sincronización gruesa basada en autocorrelación (AC), y una etapa de sincronización fina y estimación de canal a partir del cómputo de la correlación cruzada (CC) de manera eficiente. Posteriormente, se realiza un análisis del modelo de canal PLC propuesto para obtener umbrales de detección para la etapa de sincronización fina que se adaptan a las condiciones de relación señal a ruido y del canal. Finalmente, se comparan la complejidad y el desempeño de la propuesta respecto al procesamiento de distintos símbolos piloto, validando la propuesta.

- Capítulo 4: "Sincronización gruesa en sistemas DMT". En este capítulo se trata el desarrollo de la etapa de sincronización gruesa considerando una transmisión OFDM banda base o DMT. A partir del planteo de métricas de AC y CC, se 
procede a una comparación analítica de las distribuciones de las métricas para la detección primaria del símbolo piloto bajo ruido de fondo AWGN y coloreado. A partir de ello, se derivan analíticamente umbrales de detección en cada caso, y se verifica que la métrica basada en CC presenta una mayor robustez para la detección primaria que la de $\mathrm{AC}$, incluyendo efectos de ruido impulsivo y error en la frecuencia de muestreo. Finalmente, se propone una técnica de estimación de la $S N R$ a partir de las métricas de sincronización, ya que resulta de utilidad para la etapa de sincronización fina.

- Capítulo 5: “Sincronización fina y estimación de canal en sistemas DMT-PLC”. En este capítulo, como continuidad del anterior, se desarrolla la etapa de sincronización fina basada en CC y se derivan umbrales óptimos utilizados en el algoritmo de sincronización de acuerdo a las características estadísticas del canal. Luego, se plantea y evalúa la estrategia de estimación de canal que se obtiene a partir de las muestras de la correlación cruzada utilizada en las etapas de sincronización fina y gruesa, y se realiza un análisis preliminar de la complejidad asociada al cómputo del conjunto de algoritmos propuestos.

- Capítulo 6: "Sincronización y estimación de canal en sistemas DMT-PLC bajo una máscara espectral de transmisión”. En este capítulo se presenta la aplicación de una máscara de compatibilidad electromagnética al sistema DMT-PLC, y se estudia el impacto en las estrategias de sincronización y estimación de canal propuestas. Para las etapas de sincronización gruesa y fina, a partir de la inclusión de un banco de filtros notch en el receptor, y análisis del nuevo contexto, se redefinen los umbrales prácticos de detección en cada caso. Posteriormente, para la etapa de estimación de canal se propone una estrategia de baja complejidad para estimar el canal en las subportadoras inactivas y de esa manera reducir el nocivo impacto que la máscara espectral provoca en esta etapa. Finalmente, a partir del análisis de complejidad realizado en el capítulo 5, se hace lo propio incluyendo todas las modificaciones incluidas a lo largo del presente capítulo.

- Capítulo 7: “Conclusiones y trabajos futuros". En este capítulo se recopilan las conclusiones más significativas derivadas de los aportes de la presente tesis. Posteriormente, se proponen algunas líneas de investigación que podrían abordarse a partir de estas contribuciones. Por último, se listan las referencias a 
Diseño de Estrategias de Sincronización y Estimación de Canal para la Mejora de Comunicaciones en Redes Inteligentes de Energía

las publicaciones en revistas internacionales indexadas $y$ en congresos nacionales e internacionales derivadas de esta tesis. 


\section{ESTADO DEL ARTE Y OBJETIVOS PLANTEADOS}

\subsection{Introducción}

En los últimos años se ha logrado cierto consenso en todo el mundo sobre la problemática de la creciente demanda de energía, que supera el ritmo al que puede crecer la generación de energía por medios tradicionales, y la necesidad de reducir las emisiones de gases de efecto invernadero para prevenir el cambio climático. Para ello, se plantea una modernización de la red eléctrica que dé lugar a una red que integre de forma inteligente las acciones de todos los usuarios conectados a ella -generadores, consumidores, y los que hacen ambas funciones- para ofrecer un suministro eléctrico sostenible, económico y seguro [Bamberger 2006].

Así, una red eléctrica inteligente (Smart Grid - SG) facilita, por un lado, la inclusión de todas las fuentes de energías renovables, la generación distribuida, la micro generación residencial y el almacenamiento; mientras que permite la gestión eficiente de los consumidores a través de la integración de contadores inteligentes, electrodomésticos y cargas inteligentes, micro generación y almacenamiento de electricidad. A su vez, una SG gestiona el sistema de suministro de manera autónoma, redirigiendo los flujos de energía de manera dinámica, en pos del mejor balance entre lo que se necesita y cuándo se necesita, incrementando a su vez la seguridad del suministro a las perturbaciones por fallas, desastres naturales o ataques [Ekanayake 2012]. 
Diseño de Estrategias de Sincronización y Estimación de Canal para la Mejora de Comunicaciones en Redes Inteligentes de Energía

Todas estas acciones de equilibrio entre generación y demanda a una escala tan granular requieren de la integración de tecnologías adicionales de protección y control que exceden la capacidad de las actuales estructuras centralizadas de Supervisión, Control y Adquisición de Datos (SCADA). Un desarrollo esencial de la SG es establecer comunicaciones bidireccionales en todo el sistema de distribución, con clientes a través de las redes de área vecinal (Neighborhood Area Network - NAN) que cubren las áreas atendidas por las subestaciones de distribución. A su vez, las instalaciones de los clientes deben disponer de redes de área doméstica (Home Area Network - HAN), y la interfaz de las redes domésticas y vecinales se realizará a través de un contador inteligente.

La marcada demanda de comunicaciones por parte de la SG requiere de la integración de distintas tecnologías en función de los requerimientos de las distintas subredes que la componen. Así, pueden nombrarse como opciones viables para la HAN a Ethernet, Ethernet inalámbrico, Zigbee, PLC de banda angosta (Narrow Band - NB) y de banda ancha (BroadBand - BB). Por su parte, en las redes NAN resultan como variantes atractivas Digital Suscriber Line (DSL), NB-PLC, BB-PLC, Long Term Evolution (LTE), 5G y WiMax, entre otros.

\subsection{PLC como solución para redes eléctricas inteligentes}

La tecnología PLC presenta diversas ventajas en aplicaciones relacionadas a las SG [Galli 2011]. Por un lado, permite aprovechar la infraestructura de cableado existente, lo que reduce en gran medida el costo asociado al despliegue del sistema de comunicaciones. Además, las interconexiones de la red eléctrica la convierten en un canal de comunicaciones inherentemente redundante, lo que es una ventaja adicional dado los requerimientos de redundancia en las técnicas de protección y control del servicio público. En línea con la seguridad, las líneas eléctricas proporcionan una vía de comunicación que está bajo el control directo por parte de la compañía de suministro. Adicionalmente, en aplicaciones de teleprotección, donde puede ser crucial garantizar una baja latencia, las líneas eléctricas representan la ruta más directa entre los controladores y los dispositivos de protección controlados.

El uso de la tecnología PLC se remonta a la década de 1920, en la que se utilizaban las líneas eléctricas para comunicaciones telefónicas [Marumo 1920] [Slaughter 1924]. Posteriormente, con la aparición de las comunicaciones digitales, se desarrollaron 
sistemas orientados a la infraestructura de medición avanzada (Advanced Metering Infrastructure - AMR) con bajas tasas de transmisión de datos, del orden de los 100 bps [Mak 1982] [Mak 1984]. Debido a las bajas tasas de transmisión de datos y bajas frecuencias de transmisión (hasta $3 \mathrm{kHz}$ ), estas tecnologías se clasifican como PLC de banda muy angosta (Ultra Narrow Band - UNB).

En las últimas dos décadas, los sistemas PLC se han desarrollado continuamente, a partir de la implementación de nuevas tecnologías de comunicaciones en conjunto con la demanda de mayores tasas de transmisión de datos, dando lugar a los sistemas previamente mencionados NB-PLC y BB-PLC. Los sistemas NB-PLC son aquellos con frecuencias de hasta $500 \mathrm{kHz}$ y una tasa de transmisión de hasta $500 \mathrm{kbps}$ cuando se utilizan técnicas de modulación multiportadora [IEEE 2013], mientras que en los sistemas BB-PLC se alcanzan tasas de transmisión superiores a los $100 \mathrm{Mbps}$ a partir del uso de frecuencias desde $1 \mathrm{MHz}$ hasta 250MHz [Cano 2016].

Los canales PLC de banda ancha presentan una alta selectividad de frecuencia debido al efecto multicamino por las múltiples reflexiones en las distintas ramificaciones de la red, y poseen diversas fuentes de ruido, lo que lo convierte en un canal de comunicaciones muy hostil [Biglieri 2003] [Zimmermann 2002]. El ruido en el entorno PLC puede clasificarse en cinco categorías: ruido de fondo coloreado; ruido de banda estrecha; ruido impulsivo periódico, asíncrono a la frecuencia de la red; ruido impulsivo periódico, síncrono a la frecuencia de la red; y ruido impulsivo asíncrono [Meng 2005] [Cañete-Corripio 2005].

\subsection{Modulaciones multiportadora para PLC}

La multiplexación por división de frecuencias ortogonales (Orthogonal Frequency Division Multiplexing - OFDM) se ha propuesto como una solución eficiente para los sistemas BB-PLC (y NB-PLC de alta velocidad), debido a su robustez frente a la marcada selectividad en frecuencia del canal. De hecho, fue adoptado como técnica de modulación en los estándares para banda angosta PRIME, G3-PLC e IEEE 1901.2 [Hoch 2011] [IEEE 2013]; y en los estándares para banda ancha IEEE 1901, ITU-T G.hn y TIA-1113 [Galli 2010] [IEEE 2010] [Oksman 2009].

OFDM es un sistema multiportadora en el que se divide un canal de banda ancha selectivo en frecuencia en $N$ subcanales prácticamente planos, a partir de la modulación de $N$ subportadoras ortogonales. La cadena de bits a transmitir conformada a partir de 
Diseño de Estrategias de Sincronización y Estimación de Canal para la Mejora de Comunicaciones en Redes Inteligentes de Energía

pulsos de duración $T_{b}$ se mapea de acuerdo a la constelación perteneciente a una modulación por desplazamiento de fase (Phase Shift Keying - PSK), o por amplitud en cuadratura (Quadrature Amplitude Modulation - QAM). Luego del mapeo con una constelación de $M$ puntos, se obtiene una secuencia de símbolos de datos complejos, cuyos elementos tienen una duración $T_{S}=T_{b} / M$, que se multiplexan en las $N$ subportadoras. Finalmente, se realiza la suma de las señales correspondientes a cada subportadora modulada, dando lugar a un símbolo OFDM que puede expresarse como:

$$
x_{t}=\frac{1}{\sqrt{N}} \sum_{k=0}^{N-1} X_{k} \mathrm{e}^{\left(j 2 \pi f_{k} t\right)}, \quad 0<t<N T_{S}
$$

Donde $X_{k}$ son los símbolos de datos complejos de la constelación PSK o QAM, $N T_{s}$ es la duración del símbolo OFDM, y $f_{k}$ es la frecuencia correspondiente a la $k$-ésima subportadora. Para que resulten ortogonales, se definen las frecuencias de las subportadoras de acuerdo a $f_{k}=k / N T_{s}, k=0,1, \ldots, N-1$. A su vez, el símbolo OFDM de tiempo continuo puede muestrearse en los instantes $t=n T_{s}$, dando lugar a su versión discreta:

$$
x_{n}=\frac{1}{\sqrt{N}} \sum_{k=0}^{N-1} X_{k} \mathrm{e}^{\left(j \frac{2 \pi k n}{N}\right)}, \quad n=0,1, \ldots, N-1
$$

Puede observarse a partir de (2.2) que las muestras del símbolo OFDM en el dominio del tiempo se obtienen a partir de la transformada inversa de Fourier discreta (Inverse Discrete Fourier Transform - IDFT) de los $N$ símbolos de datos X. De manera análoga, es posible recuperar los símbolos de datos en el receptor a partir del proceso de DFT de las $N$ muestras del símbolo OFDM $\mathbf{x}$.

Teniendo en cuenta un canal multicamino con respuesta al impulso dada por $\mathbf{h}=$ $\left[\begin{array}{llll}h_{0} & h_{1} & \cdots & h_{L-1}\end{array}\right]$, siendo $L$ la máxima dispersión del canal discreto en muestras, debe agregarse un intervalo de guarda entre dos símbolos consecutivos para evitar que se produzca interferencia intersímbolo (Inter-Symbol Interference - ISI), y se deteriore a su vez la ortogonalidad entre subportadoras, dando lugar a interferencia entre portadoras (Inter-Carrier Interference - ICI). Por lo tanto, la longitud del intervalo de guarda debe elegirse a partir de la relación de compromiso entre ser lo suficientemente grande como para superar la máxima dispersión del canal, y lo suficientemente pequeño como para no reducir considerablemente la eficiencia espectral del sistema OFDM. El intervalo de 
guarda se suele completar a partir de la repetición cíclica de las últimas muestras del símbolo piloto, lo que se denomina prefijo cíclico (Cyclic Prefix - CP), obteniendo el símbolo OFDM extendido $\mathbf{x}_{\mathbf{e x t}}=\left[\begin{array}{ll}\mathbf{c p} & \mathbf{x}\end{array}\right]$, donde $\mathbf{c p}$ representa el prefijo cíclico de $N_{c p}$ muestras, dadas por:

$$
\mathrm{cp}_{n}=\mathrm{x}_{n+N-N_{c p}}, \quad n=0,1, \ldots, N_{c p}-1
$$

En la Fig. 2.1 se representa la extensión del símbolo OFDM a partir de la inclusión del CP. La principal ventaja del agregado del CP resulta en que, si se cumple que $N_{c p} \geq$ $L-1$, no se produce ISI ni ICI, y el símbolo recibido en el dominio de la frecuencia puede escribirse como:

$$
\mathbf{Y}=\left[\begin{array}{c}
Y_{0} \\
Y_{1} \\
\vdots \\
Y_{N-1}
\end{array}\right]=\left[\begin{array}{cccc}
X_{0} & 0 & \cdots & 0 \\
0 & X_{1} & & \vdots \\
\vdots & & \ddots & 0 \\
0 & \cdots & 0 & X_{N-1}
\end{array}\right]\left[\begin{array}{c}
H_{0} \\
H_{1} \\
\vdots \\
H_{N-1}
\end{array}\right]+\left[\begin{array}{c}
W_{0} \\
W_{1} \\
\vdots \\
W_{N-1}
\end{array}\right]=\mathbf{X H}+\mathbf{W}
$$

donde $\mathbf{X}$ es una matriz diagonal con los elementos del símbolo OFDM en el dominio de la frecuencia; H es un vector con la respuesta en frecuencia del canal (Channel Frequency Response - CFR) obtenido a partir de la DFT de $N$ puntos de la CIR h; y W es el vector de ruido aditivo en cada subportadora.

De (2.4) se desprende una ventaja fundamental de OFDM, dado que en cada subportadora el símbolo de dato $X_{k}$ se ve afectado por un único elemento de la CFR $H_{k}$, lo que confirma la subdivisión del canal en $N$ subcanales independientes. Adicionalmente, esta propiedad simplifica la tarea de ecualización del canal, a partir del uso de ecualizadores en el dominio de la frecuencia (Frequency Domain Equalizer FDE) compuestos por un simple multiplicador complejo por cada subportadora. Como ventajas adicionales, los sistemas OFDM presentan una mayor eficiencia espectral que los sistemas de portadora única, dado que la ortogonalidad entre las subportadoras permite un solapamiento espectral de las mismas; y los bloques de modulación (IDFT) y demodulación (DFT) pueden implementarse eficientemente a partir de algoritmos de transformada rápida de Fourier (Fast Fourier Transform - FFT).

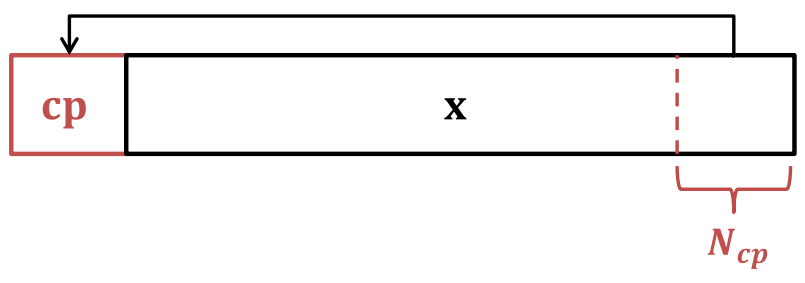

Fig. 2.1 Inserción del prefijo cíclico en símbolos OFDM. 
Diseño de Estrategias de Sincronización y Estimación de Canal para la Mejora de Comunicaciones en Redes Inteligentes de Energía

La transmisión de las señales OFDM en sistemas PLC puede realizarse a partir de dos variantes: la transmisión paso banda, en la las muestras complejas de cada símbolo OFDM de (2.4) modulan la fase y cuadratura (I/Q) de una portadora de frecuencia $f_{c}$ antes de ser transmitidas a través del canal; y la transmisión banda base, denominada también modulación por multitonos discretos (Discrete Multi-Tone - DMT), en la que se satisface la propiedad de simetría hermítica en las entradas del bloque de IDFT para obtener una salida $x_{n}$ únicamente real [Kim 2011]. Cada variante posee sus fortalezas y debilidades respecto a la otra.

En la Fig. 2.2 se presenta un diagrama en bloques básico del sistema OFDM banda base. En el transmisor, los datos binarios son mapeados de acuerdo a una determinada constelación (BPSK, QPSK, M-QAM, etc.). Los datos mapeados se multiplexan en las $N$ entradas (subportadoras) del bloque IFFT, generando un símbolo OFDM al que se le agrega un prefijo cíclico de $N_{c p}$ muestras. Luego, las muestras complejas modulan la fase y cuadratura (I/Q) de la portadora de frecuencia $f_{c}$ antes de ser transmitidas a través del canal. Por otro lado, en el receptor se realiza la demodulación I/Q a la frecuencia de portadora para obtener las muestras complejas de los símbolos OFDM en banda base. Se efectúa la etapa de sincronización de la ventana de la FFT con los símbolos recibidos, y se descarta el prefijo cíclico de los mismos. A partir de la operación FFT se obtienen los símbolos recibidos en cada subportadora, que se encuentran afectados por el canal. A partir de una etapa de estimación de canal, es posible compensar los efectos del canal por medio de un ecualizador FDE antes de demapear los datos de las constelaciones correspondientes.

Por otro lado, considerando un sistema DMT basado en una IDFT de $N$ puntos, se dispone como máximo de $N / 2$ subportadoras, ya que para obtener una salida únicamente real se debe satisfacer la propiedad de simetría hermítica en las entradas del bloque FFT. Para esto último, si en cada portadora disponible se mapean elementos complejos $X_{k}$, con $k=1, \ldots, N-1$, se debe cumplir $X_{N-k}=X_{k}^{*}$, y adicionalmente $\left\{X_{0}, X_{N / 2}\right\} \in \mathbb{R}$. Finalmente, tras efectuar la IDFT a partir de un bloque IFFT de $N$ puntos y agregar un prefijo cíclico de $N_{c p}$ muestras, la señal $x_{n}$ a transmitir puede expresarse como (2.2), garantizando que $x_{n} \in \mathbb{R}$. Luego, se agrega el CP, se realiza conversión digital-analógica (Digital to Analog Conversion - DAC), y se acondiciona la señal con un front-end analógico (Analog Front-End - AFE) antes de ser transmitida por el canal PLC. De la misma manera que en el sistema OFDM paso banda, en el receptor se efectúa la 


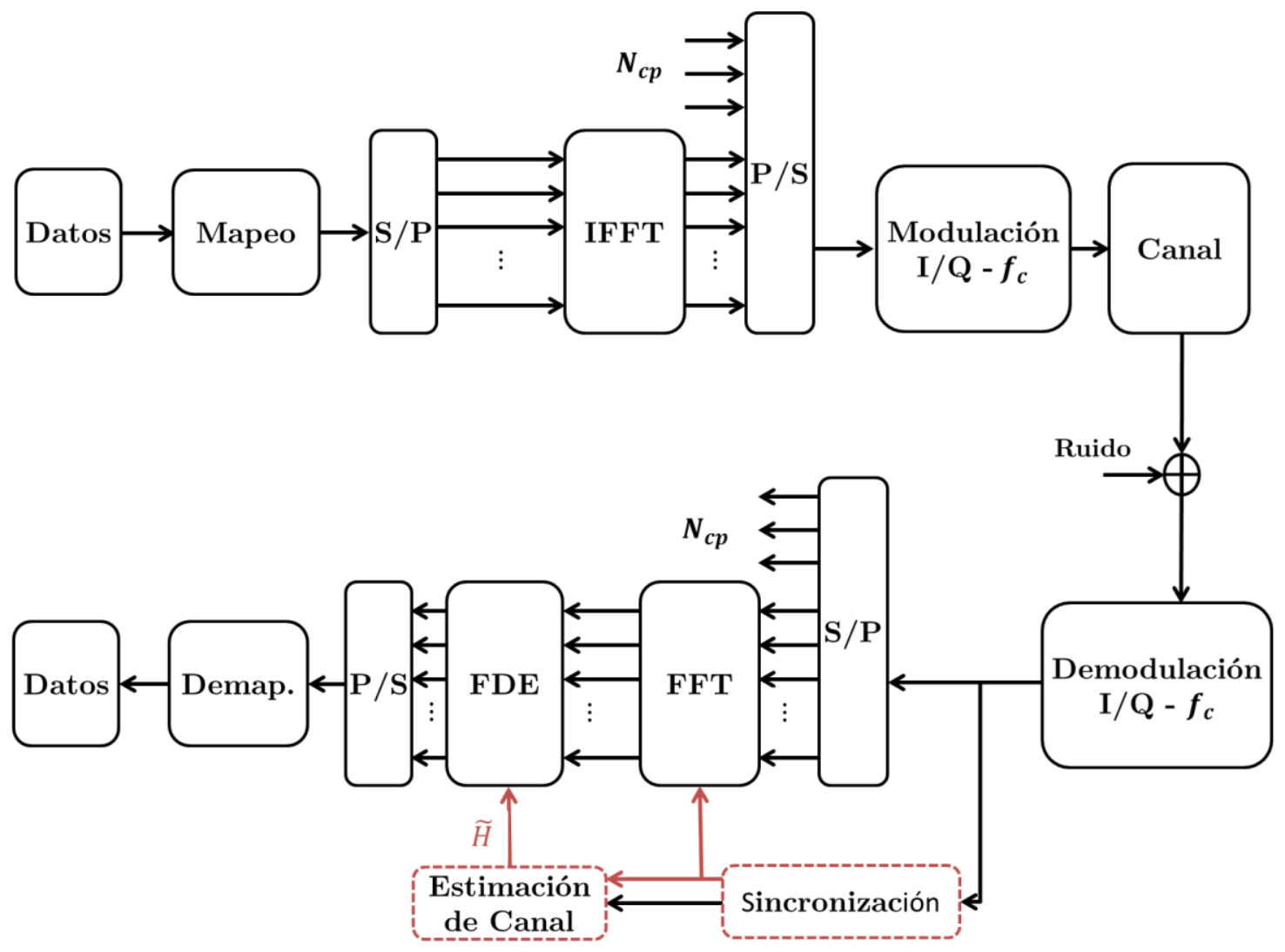

Fig. 2.2 Diagrama en bloques del transmisor y receptor del sistema OFDM-PLC.

sincronización de la ventana de FFT, la estimación de canal, el descarte del CP y la ecualización a partir de un FDE. El diagrama en bloques correspondiente se presenta en la Fig. 2.3.

Como diferencias principales, la transmisión DMT permite un procesamiento digital integral de la señal recibida sin la necesidad de incluir la modulación en fase y cuadratura en una portadora central como en la transmisión paso banda. Adicionalmente, en una transmisión paso banda, las frecuencias de portadora en el transmisor y receptor no son exactamente iguales, por lo que se introduce un error en la frecuencia de portadora (Carrier Frequency Offset - CFO) en el proceso de modulación y demodulación I/Q, fenómeno no presente en los sistemas DMT. Sin embargo, el procesamiento digital requerido por los sistemas DMT es superior a los sistemas OFDM paso banda, debido a que requiere el doble tamaño de DFT para un mismo número de subportadoras modulables (útiles).

A pesar de todas las ventajas que introducen los sistemas OFDM, éstos presentan algunas desventajas, como ser una elevada potencia pico respecto del promedio (Peak to Average Power Ratio - PAPR), una pérdida de eficiencia espectral debido a la inserción del prefijo cíclico, y son altamente vulnerables a las deficiencias de sincronización. El 


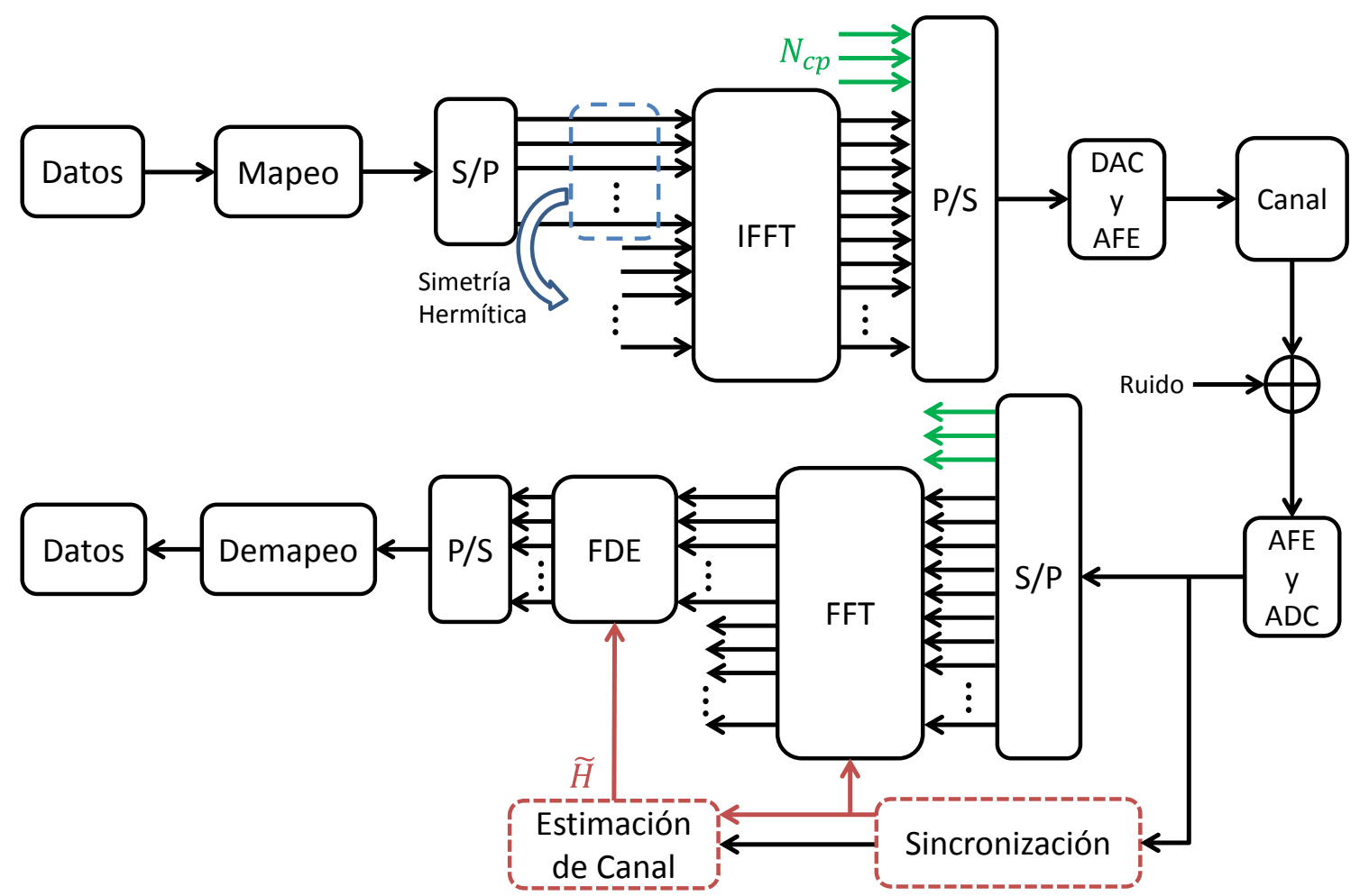

Fig. 2.3 Diagrama en bloques de un sistema DMT básico.

error en la sincronización de la ventana de la FFT puede producir ISI, y además ICI por la pérdida de ortogonalidad de las subportadoras, degradando sustancialmente el desempeño general del sistema [Mostofi 2006].

Para resolver el problema de sincronización, tanto de sistemas de transmisión DMT como OFDM paso banda, se utilizan subportadoras piloto en las que se transmite información conocida por el receptor [Morelli 2007]. Usualmente, se utilizan todas las subportadoras disponibles como piloto, para conformar lo que se denomina un símbolo piloto, y uno o varios símbolos piloto se agregan como cabecera o preámbulo a los símbolos OFDM de datos. Adicionalmente, los símbolos piloto permiten efectuar la estimación del canal que se precisa para la etapa de ecualización en el dominio de la frecuencia.

\subsection{Codificación para la sincronización y estimación de canal en sistemas OFDM.}

El presente apartado recaba distintos tipos de secuencias utilizadas para las etapas de sincronización y/o estimación de canal en sistemas OFDM. En general, se explotan las características de correlación de las secuencias en el dominio del tiempo o de la 
frecuencia para la construcción de símbolos piloto a modo de preámbulo a los símbolos de datos.

\subsubsection{Secuencias Barker}

Las secuencias Barker son un tipo de secuencias binarias con muy buenas propiedades de correlación aperiódica. Una secuencia Barker $\mathbf{s}$ de longitud $L$, con elementos $s_{l} \in\{-1,+1\}$, presenta una ACS aperiódica con lóbulos laterales menores o iguales a 1, es decir:

$$
c_{n}^{\mathbf{s}, \mathbf{s}}=\left|\sum_{m=0}^{L-n-1} s_{m} \cdot s_{m+n}\right| \leq 1, \quad n \neq 0
$$

A pesar de sus buenas propiedades de correlación, existe un pequeño número de secuencias disponibles y la longitud máxima registrada es $L=13$. Para esa longitud, la secuencia Barker es: $\mathbf{s}=[+1,+1,+1,+1,+1,-1,-1,+1,+1,-1,+1,-1,+1]$, y su ACS aperiódica se presenta en la Fig. 2.4. Las secuencias Barker se utilizan para la etapa de sincronización en OFDM en [Stantchev 1998] [Yan 2003].

\subsubsection{Secuencias pseudoaleatorias}

Las secuencias pseudoaleatorias (Pseudo-Noise - PN) componen un grupo de secuencias periódicas con comportamiento aleatorio similar al ruido blanco gaussiano, pero generadas de manera determinística. De manera general, una secuencia PN s de longitud $L$, con elementos $s_{l} \in\{-1,+1\}$, presenta una ACS periódica dada por:

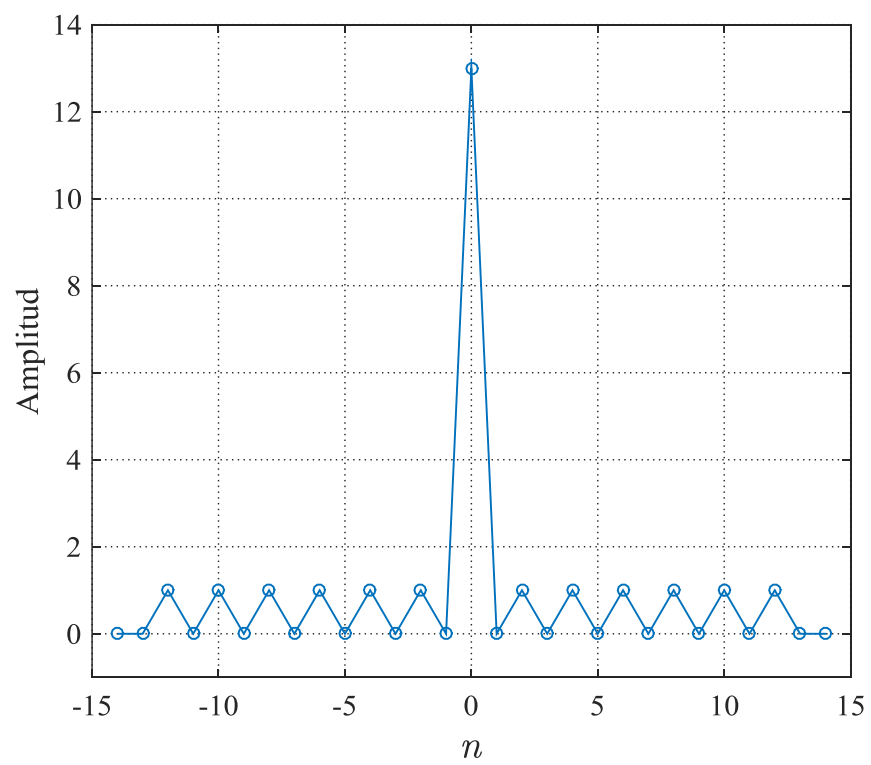

Fig. 2.4 ACS aperiódica de una secuencia Barker de longitud $L=13$ 
Diseño de Estrategias de Sincronización y Estimación de Canal para la Mejora de Comunicaciones en Redes Inteligentes de Energía

$$
c_{n}^{\mathbf{s}, \mathbf{s}}=\sum_{m=0}^{L-1} s_{m} \cdot s_{m+n}=\begin{array}{ll}
L, & n=0 \\
\varepsilon, & n \neq 0
\end{array}
$$

donde $\varepsilon$ es un número real. A su vez, la cantidad de +1 en la secuencia no excede la cantidad de -1 en más de un elemento. Dentro de las secuencias PN, las más utilizadas son las secuencias M, Gold, y Kasami [García 2015].

Las secuencias $M$ se pueden generar de manera sencilla a partir de un registro de desplazamiento con realimentación lineal (Linear Feedback Shift Register-LFSR) de $m$ etapas, y la longitud de la secuencia resulta $L=2^{m}-1$. Los coeficientes de cada etapa del LFSR se definen a partir de polinomios primitivos. En la Fig. 2.5 se presenta la ACS de una secuencia $\mathrm{M}$ con $L=63$. Las primeras (y últimas) 63 muestras corresponden a la ACS aperiódica de la secuencia, y en el resto se observa claramente la ACS periódica donde $\varepsilon=-1$. A pesar de las propiedades de ACS periódica de las secuencias M, la secuencia de correlación cruzada (Cross-Correlation Sequence - CCS) entre dos secuencias $M \mathbf{s}_{\mathbf{1}}$ y $\mathbf{s}_{\mathbf{2}}$ presenta lóbulos importantes en la correlación cruzada. Si se elige un par de secuencias "preferido", es decir, los que presentan el menor valor en el pico de la CCS, se obtiene una amplitud de pico de $2^{\frac{m+2}{2}}-1$.

Para solventar las limitaciones en el número de secuencias de un conjunto de secuencias M con bajos valores de CCS entre sí, se encuentran las secuencias Gold y Kasami que se generan a partir de las secuencias M. Permiten incrementar el número de secuencias con CSS acotada, pero a su vez el precio a pagar es un incremento en los lóbulos laterales de la ACS. Las secuencias Gold y Kasami poseen distintos algoritmos de generación a partir de las secuencias $\mathrm{M}$, y en general los lóbulos laterales de las funciones de correlación periódica de los conjuntos de secuencias Kasami son menores que las secuencias Gold, aunque el tamaño del conjunto de secuencias Kasami resulta menor. Como ejemplos del uso de secuencias PN para las etapas de sincronización en OFDM se encuentran [Minn 2000] [Schmidl 1997] [Park 2003]. 
Capítulo 2: Estado del arte y objetivos planteados

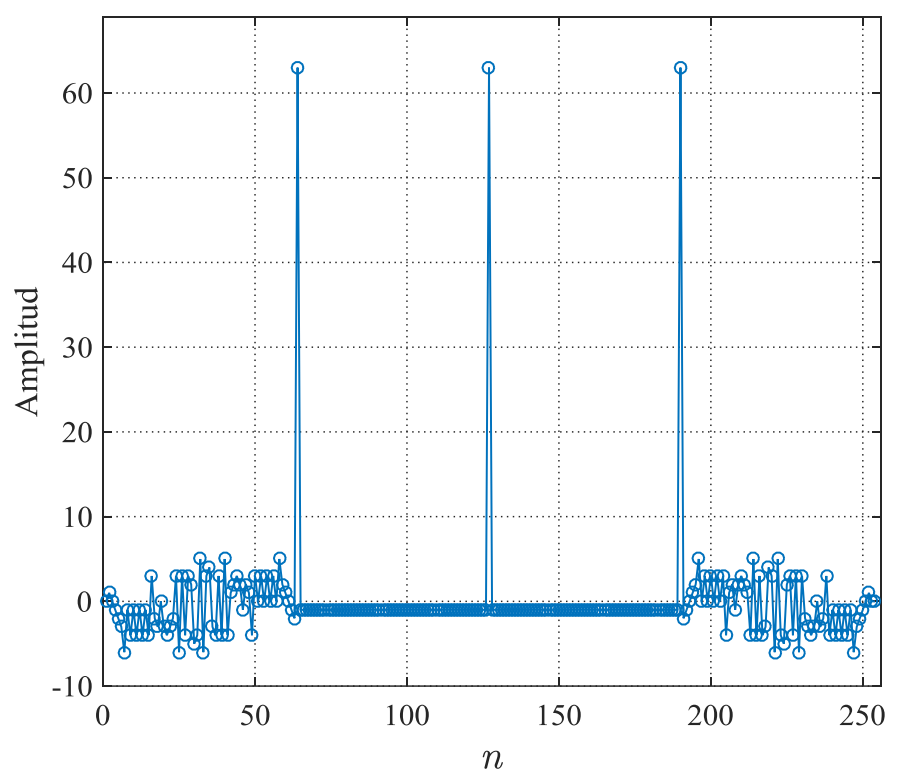

Fig. 2.5 ACS periódica y aperiódica de una secuencia $M$ de longitud $L=63$.

\subsubsection{Pares y conjuntos de secuencias complementarias}

Los pares de secuencias complementarias (CPS) fueron introducidos por M. Golay [Golay 1961], y se utilizan ampliamente en sistemas de comunicaciones. Dos secuencias binarias a y $\mathbf{b}$ de longitud $L$, cuyos elementos son $+1 \mathrm{o}-1$, componen un par complementario si la suma de las ACS aperiódicas de cada secuencia es una delta de Kronecker con amplitud 2 L. Matemáticamente, puede expresarse como:

$$
\sum_{m=0}^{L-n-1} \mathrm{a}_{m} \cdot \mathrm{a}_{m+n}+\sum_{m=0}^{L-n-1} \mathrm{~b}_{m} \cdot \mathrm{b}_{m+n}=\begin{aligned}
2 L, & n=0 \\
0, & n \neq 0
\end{aligned}
$$

Existen pares complementarios de secuencias para cualquier longitud $L=2^{N} 10^{M} 26^{P}$; donde $N, M$ y $\mathrm{P}$ son enteros positivos. La suma de las ACS para un CPS de longitud $L=32$ se presenta en la Fig. 2.6.

Un aspecto importante de los CPS es que existen algoritmos eficientes de generación y correlación que permiten su procesamiento a partir de una reducida carga computacional [Budisin 1991] [Popovic 1999] [Donato 2009]. Las fórmulas recursivas del algoritmo de generación de [Budisin 1991] pueden escribirse como:

$$
\begin{aligned}
& \mathrm{a}_{n}^{(q)}=\mathrm{a}_{n}^{(q-1)}+w^{(q)} \mathrm{b}_{n-d^{(q)}}^{(q-1)} \\
& \mathrm{b}_{n}^{(q)}=\mathrm{a}_{n}^{(q-1)}-w^{(q)} \mathrm{b}_{n-d^{(q)}}^{(q-1)}
\end{aligned}
$$


Diseño de Estrategias de Sincronización y Estimación de Canal para la Mejora de Comunicaciones en Redes Inteligentes de Energía

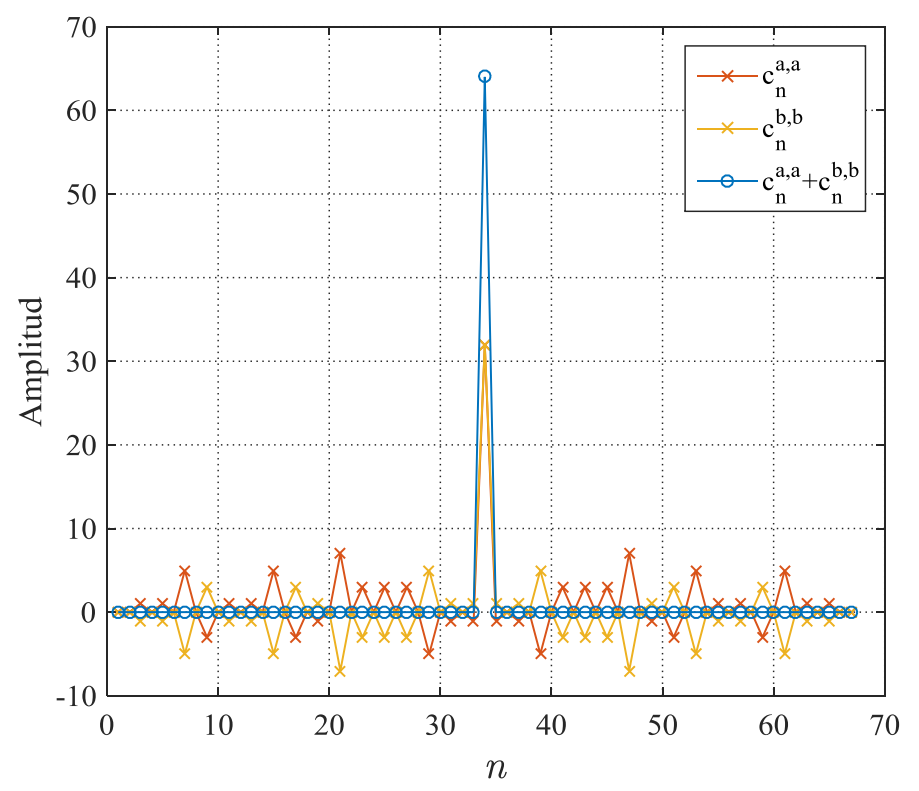

Fig. 2.6 Suma de ACS aperiódicas de un CPS de longitud $L=32$.

donde el superíndice $q$ indica el número de iteración del algoritmo recursivo, $d^{(q)}$ el retardo y $w_{q}$ la denominada semilla de generación de la $q$-ésima iteración; $\mathrm{a}_{n}^{(0)}=\mathrm{b}_{n}^{(0)}=$ $\delta_{n}$, donde $\delta_{n}$ es la delta de Kronecker. Los posibles valores para las semillas $w_{q}$ son +1 o -1 , y para una longitud $L=2^{Q}$ se precisan $Q$ recursiones, y los retardos pueden elegirse a partir de cualquier permutación del conjunto $\mathbf{d}=\left\{1,2,4, \ldots, 2^{Q-2}, 2^{Q-1}\right\}$. De manera similar, el algoritmo recursivo de correlación resulta [Popovic 1999]:

$$
\begin{aligned}
& \mathrm{a}_{n}^{\prime(q)}=\mathrm{a}_{n-d^{(q)}}^{\prime(q-1)}+w^{(q)} \mathrm{b}_{n}^{\prime(q-1)} \\
& \mathrm{b}_{n}^{\prime(q)}=\mathrm{a}_{n-d^{(q)}}^{\prime(q-1)}-w^{(q)}{\mathrm{b}_{n}^{\prime(q-1)}}^{(q)}
\end{aligned}
$$

donde $\mathrm{a}_{n}^{\prime(0)}=\mathrm{b}_{n}^{\prime(0)}=r_{n}$, y en la última recursión se obtienen las correlaciones $\mathrm{a}_{n}^{\prime(Q)}=$ $c_{n}^{\mathbf{r}, \mathbf{a}} \mathrm{y} \mathrm{b}_{n}^{\prime(Q)}=c_{n}^{\mathbf{r}, \mathbf{b}}$. A su vez, si se inicia el algoritmo con $\mathrm{a}_{n}^{\prime(0)}=\mathrm{b}_{n}^{\prime(0)}=\delta_{n}$, como resultado de la última recursión se obtienen las secuencias $\mathbf{a}^{\prime} \mathrm{y} \mathbf{b}^{\prime}$, que son las secuencias a y b invertidas, es decir: $\mathrm{a}_{n}^{\prime}=\mathrm{a}_{L-1-n}, \mathrm{~b}_{n}^{\prime}=\mathrm{b}_{L-1-n}$. Las arquitecturas equivalentes del generador y correlador eficiente de CPS se muestran en la parte superior e inferior de la Fig. 2.7, respectivamente. Si se fijan las semillas de generación del algoritmo de correlación a un valor predeterminado, la arquitectura del correlador eficiente requiere sólo $2 Q$ sumadores y elementos de memoria.

Por otro lado, en [Tseng 1972] se extendió el concepto de los CPS binarios a los Conjuntos de Secuencias Complementarias (CSS), en los que se incrementa la cantidad 

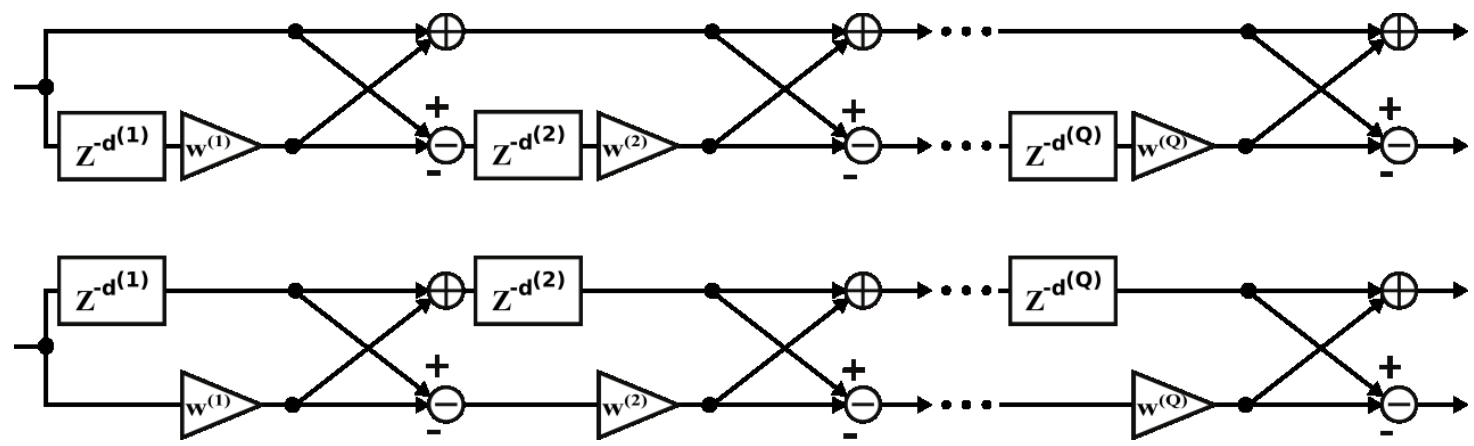

Fig. 2.7 Arquitecturas eficientes para la generación (arriba) y correlación (abajo) de un CPS.

de secuencias a $K_{C S S}=2^{k}$, con $k$ entero positivo. Así, para el caso particular en que $K_{C S S}=2$ se obtiene como resultado un CPS. Dado un CSS compuesto por secuencias $\mathbf{s}_{i}$ de longitud $L$, donde $i=0,1, \ldots, K_{C S S}-1$, se verifica que la suma de las ACS aperiódicas de cada secuencia es una delta de Kronecker con amplitud $K_{C S S} \cdot L$, y esto puede expresarse como:

$$
\sum_{i=0}^{K_{C S S^{-1}}} \sum_{m=0}^{L-n-1} s_{i_{m}} \cdot s_{i_{m+n}}=\begin{array}{rr}
K_{C S S} L, & n=0 \\
0, & n \neq 0
\end{array}
$$

Además de presentar propiedades ideales en la suma de las ACS aperiódicas, existen $K_{C S S}$ conjuntos distintos incorrelados entre sí. Es decir, la suma de las CCS aperiódicas entre las secuencias del conjunto $\mathbf{s}_{i}$ y la de otro conjunto incorrelado $\mathbf{s}_{i}^{\prime}$ es nula para todo desplazamiento:

$$
\sum_{i=0}^{K_{C S S^{-1}}} \sum_{m=0}^{L-n-1} s_{i_{m}} \cdot s^{\prime}{ }_{i_{m+n}}=0, \quad \forall n
$$

La principal ventaja de los CSS es el incremento en la cantidad de conjuntos incorrelados respecto a la cantidad de dos pares incorrelados disponibles en el caso de los CPS. Adicionalmente, existen algoritmos de generación y correlación eficiente para CSS similares a los desarrollados para CPS [De Marziani 2007].

En virtud de las propiedades de correlación ideales, a partir de la suma de las ACS y CCS, los CPS y CSS sirvieron como base para la construcción de otros tipos de secuencias en las que se relajan las propiedades de correlación ideales a zonas de correlación ideal (Zero Correlation Zone - ZCZ). Ejemplo de ello son las secuencias LS (Loosely Synchronized) [Stanczak 2001], pares T-ZCZ (Three Zero-Correlation Zones) [Zhang 2005] y secuencias GPC (Generalized Pairwise Complementary) [Chen 2005]. Asimismo, dada su estrecha relación a los CPS y CSS, existen algoritmos de correlación eficiente para estos códigos derivados [García 2012] [Pérez 2008] [Pérez 2009]. 
Diseño de Estrategias de Sincronización y Estimación de Canal para la Mejora de Comunicaciones en Redes Inteligentes de Energía

Adicionalmente, los CPS binarios fueron extendidos a secuencias reales en el alfabeto multinivel en [Budisin 1990] y posteriormente se hizo lo propio con los CSS [Kemp 1989] [García 2013]. La principal ventaja de los CSS multinivel es una mayor flexibilidad en el número y la longitud de las secuencias que componen los conjuntos.

Con respecto a las aplicaciones de los CPS y CSS en sistemas OFDM, se pueden encontrar: la reducción de PAPR a partir de CPS (Peak to Average Power Ratio) [Davis 1999] [Rezgui 2012]; sincronización temporal con CPS [Minn 2003] [Luo 2012] y CSS multinivel [Nombela 2015b]; estimación de canal en PLC [Moya 2016a]. Por su parte, las secuencias derivadas encontraron su aplicación principalmente en sincronización y estimación de canal en sistemas MIMO-OFDM (Multiple-Input Multiple-Output), gracias a la flexibilidad en la cantidad de secuencias incorreladas, y las zonas de correlación ideal que presentan tanto en su ACS como en la CCS [Akbari 2007] [Wang 2016] [Wang 2007].

\subsubsection{Secuencias CAZAC}

Las secuencias de amplitud constante y autocorrelación cero (CAZAC) conforman un conjunto de secuencias polifásicas, es decir, que sus elementos son números complejos. Como características principales, poseen una ACS periódica ideal, y magnitud unitaria en sus elementos. Dentro del grupo de secuencias CAZAC encontraron aplicación en sistemas OFDM las secuencias Frank [Frank 1962], las secuencias MOS (Modulatable Orthogonal Sequence) [Suehiro 1988], y las secuencias Zadoff-Chu (ZC) [Chu 1972]. De manera general, una secuencia ZC $\mathbf{s}$ de longitud $L$ se encuentra definida a partir de:

$$
\mathrm{s}_{n}=\exp \left(-j \frac{\pi u n(n+p)}{L}\right), \quad 0 \leq n \leq L-1
$$

donde $u$ es un número entero y primo relativo de $L, p=L \bmod$. 2. Para estas secuencias, la ACS periódica puede escribirse como:

$$
c_{n}^{\mathbf{s}, \mathbf{s}}=\sum_{m=0}^{L-1} s_{m}^{*} \cdot s_{m+n}=\begin{array}{ll}
L, & n=0 \\
0, & n \neq 0
\end{array}
$$

donde el superíndice $s_{m}^{*}$ representa el conjugado de $s_{m}$. A modo de ejemplo, en la Fig. 2.8 se presentan los elementos de una secuencia ZC $\mathbf{s}$ de longitud $L=64 \operatorname{con} u=1$, a partir de su descomposición en sus partes real e imaginaria y puede observarse además su característica de amplitud constante. A su vez, en la Fig. 2.9 se grafica la ACS de la 
misma secuencia, donde las primeras (y últimas) 64 muestras corresponden a la ACS aperiódica, y el resto corresponden a la ACS periódica.

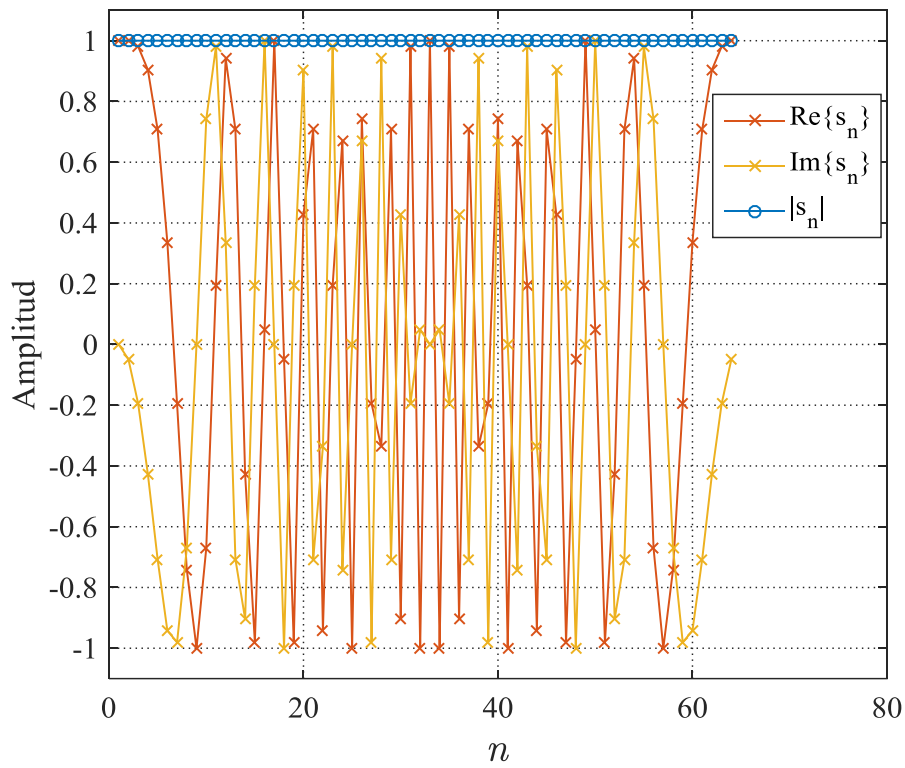

Fig. 2.8 Descomposición de los elementos de una secuencia Zadoff-Chu de longitud L=64.

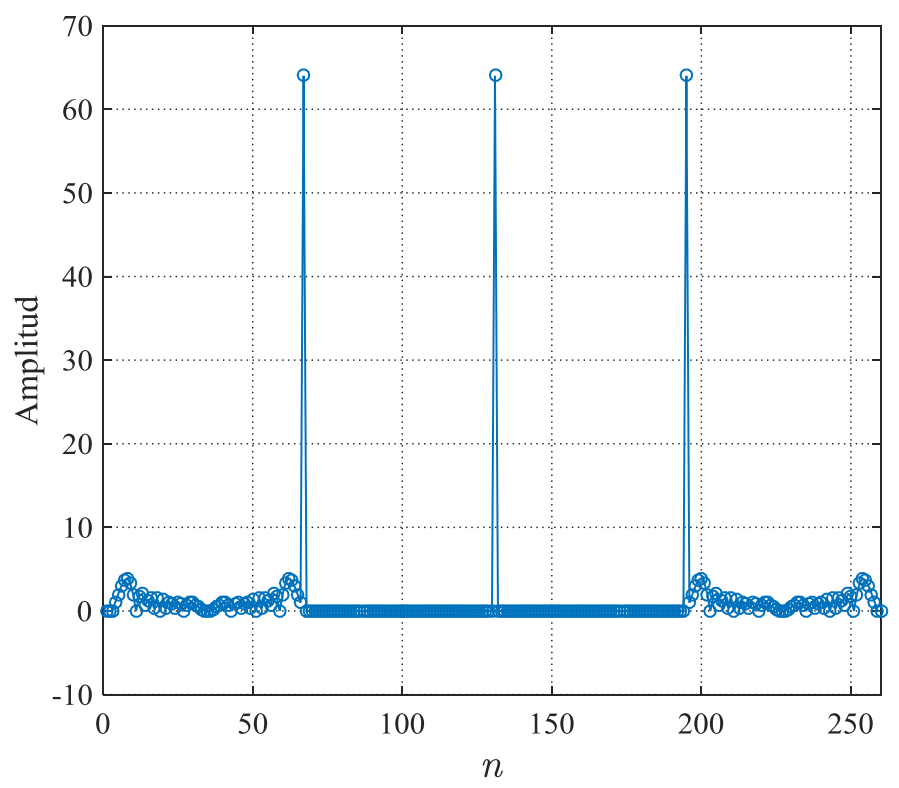

Fig. 2.9 ACS periódica y aperiódica de una secuencia Zadoff-Chu de longitud $L=64$.

Debido a sus propiedades ideales de correlación periódica, y a partir de su inclusión en los sistemas LTE (Long Term Evolution), las secuencias CAZAC en general, y las ZC en particular, fueron ampliamente utilizadas para la sincronización en sistemas OFDM [Ren 2005] [Fang 2012] [Gul 2012] [Shao 2014] [Das 2019]. 
Diseño de Estrategias de Sincronización y Estimación de Canal para la Mejora de Comunicaciones en Redes Inteligentes de Energía

\subsection{Sincronización en sistemas OFDM}

A continuación se realiza una revisión de distintas técnicas de sincronización en sistemas OFDM basadas en símbolos piloto. En general, éstas pueden clasificarse en dos grandes grupos a partir del tipo de correlación computada: las basadas en autocorrelación (AC) y las que utilizan la correlación cruzada (CC). Es importante aclarar que en el ámbito de la sincronización temporal de sistemas OFDM se entiende por $\mathrm{AC}$ a las operaciones que incluyen productos entre distintas muestras de la señal recibida, y se incluyen varios productos en una ventana deslizante. Por su parte, se entiende por CC al proceso de pasar las muestras de la señal recibida por un filtro acoplado al símbolo piloto transmitido.

\subsubsection{Sincronización basada en autocorrelación}

Las técnicas de sincronización para OFDM que utilizan la autocorrelación (AC) de la señal recibida se basan en el diseño de símbolos pilotos con una estructura de segmentos repetitivos, o bien a partir de un arreglo de segmentos con algún tipo de simetría. En base a ello, se plantea el cómputo de una métrica de temporizado normalizada, que de manera general se puede escribir de la forma:

$$
m_{n}=\frac{\left|p_{n}\right|^{2}}{e_{n}^{2}}
$$

donde $p_{n}$ es el cómputo propuesto de la autocorrelación de la señal recibida a partir de los segmentos del símbolo piloto, y $e_{n}$ es la energía de la señal recibida en una ventana determinada de muestras. Luego, el instante de sincronización gruesa se obtiene, generalmente, a partir de la ubicación del máximo valor de la métrica de temporizado. Las principales ventajas de las técnicas basadas en AC son su alta robustez al error en la frecuencia de portadora (Carrier Frequency Offset - CFO), y su baja complejidad asociada, dado que tanto $p_{n}$ como $e_{n}$ suelen computarse a partir de fórmulas recursivas simples. Adicionalmente, en algunos casos es posible obtener una estimación del CFO y/o de la condición de $S N R$ a partir de la misma métrica de sincronización.

En [Schmidl 1997] se propone un símbolo piloto compuesto de dos mitades idénticas de longitud $N / 2 \mathbf{x}=\left[\begin{array}{ll}\mathbf{a} & \mathbf{a}\end{array}\right]$, a partir del uso de una secuencia PN para modular las subportadoras pares con símbolos QPSK. El numerador y denominador de la métrica de temporizado se calculan de acuerdo a: 
Capítulo 2: Estado del arte y objetivos planteados

$$
\begin{aligned}
p_{S C_{n}} & =\sum_{m=0}^{N / 2-1} r_{n}^{*} \cdot r_{n+m+N / 2} \\
e_{S C_{n}} & =\sum_{m=0}^{N / 2-1}\left|r_{n+m+N / 2}\right|^{2}
\end{aligned}
$$

donde $N$ es la longitud del símbolo piloto, y coincide con la cantidad de subportadoras del sistema OFDM. Las fórmulas recursivas asociadas al cómputo de cada uno resultan:

$$
\begin{gathered}
p_{S C_{n+1}}=p_{S C_{n}}+r_{n+N / 2}^{*} \cdot r_{n+N}-r_{n}^{*} \cdot r_{n+N / 2} \\
e_{S C_{n+1}}=e_{S C_{n}}+\left|r_{n+N}\right|^{2}-\left|r_{n}\right|^{2}
\end{gathered}
$$

Si bien es la técnica con menor complejidad para computar la métrica de sincronización, la misma presenta una meseta en las muestras previas al instante de sincronismo óptimo, lo que introduce un elevado error de estimación. Para mitigar este fenómeno, en [Minn 2000] se propone dividir el símbolo piloto de longitud $N$ en cuatro segmentos repetitivos de acuerdo a $\mathbf{x}=\left[\begin{array}{llll}\mathbf{a} & \mathbf{a} & -\mathbf{a} & -\mathbf{a}\end{array}\right]$, utilizando también una secuencia PN en el dominio de la frecuencia para su generación. Luego en [Minn 2003] se plantea una generalización para un símbolo piloto que consta de $N_{L}$ segmentos repetidos, generados a partir de CPS. Cada segmento es multiplicado por un factor de $1 \mathrm{o}-1$ de manera convenientemente elegida, y luego se define una nueva métrica de sincronización a partir de:

$$
\begin{gathered}
p_{\text {Minn }_{n}}=\left.N_{L} \sum_{l=0}^{N_{L}-2} p_{l} \cdot p_{l+1} \sum_{m=0}^{N / N_{L}-1} r_{n+m+l}^{*}{\frac{N}{N_{L}}}_{n+m+(l+1) \frac{N}{N_{L}}} \cdot r_{n+1} \sum_{l=0} \sum_{m=0}^{N_{L}-1}\left|r_{n+m+l}\right|^{N / N_{L}-1}\right|^{2}
\end{gathered}
$$

donde $p_{l}, l=0,1, \ldots, N_{L}-1$ es el patrón de signos de los segmentos del símbolo piloto. A partir de esto, se logra una métrica con un único valor máximo y una mayor pendiente, reduciendo la varianza del error de sincronización. A pesar de que es posible aplicar fórmulas recursivas para computar la autocorrelación propuesta, la complejidad asociada se incrementa a medida que lo hace el número de segmentos $L$.

Posteriormente, en [Park 2003] se propone un nuevo símbolo piloto basado en cuatro segmentos de longitud $N / 4$ de acuerdo a $\mathbf{x}=\left[\begin{array}{llll}\mathbf{a} & \mathbf{b} & \mathbf{a}^{*} & \mathbf{b}^{*}\end{array}\right]$, donde $\mathbf{a}^{*}$ representa el 
Diseño de Estrategias de Sincronización y Estimación de Canal para la Mejora de Comunicaciones en Redes Inteligentes de Energía

conjugado de $\mathbf{a}$, y el segmento $\mathbf{b}$ es simétrico respecto a $\mathbf{a}$. El símbolo piloto completo se puede generar a partir de la modulación de las subportadoras pares con una secuencia $\mathrm{PN}$, y el numerador y denominador de la métrica propuesta son:

$$
\begin{gathered}
p_{\text {Park }_{n}}=\sum_{m=0}^{N / 2} r_{n-m} \cdot r_{n+m} \\
e_{\text {Park }_{n}}=\sum_{m=0}^{N / 2}\left|r_{n+m}\right|^{2}
\end{gathered}
$$

A partir de ellos, la métrica $m_{\text {Park }}$ presenta una característica impulsiva, gracias a que entre el instante $p_{\text {Park }_{n}}$ y Park $_{n+1}$ todos los productos realizados en (2.23) son diferentes. Por la misma razón, no se puede utilizar una fórmula recursiva para el cómputo de $p_{\text {Park }}$, y demanda $N / 2+1$ multiplicaciones complejas y $N / 2$ sumas complejas, por lo que la complejidad asociada al cómputo de la métrica se incrementa considerablemente. Una variante con un símbolo piloto compuesto de dos mitades simétricas y un cómputo de la autocorrelación similar a (2.23) se propuso en [Zhang 2004].

Luego, en [Ren 2005] se plantea una novedosa estrategia de sincronización a partir de un símbolo piloto con dos segmentos idénticos generados a partir de una secuencia CAZAC de longitud $N / 2$, que se modifican a partir de la inclusión de una secuencia PN s de longitud $N$ cuyos elementos sirven de factores de peso para la secuencia CAZAC. Así, se obtiene el símbolo piloto con muestras $x_{n}=a_{n} \cdot s_{n}$, donde $a_{n}$ es la muestra correspondiente a la secuencia CAZAC y $s_{n}$ al elemento de la secuencia PN utilizado como factor de peso. Para el cómputo de la métrica se propone:

$$
\begin{gathered}
p_{\text {Ren } n}=\sum_{m=0}^{\frac{N}{2}-1} s_{m} \cdot s_{m+N / 2} \cdot r_{n+m}^{*} \cdot r_{n+m+N / 2} \\
e_{\operatorname{Ren}_{n}}=\frac{1}{2} \sum_{m=0}^{N-1}\left|r_{n+m}\right|^{2}
\end{gathered}
$$

La mejora en el desempeño de la métrica de sincronización se consigue gracias a la adición de los factores de peso, ya que las propiedades de correlación de la secuencia PN garantizan que la métrica tenga un máximo en el instante de inicio del símbolo, y 
valores mucho menores en el resto. Aunque es una técnica que se puede clasificar dentro del grupo que utilizan AC debido a los productos de muestras de la señal recibida $r_{n+m}^{*} \cdot r_{n+m+N / 2}$ en una ventana deslizante, el cómputo de (2.25) puede entenderse como el resultado de pasar la señal $r_{n}^{*} \cdot r_{n+N / 2}$ por un filtro digital con $N / 2$ coeficientes dados por $s_{m} \cdot s_{m+N / 2}$, es decir, efectuar una correlación cruzada a partir del filtro digital. Bajo ese punto de vista, este trabajo introdujo el concepto de correlación cruzada diferencial, que se enunciará más adelante. Con respecto a la complejidad, se puede observar que para calcular (2.25) se necesita un multiplicador complejo de dos entradas y el filtro digital. Como los productos $s_{m} \cdot s_{m+N / 2}$ tienen como resultado $+1 \mathrm{o}$ -1 , finalmente se necesita un multiplicador complejo de dos entradas y $N / 2-1$ sumas complejas.

El concepto del uso de factores de peso aplicados al símbolo piloto para mejorar la característica impulsiva de la métrica de sincronización se continuó investigando posteriormente. En [Fang 2012] se propone un símbolo piloto con dos segmentos idénticos y aplicar factores de peso $s_{m}$ sólo a la primera mitad del símbolo. Los factores son elementos unitarios complejos de fase aleatoria y se calculan como $s_{m}=$ $\exp \left(j \pi b_{m}\right)$, donde $b_{m}$ es un elemento de una secuencia con distribución uniforme y valores en el intervalo $[-0,2 ; 1]$. El cómputo de $p_{n}$ es similar a $(2.25)$, pero con factores de peso complejos, lo que incrementa la complejidad a un multiplicador complejo de dos entradas, $N / 2$ multiplicadores constantes complejos y $N / 2-1$ sumadores complejos. Como ventajas introducidas, cabe destacar la ausencia de lóbulos laterales en la métrica de sincronización, como la que se presenta en la métrica de [Ren 2005], debido a la inserción del prefijo cíclico, además de una reducción del error de sincronismo en canales con considerable efecto multicamino. Finalmente, en el método propuesto en [Shao 2014] para la sincronización gruesa, la construcción del símbolo piloto también se basa en una secuencia CAZAC a de longitud $N / 4$. El preámbulo final de longitud $N$ se construye como $\mathbf{x}=\left[\begin{array}{llll}\mathbf{a} & \mathbf{b}^{*} & \mathbf{a}^{*} & \mathbf{b}\end{array}\right]$, donde $\mathbf{a}^{*}$ representa el conjugado de $\mathbf{a}$, y el segmento $\mathbf{b}$ es simétrico respecto a $\mathbf{a}$. En este método no se incluyen factores de peso, ya que, a partir del cómputo de autocorrelación propuesto, en el instante de sincronismo óptimo, quedan definidos factores de peso equivalentes a partir de los productos calculados. 
Diseño de Estrategias de Sincronización y Estimación de Canal para la Mejora de Comunicaciones en Redes Inteligentes de Energía

$$
p_{\text {Shao }_{n}}=\sum_{m=0}^{\frac{N}{4}-1} s_{m}^{*} \cdot r_{n+m}^{*} \cdot r_{n+m+N / 2}+\sum_{m=0}^{\frac{N}{4}-1} s_{N / 4-m-1} \cdot r_{n+m+N / 4}^{*} \cdot r_{n+m+3 N / 4}
$$

donde los factores de peso son ahora $s_{m}=\exp \left(-j 2 \pi \mu m^{2} / N\right), 0 \leq m \leq N / 4-1, \mathrm{y} \mu$ es un parámetro de la secuencia CAZAC utilizada. Aplicando como normalización de energía $e_{\operatorname{Shao}_{n}}=e_{\operatorname{Ren}_{n}}$, el resultado es una métrica impulsiva como en [Fang 2012]. Analizando (2.27) se puede comprobar que existe simetría en los coeficientes $s_{m}$ en ambas sumas, pero incluyendo también una conjugación. Por lo tanto, las $N / 2$ multiplicaciones complejas constantes pueden sustituirse por $N / 4$ multiplicaciones complejas constantes y $N$ sumas reales. Así, se requiere un multiplicador complejo de dos entradas, $N / 4$ multiplicadores complejos constantes, $N / 4-1$ sumas complejas y $N$ sumas reales para calcular (2.27).

Luego, en [Das 2019] se introduce un símbolo piloto simétrico basado en secuencias CAZAC, y una métrica donde $p_{\text {Das } n}$ se computa de manera idéntica a (2.23), y se propone un novedoso factor normalizador $e_{n}$ dado por:

$$
e_{\text {Das }_{n}}=\sum_{m=0}^{\frac{N}{2}-1}\left(\left|r_{n-m}\right|-\left|r_{n+m}\right|\right)^{2}
$$

A partir de ello, se obtiene una métrica que no se encuentra normalizada entre 0 y 1 , pero el valor correspondiente al instante de sincronismo óptimo es mucho mayor que para cualquier desplazamiento, obteniendo una métrica impulsiva con gran capacidad de detección de la llegada del símbolo piloto.

En la Fig. 2.10 se presentan seis variantes de métricas de sincronización normalizadas basadas en AC: $m_{S C}$ [Schmidl 1997], $m_{\text {Minn }}$ con $N_{L}=4$ y $\mathbf{p}=[-+-\quad-]$ en

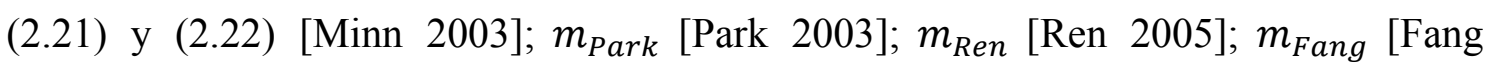
2012]; y $m_{\text {Shao }}$ [Shao 2014]. Se considera un sistema de $N=1024$ subportadoras, con longitud de prefijo cíclico $N_{c p}=128$; y un canal multicamino con una CIR compuesta por ocho coeficientes con amplitudes:

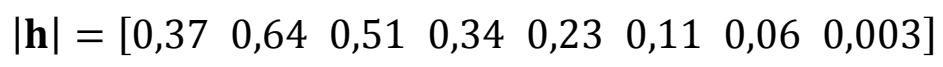

Los mismos se encuentran uniformemente espaciados cada 10 muestras, y su fase se genera a partir de un número aleatorio entre 0 y $2 \pi$. Adicionalmente, se supone una 
transmisión ideal sin ruido aditivo. En la parte derecha de la imagen se presenta un zoom en las muestras cercanas al instante de sincronismo óptimo, para una mejor visualización de las métricas de características impulsivas.

Por un lado, es importante destacar que las seis métricas presentarían un valor máximo de 1 en el instante de sincronismo óptimo, bajo un escenario de canal de un solo camino $|\mathbf{h}|=1$ ( $\sin$ selectividad en frecuencia), y sin ruido agregado. Sin embargo, puede apreciarse que la única métrica cuyo valor máximo no se ve modificado por la inclusión de un canal multicamino es $m_{S C}$, y sólo se reduce el tamaño de la meseta que la misma presenta. Luego, la métrica $m_{\text {Minn }}$ presenta una aceptable robustez al canal multicamino con una disminución a 0,94, mientras que el resto de las métricas cae por debajo de 0,5. Esto implica que, considerando a la detección del símbolo piloto como etapa preliminar de sincronización gruesa, para un mismo umbral la métrica $m_{S C}$ presenta una mayor probabilidad de éxito. Por otro lado, como las métricas impulsivas concentran sus valores máximos en una ventana más acotada de muestras que $m_{S C}$ y $m_{\text {Minn }}$, la ubicación del valor máximo puede efectuarse a partir de la búsqueda en un menor número de muestras a desde el instante de detección primaria. Adicionalmente, el error promedio en la estimación del instante de sincronización gruesa es significativamente menor para las métricas de carácter impulsivo $m_{\text {Park }}, m_{\text {Ren }}, m_{\text {Fang }}$ y $m_{\text {Shao }}$, reportando el mejor desempeño la propuesta $m_{\text {Shao }}$ [Shao 2014].
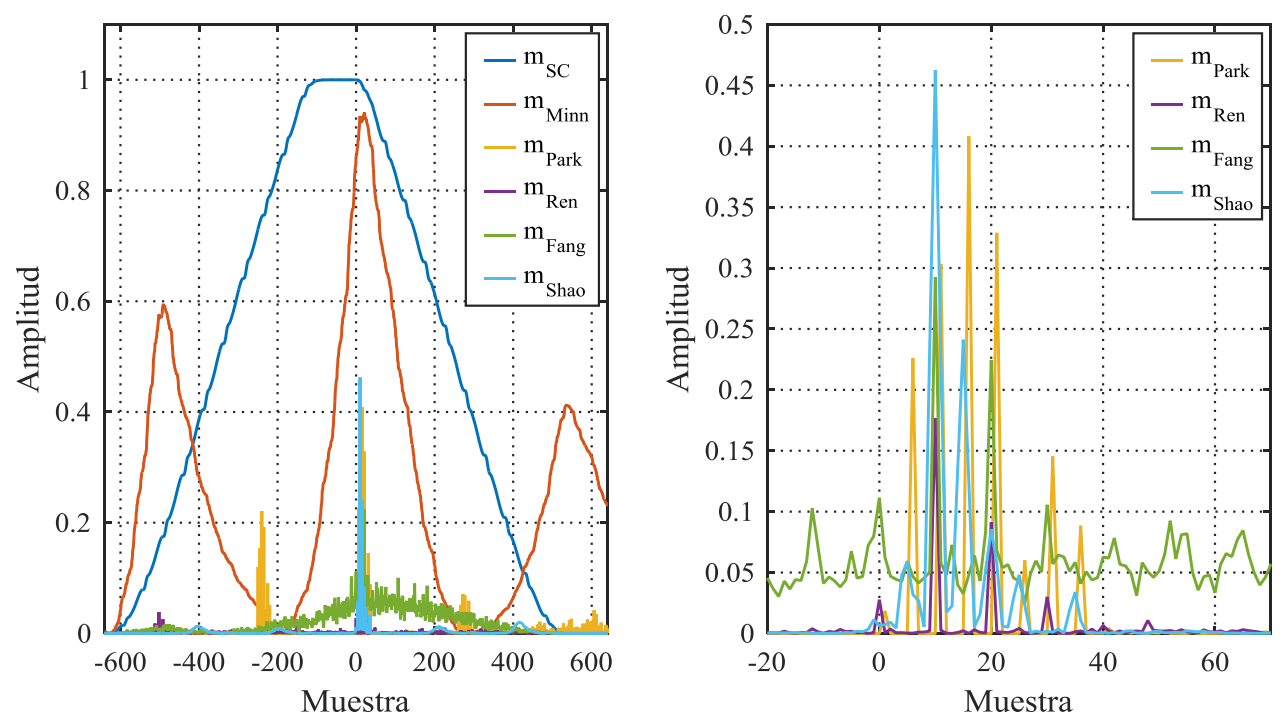

Fig. 2.10 Métricas de sincronización basadas en AC bajo un canal multicamino, sin el agregado de ruido. 
Diseño de Estrategias de Sincronización y Estimación de Canal para la Mejora de Comunicaciones en Redes Inteligentes de Energía

\subsubsection{Sincronización basada en correlación cruzada}

En general, las técnicas basadas en AC se utilizan de manera obligatoria para efectuar una sincronización gruesa en canales inalámbricos debido a su robustez al $\mathrm{CFO}$, que resulta considerable debido a la elevada frecuencia de portadora respecto al espaciamiento entre subportadoras de los sistemas OFDM inalámbricos. Como el error resultante de la etapa de sincronización gruesa no suele ser tolerable por el sistema, se suele plantear una estrategia multietapa compuesta, además, por alguna/s de las siguientes etapas: compensación gruesa de CFO, compensación fina de CFO, ajuste fino de la sincronización, y estimación de canal. El ajuste fino suele efectuarse a partir de la $\mathrm{CC}$ de la señal recibida con compensación de CFO, y en algunos casos el procesamiento resulta útil para obtener la estimación de la CIR. De manera general, la CC se basa en el conocimiento del símbolo piloto de $N$ muestras por el receptor y se computa de acuerdo a:

$$
c_{n}^{\mathbf{r}, \mathbf{x}}=\sum_{m=0}^{N-1} r_{n+m} \cdot \mathbf{x}_{m}^{*}
$$

donde $\mathrm{x}_{m}^{*}$ es la $m$-ésima muestra del símbolo piloto conjugada.

A su vez, como la $\mathrm{CC}$ resulta altamente sensible al $\mathrm{CFO}$, en algunos trabajos se utiliza una correlación cruzada diferencial (CCD) en las que se computa de manera general:

$$
c_{n}^{\mathbf{r}_{\mathbf{d}}, \mathbf{s}}=\sum_{m=0}^{N-1}\left(r_{n+m}^{*} \cdot r_{n+m+D}\right) \cdot s_{m}^{*}
$$

donde $r_{d_{n}}=r_{n}^{*} \cdot r_{n+D}$ y $D$ es un parámetro de diseño que indica el espaciamiento entre muestras multiplicadas. Los productos de una muestra conjugada por otra muestra distinta de la señal recibida es lo que le brinda robustez al CFO a las técnicas basadas en $\mathrm{AC}$, por lo que la CC entre la señal $\mathbf{r}_{\mathbf{d}} \mathrm{y}$ una determinada secuencia $\mathbf{s}$ de longitud $N$ también resulta siendo robusta. A su vez, s se diseña junto al símbolo piloto, y contempla la modificación del mismo al definir los productos de (2.31). La principal desventaja de las técnicas que utilizan la CCD radica en que la degradación de la correlación debido al ruido presente en la señal recibida resulta mayor respecto a la $\mathrm{CC}$ tradicional.

En esa línea, en [Kim 2006] se propone el uso de la CCD con $D=1$ para definir una métrica de sincronización, y el diseño del símbolo piloto tiene en cuenta una estructura 
tal que, en condiciones ideales, los productos $r_{n}^{*} \cdot r_{n+1}$ resulten en una secuencia CAZAC. De esta manera, la CCD presenta una respuesta impulsiva de acuerdo a las propiedades de correlación de la secuencia CAZAC. Por su parte, en [He 2010] se presenta una estrategia de sincronización gruesa basada en $\mathrm{AC}$, y luego se utiliza la CCD en el dominio de la frecuencia para estimar de manera robusta el CFO. Luego, en [Nasraoui 2012] los autores proponen un símbolo piloto con estructura $\mathbf{x}=\left[\begin{array}{ll}\mathbf{a} & \mathbf{a}\end{array}\right]$, donde a es una secuencia $\mathrm{M}$ de longitud $N / 2-1$. Al tratarse de un símbolo con dos mitades idénticas, se define una métrica basada en AC muy similar al método de [Schmidl 1997], para una primera etapa de sincronización gruesa. Luego, proponen una métrica de sincronización fina basada en $\mathrm{CCD}$ con parámetro $D=N / 2-1$, en la que el instante de sincronismo se obtiene a partir de la ubicación del máximo de la métrica. El cómputo de la CCD permite además obtener una estimación del CFO. Posteriormente, en [Nasraoui 2014] los mismos autores proponen la misma estrategia de sincronización, pero agregando el desempeño de distintas secuencias utilizadas para construir el símbolo piloto: secuencias M, Gold y Kasami. De acuerdo a sus resultados, concluyen que las secuencias Kasami proveen un desempeño moderadamente superior al resto.

Por otra parte, en [Awoseyila 2008] se presenta una estrategia que, a partir de un símbolo piloto con dos mitades idénticas, incluye una métrica de sincronización gruesa basada en AC para sincronización gruesa. Luego, asumiendo que el CFO se encuentra compensado en la señal recibida, se computa la CC de la señal recibida con el símbolo piloto conocido por el receptor para definir una nueva métrica que anula los lóbulos laterales principales de la CC, y se obtiene un instante de sincronismo fino inicial $n_{f_{i}}$ a partir de la ubicación del máximo. Finalmente, como el primer camino del canal no es necesariamente el de mayor amplitud, realiza una búsqueda en una determinada ventana de las muestras de la $\mathrm{CC}$ previas a $n_{f_{i}}$ tal que superen un umbral de detección. Así, el instante de sincronismo fino $n_{f}$ resulta de la muestra más alejada de $n_{f_{i}}$ que supere el umbral. Luego, los mismos autores en [Awoseyila 2009] extendieron la propuesta manteniendo la misma métrica de sincronización gruesa, y proponiendo la corrección de la parte fraccionaria del CFO a partir de la estimación obtenida mediante la misma métrica en el instante de sincronismo grueso. Como la señal presente aún contiene la parte entera del CFO, se propone una CCD con espaciamiento $D=1$ y una posterior métrica de sincronización a partir de ella. Con la ubicación del máximo se obtiene $n_{f_{i}}$, 
Diseño de Estrategias de Sincronización y Estimación de Canal para la Mejora de Comunicaciones en Redes Inteligentes de Energía

luego se propone un algoritmo, con una considerable complejidad, de estimación conjunta de la parte entera del CFO y del instante de sincronismo $n_{f}$ a partir de una métrica computada en el dominio de la frecuencia, para cada muestra posible de sincronismo. Finalmente, se compensa completamente el CFO y se realiza un último ajuste fino de sincronismo a partir de la CC con umbralización.

Luego, en [Liu 2012] los autores proponen el uso de un símbolo piloto construido a partir de una secuencia ZC de longitud $N$, y plantean la CC como estrategia de sincronización, a partir de la suposición de un CFO previamente compensando. A partir de un análisis estadístico de las propiedades de la $\mathrm{CC}$, proponen una métrica mejorada en lugar de definir el instante de sincronismo a partir de la ubicación del máximo de $\left|c_{n}^{\mathbf{r}, \mathbf{x}}\right|$ (2.30). El enfoque se basa en maximizar la probabilidad de sincronización perfecta en una determinada ventana de muestras de observación, y la métrica resulta:

$$
m_{L_{i u_{n}}}=\left|\sum_{m=0}^{N-1} r_{n+m} \cdot \mathrm{x}_{m}^{*}\right|-\sum_{m=0}^{N-1} \frac{\left|r_{n+m}\right|^{2}}{2(1+1 / S N R)}
$$

A pesar de que la métrica propuesta supera en desempeño a la CC tradicional, el algoritmo de sincronización a partir de la ubicación del máximo deriva en un error de sincronismo importante cuando el canal multicamino posee caminos secundarios de mayor amplitud que el primero, y no se presenta una derivación de umbrales de detección aplicables a la métrica propuesta en tal escenario.

En [Luo 2012] se presenta una técnica de sincronización en la que se utiliza un CPS de longitud $N / 4$ para la construcción del símbolo piloto con estructura $\mathbf{x}=$ $\left[\begin{array}{llll}\mathbf{a} & \mathbf{0} & \mathbf{b} & \mathbf{o}\end{array}\right]$, donde las secuencias a y b componen el CPS, y $\mathbf{o}$ es un arreglo de $N / 4$ ceros consecutivos. A partir de esto, los autores proponen una métrica basada en CC que se calcula como:

$$
\begin{aligned}
c_{n, l}^{\mathbf{r}, \mathbf{a}} & =\sum_{m=0}^{\frac{N}{4}-1-l} r_{n+m+l} \cdot \mathrm{a}_{m+l} \\
c_{n, l}^{\mathbf{r}, \mathbf{b}} & =\sum_{m=0}^{\frac{N}{4}-1} r_{n+m+\frac{N}{2}+l} \cdot \mathrm{b}_{m+l}
\end{aligned}
$$




$$
\begin{gathered}
p_{L u o_{n}}=\frac{1}{M} \sum_{l=0}^{M-1}\left|c_{n, l}^{\mathbf{r}, \mathbf{a}}+c_{n, l}^{\mathbf{r}, \mathbf{b}}\right|^{2} \\
e_{\text {Luo }_{n}}=\frac{1}{\frac{N}{2}-M} \sum_{l=M}^{N / 2-1}\left|c_{n, l}^{\mathbf{r}, \mathbf{a}}+c_{n, l}^{\mathbf{r}, \mathbf{b}}\right|^{2} \\
m_{\text {Luo }_{n}}=\frac{p_{\text {Luo }_{n}}^{2}}{e_{\text {Luon }_{n}}^{2}}
\end{gathered}
$$

donde $M$ es un número entero que debe elegirse de manera tal de incluir los caminos más significativos de la CIR. Gracias a las propiedades ideales de correlación de los CPS, la métrica propuesta posee un comportamiento impulsivo en condiciones ideales, y a su vez en el instante óptimo de sincronismo el pico de la métrica resulta elevado, no sólo por el valor de la correlación de (2.35), sino también porque el denominador (2.36) es el resultado de la correlación de muestras que contienen únicamente ruido (pertenecientes a los arreglos de ceros o) y el valor tiende a un mínimo que resulta entonces relativamente bajo. Los autores comparan el desempeño de la métrica propuesta con las métricas de [Ren 2005] y [Park 2003], obteniendo mejores resultados. Sin embargo, la métrica propuesta no se encuentra normalizada, y no se presenta la definición de umbrales de detección adecuados para esta circunstancia. Adicionalmente, la energía transmitida en el símbolo piloto se reduce a la mitad debido a la inserción de $N / 2$ muestras nulas, lo que implica una reducción en la ganancia del proceso de CC respecto a otras alternativas.

Si bien la problemática de sincronización temporal en OFDM fue ampliamente investigada en la década de los años 2000 para comunicaciones inalámbricas, es una temática que se continúa desarrollando con interés en la actualidad en virtud de la expansión de la utilización de OFDM como sistema de modulación en diversas aplicaciones, tales como: comunicaciones subacuáticas, comunicaciones por fibra óptica, comunicaciones por luz visible (Visible Light Communications - VLC), entre otros. También se han realizado esfuerzos en desarrollar estrategias de sincronización que se adecúen a las características distintivas de variantes de OFDM, como por ejemplo OFDM-OQAM (Offset Quadrature Amplitude Modulation) [Qian 2017b] o TDS-OFDM (Time-Domain Synchronous) [Farouk 2017].

En [Du 2019] se presenta una propuesta de sincronización para comunicaciones por fibra óptica a partir del diseño de un símbolo piloto compuesto por cuatro segmentos 
Diseño de Estrategias de Sincronización y Estimación de Canal para la Mejora de Comunicaciones en Redes Inteligentes de Energía

con particular simetría, y definiendo una métrica basada en AC cuyo valor máximo define el instante de sincronismo. En [Lee 2018] se presenta una técnica de sincronización gruesa basada en $\mathrm{AC}$ orientada a sistemas $5 \mathrm{G}$, en la que se diseña un símbolo piloto para permitir la sincronización de sistemas en que coexisten distintos tamaños de FFT. En [Offiong 2020] se hace lo propio considerando un sistema OFDM para comunicaciones ópticas en el que se propone un símbolo piloto simétrico, a partir del cual se calcula una métrica con la diferencia entre las muestras de la señal recibida y el instante de sincronismo se obtiene a partir de la ubicación del valor mínimo. En [Chen 2020] se presenta un símbolo piloto construido a partir de secuencias ZC en el que se propone una métrica basada en CC para sincronización y corrección gruesa del CFO, y luego, a partir de una técnica basada en AC, es posible estimar de manera fina el CFO para su compensación.

\subsubsection{Sincronización basada en CC incluyendo sufijo cíclico}

En algunos trabajos, el diseño del símbolo piloto incluye la inserción de un sufijo cíclico además del prefijo cíclico propio del sistema OFDM, que permiten explotar las características de correlación periódica de algunas secuencias para la etapa de sincronización. Adicionalmente, en algunos casos el diseño y procesamiento del símbolo piloto forman parte de una estrategia para obtener, además de una sincronización adecuada, una estimación de la CIR. Este enfoque tiene la principal ventaja de reducir drásticamente la complejidad asociada a la estimación de canal, porque la CIR se suele obtener a partir de los cómputos realizados en el algoritmo de sincronización.

Mientras que en los trabajos descriptos anteriormente al símbolo piloto se le añade el prefijo cíclico de la misma manera que a los símbolos de datos por requerimiento del sistema OFDM, en [Wang 2006] se presenta un diseño de símbolo piloto novedoso basado en secuencias ortogonales modulables (Modulatable Orthogonal Sequences $M O S$ ) en el que se añaden prefijo y sufijo cíclicos. La técnica multietapa consta de una sincronización gruesa y compensación de CFO, y posteriormente un algoritmo de sincronización fina y estimación de canal conjunta. El símbolo piloto propuesto tiene una estructura dada por $\mathbf{x}=\left[\begin{array}{lll}\mathbf{c p} & \mathbf{a} & \mathbf{c s}\end{array}\right]$, donde a es la secuencia MOS de longitud $N$, cp es el prefijo cíclico de $N_{c p}$ muestras, y cs el sufijo cíclico con longitud $N-N_{c p}$, y al igual que $N_{c p}$, es superior a la máxima dispersión del canal. Por un lado, la estructura 
del símbolo piloto contiene dos mitades idénticas, lo que permite como primera etapa obtener una sincronización gruesa basada en AC y la estimación y corrección del CFO. Luego, proponen la CC de la señal recibida con la secuencia MOS conocida por el receptor. Gracias al diseño del símbolo piloto, se puede obtener de manera sencilla una estimación de la CIR a partir de las muestras de la CC. El instante inicial de sincronización fina $n_{f_{i}}$ se obtiene a partir de la ubicación del máximo de la $\mathrm{CC}$, y luego se realiza un ajuste fino a partir de una técnica de detección del primer camino del canal por umbralización, donde el umbral se define como una fracción del máximo valor de la CC. Se realiza una búsqueda en $N_{b}$ muestras previas al instante de sincronismo fino inicial y el instante de sincronismo fino $n_{f}$ resulta de la muestra más alejada de $n_{f_{i}}$ que supere el umbral. Una vez finalizada la etapa de sincronismo fino, se obtiene la CIR estimada a partir de las $L$ muestras posteriores al instante de sincronismo fino $n_{f}$. La gran ventaja de esta propuesta radica en la reducida complejidad asociada a la estimación de canal, al aprovechar los recursos utilizados en la etapa de sincronización. Como principal limitación, como la secuencia MOS posee coeficientes complejos, la cantidad de operaciones requeridas para computar una muestra de la CC resulta en $N$ multiplicaciones complejas y $N-1$ sumas también complejas.

Posteriormente, los mismos autores en [Wang 2008] plantean un rediseño del símbolo piloto, reemplazando la secuencia MOS por una secuencia M. De esta manera, la complejidad del cómputo de la CC se reduce a $N-1$ sumas, sin la necesidad de multiplicadores complejos. Adicionalmente, se efectúa un análisis de la PDF de la CC en las muestras correspondientes a la CIR, y en las muestras previas al primer camino del canal. Esto permite la obtención de un umbral óptimo que maximice la probabilidad de sincronización perfecta para canales multicamino Rayleigh, en función del algoritmo de sincronización fina definido. En este caso, el algoritmo verifica si la $\mathrm{CC}$ en la muestra $n_{f}-1$ supera el umbral de detección; y, si eso sucede, entonces actualiza el instante de sincronismo fino como $n_{f}=n_{f}-1$. El proceso se repite hasta que no se supere el umbral de detección. Si bien el desempeño en simulaciones resulta satisfactorio, el algoritmo obtiene únicamente buenos resultados si los caminos del canal se encuentran contiguos, lo que no suele ser cierto en diversos modelos de canal multicamino. Finalmente, en [Wang 2009] los autores retoman el uso de secuencias MOS para el diseño del símbolo piloto, extienden la comparación hacia atrás en el algoritmo de sincronización fina incluyendo dos muestras anteriores al instante de 
Diseño de Estrategias de Sincronización y Estimación de Canal para la Mejora de Comunicaciones en Redes Inteligentes de Energía

sincronización, y derivan umbrales óptimos en consecuencia para canales multicamino tipo Rayleigh y Ricean. Además, presentan una generalización de la derivación de umbrales óptimos para estos dos canales cuando la búsqueda se realiza sobre $N_{b}$ muestras previas al instante de sincronismo en [Wang 2011].

Luego, en [Gul 2012] se presenta una novedosa estrategia de sincronización basada en CC que no precisa de una compensación previa del CFO. Para ello, los autores proponen la transmisión de dos símbolos pilotos consecutivos, y diseñan los mismos a partir de una secuencia ZC a de longitud $N$, a la que se agregan prefijo y sufijo cíclico de acuerdo a $\mathbf{x}_{1}=\left[\begin{array}{lll}\mathbf{c p} & \mathbf{a} & \mathbf{c s}\end{array}\right]$ y $\mathbf{x}_{2}=\left[\begin{array}{lll}\mathbf{c p} & \mathbf{a}^{*} & \mathbf{c s}\end{array}\right]$. La robustez al CFO se introduce gracias a las propiedades de correlación de las secuencias ZC, junto con una adecuada elección de su parámetro $\mu$, ya que se produce un desvío en la ubicación del pico de correlación, proporcional al valor de la parte entera del CFO. El diseño de cada símbolo piloto provoca un desvío en direcciones opuestas en la CC de $\mathbf{x}_{\mathbf{1}} \mathbf{y} \mathbf{x}_{\mathbf{2}}$, por lo que la ubicación del máximo permite obtener una estimación del CFO y corregir el desplazamiento en muestras correspondiente para obtener el instante de sincronismo. Posteriormente, en [Gul 2015] se realiza una extensión del trabajo anterior bajo la misma estructura de dos símbolos piloto, adicionando una etapa de detección primaria del primer símbolo piloto, la estimación y corrección del CFO fraccionario, y finalmente la sincronización fina y corrección de la parte entera del CFO del mismo modo que en [Gul 2012].

En línea con la estrategia de sincronización y estimación de canal conjunta, en [Kung 2012] se propone un símbolo piloto con estructura $\mathbf{x}=\left[\begin{array}{lll}\mathbf{a} & \mathbf{c} & \mathbf{a}\end{array}\right]$, donde $\mathbf{a}$ es una secuencia PN de longitud $N_{\mathrm{a}}$ y c es un intervalo de guarda de ceros para evitar interferencia con longitud $N_{\mathrm{c}}$, que debe ser superior a la máxima dispersión temporal del canal. A partir de la estructura del símbolo piloto propuesto, los autores plantean una estrategia multietapa con sincronización gruesa basada en AC, y luego una etapa de sincronización fina y estimación de canal. Para la estimación de canal derivan un estimador basado en el criterio de máxima verosimilitud (Maximum likelihood - ML) a partir de una ventana de observación de la señal recibida igual a la longitud del símbolo piloto. Para cada instante posible de sincronismo, se requieren cerca de $\left(2 N_{\mathrm{a}}+N_{\mathrm{c}}\right) \cdot L_{e}$ multiplicaciones complejas para obtener la estimación de la CIR, donde $L_{e}$ es un número que debe ser superior a la máxima dispersión del canal. Se obtiene un instante de sincronismo a partir de la estimación de CIR que maximiza el valor del primer 
camino del canal, y finalmente se realiza un ajuste fino a partir de una técnica de umbralización. Si bien la complejidad asociada a los algoritmos propuestos es elevada, el desempeño presenta muy buenos resultados, especialmente para bajos valores de SNR.

Finalmente, en [Qian 2018] los autores proponen la construcción de un novedoso símbolo piloto para la sincronización y estimación de canal conjunta en sistemas OFDM orientados a VLC. La innovación radica en la generación de un símbolo de amplitud constante en el dominio de la frecuencia a partir de una modulación tipo Offset Quadrature Amplitude Modulation (OQAM), y la posterior convolución en el dominio de la frecuencia con un filtro prototipo denominado PHYDYAS, y desarrollado para la capa física de modulaciones por banco de filtros (FBMC) [Bellanger 2010]. A partir de esto, se obtiene un símbolo piloto denominado por los autores como Código de Correlación Nula (ZCC por sus siglas en inglés), de amplitud constante en frecuencia pero no así en el dominio del tiempo, que posee muy buenas propiedades de correlación periódica y aperiódica. Luego, proponen una técnica de CC para obtener la sincronización y la estimación la CIR a partir de la misma, de manera similar que en [Wang 2009]. Igualmente, se plantean algunas variantes en la construcción y procesamiento de símbolos pilotos construidos a partir del filtro PHYDYAS en [Qian 2017a] y [Qian 2017b].

En la Fig. 2.11 se presentan las CC de los símbolos pilotos que incluyen prefijo y sufijo cíclico con su correspondiente versión sin extender, construidos a partir de secuencias ZC [Gul 2012] en la Fig. 2.11 a), ZCC [Qian 2018] en la Fig. 2.11 b) y secuencias M [Wang 2008] en la Fig. 2.11 c). Se considera un sistema de $N=1024$ subportadoras, con longitud de prefijo cíclico $N_{c p}=128$; y un canal multicamino con la CIR generada a partir de (2.29), sin el agregado de ruido. En la Fig. 2.11 d) se presenta un zoom de las anteriores en la vecindad del instante de sincronismo óptimo, donde se visualiza la zona de correlación ideal (casi ideal en el caso de las secuencias M), y cómo los picos de la CC se corresponden con la CIR del canal en los tres casos.

\subsection{Sincronización en PLC}

En el presente apartado se recaban distintas estrategias de sincronización y estimación de canal para PLC disponibles en la literatura. Como se ha visto anteriormente, para comunicaciones PLC es posible efectuar una transmisión tipo OFDM pasobanda en la 
Diseño de Estrategias de Sincronización y Estimación de Canal para la Mejora de Comunicaciones en Redes Inteligentes de Energía

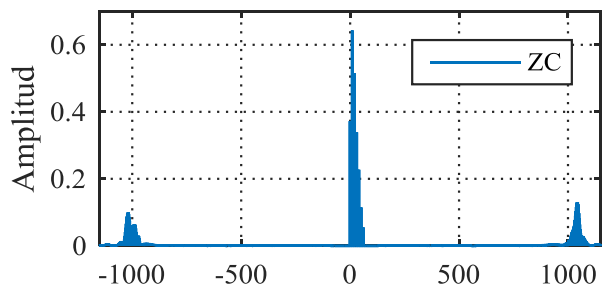

a)

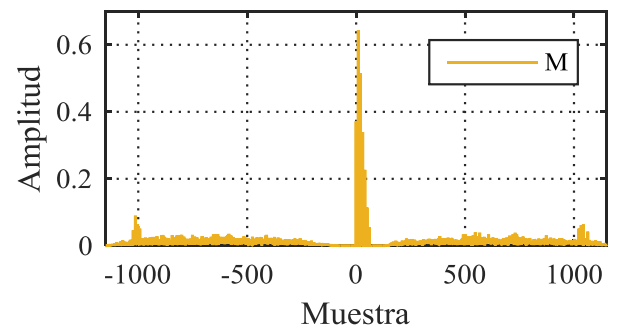

c)

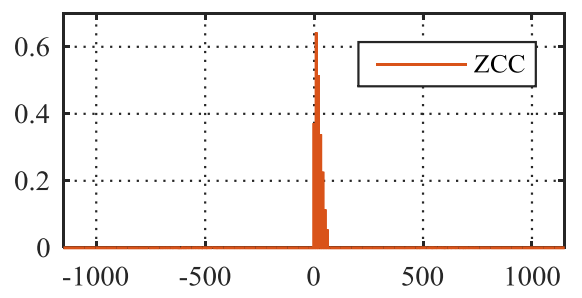

b)

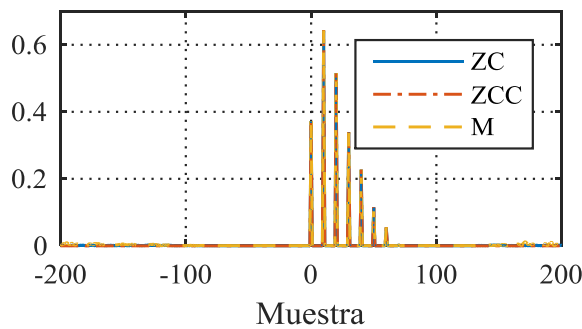

d)

Fig. 2.11 Correlación cruzada de distintos símbolos piloto extendidos con prefijo y sufijo cíclico con su correspondiente versión sin extender, bajo un canal multicamino sin el agregado de ruido.

que se requiere una modulación y demodulación en fase y cuadratura a una frecuencia de portadora determinada, obteniendo luego una señal compleja en banda base sobre la que se pueden diseñar símbolos piloto y aplicar algoritmos de sincronización y/o estimación de canal. Por otro lado, existen trabajos en los que se opta por una transmisión OFDM banda base (DMT) en la que no se requiere la modulación I/Q, y las señales transmitidas y procesadas resultan reales. Este enfoque es el adoptado además en los distintos estándares de comunicaciones PLC disponibles.

Por lo tanto, el diseño de los símbolos piloto y el planteo de métricas de sincronización y estrategias de estimación de canal requieren en cada caso un desarrollo específico. Adicionalmente, las estrategias de sincronización y/o estimación de canal desarrolladas previamente para OFDM orientado a canales inalámbricos pueden aplicarse a sistemas PLC realizando ciertas modificaciones para adecuarlas a las particularidades del canal. En esa línea, en [Xie 2013] se propone la sincronización de un sistema OFDM-PLC a partir de una métrica $\mathrm{AC}$ que, recordando (2.16), se puede escribir de manera general como:

$$
m_{n}=\frac{\left|p_{n}\right|^{2}}{e_{n}^{2}}
$$


El diseño del símbolo piloto consiste en la utilización de una secuencia ZC de longitud $N / 4$ y el arreglo $\mathbf{x}=\left[\begin{array}{llll}\mathbf{a} & \mathbf{b} & \mathbf{a} & -\mathbf{b}\end{array}\right]$, donde $\mathbf{a}$ es la secuencia $\mathrm{ZC}, \mathrm{y} \mathrm{b}_{n}=\mathrm{a}_{n} \cdot \mathrm{a}_{n}$. A partir de la estructura del símbolo piloto, se propone el cómputo la métrica $m_{X i e_{n}}$ :

$$
\begin{gathered}
p_{X i e_{n}}=\sum_{m=0}^{N / 4-1} a_{m}^{*} \cdot r_{n+m}^{*} r_{n+m+N / 4}+r_{n+m}^{*} r_{n+m+N / 2}-a_{m}^{*} \cdot r_{n+m}^{*} r_{n+m+N / 2} \\
+\sum_{m=0}^{N / 4-1} a_{m}^{*} \cdot r_{n+m+N / 4}^{*} r_{n+m+N / 2}-r_{n+m+N / 4}^{*} r_{n+m+3 N / 4}-a_{m}^{*} \cdot r_{n+m+N / 2}^{*} r_{n+m+3 N / 4} \\
e_{X i e_{n}}=\frac{3}{2} \sum_{l=0}^{3} \sum_{m=0}^{N / 4}\left|r_{n+m+l \cdot N / 4}\right|^{2}
\end{gathered}
$$

Los autores proponen además la estimación del CFO fraccionario a partir de la misma métrica de AC, y posteriormente una métrica para la estimación de la parte entera del CFO. Finalmente, comparan la métrica propuesta con las propuestas en [Schmidl 1997] y [Park 2003], obteniendo una mejora en el desempeño tanto de la etapa de sincronización como de estimación del CFO

En [Liu 2013] se presenta una estrategia de sincronización a partir de una métrica basada en AC, y el símbolo piloto propuesto tiene una longitud de $2 \mathrm{~N}$ muestras y su estructura es: $\mathbf{x}=\left[\begin{array}{llll}\mathbf{a} & -\mathbf{a} & \mathbf{a} & \mathbf{b}\end{array}\right]$, donde $\mathbf{x}^{\prime}=\left[\begin{array}{ll}-\mathbf{a} & \mathbf{a}\end{array}\right]$ tiene longitud $N$ y se obtiene a partir de la modulación de determinadas subportadoras en el dominio de la frecuencia. Luego se le agrega un prefijo cíclico de $N / 2$ muestras y, como sufijo de $N / 2$ muestras, se adiciona $\mathbf{b}$, que se obtiene como resultado de un proceso de aleatorización (scrambling) de $\boldsymbol{a}$, como: $b_{n}=(-1)^{n} \cdot a_{n}, n=0,1, \ldots, N / 4-1$. A partir del símbolo piloto, se plantean tres cómputos de AC independientes de acuerdo a (2.41) y finalmente la métrica propuesta en (2.42).

$$
\begin{gathered}
p_{\text {Liu }_{i, n}}=\sum_{m=0}^{N / 2-1}(-1)^{(n+m)} \cdot r_{n+m}^{*} \cdot r_{n+m+i \cdot N / 2} \\
m_{\text {Liu } 2_{n}}=\left|-p_{\text {Liu }_{1, n}}^{*} \cdot p_{\text {Liu }_{2, n}} \cdot p_{\text {Liu }_{3, n}}\right|
\end{gathered}
$$

La particularidad de la métrica propuesta radica en que cada uno de los cómputos de AC incluye el producto del segmento aleatorizado $\mathbf{b}$ con uno de los tres segmentos que lo anteceden, y el factor $(-1)^{(n+m)}$ se incluye para compensar la aletorización. La principal ventaja obtenida a partir de esto es una marcada robustez a interferencias de 
Diseño de Estrategias de Sincronización y Estimación de Canal para la Mejora de Comunicaciones en Redes Inteligentes de Energía

banda estrecha (Narrow Band Interference - NBI). Como resulta general en las métricas basadas en AC, se propone estimar y compensar la parte fraccionaria del CFO a partir de la métrica. Además, se propone una etapa de estimación del CFO entero en el dominio de la frecuencia. Sin embargo, la métrica no se encuentra normalizada por energía (acotada entre 0 y 1 ) y no se plantea en consecuencia un umbral práctico para la detección primaria del símbolo piloto.

Luego, en [Wu 2014] se propone un esquema de sincronización para un sistema OFDM-PLC basado en un símbolo piloto como el propuesto en [Park 2003]: $\mathbf{x}=$ $\left[\begin{array}{llll}\mathbf{a} & \mathbf{b}^{*} & \mathbf{a} & \mathbf{b}^{*}\end{array}\right]$ donde $\mathbf{a}^{*}$ representa el conjugado de $\mathbf{a}, \mathrm{y}$ el segmento $\mathbf{b}$ es simétrico respecto a a. Para la sincronización gruesa, dado que el símbolo piloto posee dos mitades idénticas, plantean una métrica similar a $m_{S C}$ [Schmidl 1997], pero extendiendo la ventana deslizante de $N / 2$ a $N / 2+N_{c p}$ muestras, para evitar el fenómeno de meseta. A partir de la ubicación del máximo, obtienen el instante de sincronismo grueso $n_{g}$. Para la sincronización fina, proponen una búsqueda en la ventana de muestras $\left[n_{g}-\right.$ $\left.N_{b}, n_{g}+N_{b}\right]$, donde $N_{b}$ se debe elegir menor a $N / 4$ y se elige a partir de una relación de compromiso entre desempeño y complejidad asociada. Así, se computa la métrica $m_{\text {Park }}$ para esa ventana reducida de muestras y se define el instante de sincronismo fino $n_{f}$ a partir de la ubicación del máximo.

Posteriormente, en [Liu 2017] se presenta una estrategia de sincronización gruesa basada en CC para un sistema OFDM-PLC, en la que se define una métrica a partir de:

$$
\begin{gathered}
p_{\text {Liu } 3_{n}}=\sum_{m=0}^{N / N_{d}-1} \alpha \cdot r_{n+m \cdot N_{d}} \cdot \mathrm{a}_{m \cdot N_{d}} \\
e_{\text {Liu }_{n}}=\sum_{m=0}^{N / N_{d}-1}\left|\alpha \cdot r_{n+m \cdot N_{d}}-\mathrm{a}_{m \cdot N_{d}}\right|^{2}
\end{gathered}
$$

donde $\mathrm{a}_{m}$ son las muestras del símbolo piloto transmitido, $N_{d}$ es un factor entero de decimación que reduce la complejidad del algoritmo, incluyendo una menor cantidad de muestras a medida que $N_{d}$ aumenta, y $\alpha$ es un factor normalizador del cociente de las energías de la señal recibida y el símbolo piloto en una ventana de $N$ muestras. La métrica presenta un comportamiento impulsivo, y es comparado con las métricas de [Schmidl 1997] y [Park 2003]. Además, el desempeño de la sincronización a partir de la ubicación del valor máximo es evaluado en pruebas experimentales. Por otro lado, la 
métrica propuesta no se encuentra acotada entre 0 y 1 , y no se deriva un umbral práctico para la detección del símbolo piloto ante esa situación.

Otra técnica de sincronización basada en CC se presenta en [Bumiller 2007] para NBPLC donde se plantea, inicialmente para un símbolo piloto genérico $\mathbf{x}$, la métrica de sincronización:

$$
m_{\text {Bum.n }}=\sqrt{\frac{\sum_{l=0}^{L-1}\left|\sum_{m=0}^{N-1} r_{n+m+l} \cdot \mathbf{x}_{m}^{*}\right|^{2}}{\sum_{m=0}^{N+L-2}\left|r_{n+m}\right|^{2}}}
$$

donde $N$ es la longitud del símbolo piloto y $L$ un número entero que estima la máxima dispersión temporal del canal. Por un lado, plantean el escenario de dos símbolos piloto distintos generados a partir de una secuencia CAZAC y una secuencia Barker, respectivamente. Luego, realizan una búsqueda exahustiva de un símbolo piloto que optimiza un factor de mérito que depende de diversos parámetros del sistema. A partir de un análisis de la métrica propuesta, derivan umbrales prácticos de detección primaria de los símbolos piloto, y mediante simulaciones verifican que la sincronización utilizando el símbolo piloto optimizado presenta un desempeño superior respecto a las variantes basadas en secuencias CAZAC y Barker.

Por su parte, en [Nikfar 2017] se propone una técnica de sincronización orientada a OFDM-PLC de banda angosta, en el que es posible transmitir en las siete distintas bandas FCC (Federal Communications Commission). Como cuatro bandas completan el espectro cubierto por las siete posibles bandas de transmisión, se propone una estrategia basada en el cómputo de cuatro métricas basadas en $\mathrm{CC}$ a partir de:

$$
m_{N i k f a r}=\sum_{l=0}^{L-1}\left|\sum_{m=0}^{N-1} r_{n+m+l} \cdot a_{i, m}^{*}\right|^{2}
$$

donde $i=1,2,3,4$ representa el número de banda, $\mathrm{y} \mathbf{a}_{i}$ es el símbolo piloto de longitud $N$ equivalente para la $i$-ésima banda, resultante de modular en frecuencia únicamente las subportadoras que le corresponden a la misma. A partir de la detección del máximo de cada métrica, es posible obtener una sincronización gruesa y además identificar en qué banda FCC se realiza la transmisión.

En lo que respecta a los sistemas de transmisión DMT-PLC, en [Godoy 2012] se presenta una estrategia de sincronización basada en AC. Por un lado, se presenta una métrica a partir de la estructura repetitiva del prefijo cíclico de los símbolos 
Diseño de Estrategias de Sincronización y Estimación de Canal para la Mejora de Comunicaciones en Redes Inteligentes de Energía

transmitidos. Luego, aprovechando la transmisión de señales reales en los sistemas DMT, proponen una métrica derivada de una modificación de las métricas $m_{S C}(2.17) \mathrm{y}$ $m_{\text {Minn }}(2.21)$ :

$$
m_{n}^{\prime}=\frac{p_{n}}{e_{n}}
$$

Puede observarse que no se computan $\left|p_{n}\right|^{2}$ ni $e_{n}^{2}$. A partir de esto, las métricas pueden adoptar valores positivos y negativos lo que resulta provechoso para reducir el error de sincronismo asociado a la ubicación del valor máximo de las métricas. Bajo la misma línea, en [Torres-Alvarado 2012] se presenta la implementación de un algoritmo de sincronización gruesa basado en la AC del prefijo cíclico, y un ajuste fino en el dominio de la frecuencia. Por su parte, en [Lalos 2016] se propone una estrategia de sincronismo para una transmisión OFDM banda base a partir de una combinación de una métrica de AC con la $\mathrm{CC}$, mediante una estructura de preámbulo compuesto por varios símbolos repetidos consecutivamente. Además, el trabajo incluye una etapa de estimación y compensación de SFO presente en la señal recibida.

En [Nombela 2015b] se presenta una estrategia de sincronización para un sistema PLC basado en Wavelet-OFDM, con restricciones en el número de subportadoras disponibles (activas). El diseño del símbolo piloto se basa en el uso de CSS multinivel para la modulación de las subportadoras, y se utiliza la CC para efectuar una etapa de sincronización gruesa. En una primera instancia, asumiendo una detección primaria del símbolo piloto resuelta, se determina la ubicación del máximo de la CC. Posteriormente, se realiza una búsqueda en una determinada ventana de muestras hacia atrás de los valores de la CC que superen un determinado umbral, definiendo el instante de sincronismo grueso $n_{g}$ a la primera muestra que cumpla la condición. Finalmente, se realiza una etapa de ajuste fino en el dominio de la frecuencia posterior a la ecualización del canal (que se asume perfectamente conocido), a partir de efectuar la correlación de la señal obtenida con la copia local de las CSS multinivel transmitidas. Este proceso se realiza para varios desplazamientos, y se elige como criterio de ajuste fino al desplazamiento para el que se obtenga el menor MSE respecto a la ACS esperada idealmente. Luego, en [Nombela 2015a] los autores proponen la implementación de la etapa de sincronización gruesa del anterior algoritmo, e incluyen secuencias ZC como símbolo piloto, además de CSS multinivel. 


\subsection{Estimación de canal en OFDM}

Con respecto a la estimación de canal en PLC, pueden aplicarse las distintas técnicas clásicas para OFDM/DMT atendiendo a las características particulares del canal PLC [Bogdanović 2014]. Para efectuar la estimación de canal en OFDM, se utilizan subportadoras piloto en las que se transmite información conocida por el receptor. La disposición de subportadoras piloto puede responder a una estructura tipo peine, en la que se utiliza sólo un número reducido de subportadoras piloto del símbolo OFDM, que pueden estar uniformemente espaciadas o no, mientras que el resto se completan con símbolos de datos. De esta manera, se reduce la transmisión de datos en todos los símbolos en pos de poder efectuar la estimación de canal en cada símbolo OFDM a partir de las subportadoras piloto. Otra opción es utilizar todas las subportadoras disponibles como piloto, para conformar lo que se denomina un símbolo piloto. Los símbolos piloto se agregan como cabecera o preámbulo a los símbolos OFDM de datos, y suelen ser utilizados para la detección de la trama, estimación de canal, y/o control automático de ganancia (Automatic Gain Control - AGC). La estructura de subportadoras piloto tipo peine se utiliza para canales de rápida variación en el tiempo, en los que se debe refinar la estimación entre un símbolo y otro, mientras que la estructura de símbolos piloto se utiliza para canales de baja variación o cuasi estáticos. El canal PLC de banda ancha suele considerarse cuasi estático, es decir, constante para toda la duración de la trama, por lo que suelen utilizarse símbolos piloto para la estimación de canal.

A continuación se detallan las técnicas más difundidas para la estimación de canal en OFDM a partir de símbolos piloto. Para analizar su desempeño, se suele considerar una situación de sincronismo perfecto (es decir, no existen ISI ni ICI), y además la dispersión máxima del canal $L$ es inferior a la longitud del prefijo cíclico $N_{c p}$. Bajo estas suposiciones, el símbolo OFDM recibido en el dominio de la frecuencia puede escribirse como:

$$
\mathbf{Y}=\left[\begin{array}{c}
Y_{0} \\
Y_{1} \\
\vdots \\
Y_{N-1}
\end{array}\right]=\left[\begin{array}{cccc}
X_{0} & 0 & \cdots & 0 \\
0 & X_{1} & & \vdots \\
\vdots & & \ddots & 0 \\
0 & \cdots & 0 & X_{N-1}
\end{array}\right]\left[\begin{array}{c}
H_{0} \\
H_{1} \\
\vdots \\
H_{N-1}
\end{array}\right]+\left[\begin{array}{c}
W_{0} \\
W_{1} \\
\vdots \\
W_{N-1}
\end{array}\right]=\mathbf{X H}+\mathbf{W}
$$

donde $\mathbf{X}$ es una matriz diagonal con los elementos del símbolo piloto en el dominio de la frecuencia; $\mathbf{H}$ es un vector con la CFR; y $\mathbf{W}$ es el vector de ruido aditivo en cada subportadora. 
Diseño de Estrategias de Sincronización y Estimación de Canal para la Mejora de Comunicaciones en Redes Inteligentes de Energía

\subsubsection{Estimación por mínimos cuadrados}

La estimación por mínimos cuadrados (Least Squares - LS) se basa en minimizar la función de error $\mathbf{e}(\widetilde{\mathbf{H}})=\|\mathbf{Y}-\mathbf{X} \widetilde{\mathbf{H}}\|^{2}$ [Van De Beek 1995] [Yong Soo 2010a]. A partir de ello, se obtiene la estimación como:

$$
\begin{gathered}
\widetilde{\mathbf{H}}_{L S}=\mathbf{X}^{-1} \mathbf{Y} \\
\widetilde{H}_{L S_{k}}=\frac{Y_{k}}{X_{k}}, \quad k=0,1, \ldots, N-1
\end{gathered}
$$

El error cuadrático medio (MSE) del estimador resulta:

$$
M S E_{L S}=E\left\{\left(\mathbf{H}-\widetilde{\mathbf{H}}_{L S}\right)^{H}\left(\mathbf{H}-\widetilde{\mathbf{H}}_{L S}\right)\right\}=\frac{1}{S N R}
$$

donde $S N R$ es la relación señal a ruido, y el superíndice $(\cdot)^{H}$ indica la operación de transposición hermitiana.

Si bien este estimador de canal en el dominio de la frecuencia presenta un considerable MSE para bajos valores de $S N R$, a su vez es uno de los más sencillos, ya que no requiere conocimiento de la condición de $S N R$ ni de las propiedades estadísticas del canal. Con respecto a su complejidad asociada, de acuerdo a (2.50), requiere únicamente $N$ multiplicaciones complejas (una por cada subportadora), lo que lo convierte en una opción de reducida complejidad.

\subsubsection{Estimación por minimización del error cuadrático medio}

Para mejorar el desempeño de la estimación de canal por LS, pueden derivarse estimadores que utilicen algún tipo de información acerca de las características del canal y del nivel de ruido presente en la señal recibida. En esa línea, el estimador lineal que minimiza el error $E\left\{\|\mathbf{H}-\widetilde{\mathbf{H}}\|^{2}\right\}$ se denomina LMMSE (Linear Minimum Mean Square Error) y su cómputo se puede escribir como [Van De Beek 1995]:

$$
\widetilde{\mathbf{H}}_{L M M S E}=\mathbf{R}_{\mathbf{H H}}\left[\mathbf{R}_{\mathbf{H H}}+\sigma_{w}^{2}\left(\mathbf{X} \mathbf{X}^{H}\right)^{-1}\right]^{-1} \widetilde{\mathbf{H}}_{L S}
$$

donde $\widetilde{\mathbf{H}}_{L S}$ es la estimación por LS de (2.50), $\sigma_{w}^{2}$ es la varianza del ruido aditivo, y $\mathbf{R}_{\mathbf{H H}}=E\left\{\mathbf{H H}^{H}\right\}$ es la matriz de autocorrelación del canal.

Esta técnica posee la principal ventaja de obtener un MSE inferior a la técnica de estimación por LS. Sin embargo, puede osbervarse que presenta una elevada complejidad para cada estimación, ya que debe realizarse la inversión de una matriz de 
$N x N$ y luego los productos matriciales restantes. A su vez, se asume el conocimiento de la matriz de autocorrelación del canal y de la varianza del ruido, que en la práctica deben estimarse o bien adoptar un valor fijo, lo que implica un incremento en la complejidad y una disminución en el desempeño.

Para reducir ligeramente la complejidad del estimador, puede evitarse el cómputo de $\left(\mathbf{X X}^{H}\right)^{-1}$ en (2.52), reemplazándolo por su valor esperado $E\left\{\left(\mathbf{X X}^{H}\right)^{-1}\right\}$ [Edfors 1998]:

$$
\widetilde{\mathbf{H}}_{L M M S E_{\text {mod }}}=\mathbf{R}_{\mathbf{H H}}\left[\mathbf{R}_{\mathbf{H H}}+\frac{\beta}{S N R}\right]^{-1} \widetilde{\mathbf{H}}_{L S}
$$

donde $\beta$ es un parámetro que depende de la constelación utilizada en la transmisión de los símbolos.

\subsubsection{Estimación de canal por transformada discreta de Fourier}

La estimación de canal por DFT es una alternativa que mejora sustancialmente la estimación por LS. La técnica se basa inicialmente en obtener la estimación de la CIR a partir de la IDFT de la estimación por LS de la CFR como:

$$
\tilde{\mathbf{h}}_{L S}=\mathbf{F} \widetilde{\mathbf{H}}_{L S}
$$

donde $\mathbf{F}$ es la matriz de IDFT de orden $N$. Asumiendo que la CIR del canal se encuentra contenida en las primeras $L$ muestras del vector $\tilde{\mathbf{h}}_{L S}$, las últimas $N-L$ muestras son muestras ruidosas, y no incluyen energía de los caminos del canal [Kang 2007]. La dispersión temporal del canal es un parámetro que suele ser desconocido y variante en el tiempo, por lo que en la práctica debe realizarse una estimación $\tilde{L}$. Adicionalmente, si el sistema OFDM se diseña adecuadamente, entonces puede asumirse que la dispersión temporal del canal es inferior a la longitud del prefijo cíclico, es decir $L<N_{c p}$. Así, la opción más simple cuando se desconoce $L$ es la estimación $\tilde{L}=N_{c p}$, porque asegura la inclusión de todas las muestras de la CIR.

Finalmente, a partir del vector $\tilde{\mathbf{h}}_{D F T}$ que contiene únicamente las $\tilde{L}$ muestras de la estimación de la CIR $\tilde{\mathbf{h}}_{L S}$, la estimación de la CFR se obtiene a partir del proceso de DFT del vector $\tilde{\mathbf{h}}_{D F T}$ :

$$
\widetilde{\mathbf{H}}_{D F T}=\mathbf{F}_{\mathbf{1}}^{H} \tilde{\mathbf{h}}_{D F T}
$$

donde $\mathbf{F}_{1}$ contiene las primeras $\tilde{L}$ filas de la matriz de IDFT, y, mediante la transposición hermitiana, $\mathbf{F}_{\mathbf{1}}^{H}$ resulta la matriz de DFT correspondiente al vector $\tilde{\mathbf{h}}_{D F T}$. 
Diseño de Estrategias de Sincronización y Estimación de Canal para la Mejora de Comunicaciones en Redes Inteligentes de Energía

El cálculo del MSE asociado a la estimación por DFT de (2.55) resulta:

$$
M S E_{D F T}=\frac{\tilde{L}}{N} \cdot \frac{1}{S N R}
$$

Así, se obtiene una reducción del MSE de la estimación por LS en (2.51) dada por el factor $\tilde{L} / N$. La mejora resulta importante porque se cumple que $\tilde{L} \leq N_{c p}<N$, y $N_{c p}$ es típicamente una fracción pequeña de $N$ en los sistemas OFDM. Adicionalmente, a pesar de presentar un MSE superior a la estimación por MMSE, es una estrategia que no precisa del conocimiento de la matriz de autocorrelación del canal $\mathbf{R}_{\mathbf{H H}}$ ni de la condición de SNR. Como contraparte, la complejidad respecto a la estimación por LS se incrementa debido a que, además de las $N$ multiplicaciones complejas, se requiere el cómputo de los procesos de IDFT y DFT, a pesar de que pueden implementarse eficientemente a partir de algoritmos de FFT.

Adicionalmente, la estimación puede mejorarse aún más si se efectúa alguna técnica avanzada de selección de los caminos más significativos de la CIR incluida en $\tilde{\mathbf{h}}_{D F T}$. Generalmente, se realiza una decisión a partir de los elementos de $\tilde{\mathbf{h}}_{D F T}$ que superan un determinado umbral de selección optimizado a partir de algún criterio, lo que requiere el conocimiento de al menos la condición de $S N R$ para un adecuado desempeño [Kang 2007] [Rosati 2012].

\subsection{Estimación de canal en OFDM-PLC}

En esta sección se realiza una reseña de trabajos en los que se han desarrollado técnicas de estimación de canal específicas para sistemas OFDM-PLC de banda ancha, haciendo foco principalmente en técnicas de estimación basadas en símbolos piloto.

En [Bogdanović 2014] se propone una estrategia de estimación en la que se utiliza un símbolo piloto para la estimación inicial de la CFR $\widetilde{\mathbf{H}}_{L S}$, y la ubicación de subportadoras piloto en los símbolos de datos siguientes con estructura tipo peine y una interpolación lineal para obtener una estimación propia en cada uno de los símbolos de datos $\widetilde{\mathbf{H}}_{P E}$. Luego, la estimación de canal final se obtiene como el promedio de las estimaciones como:

$$
\widetilde{\mathbf{H}}_{\text {Bogd. }}=\frac{\widetilde{\mathbf{H}}_{L S}+\widetilde{\mathbf{H}}_{P E}}{2}
$$


A partir de simulaciones, los autores obtienen un desempeño intermedio entre la estimación por LS y LMMSE. Sin embargo, la inclusión de subportadoras piloto en todos los símbolos de la trama disminuye sustancialmente la eficiencia espectral del sistema, además del incremento de complejidad asociado a la estimación por LS adicional, e interpolación requerida en los símbolos de datos.

Por su parte, en [Nombela 2017] se presenta una estrategia de sincronización y estimación de canal conjunta para un sistema Wavelet-OFDM, a partir de la transmisión de cuatro símbolos piloto consecutivos a modo de preámbulo a los símbolos de datos. Los dos símbolos pilotos iniciales se componen del resultado de modular la subportadoras con un par de secuencias complementarias multinivel, y se utilizan para la etapa de sincronización a partir de una técnica de CC. Los últimos dos símbolos piloto se generan a partir de modular las subportadoras con dos secuencias ZC y se utilizan para la estimación y ecualización del canal PLC. Para ello, proponen una estimación del canal dada por el promedio de las estimaciones por LS que se obtienen a partir de los dos símbolos construidos con secuencias ZC. Finalmente, realizan una comparación entre la técnica de ecualización Zero Forcing (ZF), y un ecualizador denominado Adaptive Sine-modulated/Cosine-modulated filter bank Equalizer for Transmultiplexers (ASCET). De ella se desprende que se obtienen mejores resultados con la técnica de ecualización ZF, y que su complejidad asociada es inferior al del ecualizador ASCET. Luego, en [Nombela 2019] se presenta también una estructura de cuatro símbolos piloto para sincronización y estimación de canal, pero en este caso los primeros dos símbolos piloto están compuestos por la parte real e imaginaria de una secuencia ZC, y los últimos dos símbolos piloto son una repetición de los dos primeros. La estrategia de estimación de canal se obtiene nuevamente a partir del promedio de las estimaciones por LS utilizando los últimos dos símbolos piloto, y la técnica de ecualización utilizada es ZF.

En [Moya 2015] y [Moya 2016a] se presenta una estrategia de estimación de canal para OFDM-PLC bajo la suposición de un sincronismo ideal. Se propone la transmisión de dos símbolos piloto [a $\mathbf{b}$ ], donde $\mathbf{a}$ y $\mathbf{b}$ componen un CPS de longitud $N$. A partir de las propiedades de correlación del CPS, es posible obtener la estimación del canal como:

$$
\widetilde{\mathbf{H}}_{\text {Moya }}=\frac{\mathbf{Y}_{\mathbf{A}} \mathbf{A}^{*}+\mathbf{Y}_{\mathbf{B}} \mathbf{B}^{*}}{2 N}
$$


Diseño de Estrategias de Sincronización y Estimación de Canal para la Mejora de Comunicaciones en Redes Inteligentes de Energía

donde A y $\mathbf{B}$ corresponden a la DFT de $\mathbf{a}$ y $\mathbf{b}$, respectivamente; $\mathbf{Y}_{\mathbf{A}}$ e $\mathbf{Y}_{\mathbf{B}}$ son las señales recibidas en el dominio de la frecuencia correspondientes a la ventana de cada símbolo piloto; $\mathrm{y}$ entonces la operación $\mathbf{Y}_{\mathbf{A}} \mathbf{A}^{*}$ se entiende como la correlación de la señal recibida con el símbolo piloto en el dominio de la frecuencia. Luego, los autores aplican una ecualización por ZF que puede expresarse como:

$$
Q_{C P S-Z F_{k}}=\frac{1}{\widetilde{H}_{\text {Moya }_{k}}}, \quad k=0,1, \ldots, N-1
$$

donde $k$ es el número de subportadora y $Q_{k}$ es el coeficiente ecualizador a partir del cual se obtiene el símbolo de datos ecualizado como $D_{k}=Q_{C P S-Z F_{k}} \cdot Y_{k}$. A partir de simulaciones demuestran que su propuesta presenta un desempeño superior al que se obtiene por la estrategia LS, y ligeramente inferior a la estimación por LMMSE. Esto se produce principalmente porque la transmisión se basa en dos símbolos piloto mientras la técnica por LS utiliza únicamente uno solo, y entonces la distorsión por el efecto del ruido se ve disminuida. A continuación, en [Moya 2016b] se realiza una extensión de la propuesta, manteniendo la estructura de dos símbolos piloto que componen el CPS y la estimación de canal a partir de (2.58). Adicionalmente, plantean los esquemas de ecualización por ZF de (2.59) y MMSE a partir de la estimación de canal, e introducen una novedosa estrategia de ecualización a partir de la búsqueda de los coeficientes $Q_{k}$ que optimizan por LS el resultado de la ecualización a partir de los dos símbolos piloto transmitidos y las propiedades de los CPS. De esta manera, obtienen los coeficientes de ecualización óptimos a partir de:

$$
Q_{C P S-L S_{k}}=\frac{Y_{A_{k}}^{*} \cdot A_{k}+Y_{B_{k}}^{*} \cdot B_{k}}{\left|Y_{A_{k}}\right|^{2}+\left|Y_{B_{k}}\right|^{2}}, \quad k=0,1, \ldots, N-1
$$

donde el denominador es evidentemente la suma de las energías de la señal recibida en las ventanas de cada símbolo piloto, y el numerador puede entenderse como una combinación que le da mayor peso a la estimación por un símbolo piloto u otro, en función de las distintas amplitudes de $A_{k}$ y $B_{k}$, ya que los símbolos piloto no son de amplitud constante en el dominio de la frecuencia. A partir de esta técnica, se obtiene una mejora sustancial respecto a la ecualización $\mathrm{ZF}$.

Por otro lado, también se han desarrollado recientemente estrategias de estimación de canal PLC en conjunto con mitigación de ruido impulsivo. Se basan en el desarrollo de algoritmos iterativos en los que, a partir de una estimación de canal inicial, se realizan 
sucesivas aproximaciones en la detección y mitigación de ruido impulsivo y refinamiento de la estimación del canal, derivándose distintos criterios de optimización [Chien 2015] [Yan 2017] [Shrestha 2018]. Estas alternativas presentan en general una elevada complejidad asociada que limita su implementación práctica.

\subsection{Formulación del problema y objetivos planteados}

Los sistemas PLC presentan un canal altamente selectivo en frecuencia y diversas fuentes de ruido, y se ha optado por técnicas de modulación multiportadora para lidiar con sus características hostiles. OFDM es una solución adoptada ampliamente para solventar las dificultades del canal PLC de banda ancha, pero requiere de una sincronización adecuada, en conjunto con la estimación y ecualización del canal para un desempeño correcto.

Tanto para la etapa de sincronización como para la estimación de canal, se suelen diseñar símbolos piloto a partir del uso de secuencias con buenas propiedades de correlación (CAZAC [Xie 2013], CPS [Moya 2016b], CSS [Nombela 2015b], etc.). Si bien la problemática fue estudiada ampliamente para sistemas OFDM inalámbricos, las particularidades del canal PLC condujeron recientemente al desarrollo de estrategias de sincronización y estimación de canal específicas que atiendan a sus características.

Con respecto a la etapa de sincronización en sistemas PLC, algunos trabajos optan por técnicas de sincronización basada en autocorrelación [Xie 2013] [Liu 2013] [Wu 2014]. En otros se plantean estrategias de sincronización basadas en correlación cruzada [Liu 2017] [Bumiller 2007] [Nikfar 2017] [Nombela 2015b], y no se encuentra un análisis fundamentado respecto a cuál de las dos variantes presenta mejores prestaciones ante las particularidades del canal PLC.

Por su parte, la etapa de estimación de canal suele efectuarse a partir de la adaptación al entorno PLC de alguna técnica clásica en el dominio de la frecuencia para OFDM [Bogdanović 2014] [Van De Beek 1995]. Adicionalmente, se han propuesto símbolos piloto para la estimación de canal construidos a partir de secuencias ZC [Nombela 2019] y CPS [Moya 2016a]. El diseño de los símbolos piloto es específico para la etapa de estimación de canal, y son distintos a los utilizados para la etapa de sincronización, o bien se asume que el sistema se encuentra perfectamente sincronizado.

El diseño del símbolo piloto y de la estrategia de procesamiento digital para las etapas de sincronización y/o estimación del canal resulta crucial para obtener un desempeño 
Diseño de Estrategias de Sincronización y Estimación de Canal para la Mejora de Comunicaciones en Redes Inteligentes de Energía

adecuado, y cada propuesta presenta distintas características en función de las secuencias utilizadas para la construcción de los símbolos piloto y los algoritmos propuestos en base a ellos. En particular, la marcada selectividad en frecuencia del canal PLC requiere usualmente de un sistema OFDM con un número elevado de subportadoras $-N=4096$ en el estándar IEEE 1901 [IEEE 2010]-, lo que incrementa considerablemente la complejidad de los algoritmos habitualmente utilizados en sistemas inalámbricos. Si sumado a esto se desarrollan las etapas de sincronización y estimación de canal de manera independiente, la demanda computacional puede derivar en arquitecturas de implementación de gran complejidad, incrementando el costo de fabricación y el consumo. Adicionalmente, puede dificultar la viabilidad del sistema en aplicaciones que requieran procesamiento en tiempo real.

En esa línea, la presente tesis posee los siguientes objetivos:

- Diseño de un símbolo piloto aplicable tanto a sistemas OFDM paso banda como a un esquema de transmisión DMT, que permita la sincronización del sistema a partir de técnicas de AC y CC, y la estimación del canal PLC de manera conjunta. De esta manera, los cómputos realizados en la etapa de sincronización pueden reutilizarse en la estimación de canal, y en consecuencia permiten reducir la complejidad del conjunto respecto al enfoque individual de cada etapa.

- Inclusión en la construcción del símbolo piloto de secuencias cuya correlación se pueda computar de manera eficiente, como son los CPS o CSS, y en consecuencia definir los algoritmos de sincronización y estimación de canal conjunta. Se comparará la complejidad asociada a los cómputos requeridos por ambas etapas, respecto a la utilización de otros símbolos piloto disponibles en la literatura, y se verificará por medio de simulaciones que el desempeño de la propuesta resulte competitivo.

- Realización de un análisis detallado de las características que presentan las técnicas de sincronización basadas en AC y CC, y comparar su robustez ante las condiciones del canal PLC. A partir del análisis, se derivarán estrategias de detección que garanticen una alta probabilidad de sincronización exitosa, manteniendo una baja probabilidad de falsa detección.

- Evaluación de la propuesta de sincronización y estimación de canal conjunta en un escenario con restricción en la cantidad de subportadoras habilitadas para la transmisión del símbolo piloto, a partir de la aplicación de una máscara espectral 
Capítulo 2: Estado del arte y objetivos planteados

de compatibilidad electromagnética. Se determinará la degradación del desempeño en cada una de las etapas, y se propondrán soluciones que permitan adaptar la propuesta a tal escenario. 
Diseño de Estrategias de Sincronización y Estimación de Canal para la Mejora de Comunicaciones en Redes Inteligentes de Energía

\section{SINCRONIZACIÓN Y ESTIMACIÓN DE CANAL EN SISTEMAS OFDM-PLC}

OFDM desempeña un importante papel en las comunicaciones por línea eléctrica (PLC), debido a su robustez a los canales selectivos en selectiva. No obstante, también presenta algunos inconvenientes persistentes a lo largo de los años, algunos de ellos relacionados con la sincronización y la estimación y ecualización del canal, cuyos resultados tienen un impacto importante en el desempeño global de la técnica de acceso al medio. En el Capítulo 2, hemos visto distintas variantes para la sincronización y estimación de canal conjunta en sistemas OFDM a partir de la transmisión y procesamiento de símbolos piloto, diseñados a partir de distintos tipos de secuencias y procesados en el receptor a partir de técnicas de AC y/o CC.

El presente capítulo presenta las siguientes contribuciones:

- Se realiza el diseño de un símbolo piloto a partir de pares de secuencias complementarias, de manera tal que se aprovechen sus adecuadas propiedades de correlación y la implementación eficiente del filtro acoplado de estas secuencias.

- Se presenta un método de sincronización que incluye una sincronización gruesa basada en técnicas de AC, y que, posteriormente, se complementa con una sincronización fina y estimación de canal a partir de la CC. 
Capítulo 3: Sincronización y estimación de canal en sistemas OFDM-PLC

- Se analizan los algoritmos propuestos bajo un modelo de canal PLC multicamino, en el que la amplitud de cada camino sigue una distribución de Rayleigh. A partir del análisis, se derivan umbrales prácticos para la sincronización que adaptan el sistema a la condiciones del canal y de relación señal a ruido.

Estas contribuciones forman parte del resultado de la búsqueda de una estrategia de sincronización y estimación de canal de baja complejidad, que resulte competitiva en desempeño respecto al procesamiento de símbolos pilotos diseñados a partir de otros tipos de secuencias existentes en la literatura.

\subsection{Descripción del sistema OFDM}

En esta sección se evalúa un sistema OFDM de paso banda para las comunicaciones PLC, con técnicas de sincronización y estimación de canal basadas en símbolos piloto. En la Fig. 3.1 se muestra un diagrama en bloques básico del sistema de comunicaciones. En el transmisor, los datos binarios son mapeados de acuerdo a una determinada constelación (BPSK, QPSK, M-QAM, etc.). Los datos mapeados se multiplexan en las $N$ entradas (subportadoras) del bloque IFFT, generando un símbolo OFDM al que se le agrega un prefijo cíclico de $N_{c p}$ muestras. Para el símbolo piloto únicamente, se agrega adicionalmente un sufijo cíclico de $N_{c s}$ muestras. Luego, las muestras complejas modulan la fase y cuadratura (I/Q) de la portadora de frecuencia $f_{c}$ antes de ser transmitidas a través del canal.

Por otro lado, en el receptor se realiza la demodulación I/Q a la frecuencia de portadora $f_{c}$ para obtener las muestras complejas de los símbolos OFDM en banda base. Se efectúa la etapa de sincronización de la ventana de la FFT con los símbolos recibidos, y se descarta el prefijo cíclico de los mismos. A partir de la operación FFT se obtienen los símbolos recibidos en cada subportadora, que se encuentran afectados por la respuesta del canal y posibles fuentes de ruido. Tras la etapa de estimación de canal, es posible compensar los efectos del canal por medio de un ecualizador en el dominio de la frecuencia (Frequency Domain Equalizer - FDE) antes de demapear los datos de las constelaciones correspondientes. Los bloques de contorno punteado de la Fig. 3.1 corresponden con las etapas de sincronización fina, y de la estimación del canal, a partir del procesamiento del símbolo piloto. Las muestras del símbolo piloto OFDM de banda base en el transmisor pueden expresarse como: 


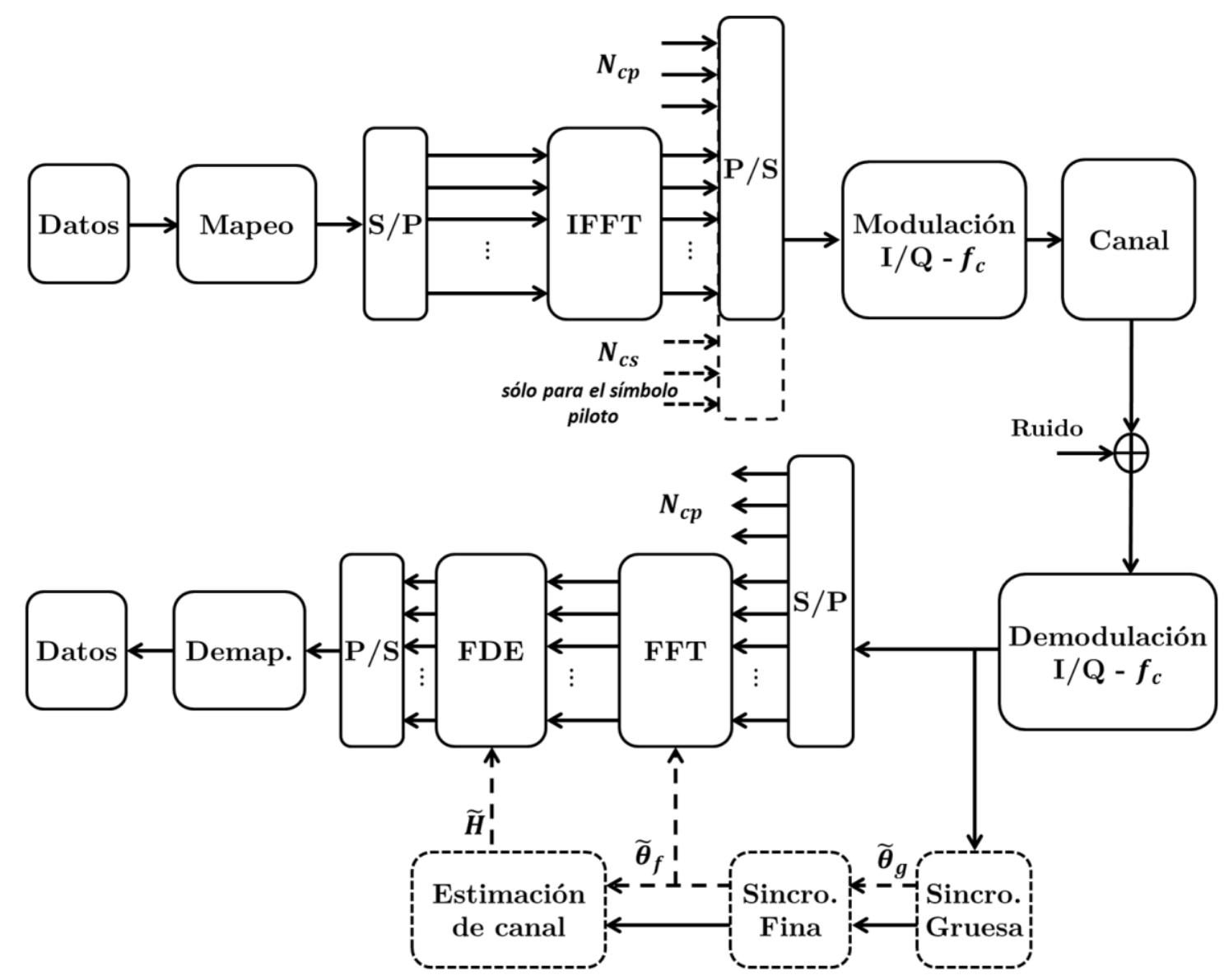

Fig. 3.1 Diagrama en bloques del transmisor y receptor del sistema OFDM-PLC.

$$
\mathrm{x}_{n}=\frac{1}{\sqrt{N}} \sum_{k=0}^{N-1} X_{k} \mathrm{e}^{\left(j \frac{2 \pi k n}{N}\right)},-N_{c p} \leq n \leq N-1+N_{c s}
$$

donde $N$ es el número total de subportadoras; $N_{c p}$ y $N_{c s}$ son las longitudes del prefijo y el sufijo cíclico, respectivamente; $X_{k}$ es el símbolo piloto complejo en la $k$-ésima subportadora en el dominio de la frecuencia; y $x_{n}$ es la muestra del símbolo piloto en el dominio del tiempo, en el instante $n$. Por otra parte, la correspondiente señal recibida $r_{n}$ puede representarse como:

$$
r_{n}=\mathrm{e}^{\left(j \frac{2 \pi \varepsilon n}{N}\right)} \sum_{l=0}^{L-1} h_{l} \sqrt{\mathrm{P}_{T}} \mathrm{x}_{n-\theta-l}+w_{n}
$$

donde $\varepsilon$ es el error en la frecuencia de portadora entre transmisor y receptor, normalizado por el espaciamiento entre subportadoras; $h_{l}$ representa el $l$-ésimo camino de la CIR; $L$ es la longitud del canal en muestras; $P_{T}$ es la potencia transmitida (sin pérdida de generalidad $E\left(\left|x_{n}^{2}\right|\right)=1$ ); $\theta$ es el error de sincronización expresado en muestras; y $w_{n}$ son las muestras complejas del ruido del canal PLC, con varianza 
$E\left(\left|w_{n}^{2}\right|\right)=\sigma_{w}{ }^{2}$. Considerando un vector de la CIR del canal $\mathbf{h}$, la SNR instantánea puede expresarse como $S N R=\|\mathbf{h}\|^{2} P_{T} / \sigma_{w}{ }^{2}$, donde $\|\mathbf{h}\|^{2}=\sum_{l=0}^{L-1}\left|h_{l}\right|^{2}$.

Los canales PLC presentan una selectividad de alta frecuencia debido al efecto multicamino y a diversas fuentes de ruido, lo que dificulta la fiabilidad del sistema de comunicaciones. Como se ha visto en el capítulo 2, el ruido en el entorno del PLC puede clasificarse en cinco categorías: ruido de fondo coloreado; ruido de banda estrecha; ruido impulsivo periódico, asincrónico a la frecuencia de la red eléctrica; ruido impulsivo periódico, sincrónico a la frecuencia de la red eléctrica; y ruido impulsivo asincrónico.

Además del ruido aditivo, el canal PLC también se ve afectado por los efectos multicamino y los desajustes de impedancia. En [Tan 2012] se ha incluido el modelo estadístico de canal PLC multicamino en el dominio del tiempo y la distribución de Rayleigh está determinada por la amplitud del primer camino de llegada. Además, diversos trabajos indican que las magnitudes de los otros caminos siguen una distribución de Rayleigh debido a la reflexión aleatoria de la señal [Tan 2012], [Liu 2008], [Rabie 2015]. Así, el modelo de canal PLC multicamino Rayleigh resulta un modelo flexible que permite sintetizar una respuesta en frecuencia del canal adecuada al ancho de banda de transmisión utilizado, ajustando la cantidad, amplitud promedio y el tiempo de llegada de cada camino. Las amplitudes de los coeficientes del canal $\left|h_{l}\right|$ son variables aleatorias independientes, con diferentes ganancias $\alpha_{l}$ según el Perfil de Potencia-Retardo (Power Delay Profile - PDP), con función de densidad de probabilidad expresada en (3.3). A su vez, el cuadrado de las amplitudes de los coeficientes del canal $\left|h_{l}\right|^{2}$ son entonces variables aleatorias exponencialmente distribuidas, con función de densidad de probabilidad dada por (3.4).

$$
\begin{gathered}
f_{\left|h_{l}\right|}(y)=\frac{y}{\alpha_{l}^{2}} e^{-\frac{y^{2}}{2 \alpha_{l}^{2}},} \quad y>0 \\
f_{\left|h_{l}\right|^{2}}(y)=\frac{e^{-\frac{y}{2 \alpha_{l}^{2}}}}{2 \alpha_{l}^{2}}, \quad y>0
\end{gathered}
$$

A lo largo de este capítulo, se evaluará un sistema OFDM-PLC con los siguientes parámetros (salvo que se indique lo contrario): la frecuencia portadora es $f_{c}=15 \mathrm{MHz}$, con un ancho de banda total de $25 \mathrm{MHz}$. El número de subportadoras es $N=1024$, y el espaciamiento de subportadoras es de $24,414 \mathrm{kHz}$, como en el estándar HomePlug 
Diseño de Estrategias de Sincronización y Estimación de Canal para la Mejora de Comunicaciones en Redes Inteligentes de Energía

Tabla 3.1 Perfil de ganancia promedio de los coeficientes para dos tipos de modelo de canal.

\begin{tabular}{|l|l|l|}
\hline & Canal A & Canal B \\
\hline $\boldsymbol{\alpha}_{\mathbf{0}}{ }^{2}$ & 0.5289 & 0.1530 \\
\hline $\boldsymbol{\alpha}_{\mathbf{1}}{ }^{2}$ & 0.2500 & 0.7095 \\
\hline $\boldsymbol{\alpha}_{\mathbf{2}}{ }^{2}$ & 0.1180 & 0.0888 \\
\hline $\boldsymbol{\alpha}_{\mathbf{3}}{ }^{2}$ & 0.0558 & 0.0213 \\
\hline $\boldsymbol{\alpha}_{\mathbf{4}}{ }^{2}$ & 0.0263 & 0.0155 \\
\hline $\boldsymbol{\alpha}_{\mathbf{5}}{ }^{2}$ & 0.0124 & 0.0090 \\
\hline $\boldsymbol{\alpha}_{\mathbf{6}}{ }^{2}$ & 0.0059 & 0.0022 \\
\hline $\boldsymbol{\alpha}_{\mathbf{7}}{ }^{2}$ & 0.0028 & 0.0007 \\
\hline
\end{tabular}

[Haniph 2013]. El prefijo y sufijo cíclico del símbolo piloto son de $N_{c p}=N_{c s}=128$ muestras cada uno. En este contexto, se consideran dos canales PLC multicamino, que contienen $L=8$ caminos, con un espaciamiento de 10 muestras entre la llegada de un camino y otro. La amplitud de cada coeficiente del canal $h_{l}$ sigue una distribución Rayleigh, la fase se encuentra uniformemente distribuida entre $[-\pi, \pi]$ y la potencia promedio de cada coeficiente se presentan en la Tabla 3.1.

\subsection{Sincronización en OFDM-PLC}

Como se ha visto en el capítulo 2, se han desarrollado varias técnicas de sincronización OFDM explotando algunas propiedades de correlación de símbolos piloto [Yong Soo 2010b], ya sea computando una AC de la señal recibida o mediante la CC entre la señal recibida y una copia de los símbolos piloto conocidos por el receptor. En las técnicas basadas en AC los símbolos piloto presentan partes simétricas, se define una métrica de tiempo normalizada en función de la simetría, y se obtiene el punto de sincronización a partir de su máximo [Park 2003], [Hlaing 2003], [Schmidl 1997]. Además, presentan una baja complejidad, ya que se utilizan fórmulas recursivas para su cómputo. Sin embargo, los métodos de AC dan lugar frecuentemente a un punto de sincronización de tiempo retardado en canales selectivos de alta frecuencia, y producen ISI si no se realiza un ajuste fino de la sincronización temporal. 
Por otra parte, en las técnicas de CC se utilizan como símbolos piloto secuencias con propiedades de correlación adecuadas y la señal recibida se compara con los símbolos piloto conocidos a través de un filtro acoplado. Si bien la complejidad que implica el cálculo de la $\mathrm{CC}$ es en general mucho mayor en comparación con las técnicas de AC, las técnicas de CC posibilitan una sincronización fina en base a la búsqueda del primer camino del canal [Wang 2009]. Además, después de la sincronización de tiempo, el símbolo piloto puede utilizarse para la estimación de la respuesta del canal PLC.

Se han propuesto diversas variantes para la construcción de símbolos piloto basados en secuencias para la sincronización y estimación de canal conjunta. En ellos, la estimación de la CIR puede obtenerse directamente de la salida del filtro acoplado utilizado en la etapa de sincronización fina. Algunas de las secuencias utilizadas para la construcción de símbolos piloto son: secuencias PN [Na 2018] [Wang 2008], Zadoff-Chu [Gul 2015], secuencias ortogonales modulables (Modulatable Orthogonal Sequence - MOS) [Wang 2009], o los pares de códigos de correlación nula (Zero Correlation Code - ZCC) [Qian 2018], [Qian 2017a].

Como la complejidad asociada con el cálculo de la $\mathrm{CC}$ es relativamente alta para estos símbolos piloto, se propone el diseño y procesamiento de un símbolo piloto novedoso para obtener una sincronización y estimación de canal de PLC de baja complejidad.

\subsubsection{Diseño del símbolo piloto}

Se propone la construcción de un símbolo piloto a partir de pares de secuencias complementarias, que tiene en cuenta una simetría adecuada para la sincronización gruesa y los algoritmos de correlación eficiente de los CPS, a fin de lograr una sincronización fina precisa y una estimación de la CIR. A partir de un par de secuencias a y $\mathbf{b}$ de longitud $N / 4$, la construcción del símbolo piloto de longitud $N$ en el dominio del tiempo se basa en concatenar el par de secuencias complementarias de la siguiente manera:

$$
\mathbf{x}^{\prime}=\left[\begin{array}{llll}
\mathbf{a} & \mathbf{b} & -\mathbf{a} & \mathbf{b}
\end{array}\right]
$$

Luego de agregar el prefijo y sufijo cíclico, el símbolo piloto extendido $\mathbf{x}$ puede escribirse como:

$$
\mathbf{x}=\left[\begin{array}{llllll}
\mathbf{c p} & \mathbf{a} & \mathbf{b} & -\mathbf{a} & \mathbf{b} & \mathbf{c s}
\end{array}\right]
$$


Diseño de Estrategias de Sincronización y Estimación de Canal para la Mejora de Comunicaciones en Redes Inteligentes de Energía

Donde cp y cs son el prefijo y sufijo cíclico, respectivamente, cuyas muestras pueden expresarse como:

$$
\begin{gathered}
\mathrm{cp}_{n}=\mathrm{b}_{n+N / 4-N_{c p}}, \quad n=0,1, \ldots, N_{c p}-1 \\
\mathrm{cs}_{n}=\mathrm{a}_{n}, \quad n=0,1, \ldots, N_{c s}-1
\end{gathered}
$$

La correlación $c_{n}^{\mathbf{x}, \mathbf{x} \prime}$ entre el símbolo piloto extendido $\mathbf{x}$ y el símbolo sin extender $\mathbf{x}^{\prime}$ es

$$
\begin{aligned}
c_{n}^{\mathbf{x}, \mathbf{x}^{\prime}}= & \sum_{l=0}^{N-1} \mathrm{x}_{l}^{\prime} \cdot \mathrm{x}_{n+l+N_{c p},}-N_{c p} \leq n \leq N_{c s} \\
c_{n}^{\mathbf{x}, \mathbf{x}^{\prime}}= & 2 \sum_{l=0}^{N / 4+n-1}\left(\mathrm{a}_{l} \cdot \mathrm{a}_{l-n}+\mathrm{b}_{l} \cdot \mathrm{b}_{l-n}\right) \\
& +\sum_{l=0}^{-n-1}\left(\mathrm{~b}_{l+n+\frac{N}{4}} \cdot \mathrm{a}_{l}-\mathrm{b}_{l+n+\frac{N}{4}} \cdot \mathrm{a}_{l}+\mathrm{a}_{l+n+\frac{N}{4}} \cdot \mathrm{b}_{l}-\mathrm{a}_{l+n+\frac{N}{4}} \cdot \mathrm{b}_{l}\right), \\
c_{n}^{\mathbf{x}, \mathbf{x}^{\prime}}= & 2 \sum_{l=n}^{N / 4-1}\left(\mathrm{a}_{l} \cdot \mathrm{a}_{l-n}+\mathrm{b}_{l} \cdot \mathrm{b}_{l-n}\right) \\
& +\sum_{l=0}^{n-1}\left(\mathrm{a}_{l-n+\frac{N}{4}} \cdot \mathrm{b}_{l}-\mathrm{a}_{l+n+\frac{N}{4}} \cdot \mathrm{b}_{l}+\mathrm{b}_{l-n+\frac{N}{4}} \cdot \mathrm{a}_{l}-\mathrm{b}_{l-n+\frac{N}{4}} \cdot \mathrm{a}_{l}\right), \\
&
\end{aligned}
$$

Dado que la suma de las ACS de un par de secuencias complementarias es cero para cualquier desplazamiento no nulo, se puede concluir que debido a la inserción del prefijo y el sufijo cíclicos, la función de correlación $c_{n}^{\mathbf{x}, \mathbf{x}^{\prime}}$ presenta una zona de correlación nula en la vecindad de $n=0$, definida por:

$$
\begin{gathered}
c_{n}^{\mathbf{x}, \mathbf{x} \prime}=N, \quad n=0 \\
c_{n}^{\mathbf{x}, \mathbf{x} \prime}=0, \quad-N_{c p} \leq n \leq N_{c s}, n \neq 0
\end{gathered}
$$

Cabe señalar que la máxima longitud alcanzable de la zona de correlación ideal es de $N / 2$ muestras, lo que implica que $N c p, N c s \leq N / 4$. Normalmente, la longitud del prefijo cíclico es una fracción menor de la longitud del símbolo en los sistemas OFDM bien diseñados, para evitar una excesiva pérdida de eficiencia espectral, y debe ser mayor que la dispersión máxima del canal $(N c p>L)$, lo que da como resultado una zona libre ISI de $N c p-L+1$ muestras. En la Fig. 3.2 se presenta a la izquierda la 
Capítulo 3: Sincronización y estimación de canal en sistemas OFDM-PLC
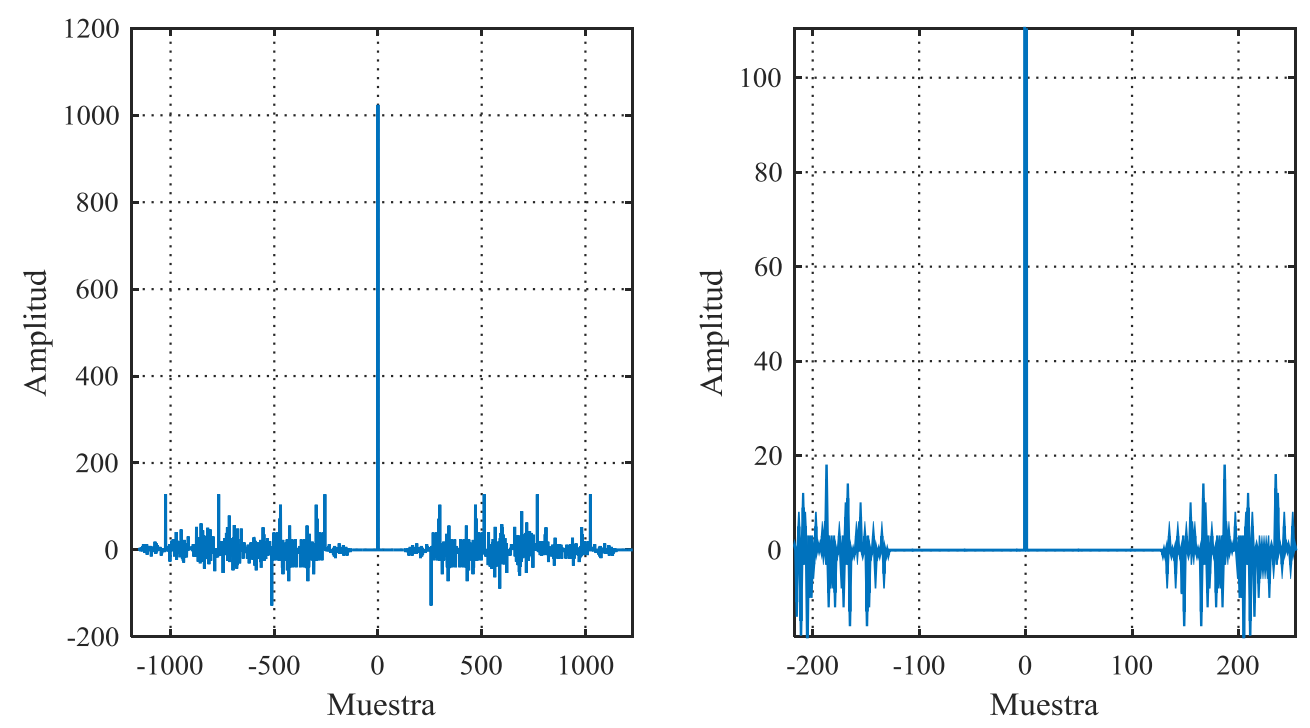

Fig. 3.2 Función de correlación cruzada entre el símbolo extendido y el filtro acoplado del símbolo sin extender.

correlación cruzada $c_{n}^{\mathbf{x}, \mathbf{x} \prime}$ para $N=1024, N_{c p}=N_{c s}=512$, y puede verificarse el valor del máximo y la longitud de la zona de correlación nula. En la parte derecha se presenta simplemente un zoom de $c_{n}^{\mathbf{x}, \mathbf{x} \prime}$ para una mejor visualización de la zona de correlación nula.

\subsubsection{Sincronización gruesa}

Como el símbolo piloto propuesto tiene una estructura repetitiva, se puede implementar una sincronización gruesa basada en la AC [Hlaing 2003]. Entonces, se define una métrica de sincronización como:

$$
m_{n}=\frac{2 \cdot \operatorname{Re}\left(p_{n}\right)}{e_{n}}
$$

donde $p_{n}$ es la autocorrelación de cuatro segmentos de la señal recibida $r_{n}$, según la simetría en (3.5), esto es, buscando $\mathbf{a} \cdot \mathbf{a}^{*}+\mathbf{b} \cdot \mathbf{b}^{*} ; \operatorname{Re}(\cdot)$ representa la parte real de su argumento; y $e_{n}$ representa la energía en la ventana de $N$ muestras correspondiente a la longitud del símbolo piloto. La AC $p_{n}$ puede definirse como (3.13), mientras que la energía $e_{n}$ se presenta en (3.14).

$$
p_{n}=\sum_{m=0}^{\frac{N}{4}-1}-r_{n+m} \cdot r_{n+m+N / 2}^{*}+r_{n+m+N / 4} \cdot r_{n+m+3 N / 4}^{*}
$$


Diseño de Estrategias de Sincronización y Estimación de Canal para la Mejora de Comunicaciones en Redes Inteligentes de Energía

$$
e_{n}=\sum_{m=0}^{\mathrm{N}-1} r_{n+m} \cdot r_{n+m}^{*}
$$

donde $r_{n}^{*}$ es el valor complejo conjugado de $r_{n}$. El punto de sincronización gruesa $n_{g}$ se obtiene a partir de la ubicación del máximo de la métrica de sincronización gruesa $m_{n}$ como (3.15):

$$
n_{g}=\underset{n}{\arg \max }\left(m_{n}\right)
$$

Así, el error de sincronismo de (3.2) está dado por $\theta=n_{\text {ópt }}-n_{g}$. En un entorno práctico, la búsqueda del máximo de la métrica debe efectuarse sobre una cantidad finita de muestras, y luego de una detección primaria de la llegada del símbolo piloto. En esta sección se supone que la detección primaria fue satisfactoria, y se incluye una ventana de $3 N$ muestras procesadas de la señal recibida para la búsqueda del máximo, que contienen muestras anteriores y posteriores (símbolos de datos) al símbolo piloto.

Por otra parte, el CFO podría estimarse a partir de la métrica de sincronización basada en AC, como se describe en [Schmidl 1997], y puede compensarse antes de las etapas de sincronización fina y estimación del canal. No obstante, para los rangos prácticos de errores de frecuencia en los sistemas PLC, en el que la frecuencia de portadora es baja respecto al ancho de banda utilizado, y menor a las frecuencias típicas de sistemas inalámbricos, el efecto del CFO es insignificante [Kim 2011]. La señal recibida $r_{n}$ en la región libre de ISI puede reescribirse como:

$$
\begin{gathered}
r_{n}=\sum_{l=0}^{L-1} h_{l} \sqrt{\mathrm{P}_{T}} \mathrm{x}_{n-l}+w_{n} \\
s_{n}=\sum_{l=0}^{L-1} h_{l} \sqrt{\mathrm{P}_{T}} \mathrm{x}_{n-l}
\end{gathered}
$$

donde $s_{n}$ es la parte útil de la señal; $P_{T}$ es la potencia transmitida; $h_{l}$ son los coeficientes del canal PLC; y $w_{n}$ son las muestras de ruido complejo del canal PLC. En muchos casos, para lograr una sincronización fina adecuada debe estimarse la condición de $S N R$ instantánea [Wang 2009] [Wang 2008]. Se puede obtener una estimación simple de la $S N R$ a partir de la métrica de sincronización $m_{n}$ en el instante de sincronización gruesa $n_{g}$, como se presenta en [Schmidl 1997]: 
Capítulo 3: Sincronización y estimación de canal en sistemas OFDM-PLC

$$
\widetilde{S N R}=\frac{m_{n_{g}}}{1-m_{n_{g}}}
$$

donde $m_{n_{g}}$ es el valor de la métrica de sincronización de (3.12) en la muestra de sincronización gruesa $n_{g}$.

\subsubsection{Correlación cruzada normalizada}

Luego de la sincronización gruesa, la CC entre la señal recibida $r_{n}$ y el símbolo piloto $\mathbf{x}^{\prime}$ se utiliza para la sincronización fina y estimación del canal. A continuación se propone y analiza una técnica de sincronización y estimación de canal teniendo en cuenta el símbolo piloto propuesto. Sin embargo, el análisis es válido para cualquier símbolo piloto cuya $\mathrm{CC}$ presente una zona de correlación nula, como por ejemplo, aquellos construidos a partir de otros tipos de secuencias de amplitud constante, como ZadoffChu, secuencias LS, o secuencias ZCZ [García 2015]. Además, la sincronización fina se basa en una selección de un umbral, cuyo valor depende de las condiciones instantáneas del canal, por lo que el análisis se basa en considerar la condición SNR instantánea como parámetro.

Para la etapa de sincronización fina, se propone computar una CC normalizada. De esta manera, el desempeño de la sincronización depende de la condición $S N R$, pero no se ve influenciado por los niveles individuales de la señal útil y el ruido presentes en la señal recibida, y no se requiere información sobre la potencia transmitida $P_{T}$. Así, se propone como factor de normalización la energía de la señal recibida $e_{n}$ en (3.14). Entonces, la expresión CC normalizada propuesta viene dada por:

$$
c_{n}^{\prime}=\frac{\left|c_{n}^{\mathbf{r}, \mathbf{x}^{\prime}}\right|^{2}}{N \cdot e_{n}}
$$

Donde $N$ es la longitud del símbolo piloto, y los términos de energía, de acuerdo a (3.14) y (3.17) se pueden escribir como:

$$
e_{n}=\sum_{m=0}^{N-1}\left(\left|s_{n+m}^{2}\right|+2 \cdot \operatorname{Re}\left\{s_{n+m} \cdot \mathrm{w}_{n+m}^{*}\right\}+\left|w_{n+m}\right|^{2}\right)
$$

El ruido del canal PLC está compuesto por muestras correladas y no gaussianas, debido a los eventos de ruido impulsivo. Por esto mismo, el nivel de ruido presente en los símbolos recibidos puede ser significativamente distinto entre una transmisión y otra, dependiendo de la ocurrencia o no de ruido impulsivo. En las simulaciones que se 
Diseño de Estrategias de Sincronización y Estimación de Canal para la Mejora de Comunicaciones en Redes Inteligentes de Energía

realizan en este capítulo, las muestras de ruido se sintetizan a partir del generador de ruido del PLC presentado en [Prasad 2016] [Prasad 2019], y se selecciona la peor condición entre las opciones disponibles. Para garantizar la condición de $S N R$, se normalizan tanto las muestras de señal útil como las de ruido para cada realización. Ante este escenario, a medida que el parámetro $N$ aumenta, el segundo y tercer término en (3.20) se convierten en gaussianos por el Teorema Central de Límite (TCL). Esta aproximación es factible porque, en los sistemas OFDM para PLC, $N$ suele ser elevado y, por lo tanto, las muestras de ruido del canal PLC después de la combinación de suficientes términos son aproximadamente Gaussianas, independientes e idénticamente distribuidas (i.i.d.), por el TCL [Chen 2013] [Guzel 2011] [Meng 2005]. Entonces, la energía en la región libre de ISI puede considerarse como una variable Gaussiana con media y varianza:

$$
e_{n} \sim \mathrm{N}\left(N\left({\sigma_{w}}^{2}+\|\boldsymbol{h}\|^{2} P_{T}\right), N{\sigma_{w}}^{4}+2 N{\sigma_{w}}^{2}\|\boldsymbol{h}\|^{2} P_{T}\right)
$$

De acuerdo a (3.2), las muestras de la CC en la zona libre de ISI puede expresarse como:

$$
\begin{gathered}
c_{n}^{\mathbf{r}, \mathbf{x}^{\prime}}=\sum_{m=0}^{N-1} \sum_{l=0}^{L-1} h_{l} \sqrt{\mathrm{P}_{\mathrm{T}}} \mathrm{x}_{n+m-l} \mathrm{x}_{m}^{\prime *}+\sum_{m=0}^{N-1} w_{n+m}{\mathrm{x}_{m}^{\prime *}}^{*} \\
n_{\mathrm{o} p t}-N_{c p}+L \leq n \leq n_{\mathrm{o} p t}+N_{c s}
\end{gathered}
$$

donde $n_{\text {ópt }}$ es la muestra correspondiente al instante óptimo de sincronismo, $h_{l}$ es el $l$ ésimo camino de la CIR del canal PLC; y $L$ es el máximo retardo del canal en las muestras. La CC del símbolo piloto propuesto exhibe una zona de correlación nula en las muestras previas al instante de sincronización óptimo, por lo que la salida del filtro acoplado resulta del filtrado de las muestras de ruido, y puede expresarse como:

$$
c_{n}^{\mathbf{r}, \mathbf{x}^{\prime}}=\sum_{m=0}^{N-1} w_{n+m} \mathbf{x}_{m}^{\prime *}, \quad n_{\text {ópt }}-N_{c p}+L-1 \leq n<n_{\text {ópt }}
$$

Como los elementos del correlador $x_{m}^{\prime *}$ tienen amplitud constante y $N$ es alto, la combinación lineal de muestras de ruido permite aplicar el TCL, y la distribución de (3.23) puede ser aproximada por una variable compleja aleatoria gaussiana con media cero y varianza $N \sigma_{w}{ }^{2}$. El cuadrado es entonces una variable aleatoria chi-cuadrado multiplicada por $N \sigma_{w}{ }^{2}$. Por otra parte, los términos de $e_{n}$ en (3.20) tienen una media 
mucho mayor que su desvío estándar [Schmidl 1997], por lo que la CC normalizada en las muestras previas al instante de sincronismo óptimo $n_{\text {ant }}$ puede aproximarse como una distribución chi-cuadrado de un grado de libertad ponderada, cuyas funciones de densidad y distribución acumulada, $f_{c^{\prime}{ }_{n_{\text {ant }}}}$ y $c d f_{c^{\prime}{ }_{n_{\text {ant }}}}$, se describen como:

$$
\begin{aligned}
& f_{c^{\prime}{ }_{n_{\text {ant }}}}(y)=\sqrt{\frac{N(1+S N R)}{2 \pi y}} e^{-\frac{N(1+S N R) y}{2}}, y>0, \quad n_{\mathrm{o} p t}-N_{c p}+L-1 \leq n<n_{\text {ópt }} \\
& c d f_{c^{\prime}{ }^{\prime}{ }_{\text {ant }}}(y)=\operatorname{erf}\left(\sqrt{\frac{y}{2 N(1+S N R)}}\right), y>0, \quad n_{\text {o } p t}-N_{c p}+L-1 \leq n<n_{\text {ó } p t}
\end{aligned}
$$

donde $\operatorname{erf}(\cdot)$ indica la función de error.

Los resultados teóricos de la distribución CC normalizada en muestras anteriores al instante de sincronismo óptimo pertenecientes a la región libre de ISI se presentan en la Fig. 3.3, a partir de evaluar la CDF de (3.25) y la CDF empírica de las muestras de simulación. Cabe señalar que la distribución es independiente del canal considerado, y los valores disminuyen a medida que el valor de $S N R$ aumenta, como era esperado. Puede además observarse que los valores no se ajustan perfectamente a la distribución propuesta, y esto es debido a que las muestras de ruido del PLC están compuestas por varios tipos de ruidos coloreados e impulsivos. No obstante, se verá próximamente que el umbral que se utilizará para la sincronización fina suele ser más alto que las muestras de la CC normalizada en las muestras previas al instante de sincronismo óptimo, por lo

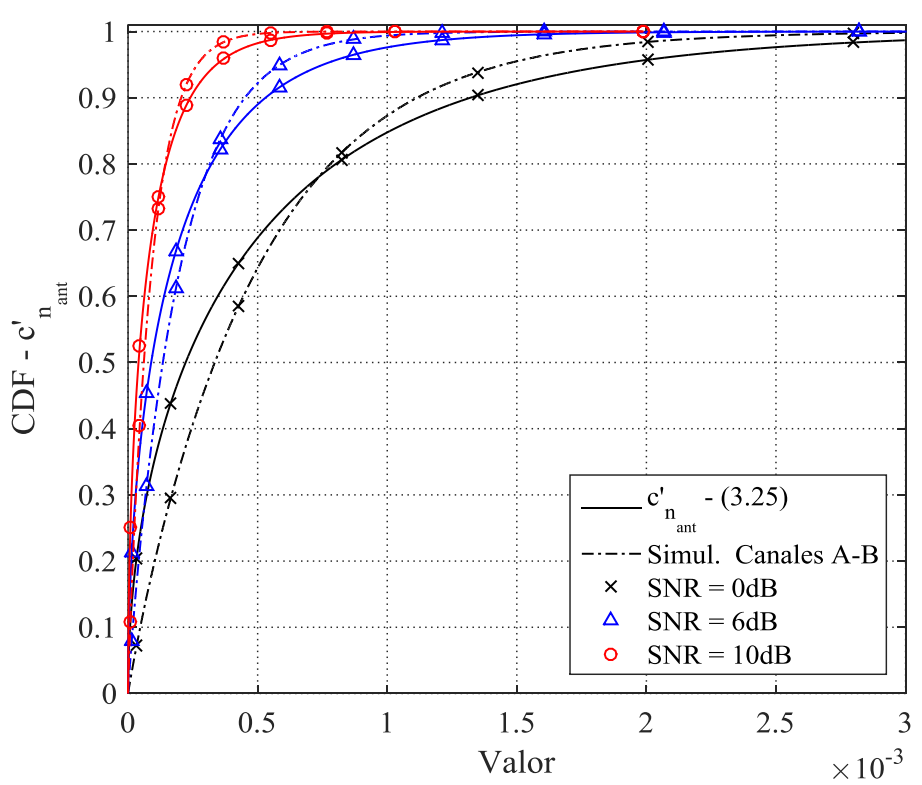

Fig. 3.3 CDF teórica y empírica de la correlación cruzada normalizada en las muestras previas al instante de sincronismo óptimo. 
Diseño de Estrategias de Sincronización y Estimación de Canal para la Mejora de Comunicaciones en Redes Inteligentes de Energía

que la aproximación será útil.

Por otro lado, gracias a la zona de correlación nula del símbolo piloto propuesto, la CC normalizada en el instante de sincronismo óptimo $c_{n_{\text {ópt }}}^{\mathbf{r}, \mathbf{x}^{\prime}}$ puede expresarse como (3.26), considerando el canal PLC selectivo en frecuencia de (3.22).

$$
c_{n_{\mathrm{o} p t}}^{\mathbf{r}, \mathbf{x} \mathbf{x}^{\prime}}=\sum_{m=0}^{N-1} h_{0} \sqrt{\mathrm{P}_{\mathrm{T}}} \mathrm{x}_{m}^{\prime}{ }_{m}^{\prime \prime}{ }_{m}^{\prime *}+\sum_{m=0}^{N-1} w_{m} \mathrm{x}_{m}^{\prime *}=N h_{0} \sqrt{\mathrm{P}_{\mathrm{T}}}+\sum_{m=0}^{N-1} w_{m} \mathrm{x}_{m}^{\prime *}
$$

El segundo término puede considerarse como una suma en que las $N$ muestras de ruido se suman aleatoriamente, mientras que el primer término resulta en el primer coeficiente del canal con una ganancia de $N$, que es la ganancia del código utilizado. Por lo tanto, resulta factible aproximar la $\mathrm{CC} c_{n_{\mathrm{o} p t}}^{\mathbf{r}, \mathbf{x}^{\prime}}$ sólo por su primer término para valores prácticos de $S N R$ y $N$. Como la media del denominador de la CC normalizada es mucho mayor que el desvío estándar, entonces la CC normalizada en $n=n_{\text {ópt }}$ puede ser aproximada por:

$$
{c^{\prime}}_{n_{\mathrm{o} p t}} \approx \frac{N^{2}\left|h_{0}\right|^{2} P_{T}}{N^{2}\left(\sigma_{w}^{2}+\|\boldsymbol{h}\|^{2} \cdot P_{T}\right)}
$$

Definiendo la variable aleatoria $\lambda_{0}$ como la ganancia del primer camino del canal normalizada por la ganancia total del canal, como se muestra en (3.28), la CC normalizada se puede escribir como (3.29).

$$
\begin{gathered}
\lambda_{0}=\frac{\left|h_{0}\right|^{2}}{\|\boldsymbol{h}\|^{2}}=\frac{\left|h_{0}\right|^{2}}{\sum_{l=0}^{L-1}\left|h_{l}\right|^{2}} \\
c_{n_{\text {o } p t}}^{\prime} \approx \frac{\lambda_{0} \cdot S N R}{(S N R+1)}
\end{gathered}
$$

Para obtener la distribución de $\lambda_{0}$, es importante tener en cuenta que el numerador y denominador de (3.28) son estadísticamente dependientes, por lo que $\lambda_{0}$ puede reescribirse como:

$$
\lambda_{0}=\frac{1}{1+\frac{\sum_{l=1}^{L-1}\left|h_{l}\right|^{2}}{\left|h_{0}\right|^{2}}}
$$

El denominador de (3.30) incluye la suma de variables aleatorias distribuidas exponencialmente e independientes, y ponderadas por la ganancia promedio de cada 
camino. Por lo tanto, definiendo la variable aleatoria $V_{3}=\sum_{l=1}^{L-1}\left|h_{l}\right|^{2}$, la función densidad de probabilidad $f_{V_{3}}(y)$ puede obtenerse como se indica en [Ali 1982] y resulta

$$
f_{V_{3}}(y)=\sum_{l=1}^{L-1} \frac{\alpha_{l}^{2(L-3)} e^{-\frac{y}{2 \alpha_{l}^{2}}}}{2 \prod_{\substack{L-1 \\ j=1}}^{L-1}\left(\alpha_{l}^{2}-\alpha_{j}^{2}\right)}, \quad y>0
$$

A su vez, la PDF del cociente entre la variable $V_{3}$ y $\left|h_{0}\right|^{2}$, que es una variable aleatoria distribuida exponencialmente de acuerdo a (3.4), puede obtenerse a partir de (3.32) [Walpole 2007], obteniendo así (3.33):

$$
\begin{gathered}
f_{V_{3} /\left|h_{0}\right|^{2}}(y)=\int_{0}^{+\infty} z f_{V_{3}}(y z) f_{\left|h_{0}\right|^{2}}(z) d z, \quad y>0 \\
f_{\frac{V_{3}}{\left|h_{0}\right|^{2}}}(y)=\sum_{l=1}^{L-1} \frac{h_{l}^{2(L-3)}}{4 h_{0}^{2} \prod_{\substack{j \neq l \\
j=1}}^{L-1}\left(h_{l}^{2}-h_{j}^{2}\right)} \cdot \frac{1}{\left(\frac{y}{2 h_{l}^{2}}+\frac{1}{2 h_{0}^{2}}\right)^{2}}, y>0
\end{gathered}
$$

Finalmente, después de sumar 1, invertir y escalar el resultado de la variable aleatoria $V_{3} /\left|h_{0}\right|^{2}$ con $K=S N R /(S N R+1)$, la distribución de $c_{n_{\text {ópt }}}^{\prime}$ puede obtenerse a partir de

$$
f_{c^{\prime} n_{\mathrm{o} p t}}(y)=\frac{K}{y^{2}} f_{V_{3} /\left|h_{0}\right|^{2}}\left(\frac{K}{y}-1\right), \quad K>y>0
$$

De esta manera, se pueden obtener la PDF y luego la CDF, $f_{c^{\prime}{ }_{n_{\mathrm{o}} p t}}$ y $c d f_{c^{\prime}{ }_{n_{\text {ó }} t}}$, de la correlación cruzada normalizada en el punto de óptimo de sincronismo para el canal Rayleigh multicamino en (3.35) y (3.36), respectivamente.

$$
\begin{aligned}
& f_{c^{\prime}{ }^{\prime}{ }_{\mathrm{o} p t}}(y) \approx \sum_{l=1}^{L-1} \frac{\alpha_{l}^{2(L-3)}}{4 \alpha_{0}^{2} \prod_{\substack{j \neq l \\
j=1}}^{L-1}\left(\alpha_{l}^{2}-\alpha_{j}^{2}\right)} \cdot \frac{S N R+1}{S N R\left(\frac{1-\frac{S N R+1}{S N R} y}{2 \alpha_{l}^{2}}+\frac{\frac{S N R+1}{S N R} y}{2 \alpha_{0}^{2}}\right)^{2}} \\
& 0<y<S N R /(S N R+1) \\
& c d f_{C^{\prime} n_{\mathrm{o} p t}}(y) \approx \sum_{l=1}^{L-1} \frac{\alpha_{l}^{2(L-1)}}{\alpha_{0}^{2} \prod_{\substack{j \neq l \\
j=1}}^{L-1}\left(\alpha_{l}^{2}-\alpha_{j}^{2}\right)} \cdot \frac{(S N R+1) y}{S N R\left[1-\left(1-\frac{\alpha_{l}^{2}}{\alpha_{0}^{2}}\right) \frac{(S N R+1) y}{S N R}\right]} \\
& 0<y<S N R /(S N R+1)
\end{aligned}
$$

El análisis de la CDF de la CC normalizada en el instante de sincronismo óptimo para los canales Rayleigh multicamino en (3.36) se confirma mediante simulaciones en la 
Diseño de Estrategias de Sincronización y Estimación de Canal para la Mejora de Comunicaciones en Redes Inteligentes de Energía

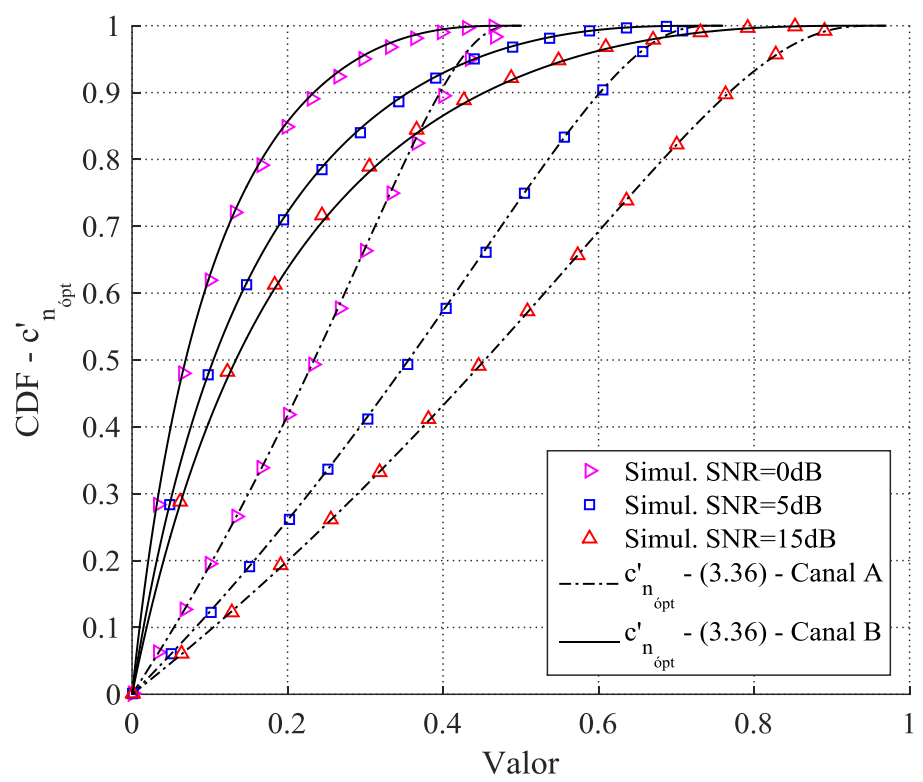

Fig. 3.4 CDF teórica y empírica de la correlación cruzada normalizada en el instante de sincronismo óptimo.

Fig. 3.4. Se puede observar que los valores están acotados entre 0 y 1 , y se hacen mayores a medida que aumenta el nivel de $S N R$. Además, los valores resultan mayores en el caso del primer canal, debido a que la relación entre la potencia promedio del primer camino y la potencia de la suma de todos los caminos es mayor que para el segundo canal. Para valores bajos de $S N R$, el resultado de simulación para la CDF difiere ligeramente del resultado analítico. Esto ocurre debido a la obviedad de los términos de ruido filtrados por el correlador en (3.26).

\subsubsection{Sincronización fina}

El proceso de sincronización fina en el caso de un canal sin multicamino (sin selectividad en frecuencia), es en general simple porque la mejor opción sería encontrar el máximo de la CC normalizada. Sin embargo, en los canales multicamino, es necesario realizar una búsqueda del primer camino del canal, para evitar ISI en los símbolos de datos y lograr una estimación de la CIR adecuada en el esquema conjunto, incluso cuando el primer camino del canal no tiene la mayor amplitud. La detección suele realizarse a partir de técnicas de umbralización, y la ubicación del instante de sincronización fina $n_{f}$ corresponderá a la misma muestra, o bien a una muestra anterior al instante resultante de la sincronización gruesa $n_{f}$ descrito anteriormente. Definiendo una ventana de búsqueda de $D$ muestras, con $D<N_{c p}-L+1$, entonces el algoritmo de sincronización fina propuesto se define como: 


$$
\begin{aligned}
& n_{f}=n_{g} \\
& \text { while } \underset{n \in\{0,1, \ldots, D\}}{\arg \max }\left\{n \mid c^{\prime}{ }_{n_{f}-n} \geq \gamma\right\}>0 \\
& n_{f}=n_{f-n} \\
& \text { end }
\end{aligned}
$$

El valor umbral $\gamma$ tiene un gran impacto en el desempeño de la sincronización, debido a que un umbral demasiado alto implicará una alta probabilidad de no detectar el primer camino del canal, mientras que un umbral bajo causará que se detecten muestras ruidosas de la zona de ISI en las que no hay caminos del canal. En esa línea, es obligatorio obtener un valor adecuado del umbral que maximice algunas condiciones de desempeño. En este trabajo se considera el umbral que maximiza la probabilidad de sincronización perfecta, es decir, la probabilidad de seleccionar el primer camino del canal como punto de sincronización. Según el esquema de sincronización fina propuesto, la probabilidad de sincronización perfecta es:

$$
P(\gamma)=\left(\int_{0}^{\gamma} f_{c_{n_{\text {ant }}}^{\prime}}(x) \mathrm{d} x\right)^{D} \cdot\left(1-\int_{0}^{\gamma} f_{c_{n_{\mathrm{o} p t}}^{\prime}}(x) \mathrm{d} x\right),-D \leq n<0
$$

El umbral $\gamma$ que maximiza la probabilidad de sincronización perfecta se obtiene a partir de $d P(\gamma) / d \gamma=0$, es decir:

$$
D f_{c_{n_{\text {ant }}}^{\prime}}(\gamma)\left(1-c d f_{c^{\prime} n_{\mathrm{o} p t}}(\gamma)\right)-\left(c d f_{c^{\prime} n_{\text {ant }}}(\gamma)\right) f_{c_{n_{\mathrm{o}} p t}^{\prime}}(\gamma)=0
$$

El umbral que maximiza (3.38) puede obtenerse a partir de algún método numérico de resolución de ecuaciones no lineales. Sin embargo, para obtener una expresión en forma cerrada del umbral, se definen algunas aproximaciones que se basan en la suposición de que los valores prácticos de $N$ y $S N R$ conducen a pequeños valores de la correlación cruzada normalizada en las zona de correlación nula, antes del instante óptimo de sincronismo. En esa línea, el umbral óptimo $\gamma$ será relativamente alto en comparación con estas muestras. Teniendo esto en cuenta, se proponen las siguientes aproximaciones:

$$
\begin{aligned}
& c d f_{c^{\prime} n_{\text {ant }}}(\gamma) \approx 1 \\
& f_{c_{n_{\text {ant }}}^{\prime}}(\gamma) \approx \frac{N(1+S N R)}{4 \sqrt{2 \pi}} e^{-\frac{N(1+S N R) \gamma}{2}}
\end{aligned}
$$


Diseño de Estrategias de Sincronización y Estimación de Canal para la Mejora de Comunicaciones en Redes Inteligentes de Energía

Por otro lado, el umbral será pequeño con respecto a los valores de la CC normalizada en el instante óptimo de sincronismo por lo que puede asumirse:

$$
\left(1-\int_{0}^{\gamma} f_{c_{n_{\text {opt }}}^{\prime}}(x) \mathrm{d} x\right) \approx 1
$$

Por último, parte del denominador de (3.35), para pequeños valores de la variable $y$, puede aproximarse como:

$$
\left(\frac{1-\frac{S N R+1}{S N R} y}{2 \alpha_{l}^{2}}+\frac{\frac{S N R+1}{S N R} y}{2 \alpha_{0}^{2}}\right)^{2} \approx \frac{1}{4 \alpha_{l}^{4}}
$$

Con esta simplificación, entonces (3.35) resulta:

$$
f_{C^{\prime} n_{\mathrm{o} p t}}(\gamma) \approx \sum_{l=1}^{L-1} \frac{\alpha_{l}^{2(L-1)}}{\alpha_{0}^{2} \prod_{\substack{j \neq l \\ j=1}}^{L-1}\left(\alpha_{l}^{2}-\alpha_{j}^{2}\right)} \cdot \frac{S N R+1}{S N R}=\frac{\sum_{l=0}^{L-1} \alpha_{l}^{2}-\alpha_{0}^{2}}{\alpha_{0}^{2}} \frac{S N R+1}{S N R}
$$

Considerando las aproximaciones enumeradas, entonces (3.38) resulta en (3.44), y el umbral subóptimo se presenta en (3.45).

$$
\begin{gathered}
D \frac{N(1+S N R)}{4 \sqrt{2 \pi}} \cdot e^{-\frac{N(1+S N R) \gamma}{2}}-\frac{\sum_{l=0}^{L-1} \alpha_{l}^{2}-\alpha_{0}^{2}}{\alpha_{0}^{2}} \cdot \frac{S N R+1}{S N R}=0 \\
\gamma_{\text {Sub }_{1}}=\frac{2}{N(1+S N R)} \ln \left(\frac{D \cdot N \cdot S N R}{4 \sqrt{2 \pi}} \frac{\alpha_{0}^{\prime 2}}{\left(1-\alpha_{0}^{\prime 2}\right)}\right)
\end{gathered}
$$

Donde $\alpha_{0}^{\prime 2}$ es la ganancia promedio del primer camino del canal, normalizada por la ganancia promedio del canal $\|\mathbf{h}\|^{2}=\sum_{l=0}^{L-1} \alpha_{l}^{2}$. Una variante simplificada del umbral, que evita la estimación de las características estadísticas del canal es la que se obtiene a partir de fijar un valor para la $\alpha_{0}^{\prime 2}$. Al igual que en [Wang 2009], si se considera $\alpha_{0}^{\prime 2}=0.5$, entonces el umbral subóptimo se puede escribir como:

$$
\gamma_{s u b_{2}}=\frac{2}{N(1+S N R)} \ln \left(\frac{D \cdot N \cdot S N R}{4 \sqrt{2 \pi}}\right)
$$

La probabilidad de sincronización perfecta de (3.37) se evalúa en la Fig. 3.5 para los canales A y B, a izquierda y derecha respectivamente. Se evalúan los resultados bajo tres umbrales $\gamma$ diferentes: el cómputo del umbral ideal denominado $\gamma_{(3.38)}$, que se obtiene a partir de resolver (3.38) a partir de un método numérico; y los umbrales subóptimos propuestos $\gamma_{s u b_{1}}$ y $\gamma_{s u b_{2}}$. El cálculo de $\gamma_{(3.38)}$ se realizó a partir del método 

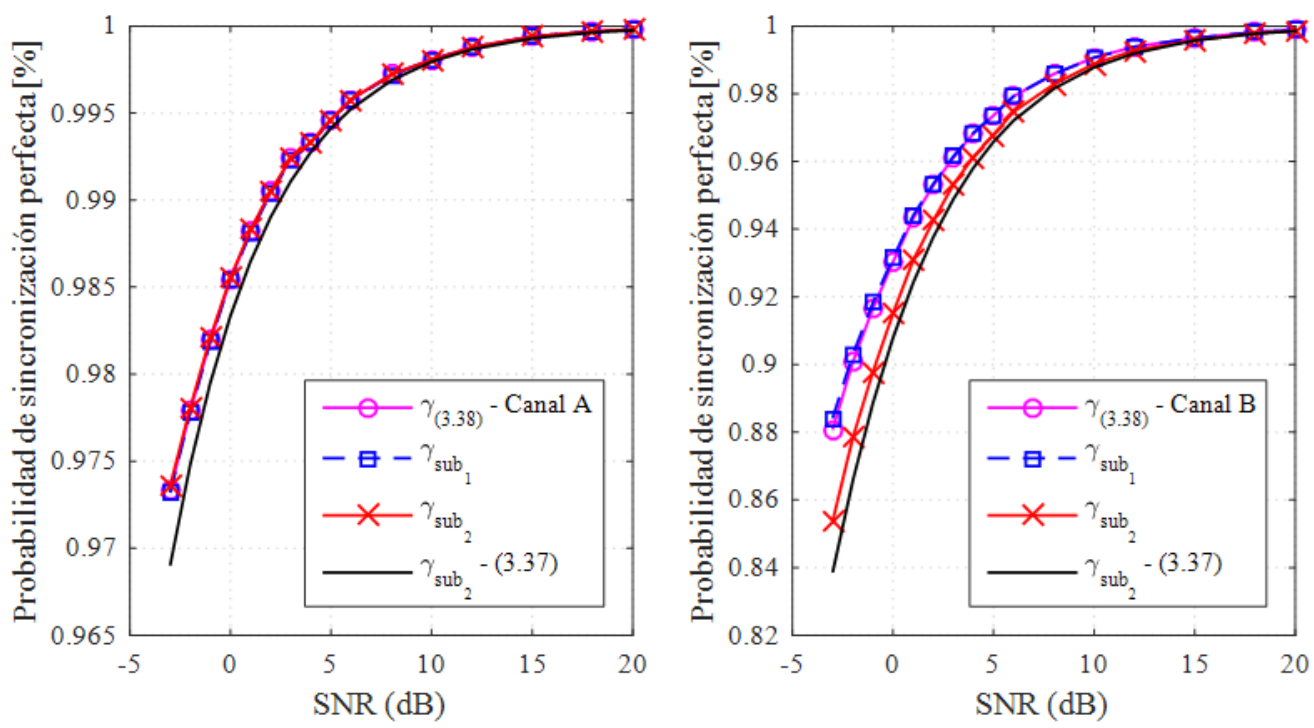

Fig. 3.5 Probabilidad de sincronización perfecta para la etapa de sincronización fina bajo distintos umbrales y condiciones de SNR para los canales $A$ y $B$.

numérico iterativo Bisección [Chapra 2010], con un error máximo admisible de $10^{-9}$ como criterio de fin. Para los dos canales considerados, la probabilidad de lograr una sincronización perfecta es casi la misma para $\gamma_{(3.38)}$ y $\gamma_{s u b_{1}}$, lo que indica que las aproximaciones efectuadas son válidas bajo las condiciones analizadas. Esto puede verificarse también a partir de los valores de los umbrales en función de la condición de SNR presentados en la Tabla 3.2, en la que puede apreciarse la semejanza entre el umbral obtenido a partir del método numérico y la aproximación realizada para obtener la fórmula cerrada de $\gamma_{s u b_{1}}$.

Sin embargo, si el número $N$ de subportadoras y el valor de $S N R$ se reducen lo suficiente, los valores de las funciones de densidad $f_{c_{n_{\text {ant }}^{\prime}}}(y)$ y $f_{c_{n_{\text {op }}}^{\prime}}(y)$ se acercan más, y el umbral óptimo no se situará cerca de la cola derecha de $f_{c_{n_{a n t}}^{\prime}}(y)$, ni cerca de la cola izquierda de $f_{c_{n_{\text {ó }}}^{\prime}}(y)$. La probabilidad de sincronización perfecta considerando el umbral subóptimo $\gamma_{s u b_{2}}$ es menor que bajo $\gamma_{s u b_{1}}$, lo que era predecible ya que $\gamma_{s u b_{2}}$ implica que no se posee información de las características del canal. La diferencia es mayor para el canal B porque la potencia promedio del primer camino es sólo el $15 \%$ de la potencia total del canal, mientras que en el canal A la relación es de casi el 53\%, mucho más cercana al 50\% del valor supuesto al derivar $\gamma_{s u b_{2}}$. Por último, se incluye el valor teórico de la probabilidad de sincronización perfecta de (3.37) bajo el umbral $\gamma_{\mathrm{Sub}_{2}}$, para su comparación. Las curvas son muy similares para valores de $S N R$ medios y altos, y difieren ligeramente para $S N R$ bajos. 
Diseño de Estrategias de Sincronización y Estimación de Canal para la Mejora de Comunicaciones en Redes Inteligentes de Energía

Adicionalmente, el desempeño del esquema de sincronización fina propuesto se muestra en la Fig. 3.6, evaluado en términos del MSE en las condiciones de ambos canales A y B. Además, se incluyen para su comparación el MSE del proceso de sincronización fina considerando los esquemas basados en distintas secuencias: PN [Wang 2008], MOS [Wang 2009], ZCC 1 [Qian 2017a] y ZCC 2 [Qian 2018]. En todos los casos se utiliza el mismo criterio de umbralización a partir de $\gamma_{s u b_{2}}$. El símbolo piloto propuesto y los basados en MOS y PN se basan en secuencias de amplitud constante, con prefijo y sufijo cíclicos adjuntos y una zona de correlación nula (cerca de cero en el caso de la secuencia PN).

Por lo tanto, las probabilidades de una sincronización perfecta similares en estos tres casos, así como el MSE de la sincronización, como puede observarse en la Fig. 3.6. También está claro que el error es mayor para el canal A que para el B. Por otra parte, el MSE de sincronización de los esquemas basados en ZCC son significativamente mayores. Esto ocurre porque se realiza un enventanado en el dominio del tiempo del símbolo piloto, lo que atenúa significativamente los valores en los extremos, dando lugar a una alta relación de potencia pico a promedio (PAPR). Como el ruido del canal PLC contiene ruido impulsivo, el alto valor de PAPR presente en el filtro acoplado a estas secuencias afecta a la salida del correlador en las muestras anteriores al primer camino del canal, generando mayores valores cuando ocurre un evento de ruido

Tabla 3.2 Valores de diferentes umbrales para la etapa sincronización fina, para los canales A y B y distintas condiciones de SNR.

\begin{tabular}{|c|c|c|c|c|c|c|}
\hline \multirow[b]{2}{*}{$S N R$} & \multicolumn{3}{|c|}{ Canal A } & \multicolumn{3}{|c|}{ Canal B } \\
\hline & $\begin{array}{l}\gamma_{(3.38)} \\
\cdot \mathbf{1 0}^{-3}\end{array}$ & $\begin{array}{l}\gamma_{s u b_{1}} \\
\cdot 10^{-3}\end{array}$ & $\begin{array}{c}\gamma_{s u b_{2}} \\
\cdot 10^{-3}\end{array}$ & $\begin{array}{l}\gamma_{(3.38)} \\
\cdot \mathbf{1 0}^{-3}\end{array}$ & $\begin{array}{l}\gamma_{s u b_{1}} \\
\cdot 10^{-3}\end{array}$ & $\begin{array}{c}\gamma_{s u b_{2}} \\
\cdot 10^{-3}\end{array}$ \\
\hline$-3 \mathrm{~dB}$ & 10,0283 & 10,0705 & 9,9197 & 7,9263 & 7,6932 & 9,9197 \\
\hline $0 \mathrm{~dB}$ & 8,1838 & 8,2334 & 8,1202 & 6,5586 & 6,4491 & 8,1202 \\
\hline $3 \mathbf{d B}$ & 5,8983 & 5,9481 & 5,8725 & 4,7932 & 4,7566 & 5,8725 \\
\hline $6 \mathrm{~dB}$ & 3,8065 & 3,8476 & 3,8022 & 3,1343 & 3,1312 & 3,8022 \\
\hline $10 \mathrm{~dB}$ & 1,8803 & 1,9058 & 1,8852 & 1,5729 & 1,5814 & 1,8852 \\
\hline $20 \mathrm{~dB}$ & 0,2475 & 0,2521 & 0,2499 & 0,2136 & 0,2168 & 0,2499 \\
\hline
\end{tabular}




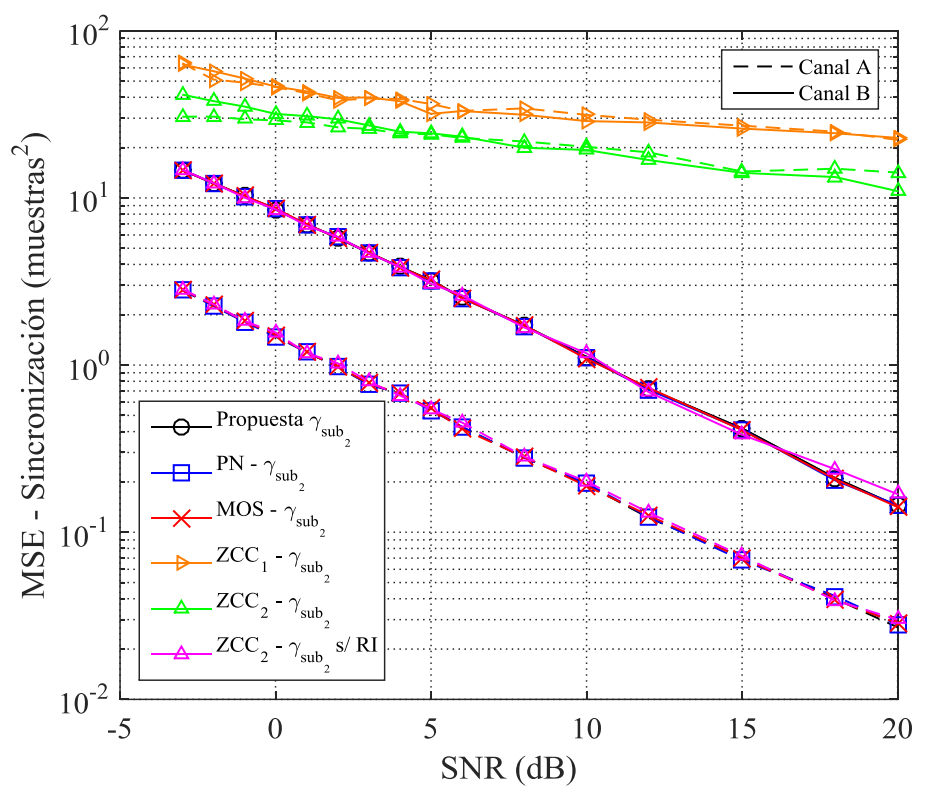

Fig. 3.6 MSE de la sincronización fina para diferentes símbolos piloto basados en secuencias en los canales A (línea punteada) y B (línea continua).

impulsivo que afecta a las muestras del símbolo piloto recibido. Por lo tanto, la probabilidad de falsa detección de cualquier muestra de ruido filtrado es mayor respecto a los símbolos piloto de amplitud constante, bajo el mismo umbral. Para aclarar esto, se muestra también el resultado utilizando el símbolo piloto $\mathrm{ZCC}_{2}$, pero sin incluir ruido impulsivo, y se observa que el desempeño es muy similar al obtenido por el resto de los símbolos pilotos en ambos canales.

\subsubsection{Estimación de canal}

De acuerdo a (3.22), la salida del filtro acoplado $c_{n}^{\mathbf{r}, \mathbf{x}^{\prime}}$ contendrá la respuesta de impulso del canal $h_{l}$ y las muestras de ruido filtradas, siempre que la dispersión del canal $L$ sea menor que la longitud del sufijo cíclico $N_{c S}$. Suponiendo una sincronización perfecta, entonces la salida del filtro acoplado puede expresarse como:

$$
c_{n}^{\mathbf{r}, \mathbf{x}^{\prime}}=N \sqrt{P_{T}} \sum_{l=0}^{L-1} h_{l} \cdot \delta_{n-l}+\sum_{m=0}^{N-1} w_{n+m} \mathbf{x}_{m}^{\prime *}, \quad n_{\text {ópt }} \leq n<n_{\text {ó } p t}+N_{c s}
$$

donde $\delta_{n}$ es la delta de Kronecker, $N$ es el número total de subportadoras; $h_{l}$ el $l$-ésimo camino de la CIR; $P_{T}$ es la potencia transmitida; y $w_{n}$ son las muestras complejas del ruido del canal PLC. Si no se estima la longitud del canal $L$, entonces deben incluirse las $N_{c S}$ muestras en la estimación de la respuesta al impulso del canal $\tilde{\mathbf{h}}$ en (3.48), y la estimación de la respuesta en frecuencia del canal $\widetilde{\mathbf{H}}$ en (3.49) se obtiene mediante la 
Diseño de Estrategias de Sincronización y Estimación de Canal para la Mejora de Comunicaciones en Redes Inteligentes de Energía

operación de DFT de $N$ puntos de $\tilde{\mathbf{h}}$, con $N-N_{C S}$ entradas con valor nulo (Zero padding).

$$
\begin{gathered}
\tilde{h}_{n}=\frac{1}{N \sqrt{P_{T}}} c_{n}^{\mathbf{r}, \mathbf{x} \prime}, \quad n_{\text {ó } p t} \leq n<n_{\text {ópt }}+N_{c S}-1 \\
\widetilde{H}_{k}=\sum_{n=0}^{N_{c S}-1} \tilde{h}_{n} \cdot \mathrm{e}^{\left(-j \frac{2 \pi k n}{N}\right)}, \quad 0 \leq k<N-1
\end{gathered}
$$

El MSE de la estimación de la CIR $\mathbf{h}$ depende del número de muestras involucradas, y puede calcularse como:

$$
\operatorname{MSE}_{\tilde{\mathbf{h}}}=E\left\{\sum_{m=0}^{N_{c s}-1}\left|\frac{\sum_{l=0}^{N-1} w_{m+l} \mathrm{x}_{l}^{\prime *}}{N \sqrt{P_{T}}}\right|^{2}\right\}
$$

Entonces, el MSE de la estimación del canal $\mathrm{MSE}_{\tilde{\mathbf{h}}}$ resulta el valor esperado de la suma de $N_{c s}$ variables aleatorias ponderadas con distribución chi-cuadrado de un grado de libertad:

$$
\mathrm{MSE}_{\tilde{\mathbf{h}}}=E\left\{\frac{\sigma_{w}{ }^{2}}{N P_{T}} \chi_{N_{c s}}^{2}\right\}=\frac{N_{c S}}{N \cdot S N R}
$$

Al igual que en la técnica de estimación de canal basada en DFT [Kang 2007], resulta proporcional al número $N_{c S}$ de muestras incluidas en la estimación de la respuesta al impulso del canal, e inversamente proporcional a $N$ y la condición de $S N R$. Además, si la longitud de la CIR es conocida o estimada de manera precisa, entonces se obtiene un menor error. Incluyendo el resultado de la etapa de sincronización fina, la estimación de la CIR de (3.48) resulta:

$$
\tilde{h}_{n}=\frac{1}{N \sqrt{P_{T}}} c_{n}^{\mathbf{r}, \mathbf{x}^{\prime} \prime}, \quad n_{f} \leq n<n_{f}+N_{c s}-1
$$

En la Fig. 3.7 se presenta el desempeño de la estimación de canal en términos del MSE de la estimación de la respuesta al impulso. Se incluye la estimación de canal después de la etapa de sincronización fina propuesta, considerando los umbrales $\gamma_{s u b_{1}}$ y $\gamma_{s u b_{2}}$. El error obtenido a partir del uso de $\gamma_{s u b_{1}}$ es ligeramente inferior al obtenido con $\gamma_{s u b_{2}}$ para ambos canales, ya que el desempeño de la sincronización es mejor. Además, el MSE es mayor en el canal B, ya que la probabilidad de sincronización perfecta es menor que la obtenida para el canal A. Adicionalmente se incluyen el MSE de las estimaciones 


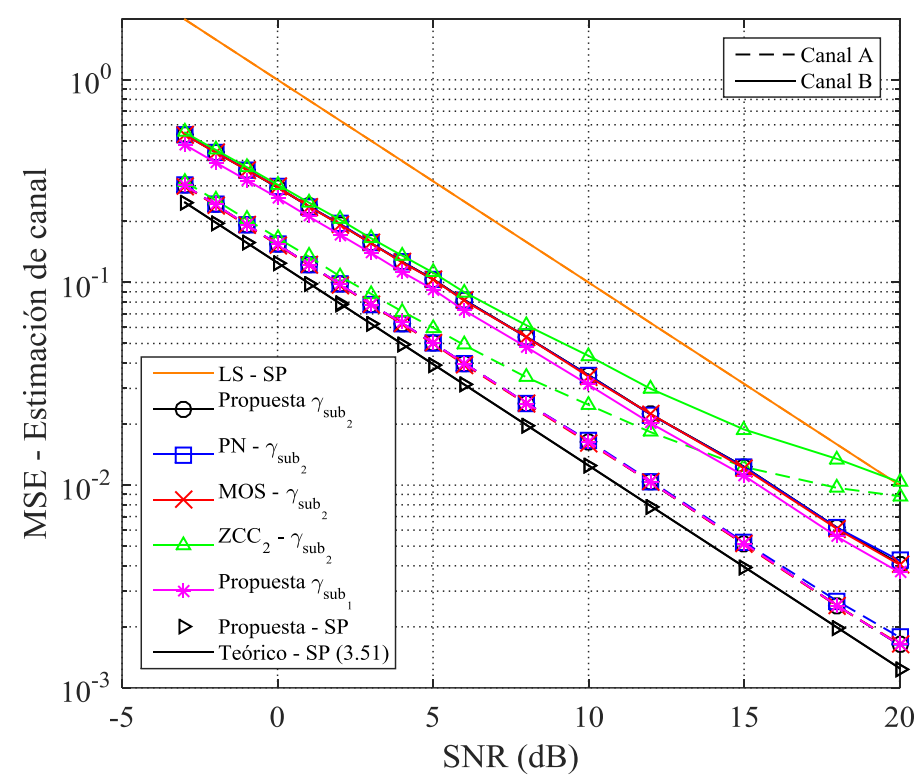

Fig. 3.7 MSE de la estimación de canal con sincronización perfecta y luego de la sincronización fina para diferentes símbolos piloto basados en secuencias en los canales A (línea punteada) y B (linea continua).

del canal a partir del resultado de la etapa de sincronización fina de cada uno de los símbolos pilotos bajo comparación. Puede observarse que el símbolo propuesto tiene un rendimiento similar al que utiliza MOS. El símbolo con secuencia PN presenta un MSE ligeramente superior para $S N R$ s por encima de $15 \mathrm{~dB}$, debido a la zona de correlación no nula. El esquema basado en $\mathrm{ZCC}_{2}$ presenta un peor desempeño en la estimación del canal, y ocurre debido al peor desempeño en la sincronización de la Fig. 3.6.

Es importante señalar que, considerando un contexto de sincronización perfecta, se alcanza el mismo valor de MSE que utilizando el símbolo basado en MOS o el propuesto. Además, el valor teórico del MSE que considera una sincronización perfecta de (3.51) se incluye como un límite inferior de error y se verifica en las simulaciones. Para comparar con una técnica sencilla de estimación de canal en el dominio de la frecuencia, se incluye el MSE que se obtiene a partir de la estimación LS considerando una sincronización perfecta, dando un mayor valor de error respecto de la propuesta incluyendo el resultado de la etapa de sincronización fina.

\subsubsection{Complejidad de la propuesta}

En esta sección se analizan algunos detalles de complejidad del esquema propuesto, en términos de las operaciones necesarias para computar los bloques principales de las etapas de sincronización y estimación del canal. El proceso de sincronización gruesa 
Diseño de Estrategias de Sincronización y Estimación de Canal para la Mejora de Comunicaciones en Redes Inteligentes de Energía

precisa del cómputo de (3.13) y (3.14), que pueden implementarse mediante las ecuaciones recursivas $(3.53)$ y (3.54).

$$
\begin{gathered}
p_{n+1}=p_{n}+r_{n} \cdot r_{n+N / 2}^{*}+r_{n+N / 2} \cdot r_{n+N}^{*}-2 r_{n+N / 4} \cdot r_{n+3 N / 4}^{*} \\
e_{n+1}=e_{n}+r_{n+N} \cdot r_{n+N}^{*}-r_{n} \cdot r_{n}^{*}
\end{gathered}
$$

Se requieren un multiplicador complejo de dos entradas con un retardo apropiado en su salida, y tres sumadores complejos para calcular (3.53). Además, se necesita un multiplicador complejo de dos entradas, elementos de retardo y dos sumadores complejos para (3.54). Luego, se precisa un multiplicador constante de factor 2, y la división correspondiente de (3.12). El diagrama en bloques de la arquitectura equivalente en operaciones reales para computar la métrica de sincronización gruesa se presenta en la Fig. 3.8.

Considerando el esquema propuesto para la sincronización fina y estimación del canal basado en la correlación cruzada, el filtro acoplado del símbolo piloto requiere del correlador del CPS de longitud N/4, y posteriormente procesamiento adicional para retardos y sumas. El CPS de longitud $N / 4$ requiere $Q$ etapas recursivas, de manera tal que $Q=\log _{2}(N / 4)$, donde cada etapa incluye dos sumadores y un elemento de memoria como se introduce en [Budisin 1991]. La concatenación propuesta de las secuencias para generar el símbolo piloto demanda dos elementos de memoria y tres sumadores. Para calcular la correlación cruzada de la señal recibida, que posee muestras complejas, se requieren dos bloques idénticos como se muestra en la Fig. 3.9 Por lo tanto, el filtro acoplado del símbolo piloto propuesto requiere sólo $4 \cdot \log _{2}(N / 2)+2$ sumadores (sin ningún multiplicador), lo que implica una importante reducción con respecto a otros tipos de símbolos piloto basados en secuencias. La correlación cruzada de un símbolo piloto basado en una secuencia compleja, como en [Wang 2009], exigiría hasta $N$ multiplicadores complejos y $N-1$ sumadores complejos. El símbolo piloto

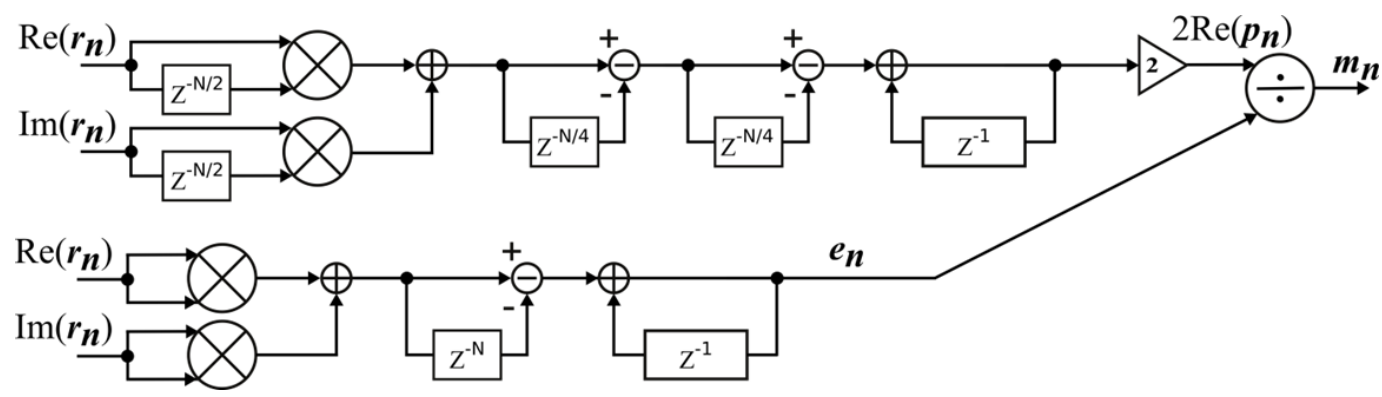

Fig. 3.8 Estructura equivalente para el cómputo de la métrica de sincronización gruesa. 
basado en el par ZCC [Qian 2018], requiere $2 N$ multiplicadores reales y $2 N-2$ sumadores reales, mientras que el basado en la secuencia binaria PN requiere $2 N-2$ sumadores reales para realizar la correlación cruzada [Wang 2008]. En la Tabla 3.3 se muestra el número de operaciones necesarias para calcular la correlación cruzada del símbolo piloto propuesto y los presentados en los trabajos mencionados, en términos de operaciones aritméticas de números reales, para una longitud $N$. Se supone que cada suma compleja equivale a dos sumas reales, mientras que el multiplicador complejo se asume compuesto por cuatro multiplicadores reales y dos sumas. Finalmente, el diagrama en bloques de la propuesta de sincronización fina y estimación de canal conjunta se presenta en la Fig. 3.9.

Tabla 3.3 Carga computacional asociada al cómputo de la correlación cruzada para distintos símbolos piloto.

\begin{tabular}{|c|c|c|}
\hline & $\begin{array}{c}\text { Multiplicadores } \\
\text { constantes }\end{array}$ & Sumadores \\
\hline MOS & $4 \mathrm{~N}$ & $4 \mathrm{~N}-2$ \\
\hline ZCC $_{2}$ & $2 \mathrm{~N}$ & $2 \mathrm{~N}-2$ \\
\hline PN & 0 & $2 \mathrm{~N}-2$ \\
\hline Propuesto & 0 & $4 \cdot \log _{2}(N / 2)+2$ \\
\hline
\end{tabular}

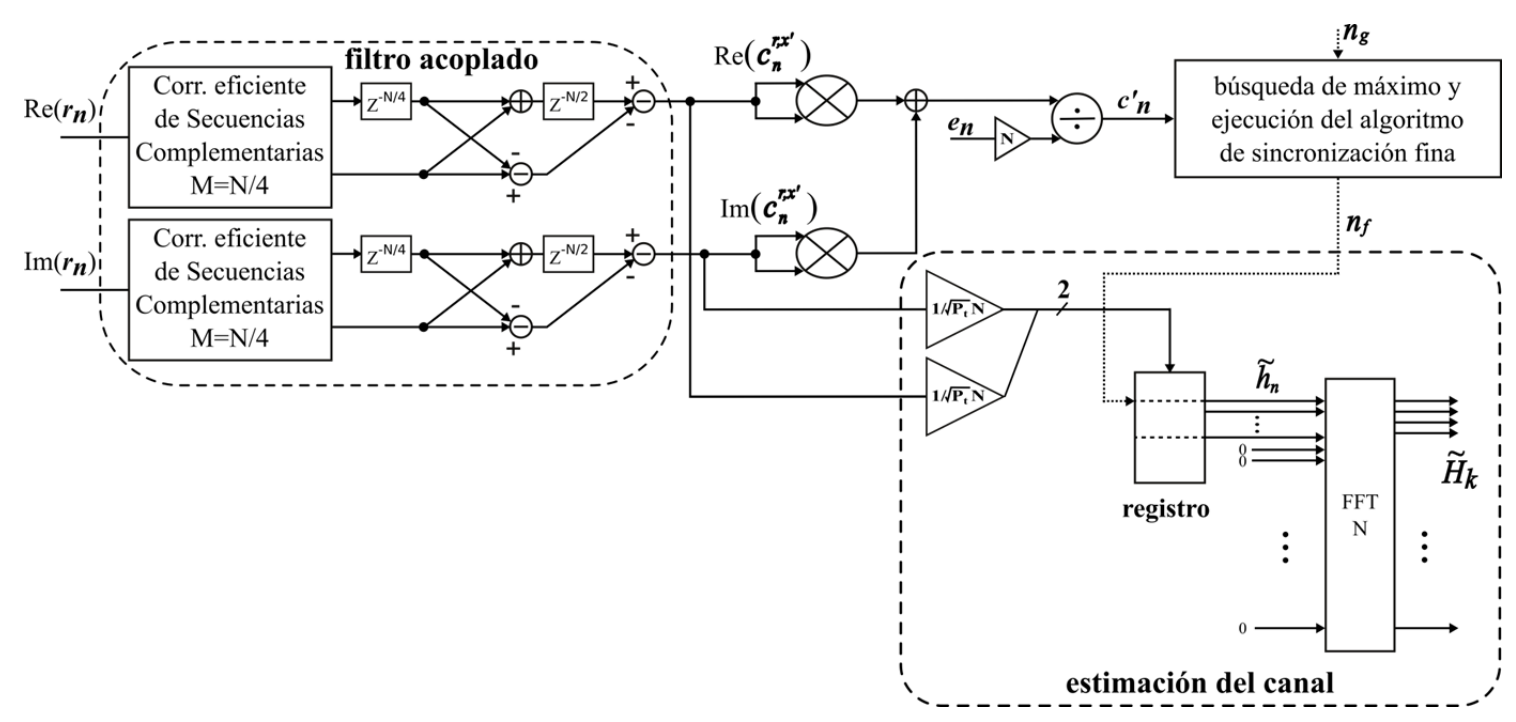

Fig. 3.9 Diagrama en bloques del procesamiento de señal requerido para la propuesta de sincronización fina y estimación de canal conjunta. 
Diseño de Estrategias de Sincronización y Estimación de Canal para la Mejora de Comunicaciones en Redes Inteligentes de Energía

\subsection{Conclusiones}

En este capítulo se planteó el problema de sincronización en los sistemas OFDM para comunicaciones en la red eléctrica, y luego, se desarrolló una técnica de sincronización y estimación de canal de baja complejidad. Se utilizaron pares de secuencias complementarias para el diseño de un símbolo piloto que asegura adecuadas propiedades de correlación y una baja complejidad asociada a su procesamiento, dado que se requieren pocas operaciones para calcular la correlación cruzada. Ésta, además, se utiliza conjuntamente no sólo para la etapa de sincronización, sino también para la estimación del canal.

Posteriormente, se propuso una correlación cruzada normalizada para la sincronización fina, y se derivaron las funciones de densidad de probabilidad de la misma en el instante de sincronismo óptimo y en las muestras previas, con el fin de obtener umbrales óptimos y subóptimos para la detección del primer camino del canal. Los umbrales obtenidos dependen de los parámetros del sistema y se ajustan en función de la condición de relación señal a ruido. El desempeño de la propuesta se verificó satisfactoriamente mediante simulaciones en un canal multicamino bajo diferentes tipos de ruidos de PLC, y se realizó una comparación de desempeño respecto a la utilización de símbolos piloto construidos a partir de otras secuencias, verificándose su competitividad.

Al igual que en otros trabajos disponibles en la literatura acerca de sincronización y estimación de canal en OFDM, se consideró un escenario simplificado del sistema, en el que la modulación en fase y cuadratura en el transmisor y el proceso de demodulación en el receptor hasta obtener las muestras en banda base se realizan de forma ideal. De esta manera, se facilitó la comparación de la estrategia de sincronización y estimación de canal con símbolos pilotos basados en secuencias, con respecto al propuesto. 


\section{SINCRONIZACIÓN GRUESA EN SISTEMAS PLC-DMT}

Los sistemas de comunicaciones de banda ancha por multitono discreto (Discrete MultiTone - DMT) son un caso particular de OFDM, en el que existe simetría hermítica en las portadoras moduladas y la salida de la DFT es únicamente real, y no se precisa de una modulación en fase y cuadratura para su transmisión por el canal [Pollet 1999]. En particular, el esquema de transmisión DMT fue incluido como sistema de modulación en los estándares IEEE 1901 [IEEE 2010] y Homeplug [Haniph 2013] para PLC. La razón fundamental se da por las bajas frecuencias de transmisión (hasta $50 \mathrm{MHz}$ ), que permiten un procesamiento digital integral de la señal recibida sin la necesidad de incluir la modulación en fase y cuadratura en una portadora central como en el sistema OFDM, que tendría un ancho de banda cercano al valor de la frecuencia de portadora como se indica en [Kim 2011]. Teniendo esta condición presente, en este capítulo se modifica el enfoque para hacer frente a la etapa de sincronización gruesa en un sistema DMT-PLC desde un punto de vista del diseño del símbolo piloto y el procesamiento digital de la señal. En esa línea, a partir del símbolo piloto propuesto, se realiza un análisis profundo de las características de la adaptación de la métrica de sincronización gruesa propuesta en el capítulo 3, y se proponen nuevas variantes. Las principales contribuciones del presente capítulo son las siguientes:

- Se proponen y estudian detalladamente dos métricas de sincronización gruesa: una basada en AC, y otra que se computa a partir de la CC de la señal recibida con el filtro acoplado al símbolo piloto. 
Diseño de Estrategias de Sincronización y Estimación de Canal para la Mejora de Comunicaciones en Redes Inteligentes de Energía

- Se realiza un análisis detallado de las distribuciones de probabilidad de las métricas en los instantes previos a la llegada del símbolo piloto y en el de sincronismo óptimo. De esta manera, resulta posible definir de forma analítica umbrales de detección primaria del símbolo piloto, tanto para un modelo de ruido de fondo AWGN como coloreado.

- Se propone y analiza una estrategia sencilla de estimación de $S N R$ a partir de las métricas, ya que, de acuerdo a lo desarrollado en el Capítulo 3, la estimación de $S N R$ puede resultar importante en el cálculo de umbrales para el proceso de sincronización fina.

A lo largo del capítulo, se valida el análisis a partir de simulaciones, y se evalúa el desempeño de las métricas, incluyendo además del ruido de fondo coloreado, el efecto del ruido impulsivo en cada métrica y el desvío en la frecuencia de muestreo.

\subsection{Modelo de canal PLC para transmisiones banda base}

En el capítulo 3, se consideró un sistema de transmisión OFDM con modulación en fase y cuadratura. Además, la distorsión del canal PLC, que depende del ancho de banda y portadora central utilizados para la transmisión, fue modelada estadísticamente a partir de un desvanecimiento multicamino, donde la amplitud de cada camino se corresponde con una distribución de Rayleigh. Esto resultó en un modelo simple y flexible, adaptable a los parámetros de transmisión utilizados. Para las trasmisiones en banda base, se considerará un modelo estadístico del canal PLC más complejo, basado también en efectos multicamino en el dominio del tiempo, generados a partir de diversos parámetros (algunos de ellos variables aleatorias independientes) relacionados con las características físicas de la red eléctrica.

Considerando el esquema de comunicaciones PLC con transmisión banda base, se utilizará un modelo estadístico de canal para obtener la respuesta del mismo en la banda de 0-50MHz. El modelo se basa en relacionar las características físicas de la red eléctrica, como son el multicamino generado por las reflexiones y transmisiones en las distintas ramificaciones de la red, la atenuación en el medio, las distancias recorridas por cada camino, etc. [Tonello 2012] [Zimmermann 2002]. La expresión para obtener la respuesta en frecuencia a partir de la superposición de los efectos de cada camino está dada por: 


$$
G_{C H}(f)=A \sum_{p=1}^{N p} g_{p} e^{-j \frac{2 \pi d_{p}}{v} f} e^{-\left(a_{0}+a_{1} f^{K}\right) d_{p}}
$$

donde $A$ es un parámetro de atenuación; $N_{p}$ es el número de caminos; $g_{p}$ es la ganancia del camino $p$, y puede considerarse como real y uniformemente distribuida $U(-1,1) ; d_{p}$ es la longitud del camino $p ; v=c / \sqrt{\varepsilon_{r}}$ con $c$ como la velocidad de la luz y $\varepsilon_{r}$ la constante dieléctrica; $a_{0}, a_{1}$ y $K$ son coeficientes de atenuación en los cables y se asumen constantes. El modelo estadístico para generar distintas funciones de transferencia se basa en asumir que los reflectores que generan los caminos se ubican a una distancia finita, de acuerdo a un proceso de Poisson con intensidad $\Lambda\left[\mathrm{m}^{-1}\right]$. La máxima longitud de la red es $L_{\max }$, y así el número de caminos sigue una distribución de Poisson con media $L_{\max } \Lambda$, mientras que las distancias entre caminos son independientes y exponencialmente distribuidas con media $1 / \Lambda$.

Si se asume $K=1$, es posible obtener la respuesta impulsiva del canal en forma cerrada de acuerdo a:

$$
\begin{gathered}
g_{C H}(t)=A \sum_{p=1}^{N p} g_{p} e^{-a_{0} d_{p}} \frac{a_{1} d_{p}+j 2 \pi\left(t-\frac{d_{p}}{v}\right)}{\left(a_{1} d_{p}\right)^{2}+4 \pi^{2}\left(t-\frac{d_{p}}{v}\right)^{2}}\left(e^{j 2 \pi B_{1}\left(t-d_{p} / v\right)-a_{1} B_{1} d_{p}}\right. \\
\left.-e^{j 2 \pi B_{2}\left(t-d_{p} / v\right)-a_{1} B_{2} d_{p}}\right)
\end{gathered}
$$

En el proyecto OMEGA, en el que se realizó una campaña de mediciones para caracterizar la red eléctrica como canal de comunicaciones, se presentó como uno de los importantes resultados un generador de modelo discreto de la respuesta al impulso del canal PLC, ajustando los parámetros de (4.2), fijando $B_{1}=0$ y $\sqrt{\varepsilon_{r}}=1,5$ [OMEGA 2008]. Además, se considera una frecuencia de muestreo $f_{s}=100 \mathrm{MHz}\left(T_{s}=10 \mathrm{~ns}\right) \mathrm{de}$ acuerdo al estándar IEEE 1901, es decir, $B_{2}=50 \mathrm{MHz}$. A partir de los resultados de las mediciones, se ajustaron los parámetros de atenuación e intensidad del proceso de Poisson. Por otro lado, [Tonello 2007] utiliza el mismo modelo de canal, considerando otros valores para los parámetros de atenuación e intensidad del proceso de Poisson, y efectuando la suposición de que el primer coeficiente del canal correspondiente al camino directo se encuentra a una distancia fija. A lo largo del presente trabajo, se denominará a estos dos modelos como canales A y B, y serán utilizados para analizar el desempeño de la propuesta de sincronización y estimación de canal conjunta. En la Tabla 4.1 se presentan los principales parámetros de los modelos de canal. 
Diseño de Estrategias de Sincronización y Estimación de Canal para la Mejora de Comunicaciones en Redes Inteligentes de Energía

En la Fig. 4.1 se presentan las respuestas al impulso para una realización de los canales A y B, respectivamente. Por un lado, puede apreciarse que el canal A posee una mayor dispersión en tiempo que el canal B. Esto se debe principalmente a que la longitud máxima considerada para generar los caminos del canal es mayor en A que en B, y el tiempo de arribo de un camino de canal depende de la distancia, la velocidad de propagación y la constante dieléctrica del medio.

Por otro lado, se observa que la respuesta al impulso del canal A presenta picos con

Tabla 4.1 Parámetros característicos para la generación de dos variantes del modelo de canal PLC.

\begin{tabular}{|c|c|c|}
\hline Parámetro & Canal A & Canal B \\
\hline $\boldsymbol{g}_{\boldsymbol{p}}$ & $U(-1,1)$ & $U(-1,1)$ \\
\hline $\boldsymbol{a}_{\mathbf{0}}$ & $3 e^{-3}$ & $1 e^{-5}$ \\
\hline $\boldsymbol{a}_{\mathbf{1}}$ & $4 e^{-10}$ & $1 e^{-9}$ \\
\hline $\boldsymbol{A}$ & $1,0864 /\left(2 B_{2}\right)$ & $6,5873 /\left(2 B_{2}\right)$ \\
\hline $\boldsymbol{d}_{\mathbf{1}}$ & Aleatoria & $30 \mathrm{~m}$ \\
\hline $\boldsymbol{\Lambda}$ & $1 / 5 \mathrm{~m}$ & $1 / 15 \mathrm{~m}$ \\
\hline $\boldsymbol{L}_{\boldsymbol{m} \text { áx }}$ & $800 \mathrm{~m}$ & $300 \mathrm{~m}$ \\
\hline
\end{tabular}
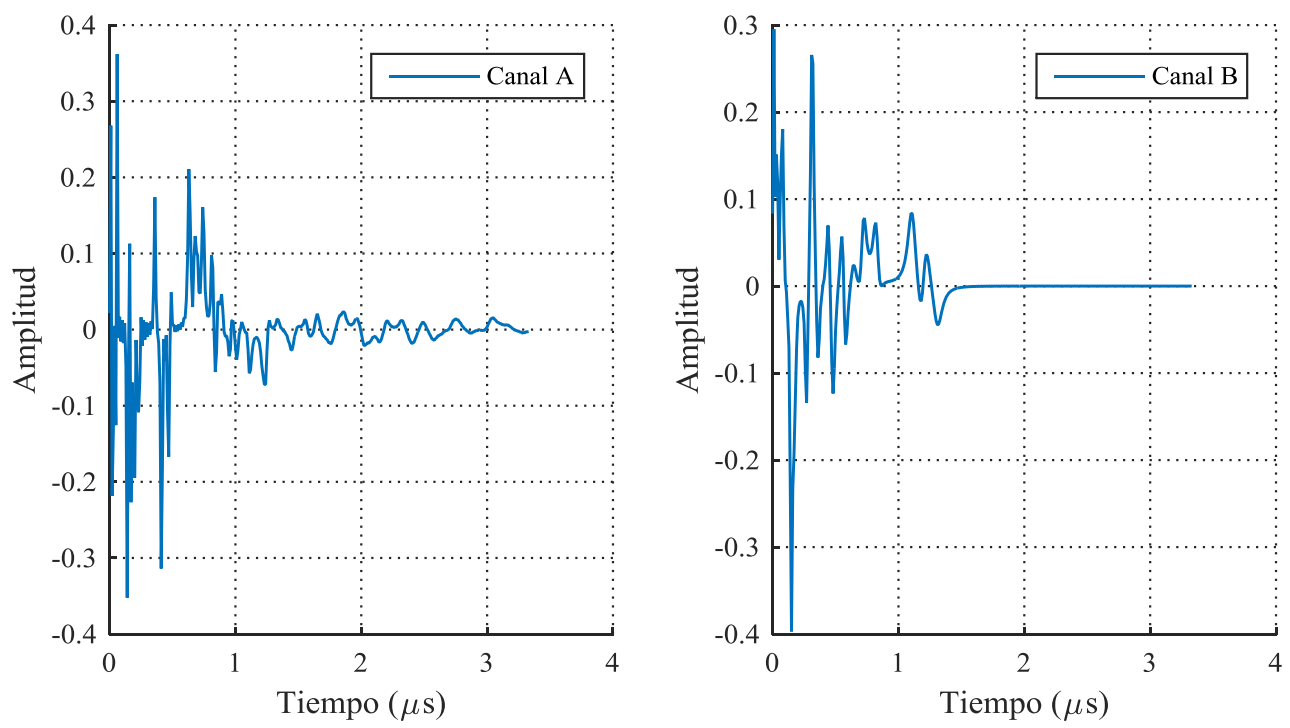

Fig. 4.1 Respuestas al impulso correspondientes a una realización de los canales A y B. 
transiciones considerablemente más rápidas respecto a aquellas del canal $\mathrm{B}, \mathrm{y}$ la diferencia se debe a que el coeficiente de atenuación $a_{1}$ es menor en el canal A. De acuerdo a (4.1), un menor valor del coeficiente implica una menor atenuación en frecuencia para cada camino generado; mientras se relaciona con transiciones más rápidas en el dominio del tiempo en (4.2). Finalmente, la intensidad $\Lambda$ del proceso de Poisson es mayor para el canal A, por lo que el espaciamiento promedio entre distintos caminos es menor en el canal A que en el B, dando lugar a una mayor cantidad de caminos en el mismo período de tiempo de la respuesta al impulso.

Adicionalmente, resulta de interés analizar la ganancia del canal, ya que define las características del desvanecimiento (fading), y depende de la cantidad de caminos y la ganancia de los mismos. El desvanecimiento en los canales PLC suele ser modelado a partir de una distribución Log-normal [Dubey 2015] [Tonello 2010]. En la Fig. 4.2 se presentan la función de distribución acumulada (CDF - Cumulative Distribution Function) empírica obtenida para 200.000 realizaciones de canal, y el ajuste a una distribución Log-normal, para ambos modelos de canal. Puede apreciarse que la ganancia del canal A presenta un buen ajuste a la distribución Log-normal esperada. Por otro lado, la ganancia correspondiente al canal B no presenta tan buen ajuste a la distribución Log-normal, siendo la distribución Gamma una mejor opción de ajuste. Finalmente, se puede observar en las figuras y resulta importante remarcar, que los dos tipos de canal se encuentran normalizados en energía, es decir, que la ganancia
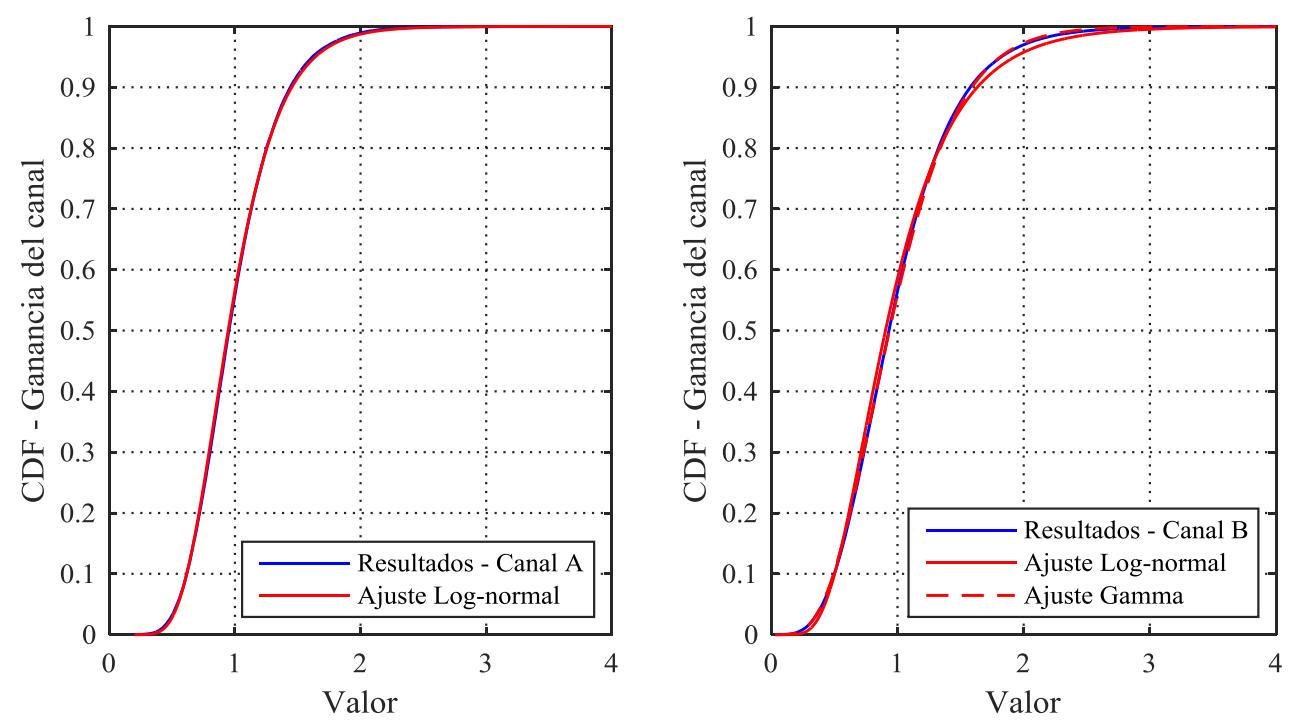

Fig. 4.2 CDFs empíricas obtenidas de la ganancia del canal A y su ajuste Log-normal, y del canal B y su ajuste Log-normal y Gamma. 
Diseño de Estrategias de Sincronización y Estimación de Canal para la Mejora de Comunicaciones en Redes Inteligentes de Energía

(desvanecimiento) promedio es uno. Los resultados del ajuste de los parámetros obtenidos para los dos tipos de canal se presentan en la Tabla 4.2.

Tabla 4.2 Parámetros de ajuste Log-normal para la ganancia del canal A, y de ajuste Log-normal y Gamma para la ganancia del canal B.

\begin{tabular}{|c|c|c|c|c|c|}
\hline \multicolumn{2}{|c|}{ Canal A } & \multicolumn{4}{c|}{ Canal B } \\
\hline \multicolumn{2}{|c|}{ Log-normal } & \multicolumn{2}{|c|}{ Log-normal } & \multicolumn{2}{c|}{ Gamma } \\
\hline $\boldsymbol{\mu}$ & $\boldsymbol{\sigma}$ & $\boldsymbol{\mu}$ & $\boldsymbol{\sigma}$ & $\boldsymbol{k}$ & $\boldsymbol{\theta}$ \\
\hline$-0,05514$ & 0,33431 & $-0,09988$ & 0,46026 & 5,16696 & 0,19354 \\
\hline
\end{tabular}

\subsection{Descripción del sistema y diseño del símbolo piloto}

Considerando un sistema DMT basado en una IDFT de $N$ puntos, implementada a partir de la FFT, se dispone como máximo de $N / 2$ subportadoras, ya que para obtener una salida únicamente real se debe satisfacer la propiedad de simetría hermítica en las entradas del bloque FFT. Para esto último, si en cada portadora disponible se mapean elementos complejos $X_{k}$, con $k=1, \ldots, N-1$, se debe cumplir $X_{N-k}=X_{k}^{*}, \mathrm{y}$ adicionalmente $\left\{X_{0}, X_{N / 2}\right\} \in \mathrm{R}$. Finalmente, tras efectuar la IDFT a partir de un bloque IFFT de $N$ puntos y agregar un prefijo cíclico de $N_{c p}$ muestras, la señal $x_{n}$ a transmitir puede expresarse como:

$$
x_{n}=\frac{1}{\sqrt{N}} \sum_{k=0}^{N-1} X_{k} \cdot \mathrm{e}^{\left(j \frac{2 \pi k n}{N}\right)}, \quad-N_{c p} \leq n \leq N-1
$$

Donde $x_{n}$ representa el $n$-ésimo elemento real del símbolo DMT en el dominio del tiempo. Por ello, la señal recibida $r_{n}$ para un canal disperso será:

$$
r_{n}=\sum_{l=0}^{L-1} h_{l} \cdot x_{n-\theta-l}+w_{n}
$$

Donde $h_{l}$ es el $l$-ésimo elemento de la respuesta impulsiva del canal y $L<N_{c p}$ la dispersión máxima del canal en muestras; $\theta$ es un desvío en muestras respecto a la sincronización temporal correcta del símbolo; y $w_{n}$ son muestras de ruido blanco Gaussiano. En el receptor, se efectúa la sincronización del símbolo y luego se descarta 
el prefijo cíclico. Posteriormente, se realiza la demodulación a partir de la FFT de $N$ puntos y se efectúa la ecualización del canal en el dominio de la frecuencia. El diagrama de bloques del sistema descrito se presenta en la Fig. 4.3.

Las estrategias de sincronización y estimación de canal propuestas, al igual que en el enfoque de transmisión OFDM, se llevan a cabo a partir de tres etapas: una etapa de sincronización gruesa basada en autocorrelación de la señal recibida; una sincronización fina basada en la correlación cruzada del símbolo piloto transmitido; y por último, la estimación de la respuesta al impulso del canal a partir de la misma correlación cruzada. Para ello, considerando el sistema DMT de $N / 2$ subportadoras se propone nuevamente el uso de un par de secuencias a y $\mathbf{b}$ de longitud $N / 4$; y la construcción del símbolo piloto de longitud $N$ en el dominio del tiempo se basa en concatenar el par de secuencias complementarias de la siguiente manera:

$$
\mathbf{x}^{\prime}=\left[\begin{array}{llll}
\mathbf{a} & \mathbf{b} & -\mathbf{a} & \mathbf{b}
\end{array}\right]
$$

Esta forma de concatenación se traduce en una restricción en los parámetros del algoritmo recursivo de generación y correlación eficientes de secuencias complementarias descripto en [Budisin 1991], en su última recursión, fijando la semilla de generación a 1 y el elemento de retardo en $N / 4$. Adicionalmente, se efectúa la misma restricción para la anteúltima etapa, esta vez fijando el valor del retardo a $N / 8$. Bajo

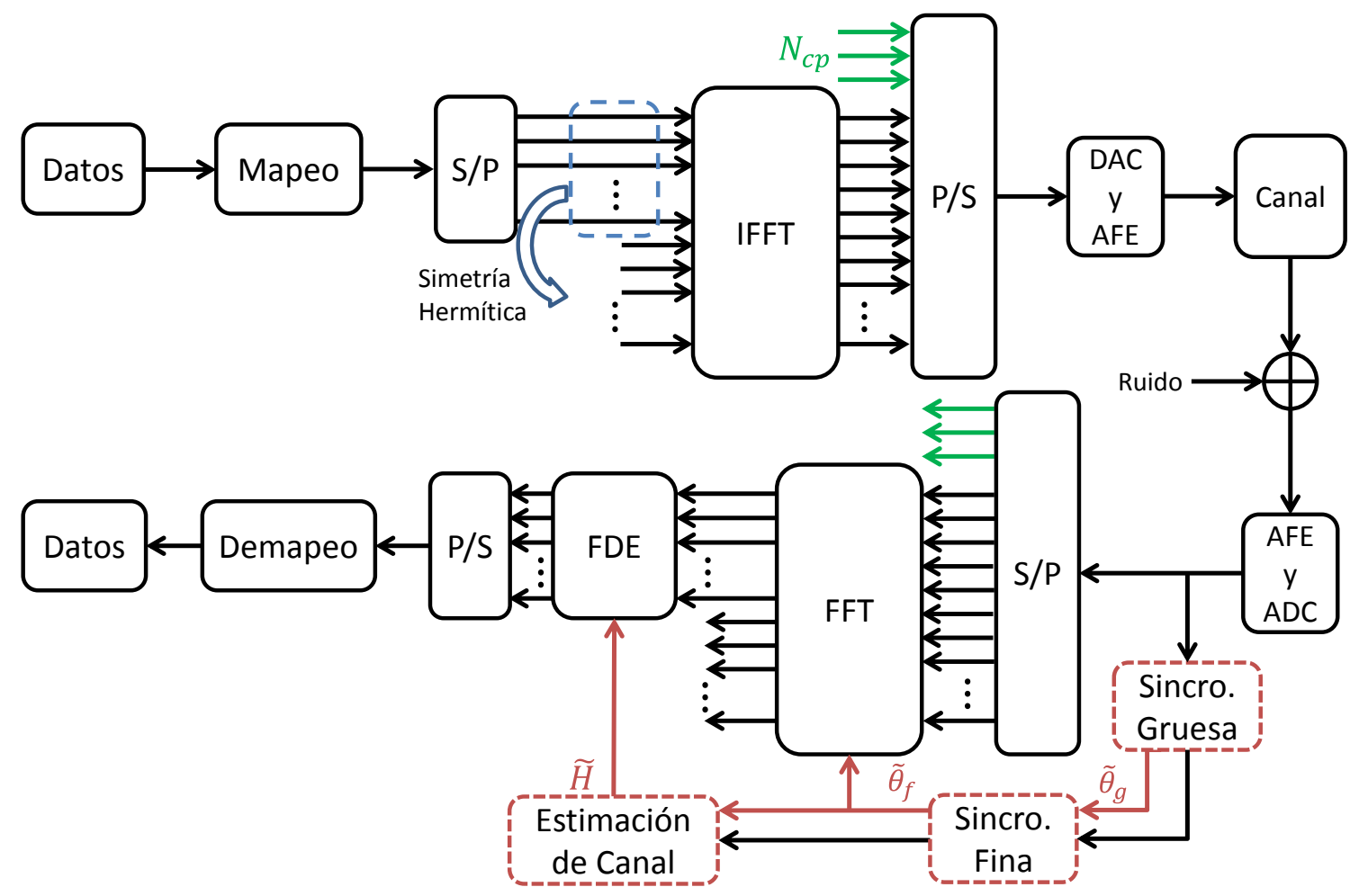

Fig. 4.3 Diagrama en bloques de un sistema DMT básico. 
Diseño de Estrategias de Sincronización y Estimación de Canal para la Mejora de Comunicaciones en Redes Inteligentes de Energía

estas consideraciones, el símbolo piloto puede expresarse entonces como una concatenación de secuencias complementarias $\mathbf{a}^{\prime}$ y $\mathbf{b}^{\prime}$ de longitud $N / 8$, de acuerdo a:

$$
\mathbf{x}^{\prime}=\left[\begin{array}{llllllll}
\mathbf{a}^{\prime} & \mathbf{b}^{\prime} & \mathbf{a}^{\prime} & -\mathbf{b}^{\prime} & -\mathbf{a}^{\prime} & -\mathbf{b}^{\prime} & \mathbf{a}^{\prime} & -\mathbf{b}^{\prime}
\end{array}\right]
$$

El símbolo piloto puede generarse en el dominio de la frecuencia, a partir de $\mathbf{X}^{\prime}=$ DFT $\left\{\mathbf{x}^{\prime}\right\}$, para luego agregar el prefijo cíclico cp de $N_{c p}$ muestras del sistema DMT. Además, se agrega un sufijo cíclico cs de $N_{c s}$ muestras, resultando en el preámbulo extendido $\mathbf{x}$ :

$$
\mathbf{x}=\left[\begin{array}{llllll}
\mathbf{c p} & \mathbf{a} & \mathbf{b} & -\mathbf{a} & \mathbf{b} & \mathbf{c s}
\end{array}\right]
$$

Tanto el prefijo cíclico cp como el sufijo cíclico cs pueden expresarse de acuerdo a:

$$
\begin{gathered}
\mathrm{cp}_{n}=\mathrm{b}_{n+N / 8-N_{c p}}, n=0,1, \ldots, N_{c p}-1 \\
\mathrm{cs}_{n}=\mathrm{a}_{n}, \quad n=0,1, \ldots, N_{c s}-1
\end{gathered}
$$

Dado que la estructura del símbolo piloto es idéntica a la utilizada en el sistema OFDM, es evidente que la correlación cruzada $c_{n}^{\mathbf{X}^{\prime}, \mathbf{x}}$ entre el símbolo extendido $\mathbf{x}$ y el filtro acoplado del símbolo sin extender $\mathbf{x}^{\prime}$ presenta una zona de correlación nula en la vecindad de $n=0$, bajo un canal ideal. En la Fig. 4.4 se presenta a la izquierda la correlación cruzada $c_{n}^{\mathbf{x}, \mathbf{x} \prime}$ para $N=4096, N_{c p}=N_{c s}=512$, y puede verificarse el valor del máximo y la longitud de la zona de correlación nula. En la parte derecha se presenta simplemente un zoom de $c_{n}^{\mathbf{x}, \mathbf{x} \prime}$ para una mejor visualización de la zona de correlación nula.

$$
\begin{gathered}
c_{n}^{\mathbf{x}, \mathbf{x} \prime}=N, \quad n=0 \\
c_{n}^{\mathbf{x}, \mathbf{x} \prime}=0, \quad-N_{c p} \leq n \leq N_{c s}, \quad n \neq 0
\end{gathered}
$$

\subsection{Sincronización gruesa}

Durante una primera etapa del proceso de sincronización, es posible explotar la repetición por segmentos del preámbulo propuesto para computar una métrica $m_{n}$ a partir de la autocorrelación de la señal (real) recibida $r_{n}$ según:

$$
m_{2, n}=\frac{p_{2, n}}{e_{n}}
$$

Donde $p_{n}$ es la autocorrelación de distintos segmentos de la señal recibida $r_{n}$; y $e_{n}$ representa la energía de una parte de la señal. La etapa de sincronización gruesa posee varios propósitos: por un lado posibilita la detección inicial de la señal recibida; por otro 

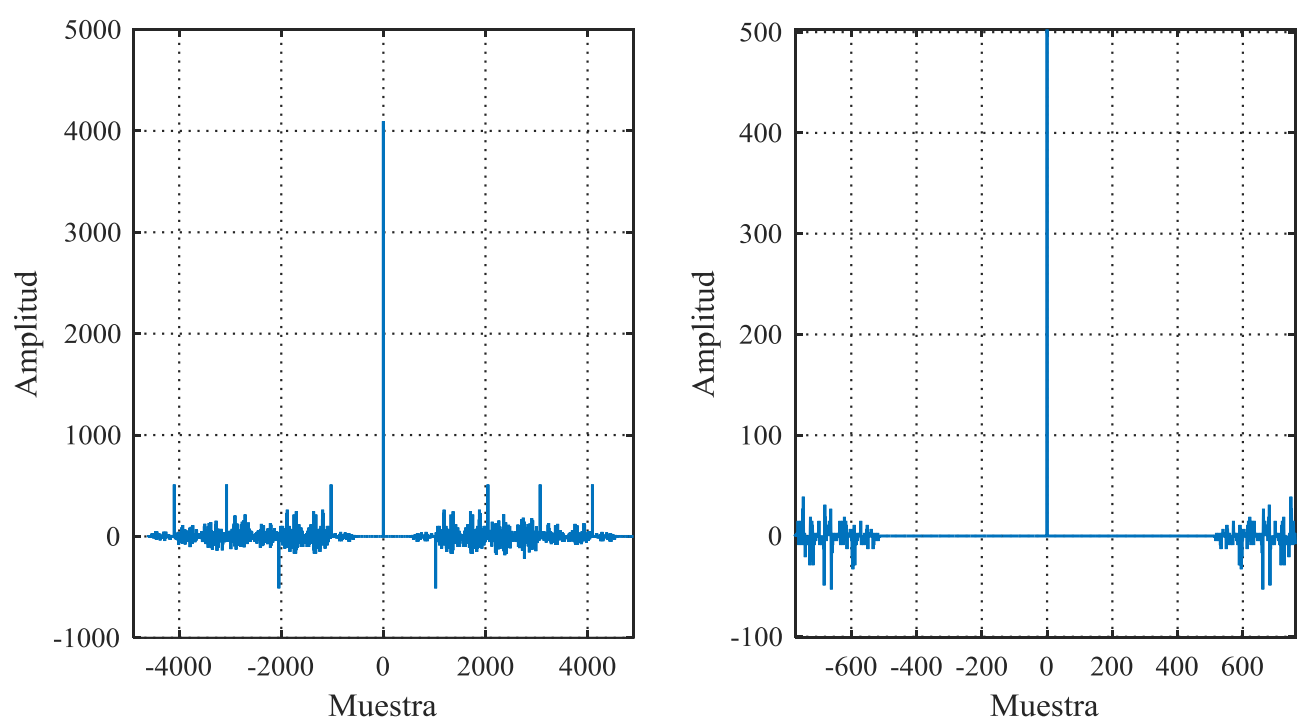

Fig. 4.4 Función de correlación cruzada entre el símbolo extendido y el filtro acoplado del símbolo sin extender.

lado, la ubicación del máximo de la métrica resulta una primera aproximación al instante de sincronismo, sirviendo como punto de partida para la etapa de sincronización fina; $y$, adicionalmente, el valor máximo de la métrica permite obtener un estimador del nivel de ruido presente en la señal, o bien de la relación señal a ruido.

La energía $e_{n}$ en (4.10) puede calcularse a partir de:

$$
e_{n}=\sum_{m=0}^{N-1} r_{n+m} \cdot r_{n+m}
$$

Por otro lado, para el cálculo de autocorrelación, se proponen dos alternativas $p_{1_{n}} \mathrm{y}$ $p_{2 n}$, de acuerdo a:

$$
\begin{gathered}
p_{1, n}=2 \cdot \sum_{m=0}^{\frac{N}{4}-1}-r_{n+m} \cdot r_{n+m+N / 2}+r_{n+m+N / 4} \cdot r_{n+\mathrm{m}+3 \mathrm{~N} / 4} \\
p_{2, n}=2 \cdot \sum_{m=0}^{\frac{N}{8}-1} r_{n+m} \cdot r_{n+m+\frac{3 \mathrm{~N}}{4}}-r_{n+m+\frac{\mathrm{N}}{8}} \cdot r_{n+m+\frac{5 \mathrm{~N}}{8}}-r_{n+m+\frac{\mathrm{N}}{4}} \cdot r_{n+m+\frac{N}{2}} \\
+r_{n+m+\frac{3 \mathrm{~N}}{8}} \cdot r_{n+m+\frac{7 \mathrm{~N}}{8}}
\end{gathered}
$$

Entonces, de acuerdo a (4.10), se definen dos alternativas para la métrica de sincronización gruesa $m_{1_{n}}$ y $m_{2 n}$ basadas en las dos alternativas de autocorrelación $p_{1, n}$ y $p_{2, n}$. En la Fig. 4.5 se presenta el resultado obtenido para las métricas de 

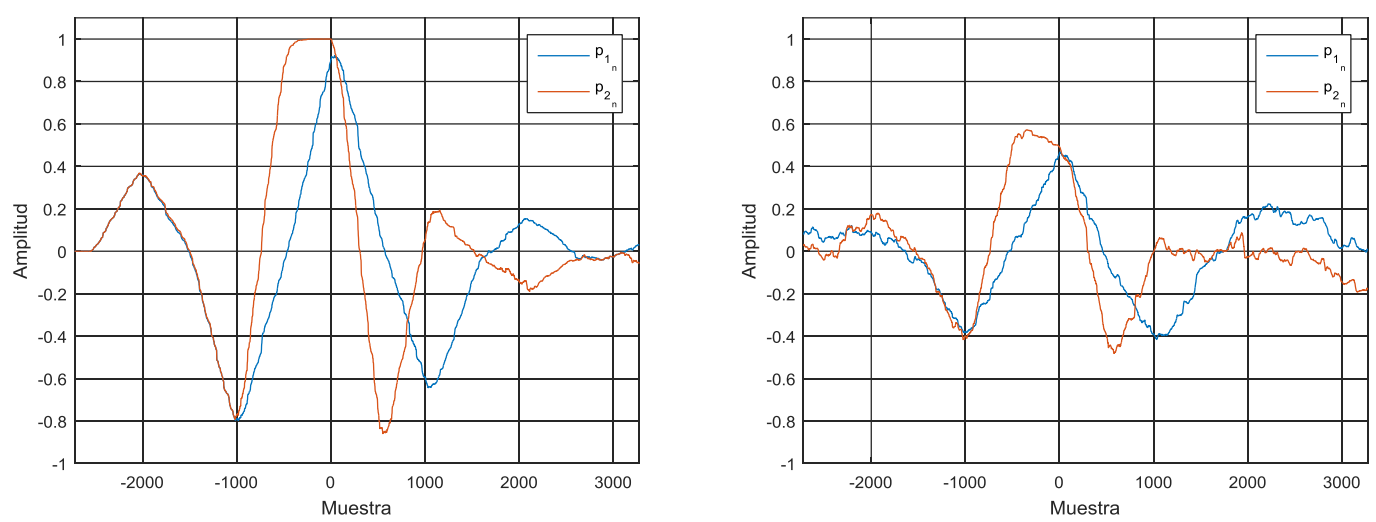

Fig. 4.5 Métricas para sincronización gruesa bajo una realización de canal A, sin agregado de ruido (izquierda) y con ruido de fondo coloreado y una SNR de $3 d B$ (derecha).

sincronización, considerando un canal PLC tipo A en una condición ideal (izquierda) y también incluyendo ruido de fondo coloreado y una $S N R=3 \mathrm{~dB}$ (derecha). El sistema DMT considerado posee $N=4096$ y $N_{c p}=N_{c s}=512$. Puede apreciarse que los valores de las métricas están comprendidos entre -1 y 1 , y que la métrica basada en $p_{1, n}$ posee un valor máximo próximo al instante óptimo de sincronismo $n=0$, mientras que la métrica basada en $p_{2, n}$ presenta una meseta en la métrica, dando lugar a una mayor zona de incerteza. Sin embargo, esta última posee una mayor robustez a la dispersión temporal del canal, lo que se evidencia en el hecho que alcanza un valor máximo mayor que la métrica basada en $p_{1, n}$, alcanzando el valor máximo 1 bajo un canal sin ruido.

Es posible reescribir las muestras de la señal recibida a partir de una parte de señal útil y otra de ruido, de acuerdo a $r_{n}=s_{n}+w_{n}$; donde $s_{n}$ es la parte útil de la señal y $w_{n}$ la parte de ruido de la muestra. A partir de ello, analizando la función de autocorrelación $p_{2, n}$ y considerando la simetría en las muestras recibidas del símbolo piloto, la ecuación (4.13) puede reescribirse como:

$$
\begin{gathered}
p_{2, n}=2 \cdot \sum_{m=0}^{N / 2-1}\left(s_{n+m}+w_{n+m}\right) \cdot\left(s_{n+m}+w_{n+m}^{\prime}\right) \\
r_{n}=s_{n}+w_{n}
\end{gathered}
$$

donde $w_{n+m}^{\prime}$ representan muestras de ruido convenientemente agrupadas, idénticamente distribuidas e independientes. Definiendo la potencia de señal como $\sigma_{s}^{2}=E\left(s_{n}^{2}\right)=P_{t} \cdot\|\mathbf{h}\|^{2}$, donde $P_{t}$ es la potencia transmitida para el símbolo piloto $\mathrm{y}$ $\|\mathbf{h}\|$ es la norma del vector que contiene los coeficientes de respuesta al impulso del 
canal, y la del ruido como $\sigma_{w}^{2}=E\left(w_{n}^{2}\right)$, entonces la relación señal a ruido se define como $S N R=\frac{\sigma_{s}^{2}}{\sigma_{w}^{2}}$. A continuación, la ecuación (4.14) se puede reescribir como:

$$
p_{2, n}=2 \cdot \sum_{m=0}^{N / 2-1} s_{n+m}^{2}+s_{n+m} \cdot\left(w_{n+m}+w^{\prime}{ }_{n+m}\right)+w_{n+m} \cdot w^{\prime}{ }_{n+m}
$$

Para simplificar el análisis de las variables aleatorias, en una primera instancia se supondrán las muestras de ruido como muestras de ruido blanco, para luego analizar el efecto del ruido de fondo coloreado.

\subsubsection{Métrica basada en autocorrelación bajo ruido de fondo blanco gaussiano.}

Dado que la cantidad de subportadoras $N$ del sistema DMT suele ser lo suficientemente grande como para que resulte válido el Teorema Central del Límite, el segundo y tercer término de (4.15) serán variables aleatorias independientes que siguen una distribución Gaussiana [Walpole 2007]. El último término de (4.15) es el producto de dos variables normales independientes con media cero y varianza $\sigma_{w}^{2}$, y la varianza del producto de dos variables independientes $\mathrm{X}$ e $\mathrm{Y}$ es:

$$
\begin{gathered}
\operatorname{Var}(X Y)=[E(X)]^{2} \operatorname{Var}(Y)+[E(Y)]^{2} \operatorname{Var}(X)+\operatorname{Var}(X) \operatorname{Var}(Y) \\
E(X Y)=E(X) E(Y)
\end{gathered}
$$

Como tanto $\mathrm{E}(\mathrm{X})=\mathrm{E}(\mathrm{Y})=0$, la media es cero y la varianza de ese término es $\sigma_{w}^{4}$. $\mathrm{Al}$ sumarlos todos, aplicar el TCL,y multiplicar por dos, la varianza resulta $2 N \sigma_{w}^{4}$. El segundo término es la suma de dos normales independientes escaladas por todos los términos $s_{i}$. La varianza de cada término Gaussiano aumenta por $s_{i}^{2}$, y al sumar todas las normales, se suman sus varianzas (tienen media cero) y resulta en una normal de media cero y varianza $4 N \sigma_{s}^{2} \sigma_{w}^{2}$. Finalmente, como se asumen independientes los términos, se suman las varianzas y la media está dada por el valor constante $N \cdot \sigma_{S}^{2}=$ $2 \sum_{m=0}^{N / 2-1} s_{n+m}^{2}$. Finalmente, como la potencia transmitida $P_{t}$ y el canal $\mathbf{h}$ se asumen constantes para toda una trama transmitida, la ecuación (4.15) puede describirse como una variable aleatoria $\mathrm{P}$ de distribución normal, con media y varianza:

$$
\begin{gathered}
\mu_{P}=N \cdot \sigma_{S}^{2} \\
\sigma_{P}^{2}=4 N \sigma_{S}^{2} \sigma_{w}^{2}+2 N \sigma_{w}^{4}
\end{gathered}
$$

Por otro lado, los términos de energía $e_{n}$ de (4.11) resultan: 
Diseño de Estrategias de Sincronización y Estimación de Canal para la Mejora de Comunicaciones en Redes Inteligentes de Energía

$$
\begin{gathered}
e_{n}=\sum_{m=0}^{N / 2-1}\left(s_{n+m}+w_{n+m}\right)^{2}+\sum_{m=0}^{N / 2-1}\left(s_{n+m}+w_{n+m}^{\prime}\right)^{2} \\
=\sum_{m=0}^{N / 2-1} 2 \cdot s_{n+m}^{2}+2 \cdot s_{n+m} \cdot\left(w_{n+m}+w^{\prime}{ }_{n+m}\right)+w_{n+m}^{2}+w_{n+m}^{\prime 2}
\end{gathered}
$$

Los últimos dos términos de (4.20) son variables aleatorias que siguen una distribución chi-cuadrado de un grado de libertad. Aplicando el TCL a cada una, y sumándolas, resultan en una variable normal de media $N \sigma_{w}^{2}$ y varianza $2 N \sigma_{w}^{4}$. El segundo es idéntico al de (4.15), resultando en una variable normal de media cero y varianza $4 N \sigma_{S}^{2} \sigma_{w}^{2}$. Por último, del primer término se desprende $\sum_{m=0}^{N / 2-1} 2 \cdot s_{n+m}^{2}=N \sigma_{S}^{2}$. Por lo tanto, la energía resulta en una variable aleatoria E, que sigue una distribución normal, con media y varianza:

$$
\begin{gathered}
\mu_{E}=N \cdot\left(\sigma_{S}^{2}+\sigma_{w}^{2}\right) \\
\sigma_{E}^{2}=4 N \sigma_{S}^{2} \sigma_{w}^{2}+2 N \sigma_{w}^{4}
\end{gathered}
$$

Finalmente, la métrica de sincronización en el instante de sincronismo óptimo $n_{\text {ópt }}$ puede expresarse de acuerdo a:

$$
m_{2, n_{\mathrm{o} p t}}=\frac{\sum_{m=0}^{N / 2-1} 2 s_{n_{\mathrm{o} p t}+m}^{2}+2 s_{n_{\mathrm{o} p t}+m}\left(w_{n_{\mathrm{o} p t}+m}+w^{\prime}{ }_{n_{\mathrm{o} p t}+m}\right)+2 w_{n_{\mathrm{o} p t}+m} w^{\prime}{ }_{n_{\mathrm{o} p t}+m}}{\sum_{m=0}^{N / 2-1} 2 s_{n_{\mathrm{o} p t}^{2}+m}^{2}+2 s_{n_{\mathrm{o} p t}+m}\left(w_{n_{\mathrm{o} p t}+m}+w^{\prime}{ }_{n_{\mathrm{o} p t}+m}\right)+w_{n_{\mathrm{o} p t}+m}^{2}+w_{n_{\mathrm{o} p t}+m}^{\prime 2}}
$$

Definiendo las variables aleatorias $X_{1}=\sum_{m=0}^{N / 2-1} 2 \cdot s_{n_{\mathrm{o} p t}+m} \cdot\left(w_{n_{\mathrm{o} p t}+m}+w^{\prime}{ }_{n_{\mathrm{o} p t}+m}\right)$, $X_{2}=\sum_{m=0}^{N / 2-1} w_{n_{\mathrm{o} p t}+m} \cdot w^{\prime}{ }_{n_{\mathrm{o} p t}+m} \mathrm{y} X_{3}=\sum_{m=0}^{N / 2-1} w_{n_{\mathrm{o} p t}+m}^{2}+w_{n_{\mathrm{o} p t}+m}^{\prime 2}$, puede reescribirse como:

$$
m_{2, n_{\mathrm{o} p t}}=\frac{\mu_{P}+X_{1}+X_{2}}{\mu_{P}+X_{1}+X_{3}}=\frac{\mu_{P}+X_{1}+X_{2}}{\mu_{E}+X_{1}+X_{3}^{\prime}}
$$

Donde $X^{\prime}{ }_{3}=X_{3}-N \sigma_{w}^{2}$. Tanto el numerador como el denominador son variables aleatorias gaussianas, con media mucho mayor que el desvío estándar, por lo que se puede utilizar la aproximación $a(1+b) /(1+c) \approx a(1+b)(1-c)$ :

$$
m_{2, n_{\mathrm{o} p t}} \approx\left[\frac{\mu_{P}}{\mu_{E}}+\frac{X_{1}+X_{2}}{\mu_{E}}\right]\left[1-\frac{\left(X_{1}+X^{\prime}{ }_{3}\right)}{\mu_{E}}\right] \approx \frac{\mu_{P}}{\mu_{E}}+\frac{X_{1}+X_{2}}{\mu_{E}}-\frac{\mu_{P}\left(X_{1}+X^{\prime}{ }_{3}\right)}{\mu_{E}^{2}}
$$


Finalmente, el valor de la métrica resulta en una variable aleatoria gaussiana $M_{2}$ ót , con media y varianza:

$$
\begin{gathered}
\mu_{M_{\mathrm{z}_{\mathrm{o}} \mathrm{p} t}}=\frac{\mu_{P}}{\mu_{E}}=\frac{\sigma_{S}^{2}}{\left(\sigma_{S}^{2}+\sigma_{w}^{2}\right)}=\frac{S N R}{1+S N R} \\
\sigma_{M_{2_{\mathrm{o}} \mathrm{p} t}}^{2}=\frac{4 S N R}{N(S N R+1)^{2}}-\frac{8 S N R^{2}}{N(S N R+1)^{3}}+\frac{4 S N R^{3}}{N(S N R+1)^{4}}+\frac{2 S N R^{2}}{N(S N R+1)^{4}} \\
+\frac{2}{N(S N R+1)^{2}}
\end{gathered}
$$

En una primera etapa de sincronización se produce la detección del símbolo piloto cuando la métrica supera un determinado umbral. Por lo tanto, la distribución de la métrica en el instante óptimo de sincronización resulta de interés debido a que permite evaluar la probabilidad de que el símbolo piloto no sea detectado, es decir, que el valor de la métrica sea inferior al umbral de detección fijado. En ese sentido, también resulta importante obtener las expresiones correspondientes a la métrica de sincronización fuera de la zona de sincronización, por ejemplo en las muestras anteriores al preámbulo, donde sólo se encuentran presentes muestras de ruido, o bien posteriores donde las muestras corresponden a otros símbolos diferentes al símbolo piloto, que pueden ser directamente los símbolos de datos aleatorios. Esto es importante debido a que permite predecir la probabilidad de falsa detección que resulta cuando la métrica supera el umbral de detección fuera de la zona del símbolo piloto.

Considerando cualquier muestra anterior al arribo del símbolo piloto $n_{\text {ant }}$ en la que no se encuentra presente señal útil, la expresión de la métrica de (4.23) resulta en:

$$
m_{2, n_{\text {ant }}}=\frac{\sum_{m=0}^{N / 2-1} 2 w_{n_{\text {ant }}+m} \cdot w^{\prime}{ }_{n_{a n t}+m}}{\sum_{m=0}^{N / 2-1} w_{n_{a n t}+m}^{2}+w_{n_{a n t}+m}^{2}}=\frac{X_{2}}{N \sigma_{w}^{2}+X_{3}^{\prime}} \approx \frac{X_{2}}{N \sigma_{w}^{2}}
$$

Como la media del numerador es cero y la del denominador es $N \sigma_{w}^{2}$, mucho mayor que su desvío estándar, entonces la métrica puede aproximarse a una variable aleatoria normal $M_{2 \text { ant }}$, con parámetros:

$$
\begin{gathered}
\mu_{M_{2_{a n t}}}=0 \\
\sigma_{M_{2_{\text {ant }}}}^{2}=2 / N
\end{gathered}
$$

A partir de este resultado, se puede fijar entonces un umbral de detección lo suficientemente alto como para mantener una baja probabilidad de falsa detección. Por 
Diseño de Estrategias de Sincronización y Estimación de Canal para la Mejora de Comunicaciones en Redes Inteligentes de Energía

ejemplo, si se desea una probabilidad de $10^{-7}$ de falsa detección de una de las muestras de la métrica cuando sólo contiene muestras de ruido, a partir de la función de distribución acumulada inversa (Inverse Cumulative Distribucion Function - ICDF) de la distribución normal, se obtiene un umbral $\gamma \approx 7,35 / \sqrt{N}$, que para $N=4096$ resulta en $\gamma \approx 0,115$.

En la Fig. 4.6 se muestran los resultados obtenidos por simulaciones para las dos métricas bajo análisis. Se grafican las PDFs empíricas de los valores en el instante óptimo de sincronismo y en instantes previos al arribo del símbolo piloto para 50.000 realizaciones de un canal PLC tipo A normalizado (se asegura $\|\mathbf{h}\|^{2}=1$ en cada realización del canal), bajo ruido blanco Gaussiano, para diferentes valores de relación señal a ruido. El sistema considerado es de $N=4096$, y el símbolo piloto transmitido
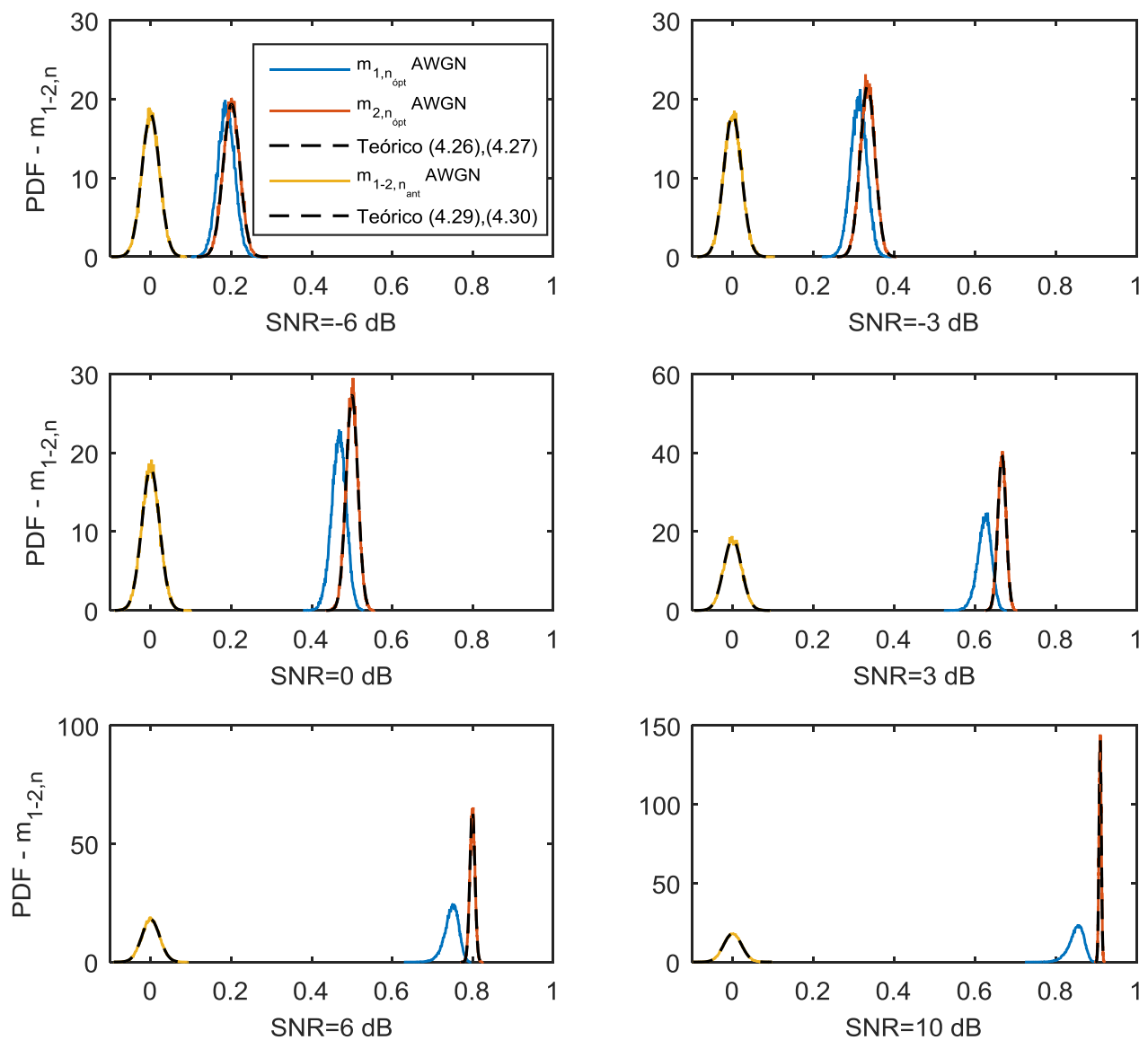

Fig. 4.6 PDFs del valor de las métricas de sincronización gruesa en el instante de sincronización óptima $y$ anteriores, obtenidas por simulaciones, bajo un canal PLC tipo A normalizado con ruido blanco Gaussiano y distintos valores de SNR. 
es el propuesto de acuerdo a (4.7). Puede apreciarse que el resultado del análisis efectuado para la métrica $m_{2}$ caracterizado por (4.26), (4.27), (4.29) y (4.30) se ajusta a lo obtenido por simulaciones. Adicionalmente, se observa que la métrica $m_{1, n_{\text {ópt }}}$ alcanza valores menores que $m_{2, n_{\text {ópt }}}$ en concordancia con lo analizado en la Fig. 4.5. Finalmente, con respecto a la capacidad de detección del símbolo piloto, se observa que las dos métricas poseen una buena capacidad de detección para el umbral $\gamma$, inclusive hasta valores de $S N R$ de $-6 \mathrm{~dB}$. La probabilidad de que la métrica en el instante óptimo de sincronización $m_{2, n_{\text {ópt }}}$ no supere el umbral para esta condición de $S N R$, a partir de la CDF de la normal descripta por los parámetros de (4.26) y (4.27), es de $1,5 \cdot 10^{-5}$. Es importante tener en cuenta que la métrica $m_{2, n}$ presenta una meseta de varias muestras independientes e idénticamente distribuidas a la del instante óptimo de sincronismo (véase la Fig. 4.5), que dependerá de la longitud efectiva del canal y su diferencia con la longitud del prefijo cíclico $N_{c p}-L$. Por lo tanto, la probabilidad de falsa detección será aún más baja.

\subsubsection{Métrica basada en autocorrelación bajo ruido de fondo coloreado}

El análisis de la métrica de sincronización basada en AC considerando ruido de fondo blanco gaussiano permitió obtener resultados preliminares de la capacidad de detección del símbolo piloto a partir de un análisis simplificado. Sin embargo, el ruido de fondo en el canal PLC es realmente coloreado, y presenta un incremento considerable a menores valores de frecuencia, por lo que la métrica de sincronización se verá afectada debido a que las muestras de ruido no serán independientes entre sí, dando lugar a incrementos en las varianzas de las variables aleatorias de (4.23) que incluyen términos de ruido en sus expresiones.

En [Benyoucef 2003], la densidad espectral de potencia del ruido coloreado se expresa a partir de un modelo de tres parámetros:

$$
S_{w_{f}}=a+b \cdot f^{c} \mathrm{dBm} / \mathrm{Hz}
$$

donde $\mathrm{a}, \mathrm{b}$ y c son parámetros que se ajustan en función de campañas de medición. En el generador de ruido introducido en [Prasad 2016], considerando el peor caso, los parámetros son $(a ; b ; c)=(-145 ; 53,23 ;-0,337)$ al igual que en [Esmailian 2003]. Bajo estos parámetros, la densidad espectral de potencia del ruido de fondo coloreado resulta en la Fig. 4.7. 
Diseño de Estrategias de Sincronización y Estimación de Canal para la Mejora de Comunicaciones en Redes Inteligentes de Energía

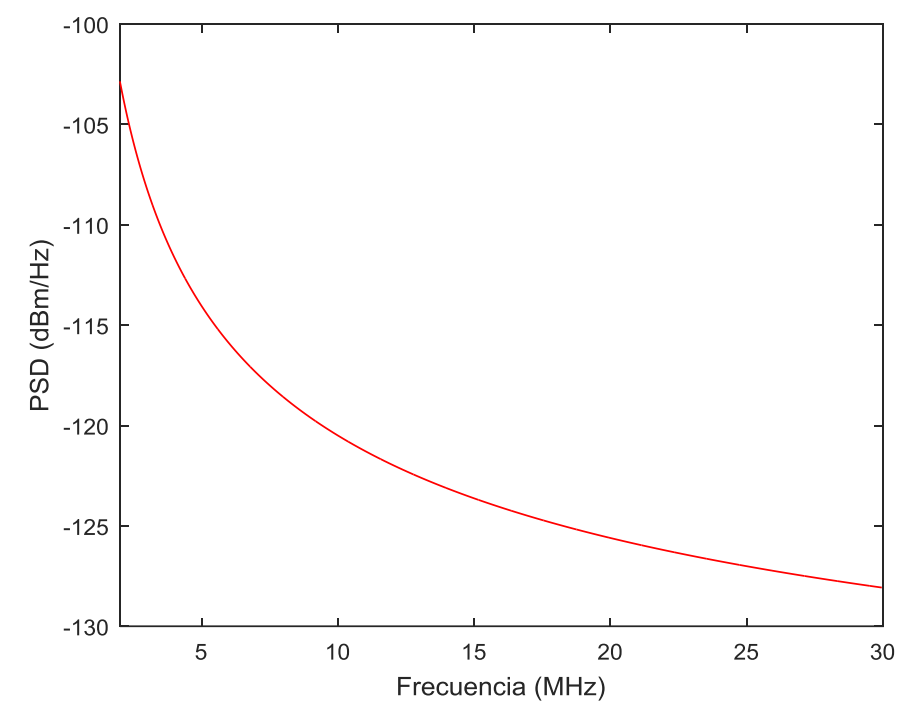

Fig. 4.7 Densidad espectral de potencia del ruido de fondo coloreado.

Es posible sintetizar el ruido de fondo coloreado a partir del filtrado de un proceso de ruido blanco gaussiano de varianza $\sigma_{w}^{2}$ con un filtro que resulta de la IDFT de la raíz cuadrada de la densidad espectral de potencia normalizada, como se presenta en (4.32) [Benyoucef 2003]. Este proceso se esquematiza en la Fig. 4.8.

$$
g_{n}=\frac{1}{N} \sum_{k=0}^{N-1} \sqrt{S_{w_{k}}} \cdot e^{i 2 \pi k n / N}
$$

Es evidente entonces que, en el modelo discreto del ruido, y para una densidad espectral de potencia en particular, es posible obtener los correspondientes coeficientes del filtro que produce ese perfil de ruido. En la Fig. 4.9 se presenta la respuesta al impulso del filtro $g_{n}$ otbenido, en el dominio del tiempo, y sus coeficientes más significativos se presentan en el Anexo A. Los coeficientes se encuentran normalizados de manera tal de no afectar la varianza resultante del proceso de ruido respecto de la varianza del proceso AWGN. Las muestras de ruido no serán independientes como en el caso de ruido blanco, y la correlación entre muestras estará descripta por la secuencia de autocorrelación (Auto-Correlation Sequence - ACS) del proceso, y estará directamente relacionada con el filtro equivalente que colorea el ruido.

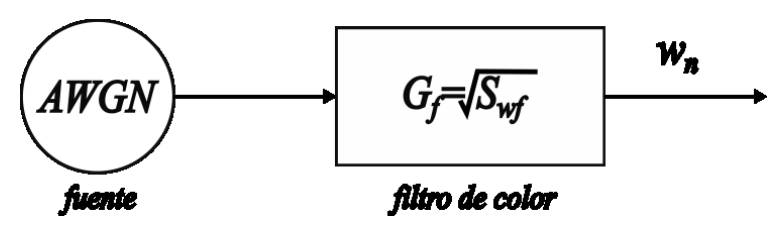

Fig. 4.8 Filtro equivalente aplicado a un proceso de ruido blanco Gaussiano para generar ruido de fondo coloreado. 


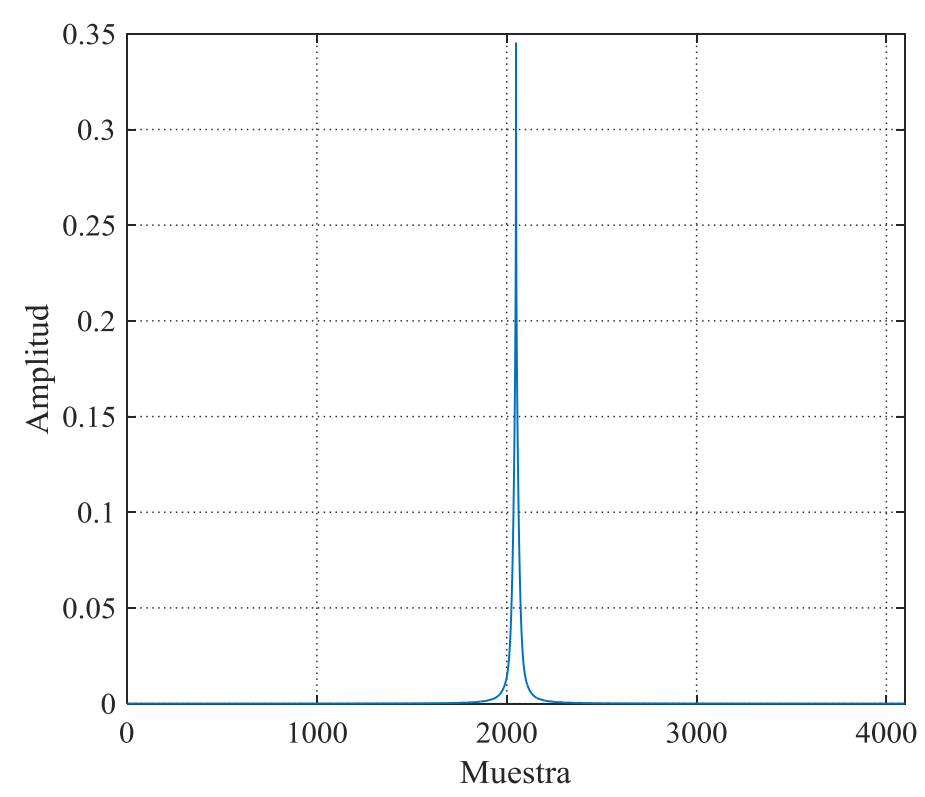

Fig. 4.9 Respuesta al impulso del filtro obtenido en el dominio del tiempo a partir de la PSD del ruido de fondo coloreado.

El resultado de una transformación lineal de un proceso estacionario en el sentido amplio (Wide-Sense Stationary - WSS) resulta útil no sólo para obtener la media y covarianza entre las muestras del proceso de ruido coloreado, ya que las sumatorias de las variables intervinientes en el cálculo de la métrica de sincronización gruesa también pueden entenderse como resultado del filtrado lineal del proceso de ruido. Adicionalmente, las operaciones no lineales de elevar al cuadrado las muestras, o el producto de distintas muestras del proceso de ruido producen otros procesos WSS, y tienen un efecto particular en la media y ACS de los mismos. El resultado de aplicar operaciones lineales y no lineales al proceso WSS Gaussiano de ruido blanco se resume en el Anexo B. A partir de aplicar el filtro equivalente $\mathbf{g}$ que colorea el proceso de ruido blanco, de acuerdo a (B.3), la media, ACS y varianza del proceso resultante de ruido coloreado resultan:

$$
\begin{gathered}
\mu_{g}=\sum_{k=-\infty}^{\infty} g_{k} \mu_{A W G N}=0 \\
\Upsilon_{g_{k}}=\sigma_{w}^{2} g_{-k} * g_{k}=\sigma_{w}^{2} \sum_{j=-\infty}^{+\infty} g_{j} g_{k+j} \\
\operatorname{Var}\left(g_{k}\right)=\Upsilon_{g_{0}}=\sigma_{w}^{2}
\end{gathered}
$$

Por lo mencionado anteriormente, se requiere un análisis particular de las variables aleatorias definidas en el cálculo de la métrica de sincronización gruesa, en (4.24): 
Diseño de Estrategias de Sincronización y Estimación de Canal para la Mejora de Comunicaciones en Redes Inteligentes de Energía

$$
\begin{aligned}
m_{2, n}= & \frac{\sum_{m=0}^{\frac{N}{2}-1} 2 \cdot s_{n+m}^{2}+2 \cdot s_{n+m} \cdot\left(w_{n+m}+w_{n+m}^{\prime}\right)+2 w_{n+m} \cdot w^{\prime}{ }_{n+m}}{\sum_{m=0}^{\frac{N}{2}-1} 2 \cdot s_{n+m}^{2}+2 \cdot s_{n+m} \cdot\left(w_{n+m}+w^{\prime}{ }_{n+m}\right)+w_{n+m}^{2}+w_{n+m}^{\prime 2}} \\
= & \frac{\sum_{m=0}^{\frac{N}{2}-1} 2 \cdot s_{n+m}^{2}+X_{1}+X_{2}}{\sum_{m=0}^{\frac{N}{2}-1} 2 \cdot s_{n+m}^{2}+X_{1}+X_{3}}
\end{aligned}
$$

Con respecto a la variable $X_{1}$, ésta puede reescribirse como $X_{1}=\sum_{m=0}^{N-1}+2 \cdot s_{n_{\text {ópt }}+m}$. $w_{n_{\text {ó } p}+m}$, y teniendo cuenta que $s_{n_{\text {ó } p t}+m}=\sum_{l=0}^{\mathrm{L}-1} \sqrt{P_{T}} h_{l} \cdot x_{m-l}^{\prime *}$, puede expandirse de acuerdo a:

$$
\begin{aligned}
& \mathrm{X}_{1}=2 \sqrt{P_{T}} h_{0}\left(w_{n_{\text {opt }}} x_{0}^{\prime *}+w_{n_{\text {opt }}+1} x_{1}^{\prime *}+\cdots+w_{n_{\text {opt }}+N-1} x_{N-1}^{\prime *}\right) \\
& +2 \sqrt{P_{T}} h_{1}\left(w_{n_{\mathrm{opt}}} x_{-1}^{\prime *}+w_{n_{\mathrm{o} p t}+1} x_{0}^{\prime^{*}}+\cdots+w_{n_{\mathrm{o} p t}+N-1} x_{N-2}^{\prime *}\right) \\
& +2 \sqrt{P_{T}} h_{\mathrm{L}-1}\left(w_{n_{\mathrm{o} p t}} x^{\prime *}{ }_{-L+1}^{*}+w_{n_{\mathrm{o} p t}+1} x_{-L+2}^{\prime *}+\cdots+w_{n_{\mathrm{o} p t}+N-1} x_{N-\mathrm{L}}^{\prime *}\right)
\end{aligned}
$$

Donde el término $\left(w_{n_{\mathrm{o} p t}} x_{0}^{\prime *}+w_{n_{\mathrm{o} p t}+1} x_{1}^{\prime *}+\cdots+w_{n_{\mathrm{o} p t}+N-1} x_{N-1}^{\prime *}\right)$ puede entenderse como una muestra resultante del filtrado lineal del proceso de ruido Gaussiano coloreado, donde el filtro es el símbolo piloto x. En la Fig. 4.10 se presentan las ACS del símbolo piloto, del filtro que colorea el ruido, y del filtro equivalente correspondiente a la convolución entre ambos. Las ACS del símbolo piloto y del filtro equivalente se encuentran normalizadas por la longitud del símbolo piloto $N$ para una adecuada

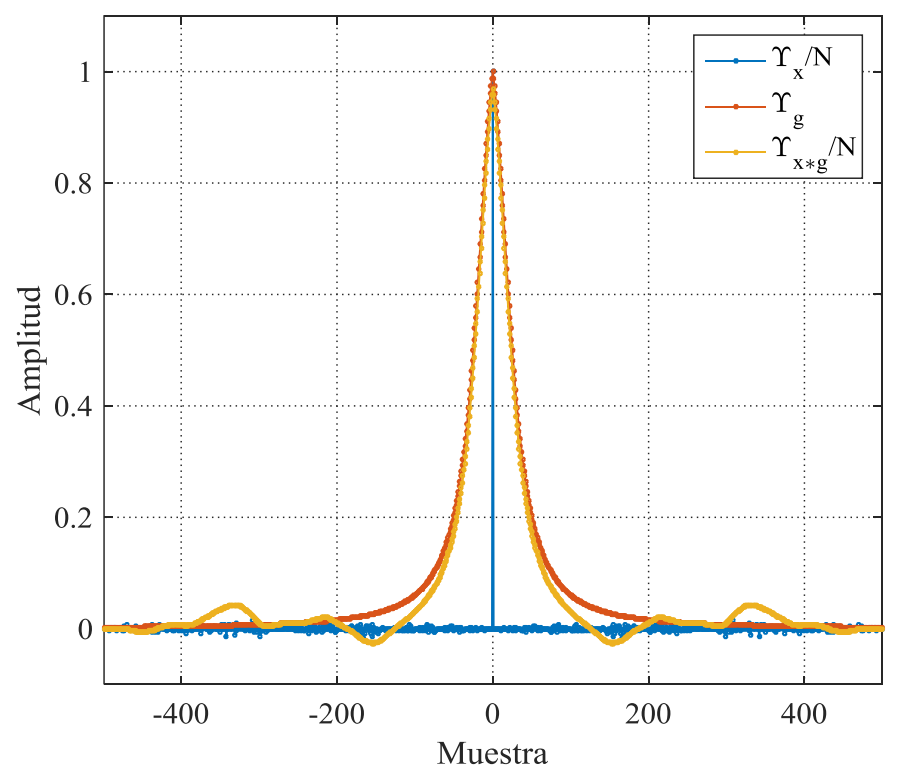

Fig. 4.10 Secuencias de autocorrelación del símbolo piloto $\mathbf{x}$, el filtro que colorea el ruido $\mathbf{g}$ y la combinación de ambos. 
representación. Como la media del proceso de ruido es cero, entonces la varianza puede obtenerse a partir de $\Upsilon_{\mathbf{g} * \mathbf{x}_{0}}$ de acuerdo a (B.2). Puede observarse que la ACS del símbolo piloto posee muy bajos lóbulos laterales, por lo que la varianza resultante es muy similar a la del proceso de ruido coloreado, multiplicada por el factor $N$, es decir $N \sigma_{w}^{2}$. En el resto de los términos de (4.58), los filtros equivalentes son versiones cíclicamente desplazadas del símbolo piloto, y sus ACS son muy similares a la del símbolo sin desplazar, por lo que las varianzas resultan muy similares.

Si además se asumen independientes los coeficientes del canal $h_{l}$, entonces la variable $X_{1}$ resulta en una variable Gaussiana de media cero con varianza $\operatorname{var}\left(X_{1}\right)=4 \sigma_{s}^{2} N \sigma_{w}^{2}$.

Con respecto a la variable $X_{2}$, ésta es el resultado de sumar productos de pares de muestras del proceso de ruido Gaussiano coloreado, donde el espaciamiento entre muestras multiplicadas se corresponde con los productos de (4.13). El mínimo espaciamiento entre muestras está dado por $N / 4$. En el sistema considerado, $N=4096$, por lo que las muestran están espaciadas al menos 1024 muestras. Puede observarse en la Fig. 4.10 que las muestras del proceso de ruido coloreado que se multiplican se encuentran prácticamente incorreladas ya que $\Upsilon_{g_{k}} \approx 0$ para $|k|>400$, y teniendo en cuenta (B.5) y el factor de 2, entonces el proceso resultante del producto de muestras de ruido $X^{\prime}{ }_{2}$, que no es Gaussiano, posee media y ACS:

$$
\begin{gathered}
\mu_{X{ }^{\prime} 2} \approx 0 \\
\Upsilon_{X^{\prime}{ }_{2} k}=4 \Upsilon_{g_{k}}^{2}=4 \sigma_{w}^{4}\left(\sum_{j=-\infty}^{+\infty} g_{j} g_{k+j}\right)^{2}
\end{gathered}
$$

Como $X_{2}$ incluye la suma de $N / 2$ productos consecutivos, $X_{2}$ puede entenderse como una muestra resultante de pasar el proceso $X^{\prime}{ }_{2 n}$ por un filtro cuya respuesta impulsiva son $N / 2$ impulsos sucesivos. Puede aplicarse el TCL por lo que la distribución de $X_{2}$ resulta Gaussiana, y a partir de (B.3) se concluye que la varianza de $X_{2}$ resulta:

$$
\operatorname{Var}\left(X_{2}\right)=\sigma_{w}^{4}\left[2 N\left(\sum_{k=-\infty}^{+\infty} g_{k}^{2}\right)^{2}+8 \sum_{j=1}^{\frac{N}{2}-1}(N / 2-j)\left(\sum_{k=-\infty}^{+\infty} g_{k} g_{k+j}\right)^{2}\right]=\sigma_{w}^{4} \Sigma_{1}
$$

donde $\Sigma_{1}$ representa la expresión entre corchetes de (4.37). Por lo tanto, la varianza de $X_{2}$ depende de la longitud del símbolo piloto $N$, la varianza del proceso de ruido $\sigma_{w}^{2} \mathrm{y}$ los coeficientes del filtro $\mathbf{g}$ que colorea el ruido. 
Diseño de Estrategias de Sincronización y Estimación de Canal para la Mejora de Comunicaciones en Redes Inteligentes de Energía

Por otro lado, la variable $X_{3}=\sum_{m=0}^{N / 2-1}\left(w_{n+m}^{2}+w_{n+m}^{2}\right)=\sum_{m=0}^{N-1} w_{n+m}^{2}$, resulta de la transformación no lineal de elevar al cuadrado las muestras del proceso de ruido coloreado, y luego sumar $N$ muestras consecutivas. A partir de (B.4), se obtiene la media y ACS del proceso resultante de elevar las muestras del proceso de ruido al cuadrado $X^{\prime}{ }_{3_{n}}$ de acuerdo a:

$$
\begin{gathered}
\mu_{X_{3}^{\prime}}=\sigma_{w}^{2} \\
\Upsilon_{X_{3}^{\prime} k}=\sigma_{w}^{4}+2 \Upsilon_{g_{k}}^{2}
\end{gathered}
$$

Luego de sumar las $N$ muestras, por el TCL la distribución de $X_{3}$ será Gaussiana, y la media y varianza de la variable, que dependen de $N$ y el filtro $\mathbf{g}$, resultan:

$$
\begin{gathered}
\mu_{X_{3}}=N \sigma_{w}^{2} \\
\operatorname{Var}\left(X_{3}\right)=\sigma_{w}^{4}\left[2 N\left(\sum_{k=-\infty}^{+\infty} g_{k}^{2}\right)^{2}+4 \sum_{j=1}^{N-1}(N-j)\left(\sum_{k=-\infty}^{+\infty} g_{k} g_{k+j}\right)^{2}\right]=\sigma_{w}^{4} \Sigma_{2}
\end{gathered}
$$

Finalmente, la métrica de sincronización en el instante óptimo de sincronismo sigue una distribución Gaussiana, con media y varianza:

$$
\begin{gathered}
\mu_{M_{2^{\prime} \mathrm{o} p t}}=\frac{\mu_{P}}{\mu_{E}}=\frac{N \sigma_{S}^{2}}{N\left(\sigma_{S}^{2}+\sigma_{w}^{2}\right)}=\frac{S N R}{1+S N R} \\
\sigma_{M_{2_{\mathrm{o}} p t}}^{2}=\frac{4 S N R}{N(S N R+1)^{2}}-\frac{8 S N R^{2}}{N(S N R+1)^{3}}+\frac{4 S N R^{3}}{N(S N R+1)^{4}} \\
+\frac{\Sigma_{1}}{N^{2}(S N R+1)^{2}}+\frac{\Sigma_{2} S N R^{2}}{N^{2}(S N R+1)^{4}}
\end{gathered}
$$

Fijando los parámetros de densidad espectral de potencia considerados para la generación del ruido de fondo a partir de (4.31) y longitud del símbolo piloto de $N=4096$, el incremento proporcional de la varianza de los últimos dos términos de (4.41) respecto al caso de ruido blanco en (4.27) es $\Sigma_{1} / 2 N \approx 37,39$ y $\Sigma_{2} / 2 N \approx$ 37,54 .

Recordando que la expresión de la métrica para cualquier muestra anterior en la que no se encuentra presente señal útil es:

$$
m_{2, n_{a n t}}=\frac{\sum_{m=0}^{N / 2-1} 2 w_{n_{a n t}+m} \cdot w_{n_{a n t}+m}^{\prime}}{\sum_{m=0}^{N / 2-1} w_{n_{a n t}+m}^{2}+w_{n_{a n t}+m}^{\prime 2}}=\frac{X_{2}}{N \sigma_{w}^{2}+X_{3}} \approx \frac{X_{2}}{N \sigma_{w}^{2}}
$$


Nuevamente, la media del numerador es cero y la del denominador es $N \sigma_{w}^{2}$, mucho mayor que su desvío estándar, por lo que la varianza de $M_{2}$ ant descripta por (4.29) y (4.30) se verá aumentada por el cambio de la varianza en $X_{2}$, resultando en una variable normal de media y varianza:

$$
\begin{gathered}
\mu_{M_{2_{a} a t}}=0 \\
\sigma_{M_{2_{\text {ant }}}^{2}}^{2}=\Sigma_{1} / N^{2}
\end{gathered}
$$

A partir de este resultado, se debe recalcular el umbral de detección para mantener la misma probabilidad de falsa detección. Nuevamente, si se desea una probabilidad de $10^{-7}$ de falsa detección de una de las muestras de la métrica cuando sólo contiene muestras de ruido, a partir de la ICDF de la distribución normal, se obtiene un umbral $\gamma \approx 7,35 \sqrt{\Sigma_{1}} / N$, que para $N=4096$ y $\Sigma_{1} / 2 N \approx 37,39$ resulta en $\gamma \approx 0,705$. El análisis realizado se ha comprobado mediante simulaciones, y en la Fig. 4.11 se grafican las PDFs empíricas de los valores de las métricas en el instante óptimo de sincronización, así como en un instante previo al arribo del símbolo piloto, para distintos valores de $S N R$ e idénticos parámetros que aquellos utilizados para generar la Fig. 4.6, con la modificación del agregado de ruido de fondo coloreado en lugar de ruido blanco. Es evidente que ahora las PDFs se solapan considerablemente a bajas $S N R$, debido a que la dependencia entre las muestras del ruido coloreado aumenta considerablemente la varianza de la métrica cuando se encuentra presente únicamente ruido en la señal procesada. Esto tiene como implicancia la definición de un umbral de detección demasiado grande $(\gamma \approx 0,705)$ para mantener una baja probabilidad de falsa detección, lo que dificulta la detección del símbolo piloto a bajas SNR. De hecho, al evaluar la CDF de la métrica en el instante óptimo de sincronización, para condiciones de $S N R$ de hasta $0 \mathrm{~dB}$, la probabilidad de que la métrica sea inferior al umbral es prácticamente 1. La detección se hace factible recién para condiciones de $S N R$ mayores a $3 \mathrm{~dB}$, ya para ese valor de $S N R$ la probabilidad de que la métrica en el instante óptimo de sincronización sea menor que el umbral es de 0,76 .

Puede concluirse entonces que las técnicas que utlizan AC se ven altamente degradadas por el ruido de fondo coloreado, lo que dificulta su aplicabilidad en los sistemas de comunicaciones PLC. Resulta de interés entonces, analizar el efecto del ruido de fondo coloreado en técnicas basadas en correlación cruzada. 
Diseño de Estrategias de Sincronización y Estimación de Canal para la Mejora de Comunicaciones en Redes Inteligentes de Energía
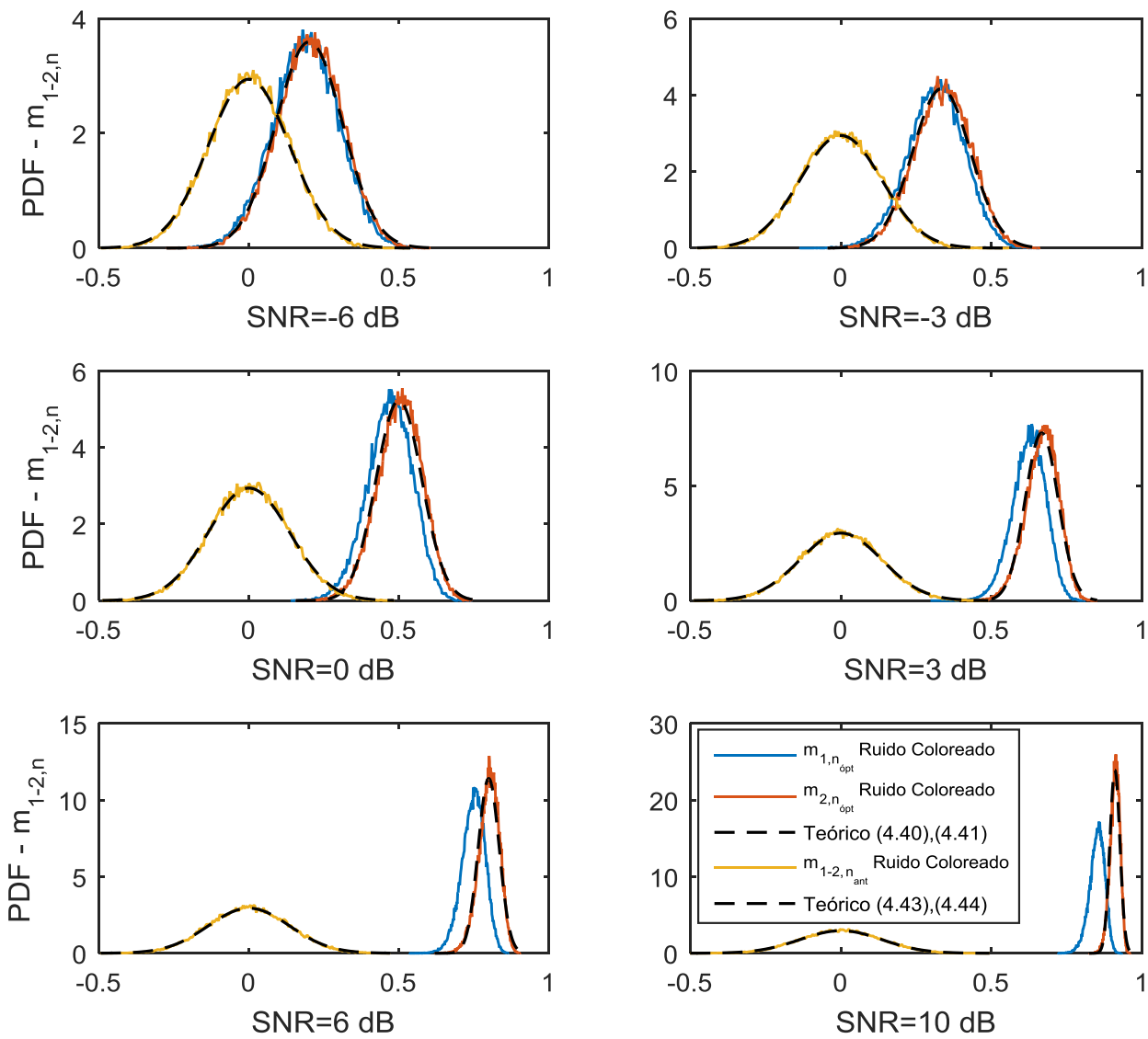

Fig. 4.11 PDFs del valor de las métricas de sincronización gruesa en el instante de sincronización óptima y anteriores, obtenidas por simulaciones, bajo un canal PLC tipo A normalizado con ruido de fondo coloreado y distintos valores de SNR.

\subsubsection{Métrica basada en correlación cruzada bajo ruido blanco gaussiano}

En las técnicas basadas en correlación cruzada, la señal recibida es filtrada por un filtro acoplado del símbolo piloto enviado. La correlación cruzada entre la señal recibida y el símbolo piloto sin extender puede expresarse de acuerdo a:

$$
c_{n}^{\mathbf{r}, \mathbf{x}^{\prime}}=\sum_{m=0}^{N-1} r_{n+m}{\mathbf{x}^{\prime}}_{m}^{*}=\sum_{m=0}^{N-1}\left(s_{n+m}+w_{n+m}\right) \mathbf{x}_{m}^{\prime *}
$$

donde $s_{n}=\sum_{l=0}^{L-1} \sqrt{P_{T}} h_{l} \mathrm{x}_{n-l}$, con $h_{l}$ como el $l$-ésimo coeficiente del canal PLC, y $P_{T}$ la potencia transmitida. Así, la relación señal a ruido en el receptor se define como $S N R=P_{T}\|\mathbf{h}\|^{2} / \sigma_{w}^{2}=\sigma_{S}^{2} / \sigma_{w}^{2}$. Teniendo en cuenta que la correlación cruzada $c_{n}^{\mathbf{x} \prime \mathbf{x}}$ entre el símbolo piloto propuesto extendido $\mathbf{x}$ y el filtro acoplado del símbolo sin extender $\mathbf{x}^{\prime}$ presenta una zona de correlación ideal de acuerdo a (4.9), entonces la 
correlación cruzada de la señal recibida con el símbolo piloto, en la vecindad del punto óptimo de sincronización puede escribirse como:

$$
c_{n}^{\mathbf{r}, \mathbf{x}^{\prime}}=\sum_{m=0}^{N-1} \sum_{l=0}^{L-1} \sqrt{P_{T}} h_{l} \cdot \mathbf{x}_{n+m-l}{\mathbf{x}^{\prime}}_{m}^{*}+\sum_{m=0}^{N-1} w_{n+m}{\mathbf{x}^{\prime *}}_{m}^{*}, \quad-N_{c p}+L \leq n \leq N_{c s}
$$

Debido a las propiedades de correlación, las muestras de la correlación cruzada contendrán los coeficientes del canal, con el agregado de ruido filtrado. Entonces, para acumular toda la energía del canal contenida en las $L$ muestras cercanas de la correlación cruzada, se propone una métrica de sincronización gruesa similar a la definida en [Bumiller 2007]:

$$
m_{3, n}=\frac{N_{2} / N \sum_{k=0}^{N_{2}-1}\left|c_{n+k}^{\mathbf{r}, \mathbf{x}^{\prime}}\right|^{2}}{\sum_{k=0}^{N_{2}-1} \sum_{m=0}^{N-1} r_{n+k+m} \cdot r_{n+k+m}}=\frac{N_{2} / N \sum_{k=0}^{N_{2}-1}\left|\sum_{m=0}^{N-1}\left(s_{n+k+m}+w_{n+k+m}\right) \mathbf{x}_{m}^{\prime *}\right|^{2}}{\sum_{k=0}^{N_{2}-1} \sum_{m=0}^{N-1}\left(s_{n+k+m}+w_{n+k+m}\right)^{2}}
$$

Donde $N_{2}$ es un número que debe ser mayor que la dispersión del canal $L$, de manera tal de acumular toda la energía del mismo, y menor o igual a la longitud del prefijo cíclico $N_{c p}$ para evitar interferencia intersímbolo. Un ejemplo de la métrica bajo un canal PLC tipo A sin el agregado de ruido se presenta en la Fig. 4.12. Teniendo en cuenta la zona libre de interferencia de la correlación cruzada, la métrica en el instante óptimo de sincronización $n_{\text {ópt }}$ puede reescribirse como:

$$
\begin{aligned}
& m_{3, n_{\text {o } p t}}=\frac{N_{2} / N \sum_{k=0}^{N_{2}-1}\left|\sqrt{P_{T}} h_{k} N+\sum_{m=0}^{N-1} w_{n_{\mathrm{o} p t}+k+m} \mathrm{x}_{m}^{\prime *}\right|^{2}}{\sum_{k=0}^{N_{2}-1} \sum_{m=0}^{N-1} s_{n_{\mathrm{o} p t}+k+m}^{2}+2 \cdot s_{n_{\mathrm{o} p t}+k+m} \cdot w_{n_{\mathrm{o} p t}+k+m}+w_{n_{\mathrm{o} p t}+k+m}^{2}} \\
& =\frac{N_{2} / N\left(P_{T}\|\mathbf{h}\|^{2} N^{2}+\sum_{k=0}^{N_{2}-1} 2 \sqrt{P_{T}} h_{k} N w_{n_{\text {o } p t+k}}^{\prime}+w_{n_{\text {ó } p t}+k}^{\prime 2}\right)}{N_{2} P_{T}\|\mathbf{h}\|^{2} N+\sum_{k=0}^{N_{2}-1} \sum_{m=0}^{N-1}\left(2 \cdot s_{n_{\text {ó } p t}+k+m} \cdot w_{n_{\text {ó } p t}+k+m}+w_{n_{\text {ó } p t}+k+m}^{2}\right)}
\end{aligned}
$$

donde $\|\mathbf{h}\|^{2}=\sum_{l=0}^{L} h_{l}^{2}, P_{T}\|\mathbf{h}\|^{2}=\sigma_{S}^{2} \mathrm{y} w_{n_{\mathrm{o} p t}+k}^{\prime}=\sum_{m=0}^{N-1} w_{n_{\mathrm{o} p t}+k+m} \mathrm{x}_{m}^{\prime *}$. Nuevamente, es factible aplicar el TCL tanto al numerador como al denominador y ambos poseen una media mucho mayor a su desvío estándar, por lo que la distribución se puede aproximar a una variable aleatoria normal. Para obtener la media y varianza, deben analizarse los términos intervinientes en el numerador y denominador, por lo que resulta conveniente definir las variables aleatorias auxiliares:

$$
Y_{1}=1 / N \sum_{k=0}^{N_{2}-1} 2 \sqrt{P_{T}} h_{k} N \sum_{m=0}^{N-1} w_{n_{o} p t+k+m} \mathrm{x}_{m}^{\prime *}
$$


Diseño de Estrategias de Sincronización y Estimación de Canal para la Mejora de Comunicaciones en Redes Inteligentes de Energía

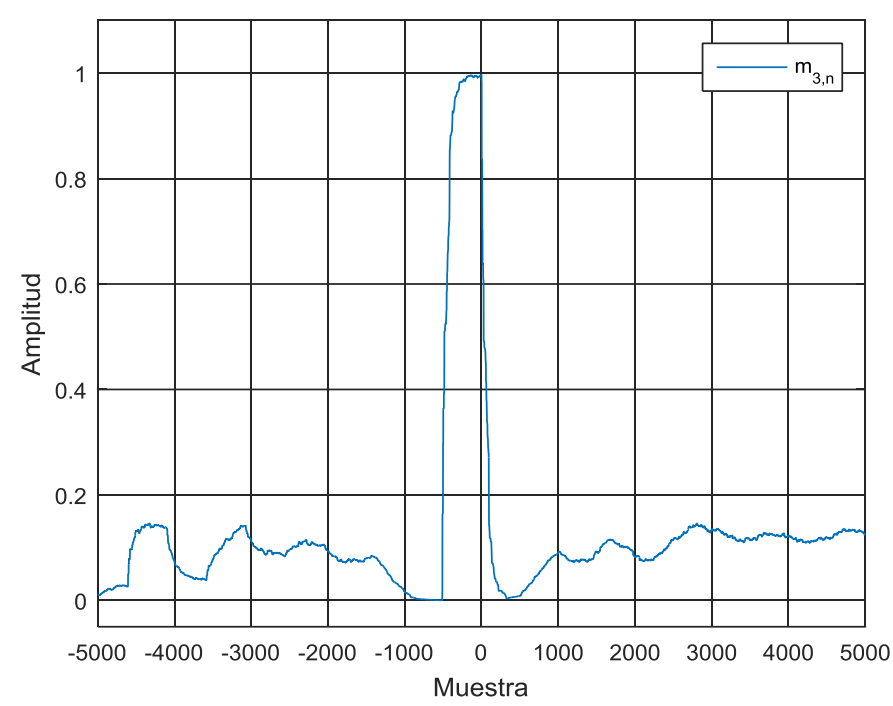

Fig. 4.12 Métrica basada en correlación cruzada del símbolo piloto propuesto, sin agregado de ruido

$$
\begin{gathered}
\text { bajo un canal PLC tipo A. } \\
Y_{2}=\sum_{k=0}^{N_{2}-1}\left|\sum_{m=0}^{N-1} w_{n_{\mathrm{o} p t}+k+m} \mathrm{x}_{m}^{\prime *}\right|^{2} \\
Y_{3}=\sum_{k=0}^{N_{2}-1} \sum_{m=0}^{N-1} 2 \cdot s_{n_{\mathrm{o} p t}+k+m} \cdot w_{n_{\mathrm{o} p t}+k+m} \\
Y_{4}=\sum_{k=0}^{N_{2}-1} \sum_{m=0}^{N-1} w_{n_{\mathrm{o} p t}+k+m}^{2}
\end{gathered}
$$

Donde $s_{n_{\mathrm{o} p t}+k+m}=\sum_{l=0}^{N_{2}-1} \sqrt{P_{T}} h_{l} \cdot x^{\prime *+m-l}$ en (4.51). Así, la métrica puede expresarse de manera simplificada como:

$$
m_{3, n_{\text {ó } p t}}=\frac{N_{2} N \sigma_{S}^{2}+N_{2} Y_{1}+\frac{N_{2}}{N} Y_{2}}{N_{2} N \sigma_{S}^{2}+Y_{3}+Y_{4}}
$$

Analizando las sumatorias intervinientes en las cuatro variables aleatorias, se observa que éstas puede expresarse como resultado de que las muestras de ruido atraviesen sucesivos filtros y bloques de ganancia, de acuerdo a la Fig. 4.13. Considerando las muestras de ruido $w_{n}$ como muestras de un proceso de ruido blanco gaussiano, la ACS del filtro $\mathbf{x}^{\prime}$ se asume prácticamente como una delta de Kronecker (véase la Fig. 4.10), y teniendo en cuenta que la amplitud de elementos $\mathrm{x}_{m}^{\prime *}$ es unitaria, entonces los valores $w_{n_{\text {opt }}+k}^{\prime}$ resultan variables aleatorias gaussianas independientes de media cero $\mathrm{y}$ 
varianza $N \sigma_{w}^{2}$. Asumiendo que $N_{2}$ es lo suficientemente grande como para aplicar el TCL, entonces el término del numerador $Y_{2}$, que es una variable aleatoria que sigue una distribución chi-cuadrado con $N_{2}$ grados de libertad, resulta aproximada por una variable aleatoria normal con media $N_{2} N \sigma_{w}^{2}$ y varianza $2 N_{2} N^{2} \sigma_{w}^{4}$. La expresión de $Y_{2}$ puede entenderse entonces en la Fig. 4.13 como la correlación entre las muestras de ruido y la secuencia $\mathbf{x}^{\prime}$, y que el resultado luego atraviesa un bloque que calcula el módulo al cuadrado. Con respecto a la variable $Y_{4}$ del denominador, expandiendo las sumatorias resulta en:

$$
\begin{aligned}
& Y_{4}=\left(\begin{array}{cccc}
w_{n_{\mathrm{o} p t}}^{2} & +w_{n_{\mathrm{o} p t}+1}^{2} & \cdots & +w_{n_{\mathrm{o} p t}+N-1}^{2} \\
+w_{n_{\mathrm{o} p t}+1}^{2} & +w_{n_{\mathrm{o} p t}+2}^{2} & \cdots & +w_{n_{\mathrm{o} p t}+N}^{2} \\
\vdots & \vdots & \ddots & \vdots \\
+w_{n_{\mathrm{o} p t}+N_{2}-1}^{2} & +w_{n_{\mathrm{o} p t}+N_{2}}^{2} & \cdots & +w_{n_{\mathrm{o}} p t+N+N_{2}-2}^{2}
\end{array}\right) \\
& =w_{n_{\mathrm{o} p t}}^{2}+2 w_{n_{\mathrm{o} p t}+1}^{2}+\cdots+N_{2} w_{n_{\mathrm{o} p t}+N_{2}-1}^{2}+\cdots+N_{2} w_{n_{\mathrm{o} p t}+N-1}^{2} \\
& +\left(N_{2}-1\right) w_{n_{\mathrm{o} p t}+N}^{2}+\cdots+w_{n_{\mathrm{o} p t}+N+N_{2}-2}^{2}
\end{aligned}
$$

Por un lado, la media de $Y_{4}$ resulta $N N_{2} \sigma_{w}^{2}$. Como la varianza de cada término de ruido al cuadrado $w_{n}^{2}$ es $2 \sigma_{w}^{4} \mathrm{y}$, en el caso de ruido blanco gaussiano los términos son independientes, la varianza de $Y_{4}$ puede expresarse de acuerdo a:

$$
\begin{aligned}
\operatorname{Var}\left[Y_{4}\right]=2 \sigma_{w}^{4}\left(2 \sum_{n=1}^{N_{2}-1} n^{2}+N_{2}^{2}\left(N-N_{2}+1\right)\right) \\
=2\left(\frac{1}{3} N_{2}\left(N_{2}-1\right)\left(2 N_{2}-1\right)+N_{2}^{2}\left(N-N_{2}+1\right)\right) \sigma_{w}^{4}=2 \Sigma_{3} \sigma_{w}^{4}
\end{aligned}
$$

Donde $\Sigma_{3}=\frac{1}{3} N_{2}\left(N_{2}-1\right)\left(2 N_{2}-1\right)+N_{2}^{2}\left(N-N_{2}+1\right)$. Teniendo en cuenta (4.54), la variable $Y_{4}$ se puede considerar como las muestras de ruido elevadas al cuadrado, que luego atraviesan dos filtros tipo escalón de longitud $N$ y $N_{2}$, de acuerdo a la Fig. 4.13. Es importante tener en cuenta que las variables $Y_{2}$ e $Y_{4}$ no son independientes. Considerando que $\left|\mathrm{x}_{m}^{\prime *}\right|^{2}=1$, entonces la variable $Y_{2}$ definida en (4.50) puede reescribirse como:

$$
Y_{2}=\sum_{k=0}^{N_{2}-1} \sum_{m=0}^{N-1} w_{n_{\mathrm{o} p t}+k+m}^{2}+\sum_{k=0}^{N_{2}-1} \sum_{m=0}^{N-1} 2 \sum_{i=0}^{m-1} x_{i} w_{n_{\mathrm{o} p t}+k+i} x_{m} w_{n_{\mathrm{o} p t}+k+m}
$$


Diseño de Estrategias de Sincronización y Estimación de Canal para la Mejora de Comunicaciones en Redes Inteligentes de Energía

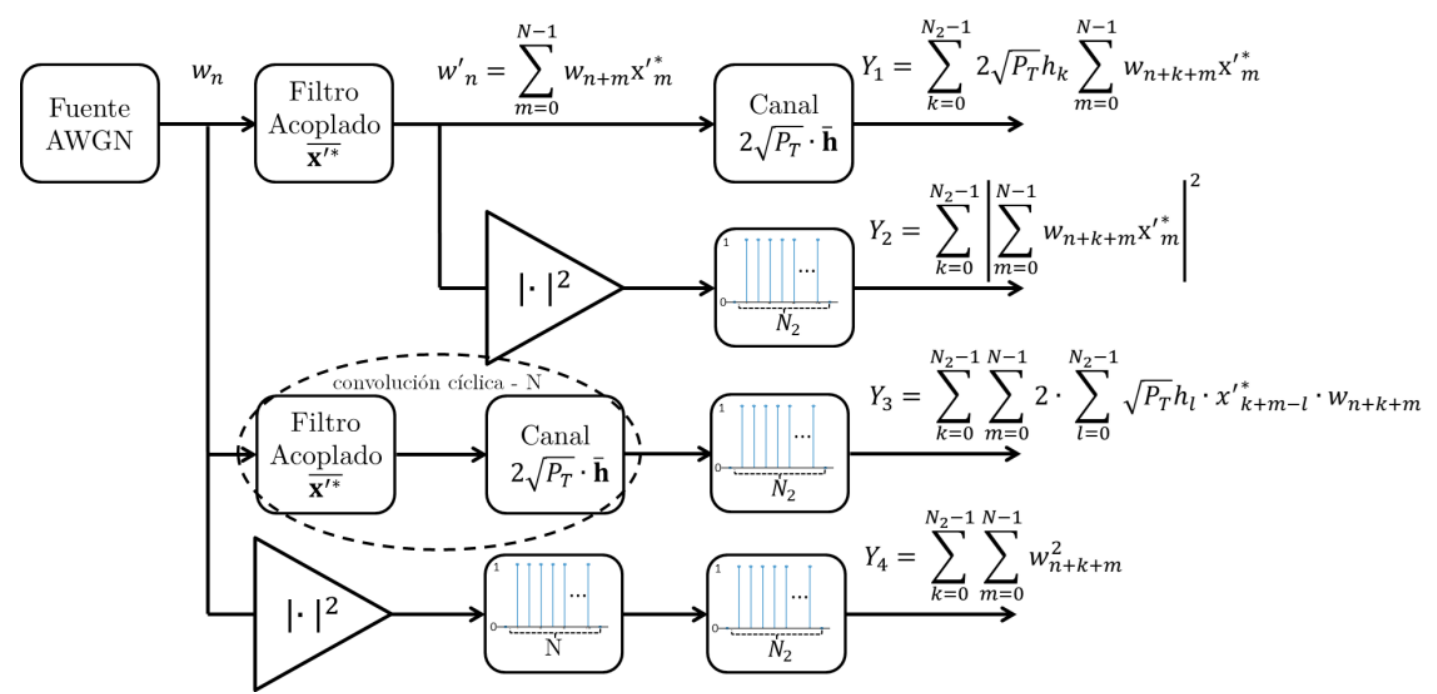

Fig. 4.13 Diagrama en bloques del cómputo equivalente de las variables aleatorias intervinientes en la métrica de sincronización bajo ruido blanco gaussiano.

Puede apreciarse que el primer término de (4.56) es igual a $Y_{4}$ (4.52). Finalmente, la covarianza entre las variables puede aproximarse directamente como la varianza de $Y_{4}$, ya que los productos $x_{i} x_{m} \in\{-1,+1\}$ aleatorizan el segundo término, tornándolo prácticamente independiente a $Y_{4}$. Entonces, $\operatorname{Cov}\left(Y_{2}, Y_{4}\right)=2 \Sigma_{3} \sigma_{w}^{4}$.

Por otro lado, la variable $Y_{1}=1 / N \sum_{k=0}^{N_{2}-1} 2 \sqrt{P_{T}} h_{k} N \sum_{m=0}^{N-1} w_{n_{\mathrm{o} p t}+k+m} \mathrm{x}_{m}^{\prime *}$ puede escribirse como:

$$
\begin{aligned}
& Y_{1}=2 \sqrt{P_{T}} h_{0} w^{\prime}{ }_{n_{\mathrm{o} p t}}+2 \sqrt{P_{T}} h_{1} w^{\prime}{ }_{n_{\mathrm{o} p t}+1}+\cdots+2 \sqrt{P_{T}} h_{N_{2}-1} w^{\prime}{ }_{n_{\mathrm{o} p t}+N_{2}-1} \\
& =2 \sqrt{P_{T}} h_{0}\left(w_{n_{\mathrm{o} p} t} x_{0}^{\prime *}+w_{n_{\mathrm{o} p t}+1} x_{1}^{\prime *}+\cdots+w_{n_{\mathrm{o} p t}+N-1} x_{N-1}^{\prime *}\right) \\
& +2 \sqrt{P_{T}} h_{1}\left(w_{n_{\mathrm{opt} t}+1} x_{0}^{\prime *}+w_{n_{\mathrm{o} p t}+2} x_{1}^{\prime *}+\cdots+w_{n_{\mathrm{o} p t}+N} x^{\prime *}{ }_{N-1}^{*}\right) \\
& +2 \sqrt{P_{T}} h_{N_{2}-1}\left(w_{n_{\mathrm{o} p t}+N_{2}-1} x_{0}^{\prime *}+w_{n_{\mathrm{o} p t}+N_{2}} x_{1}^{\prime *}+\cdots+w_{n_{\mathrm{o} p t}+N+N_{2}-2} x_{N-1}^{\prime *}\right)
\end{aligned}
$$

Como la ACS del símbolo piloto es prácticamente una delta de Kronecker, los términos $w_{n}^{\prime}$ son independientes de media cero y varianza $N \sigma_{w}^{2}$, por lo que $Y_{1}$ resulta en la suma de $N_{2}$ variables aleatorias gaussianas independientes de media cero y varianza $4 P_{T} h_{k}^{2} N \sigma_{w}^{2}$, y el resultado es una variable normal de media cero y varianza $4 \sigma_{s}^{2} N \sigma_{w}^{2}$. La variable $Y_{1}$ entonces puede entenderse en la Fig. 4.13 como la correlación entre las muestras de ruido y un filtro resultante de la convolución entre la secuencia $\mathbf{x}^{\prime}$ y el canal h. A su vez, la variable $Y_{3}=\sum_{k=0}^{N_{2}-1} \sum_{m=0}^{N-1}+2 \cdot s_{n_{\mathrm{o} p t}+k+m} \cdot w_{n_{\mathrm{o} p t}+k+m}$, teniendo cuenta que $s_{n_{\text {o } p t}+k+m}=\sum_{l=0}^{N_{2}-1} \sqrt{P_{T}} h_{l} \cdot x^{\prime *+m-l}$, puede expandirse de acuerdo a: 


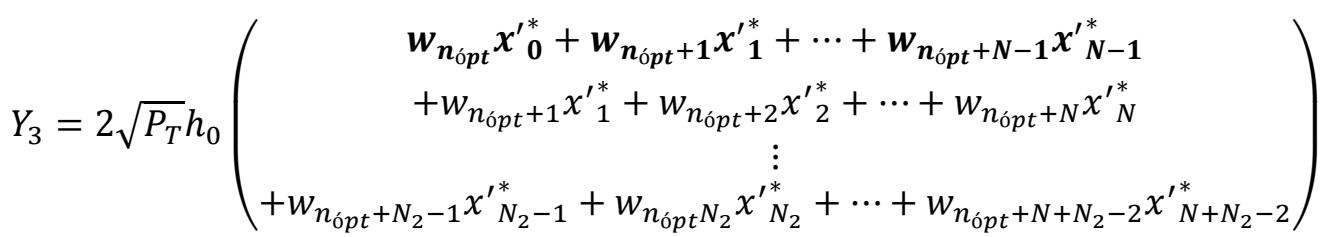

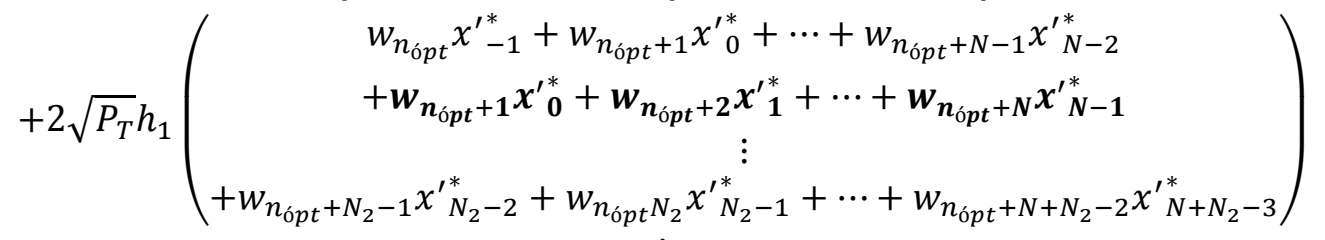

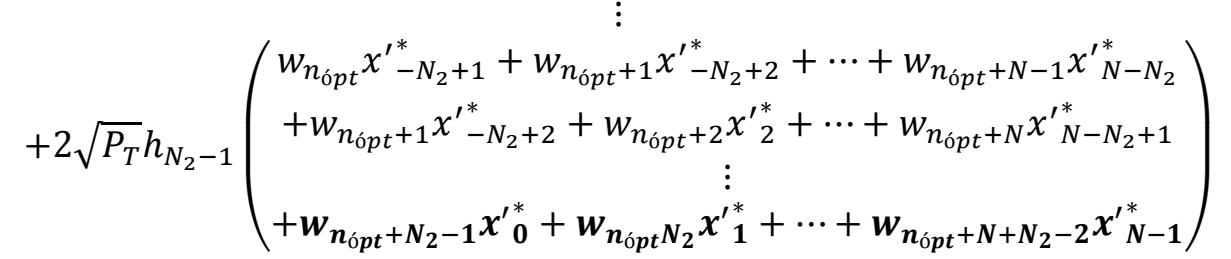

Las sumas entre paréntesis de esta ecuación implican la suma de variables dependientes, que pueden reescribirse como:

$$
\begin{aligned}
& 2 \sqrt{P_{T}} h_{0}\left(\begin{array}{c}
w_{n_{\mathrm{o} p t}} x_{0}^{\prime *}+2 w_{n_{\mathrm{o} p t}+1} x_{1}^{\prime *}+\cdots+N_{2} w_{n_{\mathrm{op} t}+N_{2}-1} x_{N_{2}-1}^{\prime *}+\cdots+N_{2} w_{n_{\mathrm{o} p t}+N-1} x_{N-1}^{\prime *} \\
+\left(N_{2}-1\right) w_{n_{\mathrm{oppt}}+N} x_{N}^{\prime *}+\cdots+w_{n_{\mathrm{oppt}}+N+N_{2}-2} x_{N+N_{2}-2}^{\prime *}
\end{array}\right) \\
& +2 \sqrt{P_{T}} h_{1}\left(\begin{array}{c}
w_{n_{\mathrm{o} p t}} x_{-1}^{\prime *}+2 w_{n_{\mathrm{o} p t}+1} x_{0}^{\prime *}+\cdots+N_{2} w_{n_{\mathrm{op} t}+N_{2}-1} x_{N_{2}-2}^{\prime *}+\cdots+ \\
+N_{2} w_{n_{\mathrm{o} p t}+N-1} x_{N-2}^{\prime *}+\left(N_{2}-1\right) w_{n_{\mathrm{o} p t}+N} x_{N-1}^{\prime *}+\cdots+w_{n_{\mathrm{o} p t}+N+N_{2}-2} x_{N+N_{2}-3}^{\prime *}
\end{array}\right)
\end{aligned}
$$

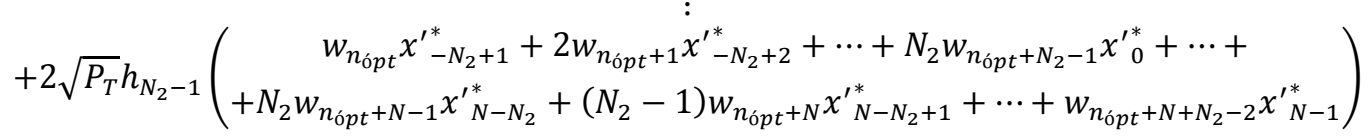

Análogamente a lo desarrollado en (4.55), la variable $Y_{3}$ resulta en la suma de $N_{2}$ variables independientes con varianza $4 P_{T} h_{k}^{2} \sigma_{w}^{2}\left[\frac{1}{3} N_{2}\left(N_{2}-1\right)\left(2 N_{2}-1\right)+N_{2}^{2}\left(N-N_{2}+\right.\right.$ 1)]. Finalmente la varianza de $Y_{3}$ es $4 \sigma_{s}^{2} \sigma_{w}^{2} \Sigma_{3}$. En la Fig. 4.13, la variable $Y_{3}$ puede entenderse a partir de muestras de ruido, atravesando un filtro de orden $N$, que calcula la correlación de las muestras con la convolución cíclica de la secuencia $\mathbf{x}^{\prime}$ y el canal $\mathbf{h}$. Es importante notar que los términos resaltados en (4.58) son los que definen a la variable aleatoria $Y_{1}$ en (4.57), es decir que $Y_{1}$ del numerador está incluida en $Y_{3}$ del denominador, por lo que la covarianza entre ellas será elevada y aproximadamente igual a $4 \sigma_{s}^{2} \sigma_{w}^{2} N_{2}\left(N-\frac{\left(N_{2}-1\right)}{2}\right)$.

Una vez analizadas las variables aleatorias, y análogamente a (4.24) y (4.25) la métrica puede reescribirse como:

$$
m_{3, n_{\mathrm{o} p t}}=\frac{\mu_{P_{3}}+N_{2} Y_{1}+\frac{\mathrm{N}_{2}}{N} Y_{2}^{\prime}}{\mu_{E_{3}}+Y_{3}+Y^{\prime}{ }_{4}}
$$


Diseño de Estrategias de Sincronización y Estimación de Canal para la Mejora de Comunicaciones en Redes Inteligentes de Energía

Donde $\mu_{P_{3}}=N_{2}\left(N \sigma_{S}^{2}+N_{2} \sigma_{w}^{2}\right)$ y $\mu_{E_{3}}=N_{2} N\left(\sigma_{S}^{2}+\sigma_{w}^{2}\right)$ son la media del numerador $\mathrm{y}$ denominador respectivamente, e $Y^{\prime}{ }_{2}=Y_{2}-N_{2} N \sigma_{w}^{2} ; Y^{\prime}{ }_{4}=Y_{4}-N_{2} N \sigma_{w}^{2}$. Teniendo en cuenta que la media del denominador es mucho mayor que su desvío estándar, entonces se puede aproximar la métrica de acuerdo a:

$$
m_{3, n_{\mathrm{o} p t}} \approx \frac{\mu_{P_{3}}}{\mu_{E_{3}}}+\frac{N_{2} Y_{1}+\frac{\mathrm{N}_{2}}{N} Y_{2}^{\prime}}{\mu_{E_{3}}}-\frac{\mu_{P_{3}}\left(Y_{3}+Y_{4}^{\prime}\right)}{\mu_{E_{3}}^{2}}
$$

Debido a que la varianza de la resta de dos variables dependientes ponderadas es $\operatorname{Var}(a X-b Y)=a^{2} \operatorname{Var}(X)+b^{2} \operatorname{Var}(Y)-2 a b \operatorname{Cov}(X, Y)$, entonces:

$$
\begin{array}{r}
\operatorname{Var}\left(\frac{N_{2} Y^{\prime}{ }_{2}}{N \mu_{E_{3}}}-\frac{\mu_{P_{3}} Y^{\prime}{ }_{4}}{\mu_{E_{3}}^{2}}\right)=\frac{N_{2}^{2}}{N^{2} \mu_{E_{3}}^{2}} 2 N_{2} N^{2} \sigma_{w}^{4}+2 \Sigma_{3} \sigma_{w}^{4}\left(\frac{\mu_{P_{3}}^{2}}{\mu_{E_{3}}^{4}}-\frac{2 N_{2} \mu_{P_{3}}}{N \mu_{E_{3}}^{3}}\right) \\
\operatorname{Var}\left(\frac{N_{2} Y_{1}}{\mu_{E_{3}}}-\frac{\mu_{P_{3}} Y_{3}}{\mu_{E_{3}}^{2}}\right)=\frac{N_{2}^{2}}{\mu_{E_{3}}^{2}} 4 \sigma_{S}^{2} N \sigma_{w}^{2}+\frac{\mu_{P_{3}}^{2}}{\mu_{E_{3}}^{4}} 4 \sigma_{S}^{2} \sigma_{w}^{2} \Sigma_{3}-2 \frac{N_{2} \mu_{P_{3}}}{\mu_{E_{3}}^{3}} 4 \sigma_{S}^{2} \sigma_{w}^{2} N_{2}\left(N-\frac{\left(N_{2}-1\right)}{2}\right) \\
=\frac{4 S N R}{N(S N R+1)^{2}}+\frac{4 S N R \Sigma_{3}\left(S N R+\frac{N_{2}}{N}\right)^{2}}{N_{2}^{2} N^{2}(S N R+1)^{4}}-\frac{8 S N R\left(N-\frac{\left(N_{2}-1\right)}{2}\right)\left(S N R+\frac{N_{2}}{N}\right)}{N^{2}(S N R+1)^{3}}
\end{array}
$$

Finalmente, la métrica en el instante óptimo de sincronismo puede expresarse como una variable normal $M_{3 \text { ópt }}$ con media y varianza:

$$
\begin{gathered}
\mu_{M_{3 \text { o } p t}}=\frac{\mu_{P}}{\mu_{E}}=\frac{N_{2}\left(N \sigma_{S}^{2}+N_{2} \sigma_{w}^{2}\right)}{N_{2} N\left(\sigma_{S}^{2}+\sigma_{w}^{2}\right)}=\frac{S N R+N_{2} / N}{S N R+1} \\
\sigma_{M_{3 \text { op } t}}^{2}=\frac{4 S N R}{N(S N R+1)^{2}}+\frac{4 S N R \Sigma_{3}\left(S N R+\frac{N_{2}}{N}\right)^{2}}{N_{2}^{2} N^{2}(S N R+1)^{4}}-\frac{8 S N R\left(N-\frac{\left(N_{2}-1\right)}{2}\right)\left(S N R+\frac{N_{2}}{N}\right)}{N^{2}(S N R+1)^{3}} \\
+\frac{2 N_{2}}{N^{2}(S N R+1)^{2}}+\frac{2 \Sigma_{3}\left(S N R+\frac{N_{2}}{N}\right)^{2}}{N_{2}^{2} N^{2}(S N R+1)^{4}}-\frac{4 \Sigma_{3}\left(S N R+\frac{N_{2}}{N}\right)}{N_{2} N^{3}(S N R+1)^{3}}
\end{gathered}
$$

Posteriormente, resulta de interés analizar la métrica en muestras previas a la llegada del símbolo piloto, en las que sólo se encuentra presente ruido, de forma que la ecuación (4.47) puede reescribirse como:

$$
m_{3, n_{\text {ant }}}=\frac{N_{2} / N \sum_{k=0}^{N_{2}-1}\left|\sum_{m=0}^{N-1} w_{n_{a n t}+k+m} \mathrm{x}_{m}^{\prime *}\right|^{2}}{\sum_{k=0}^{N_{2}-1} \sum_{m=0}^{N-1} w_{n_{a n t}+k+m}^{2}}=\frac{\frac{N_{2}}{N} Y_{2}}{Y_{4}}
$$


Aplicando el teorema del límite central en el denominador, resulta $Y_{4}$ una variable normal de media $N N_{2} \sigma_{w}^{2}$ y varianza $2 \Sigma_{3} \sigma_{w}^{4}$. En el numerador, se ha analizado que la variable $Y_{2}$ resulta en una variable normal de media $N_{2} N \sigma_{w}^{2}$ y varianza $2 N_{2} N^{2} \sigma_{w}^{4}$. Como la media del denominador es mucho mayor que su desvío estándar, entonces reemplazando $S N R=0$ en (4.64) y (4.65) la métrica puede aproximarse a una variable normal de parámetros:

$$
\begin{gathered}
\mu_{M_{3 \text { ant }}}=\frac{N_{2}}{N} \\
\sigma_{M_{3 \text { ant }}}^{2}=\frac{2 \cdot N_{2}}{N^{2}}-\frac{2 \Sigma_{3}}{N^{4}}
\end{gathered}
$$

Los resultados obtenidos para la distribución de la métrica en las muestras previas a la llegada del símbolo piloto se verificaron mediante simulaciones, y se presentan en la Fig. 4.14.

A partir de los resultados obtenidos de la distribución de la métrica, se puede determinar entonces un umbral de detección lo suficientemente alto como para mantener una baja probabilidad de falsa detección. Por ejemplo, si se desea una probabilidad de $10^{-7}$ de falsa detección de una de las muestras de la métrica cuando sólo contiene muestras de ruido, a partir de ICDF de la distribución normal, se obtiene el umbral:

$$
\gamma \approx N_{2} / N+5,2 \sqrt{\frac{2 \cdot N_{2}}{N^{2}}-\frac{2 \Sigma_{3}}{N^{4}}}
$$

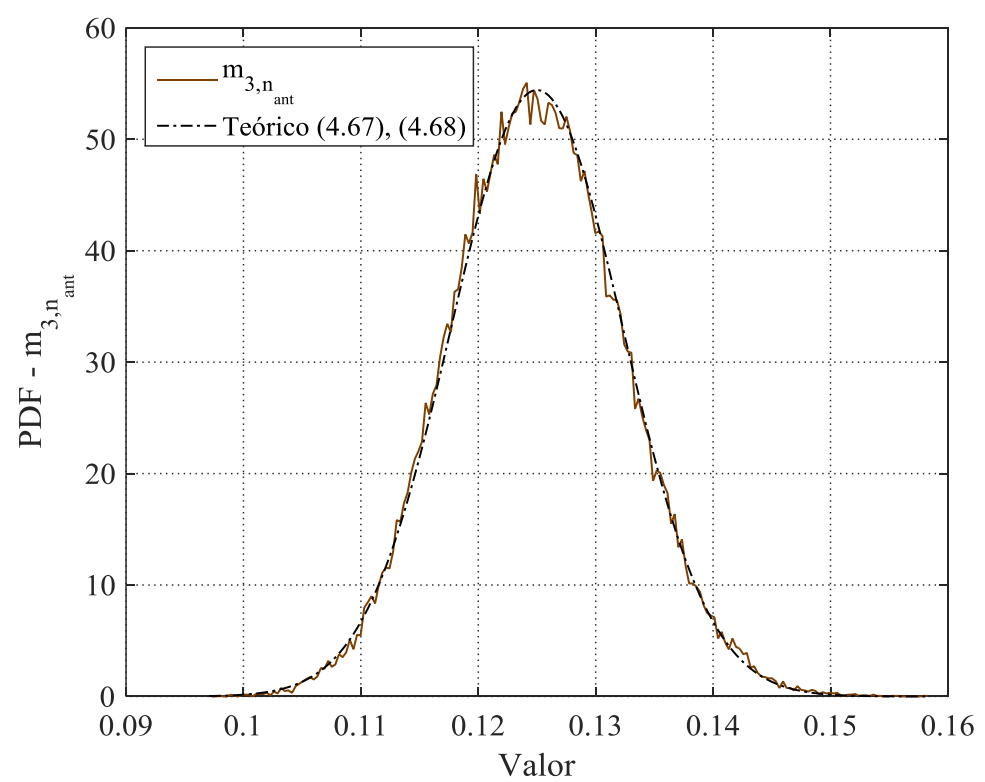

Fig. 4.14 PDFs empírica y teórica del valor de la métrica de sincronización gruesa $m_{3, n}$ en las muestras previas a la llegada del símbolo piloto con ruido de fondo blanco Gaussiano. 
Diseño de Estrategias de Sincronización y Estimación de Canal para la Mejora de Comunicaciones en Redes Inteligentes de Energía

Para los parámetros bajo estudio $N=4096$ y $N_{2}=512$ resulta en un umbral $\gamma \approx$ 0,163. En la Fig. 4.15 se muestran los resultados obtenidos por simulaciones para la métrica bajo análisis. Se grafican las PDFs empíricas de los valores en el instante óptimo de sincronismo y en instantes previos al arribo del símbolo piloto para 50.000 realizaciones de un canal PLC tipo A normalizado (se asegura $\|\mathbf{h}\|^{2}=1$ en cada realización del canal), bajo ruido blanco Gaussiano, para diferentes valores de relación señal a ruido. Puede apreciarse que los parámetros obtenidos para describir la métrica $m_{3}$ de (4.64), (4.65), (4.67) y (4.68) se ajustan a lo obtenido por simulaciones. Respecto a la capacidad de detección del símbolo piloto, se observa que la métrica basada en correlación cruzada posee una buena capacidad de detección para el umbral $\gamma$, inclusive hasta valores de $S N R$ de $-6 \mathrm{~dB}$. La probabilidad de que la métrica en el instante óptimo de sincronización $m_{3, n_{\text {ó }} t}$ no supere el umbral para esta condición de $S N R$, a partir de la CDF de la normal descripta por los parámetros de (4.64) y (4.65), es de $6 \cdot 10^{-30}$ es decir, prácticamente nula.

\subsubsection{Métrica basada en correlación cruzada bajo ruido de fondo coloreado}

Si ahora se considera un proceso de ruido coloreado con densidad espectral de potencia de acuerdo a (4.31), en lugar de ruido blanco, el desempeño de la sincronización puede verse afectado. Recordando la métrica de sincronización en el instante óptimo de sincronización:

$$
\begin{gathered}
m_{3, n_{\mathrm{oppt}}}=\frac{N_{2} / N\left(P_{T}\|\mathbf{h}\|^{2} N^{2}+\sum_{k=0}^{N_{2}-1} 2 \sqrt{P_{T}} h_{k} N w_{n_{\mathrm{op} t}+k}^{\prime}+w_{n_{\mathrm{op} t}+k}^{\prime 2}\right)}{N_{2} P_{T}\|\mathbf{h}\|^{2} N+\sum_{k=0}^{N_{2}-1} \sum_{m=0}^{N-1} 2 \cdot s_{n_{\mathrm{o} p t}+k+m} \cdot w_{n_{\mathrm{o} p t}+k+m}+w_{n_{\mathrm{op} t}+k+m}^{2}} \\
=\frac{N_{2} N \sigma_{S}^{2}+N_{2} Y_{1}+\frac{N_{2}}{N} Y_{2}}{N_{2} N \sigma_{S}^{2}+Y_{3}+Y_{4}}
\end{gathered}
$$

es evidente que las cuatro variables aleatorias se verán afectadas por el efecto del ruido coloreado. Tanto $Y_{1}$ como $Y_{3}$ ahora pueden entenderse como muestras de un proceso de ruido blanco gaussiano que atraviesan una sucesión de filtros lineales de acuerdo a la Fig. 4.16, comenzando por el filtro que colorea el ruido de acuerdo a la densidad espectral de potencia de (4.31), y atravesando luego el filtro acoplado de la secuencia $\mathbf{x}^{\prime}$ y el canal $\mathbf{h}$. Al igual que el caso previamente analizado en el que se consideraron muestras de un proceso de ruido blanco gaussiano, las variables se pueden escribir de 


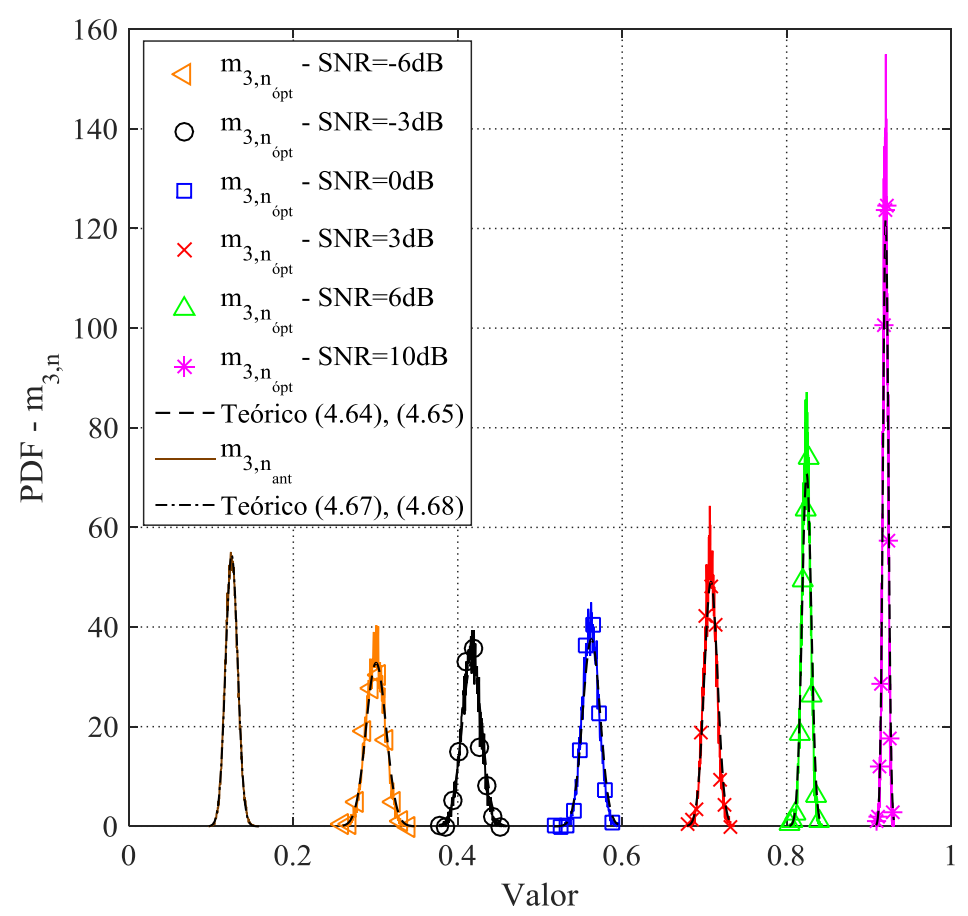

Fig. 4.15 PDFs del valor de la métrica de sincronización gruesa $m_{3, n}$ en los instantes de sincronización óptimo y anteriores, obtenidos por simulaciones bajo un canal PLC tipo A normalizado con ruido de fondo blanco Gaussiano y distintos valores de SNR.

acuerdo a (4.57) y (4.58), y están fuertemente correladas. Sin embargo, al incluir el efecto del ruido coloreado, la varianza de las variables se verá influenciada no sólo por el filtro que colorea el ruido, sino también por la ACS del canal que, en principio, se desconoce. Sin embargo, para una primera aproximación de la varianza de estas variables, se considera que los coeficientes del canal son independientes, por lo que la ACS del canal es simplemente una delta de Kronecker $\|\mathbf{h}\|^{2} \delta_{k}$. Además, el valor esperado de los coeficientes del canal es cero $E\left(h_{i}\right)=0$, de acuerdo al modelo descripto en (4.2). Bajo estas consideraciones, y teniendo en cuenta que la media del proceso de ruido es cero, entonces la media resultante de la variable $Y_{1}$ desarrollada en (4.57) es nuevamente cero, y su varianza resulta $\operatorname{Var}\left(Y_{1}\right)=4 N \sigma_{s}^{2} \sigma_{w}^{2}$.

Con respecto a la variable $Y_{3}$, su media resulta cero, y analizando su expresión en (4.59), su varianza resulta $\operatorname{Var}\left(Y_{3}\right)=4 \sigma_{s}^{2} \sigma_{w}^{2} \Sigma_{3}$, donde $\Sigma_{3}=\left(\frac{1}{3} N_{2}\left(N_{2}-1\right)\left(2 N_{2}-1\right)+\right.$ $\left.N_{2}^{2}\left(N-N_{2}+1\right)\right)$. La covarianza entre $Y_{1}$ e $Y_{3}$ es, nuevamente, aproximadamente igual a $4 \sigma_{s}^{2} \sigma_{w}^{2} N_{2}\left(N-\frac{\left(N_{2}-1\right)}{2}\right)$.

Por otro lado, las variables aleatorias $Y_{2}$ e $Y_{4}$ pueden entenderse como el filtrado lineal del proceso de ruido, con el adicional de que las muestras del proceso además son elevadas al cuadrado en alguna de las etapas de filtros, como puede observarse en la 
Diseño de Estrategias de Sincronización y Estimación de Canal para la Mejora de Comunicaciones en Redes Inteligentes de Energía

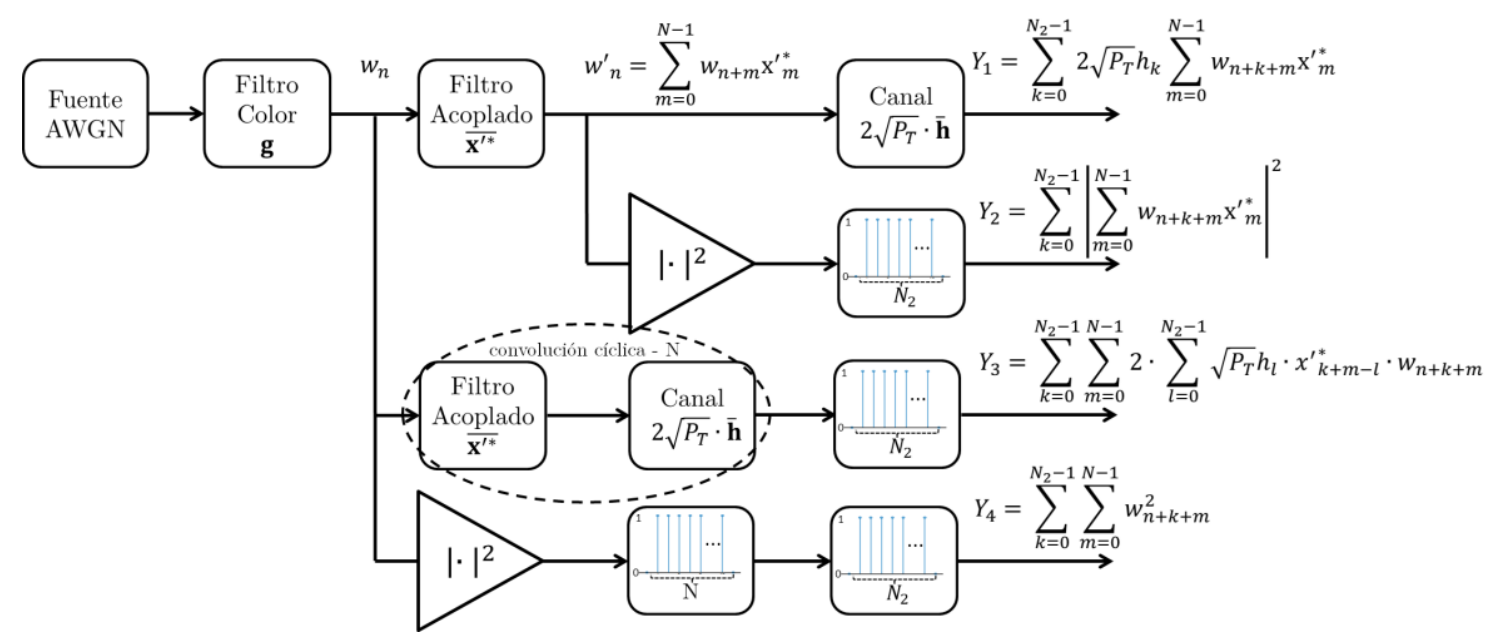

Fig. 4.16 Diagrama en bloques del cómputo equivalente de las variables aleatorias intervinientes en la métrica de sincronización bajo ruido de fondo coloreado.

Fig. 4.16. Con respecto a $Y_{2}$, puede calcularse un filtro equivalente resultante entre el filtro que colorea el ruido $\mathbf{g}$ y el filtro acoplado del símbolo piloto $\mathbf{x}^{\prime}$, como $f_{1_{k}}=$ $\sum_{j=-\infty}^{+\infty} g_{j} x^{\prime}{ }_{k+j}=g_{k} * x_{-k}^{\prime}$. Luego de elevar al cuadrado y finalmente sumar las $N_{2}$ muestras del proceso resultante, la media y la varianza resultan:

$$
\begin{gathered}
\mu_{Y_{2}}=E\left(Y_{2}\right)=N_{2} \sigma_{w}^{2} \sum_{k=-\infty}^{\infty} f_{1_{k}}^{2}=N_{2} \sigma_{w}^{2} \Sigma_{4} \\
\operatorname{Var}\left(Y_{2}\right)=\sigma_{w}^{4}\left[2 N_{2}\left(\sum_{k=-\infty}^{+\infty} f_{1_{k}}^{2}\right)^{2}+4 \sum_{j=1}^{N_{2}-1}\left(N_{2}-j\right)\left(\sum_{k=-\infty}^{+\infty} f_{1_{k}} f_{1_{k+j}}\right)^{2}\right]=\sigma_{w}^{4} \Sigma_{5}
\end{gathered}
$$

Puede apreciarse entonces, que tanto la media como la varianza de $Y_{2}$ dependen del filtro que colorea el ruido (equivalentemente la PSD del ruido coloreado), la longitud $N$ y de la ACs del símbolo piloto, y el tamaño de la ventana de $N_{2}$.

Con respecto a la variable $Y_{4}$, teniendo en cuenta el efecto del filtro que colorea el ruido, la operación no lineal de elevar al cuadrado, el filtrado por un escalón de $N$ muestras, y la suma final de las $N_{2}$ muestras, pueden calcularse la media y varianza como:

$$
\begin{gathered}
\mu_{Y_{4}}=E\left(Y_{4}\right)=N_{2} N \sigma_{w}^{2} \\
\operatorname{Var}\left(Y_{4}\right)=\sigma_{w}^{4}\left[2 N_{2} \sum_{k=-\infty}^{+\infty} f_{2_{k}}^{2}+4 \sum_{j=1}^{N_{2}-1}\left(N_{2}-j\right) \sum_{k=-\infty}^{+\infty} f_{2_{k}} f_{2}\right]=\sigma_{w+j}^{4} \Sigma_{6}
\end{gathered}
$$

donde $f_{2 k}=\left(g_{k} * g_{-k}\right)^{2} *\left(\operatorname{rect}_{k / N} * \operatorname{rect}_{k / N}\right)$ y rect ${ }_{k / N}$ es una función rectangular discreta de longitud $N$. Nuevamente, las variables $Y_{2}$ e $Y_{4}$ son dependientes, debido a 
que $Y_{4}$ está incluída en $Y_{2}$ de acuerdo a (4.56), y la covarianza puede también aproximarse por la varianza de $Y_{4}$, es decir, $\operatorname{Cov}\left(Y_{2}, Y_{4}\right)=\sigma_{w}^{4} \Sigma_{6}$. Una vez caracterizadas las variables intervinientes, la métrica de sincronización en el instante óptimo de sincronismo puede expresarse como una variable aleatoria gaussiana al igual que en (4.61), de acuerdo a:

$$
m_{3, n_{\mathrm{o} p t}} \approx \frac{\mu_{P_{3}}}{\mu_{E_{3}}}+\frac{N_{2} Y_{1}+\frac{\mathrm{N}_{2}}{N} Y_{2}^{\prime}}{\mu_{E_{3}}}-\frac{\mu_{P_{3}}\left(Y_{3}+Y_{4}^{\prime}\right)}{\mu_{E_{3}}^{2}}
$$

y sus parámetros resultan:

$$
\begin{gathered}
\mu_{M_{3 o p t}}=\frac{N_{2}\left(N \sigma_{S}^{2}+\frac{N_{2} \Sigma_{4}}{N} \sigma_{w}^{2}\right)}{N_{2} N\left(\sigma_{S}^{2}+\sigma_{w}^{2}\right)}=\frac{S N R+N_{2} \Sigma_{4} / N^{2}}{S N R+1} \\
\sigma_{M_{3 o p t}}^{2}=\frac{4 S N R}{N(S N R+1)^{2}}+\frac{4 S N R \Sigma_{3}\left(S N R+\frac{N_{2} \Sigma_{4}}{N^{2}}\right)^{2}}{N_{2}^{2} N^{2}(S N R+1)^{4}}-\frac{8 S N R\left(N-\frac{\left(N_{2}-1\right)}{2}\right)\left(S N R+\frac{\left.N_{2} \Sigma_{4}\right)}{N^{2}}\right)}{N^{2}(S N R+1)^{3}} \\
+\frac{\Sigma_{5}}{N^{4}(S N R+1)^{2}}+\frac{\left(S N R+\frac{N_{2} \Sigma_{4}}{N^{2}}\right)^{2} \Sigma_{6}}{N_{2}^{2} N^{2}(S N R+1)^{4}}-\frac{2\left(S N R+\frac{N_{2} \Sigma_{4}}{N^{2}}\right) \Sigma_{6}}{N_{2} N^{3}(S N R+1)^{3}}
\end{gathered}
$$

Analizando la métrica en muestras previas a la llegada del símbolo piloto, en las que sólo se encuentra presente ruido, la ecuación (4.70) puede reescribirse como:

$$
m_{3, n_{a n t}}=\frac{N_{2} / N \sum_{k=0}^{N_{2}-1}\left|\sum_{m=0}^{N-1} w_{n_{a n t}+k+m} \mathrm{x}_{m}^{\prime *}\right|^{2}}{\sum_{k=0}^{N_{2}-1} \sum_{m=0}^{N-1} w_{n_{a n t}+k+m}^{2}} \approx \frac{N_{2}}{N \mu_{Y_{4}}} Y_{2}-\frac{N_{2} \mu_{Y_{2}}}{N \mu_{Y_{4}}^{2}} Y_{4}^{\prime}
$$

Reemplazando $S N R=0$ en (4.76) y (4.77), la media y varianza de la métrica resulta:

$$
\begin{gathered}
\mu_{M_{3} \text { ant }}=\frac{N_{2} \Sigma_{2}}{N^{2}} \\
\sigma_{M_{3} \text { ant }}^{2}=\frac{\Sigma_{5}}{N^{4}}-\frac{\frac{\Sigma_{4}}{N}\left(2-\frac{\Sigma_{4}}{N}\right) \Sigma_{6}}{N^{4}}
\end{gathered}
$$

Sin embargo, la aproximación a variable gaussiana no resulta válida como en el análisis de la métrica bajo ruido blanco gaussiano de (4.67) y (4.68). En el caso de ruido blanco gaussiano, la suma de $N_{2}$ términos en $Y_{2}=\sum_{k=0}^{N_{2}-1}\left|\sum_{m=0}^{N-1} w_{n_{a n t}+k+m} \mathrm{x}_{m}^{\prime *}\right|^{2}$ podía asumirse como la suma $N_{2}$ variables aleatorias independientes con distribución chicuadrado, al considerar la ACS del símbolo piloto $\mathbf{x}^{\prime}$ prácticamente como una delta de Kronecker. La diferencia radica en que bajo ruido de fondo coloreado tanto $Y_{2}$ como $Y_{4}$ 
Diseño de Estrategias de Sincronización y Estimación de Canal para la Mejora de Comunicaciones en Redes Inteligentes de Energía

contienen la suma de muestras del proceso de ruido que no son independientes elevadas al cuadrado, de acuerdo a la Fig. 4.16. Así, no resulta factible aplicar el TCL a $Y_{2}$ ya que la suma de variables aleatorias dependientes con distribución chi-cuadrado no resulta necesariamente gaussiana, sino que puede aproximarse a partir de una distribución Gamma $\Gamma(k, \theta)$, como se muestra en [Ferrari 2019], donde $k$ y $\theta$ son los parámetros de forma y escala, respectivamente. Así, teniendo en cuenta la media (4.79) y varianza (4.80) obtenidas y que la media de la distribución Gamma es $k \theta$ y la varianza $k \theta^{2}$, los parámetros de la variable $M_{3 a n t}$ con distribución Gamma que define la métrica en los instantes anteriores a la llegada del símbolo piloto pueden escribirse como:

$$
\begin{aligned}
& k_{M_{3} \text { ant }}=\frac{N_{2}^{2} \Sigma_{4}^{2}}{\Sigma_{5}-\frac{\Sigma_{4}}{N}\left(2-\frac{\Sigma_{4}}{N}\right) \Sigma_{6}} \\
& \theta_{M_{3 \text { ant }}}=\frac{\Sigma_{5}-\frac{\Sigma_{4}}{N}\left(2-\frac{\Sigma_{4}}{N}\right) \Sigma_{6}}{N^{2} N_{2} \Sigma_{4}}
\end{aligned}
$$

El ajuste de la métrica a la distribución Gamma se evaluó a partir de simulaciones a través de la PDF empírica, y los resultados se presentan en la Fig. 4.17. Puede apreciarse cierto error para valores pequeños de la variable, pero un buen ajuste en la cola derecha de la distribución, es decir, para los mayores valores de la variable.

A partir de los resultados obtenidos para la distribución de la métrica, nuevamente se

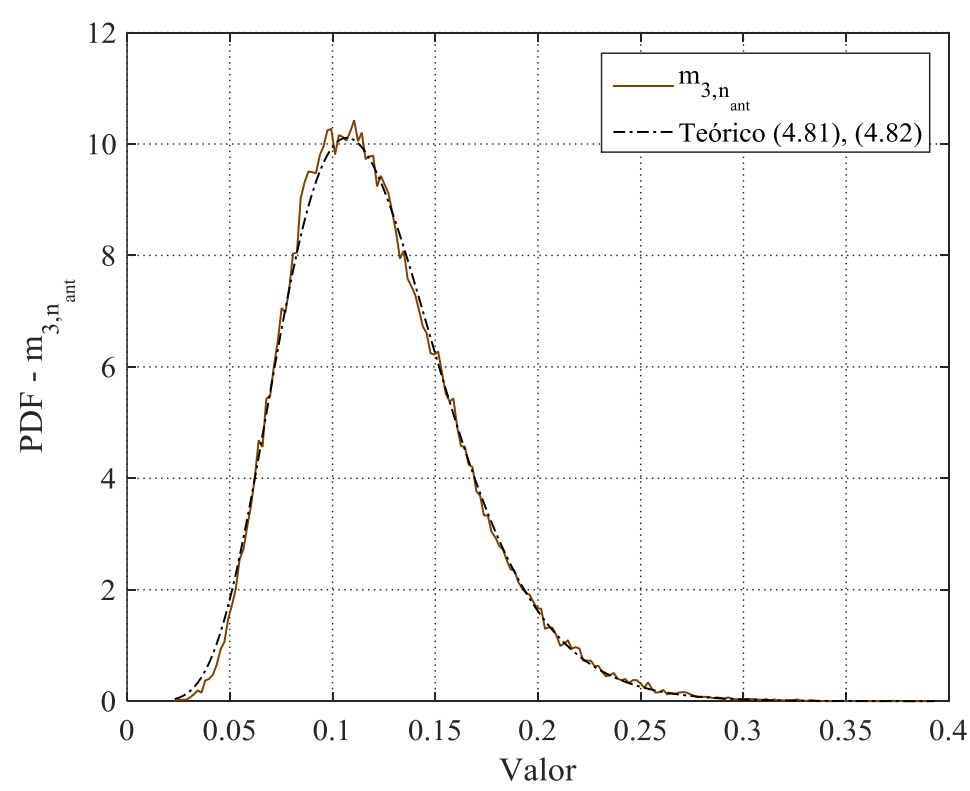

Fig. 4.17 PDFs empírica y teórica del valor de la métrica de sincronización gruesa $m_{3, n}$ en las muestras previas a la llegada del símbolo piloto con ruido de fondo coloreado. 
puede determinar un umbral de detección lo suficientemente alto como para mantener una baja probabilidad de falsa detección. Así, para una probabilidad de $10^{-7}$ de falsa detección de una de las muestras de la métrica cuando sólo contiene muestras de ruido, a partir de la función de la ICDF de la distribución Gamma y considerando los parámetros del sistema $N=4096, N_{2}=512$, se obtiene el umbral $\gamma \approx 0,47$.

En la Fig. 4.18 se muestran los resultados obtenidos por simulaciones para la métrica bajo análisis.Se grafican las PDFs empíricas de los valores en el instante óptimo de sincronismo y en instantes previos al arribo del símbolo piloto, y puede apreciarse como los parámetros obtenidos para describir la métrica $m_{3}$ de (4.76), (4.77), (4.81) y (4.82) se ajustan a lo obtenido por simulaciones. Respecto a la capacidad de detección del símbolo piloto, se observa, para el umbral fijado, la probabilidad de detección a $S N R$ inferiores a $0 \mathrm{~dB}$ es muy baja, debido a la degradación producida por el efecto del ruido de fondo coloreado. Para la condición de $S N R$ de $0 \mathrm{~dB}$, la probabilidad de que la métrica sea inferior al umbral es de $7 \cdot 10^{-3}$.

Comparando la Fig. 4.18 con la Fig. 4.11, puede apreciarse que la capacidad de detección del símbolo piloto bajo ruido de fondo coloreado es superior en la métrica basada en correlación cruzada $m_{3, n}$, con respecto a la métrica basada en AC $m_{2, n}$. Este resultado se reafirma en la Fig. 4.19, en la que se representa la probabilidad de

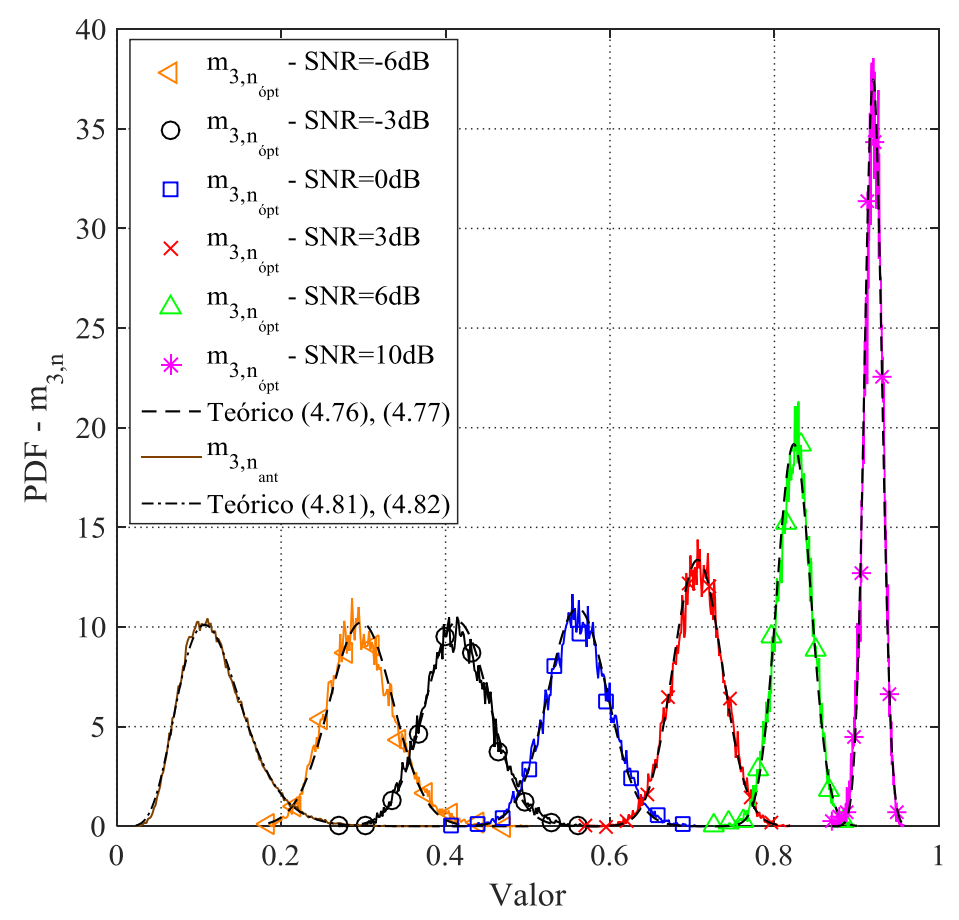

Fig. 4.18 PDFs del valor de la métrica de sincronización gruesa $m_{3, n}$ en los instantes de sincronización óptimo y anteriores, obtenidos por simulaciones bajo un canal PLC tipo A normalizado con ruido de fondo coloreado y distintos valores de SNR. 
Diseño de Estrategias de Sincronización y Estimación de Canal para la Mejora de Comunicaciones en Redes Inteligentes de Energía

detección del símbolo piloto a partir de las mencionadas métricas considerando el canal PLC tipo A y ruido de fondo coloreado, para distintas condiciones de SNR. Parece claro que la métrica basada en correlación cruzada presenta una capacidad de detección mayor, con una diferencia de $5 \mathrm{~dB}$ respecto de la métrica basada en AC. Por otro lado, la probabilidad de detección de las métricas bajo ruido blanco Gaussiano es prácticamente 1 para todos los valores de $S N R$, y en simulaciones se verificó la capacidad de detección del 100\%, en todas las condiciones de SNR consideradas.

\subsubsection{Estimación de SNR a partir de las métricas de sincronización gruesa}

La etapa de sincronización fina se basa en una detección de los caminos del canal a partir de técnicas de umbralización, y resulta evidente que el cálculo de un umbral que optimice la probabilidad de un sincronismo óptimo dependerá de la condición de $S N R$. Por lo tanto, la estimación de la relación señal a ruido resulta de vital importancia para la etapa de sincronización, y en ese sentido, las métricas de sincronización gruesa permiten obtener una estimación de la condición de $S N R$, partiendo de la propuesta de [Schmidl 1997].

4.3.5.1 Estimación a partir de las métricas basadas en autocorrelación

Considerando que la métrica basada en $\mathrm{AC} m_{2, n}$ en el instante óptimo de sincronismo $n_{\text {ópt }}$ tiene por distribución una variable aleatoria gaussiana $M_{2_{n_{\mathrm{o}} p t}}$, con media $\mu_{M_{2_{\mathrm{o}} p t}}=$

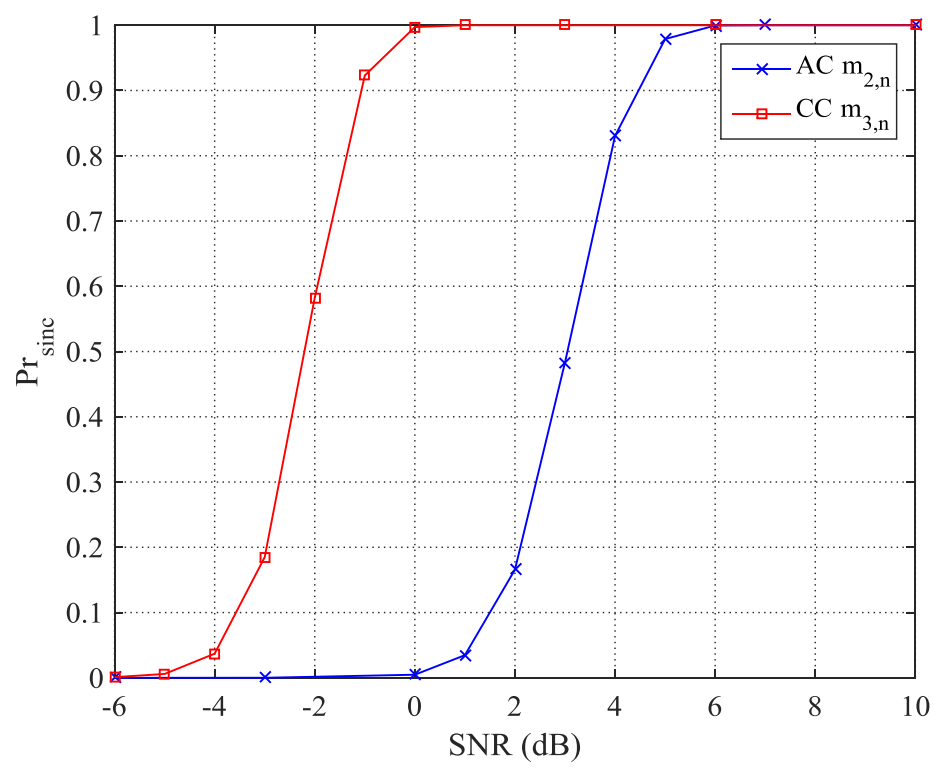

Fig. 4.19 Probabilidad de sincronización en términos de la detección del símbolo piloto para las métricas basadas en autocorrelación y correlación cruzada bajo ruido de fondo coloreado. 
$S N R /(S N R+1)$ de acuerdo a (4.26) y (4.40), puede obtenerse un estimador de la $S N R$ como:

$$
\widetilde{S N R}_{A C}=\frac{m_{2, n_{\mathrm{o} p t}}}{1-m_{2, n_{\mathrm{o}} p t}}
$$

Teniendo en cuenta las varianzas $\sigma_{M_{2 o ́} p t}^{2}$ con ruido de fondo blanco (4.27) y coloreado (4.41), es posible obtener la distribución del estimador de la $S N R$. Dado que la media de $M_{2_{n_{\text {ó }} t}}$ es mucho mayor que su desvío estándar, es factible aproximar la distribución del estimador de la condición de $S N R$ como gaussiana. Definiendo la variable normal

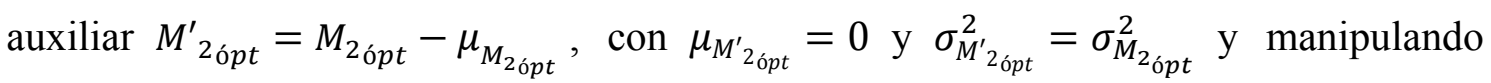
(4.83) se obtiene:

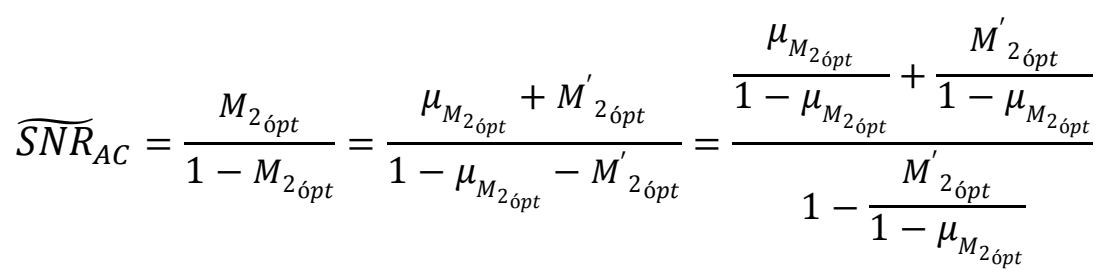

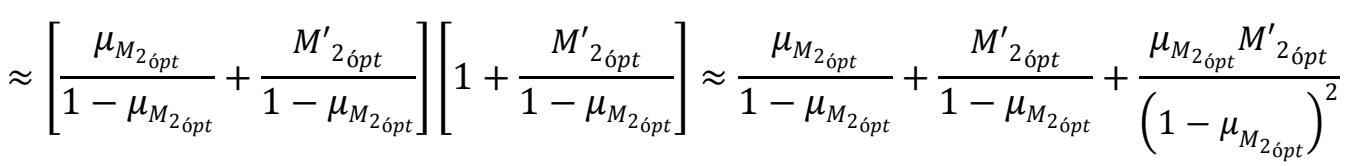

Luego, pueden obtenerse la media y la varianza del estimador de la relación señal a ruido, de acuerdo a:

$$
\begin{aligned}
& \mu_{\widetilde{S N R}_{A C}}=\frac{\mu_{M_{2_{o ́} p t}}}{1-\mu_{M_{2_{o p t}}}}=S N R \\
& \sigma_{\overline{S N R}_{A C}}^{2}=\frac{\sigma_{M_{2^{\circ} p t}}^{2}}{\left(1-\mu_{M_{2^{\prime} p t}}\right)^{4}}=\sigma_{M_{2^{\prime} p t}}^{2}(S N R+1)^{4}
\end{aligned}
$$

Y la varianza del estimador para el caso de ruido blanco resulta en (4.87), mientras que para el caso de ruido de fondo coloreado es (4.88). Como el estimador basado en $m_{2, n}$ es insesgado, entonces la varianza coincide con el error cuadrático medio (Mean square error-MSE) del estimador.

$$
\sigma_{\overline{S N R}_{A C, B}}^{2}=\frac{4 S N R(S N R+1)^{2}}{N}-\frac{8 S N R^{2}(S N R+1)}{N}+\frac{4 S N R^{3}}{N}+\frac{2 S N R^{2}}{N}+\frac{2(S N R+1)^{2}}{N}
$$


Diseño de Estrategias de Sincronización y Estimación de Canal para la Mejora de Comunicaciones en Redes Inteligentes de Energía

$$
\begin{gathered}
\sigma_{\overline{S N R}_{A C, C}}^{2}=\frac{4 S N R(S N R+1)^{2}}{N}-\frac{8 S N R^{2}(S N R+1)}{N}+\frac{4 S N R^{3}}{N}+\frac{\Sigma_{1}(S N R+1)^{2}}{N^{2}} \\
+\frac{\Sigma_{2} S N R^{2}}{N^{2}}
\end{gathered}
$$

En la Fig. 4.20 se presenta la comparación entre los resultados analíticos obtenidos a partir del análisis realizado para el estimador de $S N R$, y los resultados obtenidos por simulación, para las dos métricas basadas en AC $m_{1, n}$ y $m_{2, n}$. Puede observarse cómo la media del estimador basado en $m_{2, n}$ coincide con el valor de $S N R$ (estimador insesgado). Por otro lado, el estimador obtenido a partir de $m_{1, n}$ es un estimador sesgado debido a que la métrica no posee una meseta, y su valor máximo queda sujeto a la dispersión del canal, disminuyendo a medida que la dispersión del canal aumenta. Además, el análisis del estimador realizado para el estimador basado en $m_{2, n}$ se ajusta perfectamente a las simulaciones bajo ruido blanco, mientras que, en el caso de ruido coloreado, en el resultado de simulaciones se aprecia una varianza mayor respecto al
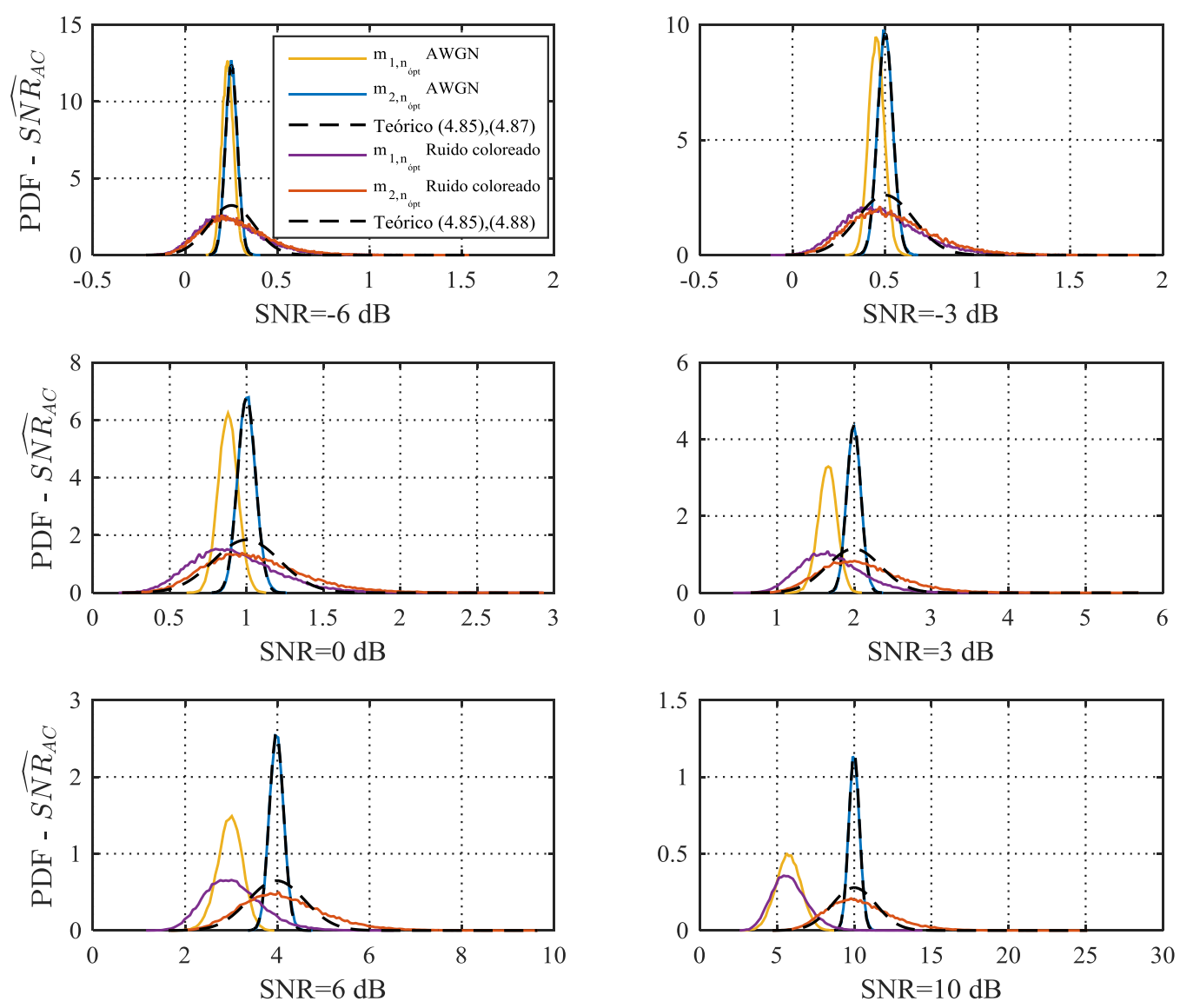

Fig. 4.20 PDFs del estimador de SNR obtenido a partir de las métricas basadas en autocorrelación. 
esperado de acuerdo a (4.88), debido a las aproximaciones realizadas. Lógicamente, la varianza (y MSE) del estimador resulta mayor cuando se considera ruido de fondo coloreado en lugar de ruido blanco, debido a que las muestras de ruido correladas incrementan la varianza de la métrica en el instante óptimo de sincronismo.

\subsubsection{Estimación a partir de la métrica basada en correlación cruzada}

La métrica basada en correlación cruzada $m_{3, n}$ en el instante óptimo de sincronismo $n_{\text {ópt }}$ tiene por distribución una variable aleatoria gaussiana $M_{3_{n_{\text {ó }} t}}$, con media $\mu_{M_{3 o ́ p t}}=$ $\left(S N R+N_{2} / N\right) /(S N R+1)$ de acuerdo a (4.64) y (4.76), y en este caso, se obtiene el estimador de $S N R$ de acuerdo a:

$$
S \widetilde{N R_{C C}}=\frac{m_{3, n_{\text {ópt }}}-N_{2} / N}{1-m_{3, n_{\text {ópt }}}}
$$

Teniendo en cuenta las varianzas $\sigma_{M_{3 o ́} p}^{2}$ con ruido de fondo blanco (4.65) y coloreado (4.77), es posible obtener la distribución del estimador de $S N R$. Como la media de $M_{3_{n_{\text {ó }} t}}$ es mucho mayor que su desvío estándar, es factible aproximar la distribución del estimador de la condición de $S N R$ como gaussiana. Definiendo la variable normal

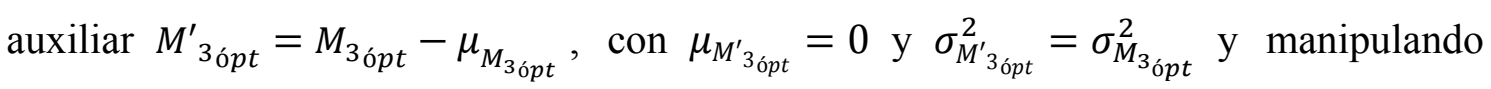
(4.89) de la misma manera que en (4.84), se obtiene:

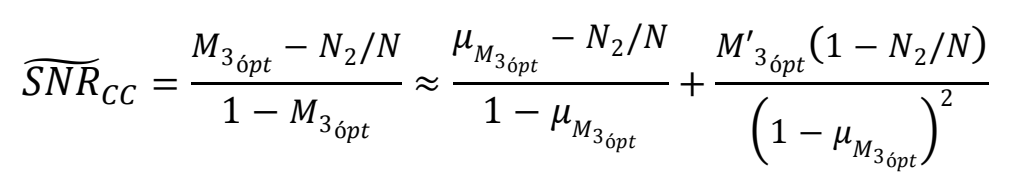

Luego, pueden obtenerse la media y la varianza del estimador de la relación señal a ruido, de acuerdo a:

$$
\begin{gathered}
\mu_{\overline{S N R} C C}=\frac{\mu_{M_{3_{\mathrm{o} p t}}}-N_{2} / N}{1-\mu_{M_{3_{\mathrm{o}} p t}}}=S N R \\
\sigma_{\overline{S N R}_{C C}}^{2}=\frac{\sigma_{M_{3_{\mathrm{o}} p t}}^{2}\left(1-N_{2} / N\right)}{\left(1-\mu_{M_{3_{\mathrm{o}} p t}}\right)^{4}}=\sigma_{M_{3_{\mathrm{o}} p t}}^{2} \frac{(S N R+1)^{4}}{\left(1-\frac{N_{2}}{N}\right)^{2}}
\end{gathered}
$$

Luego, la varianza (y MSE) del estimador para el caso de ruido blanco resulta en (4.93), mientras que para el caso de ruido de fondo coloreado es (4.94). 


$$
\begin{aligned}
& \sigma_{\overline{S N R}_{C C, B}}^{2}=\frac{4 S N R(S N R+1)^{2}}{N\left(1-\frac{N_{2}}{N}\right)^{2}}+\frac{4 S N R \Sigma_{3}\left(S N R+\frac{N_{2}}{N}\right)^{2}}{N_{2}^{2} N^{2}\left(1-\frac{N_{2}}{N}\right)^{2}}-\frac{8 S N R\left(N-\frac{\left(N_{2}-1\right)}{2}\right)\left(S N R+\frac{N_{2}}{N}\right)(S N R+1)}{N^{2}\left(1-\frac{N_{2}}{N}\right)^{2}} \\
& +\frac{2 N_{2}(S N R+1)^{2}}{N^{2}\left(1-\frac{N_{2}}{N}\right)^{2}}+\frac{2 \Sigma_{3}\left(S N R+\frac{N_{2}}{N}\right)^{2}}{N_{2}^{2} N^{2}\left(1-\frac{N_{2}}{N}\right)^{2}}-\frac{4 \Sigma_{3}\left(S N R+\frac{N_{2}}{N}\right)(S N R+1)}{N_{2} N^{3}\left(1-\frac{N_{2}}{N}\right)^{2}} \\
& \sigma_{\overline{S N R} C C, C}^{2}=\frac{4 S N R(S N R+1)^{2}}{N\left(1-\frac{N_{2}}{N}\right)^{2}}+\frac{4 S N R \Sigma_{3}\left(S N R+\frac{N_{2}}{N}\right)^{2}}{N_{2}^{2} N^{2}\left(1-\frac{N_{2}}{N}\right)^{2}}-\frac{8 S N R\left(N-\frac{\left(N_{2}-1\right)}{2}\right)\left(S N R+\frac{N_{2}}{N}\right)(S N R+1)}{N^{2}\left(1-\frac{N_{2}}{N}\right)^{2}} \\
& +\frac{\Sigma_{5}(S N R+1)^{2}}{N^{4}\left(1-\frac{N_{2}}{N}\right)^{2}}+\frac{\left(S N R+\frac{N_{2} \Sigma_{4}}{N^{2}}\right)^{2} \Sigma_{6}}{N_{2}^{2} N^{2}\left(1-\frac{N_{2}}{N}\right)^{2}}-\frac{2\left(S N R+\frac{N_{2} \Sigma_{4}}{N^{2}}\right) \Sigma_{6}(S N R+1)}{N_{2} N^{3}\left(1-\frac{N_{2}}{N}\right)^{2}}
\end{aligned}
$$

En la Fig. 4.21 se presenta la comparación entre los resultados analíticos obtenidos a partir del análisis y los resultados obtenidos por simulación, para el estimador de $S N R$ de la métrica basada en correlación cruzada $m_{3, n}$. Puede observarse que, si bien el ajuste es aceptable para el rango de $S N R$ de -6 a $10 \mathrm{~dB}$, la varianza teórica del estimador es ligeramente superior a la empírica obtenida a través de simulaciones, tanto para la métrica bajo ruido blanco como bajo ruido coloreado.

\subsubsection{Métricas de sincronización gruesa bajo ruido impulsivo}

Como se ha descrito en el capítulo 2, el ruido del canal PLC está compuesto no sólo por ruido de fondo coloreado, sino también por diversas fuentes de ruido impulsivo. Los eventos de ruido impulsivo suelen ser de corta duración y de mucha mayor amplitud que las muestras del ruido de fondo coloreado. Además, en lo que respecta al análisis del impacto en la técnica de sincronización gruesa, no afectarán a todos los símbolos pilotos que se transmitan, sino que lo harán en función de una determinada probabilidad de ocurrencia. En la Fig. 4.22 se presenta la descomposición del modelo de ruido del canal PLC obtenido a partir del sintetizador de [Prasad 2019], en ruido de fondo coloreado, ruido impulsivo periódico asíncrono con la red, impulsivo periódico síncrono con la red, e impulsivo aperiódico. Puede apreciarse que, en general, la amplitud del ruido impulsivo es mucho mayor que la amplitud de las muestras del ruido de fondo coloreado. Por otro lado, sólo algunas de las transmisiones se verán afectadas por un evento de ruido impulsivo. La probabilidad de ocurrencia de un evento de ruido impulsivo en la señal recibida que contiene el símbolo piloto fue obtenida a partir de 

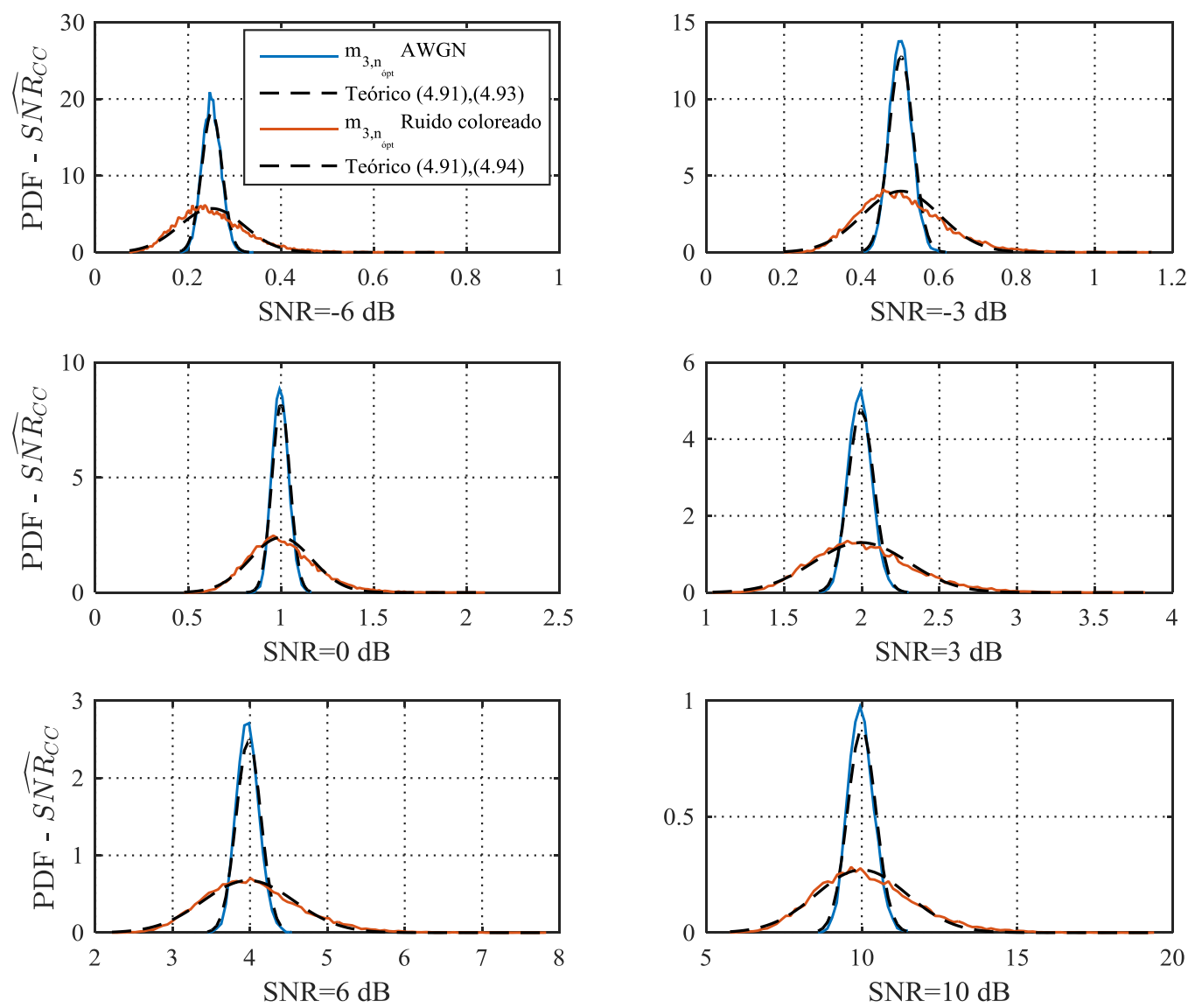

Fig. 4.21 PDFs del estimador de SNR obtenido a partir de la métrica basada en correlación cruzada. simulaciones y resultó de aproximadamente un $8 \%$. El resultado resulta comparable a probabilidades de ocurrencia de hasta 10\% consideradas en trabajos previos [Alsusa 2013] [Juwono 2014] [Juwono 2016].

\subsubsection{Efecto del ruido impulsivo en la sincronización gruesa}

Un evento de ruido impulsivo afectará severamente las métricas de sincronización gruesa basadas en $\mathrm{AC}$ y en $\mathrm{CC}$, debido a que la energía que normaliza las métricas sufre un gran incremento. Por otro lado, el valor resultante en el numerador de ambas métricas no se incrementa considerablemente. En el caso de la métrica basada en AC $m_{2, n_{\text {ópt }}}$ (4.23), es debido a que las muestras de ruido que contienen ruido impulsivo se multiplican por muestras de ruido que se encuentran alejadas del evento de ruido, y con muy baja probabilidad de que se vean afectadas por otro evento de ruido impulsivo. En el caso de la métrica basada en correlación cruzada $m_{3, n_{\text {ópt }}}$ (4.48), en el cálculo del numerador las muestras de ruido atraviesan el filtro acoplado correspondiente al 
Diseño de Estrategias de Sincronización y Estimación de Canal para la Mejora de Comunicaciones en Redes Inteligentes de Energía
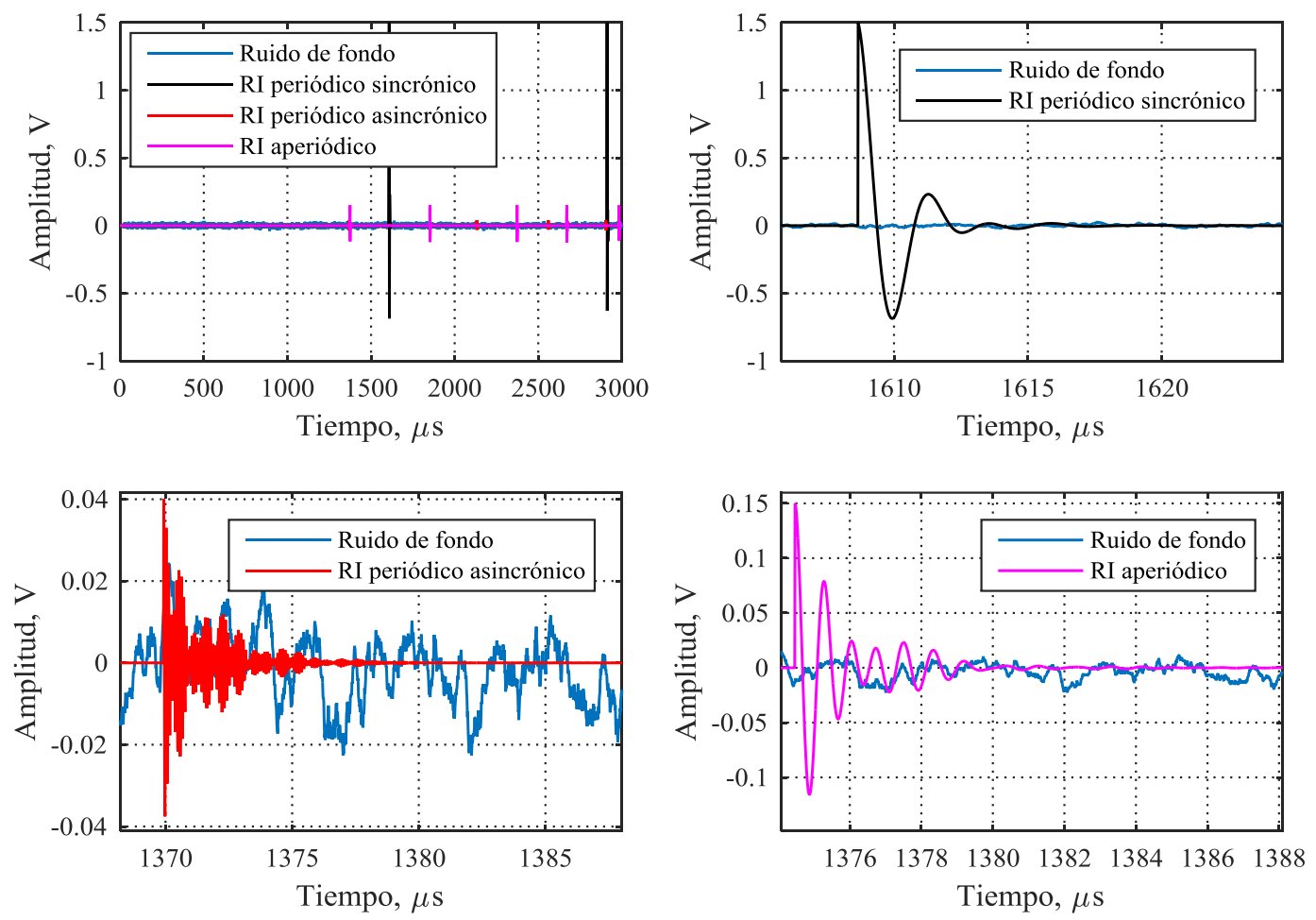

Fig. 4.22 Descomposición del modelo de ruido del canal PLC en ruido de fondo y diversas fuentes de ruido impulsivo.

símbolo piloto, que aleatoriza el signo de las muestras del evento de ruido impulsivo, que al sumarse y luego elevarse al cuadrado dan por resultado un valor relativamente bajo respecto al incremento de energía del denominador. Así, puede concluirse que los valores resultantes de las métricas en el instante óptimo de sincronismo disminuyen considerablemente ante la ocurrencia de un evento de ruido impulsivo. Bajo las mismas consideraciones, es esperable que las métricas en las muestras previas a la llegada del símbolo piloto $m_{2, n_{a n t}}$ y $m_{3, n_{a n t}}$ también disminuyan sus valores. En la Fig. 4.23 se presentan los resultados obtenidos para las métricas $m_{2, n_{\text {ó }} t}$ y $m_{3, n_{\text {ó }} t}$, evaluando las PDFs empíricas para el instante óptimo de sincronismo con y sin ruido impulsivo, y una SNR 0 $\mathrm{dB}^{1}$. Puede observarse que, bajo ruido impulsivo, los valores de las métricas se ven reducidos respecto a una condición sin ruido impulsivo, y acorde a la probabilidad de ocurrencia mencionada.

\footnotetext{
${ }^{1}$ La SNR continúa siendo definida por la relación de señal a ruido de fondo coloreado, es decir, sin incluir la potencia del ruido impulsivo.
} 

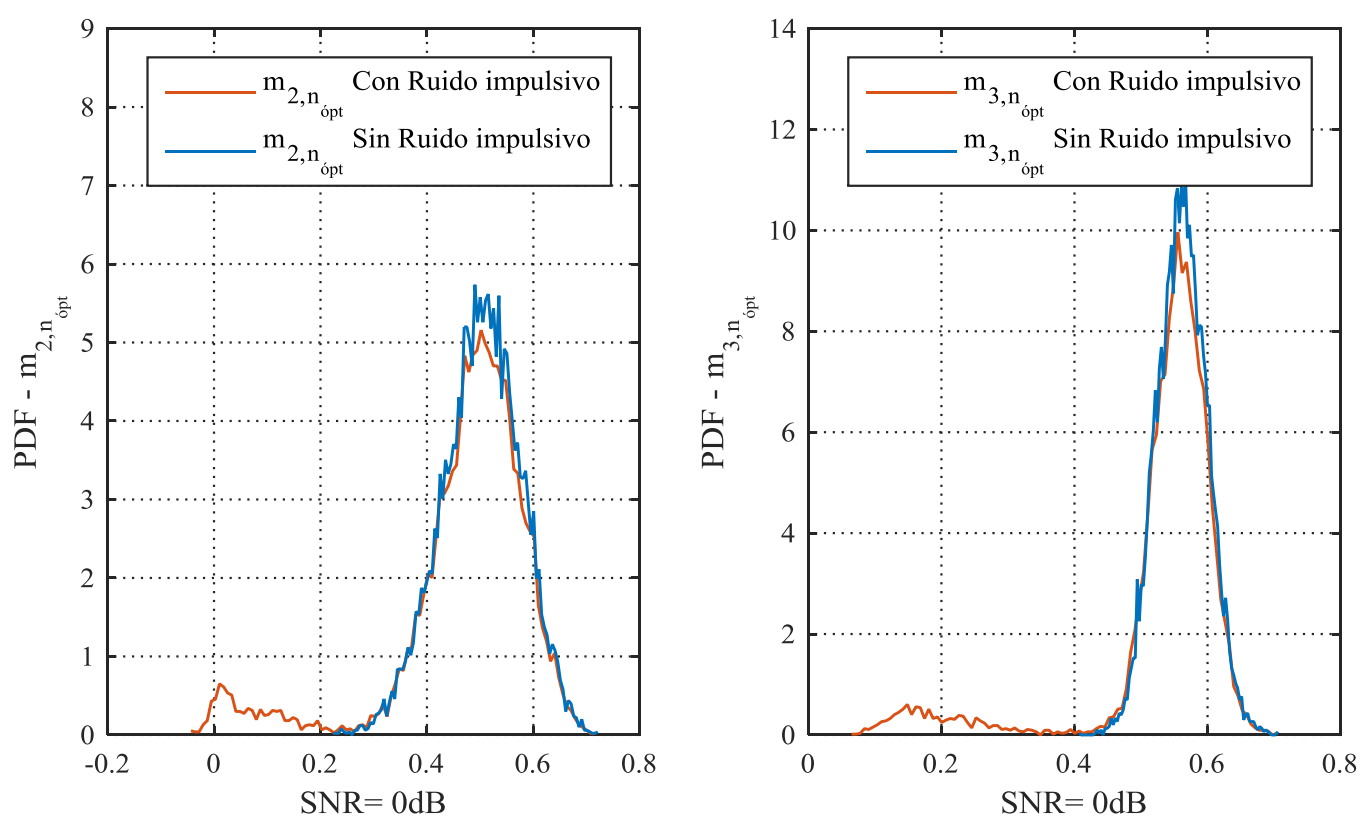

Fig. 4.23 PDF empirica de las métricas de sincronización gruesa en el instante óptimo de sincronismo, con y sin eventos de ruido impulsivo con probabilidad de ocurrencia del $8 \%$ y una $S N R=0 d B$.
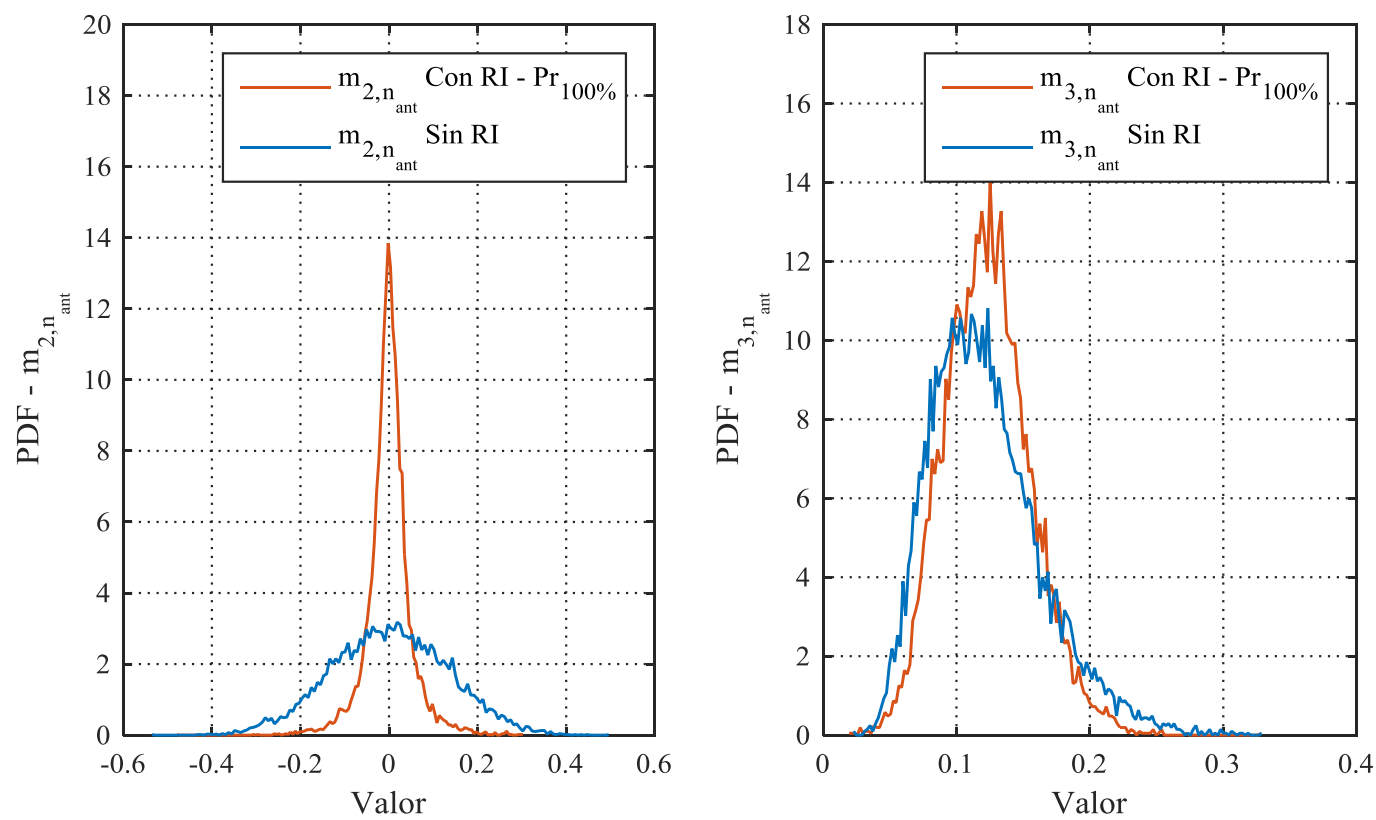

Fig. 4.24 PDFs empíricas de las métricas de sincronización gruesa en los instantes previos a la llegada del símbolo piloto, con y sin eventos de ruido impulsivo con probabilidad de ocurrencia del 100\%.

Con respecto a los valores de las métricas en muestras previas a la llegada del símbolo piloto, en la Fig. 4.24 se presenta el efecto del ruido impulsivo. Para hacerlo más notorio, en lugar de considerar la probabilidad de ocurrencia esperable de $8 \%$ de un evento de ruido impulsivo en el canal, se computan las PDFs empíricas considerando una probabilidad de ocurrencia hipotética del 100\%. Es decir, en todas las transmisiones 
Diseño de Estrategias de Sincronización y Estimación de Canal para la Mejora de Comunicaciones en Redes Inteligentes de Energía

al menos un evento de ruido impulsivo afecta a las muestras procesadas en el cálculo de las métricas.

Puede observarse claramente que, bajo ruido impulsivo, las métricas en las muestras previas a la llegada del símbolo piloto presentan una menor varianza. Sin embargo, como la probabilidad real de ocurrencia de los eventos de ruido impulsivo es mucho menor, los umbrales de detección deben mantenerse de acuerdo a los valores obtenidos a partir de (4.43), (4.44), (4.81) y (4.82), para mantener baja la probabilidad de falsa detección cuando no ocurre un evento de ruido impulsivo.

Como el ruido impulsivo implica una disminución en los valores efectivos de las métricas, y no es factible disminuir el umbral de detección, la probabilidad de detección del símbolo piloto se verá disminuida. En la Fig. 4.25 se presenta la probabilidad de detección del símbolo piloto para las métricas de sincronización para distintos valores de $S N R$, con y sin eventos de ruido impulsivo con probabilidad de ocurrencia típica del $8 \%$, y para el hipotético caso de probabilidad de ocurrencia del 100\%. Puede apreciarse que bajo ruido impulsivo la capacidad de detección disminuye, degradando todo el rango de $S N R$ bajo estudio, y reduciendo su efecto recién a muy altas $S N R$ s. El caso de probabilidad de ocurrencia de eventos de ruido impulsivo del 100\% resulta útil para analizar el efecto del mismo en la métrica de sincronización. Puede apreciarse que, condicionada a la ocurrencia de un evento de ruido impulsivo, la probabilidad de detección en ambas métricas se degrada sustancialmente. La capacidad de detección para la métrica $m_{2, n}$ se reduce de casi $100 \%$ a $23 \%$ para una $S N R$ de $6 \mathrm{~dB}$, y de $100 \%$ a $31 \%$ para una de $10 \mathrm{~dB}$. Por su parte, la métrica $m_{3, n}$ reduce su capacidad de detección de $100 \%$ al $13 \%$ para una $S N R$ de $0 \mathrm{~dB}$, y de $100 \%$ a $64 \%$ para una de $10 \mathrm{~dB}$.

4.3.6.2 Mitigación de ruido impulsivo en la etapa de sincronización gruesa Como el ruido impulsivo afecta sustancialmente la capacidad de detección del símbolo piloto, deben implementarse técnicas para mitigar su presencia en la señal recibida. Existen técnicas de procesamiento no lineal que permiten reducir el impacto del ruido impulsivo [Juwono 2014]. Dentro de las más difundidas se encuentran el recorte (clipping), que implica recortar las muestras de la seña recibida que superen un determinado umbral, que debe definirse convenientemente. Así, los altos picos producidos por el ruido impulsivo se verán limitados, y las muestras de la señal recibida presentarán un fenómeno de saturación. Otra de las técnicas más difundidas es el 


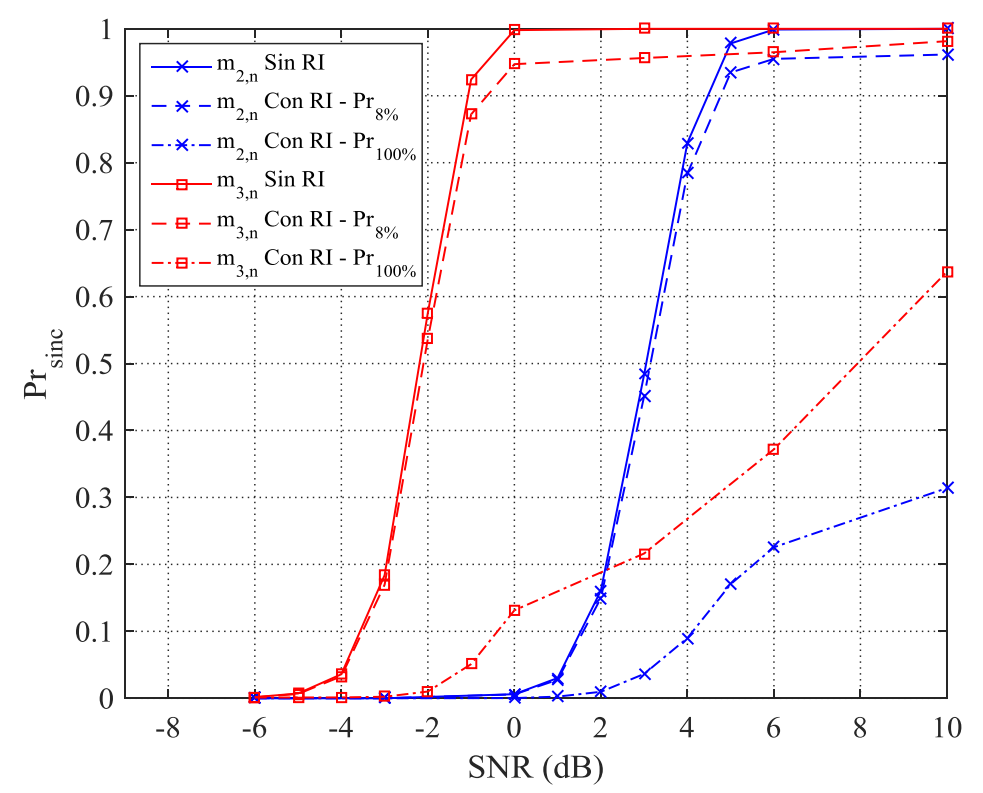

Fig. 4.25 Probabilidad de detección del símbolo piloto a partir de las métricas de sincronización gruesa, con y sin eventos de ruido impulsivo con probabilidad de ocurrencia de 8 y $100 \%$.

blanqueo (blanking), en el que las muestras de la señal recibida que superan un determinado umbral son anuladas, adoptando un valor de cero. El fundamento en la técnica de blanqueo radica en que, si el ruido impulsivo afecta tan severamente a las muestras de la señal, conservar las muestras puede introducir más interferencia que descartarlas. La elección de los umbrales, sea estática o dinámicamente, resulta de vital importancia en este tipo de técnicas. Si el umbral se fija con un valor relativamente alto, sólo una pequeña parte de las muestras que contienen ruido impulsivo se verán recortadas o anuladas por el proceso; y, si por el contrario se fija con un valor relativamente bajo, se recortará o blanqueará la señal útil en las muestras donde no existe influencia de ruido impulsivo. Adicionalmente, puede aplicarse una combinación de estas técnicas para mejorar el rendimiento, de acuerdo a [Juwono 2016].

Como la detección de la señal en la etapa de sincronización gruesa es una de las primeras etapas de procesamiento, debe definirse el umbral de recorte o blanqueo a partir del procesamiento de la misma señal recibida, por ejemplo a partir de una estimación de la condición de $S N R$, o bien definir un umbral dinámico a partir de la evaluación de la amplitud de las muestras recibidas en una determinada ventana de tiempo, lo que puede ser adecuado siempre que la parte útil de la señal recibida no tenga una potencia pico a promedio (Peak to average power ratio - PAPR) elevada. Si bien el desarrollo de técnicas que permitan obtener umbrales de blanqueo o recorte excede el alcance de esta tesis, se realizaron simulaciones para evaluar las potencialidades de aplicar este tipo de técnicas a la señal recibida para mitigar el efecto del ruido 
Diseño de Estrategias de Sincronización y Estimación de Canal para la Mejora de Comunicaciones en Redes Inteligentes de Energía
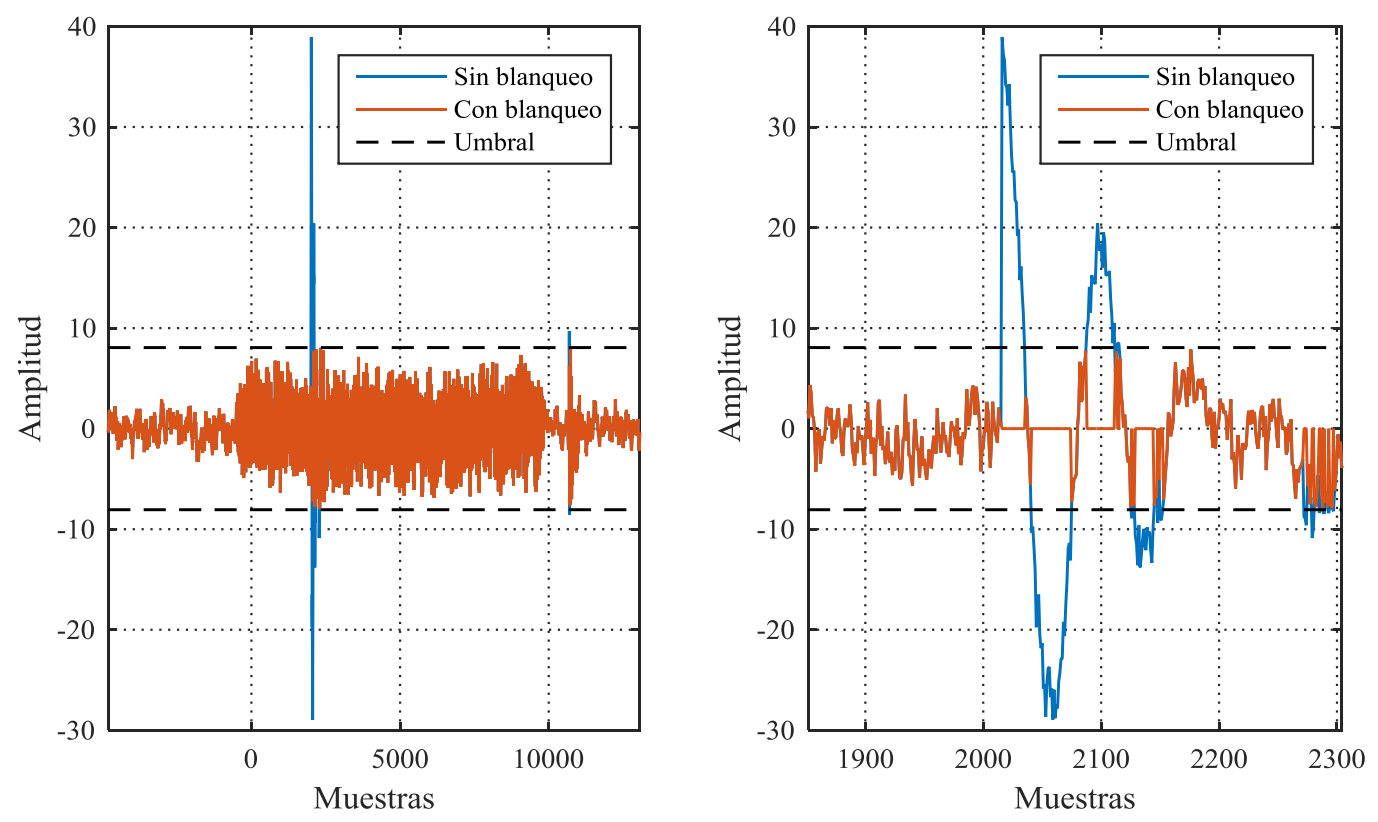

Fig. 4.26 Muestras de la señal recibida antes y después de aplicar el procesamiento no lineal de blanqueo, con SNR de $6 d B$.

impulsivo. Se implementó la técnica de blanqueo, considerando un umbral estático definido arbitrariamente, pero que garantiza un blanqueo importante del ruido impulsivo, sin anular muestras de la señal que no se vean afectadas por dicho ruido. En la Fig. 4.26 se presenta a la izquierda una realización correspondiente a la señal recibida con una condición de $S N R$ de $6 \mathrm{~dB}$ afectada por ruido impulsivo, el umbral de blanqueo fijado, y la señal luego de la aplicación del procesamiento no lineal. A la derecha se presenta un zoom de la anterior en las muestras que se ven afectadas por un evento de ruido impulsivo.

Otra estrategia para mitigar el ruido impulsivo descripta en [Bumiller 2007] se basa en el hecho de que la señal recibida es amplificada en una etapa previa al convertidor analógico-digital (Analog to Digital Converter - ADC), a partir de un amplificador de ganancia variable cuya ganancia es modificada por un módulo de control automático de ganancia (Automatic Gain Control - AGC). Con una dinámica de la etapa de AGC adecuada puede garantizarse el uso del rango dinámico del amplificador para la señal útil, mientras que las muestras que se ven distorsionadas por un evento de ruido impulsivo se verán limitadas por el rango dinámico del amplificador, lo que equivaldría a la aplicación de una técnica no lineal de recorte de la señal. Puede realizarse un recorte adicional en el procesamiento digital si la ganancia utilizada para la amplificación de la señal no cubre todo el rango dinámico del amplificador. 
Nuevamente, a pesar de que la dinámica de la etapa de AGC requiere de un diseño y estudio específico, en nuestro trabajo se considera una etapa de AGC simple, en el que el valor de la ganancia para una muestra se obtiene a partir de la media móvil de la amplitud de las muestras de la señal recibida anteriores, en una ventana de longitud $N_{A G C}$ muestras. Debido a que la ganancia tiene un retardo para estabilizarse, para evitar distorsiones en la recepción de la señal útil, se ha considerado la extensión del prefijo cíclico del símbolo piloto de $N_{c p}$ a $N_{c p}+N_{A G C}$ muestras.

El cálculo de la ganancia puede efectuarse a partir de:

$$
K_{A G C_{n}}=\frac{N_{A G C}}{\sum_{m=0}^{N_{A G C^{-1}}\left|r_{n-m}\right|}}
$$

Luego, los cambios en la ganancia $K_{A G C_{n}}$ pueden cuantificarse para evitar oscilaciones o efecto serrucho. En la Fig. 4.27 se presenta a la izquierda el resultado obtenido por simulaciones al aplicar la etapa de AGC a la señal recibida, mientras que a la derecha se incluye un zoom de la anterior en las muestras que se ven afectadas por un evento de ruido impulsivo. La condición de $S N R$ es de $6 \mathrm{~dB}, N_{A G C}=512$, y los valores de saturación se fijaron a \pm 4 . Puede apreciarse cómo la ganancia disminuye ante la llegada del símbolo piloto respecto de las muestras que sólo contienen ruido. Además, cuando se produce un evento de ruido impulsivo, la distorsión se ve limitada por el efecto de saturación en las primeras muestras del impulso, y luego por la disminución de la
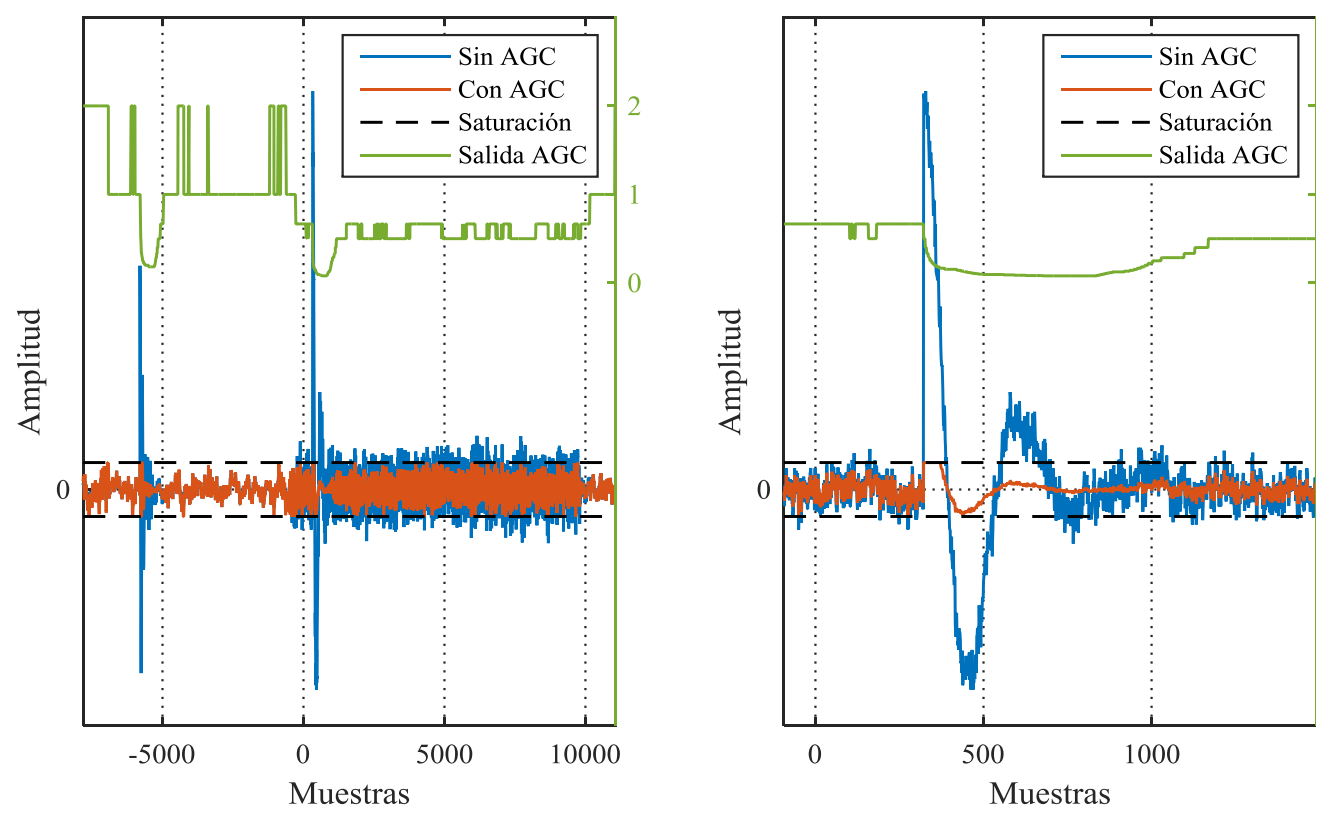

Fig. 4.27 Resultado del uso de la etapa de AGC como medio para mitigar el efecto del ruido impulsivo. 
Diseño de Estrategias de Sincronización y Estimación de Canal para la Mejora de Comunicaciones en Redes Inteligentes de Energía

ganancia.

Comparada con la técnica basada en blanqueo, puede apreciarse que la ganancia continúa en un nivel bajo a pesar de la extinción del impulso durante algunas muestras, debido a la dinámica propia de la ganancia que depende de la ventana de $N_{A G C}$ muestras incluidas en la ventana de media móvil. Una ventaja de esta técnica radica en que, a partir de valores de saturación conocidos, la etapa de AGC permite que las señales procesadas se encuentren siempre dentro de los niveles de saturación, independientemente de la condición de $S N R$.

Sin embargo, las técnicas mencionadas no son excluyentes, en el sentido que el bloque de AGC es obligatorio en la práctica, y a su vez puede complementarse con un procesamiento no lineal posterior a la conversión analógico-digital. Finalmente, se efectuaron simulaciones para evaluar las técnicas basadas en blanqueo y AGC para la mitigación del ruido impulsivo. En las Fig. 4.28 y Fig. 4.29 se presentan los resultados obtenidos para la métrica basada en $\mathrm{AC} m_{2, n}$ y en correlación cruzada $m_{3, n}$ respectivamente. En ambos casos, se incluyen las PDFs de las métricas sin ruido impulsivo, con ruido impulsivo, y luego de aplicar las técnicas de mitigación. Además, se consideró una probabilidad de ocurrencia de los eventos de ruido impulsivo de $100 \%$ y diferentes niveles de $S N R$. Puede observarse en ambas que las técnicas reducen significativamente el impacto del ruido impulsivo en las métricas de sincronización y, bajo los umbrales fijados, la técnica de blanqueo logra resultados ligeramente superiores a la basada en AGC. En ningún caso se alcanzan los valores obtenidos sin ruido impulsivo, y esto se debe a que un impulso puede afectar hasta un cuarto de la longitud del símbolo piloto, deteriorando las propiedades de correlación del símbolo piloto a pesar de ser mitigado, además de incrementar el valor de $S N R$ efectiva.

En la Fig. 4.30 se presenta la probabilidad de detección del símbolo piloto con y sin ruido impulsivo con probabilidad de ocurrencia del 100\%, y luego de aplicar las técnicas de mitigación. La capacidad de detección para la métrica $m_{2, n}$ mejora significativamente recién para condiciones de $S N R$ mayores a $5 \mathrm{~dB}$, alcanzando a $10 \mathrm{~dB}$ una probabilidad de detección de 60\% con el método basado en AGC y $66 \%$ para el método de blanqueo, respecto del 31\% sin aplicar técnicas de mitigación. Por su parte, la métrica basada en correlación cruzada $m_{3, n}$ ve mejorada considerablemente su capacidad de detección, aumentando la probabilidad de detección, a una $S N R$ de $3 \mathrm{~dB}$, 

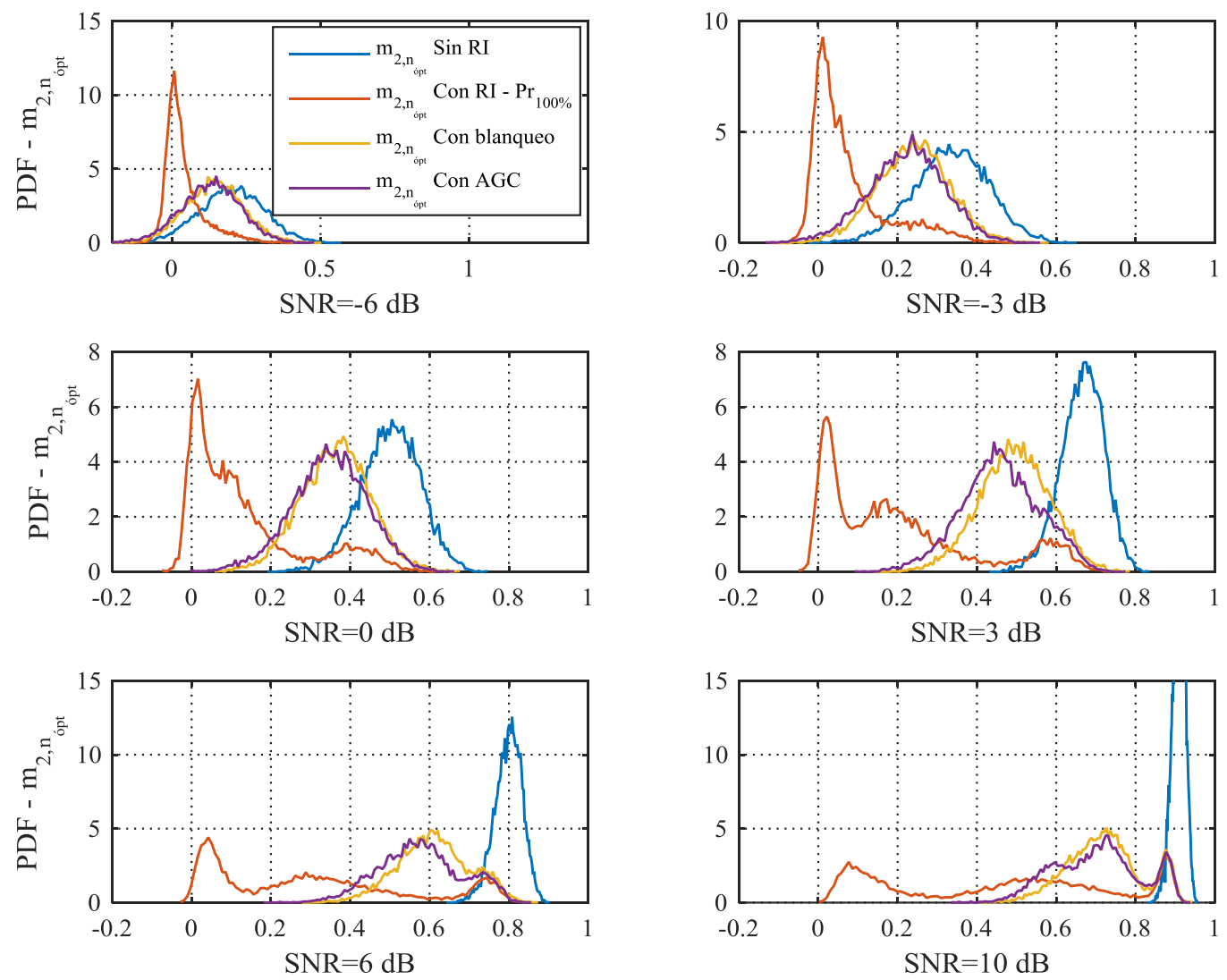

Fig. 4.28 Efecto de las técnicas de blanqueo y AGC en las PDFs de la métrica $m_{2, n}$ bajo eventos de ruido impulsivo con probabilidad de ocurrencia de $100 \%$.

de un 22\% sin mitigación, a $88 \%$ y $94 \%$ para las técnicas basadas en AGC y blanqueo, respectivamente.

A partir de una $S N R$ de $6 \mathrm{~dB}$, se obtiene una capacidad de detección del 100\%. Si bien estos resultados resultan útiles para verificar la capacidad de atenuación del efecto del ruido impulsivo al aplicar las técnicas propuestas, como la probabilidad de ocurrencia real de los eventos de ruido impulsivo es del $8 \%$, la probabilidad de sincronización del sistema resulta superior, y se encuentra en la Fig. 4.31.

\subsubsection{Efecto del desvío en la frecuencia de muestreo en la sincronización gruesa}

De acuerdo a lo estudiado el capítulo 3, en los sistemas OFDM el CFO no afecta a las métricas basadas en AC, mientras que degrada la correlación cruzada del símbolo piloto. Por otra parte, en los sistemas DMT no se produce CFO debido a que se trata de transmisiones banda base, sin las etapas de modulación de fase y cuadratura en una portadora central. Sin embargo, se presenta el fenómeno de desvío en la frecuencia de muestreo (SFO - Sampling Frequency Offset) que afecta el desempeño de las técnicas 
Diseño de Estrategias de Sincronización y Estimación de Canal para la Mejora de Comunicaciones en Redes Inteligentes de Energía
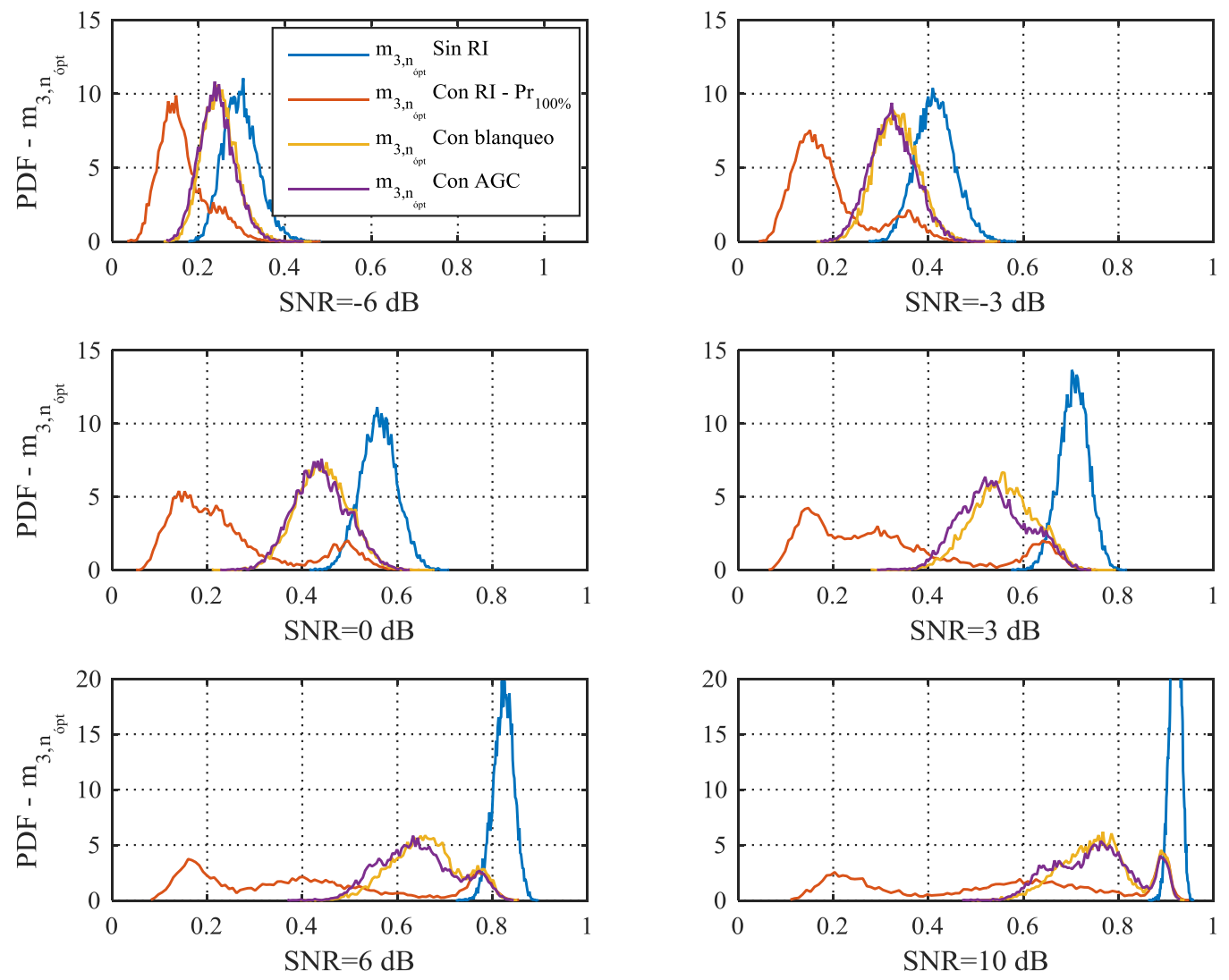

Fig. 4.29 Efecto de las técnicas de blanqueo y AGC en las PDFs de la métrica $m_{3, n}$ bajo eventos de ruido impulsivo con probabilidad de ocurrencia de $100 \%$.

de sincronización, tanto para las métricas basadas en AC como en correlación cruzada. En el estándar IEEE 1901 [IEEE 2010] se recomienda una tolerancia máxima en la frecuencia de muestreo de $\pm 25 \mathrm{ppm}$, mientras que en [Kim 2011] se considera una tolerancia máxima de $\pm 100 \mathrm{ppm}$.

Por lo tanto, el valor de SFO máximo resulta en $\pm 200 \mathrm{ppm}$. A partir de esto, se efectuaron simulaciones para determinar el impacto del SFO en las técnicas de sincronización gruesa propuestas. En la Fig. 4.32 se grafican las PDFs de la métrica basada en $\mathrm{AC} m_{2, n}$ (izquierda) y de la métricas basada en correlación cruzada $m_{3, n}$ (derecha), bajo ruido de fondo coloreado, una condición de $S N R$ de $6 \mathrm{~dB}$ y tres variantes de SFO: sin SFO, con un SFO intermedio de 100ppm, y con un SFO máximo de 400ppm. Puede observarse que, para un SFO de100ppm, las dos métricas prácticamente no se modifican, y, para el caso de SFO de 400ppm, las dos métricas se degradan de manera similar.

En la Fig. 4.33 se presenta la probabilidad de detección del símbolo piloto para las mismas variantes de SFO utilizadas en la Fig. 4.32. Puede observarse cómo la 


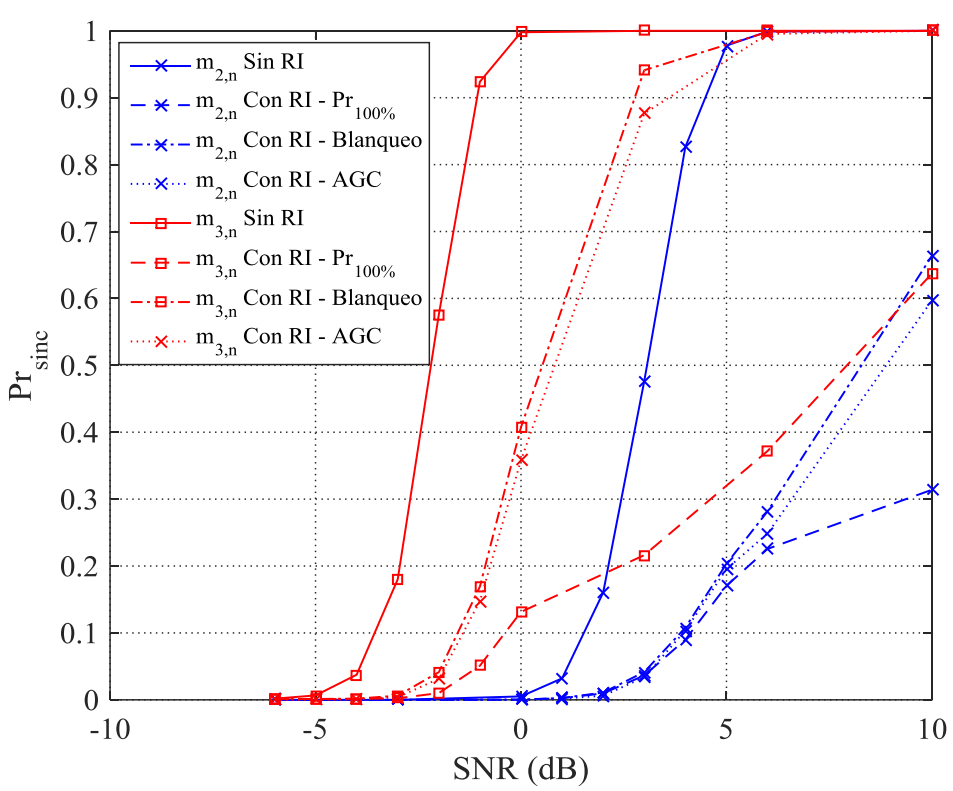

Fig. 4.30 Probabilidad de detección del símbolo piloto con y sin mitigación de eventos de ruido impulsivo con probabilidad de ocurrencia de 100\%.

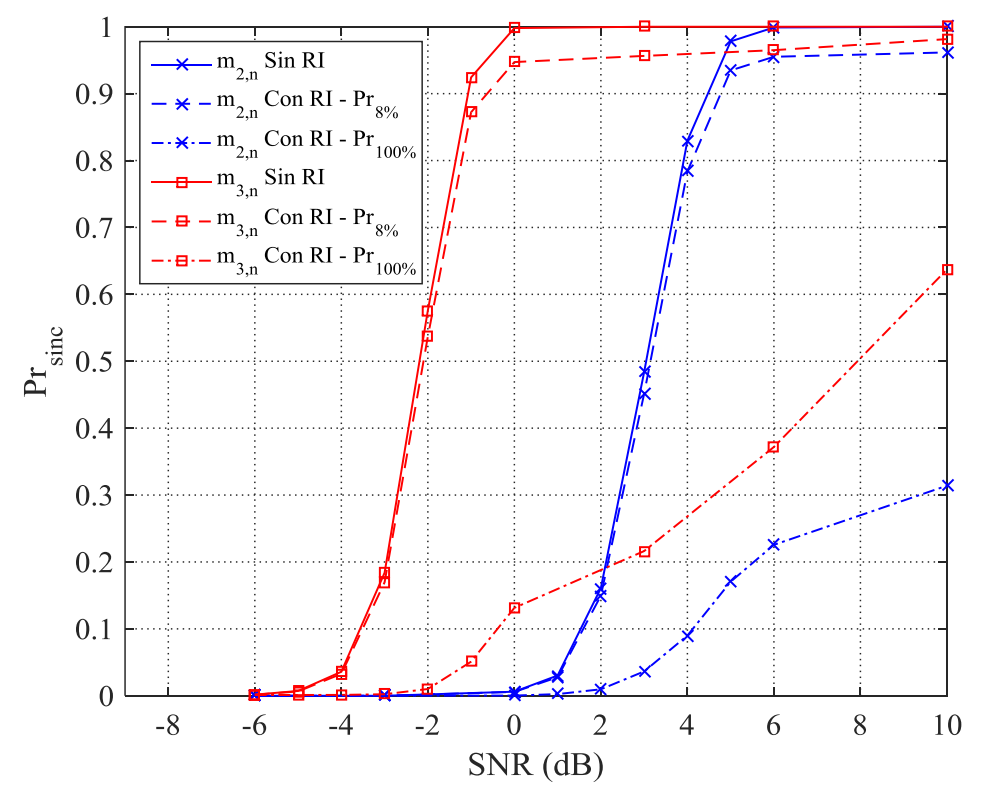

Fig. 4.31 Probabilidad de detección del símbolo piloto con eventos de ruido impulsivo con probabilidad de ocurrencia de $8 \%$ y $100 \%$.

probabilidad de detección disminuye ligeramente a medida que se incrementa el valor de SFO hasta 400ppm, y que para la métrica basada en correlación cruzada $m_{3, n}$ el impacto del SFO es menor que en la métrica basada en $\mathrm{AC} m_{2, n}$. Puede concluirse que el efecto del SFO en la etapa de sincronización gruesa es relativamente bajo, siempre que el SFO se mantenga por debajo de los valores máximos recomendados.

\subsubsection{Resumen de desempeño de la etapa de sincronización gruesa}


Diseño de Estrategias de Sincronización y Estimación de Canal para la Mejora de Comunicaciones en Redes Inteligentes de Energía
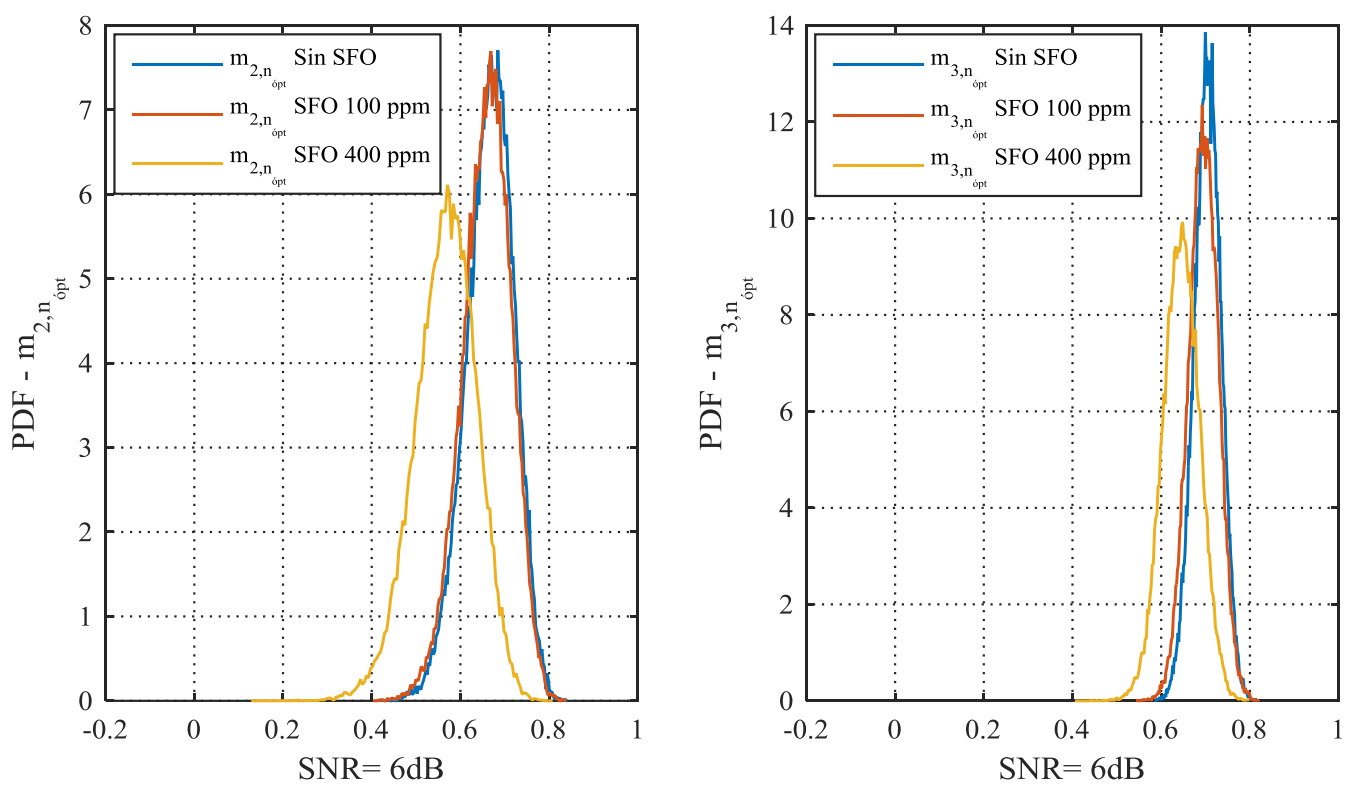

Fig. 4.32 PDFs empiricas de las métricas de sincronización gruesa en el instante óptimo de sincronismo, con y sin SFO, bajo de ruido de fondo coloreado y una SNR de $6 d B$.

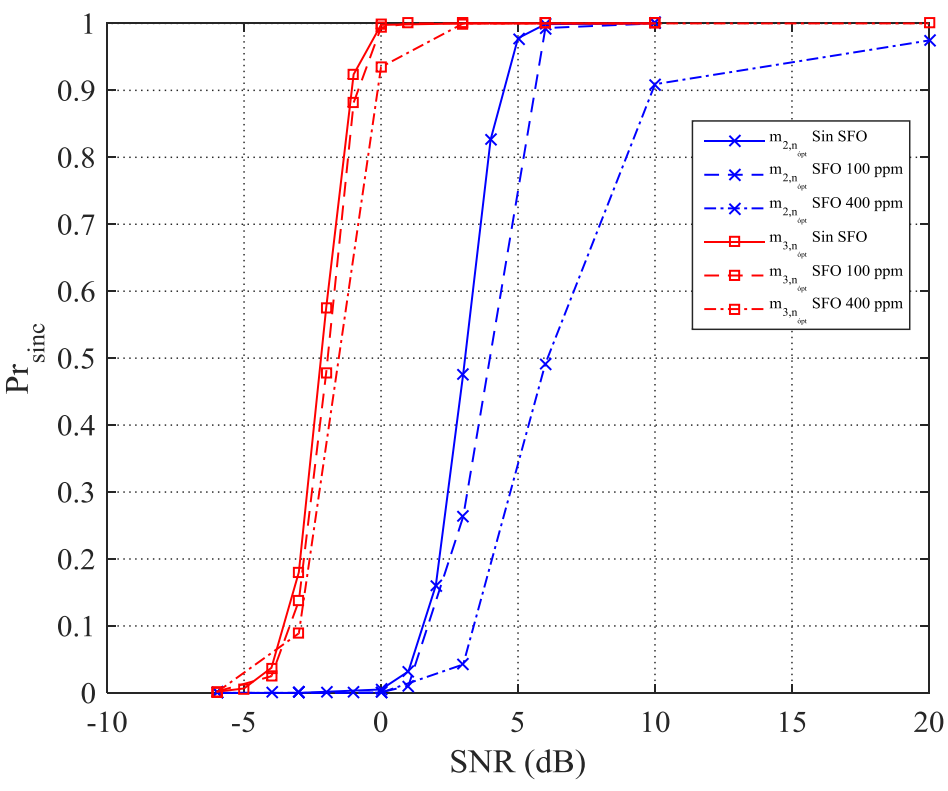

Fig. 4.33 Probabilidad de detección del símbolo piloto con y sin SFO bajo ruido de fondo coloreado.

Luego de analizar las métricas de sincronización gruesa propuestas, y la degradación en éstas producida por el ruido de fondo coloreado, el ruido impulsivo y el desvío en la frecuencia de muestreo de manera individual, es posible evaluar el desempeño de la etapa de sincronización gruesa de manera global. En una primera (y crítica) instancia se encuentra la capacidad de sincronizar, es decir, de ser capaz de detectar el arribo del símbolo piloto en recepción. En ese sentido, la Fig. 4.34 presenta la probabilidad de detección para las dos métricas propuestas en tres condiciones: bajo ruido de fondo coloreado únicamente; incluyendo además ruido impulsivo con probabilidad de 


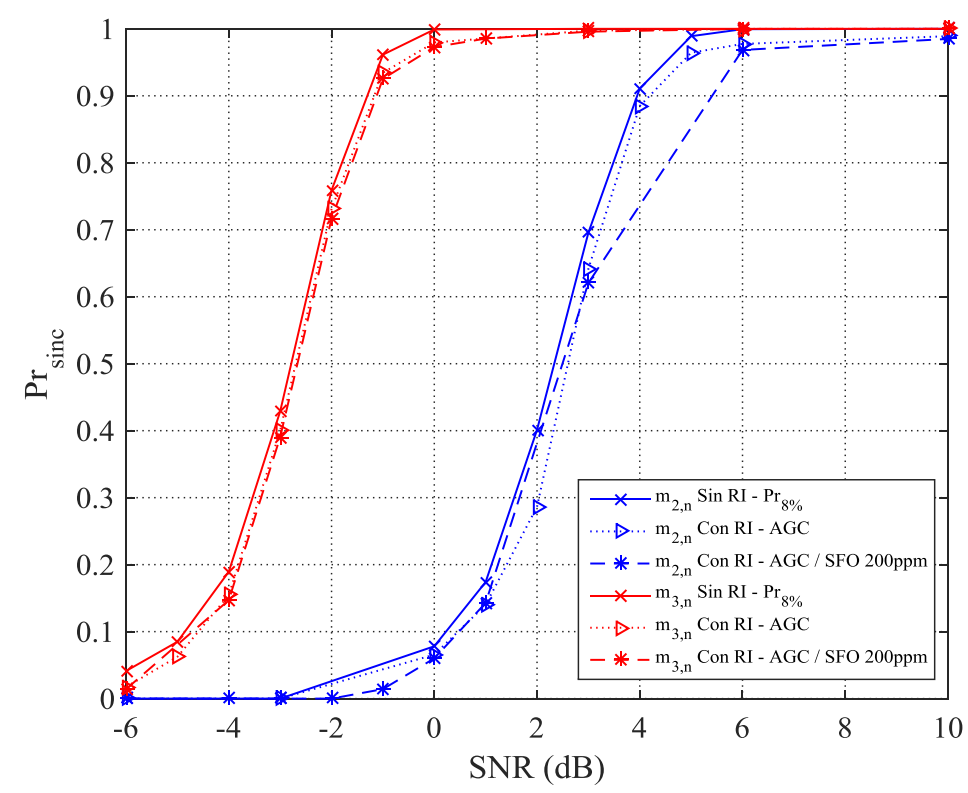

Fig. 4.34 Probabilidad de detección del símbolo bajo ruido de fondo coloreado con y sin ruido impulsivo y SFO.

ocurrencia típica de $8 \%$ y la técnica de mitigación basada en AGC; y el caso anterior pero además incluyendo un SFO de 200ppm. Puede observarse la pequeña degradación al incluir el ruido impulsivo y luego el SFO. La métrica basada en correlación cruzada permite detectar el símbolo piloto en un rango mayor de $S N R \mathrm{~s}$, con unos $5 \mathrm{~dB}$ de diferencia respecto a la métrica basada en AC.

Luego del proceso de detección del símbolo piloto, debe definirse el instante de sincronismo grueso $n_{g}$. Un procedimiento básico y ampliamente utilizado consiste en fijar ese instante a partir de la posición del máximo de la métrica [Schmidl 1997], [Minn 2004]:

$$
n_{g}=\underset{n}{\arg \max }\left(m_{1, n}\right)
$$

En la Fig. 4.35 se presentan los resultados obtenidos por simulaciones para el desempeño de la etapa de sincronización gruesa una vez que se ha logrado la detección del símbolo piloto, en términos de la raíz cuadrada del error cuadrático medio (RMSE Root Mean Square Error). Como el máximo de cualquiera de las métricas $m_{2, n} \mathrm{y} m_{3, n}$ no se corresponde necesariamente con el inicio del símbolo piloto, y debido a que las métricas poseen una meseta, el error cometido en la estimación del instante óptimo de sincronismo resulta considerable. Puede apreciarse que la métrica basada en $\mathrm{AC} m_{2, n}$ no sólo presenta una menor probabilidad de detección del símbolo piloto de acuerdo a la Fig. 4.34, sino que además, el valor de RMSE de la estimación es considerablemente 
Diseño de Estrategias de Sincronización y Estimación de Canal para la Mejora de Comunicaciones en Redes Inteligentes de Energía

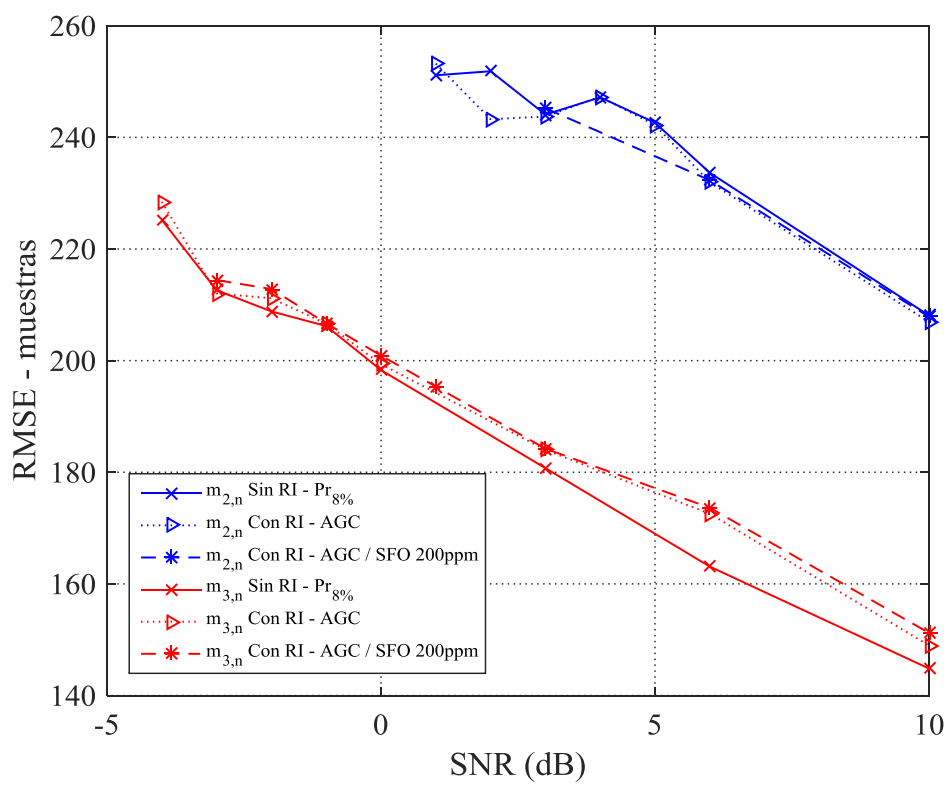

Fig. 4.35 Desempeño de las técnicas de sincronización propuestas en términos del RMSE, para distintas condiciones de SNR.

mayor que en la sincronización obtenida a partir de la métrica basada en correlación cruzada $m_{3, n}$. Lógicamente, para ambas métricas, los valores de RMSE de la estimación se encuentran en el rango $0<R M S E<N_{p c}=512$, y tienden a disminuir a medida que mejora la condición de $S N R$.

De acuerdo a lo expuesto en [Morelli 2007], el error de sincronización debería acotarse a unas pocas unidades porcentuales de la longitud del símbolo $N$ para obtener una pérdida de $S N R$ tolerable. Si se considera un 1\% de error porcentual para el sistema de $N=4096$, esto significa un error de sincronización de aproximadamente 40 muestras. Es decir, aun utilizando la estrategia de sincronización con la métrica $m_{3, n}$, el error promedio cometido es elevado, por lo que se debe desarrollar una etapa de ajuste fino del sincronismo para evitar ISI e ICI en los símbolos de datos.

\subsection{Conclusiones}

En este capítulo se desarrolló la etapa de sincronización gruesa en sistemas PLC-DMT, a partir del planteo y análisis detallado de métricas de sincronización gruesa computadas en base a técnicas de AC y CC. Ante la falta en la literatura de una comparación exhaustiva entre las técnicas de sincronización gruesa basadas en AC y CC en las condiciones particulares del canal PLC, a nuestro leal saber y entender, se demostró analíticamente y por simulaciones que la métrica basada en CC presenta un comportamiento más favorable para la detección primaria del símbolo piloto, en 
diversos escenarios planteados: con AWGN, ruido de fondo coloreado, ruido impulsivo, con técnicas de mitigación de ruido impulsivo, y error en la frecuencia de muestreo.

El análisis de las variables aleatorias intervinientes en el cómputo de las métricas tanto bajo AWGN como ruido de fondo coloreado resultó fundamental para definir umbrales de detección primaria, así como para la comparación de las métricas planteadas a partir del símbolo piloto propuesto. Pero, además, el análisis efectuado puede resultar útil como punto inicial para el estudio de métricas de sincronización definidas a partir de distintos prototipos de símbolo piloto, dado que las variables aleatorias planteadas se encuentran caracterizadas por las propiedades de correlación del símbolo piloto, del modelo de canal y las propiedades del proceso de ruido adicionado a la señal, lo que posibilita la extensión del análisis presentado con relativa facilidad. 
Diseño de Estrategias de Sincronización y Estimación de Canal para la Mejora de Comunicaciones en Redes Inteligentes de Energía

\section{SINCRONIZACIÓN FINA Y ESTIMACIÓN DE CANAL EN SISTEMAS DMT-PLC}

En el capítulo 4 se realizó un estudio detallado de la etapa de sincronización gruesa en el sistema DMT-PLC, en la que se realiza la detección primaria del símbolo piloto a partir de una métrica de sincronismo, y se define como instante de sincronismo inicial la ubicación del máximo de la métrica. Como el error promedio cometido por esta técnica resulta elevado, en el presente capítulo se presenta una etapa de sincronización fina, y una posterior estimación de canal.

Se plantea un algoritmo de sincronización fina y estimación de canal conjunta similar al presentado en el capítulo 3, pero incluyendo un modelo estadístico del canal PLC más complejo, basado en efectos multicamino en el dominio del tiempo, generados a partir de diversos parámetros relacionados con las características físicas de la red eléctrica.

Los principales aportes del presente capítulo se detallan a continuación:

- Se realiza un análisis del modelo estadístico del canal que resulta útil para obtener de manera analítica umbrales aplicables en el algoritmo de sincronización fina.

- Se plantea el algoritmo de sincronización fina a partir de una correlación cruzada normalizada, se derivan umbrales prácticos a partir del análisis efectuado sobre 
Capítulo 5: Sincronización fina y estimación de canal en sistemas DMT-PLC

la distribución del primer camino del modelo del canal, y se evalúa el desempeño a partir de simulaciones. Luego, se propone una modificación novedosa en el cómputo de la correlación cruzada normalizada en la que se verifica una mejora en el desempeño, tanto de manera analítica como por simulaciones.

- Se plantea y evalúa una estrategia de estimación de canal que se obtiene a partir de las muestras de la correlación cruzada utilizada en las etapas de sincronización fina y gruesa.

- Finalmente, se realiza un análisis preliminar de la complejidad asociada al cómputo de los algoritmos propuestos para la sincronización y estimación de canal conjunta, a partir del planteo de una arquitectura de procesamiento de señal con bloques multiplicadores, sumadores, elementos de retardo y lógica combinacional y secuencial.

Tanto para la etapa de sincronización fina como la de estimación de canal, se realiza un estudio detallado considerando distintos escenarios: con ruido de fondo AWGN, ruido de fondo coloreado, e incluyendo eventos de ruido impulsivo y técnicas de mitigación del mismo.

\subsection{Sincronización fina}

Una vez obtenido el instante de sincronización gruesa a partir de la métrica basada en correlación cruzada $m_{3, n}$ del capítulo 4 , debe realizarse un ajuste fino del sincronismo. Para ello se propone, de manera similar a la técnica desarrollada en el capítulo 3, utilizar las muestras de la salida del filtro acoplado utilizado para calcular la correlación cruzada. Como el principio de la sincronización fina se basa en elaborar una técnica para detectar el primer coeficiente de la respuesta al impulso de canal, resulta de interés analizar sus características desde un punto de vista estadístico.

\subsubsection{Análisis del canal PLC para la sincronización fina}

Para continuar el estudio en el mismo contexto que la etapa de sincronización gruesa para el sistema DMT-PLC, se incluyen los dos tipos de canales presentados en el capítulo 4.

Recordando que la CIR del modelo de canal PLC de (4.2) está dada por: 
Diseño de Estrategias de Sincronización y Estimación de Canal para la Mejora de Comunicaciones en Redes Inteligentes de Energía

$$
\begin{gathered}
g_{C H}(t)=A \sum_{p=1}^{N p} g_{p} e^{-a_{0} d_{p}} \frac{a_{1} d_{p}+j 2 \pi\left(t-\frac{d_{p}}{v}\right)}{\left(a_{1} d_{p}\right)^{2}+4 \pi^{2}\left(t-\frac{d_{p}}{v}\right)^{2}}\left(e^{j 2 \pi B_{1}\left(t-d_{p} / v\right)-a_{1} B_{1} d_{p}}\right. \\
\left.-e^{j 2 \pi B_{2}\left(t-d_{p} / v\right)-a_{1} B_{2} d_{p}}\right)
\end{gathered}
$$

donde $A$ es un parámetro de atenuación, $N_{p}$ es el número de caminos, $g_{p}$ es la ganancia del camino $p$, y puede considerarse como real y uniformemente distribuida $U(-1,1) ; d_{p}$ es la longitud del camino $p, v=c / \sqrt{\varepsilon_{r}}$ con $c$ como la velocidad de la luz y $\varepsilon_{r}$ la constante dieléctrica; $a_{0}$ y $a_{1}$ son coeficientes de atenuación en los cables y se asumen constantes; $B_{1}=0$ y $B_{2}=50 \mathrm{MHz}$ son las frecuencias mínima y máxima de síntesis del canal. La CIR en el instante de llegada del primer camino, es decir en el instante $t_{1}=d_{1} / v$ resulta:

$g_{C H}\left(t_{1}\right)=A \sum_{p=1}^{N p} g_{p} e^{-a_{0} d_{p}} \frac{a_{1} d_{p}+j 2 \pi\left(\frac{d_{1}}{v}-\frac{d_{p}}{v}\right)}{\left(a_{1} d_{p}\right)^{2}+4 \pi^{2}\left(\frac{d_{1}}{v}-\frac{d_{p}}{v}\right)^{2}}\left(1-e^{j 2 \pi B_{2}\left(d_{1} / v-d_{p} / v\right)-a_{1} B_{2} d_{p}}\right)$

Considerando únicamente el efecto del primer camino en $g_{C H}\left(t_{1}\right)$, se obtiene la aproximación:

$$
g_{C H}\left(t_{1}\right) \approx \frac{A^{\prime}}{2 B_{2}} g_{1} e^{-a_{0} d_{1}} \frac{\left(1-e^{-a_{1} B_{2} d_{1}}\right)}{a_{1} d_{1}}
$$

donde se efectuó el reemplazo $A=\frac{A^{\prime}}{2 B_{2}}$. Es importante notar que en (5.3) están involucradas las variables aleatorias $g_{1} \mathrm{y} d_{1}$; donde $g_{1}$ se encuentra uniformemente distribuida en el intervalo $(-1,1)$, y $d_{1}$ sigue una distribución exponencial de parámetro $\Lambda$. Teniendo en cuenta que la variable aleatoria $d_{1}$ con distribución exponencial se puede expresar a partir de la transformación de una variable aleatoria $X$ uniformemente distribuida en el intervalo $(0,1)$, a partir de $d_{1}=-\Lambda^{-1} \ln (X)$ [Walpole 2007], la respuesta al impulso en el instante de arribo del primer camino del canal puede expresarse como una función de las variables $g_{1}$ y $X$, de acuerdo a:

$$
g_{C H}\left(t_{1}\right) \approx A^{\prime} g_{1} e^{-a_{0} d_{1}} \frac{\left(1-e^{-a_{1} B_{2} d_{1}}\right)}{2 B_{2} a_{1} d_{1}}=A^{\prime} g_{1} \cdot \frac{X^{a_{0} / \Lambda}\left(1-X^{\frac{a_{1} B_{2}}{\Lambda}}\right)}{\ln \left(X^{-\frac{2 a_{1} B_{2}}{\Lambda}}\right)}
$$

En una primera instancia, resulta de interés cuantificar los valores mínimos y máximos que se esperan en la amplitud del canal en el instante $t_{1}$. El producto $A^{\prime} g_{1}$ tendrá como 
valores límites a $-A^{\prime}$ y $A^{\prime}$, respectivamente, dado que $g_{1}$ es una variable aleatoria con distribución uniforme. Por otro lado, el término producto que depende de la variable aleatoria $X$ es una función creciente y los valores límites se pueden obtener a partir de:

$$
\begin{gathered}
\lim _{X \rightarrow 0} \frac{X^{a_{0} / \Lambda}\left(1-X^{\frac{a_{1} B_{2}}{\Lambda}}\right)}{\ln \left(X^{-\frac{2 a_{1} B_{2}}{\Lambda}}\right)}=\lim _{X \rightarrow 0} \frac{\frac{a_{0}}{\Lambda} X^{a_{0} / \Lambda-1}-\left(\frac{a_{1} B_{2}}{\Lambda}+\frac{a_{0}}{\Lambda}\right) X^{\left(a_{1} B_{2} / \Lambda+a_{0} / \Lambda-1\right)}}{\frac{-\frac{2 a_{1} B_{2}}{\Lambda}}{X}}=0 \\
\lim _{X \rightarrow 1} \frac{X^{a_{0} / \Lambda}\left(1-X^{\frac{a_{1} B_{2}}{\Lambda}}\right)}{\ln \left(X^{-\frac{2 a_{1} B_{2}}{\Lambda}}\right)}=\lim _{X \rightarrow 1} \frac{\frac{a_{0}}{\Lambda} X^{a_{0} / \Lambda-1}-\left(\frac{a_{1} B_{2}}{\Lambda}+\frac{a_{0}}{\Lambda}\right) X^{\left(a_{1} B_{2} / \Lambda+a_{0} / \Lambda-1\right)}}{\frac{-\frac{2 a_{1} B_{2}}{\Lambda}}{X}} \\
=\frac{\frac{a_{0}}{\Lambda}-\left(\frac{a_{1} B_{2}}{\Lambda}+\frac{a_{0}}{\Lambda}\right)}{-\frac{2 a_{1} B_{2}}{\Lambda}}=\frac{1}{2}
\end{gathered}
$$

Por lo tanto, los valores máximo y mínimo $\pm K_{A}$, en el instante de llegada del primer camino del canal $g_{C H}\left(t_{1}\right)$ en (5.4) estarán dados por:

$$
K_{A}=\frac{A^{\prime}}{2}=0,5432
$$

con $A^{\prime}=1,0864$ de acuerdo a los parámetros de la Tabla 4.1. Para pequeños valores de la variable, $g_{C H}\left(t_{1}\right)$ puede aproximarse a partir de una distribución uniforme en el intervalo $\left(-K_{A}, K_{A}\right)$. En la Fig. 5.1, a la izquierda, se presenta la CDF empírica de $g_{C H}\left(t_{1}\right)$ obtenido a partir del generador del canal, es decir, que incluye el efecto en el instante de arribo de todos los demás caminos del canal de acuerdo a (5.2). Además, se incluye la aproximación uniforme obtenida en (5.7). Por su parte, a la derecha, se presenta la CDF empírica de la aproximación de $g_{C H}\left(t_{1}\right)$ a partir de la evaluación de (5.3) efectuando $10^{6}$ realizaciones, donde se puede apreciar que los valores límites se corresponden con lo analizado, y que la distribución uniforme que sigue el coeficiente del canal $g_{1}$ se ve afectada por el proceso de Poisson que define la distancia del primer camino $d_{1}$ en cada realización. Puede concluirse que, si bien los siguientes caminos afectan la amplitud del canal en el instante $t_{1}$, la preponderancia del primer camino del canal es significativa.

5.1.1.1 Distribución de la respuesta al impulso en el instante de llegada del primer camino del canal B

Para el canal B, la respuesta al impulso en el instante de arribo del primer camino considerando el efecto únicamente del primer camino es: 
Diseño de Estrategias de Sincronización y Estimación de Canal para la Mejora de Comunicaciones en Redes Inteligentes de Energía
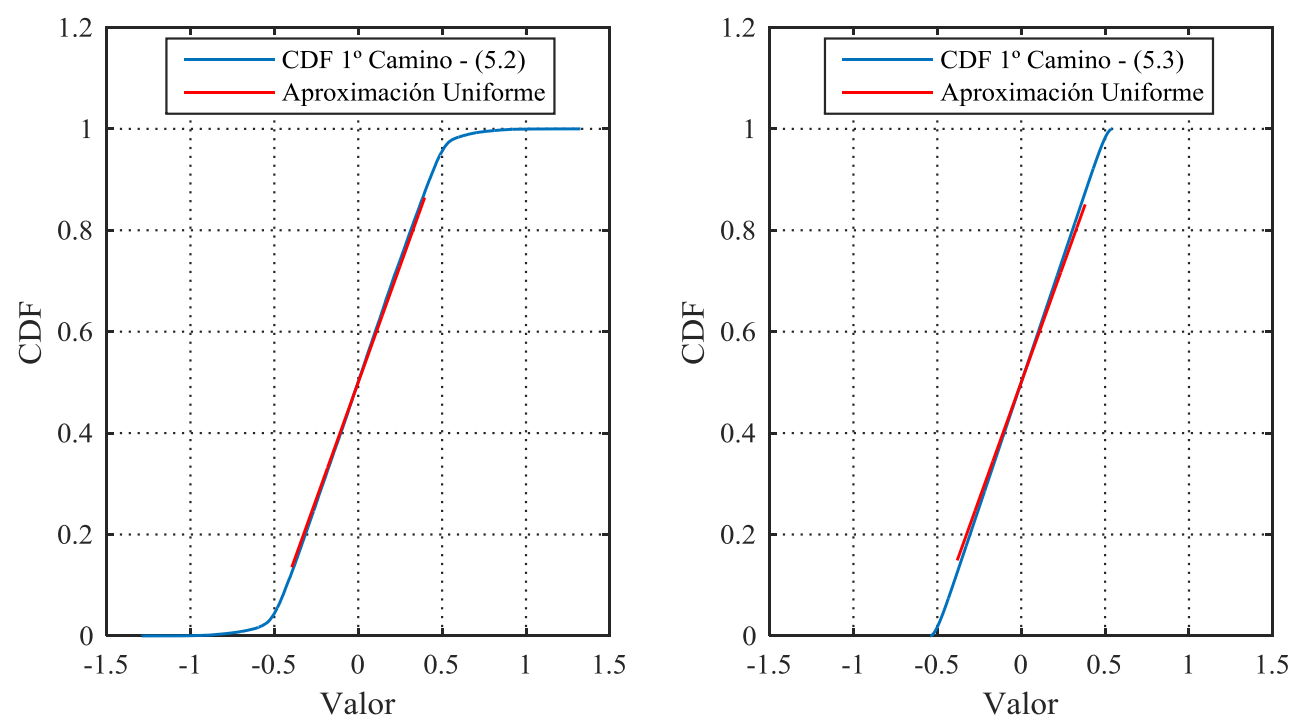

Fig. 5.1 CDFs empiricas de $g_{C H}\left(t_{1}\right)$ a partir del generador de canal (izquierda) y de la aproximación considerando sólo el efecto del primer camino (derecha), para el canal A.

$$
g_{C H}\left(t_{1}\right) \approx A g_{1} e^{-a_{0} d_{1}} \frac{\left(1-e^{-a_{1} B_{2} d_{1}}\right)}{a_{1} d_{1}}
$$

En contraste con el canal A, la distancia del primer camino es fija, por lo que la única variable aleatoria interviniente en (5.8) es $g_{1}$, uniformemente distribuida en el intervalo $(-1,1)$. Por lo tanto, la amplitud del primer camino del canal será una variable aleatoria uniformemente distribuida.

Los valores mínimos y máximos estarán fijados por una constante $\pm K_{B}$, que depende de los parámetros fijos $d_{1}, a_{0}, a_{1} y A$ de la Tabla 4.1:

$$
K_{B}=A e^{-a_{0} d_{1}} \frac{\left(1-e^{-a_{1} B_{2} d_{1}}\right)}{a_{1} d_{1}}=0,6615
$$

En la Fig. 5.2, a la izquierda, se presenta el resultado de $g_{C H}\left(t_{1}\right)$ obtenido a partir del generador del canal, es decir, que incluye el efecto en el instante de arribo de todos los demás caminos del canal de acuerdo a (5.2), para el canal B. Al igual que el caso del canal A, puede apreciarse que la aproximación a una distribución uniforme es acertada para el canal B, para valores pequeños de la variable. En la derecha, se presenta la CDF empírica de la aproximación de $g_{C H}\left(t_{1}\right)$ a partir de la evaluación de (5.8) efectuando $10^{6}$ realizaciones, donde se puede apreciar que los valores límites se corresponden con lo analizado, y que la distribución que sigue el primer coeficiente del canal es uniforme en el intervalo $\left(-K_{B}, K_{B}\right)$. 


\subsubsection{Sincronización fina a partir de la correlación cruzada normalizada}

Como la etapa de sincronización fina utiliza las propiedades de correlación del símbolo piloto propuesto, desarrollado para la sincronización gruesa en el capítulo 4, resulta útil recordar que su estructura está dada por:

$$
\mathbf{x}=\left[\begin{array}{llllll}
\mathbf{c p} & \mathbf{a} & \mathbf{b} & -\mathbf{a} & \mathbf{b} & \mathbf{c s}
\end{array}\right]
$$

donde $\mathbf{a}$ y $\mathbf{b}$ son secuencias binarias que componen un CPS, con longitud $M=N / 4$, donde $N$ es la cantidad de puntos de la DFT del sistema DMT; cp es el prefijo cíclico de $N_{c p}$ muestras; y ; cs es el sufijo cíclico de $N_{c s}$ muestras. A su vez, la correlación del símbolo piloto extendido $\mathbf{x}$ con el símbolo piloto sin extender $\mathbf{x}^{\prime}=\left[\begin{array}{llll}\mathbf{a} & \mathbf{b} & -\mathbf{a} & \mathbf{b}\end{array}\right]$, es decir, $c_{n}^{\mathbf{x}, \mathbf{x}^{\prime}}$ posee una zona de correlación ideal de $N_{c p}+N_{c s}$ muestras. Por último, la métrica de sincronización gruesa basada en CC se definió como (4.47):

$$
m_{3, n}=\frac{N_{2} / N \sum_{k=0}^{N_{2}-1}\left|c_{n+k}^{\mathbf{r}, \mathbf{x}^{\prime}}\right|^{2}}{\sum_{k=0}^{N_{2}-1} \sum_{m=0}^{N-1} r_{n+k+m} \cdot r_{n+k+m}}
$$

Reutilizando parte de los cálculos efectuados para el cómputo de la métrica de sincronización gruesa $m_{3, n}$, se propone evaluar la correlación cruzada normalizada $(\mathrm{CCN})$ que se expresa a continuación:

$$
c_{1, n}^{\prime}=\frac{1 / N\left|c_{n}^{\mathbf{r}, \mathbf{x}^{\prime}}\right|^{2}}{\sum_{m=0}^{N-1} r_{n+m} \cdot r_{n+m}}
$$
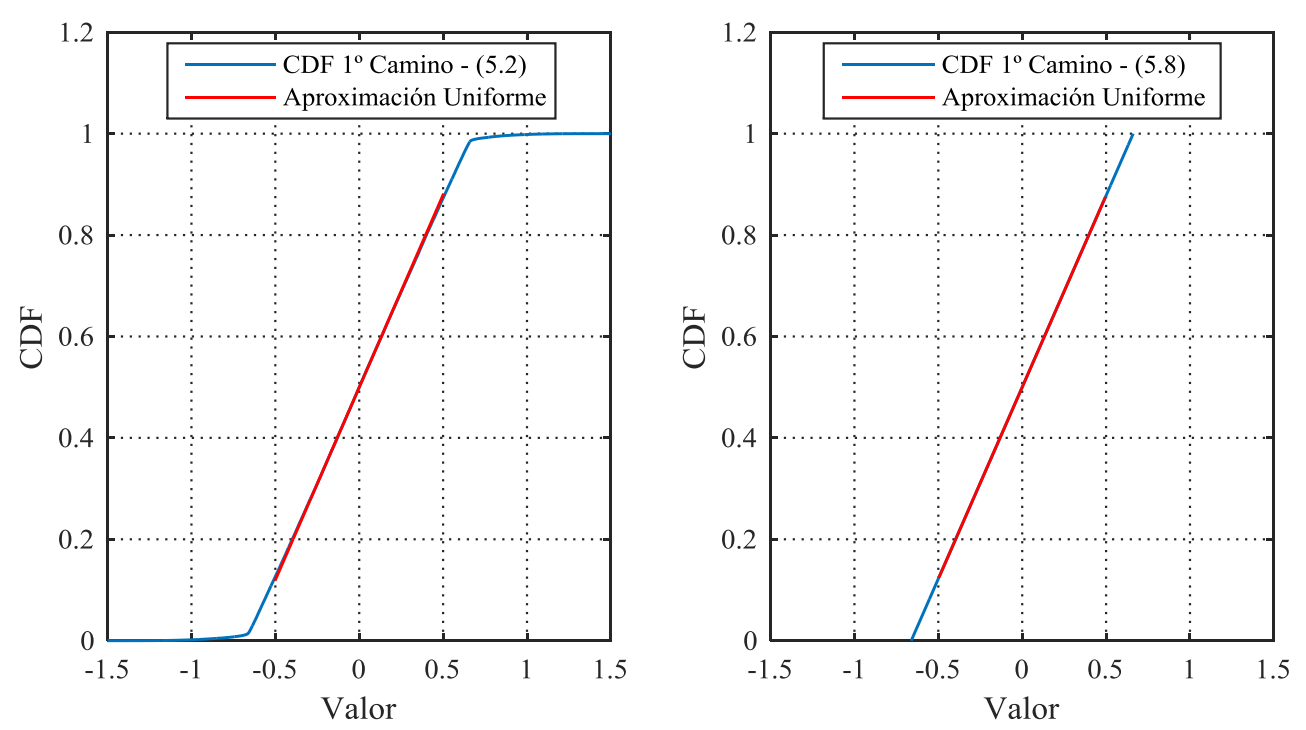

Fig. 5.2 CDFs empíricas de $g_{C H}\left(t_{1}\right)$ a partir del generador de canal (izquierda) y de la aproximación considerando sólo el efecto del primer camino (derecha), para el canal A. 
Diseño de Estrategias de Sincronización y Estimación de Canal para la Mejora de Comunicaciones en Redes Inteligentes de Energía

donde $r_{n}$ es la $n$-ésima muestra de la señal recibida, y $c_{n}^{\mathbf{r}, \mathbf{x}^{\prime}}$ es entonces la CC entre la señal recibida y el símbolo piloto sin extender. En ausencia de ruido, $c_{n}^{\mathbf{r}, \mathbf{x}^{\prime}}$ posee una zona de correlación nula en las $N_{c p}-L$ muestras previas al primer coeficiente de la respuesta impulsiva del canal, de acuerdo a (4.46). El algoritmo de sincronización fina comienza en la muestra $n_{g}$ como valor inicial, que es el resultado obtenido en la etapa de sincronización gruesa. Luego, se busca el máximo de la CCN y se obtiene el instante de sincronización fina preliminar $n_{f_{1}}$.

$$
n_{f_{1}}=\underset{n}{\arg \max }\left(c_{1, n}^{\prime}\right)
$$

Como el coeficiente del canal de máxima amplitud no es necesariamente el primero, se realiza una búsqueda en las muestras previas a $n_{f_{1}}$ para determinarlo. Para ello, el algoritmo verifica si algún valor en la ventana de búsqueda de $D$ muestras anteriores es mayor que un determinado umbral $\alpha$. Si esto sucede, se actualiza el índice de sincronización fina $n_{f}$ con la muestra más alejada, y se repite el proceso hasta que las anteriores $D$ muestras previas se encuentren por debajo del umbral. El algoritmo se puede escribirse como:

$$
\begin{aligned}
& n_{f}=n_{f_{1}} \\
& \text { while } \underset{n \in\{0,1, \ldots, D\}}{\arg \max }\left(n \mid c^{\prime}{ }_{n_{f}-n} \geq \alpha\right)>0 \\
& n_{f}=n_{f-n} \\
& \text { end }
\end{aligned}
$$

En la Fig. 5.3 se presenta la CCN junto con la métrica de sincronización gruesa y la aplicación del algoritmo de sincronización fina, bajo el canal PLC tipo A con una condición de $S N R$ de $3 \mathrm{~dB}$. Puede observarse como el máximo de la métrica de sincronización gruesa se encuentra alejada del instante de sincronismo óptimo, y que la ubicación del máximo de la CCN no se corresponde con el primer camino del canal. Adicionalmente, se observan que los valores en las muestras previas al primer camino de canal son bajos, debido a la zona de correlación nula diseñada para el símbolo piloto. La longitud de la ventana de búsqueda debe elegirse de manera tal de ser menor que la longitud de la zona libre de interferencia, $D<N_{c p}-L$. Además, $D$ debe ser lo suficientemente grande como para incluir la máxima distancia en muestras entre caminos significativos del canal. Por otro lado, es evidente que el valor del umbral $\alpha$ 


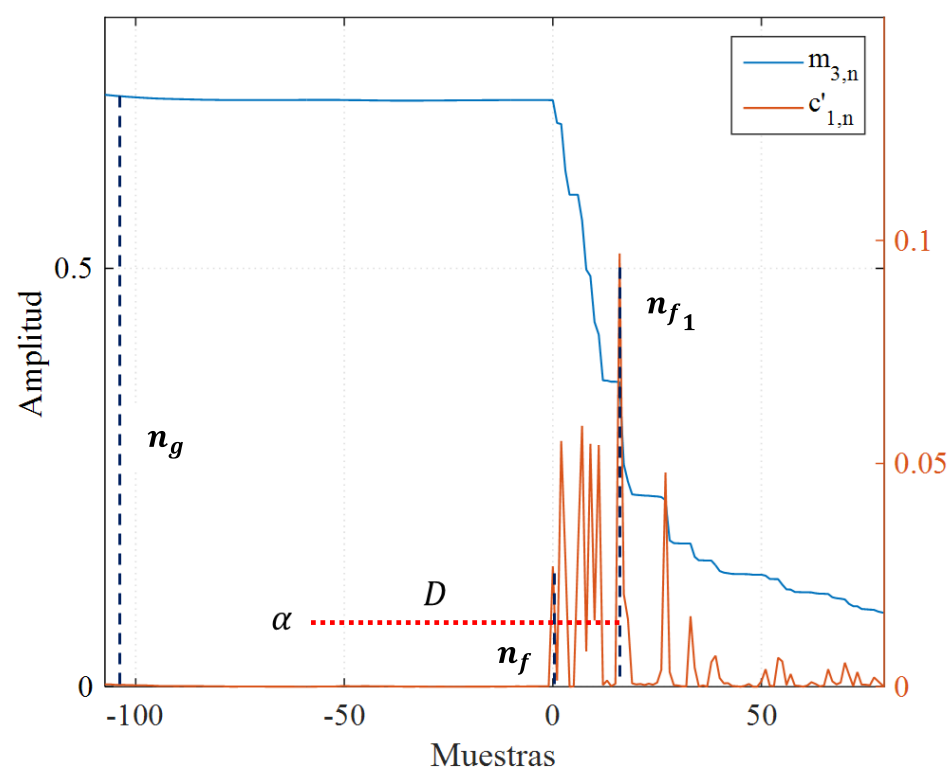

Fig. 5.3 Algoritmo de sincronización fina basado en la correlación cruzada normalizada, sin ruido agregado.

tiene un alto impacto en el desempeño del algoritmo de sincronización fina. Un valor demasiado bajo incrementaría la probabilidad de falsa detección de muestras ruidosas como coeficientes del canal, mientras que un valor demasiado alto aumentaría la probabilidad de no detectar coeficientes del canal. Como la elección del umbral resulta crucial en la etapa de sincronización fina, es necesario analizar las distribuciones de la CCN para obtener un umbral adecuado.

La CCN está estrechamente relacionada con la métrica de sincronización gruesa basada en CC, ya que la ecuación (5.12) puede obtenerse a partir de (4.48) considerando $N_{2}=1$. Por lo tanto, la CCN en el instante óptimo de sincronismo resulta:

$$
c_{1, n_{\mathrm{o} p t}}^{\prime}=\frac{1 / N\left|\sqrt{P_{T}} h_{0} N+\sum_{m=0}^{N-1} w_{n_{\mathrm{o} p t}+m^{\prime}} \mathrm{x}_{m}^{\prime *}\right|^{2}}{\sum_{m=0}^{N-1} s_{n_{\mathrm{o} p t}+m}^{2}+2 \cdot s_{n_{\mathrm{o} p t}+m} \cdot w_{n_{\mathrm{o} p t}+m}+w_{n_{\mathrm{o} p t}+m}^{2}}
$$

Para el análisis de la métrica, teniendo en cuenta que el coeficiente del canal $h_{0}$ es una variable aleatoria, analizada a partir del modelo estadístico del canal PLC en (5.4) y (5.8), si la longitud del símbolo piloto $N$ es lo suficientemente grande, entonces en el numerador la variable aleatoria preponderante será la correspondiente al término del coeficiente del canal. Además, como en el numerador la media es mucho mayor que su desvío estándar, una aproximación sencilla de la CCN puede escribirse como:

$$
c_{1, n_{\mathrm{o} p t}}^{\prime} \approx \frac{1 / N\left|\sqrt{P_{T}} h_{0} N\right|^{2}}{N\left(\sigma_{S}^{2}+\sigma_{w}^{2}\right)}
$$


Diseño de Estrategias de Sincronización y Estimación de Canal para la Mejora de Comunicaciones en Redes Inteligentes de Energía

Donde $\sigma_{S}^{2}=P_{T}\|\mathbf{h}\|^{2}$ es la potencia de la parte útil de la señal recibida, que es una variable aleatoria dado que la ganancia del canal $\|\mathbf{h}\|^{2}$ es una variable aleatoria que sigue una distribución log-normal debido al fenómeno de desvanecimiento. Para analizar la probabilidad de detección del primer camino del canal $c_{1, n_{\text {ópt }}}$ a partir de un umbral $\alpha$, puede considerarse la probabilidad de que la variable aleatoria $\sqrt{c_{1, n_{\text {opt }}}}$ supere el umbral $\sqrt{\alpha}$. Además, para simplificar el análisis, puede considerarse que el efecto del desvanecimiento del canal no afecta significativamente la PDF $\left(\|\mathbf{h}\|^{2}=\right.$ $E\left[\|\mathbf{h}\|^{2}\right]=1$ ), y entonces la variable aleatoria puede escribirse como:

$$
\sqrt{c^{\prime}{ }_{1, n_{\mathrm{o} p t}}} \approx \frac{\left|\sqrt{P_{T}} h_{0}\right|}{\sqrt{\left(\mathrm{P}_{\mathrm{T}}+\sigma_{w}^{2}\right)}}=\frac{\sqrt{S N R}\left|h_{0}\right|}{\sqrt{(S N R+1)}}
$$

Donde $S N R=\mathrm{P}_{\mathrm{T}} / \sigma_{w}^{2}$ es la relación señal a ruido media en el canal multicamino con desvanecimiento. Como $h_{0}$ puede aproximarse a partir de una variable aleatoria de distribución uniforme para valores pequeños de la variable, y posee una PDF simétrica con media cero, entonces el valor constante $K_{P D F}$ que aproxima la PDF de $\sqrt{c^{\prime}{ }_{1, n_{\text {op }}}}$ para valores pequeños de la variable puede obtenerse como:

$$
K_{P D F}=\frac{1}{K_{A / B}} \frac{\sqrt{(S N R+1)}}{\sqrt{(S N R)}}
$$

Donde $K_{A / B}$ adopta el valor $K_{A}(5.7)$ para el canal PLC tipo A y $K_{B}(5.9)$ para el tipo B. En la Fig. 5.4 se presentan los resultados obtenidos por simulación para $\sqrt{c^{\prime}{ }_{1, n_{o} p t}}$ bajo los canales PLC tipo A y B con ruido de fondo coloreado ${ }^{2}$ y distintas condiciones de SNR. Además, se incluye la aproximación uniforme de (5.17) correspondiente en cada caso. Puede apreciarse que la aproximación de la PDF para el canal A se ajusta considerablemente mejor a altas $S N R \mathrm{~s}$, mientras que en el canal B el ajuste es mejor a bajas $S N R$ s.

\footnotetext{
${ }^{2}$ Los resultados para el caso de ruido blanco Gaussiano no se incluyen debido a que no se aprecia diferencia con los obtenidos para ruido coloreado.
} 

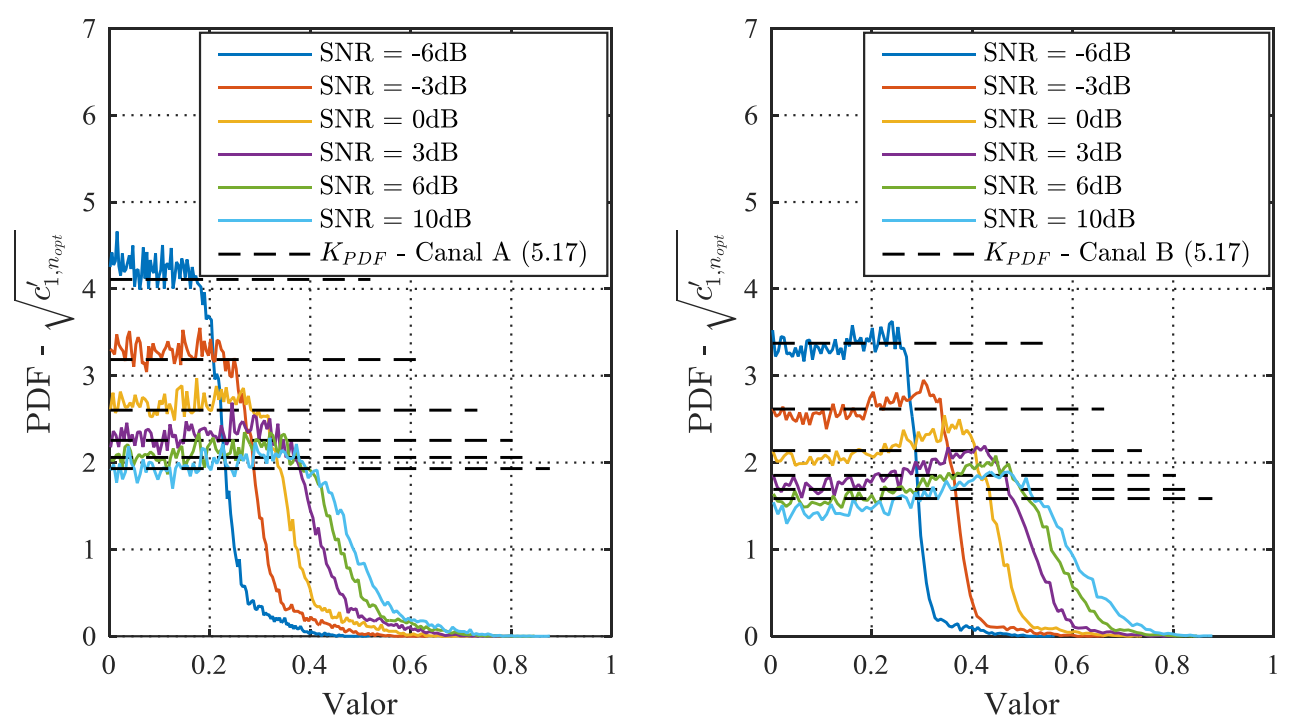

Fig. 5.4 PDFs empíricas de la raíz de la correlación cruzada normalizada en el instante óptimo de sincronismo para el canal A (izquierda) y B (derecha), bajo ruido de fondo coloreado y diferentes condiciones de SNR.

En las muestras previas al primer camino del canal $n_{\text {ant }} \in\left\{n_{\text {ó } p t}-N_{c p}+L, n_{\text {ó } p t}-\right.$ $\left.N_{c p}+L-1, \ldots, n_{\text {ópt }}-1\right\}$, la CCN resulta:

$$
\begin{aligned}
& c^{\prime}{ }_{1, n_{\text {ant }}}=\frac{1 / N\left|\sum_{m=0}^{N-1} w_{n_{\text {ant }}+m} \mathrm{x}_{m}^{\prime *}\right|^{2}}{\sum_{m=0}^{N-1} s_{n_{a n t}+m}^{2}+2 \cdot s_{n_{\text {ant }}+m} \cdot w_{n_{\text {ant }}+m}+w_{n_{\text {ant }}+m}^{2}} \\
& \approx \frac{1 / N\left|\sum_{m=0}^{N-1} w_{n_{a n t}+m} \mathrm{x}_{m}^{\prime *}\right|^{2}}{N\left(\sigma_{S}^{2}+\sigma_{w}^{2}\right)}
\end{aligned}
$$

\subsubsection{Sincronización fina bajo ruido blanco gaussiano}

Considerando muestras de ruido blanco gaussiano, la variable aleatoria del numerador de (5.18) resulta una variable aleatoria que sigue una distribución chi-cuadrado de un grado de libertad, con media $N \sigma_{w}^{2}$ y varianza $2 N^{2} \sigma_{w}^{4}$, resultado que se desprende de utilizar $N_{2}=1$ en el resultado obtenido para la variable aleatoria $Y_{2}$ de (4.50). Por lo tanto, la variable $\sqrt{c^{\prime}{ }_{1, n_{\text {ant }}}}$ tiene una distribución normal doblada con PDF:

$$
f_{\sqrt{c_{1, n_{\text {ant }}}}}(x) \approx \frac{\sqrt{2}}{\sigma \sqrt{\pi}} e^{\frac{-x^{2}}{2 \sigma^{2}}}, \quad x>0
$$

Con $\sigma^{2}=1 /(N(S N R+1))$. Los resultados obtenidos por simulaciones para la PDF de $\sqrt{c^{\prime}{ }_{1, n_{a n t}}}$ se contrastan con la PDF analítica en la Fig. 5.5. Se produce un buen ajuste a 
Diseño de Estrategias de Sincronización y Estimación de Canal para la Mejora de Comunicaciones en Redes Inteligentes de Energía
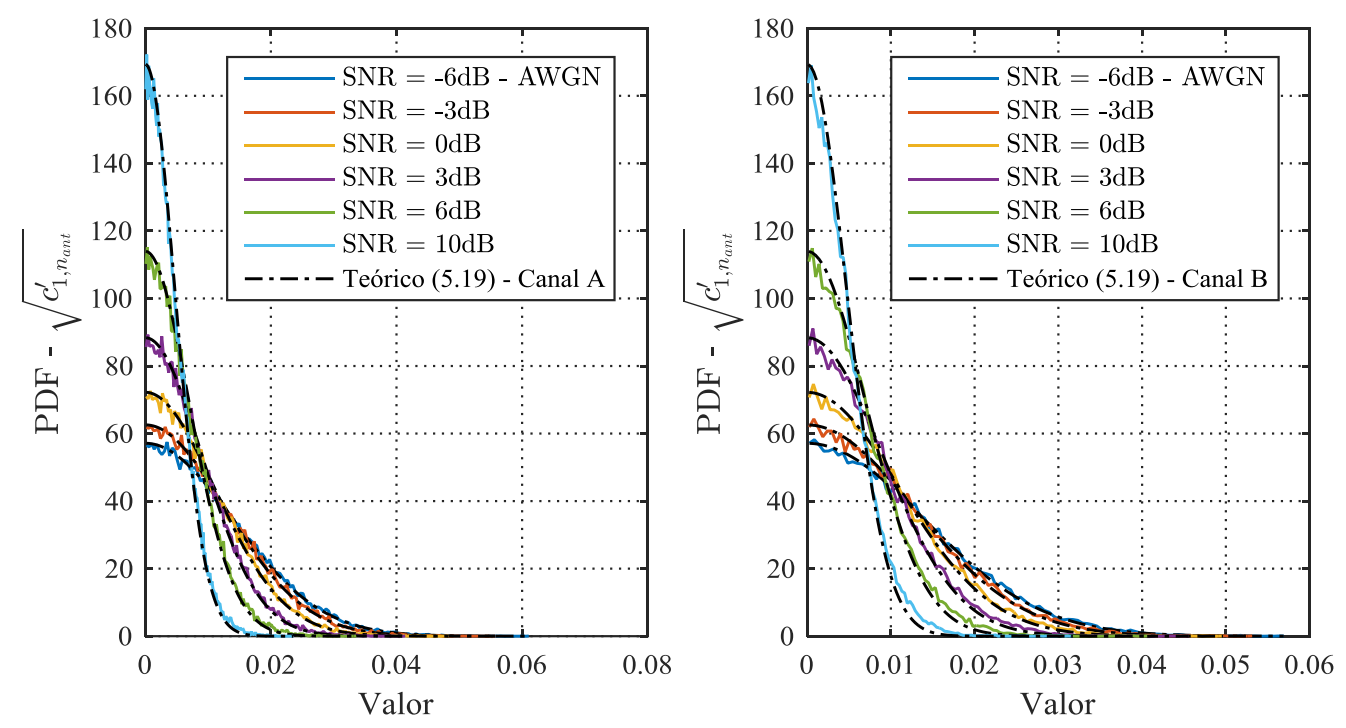

Fig. 5.5 PDFs empiricas de la raiz de la correlación cruzada normalizada en la zona de correlación nula para el canal A (izquierda) y B (derecha), bajo ruido blanco gaussiano y diferentes condiciones de SNR. bajas $S N R \mathrm{~s}$, mientras que la varianza obtenida por simulaciones es levemente superior a la analítica para altas $S N R$ s, siendo más notoria la diferencia en el caso del canal B. A partir del resultado analítico de la PDF, puede definirse un umbral $\alpha$ de manera tal que la probabilidad de falsa detección de una muestra previa al instante de sincronismo óptimo sea lo suficientemente baja. Por ejemplo, para una probabilidad de falsa detección de $10^{-7}$, evaluando la ICDF se obtiene $\sqrt{\alpha_{1}}$, y luego el umbral $\alpha_{1}$ resulta:

$$
\alpha_{1}=\frac{28,374}{N(S N R+1)}
$$

Un evento de sincronización perfecta implica que la CCN en el instante de sincronismo óptimo $n_{\text {ópt }}$ supera un umbral $\alpha$, mientras que no lo haga en ninguna de las $D$ muestras previas $n_{\text {ant }}$. Como para el caso de ruido blanco gaussiano las muestras previas pueden considerarse independientes, entonces la probabilidad de sincronización perfecta puede expresarse de acuerdo a:

$$
P_{\text {sinc }}(\alpha)=\left(\int_{0}^{\sqrt{\alpha}} f_{\sqrt{c_{1, n_{\text {ant }}}^{\prime}}}(x)\right)^{D} \cdot\left(1-\int_{0}^{\sqrt{\alpha}} f \sqrt{\sqrt{c_{1, n o p t}^{\prime}}}(x)\right)
$$

Resulta importante mencionar que, para evaluar el desempeño de la etapa de sincronización fina exclusivamente, se considera una detección exitosa del símbolo piloto en la etapa previa de sincronización gruesa. A partir de (5.21), es posible evaluar el desempeño de la etapa de sincronización fina bajo un determinado umbral de 
detección. Adicionalmente, puede obtenerse a partir de ella un umbral que maximice la probabilidad de detección, es decir el umbral $\alpha_{\text {ópt }}$ que satisface la ecuación:

$$
\begin{gathered}
\frac{d P_{\operatorname{sinc}}(\alpha)}{d \alpha}=0=D f \sqrt{\sqrt{c_{1, n_{\text {ant }}}^{\prime}}}(\sqrt{\alpha}) \cdot\left(1-\int_{0}^{\sqrt{\alpha}} f \sqrt{\sqrt{c_{1, n_{\text {op }}}^{\prime}}}(x)\right) \\
-\left(\int_{0}^{\sqrt{\alpha}} f_{\sqrt{c_{1, n_{\text {ant }}}^{\prime}}}(x)\right) \cdot f \sqrt{\sqrt{c_{1, n_{\text {opt }}}}}(\sqrt{\alpha}) \\
\frac{d P_{\operatorname{sinc}}(\alpha)}{d \alpha}=0=D \frac{2 \sqrt{N(S N R+1)}}{\sqrt{2 \pi}} e^{-\alpha \frac{N(S N R+1)}{2}}\left(1-\frac{1}{K_{A / B}} \frac{\sqrt{\alpha(S N R+1)}}{\sqrt{(S N R)}}\right) \\
-\operatorname{erf}(\sqrt{\alpha N(S N R+1) / 2}) \frac{1}{K_{A / B}} \frac{\sqrt{(S N R+1)}}{\sqrt{(S N R)}}
\end{gathered}
$$

Los valores del umbral $\alpha_{\text {ópt }}$ dependen entonces de la condición de $S N R$, la longitud del símbolo piloto $N$, la ventana de búsqueda $D$, y de la distribución del primer camino del canal PLC $h_{0}$. El umbral es óptimo en términos de maximizar la probabilidad de sincronización perfecta de (5.21) en particular, pero en términos globales es subóptimo dado que las PDFs intervinientes son aproximadas. En la Tabla 5.1 se enseñan los resultados teóricos obtenidos para los valores de los umbrales $\alpha_{1}$ y $\alpha_{\text {ópt }}$, y la probabilidad de sincronización perfecta en cada caso, considerando una ventana de búsqueda de $D=50$ muestras, para los canales PLC tipo A y B bajo diferentes niveles de ruido. Dado que (5.23) no posee solución analítica, los valores de $\alpha_{\text {ópt }}$ se calcularon a partir del método numérico iterativo Bisección [Chapra 2010], con un error máximo admisible de $10^{-9}$ como criterio de fin. Puede apreciarse que el umbral $\alpha_{1}$ que garantiza una probabilidad de falsa detección de $10^{-7}$ resulta menor que el umbral óptimo, y se verifica que la probabilidad de sincronización perfecta teórica es superior para el umbral óptimo obtenido. Así mismo, para el umbral $\alpha_{1}$ la probabilidad de sincronización perfecta es mayor en el caso del canal tipo B respecto del tipo A. Esto se debe a que, bajo un mismo valor de umbral, la probabilidad de falsa detección es la misma para ambos casos, pero la probabilidad de no detectar el primer camino del canal es menor para el canal B. Esto puede observarse sencillamente comparando las PDFs de la Fig. 5.4. Es evidente además que la probabilidad de sincronización perfecta aumenta a medida que aumenta la condición de $S N R$. 
Diseño de Estrategias de Sincronización y Estimación de Canal para la Mejora de Comunicaciones en Redes Inteligentes de Energía

Tabla 5.1 Umbrales de sincronización fina y probabilidades de sincronización perfecta teóricas para los canales PLC tipo A y B bajo ruido blanco gaussiano.

\begin{tabular}{|c|c|c|c|c|c|c|}
\hline & \multicolumn{6}{|c|}{ SNR } \\
\hline & $-6 \mathrm{~dB}$ & $-3 \mathrm{~dB}$ & $0 \mathrm{~dB}$ & $3 \mathrm{~dB}$ & $6 \mathrm{~dB}$ & $10 \mathrm{~dB}$ \\
\hline$\alpha_{1}\left(* 10^{-3}\right)$ & 5,54 & 4,61 & 3,46 & 2,31 & 1,39 & 0,63 \\
\hline$\alpha_{\text {ópt } / A}\left(* 10^{-3}\right)$ & 2,46 & 2,19 & 1,74 & 1,22 & 0,77 & 0,37 \\
\hline$\alpha_{\mathrm{o} p t / B}\left(* 10^{-3}\right)$ & 2,56 & 2,26 & 1,79 & 1,26 & 0,79 & 0,38 \\
\hline$P_{\text {sinc }}\left(\alpha_{1 / \mathrm{A}}\right)$ & $69,4 \%$ & $78,4 \%$ & $84,7 \%$ & $89,2 \%$ & $92,3 \%$ & $95,2 \%$ \\
\hline$P_{\text {sinc }}\left(\alpha_{1 / \mathrm{B}}\right)$ & $74,9 \%$ & $82,2 \%$ & $87,4 \%$ & $91,1 \%$ & $93,7 \%$ & $96 \%$ \\
\hline$P_{\text {sinc }}\left(\alpha_{\mathrm{o} p t / A}\right)$ & $78,1 \%$ & $84,1 \%$ & $88,4 \%$ & $91,6 \%$ & $93,9 \%$ & $96,1 \%$ \\
\hline$P_{s i n c}\left(\alpha_{\text {ópt/B }}\right)$ & $81,7 \%$ & $86,7 \%$ & $90,4 \%$ & $93 \%$ & $95 \%$ & $96,7 \%$ \\
\hline
\end{tabular}

Finalmente, se intentó verificar mediante simulaciones el análisis teórico realizado. En la Fig. 5.6 se presentan los resultados obtenidos por simulación en cada caso, y los resultados teóricos analizados para contrastar. Puede apreciarse que los resultados de simulaciones se ajustan adecuadamente al análisis teórico para el umbral $\alpha_{1}$, mientras que para el umbral $\alpha_{\text {ópt }}$ los resultados no se corresponden con el análisis, salvo para bajos valores de $S N R$; y la diferencia es mayor en el caso del canal B respecto del canal A para altos valores de $S N R$. Esto se debe a que el efecto de desvanecimiento del canal influye considerablemente en la $\mathrm{CCN}$, y la aproximación aplicada no resulta apropiada. Además, la varianza de la ganancia del canal $\|\mathbf{h}\|^{2}$ es mayor para el canal B que para el A (Fig. 4.2), por lo que el desvío resulta mayor. El error en el cálculo de la probabilidad de sincronización perfecta se produce en mayor medida por el error en el cálculo de la probabilidad de falsa detección, ya que $f_{\sqrt{c^{\prime}{ }_{1, \text { ant }}}}(x)$ describe valores más pequeños que los obtenidos por simulación, especialmente a altas $S N R$, de acuerdo a la Fig. 5.5. Esto conlleva a que, para un determinado umbral, la probabilidad de falsa detección sea en realidad superior respecto del valor analítico, incrementándose el error especialmente debido al exponente $D$ en (5.21). En el caso del umbral $\alpha_{1}$ no se produce el fenómeno debido a que la probabilidad de falsa detección a partir de la que se obtiene su valor es muy baja $\left(10^{-7}\right)$, por lo que un pequeño incremento en esa probabilidad no afecta considerablemente el resultado final de la probabilidad de sincronización perfecta. 

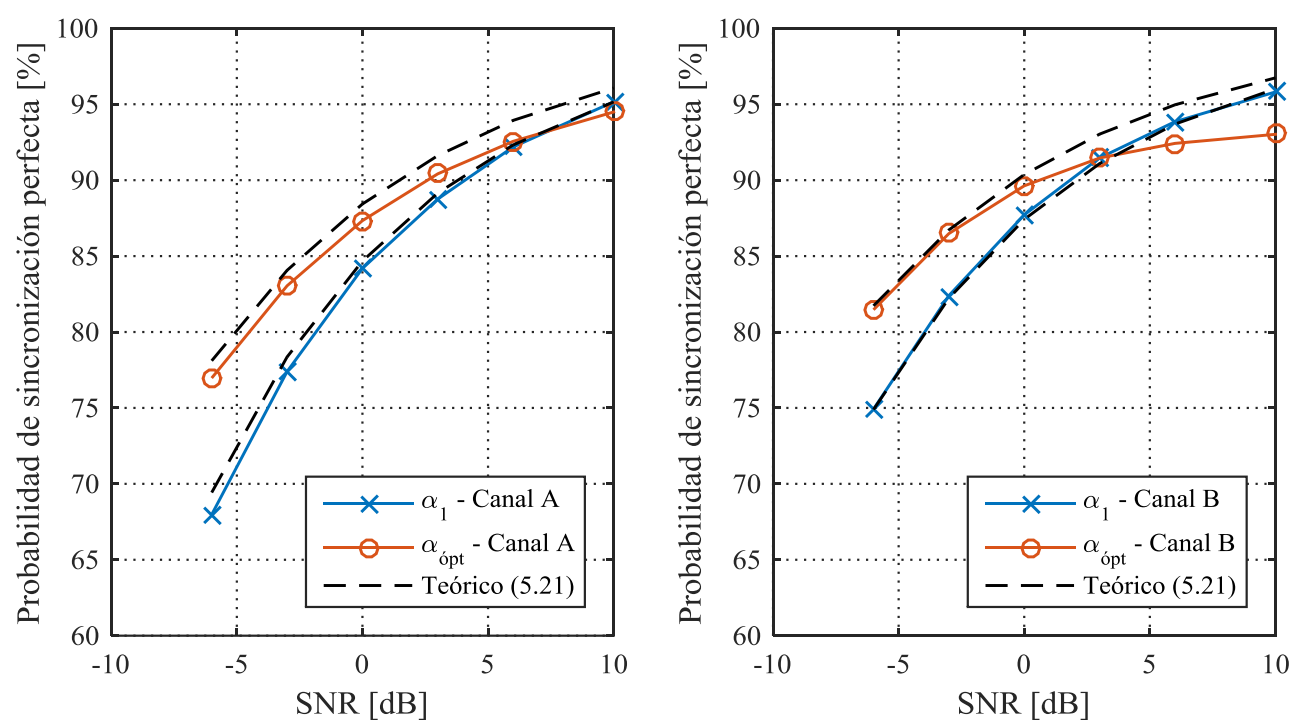

Fig. 5.6 Resultados de simulación de la probabilidad de sincronización perfecta bajo los canales PLC tipo A (izquierda) y B (derecha) con ruido blanco Gaussiano.

Como conclusión, para obtener un desempeño adecuado de la etapa de sincronización fina basada en la $\mathrm{CCN} c^{\prime}{ }_{1, n}$, los umbrales de detección deben ser dinámicos, y para cada ejecución del algoritmo de sincronización se precisa conocer la condición de SNR para los dos umbrales $\alpha_{1}$ y $\alpha_{\text {ópt }}$. Esto podría cumplimentarse a partir del estimador de la condición de $S N R$ propuesto en el capítulo 4, sección 4.3.5, con un error de estimación asociado. Adicionalmente, el umbral $\alpha_{\text {ópt }}$ que maximiza la probabilidad de sincronización perfecta precisa para su cómputo además el conocimiento estadístico del canal, a partir de la distribución del primer camino del canal $h_{0}$. Debido a que no se incluyó el efecto del desvanecimiento del canal, y a simplificaciones de algunos términos del denominador en (5.18), las PDFs obtenidas no resultan completamente útiles para definir un umbral óptimo $\alpha_{\text {ópt }}$ que supere el desempeño del umbral $\alpha_{1}$ en todo el rango de condiciones de $S N R$. Por lo tanto, resulta interesante derivar una variante de normalización de la CC que evite alguna de estas limitaciones.

\subsubsection{Sincronización fina a partir de una correlación cruzada normalizada modificada}

Para intentar solventar los inconvenientes mencionados, se propone modificar el cálculo de la CCN de acuerdo a:

$$
c_{2, n}^{\prime}=\frac{c_{1, n}^{\prime}}{1-m_{3, n}}
$$


Diseño de Estrategias de Sincronización y Estimación de Canal para la Mejora de Comunicaciones en Redes Inteligentes de Energía

La estrategia se basa principalmente en intentar evitar el término $\sigma_{S}^{2}$ en el denominador de (5.18) que incluye el fenómeno de desvanecimiento del canal. Considerando a $\sigma_{S}^{2}$ como un término constante en principio para un análisis sencillo, la media de $m_{3, n}$ en la zona libre de interferencia resultaría en

$$
\mu_{m_{3, n}}=\frac{\left(\sigma_{s}^{2}+\frac{N_{2}}{N} \sigma_{w}^{2}\right)}{\left(\sigma_{S}^{2}+\sigma_{w}^{2}\right)}, \quad n_{\text {ópt }}-N_{p c}+L<n \leq n_{\text {ópt }}
$$

Luego, la media de $1-m_{3, n}$ resulta:

$$
1-\mu_{m_{3, n}}=\frac{\left(1-\frac{N_{2}}{N}\right) \sigma_{w}^{2}}{\left(\sigma_{S}^{2}+\sigma_{w}^{2}\right)}
$$

Así, la CCN modificada en el instante de sincronismo óptimo puede expresarse aproximadamente como:

$$
c^{\prime}{ }_{2, n_{\text {o } p t}} \approx \frac{P_{T}\left|h_{0}\right|^{2}}{\left(\sigma_{S}^{2}+\sigma_{w}^{2}\right)} \frac{\left(\sigma_{S}^{2}+\sigma_{w}^{2}\right)}{\left(1-\frac{N_{2}}{N}\right) \sigma_{w}^{2}}=\frac{P_{T}\left|h_{0}\right|^{2}}{\left(1-\frac{N_{2}}{N}\right) \sigma_{w}^{2}}=\frac{S N R\left|h_{0}\right|^{2}}{\left(1-\frac{N_{2}}{N}\right)}
$$

Donde es evidente la ausencia del término $\sigma_{S}^{2}$. Entonces, la raíz cuadrada y su PDF se pueden aproximar de acuerdo a:

$$
\begin{gathered}
\sqrt{c^{\prime}{ }_{2, n_{\mathrm{o} p t}}} \approx \frac{\sqrt{S N R}\left|h_{0}\right|}{\sqrt{\left(1-\frac{N_{2}}{N}\right)}} \\
K_{P D F}=\frac{1}{K_{A / B}} \frac{\sqrt{\left(1-\frac{N_{2}}{N}\right)}}{\sqrt{(S N R)}}
\end{gathered}
$$

Los resultados obtenidos por simulación se contrastan con la aproximación teórica realizada en la Fig. 5.7, y se evidencia que la aproximación uniforme continúa siendo válida para valores pequeños de la variable.

\subsubsection{Sincronización fina bajo ruido blanco gaussiano}

Por otro lado, la CCN modificada en las muestras previas al instante de sincronismo óptimo resulta:

$$
c_{2, n_{a n t}}^{\prime} \approx \frac{1 / N\left|\sum_{m=0}^{N-1} w_{n_{a n t}+m} \mathrm{x}_{m}^{\prime *}\right|^{2}}{N\left(\sigma_{S}^{2}+\sigma_{w}^{2}\right)} \frac{\left(\sigma_{S}^{2}+\sigma_{w}^{2}\right)}{\left(1-\frac{N_{2}}{N}\right) \sigma_{w}^{2}}=\frac{1 / N\left|\sum_{m=0}^{N-1} w_{n_{a n t}+m} \mathrm{x}_{m}^{\prime *}\right|^{2}}{N\left(1-\frac{N_{2}}{N}\right) \sigma_{w}^{2}}
$$



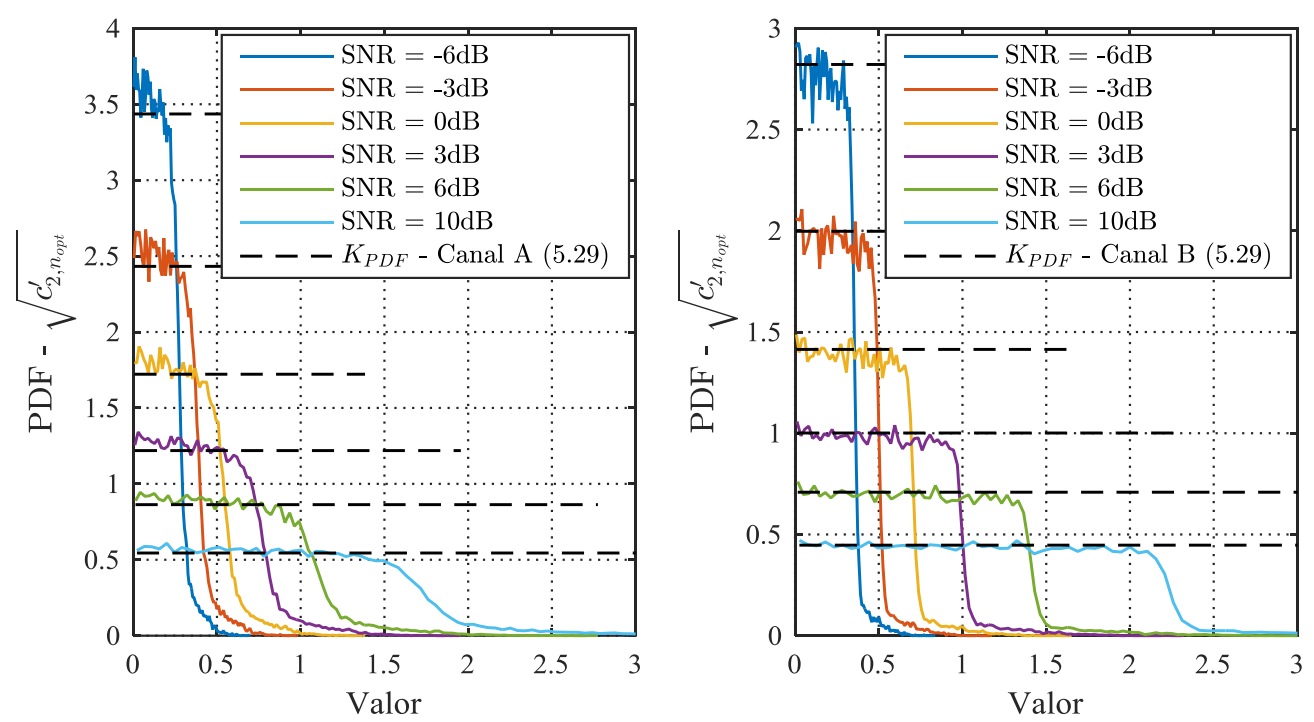

Fig. 5.7 PDFs empíricas de la raíz de la correlación cruzada normalizada modificada en el instante óptimo de sincronismo para el canal A (izquierda) y B (derecha), bajo ruido de fondo coloreado y

diferentes condiciones de SNR.

Al igual que en el caso anterior, la PDF de la raíz cuadrada se puede aproximar de acuerdo a:

$$
f_{\sqrt{c_{2, n_{n n t}}^{\prime}}}(x) \approx \frac{\sqrt{2}}{\sigma \sqrt{\pi}} e^{\frac{-x^{2}}{2 \sigma^{2}},} \quad x>0
$$

Con $\sigma^{2}=1 /\left(N\left(1-\frac{N_{2}}{N}\right)\right)$. Los resultados obtenidos por simulaciones para la PDF de $\sqrt{c^{\prime}{ }_{2, n_{\text {ant }}}}$ se contrastan con la PDF analítica en la Fig. 5.8. El resultado obtenido por simulaciones se ajusta adecuadamente a la PDF propuesta, y queda en evidencia que los valores resultan independientes de la condición de $S N R$ con la modificación aplicada. Esto permite que el umbral de detección $\alpha_{1}$ no dependa del nivel de $S N R$ ni de las propiedades estadísticas del canal, y por lo tanto resulte en un valor fijo para una determinada probabilidad de falsa detección. Por ejemplo, para una probabilidad de falsa detección de $10^{-7}$, evaluando la ICDF se obtiene $\sqrt{\alpha_{1}}$, y luego el umbral $\alpha_{1}$ resulta:

$$
\alpha_{1}=\frac{28,374}{N\left(1-\frac{N_{2}}{N}\right)}
$$

En este caso, la probabilidad de sincronización perfecta para un umbral $\alpha$ está dada por:

$$
P_{\text {sinc }}(\alpha)=\left(\int_{0}^{\sqrt{\alpha}} f_{\sqrt{c_{2, n_{\text {ant }}}^{\prime}}}(x)\right)^{D} \cdot\left(1-\int_{0}^{\sqrt{\alpha}} f \sqrt{\sqrt{c_{2, n o p t}^{\prime}}}(x)\right)
$$


Diseño de Estrategias de Sincronización y Estimación de Canal para la Mejora de Comunicaciones en Redes Inteligentes de Energía
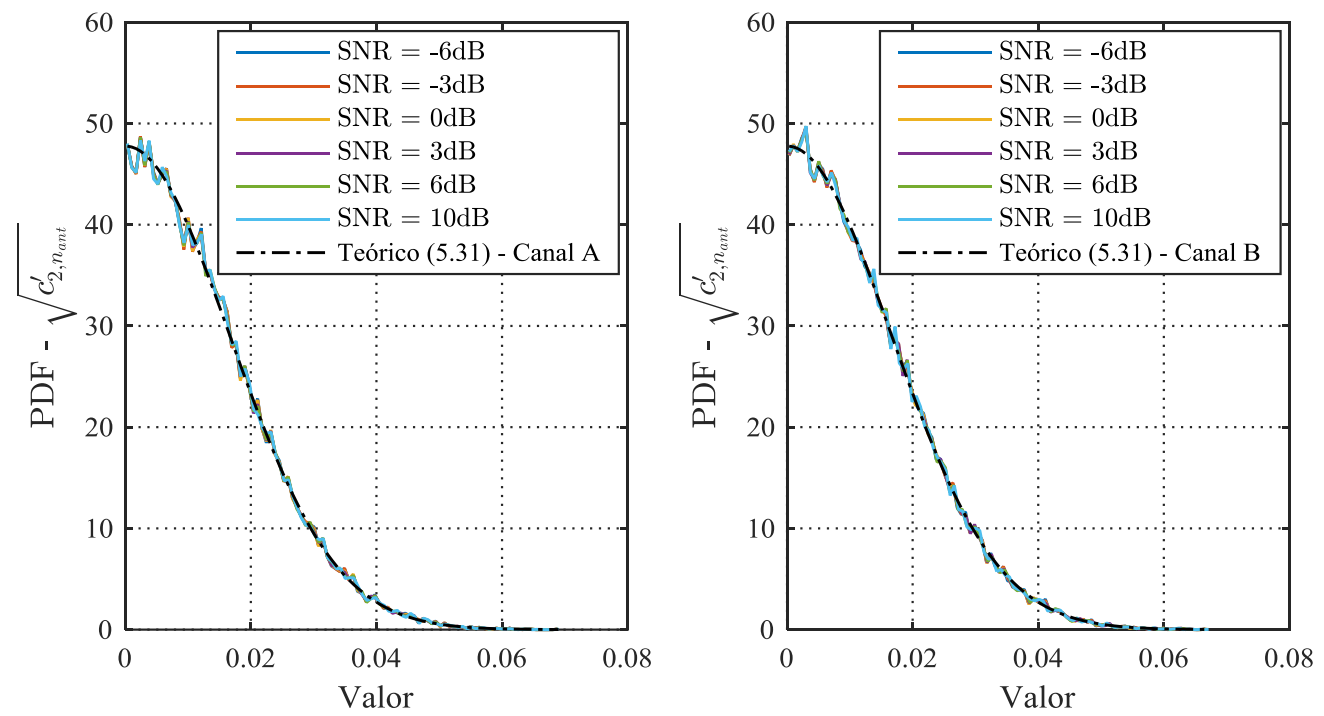

Fig. 5.8 PDFs empíricas de la raíz de la correlación cruzada normalizada modificada en la zona de correlación nula para el canal A (izquierda) y B (derecha), bajo ruido blanco gaussiano y diferentes condiciones de SNR.

Y el umbral que maximiza la probabilidad puede obtenerse a partir de:

$$
\begin{aligned}
\frac{d P_{\operatorname{sinc}}(\alpha)}{d \alpha}=0 & =D \frac{2 \sqrt{N\left(1-\frac{N_{2}}{N}\right)}}{\sqrt{2 \pi}} e^{-\alpha \frac{N\left(1-\frac{N_{2}}{N}\right)}{2}}\left(1-\frac{1}{K_{A / B}} \frac{\sqrt{\alpha\left(1-\frac{N_{2}}{N}\right)}}{\sqrt{(S N R)}}\right) \\
& -\operatorname{erf}\left(\sqrt{\alpha N\left(1-\frac{N_{2}}{N}\right) / 2}\right) \frac{1}{K_{A / B}} \frac{\sqrt{\left(1-\frac{N_{2}}{N}\right)}}{\sqrt{(S N R)}}
\end{aligned}
$$

Los valores del umbral $\alpha_{\text {ópt }}$ dependen entonces de la condición de $S N R$, los parámetros $N, N_{2}$, las ventanas de búsqueda $D$, y de la distribución del primer camino del canal PLC; pero no depende de la ganancia del canal, por lo que no se ve afectado por el efecto de desvanecimiento del mismo. En la Tabla 5.2 se presentan los resultados teóricos obtenidos para los valores de los umbrales $\alpha_{1}$ y $\alpha_{\text {ópt }}$, y la probabilidad de sincronización perfecta para la CCN modificada. Puede verificarse que, si bien los valores de los umbrales son diferentes a los obtenidos en la Tabla 5.1, la probabilidad de sincronización perfecta teórica resulta idéntica en todos los casos.

Luego, se verificó si la modificación en el cálculo de la CCN mejora el ajuste entre el análisis teórico realizado y las pruebas de simulaciones. En la Fig. 5.9 se presentan los resultados obtenidos por simulación y los resultados teóricos analizados para contrastar. 
Capítulo 5: Sincronización fina y estimación de canal en sistemas DMT-PLC

Tabla 5.2 Umbrales de sincronización fina y probabilidades de sincronización perfecta teóricas para los canales PLC tipo A y B bajo ruido blanco gaussiano.

\begin{tabular}{|c|c|c|c|c|c|c|}
\hline & \multicolumn{6}{|c|}{$S N R$} \\
\hline & $-6 \mathrm{~dB}$ & $-3 \mathrm{~dB}$ & $0 \mathrm{~dB}$ & $3 \mathrm{~dB}$ & $6 \mathrm{~dB}$ & $10 \mathrm{~dB}$ \\
\hline$\alpha_{1}\left(* 10^{-3}\right)$ & 7,92 & 7,92 & 7,92 & 7,92 & 7,92 & 7,92 \\
\hline$\alpha_{\text {ópt } / A}\left(* 10^{-3}\right)$ & 3,52 & 3,75 & 3,97 & 4,18 & 4,39 & 4,66 \\
\hline$\alpha_{\text {ópt } / B}\left(* 10^{-3}\right)$ & 3,65 & 3,88 & 4,09 & 4,3 & 4,51 & 4,77 \\
\hline$P_{\text {sinc }}\left(\alpha_{1 / \mathrm{A}}\right)$ & $69,4 \%$ & $78,4 \%$ & $84,7 \%$ & $89,2 \%$ & $92,3 \%$ & $95,2 \%$ \\
\hline$P_{\operatorname{sinc}}\left(\alpha_{1 / \mathrm{B}}\right)$ & $74,9 \%$ & $82,2 \%$ & $87,4 \%$ & $91,1 \%$ & $93,7 \%$ & $96 \%$ \\
\hline$P_{\text {sinc }}\left(\alpha_{\text {ópt } / A}\right)$ & $78,1 \%$ & $84,1 \%$ & $88,4 \%$ & $91,6 \%$ & $93,9 \%$ & $96,1 \%$ \\
\hline$P_{\text {sinc }}\left(\alpha_{\circ p t / B}\right)$ & $81,7 \%$ & $86,7 \%$ & $90,4 \%$ & $93 \%$ & $95 \%$ & $96,7 \%$ \\
\hline
\end{tabular}

Puede apreciarse que los resultados de simulaciones para $c_{2, n}^{\prime}$ se ajustan mucho mejor al análisis teórico respecto al resultado obtenido para la $\mathrm{CCN} c_{1, n}^{\prime}$ en la Fig. 5.6. Adicionalmente, la sincronización fina basada en $c_{2, n}^{\prime}$ posee la ventaja que el valor del umbral $\alpha_{1}$, que fija la probabilidad de falsa detección, es independiente de la condición de $S N R$ y de las estadísticas del canal. Los resultados obtenidos para el caso del canal B son ligeramente superiores a aquellos del canal A, debido a que el ajuste de la aproximación uniforme a la PDF de $c_{2, n_{\text {ó }} \text { es }}$ esejor para el caso del canal $\mathrm{B}$, de acuerdo a la Fig. 5.7.

Finalmente, se evaluó el algoritmo de sincronización fina en términos del RMSE, como una medida de desempeño adicional a la probabilidad de sincronización perfecta. En la Fig. 5.10 se presentan los resultados obtenidos bajo los umbrales de detección $\alpha_{1}$ y $\alpha_{\text {ópt }}$ para las dos variantes de CCN planteadas, y además el resultado obtenido a partir de la posición del máximo de cada $\mathrm{CCN} n_{f_{1}}$ de (5.13). En todos los casos, el error obtenido para la $\mathrm{CCN} c_{2, n}^{\prime}$ es menor al obtenido para $c_{1, n}^{\prime}$.

Por otro lado, el umbral que maximiza la probabilidad de sincronización perfecta no necesariamente minimiza el RMSE del algoritmo de sincronización fina. Esto se evidencia en el caso del canal A, donde el error obtenido bajo el umbral $\alpha_{1}$ es inferior al obtenido bajo $\alpha_{\text {ópt }}$. 
Diseño de Estrategias de Sincronización y Estimación de Canal para la Mejora de Comunicaciones en Redes Inteligentes de Energía
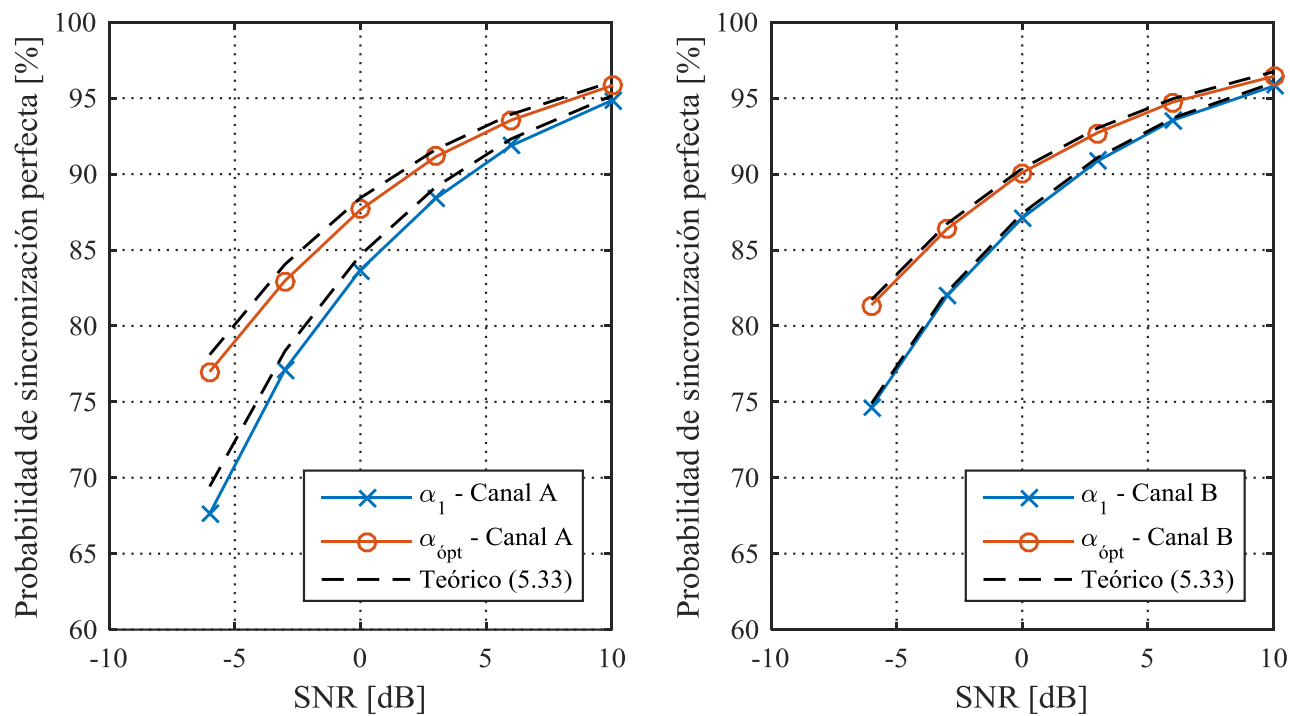

Fig. 5.9 Resultados de simulación de la probabilidad de sincronización perfecta para la correlación cruzada normalizada modificada, bajo los canales PLC tipo A (izquierda) y B (derecha) con ruido blanco gaussiano.
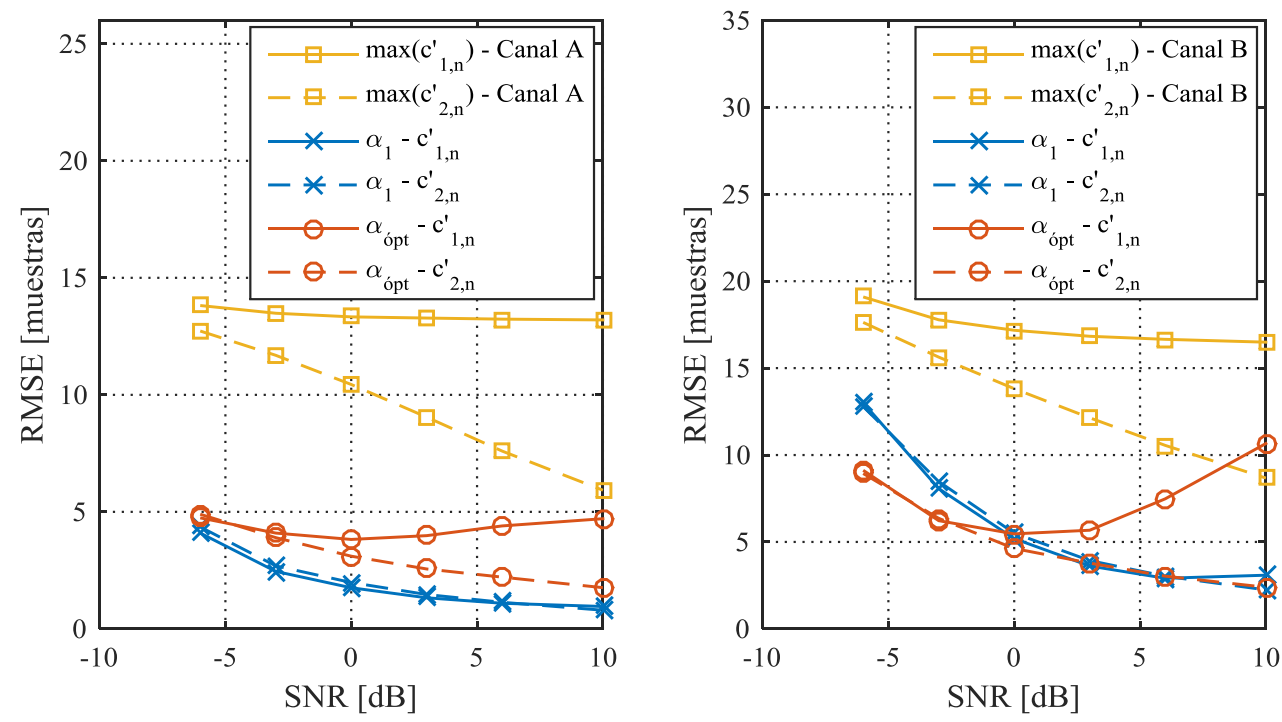

Fig. 5.10 Desempeño de la etapa de sincronización fina propuesta en términos del RMSE, bajo los canales PLC tipo A (izquierda) y B (derecha) con ruido blanco gaussiano.

Esto se produce debido a que el umbral $\alpha_{\text {ópt }}$ es menor a $\alpha_{1}$, aumentando la probabilidad de falsa detección, cuya ubicación puede ser cualquiera de las $D$ muestras previas al instante de sincronización óptimo de manera equiprobable.

Adicionalmente, como las ganancias promedio de los caminos del canal disminuyen exponencialmente con la distancia de acuerdo a (5.1), ante un evento de no detección del primer camino del canal, es más probable detectar caminos cercanos al mismo. Este 
fenómeno no se produce para el canal B, porque los arribos de los caminos del canal se encuentran más distanciados en el tiempo (Fig. 4.1), lo que implica un aumento relativo del RMSE cuando no se detecta el primer camino del canal, sino alguno de los siguientes.

Puede concluirse entonces que la CCN modificada implica una mejora en el proceso de definición de umbrales para el proceso de sincronización fina, respecto a la solución planteada en una primera instancia. Por lo tanto, el estudio posterior de los efectos adversos de las distintas fuentes de ruido presentes en el canal PLC se centra en esta técnica.

\subsubsection{Sincronización fina bajo ruido de fondo coloreado}

Considerando un escenario con ruido de fondo del canal PLC coloreado, el desempeño de la estrategia de sincronización fina se verá afectada, y las variables aleatorias en las que interviene el proceso de ruido verán modificadas sus varianzas, por lo que resulta de interés caracterizar estos cambios, y definir umbrales de sincronización adecuados para este contexto. Por un lado, la variable aleatoria correspondiente al instante de sincronismo óptimo $c_{2, n_{\text {ó }} \text { no }}^{\prime}$ ne ve significativamente influenciada por el ruido de fondo, de acuerdo a (5.27) y a las simulaciones presentadas en la sección anterior. Por otro lado, la variable aleatoria que representa la $\mathrm{CCN}$ en las muestras anteriores al instante óptimo de sincronismo dentro de la zona libre de interferencia $c^{\prime}{ }_{2, n_{\text {ant }}}$ posee una mayor varianza dado que:

$$
c_{2, n_{\text {ant }}}^{\prime} \approx \frac{\frac{1}{N} \mid \sum_{m=0}^{N-1} w_{n_{\mathrm{an} t}+\left.m^{\prime} \mathrm{x}_{m}^{\prime *}\right|^{2}}}{N\left(1-\frac{N_{2}}{N}\right) \sigma_{w}^{2}}=\frac{\frac{1}{N} \mathrm{Y}_{2}}{N\left(1-\frac{N_{2}}{N}\right) \sigma_{w}^{2}}
$$

Donde $Y_{2}$ es la variable aleatoria analizada en (4.71) y (4.72) modificada, considerando el valor $N_{2}=1$-únicamente para $Y_{2}$, no para el denominador de (5.35)-. A partir del análisis previo, puede derivarse que la distribución de $Y_{2}$ es una Chi-cuadrado de un grado de libertad, con media y varianza:

$$
\begin{aligned}
& \mu_{Y_{2}}=E\left(Y_{2}\right)=\sigma_{w}^{2} \sum_{k=-\infty}^{\infty} f_{1_{k}}^{2} \\
& \operatorname{Var}\left(Y_{2}\right)=\sigma_{w}^{4} 2\left(\sum_{k=-\infty}^{+\infty} f_{1_{k}}^{2}\right)^{2}
\end{aligned}
$$


Diseño de Estrategias de Sincronización y Estimación de Canal para la Mejora de Comunicaciones en Redes Inteligentes de Energía

Donde $f_{1_{k}}$ es la secuencia de correlación cruzada entre el filtro que colorea el ruido $g_{k}$ y el símbolo piloto $x_{-k}^{\prime}: f_{1_{k}}=\sum_{j=-\infty}^{+\infty} g_{j} x_{k+j}^{\prime}=g_{k} * x_{-k}^{\prime}$. Teniendo en cuenta este resultado y las constantes adicionales en (5.35), la media y varianza de $c^{\prime}{ }_{2, n_{\text {ant }}}$ resultan:

$$
\begin{gathered}
\mu_{c^{\prime}{ }_{2, n_{\text {ant }}}}=\frac{\sum_{k=-\infty}^{\infty} f_{1_{k}}^{2}}{N^{2}\left(1-\frac{N_{2}}{N}\right)} \\
\operatorname{Var}\left(c_{2, n_{\text {ant }}}^{\prime}\right)=\frac{2\left(\sum_{k=-\infty}^{+\infty} f_{1_{k}}^{2}\right)^{2}}{N^{4}\left(1-\frac{N_{2}}{N}\right)^{2}}
\end{gathered}
$$

Al aplicar la raíz cuadrada, se puede obtener la PDF de $\sqrt{c^{\prime}{ }_{2, n_{a n t}}}$, de acuerdo a la distribución normal doblada:

$$
f_{\sqrt{c^{\prime}, n_{\text {ant }}}}(x) \approx \frac{\sqrt{2}}{\sigma \sqrt{\pi}} e^{\frac{-x^{2}}{2 \sigma^{2}}}, \quad \sigma^{2}=\frac{\sum_{k=-\infty}^{\infty} f_{1 k}^{2}}{N^{2}\left(1-\frac{N_{2}}{N}\right)}, \quad x>0
$$

Por lo tanto, la distribución de $c^{\prime}{ }_{2, n_{a n t}}$ depende de la longitud del símbolo piloto $N$, la cantidad de muestras acumuladas en la métrica de sincronización gruesa $N_{2}$, el símbolo piloto utilizado $\mathbf{x}^{\prime} \mathbf{y}$ el filtro del proceso de ruido $\mathbf{g}$ que depende de la densidad espectral de potencia del proceso. La PDF de (5.40) fue verificada por simulaciones bajo los canales PLC A y B, y el proceso de ruido coloreado descrito en el capítulo 4, sección 4.3.2, y los resultados se presentan en la Fig. 5.11. El umbral $\alpha_{1}$ que garantiza una probabilidad de falsa detección de $10^{-7}$ de cada muestra de $c^{\prime}{ }_{2, n_{\text {ant }}}$ resulta:

$$
\alpha_{1}=\frac{28,374 \sum_{k=-\infty}^{\infty} f_{1_{k}}^{2}}{N^{2}\left(1-\frac{N_{2}}{N}\right)}
$$

Suponiendo que las muestras correspondientes a $c_{2, n_{a n t}}^{\prime}$ son prácticamente independientes, la probabilidad de sincronización perfecta para un umbral $\alpha$ está dada por:

$$
P_{\text {sinc }}(\alpha)=\left(\int_{0}^{\sqrt{\alpha}} f_{\sqrt{c_{2, n_{\text {ant }}}^{\prime}}}(x)\right)^{D} \cdot\left(1-\int_{0}^{\sqrt{\alpha}} f \sqrt{\sqrt{c_{2, n o p t}^{\prime}}}(x)\right)
$$

Y el umbral que maximiza la probabilidad puede obtenerse a partir de: 

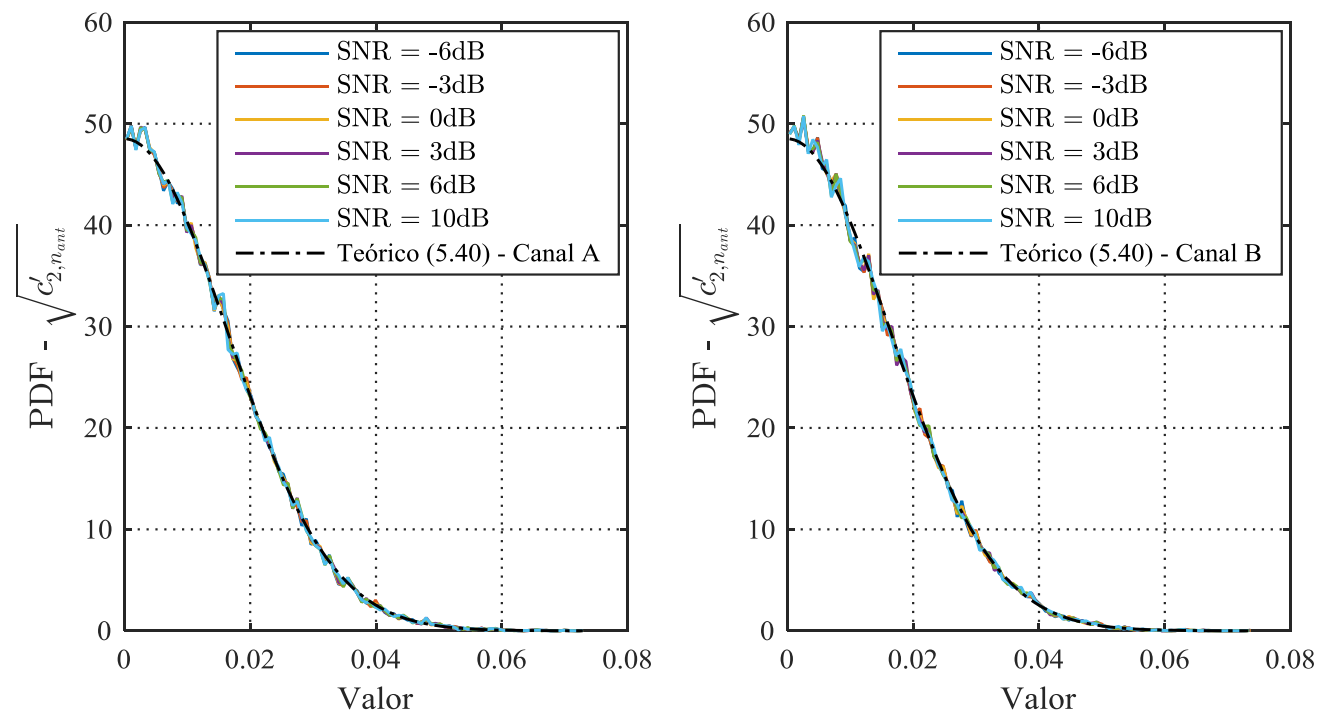

Fig. 5.11 PDFs empíricas de la raíz de la correlación cruzada normalizada en zona de correlación nula para el canal A (izquierda) y B (derecha), bajo ruido de fondo coloreado y diferentes condiciones de SNR.

$$
\begin{aligned}
\frac{d P_{\operatorname{sinc}}(\alpha)}{d \alpha}=0 & =D \frac{2 \sqrt{N^{2}\left(1-\frac{N_{2}}{N}\right)}}{\sqrt{2 \pi \sum_{k=-\infty}^{\infty} f_{1 k}^{2}}} e^{-\alpha \frac{N^{2}\left(1-\frac{N_{2}}{N}\right)}{2 \sum_{k=-\infty}^{\infty} f_{1 k}^{2}}\left(1-\frac{1}{K_{A / B}} \frac{\sqrt{\alpha\left(1-\frac{N_{2}}{N}\right)}}{\sqrt{(S N R)}}\right)} \\
& -\operatorname{erf}\left(\sqrt{\frac{\alpha N^{2}\left(1-\frac{N_{2}}{N}\right)}{2 \sum_{k=-\infty}^{\infty} f_{1 k}^{2}}}\right) \frac{1}{K_{A / B}} \frac{\sqrt{\left(1-\frac{N_{2}}{N}\right)}}{\sqrt{(S N R)}}
\end{aligned}
$$

Los valores del umbral $\alpha_{\text {ópt }}$ dependen de los parámetros $S N R, N, N_{2}, D$, la distribución del primer camino del canal PLC, la densidad espectral de potencia del ruido coloreado, y el símbolo piloto transmitido. En la Tabla 5.3 se presentan los resultados teóricos obtenidos para los valores de los umbrales $\alpha_{1}$ y $\alpha_{\text {ópt }}$ y la probabilidad de sincronización perfecta para la CCN modificada.

En la Fig. 5.12 se contrastan las probabilidades de sincronización perfecta obtenidas por simulación y los resultados teóricos planteados. También en este caso, el ajuste es mejor en el canal B que en el tipo A. La probabilidad de sincronización perfecta obtenida por simulaciones bajo el umbral $\alpha_{\text {ópt }}$ resulta ligeramente superior a la esperada a partir del análisis para el canal tipo B. Esto se debe a que, en realidad, las muestras previas al instante de sincronismo óptimo son dependientes, por lo que la probabilidad de falsa detección acumulada por las $D$ muestras es ligeramente menor a la supuesta en (5.42). 
Diseño de Estrategias de Sincronización y Estimación de Canal para la Mejora de Comunicaciones en Redes Inteligentes de Energía

Tabla 5.3 Umbrales de sincronización fina y probabilidades de sincronización perfecta teóricas para los canales PLC tipo A y B bajo ruido de fondo coloreado.

\begin{tabular}{|c|c|c|c|c|c|c|}
\hline & \multicolumn{6}{|c|}{ SNR } \\
\hline & $-6 \mathrm{~dB}$ & $-3 \mathrm{~dB}$ & $0 \mathrm{~dB}$ & $3 \mathrm{~dB}$ & $6 \mathrm{~dB}$ & $10 \mathrm{~dB}$ \\
\hline$\alpha_{1}\left(* 10^{-3}\right)$ & 7,67 & 7,67 & 7,67 & 7,67 & 7,67 & 7,67 \\
\hline$\alpha_{\text {ópt } / A}\left(* 10^{-3}\right)$ & 3,43 & 3,65 & 3,86 & 4,06 & 4,26 & 4,52 \\
\hline$\alpha_{\text {ópt } / B}\left(* 10^{-3}\right)$ & 3,56 & 3,77 & 3,98 & 4,18 & 4,38 & 4,63 \\
\hline$P_{\text {sinc }}\left(\alpha_{1 / \mathrm{A}}\right)$ & $69,9 \%$ & $78,7 \%$ & $84,9 \%$ & $89,3 \%$ & $92,4 \%$ & $95,2 \%$ \\
\hline$P_{\text {sinc }}\left(\alpha_{1 / \mathrm{B}}\right)$ & $75,3 \%$ & $82,5 \%$ & $87,6 \%$ & $91,2 \%$ & $93,8 \%$ & $96,1 \%$ \\
\hline$P_{\text {sinc }}\left(\alpha_{\text {ópt } / A}\right)$ & $78,4 \%$ & $84,3 \%$ & $88,6 \%$ & $91,7 \%$ & $94 \%$ & $96,1 \%$ \\
\hline$P_{\text {sinc }}\left(\alpha_{\mathrm{o} p t / B}\right)$ & $82 \%$ & $86,9 \%$ & $90,5 \%$ & $93,1 \%$ & $95 \%$ & $96,8 \%$ \\
\hline
\end{tabular}

Por lo tanto, para un determinado umbral, la probabilidad de falsa detección disminuye y entonces la probabilidad de sincronización perfecta se ve incrementada. Comparando las figuras Fig. 5.9 y Fig. 5.12 puede concluirse que el ruido de fondo coloreado no afecta significativamente el proceso de sincronización fina respecto al caso en el que se consideró ruido blanco gaussiano.

\subsubsection{Sincronización fina bajo ruido impulsivo}

Al igual que en el caso de la métrica de sincronización gruesa, un evento de ruido impulsivo afectará la CCN y el proceso de sincronización fina. En las Fig. 5.13 y Fig. 5.14 se presentan los resultados de las PDFs de $\sqrt{c_{2, n_{\text {opt }}}^{\prime}} \mathrm{y} \sqrt{c^{\prime}{ }_{2, n_{\text {ant }}}}$ para una $S N R$ de $0 \mathrm{~dB}$, respectivamente. En ambos casos, se considera ruido de fondo coloreado, el modelo de ruido impulsivo utilizado en la Sección 4.3.6, con eventos de ruido impulsivo con probabilidad de ocurrencia del $8 \%$. Se presentan además, las respectivas PDFs en un contexto sin ruido impulsivo, y también luego de aplicar la técnica de mitigación basada en el control automático de ganancia de la sección 4.3.6.2. Por un lado, se puede observar en la Fig. 5.13 que cuando ocurre un evento de ruido impulsivo, los valores que adopta la $\mathrm{CCN}$ en el instante de sincronismo óptimo $c^{\prime}{ }_{2, n_{\text {ó }} t}$ disminuyen significativamente. Además, puede observarse cómo, luego de aplicar la técnica de mitigación basada en AGC, la PDF obtenida se aproxima a la de un escenario sin ruido 

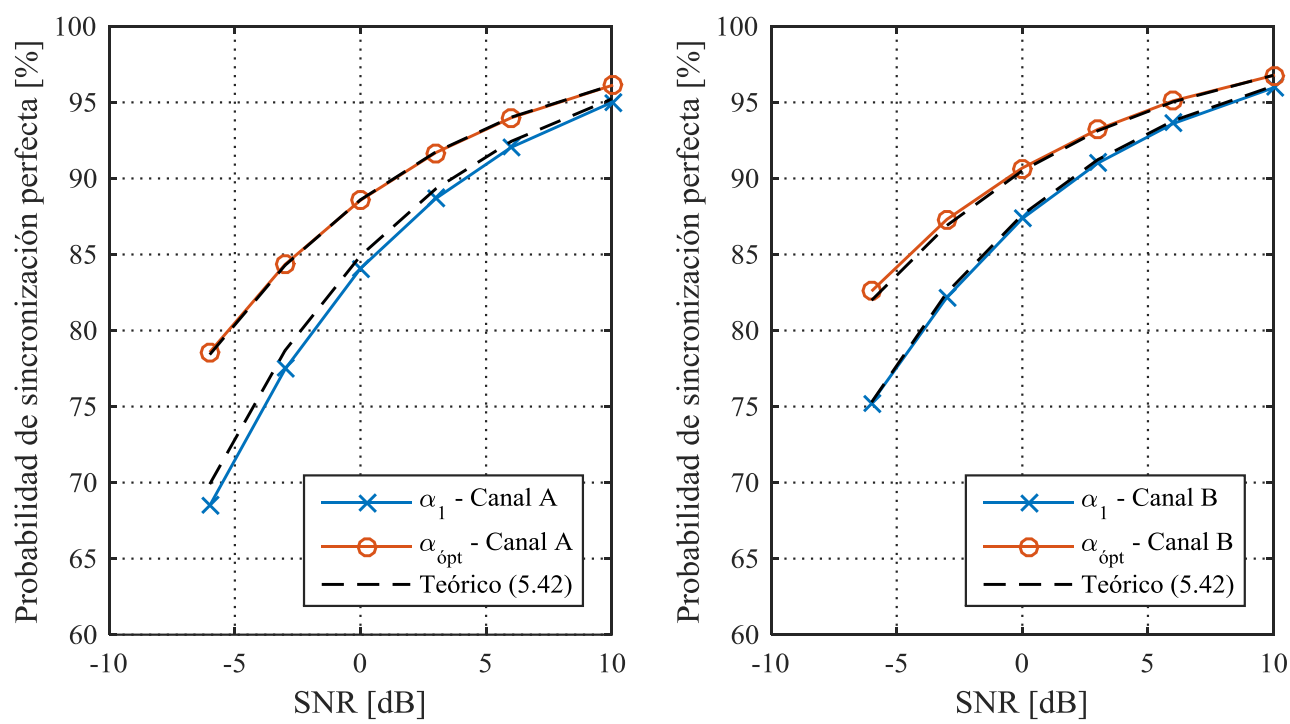

Fig. 5.12 Resultados de simulación de la probabilidad de sincronización perfecta para la correlación cruzada normalizada modificada, bajo los canales PLC tipo A (izquierda) y B (derecha) con ruido de fondo coloreado.
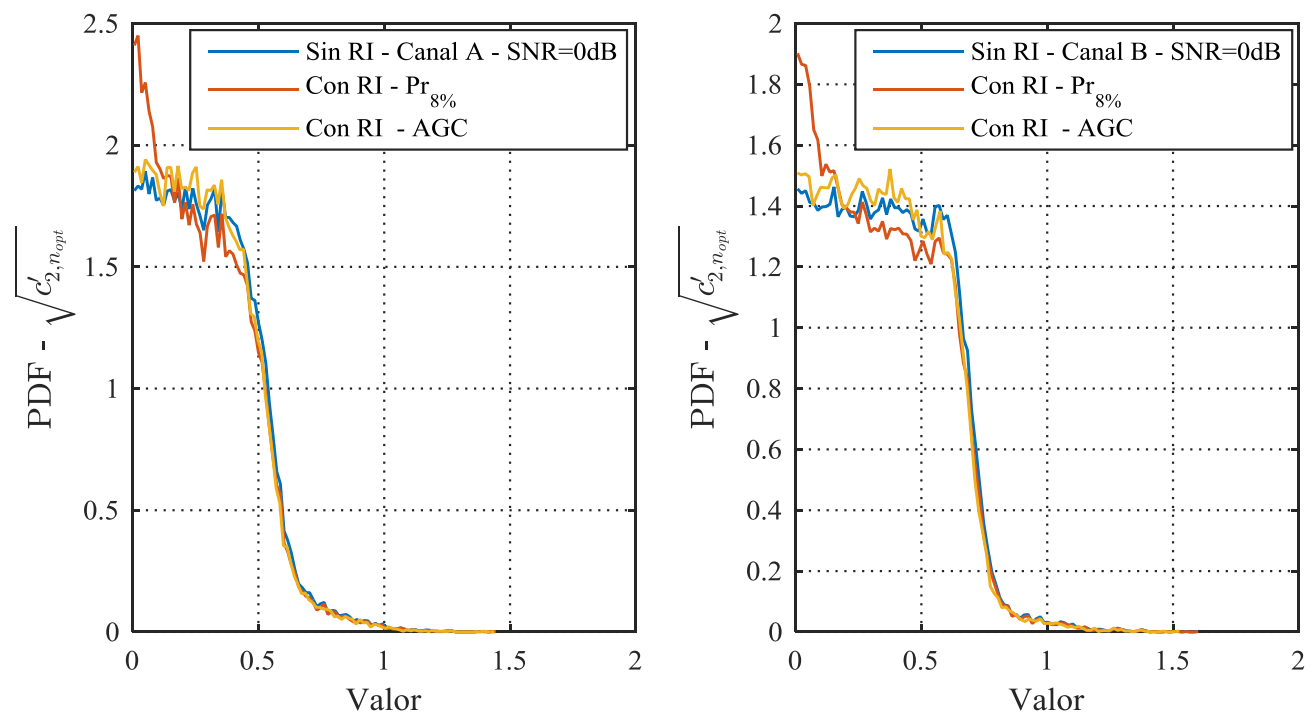

Fig. 5.13 PDFs empiricas de la raiz de la correlación cruzada normalizada modificada en el instante óptimo de sincronismo para el canal A (izquierda) y B (derecha), bajo ruido de fondo coloreado e impulsivo, y una condición de $S N R=0 d B$.

impulsivo. Por otro lado, la Fig. 5.14 indica que prácticamente no se modifica la PDF correspondiente a la CCN en las muestras previas al instante de sincronismo óptimo.

Para analizar el desempeño de la etapa de sincronización fina bajo ruido impulsivo, se incluye el resultado de simulaciones en las que se evalúa la probabilidad de sincronización perfecta, en la Fig. 5.15. En ella se observa que, para los dos tipos de canales, el ruido impulsivo disminuye la probabilidad de sincronización perfecta. 
Diseño de Estrategias de Sincronización y Estimación de Canal para la Mejora de Comunicaciones en Redes Inteligentes de Energía

Adicionalmente, al aplicar la técnica de mitigación basada en AGC se observa como la probabilidad de sincronización perfecta se recupera, a valores ligeramente inferiores al caso en el que no se incluye ruido impulsivo.

Finalmente, se evaluó el algoritmo de sincronización fina bajo ruido de fondo coloreado e impulsivo en términos del RMSE. En la Fig. 5.16 se presentan los resultados obtenidos bajo los umbrales de detección $\alpha_{1}$ y $\alpha_{\text {ópt }}$ para los casos A y B, y tres escenarios respecto del ruido: con ruido de fondo coloreado sin ruido impulsivo; ruido de fondo coloreado con eventos de ruido impulsivo con probabilidad de ocurrencia típica del $8 \%$; y ruido de fondo coloreado con eventos de ruido impulsivo mitigados a partir de la técnica basada en AGC.

Puede observarse cómo el error se dispara cuando se incluye el ruido impulsivo, y luego la técnica de mitigación reduce el error acercándolo al RMSE del escenario sin ruido impulsivo. En todos los casos, el RMSE obtenido a partir del umbral $\alpha_{\text {ópt }}$ es menor que el obtenido bajo el umbral $\alpha_{1}$. De acuerdo a lo expuesto en [Morelli 2007], el error de sincronización debe mantenerse a unas pocas unidades porcentuales de la longitud del símbolo $N$ para obtener una pérdida de $S N R$ tolerable. Si se considera un $1 \%$ de error porcentual para el sistema de $N=4096$, significa un error de sincronización de aproximadamente 40 muestras. De acuerdo a los resultados obtenidos en la etapa de
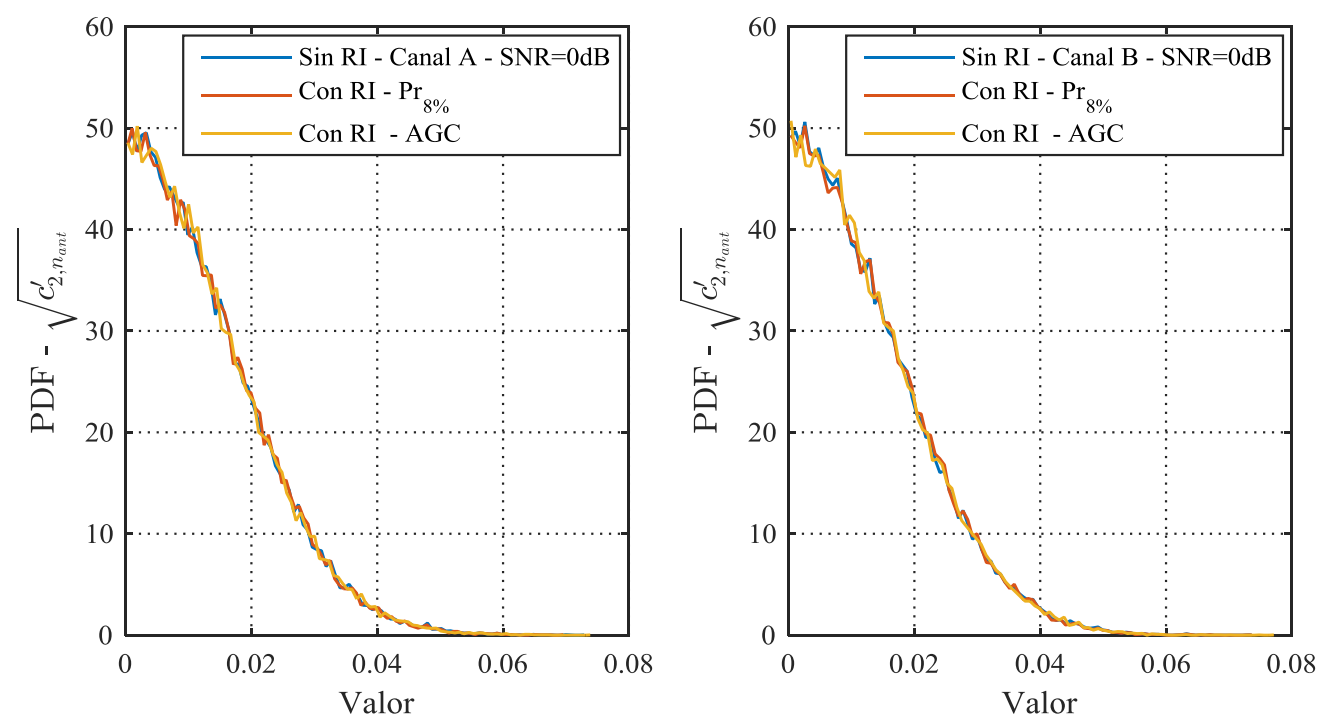

Fig. 5.14 PDFs empíricas de la raíz de la correlación cruzada normalizada en la zona de correlación nula para el canal A (izquierda) y B (derecha), bajo ruido de fondo coloreado e impulsivo, y una condición de $S N R=O d B$. 
sincronización fina, para valores de $S N R$ por encima de $-3 \mathrm{~dB}$, el RMSE se mantiene por debajo de las 10 muestras lo que indica a priori que la pérdida de $S N R$ se encontrará dentro de niveles tolerables.

\subsection{Estimación de canal}

Al igual que la técnica de estimación de canal presentada en el capítulo 3, sección 3.2.5,
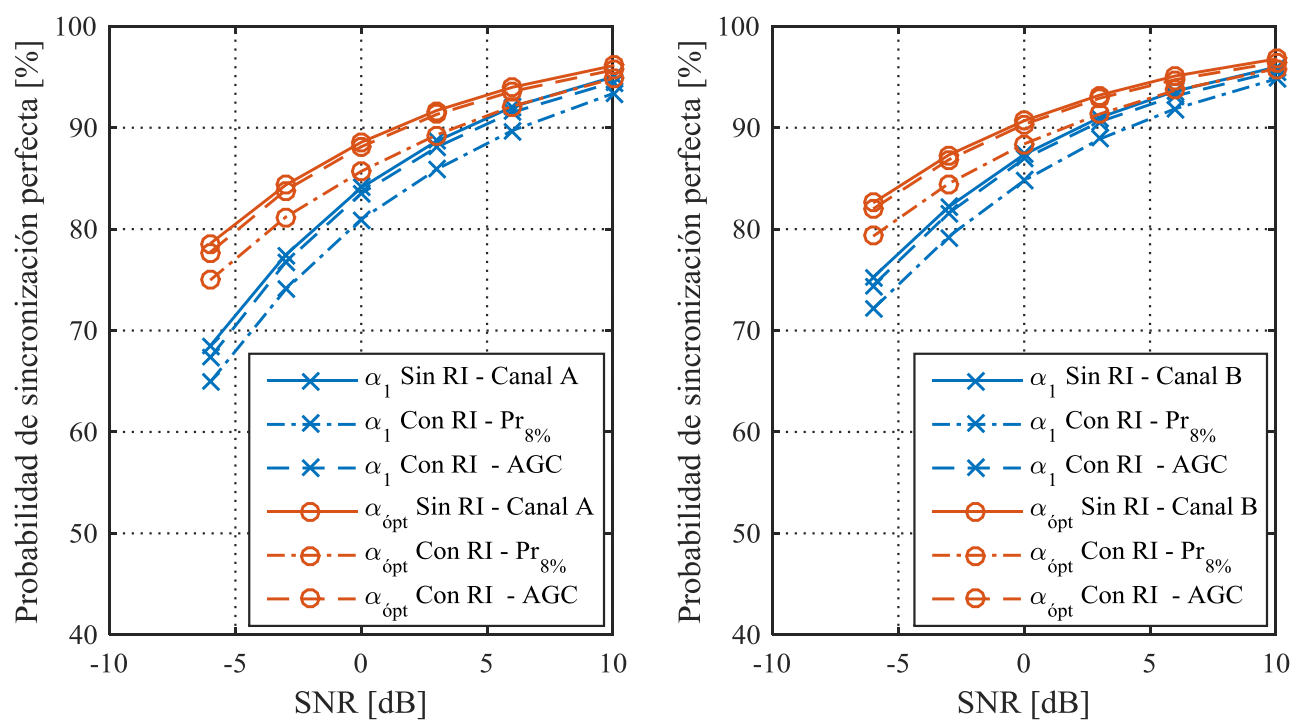

Fig. 5.15 Resultados de simulación de la probabilidad de sincronización perfecta para la correlación cruzada normalizada modificada, bajo los canales PLC tipo A (izquierda) y B (derecha) con ruido de fondo coloreado e impulsivo.
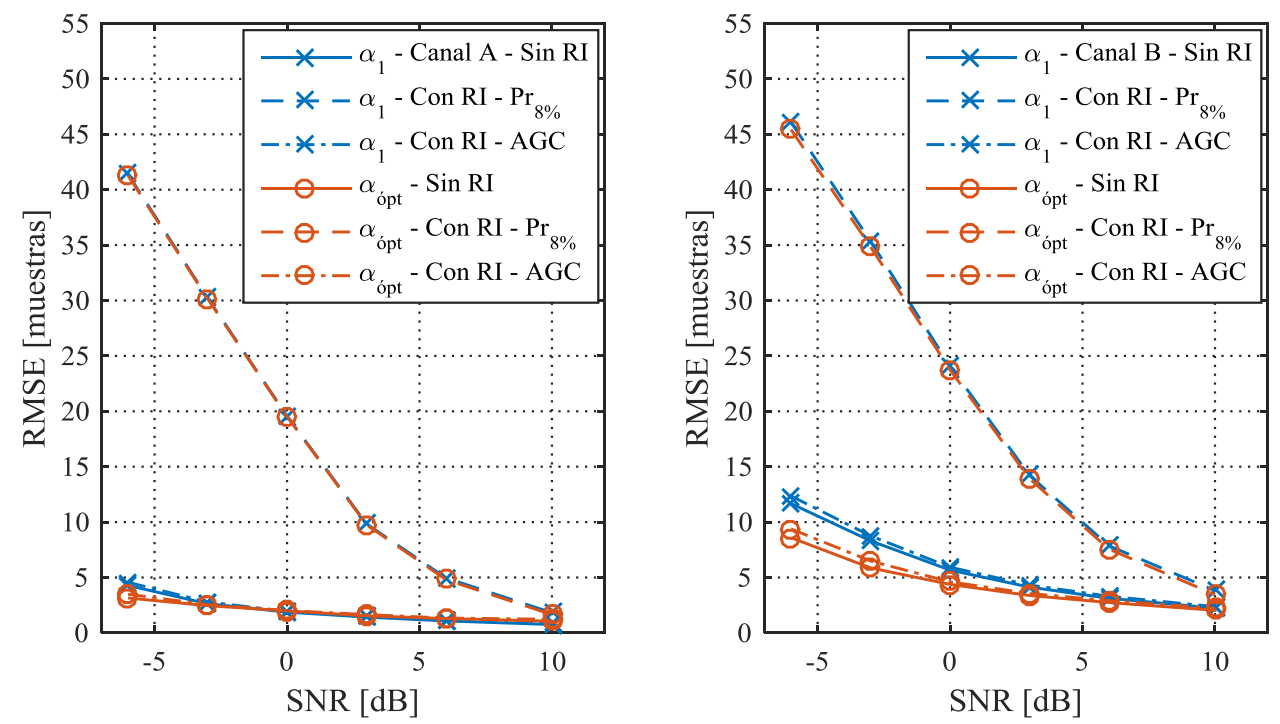

Fig. 5.16 Desempeño de la etapa de sincronización fina propuesta en términos del RMSE, bajo los canales PLC tipo A (izquierda) y B(derecha) con ruido de fondo coloreado e impulsivo. 
Diseño de Estrategias de Sincronización y Estimación de Canal para la Mejora de Comunicaciones en Redes Inteligentes de Energía

la salida del filtro acoplado del símbolo piloto propuesto puede utilizarse para obtener una estimación de la respuesta al impulso del canal. Teniendo en cuenta que la correlación cruzada $c_{n}^{\mathbf{x} \prime, \mathbf{x}}$ entre el símbolo piloto propuesto extendido $\mathbf{x}$ y el fíltro acoplado del símbolo sin extender $\mathbf{x}^{\prime}$ presenta una zona de correlación ideal, entonces la correlación cruzada de la señal recibida con el símbolo piloto, en la vecindad del instante óptimo de sincronismo, puede escribirse como:

$$
c_{n}^{\mathbf{r}, \mathbf{x}^{\prime}}=\sqrt{P_{T}} N \sum_{l=0}^{L-1} h_{l} \cdot \delta_{n-n_{\mathrm{o} p t}-l}+\sum_{m=0}^{N-1} w_{n+m} \mathbf{x}_{m}^{\prime *}, \quad n_{\text {ó } p t} \leq n<n_{\text {ópt }}+N_{c s}
$$

donde $P_{T}$ es la potencia transmitida; $N$ es la longitud del símbolo piloto; $h_{l}$ representa el $l$-ésimo camino de la CIR y $L$ es la longitud del canal en muestras; $w_{n}$ son las muestras complejas del ruido del canal PLC. Se deben incluir la cantidad suficiente de muestras posteriores al instante óptimo de sincronismo para incluir todos los coeficientes del canal. Si no se conoce (o estima) la longitud del canal $L$, entonces se incluyen las $N_{c s}-1$ muestras posteriores a la muestra resultante del proceso de sincronización fina $n_{f}$, obteniendo la estimación de la respuesta al impulso del canal de acuerdo a:

$$
\tilde{h}_{n}=\frac{1}{\sqrt{P_{T}} N} c_{n+n_{f}}^{\mathbf{r}, \mathbf{x}^{\prime}}, \quad 0 \leq n \leq N_{c s}-1
$$

A partir de este resultado, se puede obtener la respuesta en frecuencia del canal mediante la DFT de tamaño $N$ completada con ceros de acuerdo a:

$$
\widetilde{H}_{k}=\sum_{n=0}^{N_{c S}-1} \tilde{h}_{n} \cdot e^{-j \frac{2 \pi k n}{N}}, \quad 0 \leq k \leq N-1
$$

Si se considera una sincronización óptima, entonces puede obtenerse el límite inferior del MSE de la estimación del canal, que puede expresarse a partir de:

$$
M S E_{\widetilde{\boldsymbol{h}}}=E\left\{\|\mathbf{h}-\tilde{\mathbf{h}}\|^{2}\right\}=E\left\{\sum_{n=n_{\text {ó } p t}}^{n_{\mathrm{o} p} t+N_{c S}-1}\left|\frac{\sum_{m=0}^{N-1} w_{n+m} \mathrm{x}_{m}^{\prime *}}{\sqrt{P_{T}} N}\right|^{2}\right\}
$$

Entonces, el MSE de la estimación depende directamente del proceso de ruido, la longitud del símbolo piloto y la $S N R$. 


\subsubsection{Estimación de canal bajo ruido blanco gaussiano}

Para analizar el error cuadrático medio en el caso en el que se consideran las muestras de ruido a partir de un proceso de ruido blanco gaussiano, se aprovecha el análisis de las variables aleatorias analizadas en la sección 4.3.3. La variable $\sum_{n=n_{\text {ópt }}}^{n_{\text {ó } t}+N_{c s}-1}\left|\sum_{m=0}^{N-1} w_{n+m} \mathrm{x}_{m}^{\prime *}\right|^{2}$ coincide exactamente con la variable $Y_{2}$ planteada en (4.50) con $N_{2}=N_{c S}$, y tiene un valor medio $N_{c S} N \sigma_{w}^{2}$. Por lo tanto, el MSE bajo ruido blanco gaussiano se puede expresar como:

$$
M S E_{\widetilde{\boldsymbol{h}}, B}=\frac{N_{c S}}{N \cdot S N R}
$$

Comparando el desempeño con sincronización perfecta respecto a la técnica de estimación de canal por LS, como esta última presenta un MSE dado por $1 / S N R$, se obtiene una reducción del MSE dada por $N / N_{c S}$, que en función de los parámetros del sistema bajo estudio es 8 . El desempeño que se obtiene en este caso es el mismo que si se aplica la técnica de estimación de canal por DFT, pero con la ventaja de obtener la estimación a partir de las muestras del filtro acoplado utilizado en la etapa de sincronización.

En la Fig. 5.17 se presentan los resultados obtenidos por simulaciones para el MSE de la estimación del canal para los canales A y B con ruido blanco gaussiano. Por un lado, para los canales A y B se verifica que la cota inferior de MSE, es decir, considerando una sincronización fina perfecta, es la obtenida en (5.48). Adicionalmente, se agregan las simulaciones correspondientes a la estimación de canal a partir del resultado de la etapa de sincronización fina para los umbrales $\alpha_{1}$ y $\alpha_{\text {ópt }}$; y para la sincronización previa obtenida a partir de la ubicación del máximo de la CCN. Puede observarse cómo el MSE obtenido es menor bajo el canal B que el A, en todos los casos. El error obtenido a partir de la sincronización fina con el umbral $\alpha_{1}$ es superior al obtenido considerando el umbral $\alpha_{\text {ópt }}$. Como es de esperarse, el peor resultado de la estimación de canal se obtiene sin aplicar el algoritmo de sincronización fina propuesto, es decir, considerando el instante de sincronismo a partir de la ubicación del máximo de la $\mathrm{CCN}$.

\subsubsection{Estimación de canal bajo ruido de fondo coloreado}

Para analizar el error cuadrático medio en el escenario en el que las muestras de ruido pertenecen al proceso de ruido coloreado, se aprovecha el análisis de las variables aleatorias analizadas en la sección 4.3.4. La variable $\sum_{n=n_{\text {ó } p t}}^{n_{\text {ó }}+N_{c s}-1}\left|\sum_{m=0}^{N-1} w_{n+m} \mathrm{x}_{m}^{\prime *}\right|^{2}$ 
Diseño de Estrategias de Sincronización y Estimación de Canal para la Mejora de Comunicaciones en Redes Inteligentes de Energía

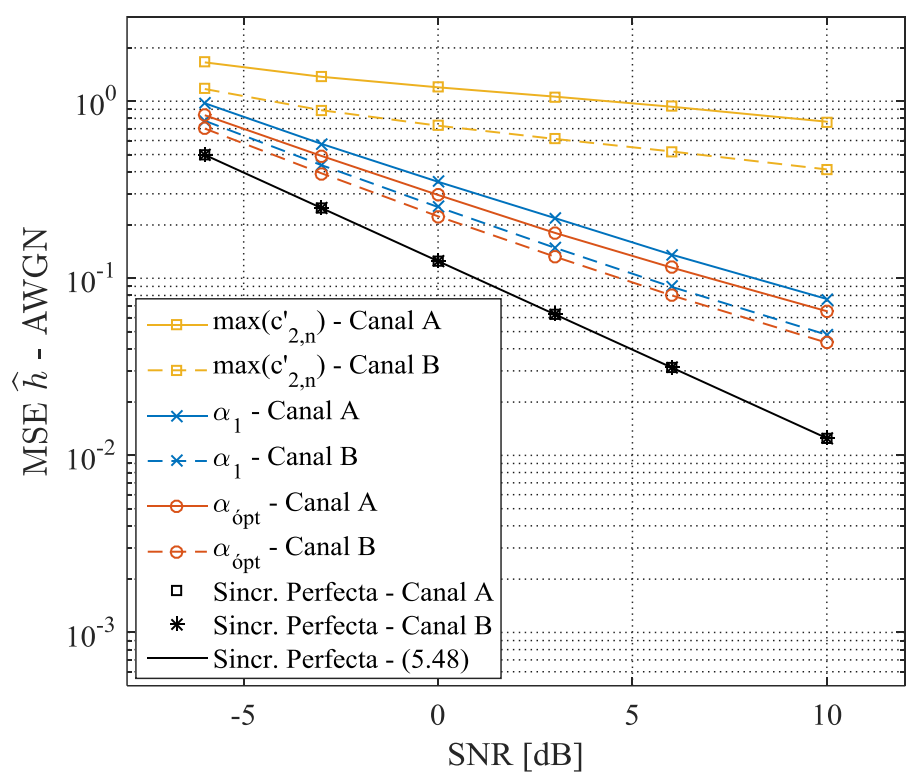

Fig. 5.17 MSE de la estimación del canal PLC bajo ruido blanco gaussiano.

coincide exactamente con la variable $Y_{2}$ planteada en (4.71) y (4.72), con $N_{2}=N_{c S}$, y tiene un valor medio $N_{c S} \sigma_{w}^{2} \sum_{k=-\infty}^{\infty} f_{1_{k}}^{2}$; donde $f_{1_{k}}$ es la secuencia de correlación cruzada entre el filtro que colorea el ruido $g_{k}$ y el símbolo piloto $x_{k}^{\prime}: f_{1_{k}}=$ $\sum_{j=-\infty}^{+\infty} g_{j} x_{k+j}^{\prime}=g_{k} * x_{-k}^{\prime}$. Por lo tanto, el MSE bajo ruido de fondo coloreado resulta:

$$
M S E_{\widetilde{\boldsymbol{h}}, C}=\frac{N_{c S} \sum_{k=-\infty}^{\infty} f_{1_{k}}^{2}}{N^{2} \cdot S N R}
$$

De manera similar que para el caso bajo ruido blanco gaussiano, en la Fig. 5.18 se presentan los resultados obtenidos por simulaciones para el MSE de la estimación del canal PLC bajo ruido de fondo coloreado. Por un lado, para los canales A y B se verifica que la nueva cota inferior de MSE es la obtenida en (5.49). Nuevamente, el MSE obtenido es menor bajo el canal B que el A, en todos los casos, y el error obtenido a partir de la sincronización fina con el umbral $\alpha_{1}$ es superior al obtenido considerando el umbral $\alpha_{\text {ópt }}$. Los resultados obtenidos resultan muy similares al escenario de ruido blanco gaussiano, debido a que en el caso particular de PSD evaluado en las simulaciones, la sumatoria de las muestras $\sum_{k=-\infty}^{\infty} f_{1}^{2}$ resulta muy cercana a $N$, siendo ligeramente inferior $\left(\sum_{k=-\infty}^{\infty} f_{1}^{2} / N \approx 0,97\right)$, por lo que el MSE bajo una sincronización perfecta resulta ligeramente menor.

Finalmente, resulta importante mencionar que el MSE calculado de acuerdo a (5.47) se ve directamente influenciado por el error de sincronismo, ya que en ese caso la 


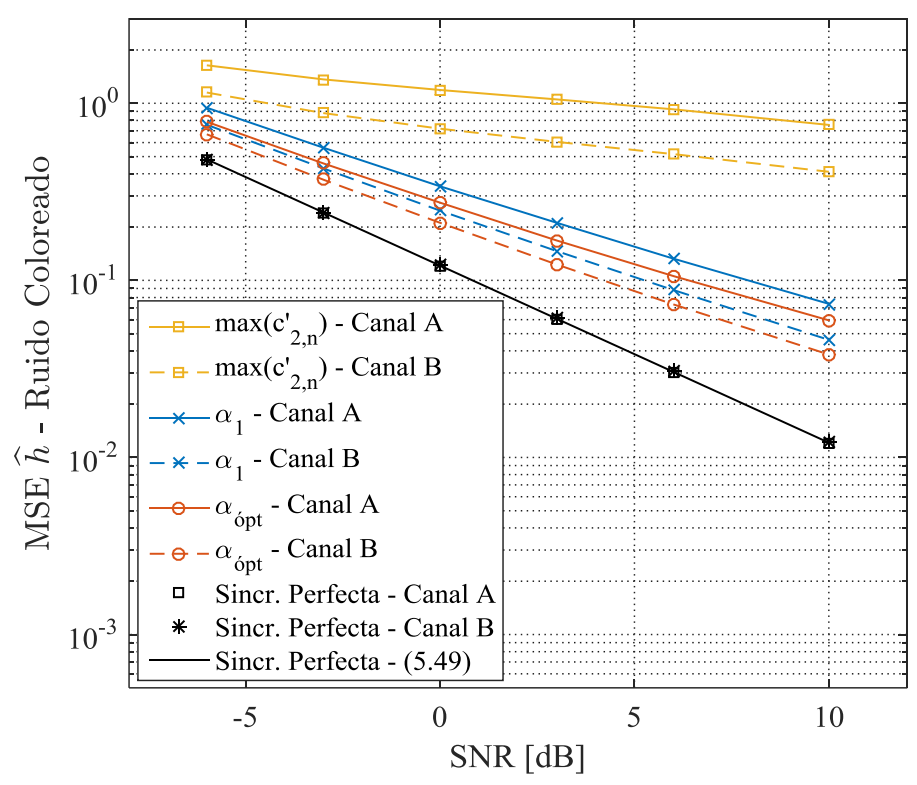

Fig. 5.18 MSE de la estimación del canal PLC bajo ruido de fondo coloreado.

respuesta al impulso estimada $\tilde{\mathbf{h}}$ se encuentra retardada o adelantada respecto a la respuesta al impulso del canal real $\mathbf{h}$. Entonces, el MSE $E\left\{\|\mathbf{h}-\tilde{\mathbf{h}}\|^{2}\right\}$ se incrementa debido a que no coincide el orden de las componentes de los vectores. El efecto de retardo o adelanto en la respuesta impulsiva del canal, no degrada significativamente el desempeño del sistema de comunicaciones, ya que el error en el sincronismo del símbolo piloto es el mismo que en los símbolos de datos. Esto sucede debido a que el mismo retardo o adelanto provocado por el error de sincronismo en la estimación del canal se presenta también en la ventana de FFT aplicada a cada símbolo de datos. Sin embargo, un error de sincronismo por atraso, que implica no haber detectado el primer (o los primeros) caminos del canal en la etapa de sincronismo fino, puede introducir ISI deteriorando el desempeño del sistema si las muestras que se incluyen superan el límite de zona de correlación ideal de $N_{C S}$ muestras. Así mismo, un error de sincronismo en adelanto lo suficientemente elevado, también puede producir interferencia si el error absoluto en muestras supera la longitud de la zona libre de interferencia $N_{p c}-L+2$. Como los umbrales de detección propuestos para la sincronización fina $\alpha_{1} \mathrm{y} \alpha_{\text {ópt }}$ son relativamente bajos, el nivel de ISI se verá acotado dado que los caminos del canal que no pueden ser detectados tendrán una baja amplitud, dependiendo además de la probabilidad de que sucesivos caminos del canal tengan una amplitud que resulte en una muestra de la CCN inferior al umbral de detección. 
Diseño de Estrategias de Sincronización y Estimación de Canal para la Mejora de Comunicaciones en Redes Inteligentes de Energía

\subsubsection{Efecto del ruido impulsivo en la estimación de canal}

Por último, resulta de interés evaluar el efecto del ruido impulsivo a la estimación de canal, al adicionar fuentes de ruido impulsivo al ruido de fondo coloreado. En la Fig. 5.19 se presentan los resultados obtenidos para las estimaciones de canal, luego de efectuar la sincronización fina a partir de los umbrales $\alpha_{1} \mathrm{y} \alpha_{\text {ópt }}$. Se incluyen los tres escenarios de ruido: ruido de fondo coloreado sin ruido impulsivo, ruido de fondo coloreado con eventos de ruido impulsivo con probabilidad de ocurrencia típica del $8 \%$; y ruido de fondo coloreado con eventos de ruido impulsivo mitigados a partir de la técnica de procesamiento no lineal de blanqueo. No se incluye la estimación de canal bajo un escenario de mitigación de ruido impulsivo a partir de AGC porque, para obtener una estimación adecuada, debe realizarse un procesamiento adicional de la señal, para compensar la ganancia variable aplicada a cada una de las muestras de la señal recibida. Puede entenderse que la señal recibida modificada por el AGC se ve afectada por un canal equivalente, que es variante en el tiempo por los cambios en la ganancia, y esa distorsión debe ser compensada.

Puede observarse en la Fig. 5.19 que el error se incrementa considerablemente al incluir el ruido impulsivo respecto del caso sin ruido impulsivo. Esto se debe principalmente a dos factores: por un lado, el error se incrementa debido a que el desempeño de la sincronización se deteriora de acuerdo a la Fig. 5.16. Además, un evento de ruido impulsivo incrementa el nivel de ruido presente en la señal, y distorsiona las muestras
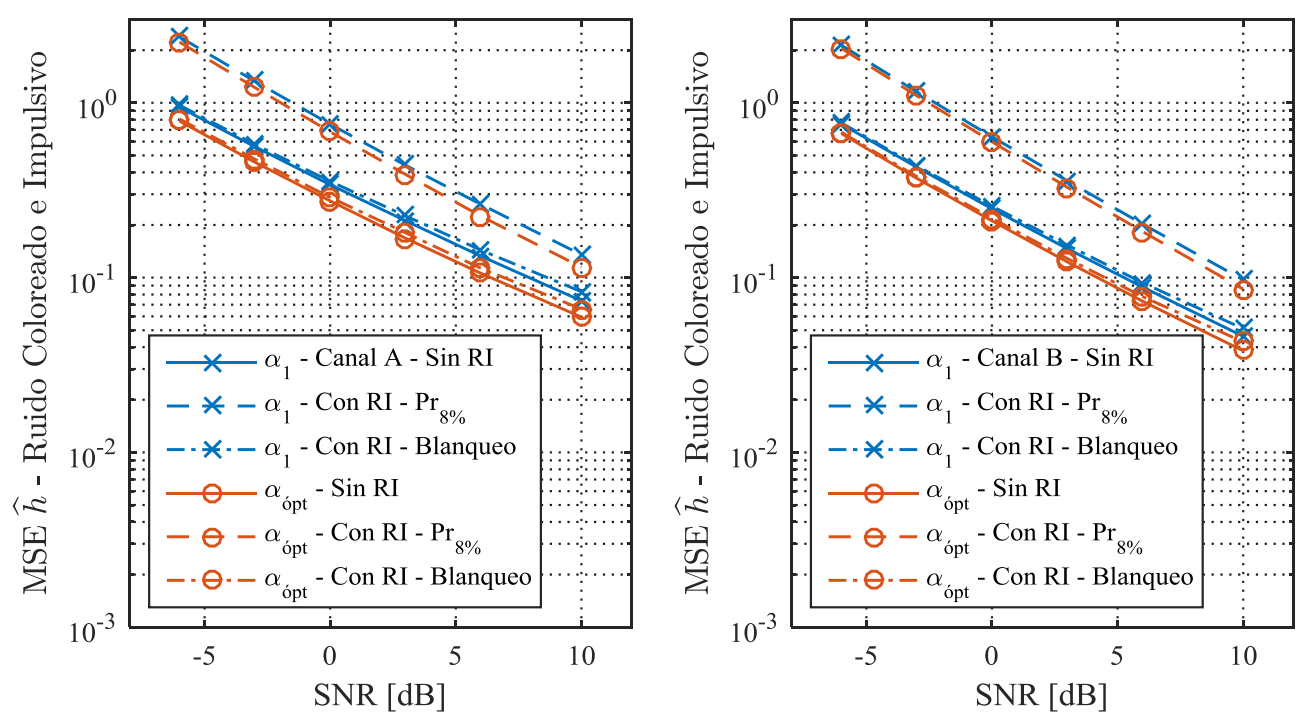

Fig. 5.19 MSE de la estimación del canal PLC tipo A (izquierda) y B (derecha), bajo ruido de fondo coloreado e impulsivo. 
del símbolo piloto, por lo que la correlación se ve degradada y en consiguiente la estimación de la respuesta al impulso.

\subsection{Complejidad de la propuesta de sincronización y estimación de canal}

A continuación, se evalúa de manera simplificada la complejidad de la propuesta de sincronización y estimación de canal, a partir de las operaciones que deben efectuarse en cada etapa. Por un lado, teniendo en cuenta el sistema DMT con tamaño de DFT igual a $N$, deben computarse el numerador y denominador de la métrica de sincronización gruesa $m_{3, n}$ :

$$
m_{3, n}=\frac{N_{2} \sum_{k=0}^{N_{2}-1}\left|c_{n+k}^{\mathbf{r}, \mathbf{x}^{\prime}}\right|^{2}}{N \sum_{k=0}^{N_{2}-1} \sum_{m=0}^{N-1}\left|r_{n+k+m}\right|^{2}}=\frac{P_{3, n}}{E_{3, n}}
$$

El numerador $P_{3, n}$ contiene muestras de la correlación de la señal recibida con el símbolo piloto elevadas al cuadrado, y se efectúa la suma de $N_{2}$ muestras. Esa suma puede computarse a partir de la fórmula recursiva:

$$
P_{3, n+1}=P_{3, n}+N_{2}\left|c_{n+N_{2}}^{\mathbf{r}, \mathbf{x}^{\prime}}\right|^{2}-N_{2}\left|c_{n}^{\mathbf{r}, \mathbf{x}^{\prime}}\right|^{2}
$$

Además, considerando la estructura del símbolo piloto de (4.6), y la arquitectura equivalente modular del correlador de pares de secuencias complementarias de [Popovic 1999], el numerador puede computarse a partir de la estructura de la Fig. 5.20.

El denominador resulta de la suma de muestras de la señal recibida elevadas al cuadrado, considerando las ventanas de muestras de longitud $N$ y $N_{2}$ sucesivas. Aplicando el mismo esquema de recursividad que para el numerador, el denominador puede computarse a partir de la estructura de la Fig. 5.21.

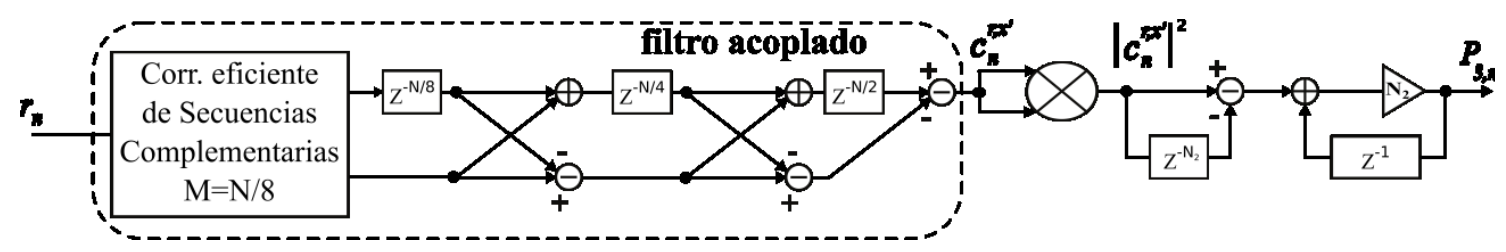

Fig. 5.20 Estructura equivalente para el cómputo del numerador de la métrica de sincronización gruesa.

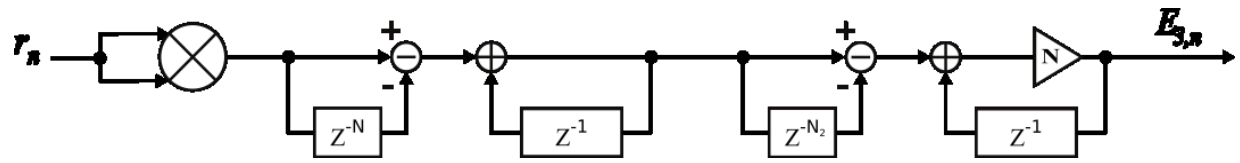

Fig. 5.21 Estructura equivalente para el cómputo del denominador de la métrica de sincronización gruesa. 
Diseño de Estrategias de Sincronización y Estimación de Canal para la Mejora de Comunicaciones en Redes Inteligentes de Energía

Para la detección inicial de símbolo piloto cuando la métrica $m_{3, n}$ supera el umbral $\gamma$, pueden utilizarse los cómputos del numerador y denominador a partir de un comparador, de acuerdo a:

$$
m_{3, n}>\gamma \rightarrow P_{3, n}>\gamma \cdot E_{3, n}
$$

Luego de la detección inicial, se realiza la búsqueda del máximo de la métrica de sincronización gruesa para obtener el instante de sincronización gruesa $\tilde{\theta}_{g}$, por lo tanto debe computarse el cociente $P_{3, n} / E_{3, n}$.

A partir del resultado de la sincronización gruesa, se activará la etapa de sincronización fina a partir de la CCN modificada $c^{\prime}{ }_{2, n}$ :

$$
c_{2, n}^{\prime}=\frac{c^{\prime}{ }_{1, n}}{1-m_{3, n}}=\frac{P_{2, n}^{\prime}}{E_{2, n}^{\prime}}
$$

Por un lado, $c^{\prime}{ }_{1, n}$ puede expresarse de acuerdo a:

$$
c_{1, n}^{\prime}=\frac{\left|c_{n}^{\mathbf{r}, \mathbf{x}^{\prime}}\right|^{2}}{N \sum_{m=0}^{N-1} r_{n+m} \cdot r_{n+m}}=\frac{P_{1, n}^{\prime}}{E_{1, n}^{\prime}}
$$

Tanto el numerador como el denominador de $c_{1, n}^{\prime}$ pueden obtenerse de resultados intermedios del cálculo del numerador y denominador de la métrica de sincronización gruesa $m_{3, n}$. Luego, $c_{2, n}^{\prime}$ resulta:

$$
c^{\prime}{ }_{2, n}=\frac{c^{\prime}{ }_{1, n}}{1-m_{3, n}}=\frac{P^{\prime}{ }_{1, n}}{E_{1, n}^{\prime}} \cdot \frac{E_{3, n}}{E_{3, n}-P_{3, n}}
$$

Luego de obtener la ubicación del máximo, se ejecuta el algoritmo de sincronización fina, identificando como coeficientes del canal a las muestras de $c^{\prime}{ }_{2, n}$ que superan el umbral de detección $\alpha$. Esta prueba puede realizarse a partir de los cómputos previos, a partir de la comparación:

$$
c^{\prime}{ }_{2, n}>\alpha \rightarrow P_{1, n}^{\prime} \cdot E_{3, n}>\alpha \cdot E_{1, n}^{\prime} \cdot\left(E_{3, n}-P_{3, n}\right)
$$

Finalmente, para la estimación de canal se almacenan las muestras correspondientes a la salida del filtro acoplado multiplicadas por $1 / \sqrt{P_{T}} N$, de acuerdo a (5.45). A partir del resultado de sincronización fina, se seleccionan las $N_{c p}$ muestras que se consideran como muestras de la respuesta al impulso del canal $\tilde{h}_{n}$. Luego, se efectúa la DFT correspondiente para obtener la estimación de la respuesta en frecuencia del canal. 
El diagrama en bloques que resume el procesamiento digital de señal requerido por la propuesta de sincronización y estimación de canal conjunta se presenta en la Fig. 5.22. Por un lado, la correlación cruzada, que demandaría una gran cantidad de recursos si se utilizaran otros tipos de secuencias en la construcción del símbolo piloto, precisa únicamente $2 \log _{2}(N)-1$ bloques sumadores/restadores, y elementos de memoria. Adicionalmente, los términos que normalizan las métricas pueden computarse a partir de fórmulas recursivas que requieren un número reducido de bloques sumadores/restadores y elementos de memoria. Se requieren, además, bloques multiplicadores por constantes con el valor de los umbrales $\gamma$ y $\alpha$, y sendos comparadores; deben implementarse multiplicadores de dos entradas para calcular el cuadrado de las muestras requeridas, y calcularse el cociente interviniente en las métricas. A pesar de no haber sido detallado en la Fig. 5.22 ni en el análisis de la complejidad, las búsquedas de valores máximos y la ejecución del algoritmo de sincronización fina pueden implementarse a partir de bloques de lógica combinacional y secuencial. Además, es necesario incluir el bloque de procesamiento no lineal para mitigar el ruido impulsivo. Un diagrama en bloques de las arquitecturas equivalentes correspondientes al procesamiento basado en blanqueo y en el control automático de ganancia se presenta en la Fig. 5.23. Por un lado, en la parte superior de la figura, se presenta el diagrama de la estrategia que utiliza el AGC, que precisa obtener el valor absoluto de las muestras de la señal recibida y efectuar una sumatoria de una ventana de $N_{A G C}$ muestras, que nuevamente puede obtenerse a partir de fórmulas recursivas. Luego, se multiplica el resultado por $1 / N_{A G C}$, obteniendo el valor inverso de la ganancia $1 / K_{A G C, n}$ que divide a la correspondiente muestra de la señal recibida a partir de un divisor de dos entradas. En la parte inferior de la Fig. 5.23, se presenta el diagrama en bloques correspondiente a la técnica de blanqueo. A partir de comparar el valor absoluto de las muestras de la señal recibida con el umbral de blanqueo $\beta$, a partir de un multiplexador se selecciona la muestra correspondiente de la señal sin alteraciones, o bien un cero si se efectúa el blanqueo. Evidentemente, el umbral de blanqueo debe definirse cuidadosamente y puede ser de un valor fijo o actualizarse dinámicamente. El estudio detallado de la selección del umbral de blanqueo excede el alcance de esta Tesis. 
Diseño de Estrategias de Sincronización y Estimación de Canal para la Mejora de Comunicaciones en Redes Inteligentes de Energía

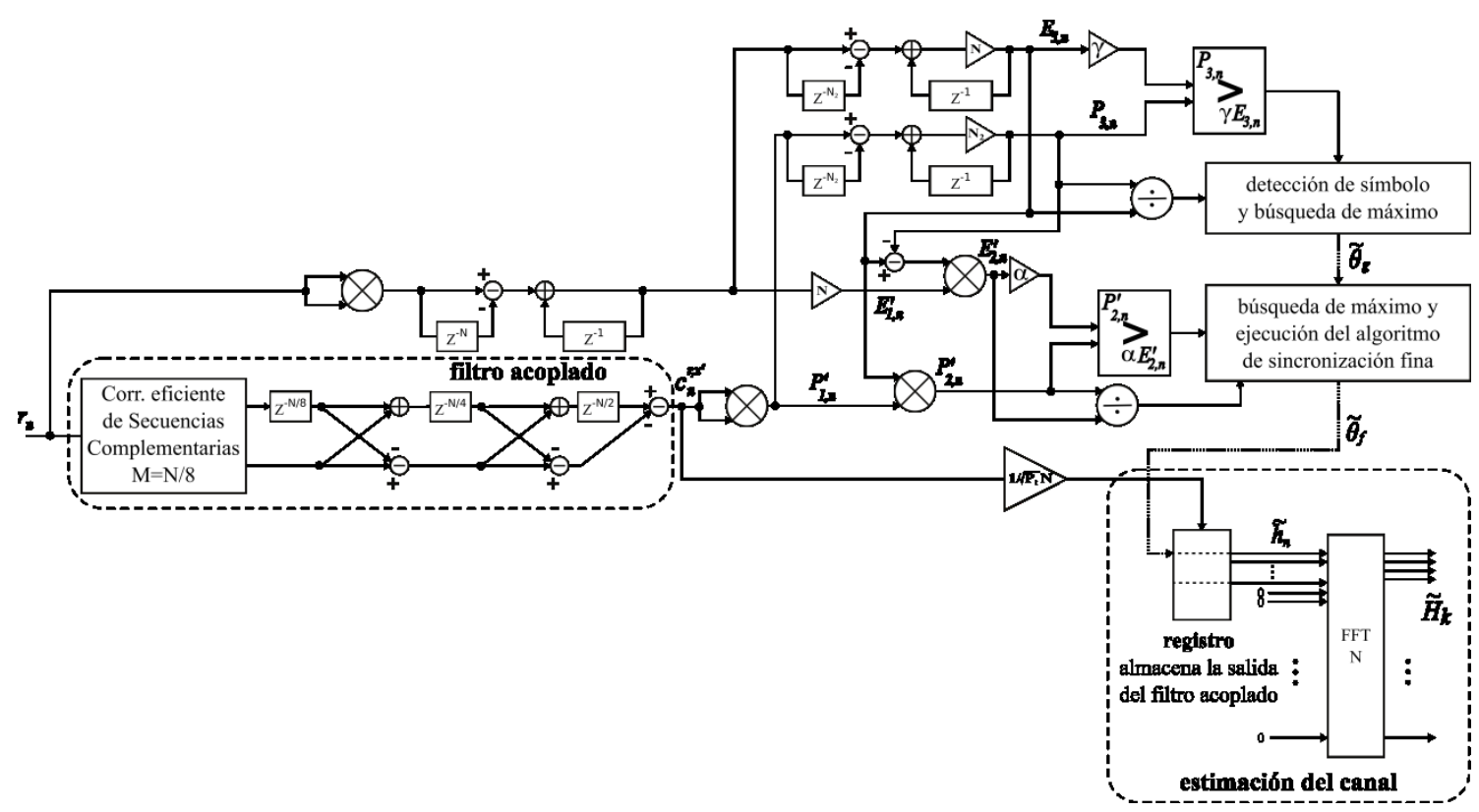

Fig. 5.22 Diagrama en bloques del procesamiento de señal requerido para la propuesta de sincronización y estimación de canal conjunta.
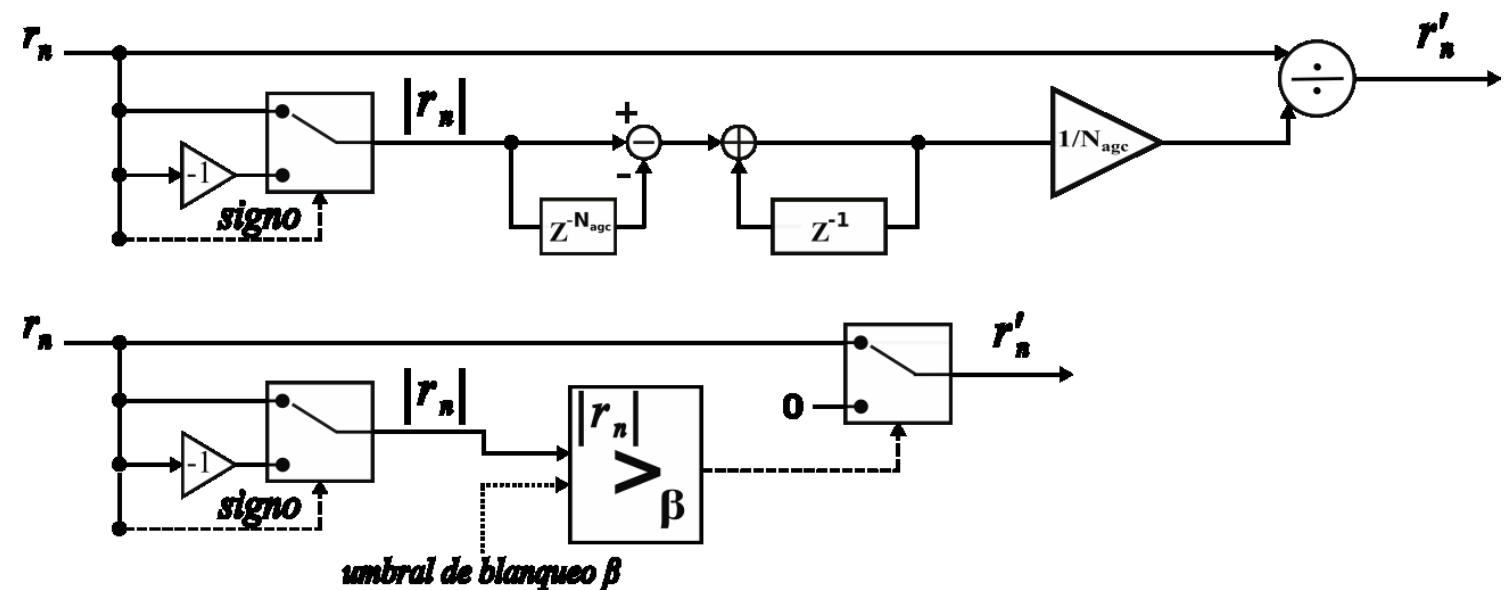

Fig. 5.23 Diagrama en bloques del procesamiento no lineal para la mitigación del ruido impulsivo.

\subsection{Conclusiones}

En este capítulo, como continuidad del capítulo 4 que trató la etapa de sincronización gruesa, a partir del símbolo piloto propuesto, se desarrolló la etapa de sincronización fina y estimación de canal conjunta para el sistema PLC-DMT.

A partir de un análisis del modelo estadístico del canal PLC, se verificó que la PDF del primer camino del canal se puede aproximar a una distribución uniforme, cuyos valores mínimo y máximo se obtienen a partir de los parámetros de transmisión del canal. Esto permitió evaluar la PDF de la CCN propuesta para ejecutar el algoritmo de sincronización fina en el instante de sincronismo óptimo, tanto para una primera aproximación considerando AWGN, como incluyendo el modelo de ruido de fondo 
coloreado. Adicionalmente, se caracterizó de manera aproximada la PDF de la CCN en las muestras anteriores al de sincronismo óptimo, para poder evaluar analíticamente la probabilidad de sincronización perfecta del algoritmo.

Mediante simulaciones se verificó que la variación en la ganancia del canal, que sigue una distribución Log-Normal, tiene una influencia importante en la PDF de la CCN. En base a eso, se definió una CCN modificada para reducir la influencia de la ganancia del canal en la PDF. Para las dos variantes de la CCN, a partir de las PDFs se derivaron un umbral con una baja probabilidad de falsa detección, y un umbral que maximiza la probabilidad de sincronización perfecta. A partir de simulaciones se contrastó el resultado obtenido en cada caso con el esperado a partir del análisis realizado y se evaluó el desempeño del algoritmo de sincronización fina, obteniendo un valor de RMSE muy inferior respecto al que se obtiene en la sincronización gruesa, aún bajo la presencia de ruido impulsivo.

Luego, a partir de las muestras de la salida del filtro acoplado utilizado para la sincronización fina, se planteó la misma estrategia de estimación de la CIR que en el capítulo 3. Particularmente el análisis de la cota superior de error bajo un escenario de sincronismo perfecto, tanto para el escenario con AWGN como con ruido de fondo coloreado, se ajustó adecuadamente al resultado de las simulaciones. Además, se evaluó el desempeño al incluir los resultados de la etapa de sincronismo fino.

Finalmente, a partir del análisis de complejidad asociada al cómputo de los algoritmos para la sincronización y estimación de canal conjunta, se verifica que la propuesta representa una opción de reducida complejidad. Esto se debe principalmente a que las tres etapas (sincronización gruesa, sincronización fina y estimación de canal) utilizan fundamentalmente las muestras correspondientes a la correlación cruzada, y ésta requiere únicamente algunos bloques sumadores y elementos de memoria. Esto es posible debido al diseño del símbolo piloto propuesto y su arquitectura de correlación eficiente. 
Diseño de Estrategias de Sincronización y Estimación de Canal para la Mejora de Comunicaciones en Redes Inteligentes de Energía

\section{SINCRONIZACIÓN Y ESTIMACIÓN DE CANAL EN SISTEMAS DMT-PLC BAJO UNA MÁSCARA ESPECTRAL DE TRANSMISIÓN}

En los capítulos 3, 4 y 5, se desarrollaron estrategias de sincronización y estimación de canal para sistemas PLC, considerando esquemas multiportadora OFDM y DMT en los que se dispone de todas las subportadoras para la generación, transmisión y procesamiento del símbolo piloto. Ese enfoque facilitó el análisis del desempeño de las técnicas desarrolladas en las condiciones impuestas por los modelos de canal PLC, resultando de gran utilidad para analizar cotas superiores de funcionamiento. Adicionalmente, permitió la comparación de las técnicas propuestas con otras existentes en la literatura con similares consideraciones, en las que se asume la posibilidad de transmitir de manera completa las secuencias utilizadas como símbolos piloto.

En el presente capítulo se introduce un escenario más realista de transmisión del sistema DMT-PLC en el que, tanto por necesidades de los sistemas prácticos como por cuestiones de compatibilidad electromagnética, no es posible utilizar todas las 
subportadoras teóricamente disponibles para la transmisión de información. En ese contexto, los aportes del presente capítulo se pueden resumir a los siguientes:

- Se evalúa el impacto de la inactivación de subportadoras, al aplicar una máscara espectral de transmisión, en las propiedades de correlación del símbolo propuesto y su impacto en la estrategia de sincronización gruesa, fina $\mathrm{y}$ estimación de canal.

- Para la etapa de sincronización gruesa, se realiza el análisis de las PDFs de las métricas presentadas en el capítulo 4, teniendo en cuenta la máscara espectral de transmisión y un filtro notch incluido en el receptor. De esta manera, resulta posible redefinir de forma analítica umbrales de detección primaria del símbolo piloto bajo el nuevo escenario. Adicionalmente, se verifica por simulaciones la capacidad de detección del símbolo piloto y se compara con los resultados obtenidos en el capítulo 4.

- Se evalúa por simulaciones el desempeño de los umbrales derivados en el capítulo 5 para la ejecución del algoritmo de sincronización fina en el nuevo escenario, y posteriormente se presenta un nuevo umbral práctico que mejora sustancialmente el desempeño. A su vez, se compara con el resultado de la sincronización fina a partir de la utilización de umbrales óptimos que se obtienen de manera empírica.

- Con respecto a la estimación de canal, se evalúa el (severo) impacto de la introducción de la máscara espectral de transmisión en el desempeño de la propuesta respecto a los resultados obtenidos en el capítulo 5. A partir de ello, se plantean variantes de estimación de canal bajo el escenario de subportadoras inactivas disponibles en la literatura, y se presenta una alternativa novedosa bajo la premisa de mantener una complejidad asociada lo más reducida posible, pero con un desempeño competitivo. En ese sentido, se propone la modificación de las muestras de la correlación cruzada de manera tal que el resultado se traduce en completar las subportadoras inactivas a partir de interpolación (y extrapolación) de la correlación cruzada en el dominio de la frecuencia.

- Finalmente, a partir del análisis de complejidad realizado en el capítulo 5, se hace lo propio incluyendo todas las modificaciones incluidas a lo largo del presente capítulo. 
Diseño de Estrategias de Sincronización y Estimación de Canal para la Mejora de Comunicaciones en Redes Inteligentes de Energía

Tanto para las etapas de sincronización gruesa y fina como la de estimación de canal, se realizan diversas simulaciones considerando distintos escenarios con ruido de fondo coloreado, incluyendo eventos de ruido impulsivo y técnicas de mitigación del mismo.

\subsection{Definición de la máscara espectral de transmisión y su efecto en las propiedades del símbolo piloto propuesto}

La porción del espectro disponible para los sistemas PLC de banda ancha depende de las regulaciones de los distintos continentes o países en los que se desarrollan, definidas por comités locales o regionales tales como, CENELEC (Comité Européen de Normalisation Électrotechnique) en Europa y FCC (Federal Communications Commission) en Estados Unidos [Girotto 2017]. Una de las principales limitaciones en la asignación del espectro a los sistemas PLC resulta de asegurar la compatibilidad electromagnética (Electromagnetic Compatiblity - EMC), y esto se debe a que las instalaciones de distribución eléctrica no fueron diseñadas para la transmisión de señales de alta frecuencia, por lo que la red se convierte en un potencial radiador no intencional que puede interferir con otros sistemas de comunicaciones. En ese sentido, otra razón por la que los estándares para sistemas PLC adoptaron modulaciones multiportadora es para asegurar un uso flexible del espectro y cumplir con las normas de EMC. En particular, el estándar IEEE P1901 adopta la utilización de una máscara espectral en la transmisión para la capa física, limitando la cantidad de subportadoras que se pueden utilizar en el sistema. Para cubrir la banda de 1,8 - 28 MHz con una frecuencia de muestreo de $100 \mathrm{MHz}$, utiliza un tamaño de FFT $N=4096$ que permite un máximo de 2048 subportadoras, de las cuales se permiten utilizar sólo 917 [IEEE 2010]. La máscara anula las subportadoras necesarias para no interferir con radio AM en bajas frecuencias, con radio amateur en frecuencias intermedias, y además presenta un marcado sobremuestreo al anular las subportadoras correspondientes al intervalo 28$50 \mathrm{MHz}$, como se puede observar en la Fig. 6.1. El detalle de las nueve bandas de subportadoras activas se presenta en la Tabla 6.1.

La aplicación de la máscara espectral en el transmisor se esquematiza en la Fig. 6.2. Por cada símbolo, se modulan sólo las $N_{a}=917$ subportadoras activas mientras el resto se anula. Debido a que el símbolo piloto, al igual que los símbolos de datos, debe ajustarse 
Capítulo 6: Sincronización y estimación de canal en sistemas DMT-PLC bajo una máscara espectral de transmisión

a la máscara de transmisión, resulta necesario verificar el impacto de la aplicación de la máscara en la propuesta de sincronización y estimación de canal.

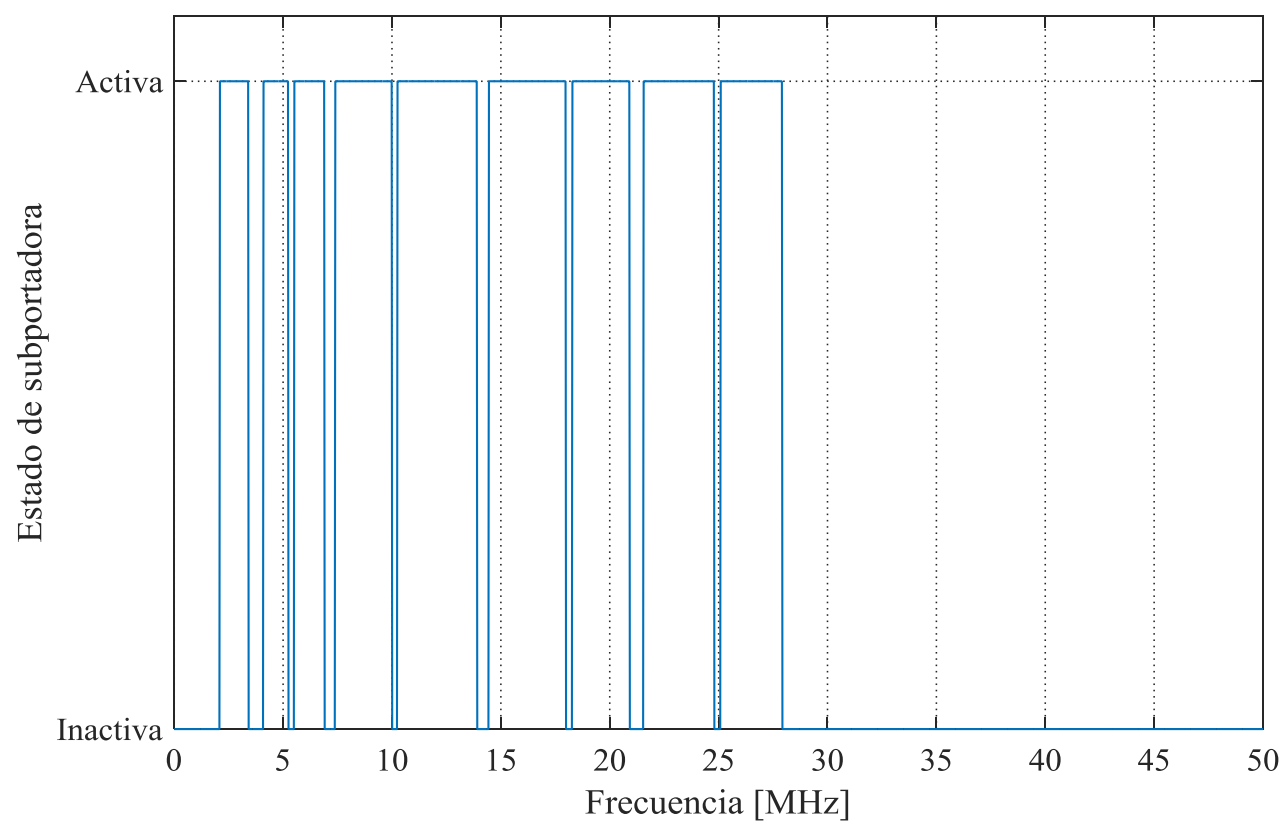

Fig. 6.1 Máscara espectral de compatibilidad electromagnética para transmisiones PLC en el estándar IEEE P1901 [IEEE 2010].

Tabla 6.1 Detalle de portadoras activas al aplicar la máscara espectral de transmisión.

\begin{tabular}{|c|c|}
\hline \hline Frecuencia [MHz] & Subportadoras activas \\
\hline $2,1<f<3,4$ & $86-139$ \\
\hline $4,1<f<5,2$ & $168-214$ \\
\hline $5,5<f<6,9$ & $226-282$ \\
\hline $7,4<f<10,0$ & $303-409$ \\
\hline $10,3<f<13,9$ & $420-569$ \\
\hline $14,5<f<19,0$ & $592-736$ \\
\hline $18,3<f<20,9$ & $749-856$ \\
\hline $21,6<f<24,8$ & $883-1015$ \\
\hline $25,1<f<27,9$ & $1028-1143$ \\
\hline \hline
\end{tabular}




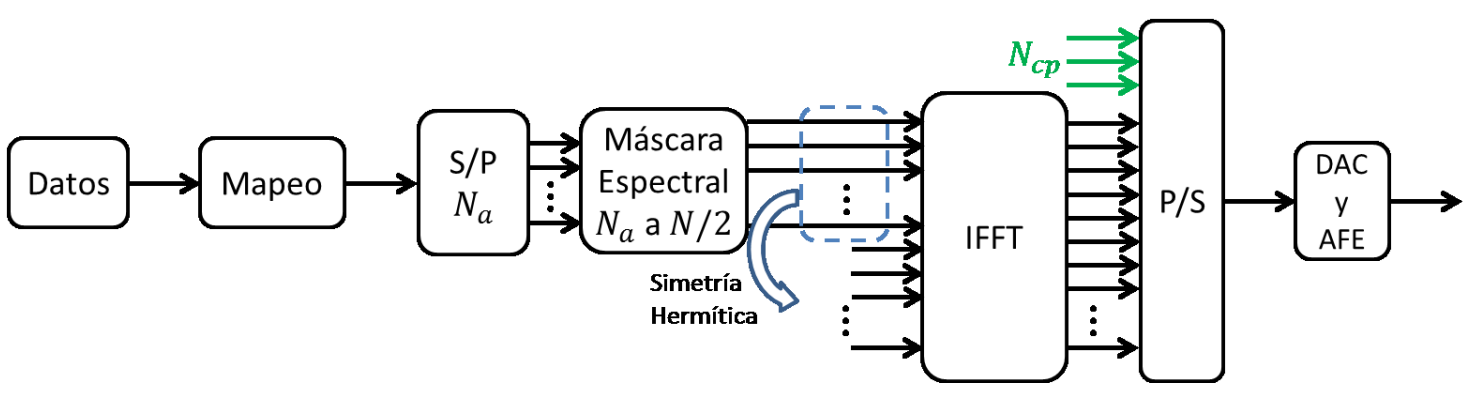

Fig. 6.2 Transmisor del sistema DMT modificado incluyendo la máscara espectral de emisión.

Considerando el símbolo piloto propuesto basado en los CPS y su correlación eficiente, para la sincronización y estimación de canal de baja complejidad, deben anularse en el transmisor las subportadoras que correspondan de acuerdo a la máscara espectral. Es evidente que el desempeño de la propuesta se verá afectado, tanto en la etapa de sincronismo como en la estimación del canal, debido a las distorsiones introducidas al anular subportadoras. Recordando que para un sistema DMT de $N / 2$ subportadoras se propuso la construcción del símbolo piloto $\mathbf{x}^{\prime}$ a partir del uso de un par de secuencias $\mathbf{a}$ y b de longitud $N / 4$; de acuerdo a:

$$
\mathbf{x}^{\prime}=\left[\begin{array}{llll}
\mathbf{a} & \mathbf{b} & -\mathbf{a} & \mathbf{b}
\end{array}\right]
$$

Entonces, el símbolo piloto puede generarse en el dominio de la frecuencia, a partir de $\mathbf{X}^{\prime}=\operatorname{DFT}\left\{\mathbf{x}^{\prime}\right\}$. Sin embargo, al aplicar la máscara de transmisión, se produce el recorte espectral del símbolo piloto obteniendo el símbolo piloto $\mathbf{X}_{2}^{\prime}$ en el dominio de la frecuencia. Así, puede obtenerse el símbolo piloto modificado que respeta la máscara de trnamisión como $\mathbf{x}_{2}^{\prime}=I \mathrm{DFT}\left\{\mathbf{X}_{2}^{\prime}\right\}$. Luego, se insertan el prefijo y sufijo cíclicos cp y cs de $N_{c p}$ y $N_{c s}$ muestras respectivamente, obteniendo el símbolo extendido $\mathbf{x}_{2}$ que efectivamente se transmite. En la Fig. 6.3 se presenta en la parte izquierda la correlación cruzada $c_{n}^{\mathbf{x}_{2}, \mathbf{x}^{\prime}}$ entre el símbolo extendido modificado por la máscara espectral $\mathbf{x}_{2}, \mathrm{y}$ el filtro acoplado al símbolo ideal $\mathbf{x}^{\prime}$. Adicionalmente, se agrega la correlación del símbolo sin aplicar la máscara de transmisión $c_{n}^{\mathbf{x}^{\prime}, \mathbf{x}}$. En ambos casos se considera $N=$ 4096, $N_{c p}=N_{c s}=512$. En la parte derecha se presenta simplemente un zoom de $c_{n}^{\mathbf{x}_{2}, \mathbf{x}^{\prime}}$ para una mejor visualización de la zona de interés. Se observa claramente que se degrada la zona de correlación ideal del símbolo piloto, y el valor máximo en $n=0$ se reduce significativamente. Esto se produce ya que de las $N / 2$ subportadoras disponibles se activan sólo $N_{a}=917$; aplicando el Teorema de Parseval se obtiene un máximo en la correlación cruzada de $2 N_{a}=1834$. 
Capítulo 6: Sincronización y estimación de canal en sistemas DMT-PLC bajo una máscara espectral de transmisión
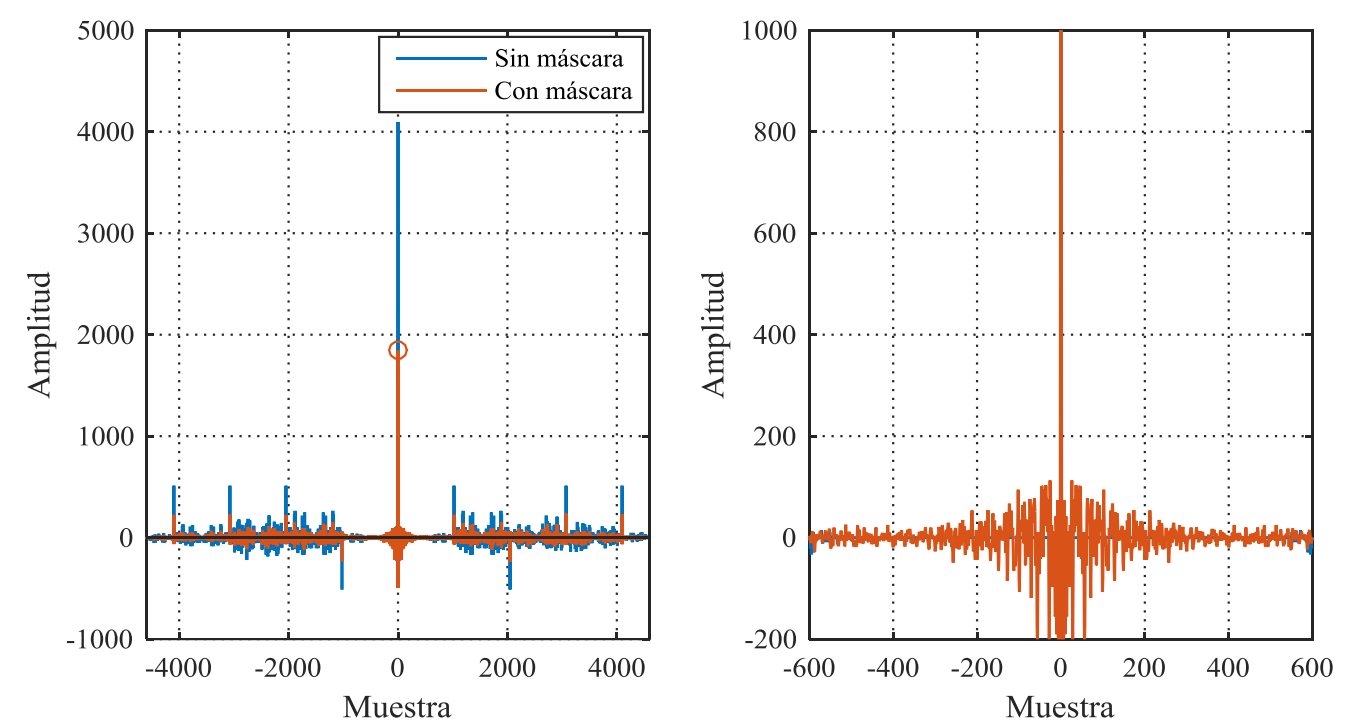

Fig. 6.3 Función de correlación cruzada entre el símbolo piloto extendido con y sin máscara aplicada y el símbolo ideal mediante el filtro acoplado.

\subsection{Sincronización gruesa}

En el capítulo 4 se definieron y compararon tres métricas de sincronización gruesa, dos basadas en las denominadas técnicas de autocorrelación $m_{1, n} \mathrm{y} m_{2, n}$, y otra calculada a partir de la correlación cruzada $m_{3, n}$. Si no se aplica una máscara espectral de emisión, entonces la métrica basada en $\mathrm{CC} m_{3, n}$ es más conveniente que las que utilizan $\mathrm{AC}$, en cuanto a la probabilidad de detectar correctamente el símbolo piloto y al error en la sincronización gruesa, obtenida a partir del máximo de las métricas. El lector puede referirse, por ejemplo, a la Fig. 4.34 y Fig. 4.35. Sin embargo, debido a que las operaciones involucradas en las métricas son muy diferentes, debe reevaluarse la capacidad de detección y sincronización gruesa bajo la máscara espectral de transmisión.

El efecto de la máscara espectral en la métrica de sincronización gruesa $m_{2, n}$ considerando el canal PLC multicamino tipo A y ruido de fondo coloreado se presenta en la Fig. 6.4, incluyendo las PDFs empíricas en el instante de sincronismo óptimo y anteriores, a partir de la transmisión del símbolo piloto con y sin aplicar la máscara. Por un lado, puede observarse que los valores de la métrica en el instante de sincronismo óptimo $m_{2, n_{\text {ópt }}}$ resultan menores en el caso de transmisión con máscara. Esto sucede debido a que el recorte espectral del símbolo piloto disminuye la ganancia de $\mathrm{AC}$ en el instante de sincronismo óptimo según el factor $2 N_{a} / N$, y distorsiona la simetría original 
Diseño de Estrategias de Sincronización y Estimación de Canal para la Mejora de Comunicaciones en Redes Inteligentes de Energía

entre los distintos segmentos de la señal recibida. En las muestras previas a la llegada del símbolo piloto $m_{2, n_{a n t}}$, como el cómputo depende únicamente de las muestras de ruido y el receptor mantiene su estructura, entonces el resultado es idéntico aplicando o no la máscara espectral de transmisión. Cabe aclarar que los parámetros para generar el símbolo piloto y efectuar las simulaciones a lo largo de este capítulo son $N=4096$, $N_{c p}=1024$ y $N_{c s}=512$, salvo que se indique lo contrario.

Por otro lado, la métrica basada en $\mathrm{CC}$ se ve afectada por el recorte espectral del símbolo piloto ya que la zona de correlación ideal se deteriora, el máximo de la CC entre el símbolo piloto y el filtro acoplado disminuye de $N$ a $2 N_{a}$, y además la zona de correlación nula presenta ahora lóbulos laterales de acuerdo a la Fig. 6.3. La disminución de la métrica en el instante de sincronismo óptimo $m_{3, n_{\mathrm{o} p t}}$ depende de los valores que adopta la $\mathrm{CC}$, pero además de la amplitud y correlación existente entre los distintos coeficientes del canal. Al igual que en el caso anterior, para la métrica en las muestras previas a la llegada del símbolo piloto $m_{3, n_{\text {ant }}}$ el resultado es el mismo
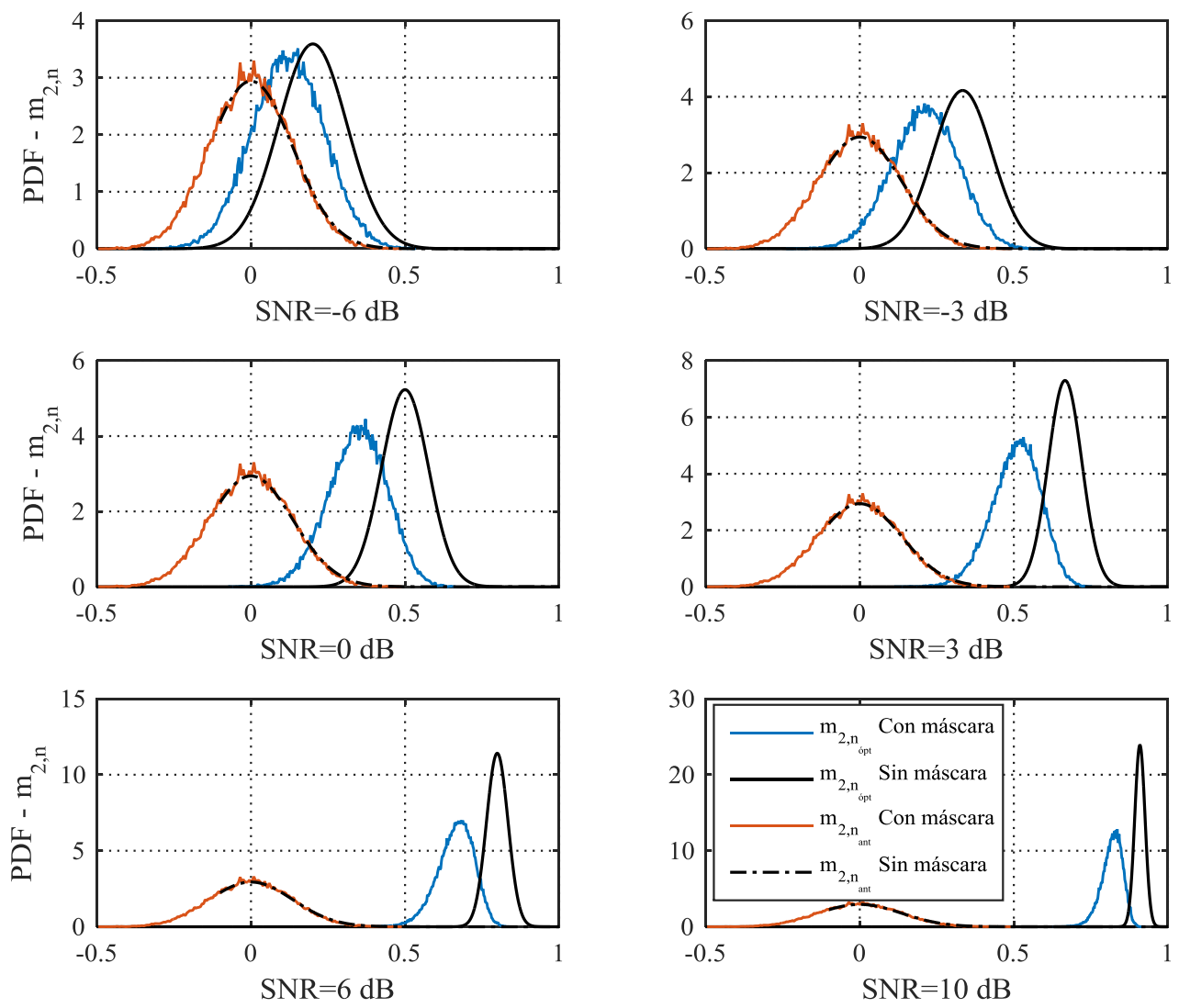

Fig. 6.4 PDFs del valor de la métrica de sincronización gruesa basada en AC, en el instante de sincronización óptimo y anteriores, aplicando la máscara espectral de transmisión 
Capítulo 6: Sincronización y estimación de canal en sistemas DMT-PLC bajo una máscara espectral de transmisión

aplicando o no la máscara de transmisión. Los resultados de las PDFs correspondientes se encuentran en la Fig. 6.5.

Como los valores de las métricas en las muestras previas a la llegada del símbolo son los mismos aplicando la máscara de transmisión o no, entonces los valores de los umbrales $\gamma$ obtenidos en el capítulo 4 no se ven modificados. Así, considerando una probabilidad de falsa detección de $10^{-7}$, el umbral para la métrica $m_{2, n}$ es $\gamma \approx 0,705 \mathrm{y}$ para $m_{3, n}$ es $\gamma \approx 0,471$. Como los valores de las métricas en el instante de sincronismo óptimo se ven reducidos al aplicar la máscara, entonces la probabilidad de detectar correctamente los símbolos pilotos transmitidos se ve disminuida. Esto se verifica en la Fig. 6.6 donde se presenta la probabilidad de detectar el símbolo piloto para ambas métricas, con y sin la aplicación de la máscara espectral. Puede observarse también que la métrica basada en $\mathrm{CC} m_{3, n}$ continúa siendo ventajosa respecto de la métrica basada en $\mathrm{AC} m_{2, n}$.
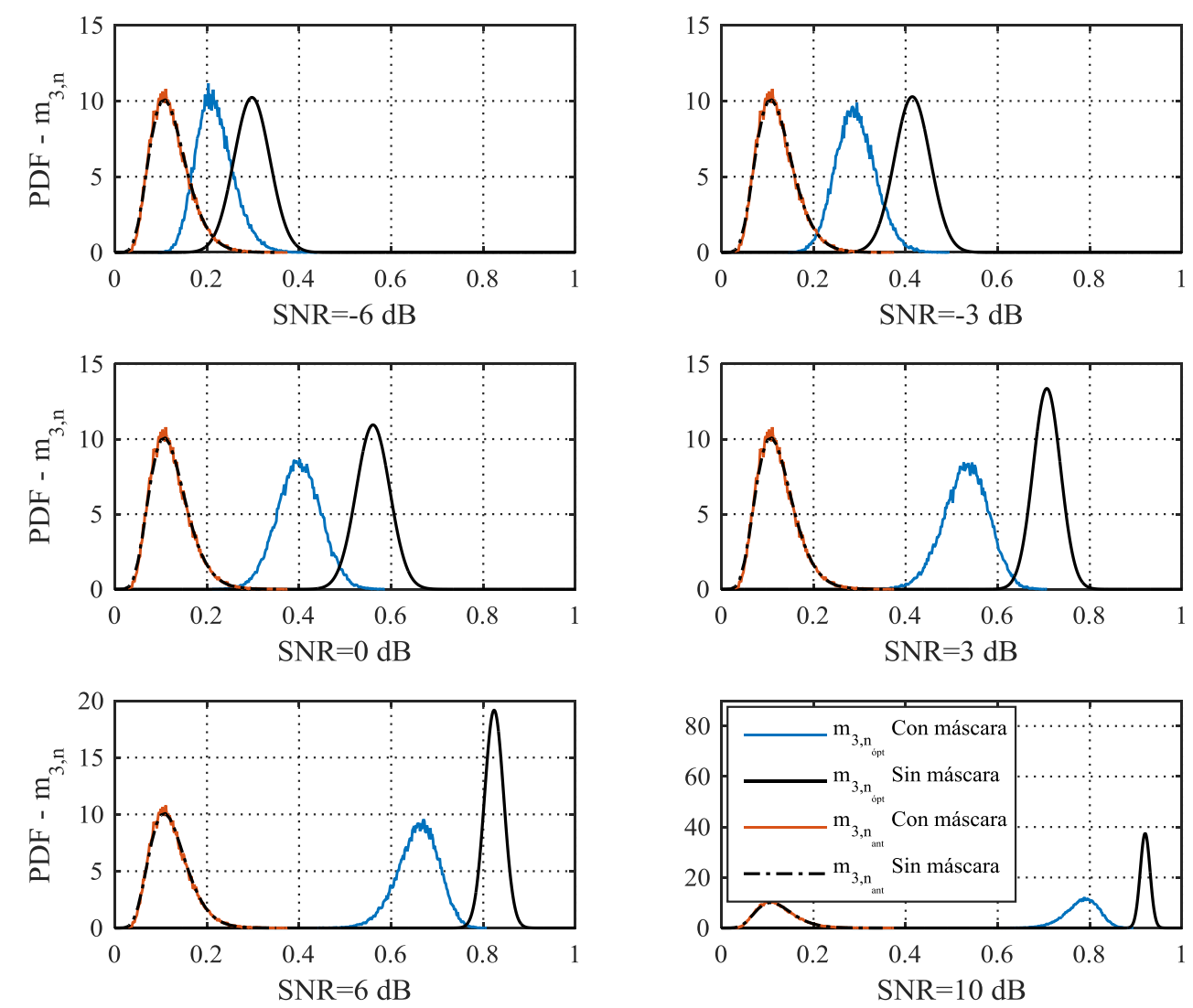

Fig. 6.5 PDFs del valor de la métrica de sincronización gruesa basada en CC, en el instante de sincronización óptimo y anteriores, aplicando la máscara espectral de transmisión. 
Diseño de Estrategias de Sincronización y Estimación de Canal para la Mejora de Comunicaciones en Redes Inteligentes de Energía

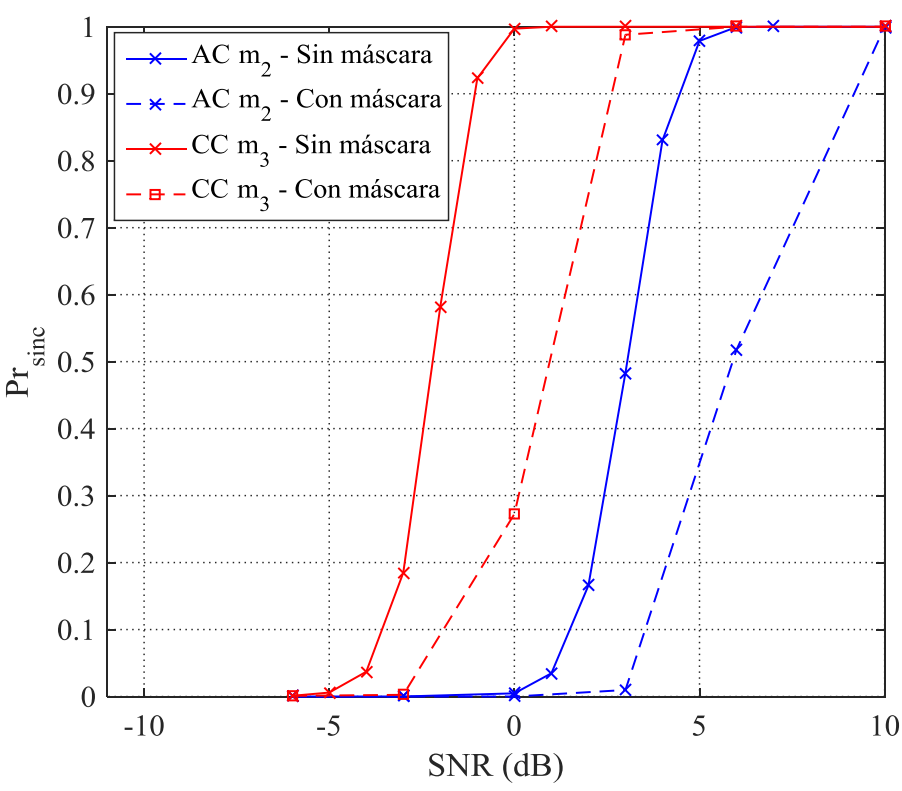

Fig. 6.6 Probabilidad de sincronización en términos de la detección del símbolo piloto para las métricas basadas en AC y CC bajo ruido de fondo coloreado y máscara espectral de transmisión.

\subsubsection{Inclusión de filtro notch en recepción}

El efecto de la máscara de transmisión en la sincronización gruesa implica un deterioro en la capacidad de detectar el símbolo piloto si se aplica la máscara en el transmisor y no se modifica la estructura del receptor, respecto al escenario sin máscara en el que se pueden utilizar todas las subportadoras para generar y transmitir el símbolo piloto. Sin embargo, dado que una gran parte del ancho de banda de procesamiento digital (0$50 \mathrm{MHz})$ no se utiliza efectivamente para la transmisión de información, puede incluirse un filtro que atenúe el ruido o interferencias que se produzcan en las bandas en que las subportadoras se encuentran inactivas. Adicionalmente, como el ruido de fondo coloreado presenta una PSD decreciente con la frecuencia, puede reducirse una gran parte del ruido presente en la señal recibida si se suprime la banda de baja frecuencia de $0-2 \mathrm{MHz}$ aproximadamente. En la Fig. 6.7 se grafican conjuntamente la máscara espectral de transmisión y la PSD normalizada del ruido de fondo coloreado.

Por lo mencionado, resulta importante incluir en el receptor uno o varios filtros que supriman el ruido presente en las bandas inactivas donde no se transmite señal útil. A los filtros que suprimen determinadas bandas se los denomina también filtros notch. En una implementación práctica, resultaría conveniente implementar un filtro analógico de manera tal que el nivel de ruido se reduzca previo a la etapa de conversión $\mathrm{A} / \mathrm{D}$ de la 


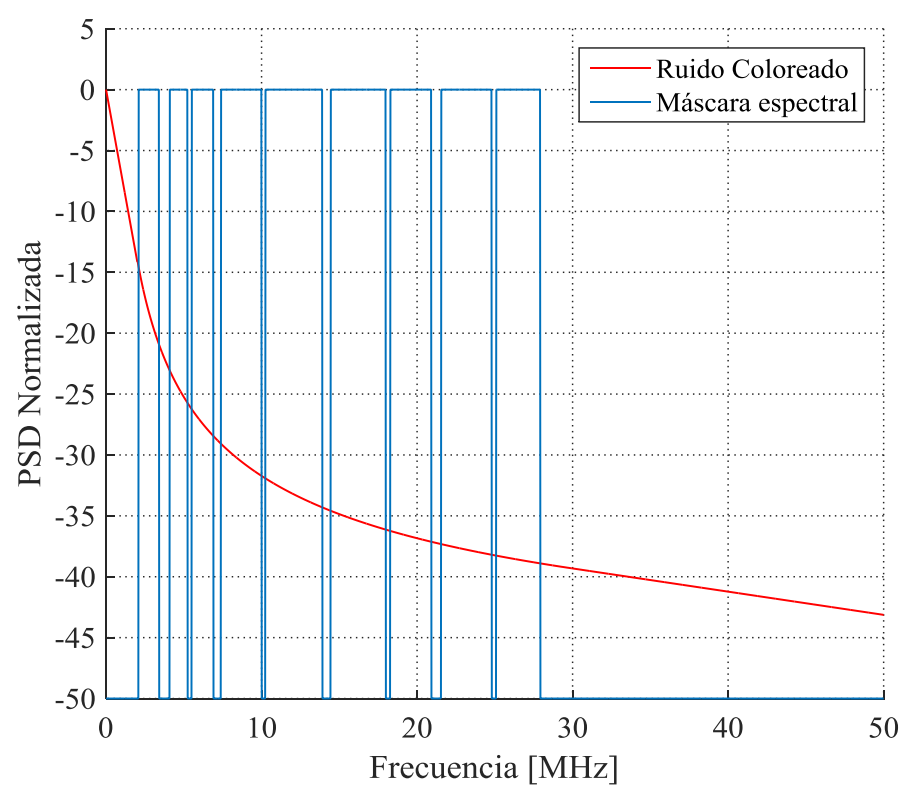

Fig. 6.7 Máscara espectral de transmisión y PSD normalizada del ruido de fondo coloreado.

señal recibida. Puede, por ejemplo, diseñarse un filtro pasabanda analógico desde la frecuencia correspondiente a la primera subportadora activa hasta la última subportadora activa, es decir, en la banda $2-28 \mathrm{MHz}$ aproximadamente. Luego, puede adicionalmente diseñarse un filtro digital para reducir el ruido en todas las bandas intermedias con subportadoras inactivas.

Para analizar la mejora que puede obtenerse a partir de la implementación de un filtro notch que distorsione lo menor posible la señal en el receptor como etapa previa a los algoritmos de sincronización y estimación de canal, se diseñó un filtro digital de alto orden con respuesta al impulso c finita (Finite Impulse Response - FIR). El orden del filtro diseñado es $N_{c}=2048$, y fue sintetizado a partir de 9 filtros pasabanda del mismo orden utilizando la herramienta de Matlab ${ }^{\circledR}$ fdatool, con el método de diseño basado en mínimos cuadrados. La magnitud de la respuesta en frecuencia del filtro resultante se encuentra en la Fig. 6.8, y los coeficientes más significativos de la respuesta al impulso se presentan en el Anexo C. Es importante tener en cuenta que al implementar un filtro de este tipo se agrega al sistema un retardo directamente relacionado con el orden del filtro, y, sumado al retardo del resto de las etapas de procesamiento digital, puede superar el retardo máximo admisible por el sistema de comunicaciones. En particular, si se implementara un filtro como el diseñado de orden 2048, se agregaría al sistema un retardo de 1024 muestras. La inclusión del filtro notch en el receptor se esquematiza en la Fig. 6.9. 
Diseño de Estrategias de Sincronización y Estimación de Canal para la Mejora de Comunicaciones en Redes Inteligentes de Energía

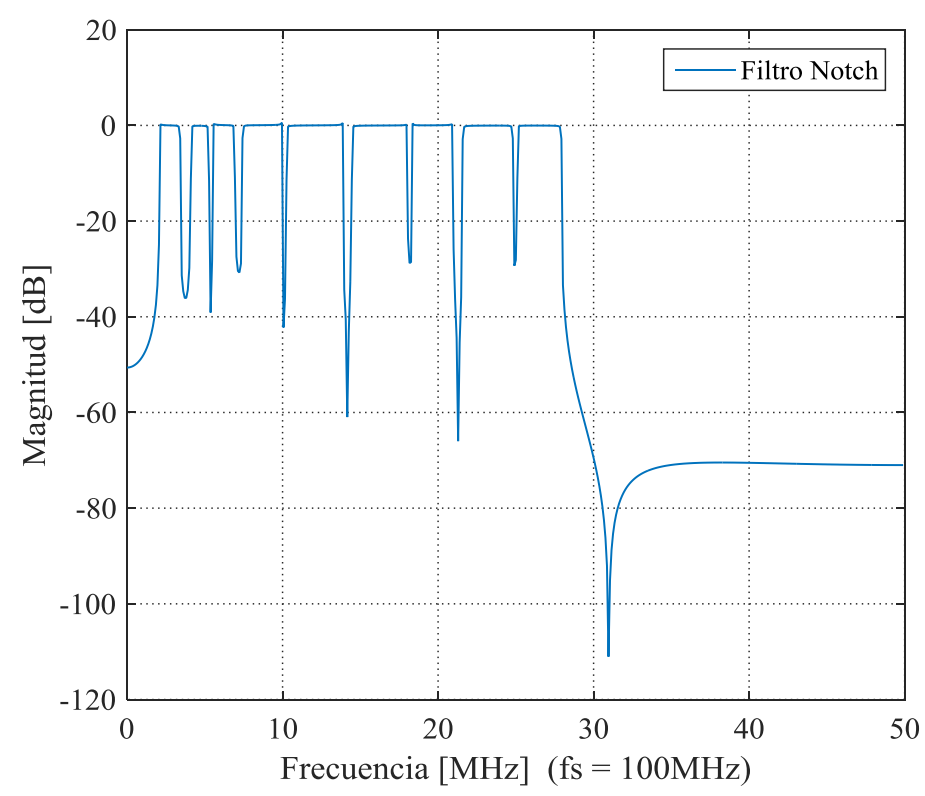

Fig. 6.8 Respuesta en frecuencia del filtro notch de orden 2048.

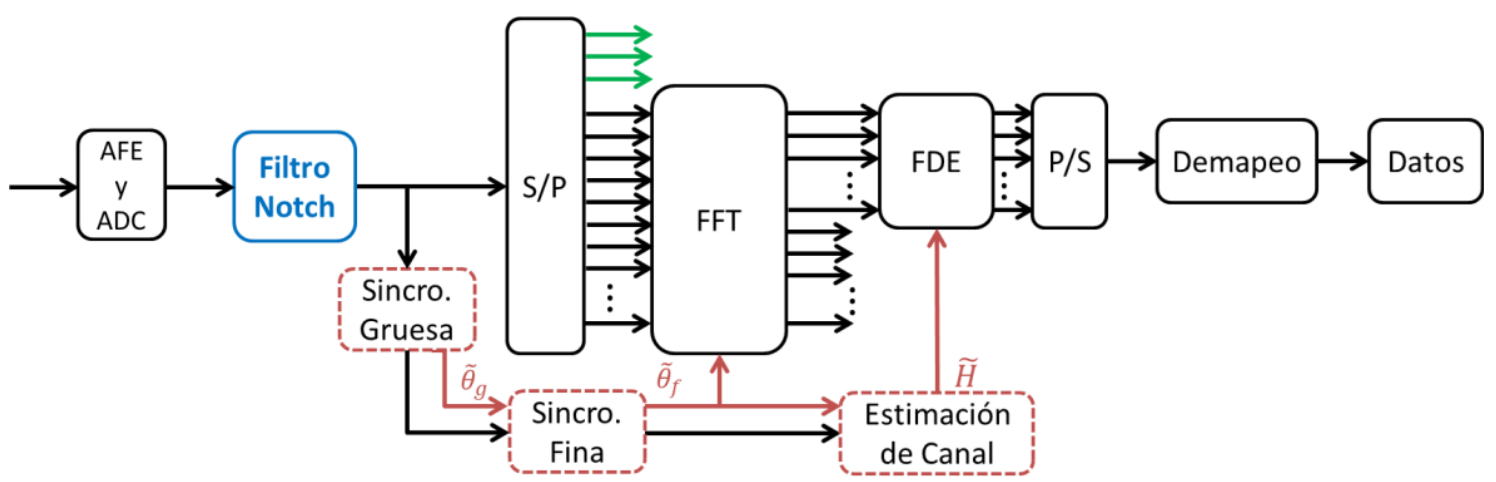

Fig. 6.9 Receptor del sistema DMT modificado incluyendo el filtro notch

Para analizar el impacto de la inclusión del filtro notch en la métrica de sincronización gruesa basada en $\mathrm{AC} m_{2, n}$, en la Fig. 6.10 se presentan las PDFs correspondientes al instante de sincronismo óptimo y en los anteriores. Puede observarse que, aplicando la máscara espectral de transmisión y el filtro notch en recepción, los valores de la métrica en el instante de sincronismo óptimo son superiores a la transmisión sin máscara espectral ni filtro, para todos los niveles de $S N R$. Esto se produce debido a que el filtro notch reduce la mayor parte del ruido presente en la señal recibida, de acuerdo a la Fig. 6.7, por lo que la relación señal a ruido efectiva es mayor respecto a la situación en la que se transmite en todo el ancho de banda teóricamente disponible. Para comparar las dos situaciones, se considera como referencia el valor de $S N R$ de la transmisión sin máscara, es decir, el nivel de potencia de señal útil cuando se transmite el símbolo completo, respecto a la potencia del proceso de ruido coloreado en todo el ancho de 

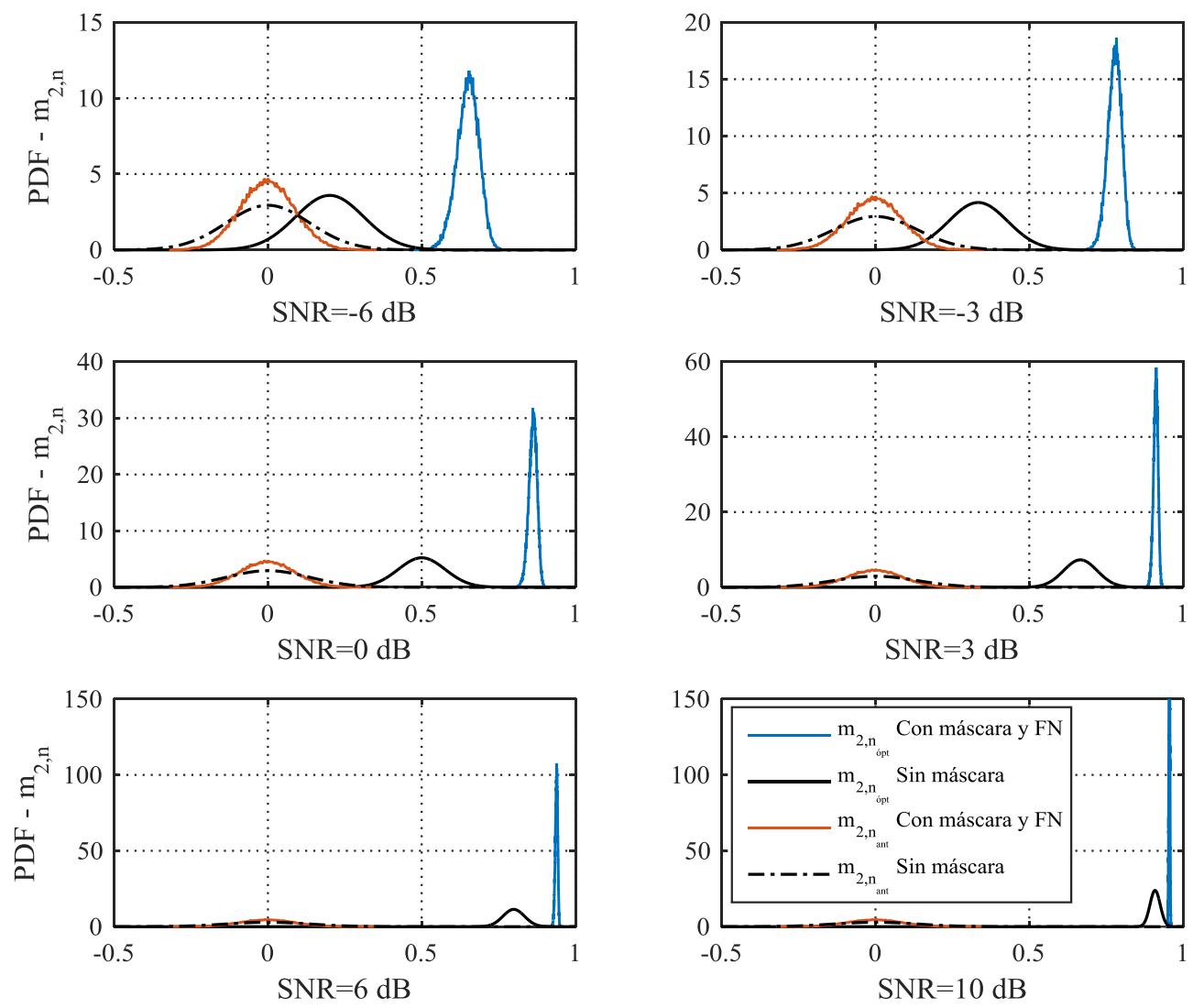

Fig. 6.10 PDFs del valor de la métrica de sincronización gruesa basada en AC, en el instante de sincronización óptimo y anteriores, aplicando la máscara espectral de transmisión y el filtro notch en recepción para distintos valores de SNR.

banda entre $0-50 \mathrm{MHz}$. La potencia asignada a cada subportadora activa y el proceso de ruido son los mismos en ambos casos. Al aplicar la máscara y dejar sólo $N_{a}$ subportadoras activas, la potencia transmitida se reduce en $2 N_{a} / N$, mientras que el nivel de ruido al utilizar el filtro notch disminuye considerablemente más.

Adicionalmente, a diferencia del caso analizado para la métrica aplicando la máscara espectral pero sin el filtro notch (Fig. 6.4), la PDF en las muestras previas a la llegada del símbolo piloto $m_{2, n_{a n t}}$ presenta ahora una menor varianza respecto al caso en el que no se aplica la máscara de transmisión. Por esto, si se mantiene el valor del umbral derivado para el caso de la métrica sin aplicar la máscara espectral de transmisión, entonces la probabilidad de falsa detección será menor a $10^{-7}$. Sin embargo, puede ajustarse el valor del umbral si se deriva la PDF correspondiente, que se ve afectada por la inclusión del filtro notch en recepción. Para ello, puede efectuarse el mismo análisis realizado para la métrica en las muestras previas a la llegada del símbolo piloto presentada en el capítulo 4, pero reemplazando el filtro que colorea el ruido $\mathbf{g}$ por un 
Diseño de Estrategias de Sincronización y Estimación de Canal para la Mejora de Comunicaciones en Redes Inteligentes de Energía

filtro equivalente $\mathbf{f}_{\mathbf{1}}$ a partir de la convolución entre $\mathbf{g}$ y el filtro notch $\mathbf{c}$ es decir $\mathbf{f}_{\mathbf{1}}=\mathbf{g} * \mathbf{c}$. En la Fig. 6.11 se presentan las ACs de los filtros $\mathbf{g}, \mathbf{c}$ y $\mathbf{f}_{\mathbf{1}}$, y en el caso de $\mathbf{f}_{\mathbf{1}}$ se encuentra magnificada por un factor de 10 para una visualización más clara. Así, la métrica puede escribirse como:

$$
m_{2, n_{\text {ant }}}=\frac{\sum_{m=0}^{N / 2-1} 2 z_{n_{a n t}+m} \cdot z^{\prime}{ }_{n_{a n t}+m}}{\sum_{m=0}^{N-1} z_{n_{a n t}}^{2}+m}=\frac{Z_{1}}{Z_{2}} \approx \frac{Z_{1}}{\mu_{Z_{2}}}
$$

donde $Z_{1}$ y $Z_{2}$ son variables aleatorias auxiliares que se analizarán a continuación, mientras que $z_{n}$ son los términos resultantes de filtrar las muestras del proceso de ruido coloreado por el filtro notch, y las muestras resultantes pueden entenderse como el sistema LTI (Linear Time-Invariant) de la Fig. 6.12.

Realizando un análisis idéntico al efectuado en (4.36) y (4.37), la variable aleatoria $Z_{1}$ resulta en una variable Gaussiana de acuerdo al TCL, con media y varianza:

$$
\mu_{Z_{1}} \approx 0
$$

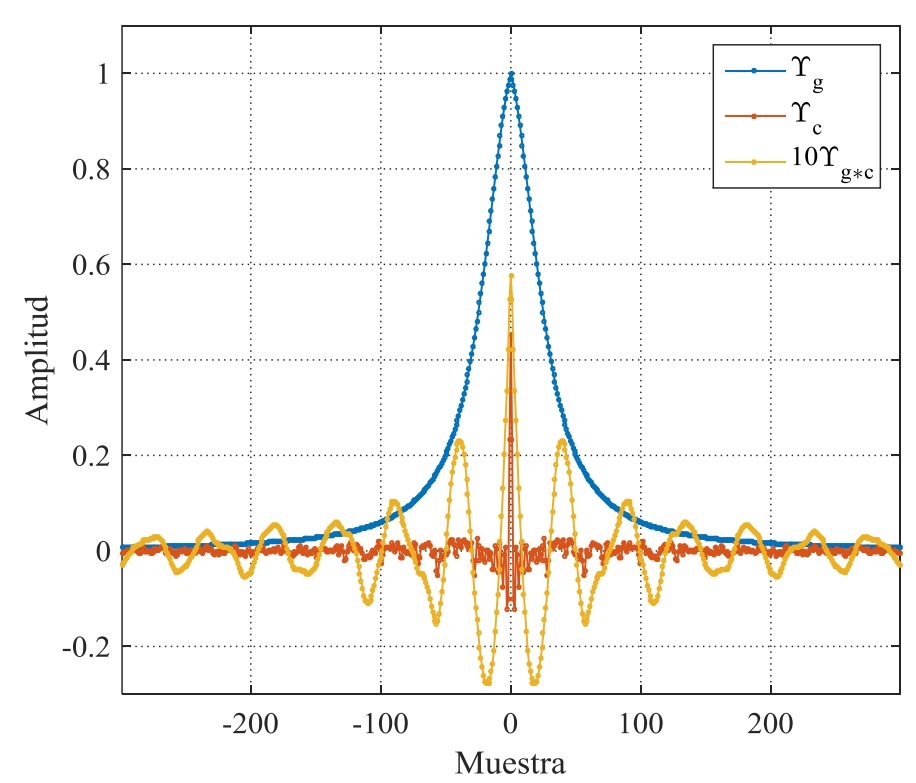

Fig. 6.11 Secuencias de autocorrelación del filtro que colorea el ruido $\mathbf{g}$, el filtro notch c y la combinación de ambos.

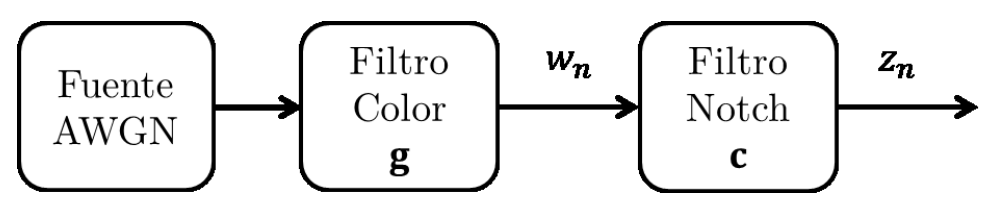

Fig. 6.12 Diagrama equivalente del filtrado de muestras de ruido coloreado a partir del filtro notch en recepción. 


$$
\operatorname{Var}\left(Z_{1}\right)=\sigma_{w}^{4}\left[2 N\left(\sum_{k=-\infty}^{+\infty} f_{1_{k}}^{2}\right)^{2}+8 \sum_{j=1}^{\frac{N}{2}-1}(N / 2-j)\left(\sum_{k=-\infty}^{+\infty} f_{1_{k}} f_{1_{k+j}}\right)^{2}\right]=\sigma_{w}^{4} \Sigma_{1}
$$

donde $\Sigma_{1}$ representa la expresión entre corchetes de (6.4). Por su parte, a partir del resultado obtenido en (4.39), puede obtenerse el valor medio de la variable $Z_{2}$ en función del filtro equivalente $\mathbf{f}_{\mathbf{1}}$ y la longitud del símbolo piloto $N$ como:

$$
\mu_{Z_{2}}=N \sigma_{w}^{2} \sum_{k=-\infty}^{+\infty} f_{1_{k}}^{2}
$$

Finalmente, la métrica basada en AC en las muestras previas a la llegada del símbolo piloto $m_{2, n_{\text {ant }}}$ resulta en una variable aleatoria $M_{2}$, gaussiana con media y varianza:

$$
\begin{gathered}
\mu_{M_{2 a n t}}=0 \\
\sigma_{M_{2 a n t}}^{2}=\frac{\Sigma_{1}}{N^{2} \sum_{k=-\infty}^{+\infty} f_{1_{k}}^{2}}
\end{gathered}
$$

Considerando los parámetros del sistema bajo estudio y el filtro notch, la varianza obtenida es $\sigma_{M_{2 a n t}}^{2} \approx 8,59 \mathrm{e}-3$, mientras que en el caso sin incluir el filtro notch resulta $18,26 \cdot 10^{-3}$. Los resultados de la PDF analítica junto a los resultados obtenidos por simulaciones se encuentran en la Fig. 6.13. A partir de este resultado, para una misma probabilidad de falsa detección de $10^{-7}$, el umbral se reduce de $\gamma \approx 0,705$ a 0,482 cuando se incluye el filtro notch en recepción.

Por otro lado, el impacto de la inclusión del filtro notch en la métrica de sincronización gruesa basada en $\mathrm{CC} m_{3, n}$ se presenta en la Fig. 6.14, a partir de las PDFs correspondientes al instante de sincronismo óptimo y en los anteriores. Nuevamente, al aplicar la máscara espectral de transmisión y el filtro notch en recepción, los valores de la métrica en el instante de sincronismo óptimo son superiores a la transmisión sin máscara espectral ni filtro, para todos los niveles de SNR. Además, la distribución de la métrica en las muestras previas a la llegada del símbolo piloto $m_{3, n_{a n t}}$ presenta también una menor varianza respecto al caso en el que no se aplica la máscara de transmisión. 
Diseño de Estrategias de Sincronización y Estimación de Canal para la Mejora de Comunicaciones en Redes Inteligentes de Energía

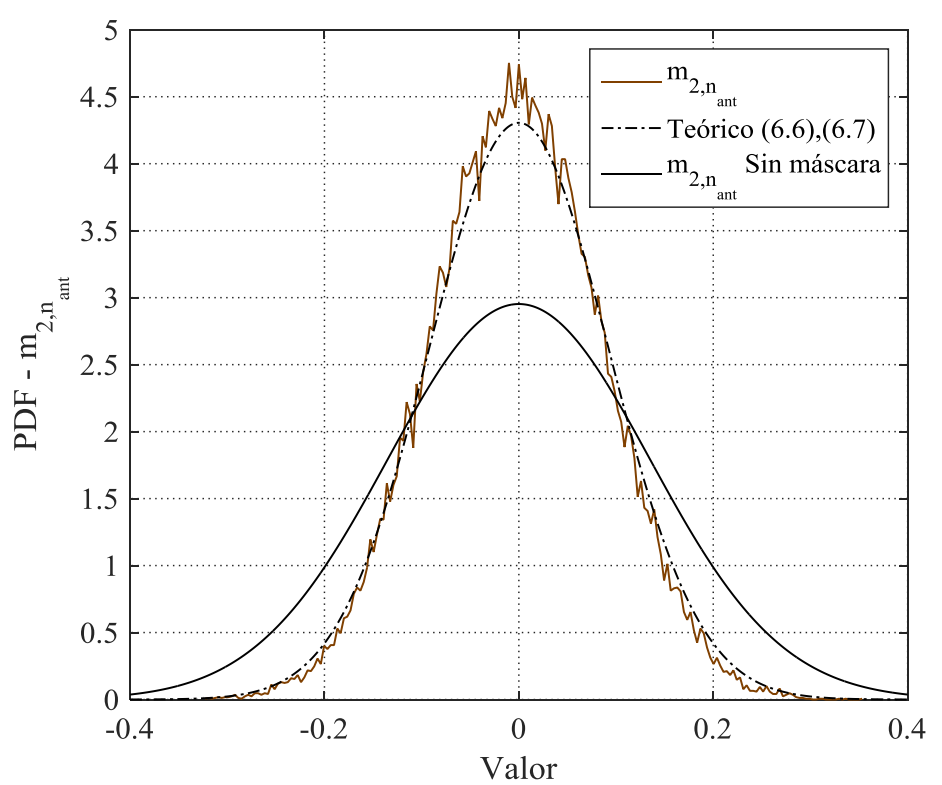

Fig. 6.13 PDFs empírica y teórica del valor de la métrica de sincronización gruesa basada en AC en las muestras previas a la llegada del simbolo piloto con máscara espectral de transmisión y filtro notch en recepción.

La métrica en las muestras previas a la llegada del símbolo piloto, de acuerdo a (4.66), se computa ahora de la forma:

$$
m_{3, n_{a n t}}=\frac{N_{2} / N \sum_{k=0}^{N_{2}-1}\left|\sum_{m=0}^{N-1} Z_{n_{a n t}+k+m} \mathrm{x}_{m}^{\prime *}\right|^{2}}{\sum_{k=0}^{N_{2}-1} \sum_{m=0}^{N-1} z_{n_{a n t}+k+m}^{2}}=\frac{\frac{N_{2}}{N} Z_{3}}{Z_{4}}
$$

Con respecto a $Z_{3}$, debe considerarse el filtro equivalente $\mathbf{f}_{2}$ que incluye la conexión en cascada del filtro que colorea el ruido $\mathbf{g}$, el filtro notch $\mathbf{c}$ y el filtro acoplado al símbolo piloto $\overline{\mathbf{x}^{\prime}}$, donde $\mathbf{f}_{2}=\mathbf{g} * \mathbf{c} * \overline{\mathbf{x}}^{\prime}$. En la Fig. 6.15 se presentan las ACS de los filtros $\overline{\mathbf{x}}^{\prime}, \mathbf{f}_{\mathbf{1}}=\mathbf{g} * \mathbf{c}$ y $\mathbf{f}_{\mathbf{2}}$, escaladas para una correcta representación.

Con el filtro equivalente modificado, y el mismo análisis realizado para (4.71) y (4.72), se obtienen la media y varianza de $Z_{3}$ como:

$$
\begin{gathered}
\mu_{Z_{3}}=N_{2} \sigma_{w}^{2} \sum_{k=-\infty}^{\infty} f_{2_{k}}^{2}=N_{2} \sigma_{w}^{2} \Sigma_{2} \\
\operatorname{Var}\left(Z_{3}\right)=\sigma_{w}^{4}\left[2 N_{2}\left(\sum_{k=-\infty}^{+\infty} f_{2_{k}}^{2}\right)^{2}+4 \sum_{j=1}^{N_{2}-1}\left(N_{2}-j\right)\left(\sum_{k=-\infty}^{+\infty} f_{2_{k}} f_{2_{k+j}}\right)^{2}\right]=\sigma_{w}^{4} \Sigma_{3}
\end{gathered}
$$


Capítulo 6: Sincronización y estimación de canal en sistemas DMT-PLC bajo una máscara espectral de transmisión
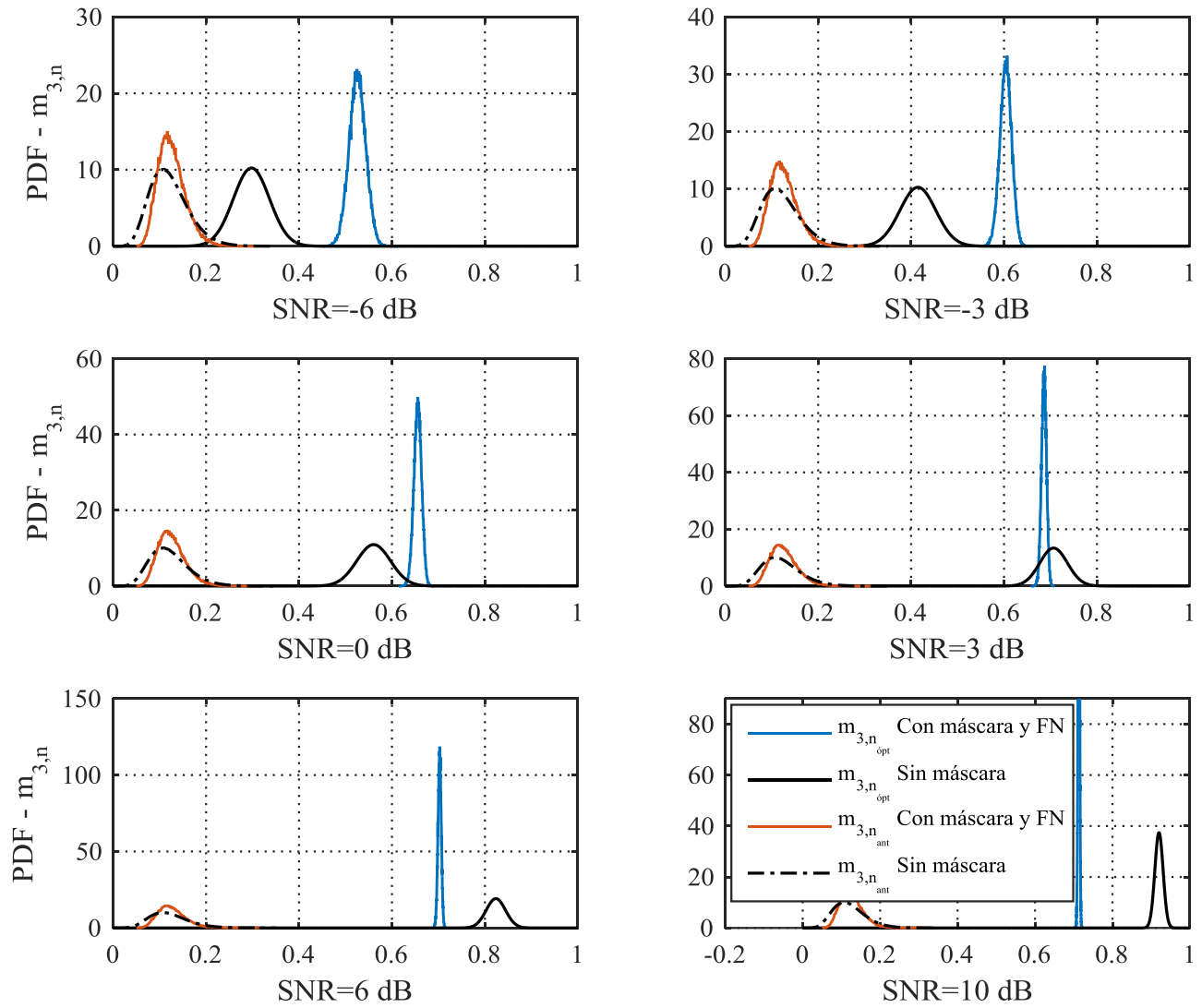

Fig. 6.14 PDFs del valor de la métrica de sincronización gruesa basada en CC, en el instante de sincronización óptimo y anteriores, aplicando la máscara espectral de transmisión y el filtro notch en recepción para distintos valores de SNR.

Por su parte, la media y varianza de la variable $Z_{4}$ pueden obtenerse análogamente a lo desarrollado en (4.73) y (4.74), de acuerdo a:

$$
\begin{gathered}
\mu_{Z_{4}}=N_{2} N \sigma_{w}^{2} \sum_{k=-\infty}^{+\infty} f_{1_{k}}^{2}=N_{2} N \sigma_{w}^{2} \Sigma_{4} \\
\operatorname{Var}\left(Z_{4}\right)=\sigma_{w}^{4}\left[2 N_{2} \sum_{k=-\infty}^{+\infty} f_{3_{k}}^{2}+4 \sum_{j=1}^{N_{2}-1}\left(N_{2}-j\right) \sum_{k=-\infty}^{+\infty} f_{3_{k}} f_{3_{k+j}}\right]=\sigma_{w}^{4} \Sigma_{5}
\end{gathered}
$$

donde $f_{3_{k}}=\left(f_{1_{k}} * f_{1_{-k}}\right)^{2} *\left(\operatorname{rect}_{k / N} * \operatorname{rect}_{k / N}\right)$ y rect $_{k / N}$ es una función rectangular discreta de longitud $N$. Como resultado, la métrica en las muestras previas a la llegada del símbolo piloto resulta en una variable aleatoria $M_{3_{a n t}}$ con media y varianza:

$$
\mu_{M_{3 \text { ant }}}=\frac{N_{2} \Sigma_{2}}{N^{2} \Sigma_{4}}
$$


Diseño de Estrategias de Sincronización y Estimación de Canal para la Mejora de Comunicaciones en Redes Inteligentes de Energía

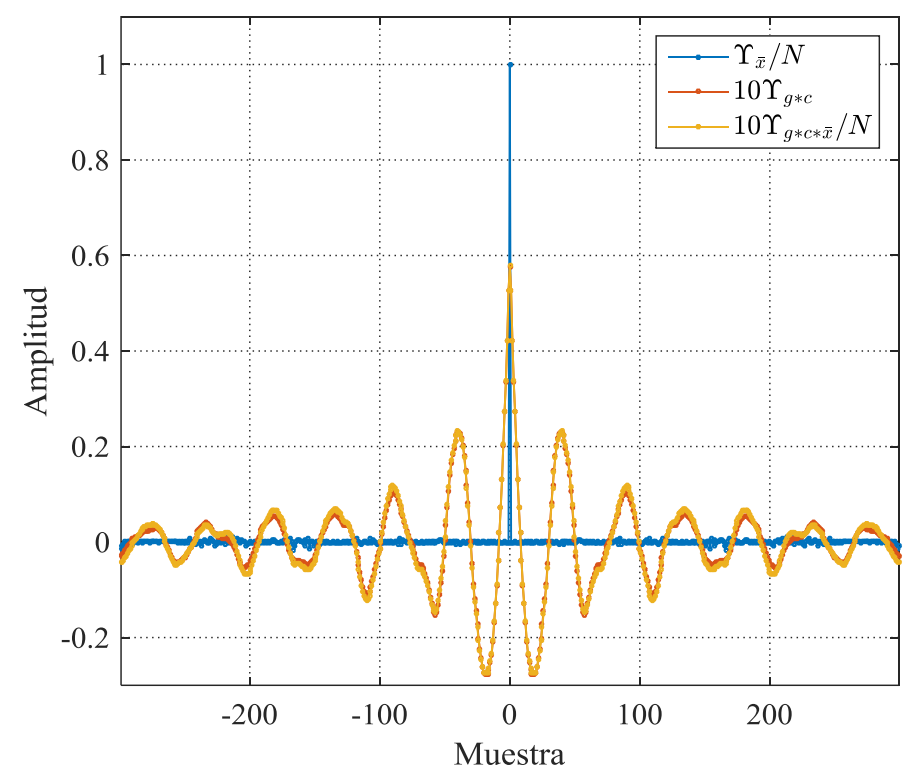

Fig. 6.15 Secuencias de autocorrelación del filtro $\boldsymbol{f}_{\mathbf{1}}$, el filtro acoplado del símbolo piloto $\boldsymbol{x}^{\prime} y$ la combinación de ambos.

$$
\sigma_{M_{3 \text { ant }}}^{2}=\frac{\Sigma_{3}-\frac{\Sigma_{2}}{N}\left(\frac{2}{\Sigma_{4}}-\frac{\Sigma_{2}}{N \Sigma_{4}^{2}}\right) \Sigma_{5}}{N^{4} \Sigma_{4}^{2}}
$$

Al igual que el resultado obtenido en (4.81) y (4.82), la distribución de la métrica $m_{3, n_{a n t}}$ resulta en una distribución Gamma, con parámetros $k$ y $\theta$ :

$$
\begin{aligned}
k_{M_{3} \text { ant }} & =\frac{N_{2}^{2} \Sigma_{2}^{2}}{\Sigma_{3}-\frac{\Sigma_{2}}{N}\left(\frac{2}{\Sigma_{4}}-\frac{\Sigma_{2}}{N \Sigma_{4}^{2}}\right) \Sigma_{5}} \\
\theta_{M_{3 \text { ant }}} & =\frac{\Sigma_{3}-\frac{\Sigma_{2}}{N}\left(\frac{2}{\Sigma_{4}}-\frac{\Sigma_{2}}{N \Sigma_{4}^{2}}\right) \Sigma_{5}}{N^{2} N_{2} \Sigma_{2}}
\end{aligned}
$$

A partir de este resultado, para una misma probabilidad de falsa detección de $10^{-7}$, el umbral se reduce de $\gamma \approx 0,471$ a 0,349 cuando se incluye el filtro notch en recepción. Los resultados de la PDF analítica junto a los resultados obtenidos por simulaciones se encuentran en la Fig. 6.16.

Luego de obtener los respectivos umbrales de detección $\gamma$ para las métricas $m_{2, n} \mathrm{y}$ $m_{3, n}$, se está en condiciones de evaluar la capacidad de detección del símbolo piloto al incluir la máscara espectral de transmisión y el filtro notch en el receptor. En la Fig. 6.17 se presenta la probabilidad de detección del símbolo para ambas métricas en tres condiciones: sin la aplicación de la máscara espectral, con máscara espectral sin incluir 


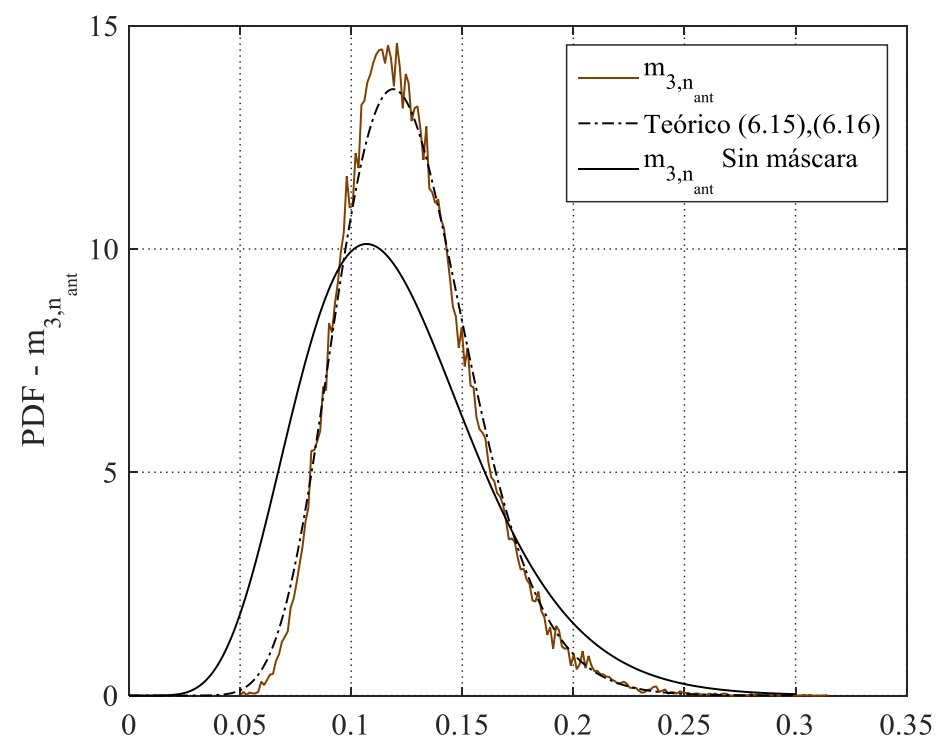

Fig. 6.16 PDFs empírica y teórica del valor de la métrica de sincronización gruesa basada en CC en las muestras previas a la llegada del simbolo piloto con máscara espectral de transmisión y filtro notch en recepción.

el filtro notch, y con máscara espectral incluyendo el filtro notch. Puede observarse que al incluir el filtro en recepción se mejora significativamente la capacidad de detectar el símbolo piloto respecto a la transmisión sin máscara, para ambas métricas y en todo el rango de $S N R$ considerado. Además, la capacidad de detección de la métrica basada en $\mathrm{CC}$ es ligeramente superior a la métrica de $\mathrm{AC}$, lo que permite la detección del símbolo piloto aún a muy baja SNR. La mejora se produce debido a que, como la PSD del ruido coloreado (Fig. 6.7) es decreciente con la frecuencia, el filtro notch reduce significativamente el nivel de ruido presente en las muestras de la señal recibida al anular las subportadoras correspondientes al rango $0-2 \mathrm{MHz}$.

La condición de $S N R$ efectiva que resulta de la relación entre el nivel de señal en las subportadoras activas y la PSD del ruido en las bandas de frecuencia definidas por las subportadoras activas es significativamente mayor porque una gran porción de ruido resulta mitigado. El factor de disminución del nivel de ruido puede obtenerse a partir de la varianza del proceso de ruido resultante de pasar las muestras del ruido coloreado por el filtro notch. Para el caso bajo estudio se obtiene directamente de $\Sigma_{4} \approx 0,056$, lo que se traduce en una reducción de $12,5 \mathrm{~dB}$. Como se trata de un filtro notch de alto orden, se puede obtener prácticamente el mismo resultado a partir de la integral de la PSD del proceso de ruido coloreado en las bandas correspondientes a las subportadoras activas (Fig. 6.7). 
Diseño de Estrategias de Sincronización y Estimación de Canal para la Mejora de Comunicaciones en Redes Inteligentes de Energía

Por último, como la potencia de señal útil transmitida al aplicar la máscara espectral se reduce en la proporción $2 N_{a} / N=0,4478$ (casi-3,5dB), y a lo largo del capítulo se comparan las condiciones de transmisión con y sin máscara espectral, se define la relación señal a ruido equivalente en las bandas activas como $S N R_{\text {act }} \approx S N R+$ $12,5 \mathrm{~dB}-3,5 \mathrm{~dB}=S N R+9 \mathrm{~dB}$, donde $S N R$ es la relación señal a ruido cuando se transmite en todo el ancho de banda teóricamente disponible, es decir, sin aplicar la máscara.

\subsubsection{Efecto del ruido impulsivo en la etapa de sincronización gruesa}

De manera similar al estudio realizado en la sección 4.3.6 para la etapa de sincronización gruesa sin la aplicación de la máscara espectral de transmisión, un evento de ruido impulsivo degradará la métrica de sincronización gruesa propuesta en el sistema que incluye la máscara espectral de transmisión y el filtro notch en recepción. Ante un evento de ruido impulsivo, la energía que normaliza a $m_{3, n}$ sufre un gran incremento mientras que el valor resultante en el numerador no se incrementa considerablemente. Por lo tanto, los valores resultantes de la métrica en el instante óptimo de sincronismo (y cercanos) disminuyen ante la ocurrencia de un evento de ruido impulsivo.

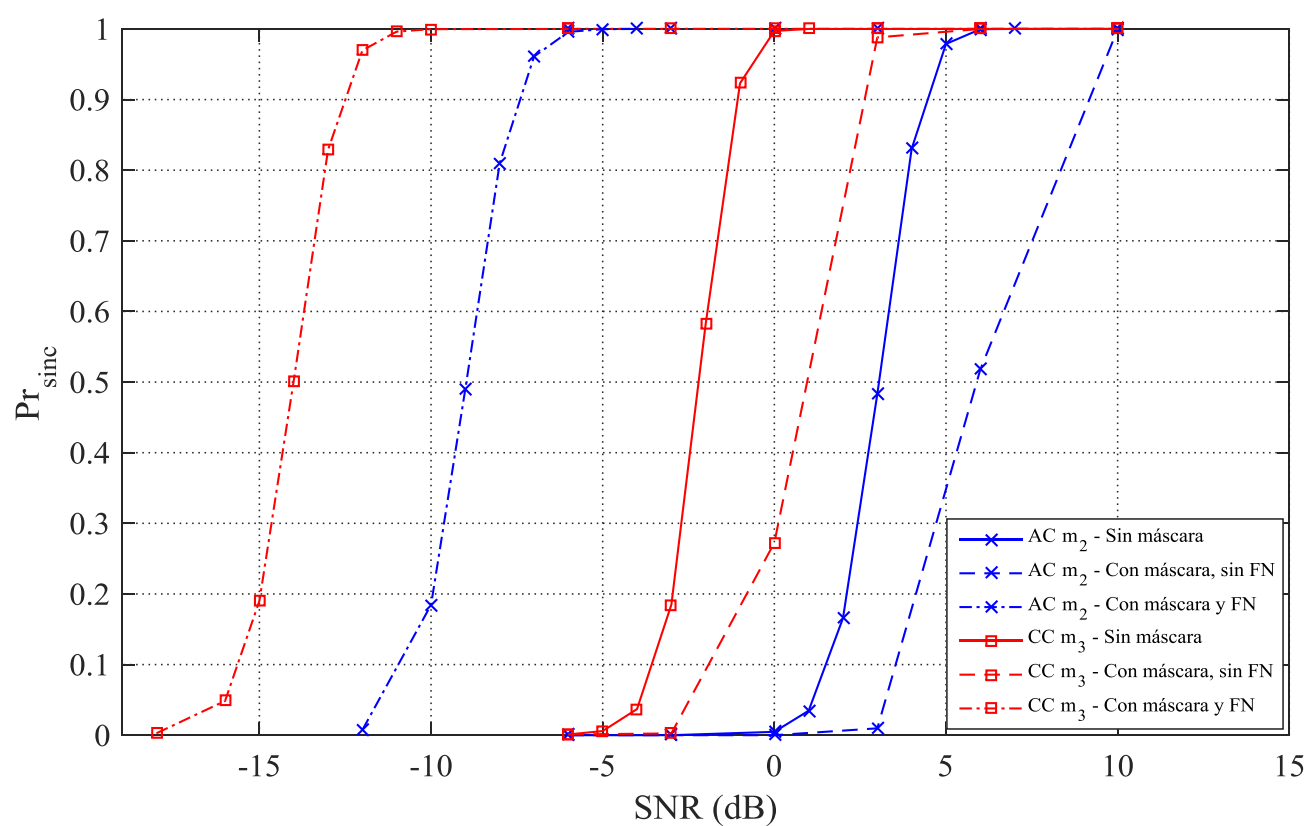

Fig. 6.17 Probabilidad de sincronización en términos de la detección del símbolo piloto para las métricas basadas en AC y CC bajo ruido de fondo coloreado, máscara espectral de transmisión y filtro notch en recepción para distintos valores de SNR. 


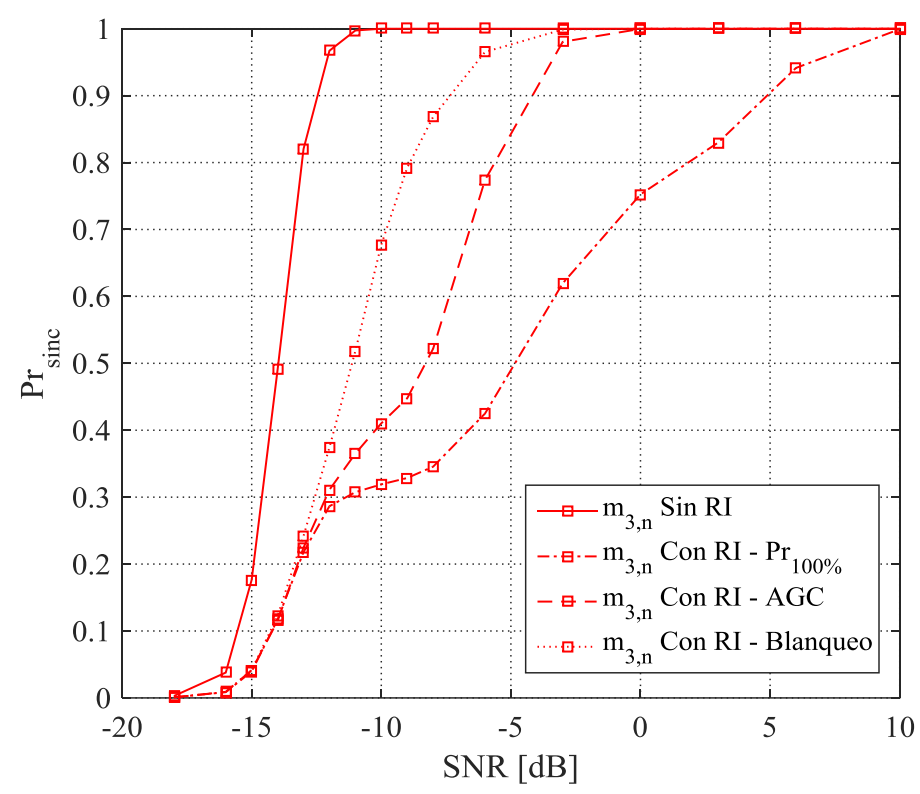

Fig. 6.18 Probabilidad de detección del símbolo piloto con y sin mitigación de eventos de ruido impulsivo con probabilidad de ocurrencia de $100 \%$.

Bajo las mismas consideraciones, la métrica en las muestras previas a la llegada del símbolo piloto $m_{3, n_{a n t}}$ también disminuye. Sin embargo, como la probabilidad de ocurrencia de los eventos de ruido impulsivo es del 8\%, el umbral de detección debe mantenerse de acuerdo al valor $\gamma \approx 0,349$ obtenido a partir de (6.15) y (6.16), para mantener baja la probabilidad de falsa detección cuando no ocurre un evento de ruido impulsivo.

Para reducir el efecto del ruido impulsivo, se incluyen las mismas técnicas introducidas en la sección 4.3.6.2 de blanqueo no lineal y mitigación a partir de la etapa de AGC. En la Fig. 6.18 se presenta la probabilidad de detección del símbolo piloto con y sin ruido impulsivo con probabilidad de ocurrencia del 100\%, y luego de aplicar las dos técnicas de mitigación. La capacidad de detección para la métrica de sincronización gruesa $m_{3, n}$ ve mejorada considerablemente su capacidad de detección, aumentando la probabilidad de detección, a una $S N R$ de $-6 \mathrm{~dB}$, de un $42 \%$ sin mitigación, a 77\% y $97 \%$ para las técnicas basadas en AGC y blanqueo, respectivamente. A partir de una $S N R$ de $-3 \mathrm{~dB}$, se obtiene una capacidad de detección del 100\% con la técnica de blanqueo, logrando lo mismo con $\mathrm{AGC}$ a partir de $0 \mathrm{~dB}$, mientras que sin aplicar ninguna técnica de mitigación se logra a partir de $10 \mathrm{~dB}$. Para valores de $S N R$ de $-13 \mathrm{~dB}$ e inferiores, además de presentar una probabilidad de sincronización inferior al 25\%, las técnicas de mitigación del ruido impulsivo no presentan una mejora significativa en el desempeño. 
Diseño de Estrategias de Sincronización y Estimación de Canal para la Mejora de Comunicaciones en Redes Inteligentes de Energía

Si bien estos resultados resultan útiles para verificar la capacidad de atenuación del efecto del ruido impulsivo al aplicar las técnicas de mitigación, como la probabilidad de ocurrencia real de los eventos de ruido impulsivo es cercana al 8\%, la probabilidad de sincronización del sistema resulta superior. Las curvas correspondientes de probabilidad de sincronización perfecta versus $S N R$ se presentan en la Fig. 6.19.

\subsection{Sincronización Fina}

En la sección 5.4 se propuso una estrategia de sincronización fina a partir de dos variantes de correlación cruzada normalizada del símbolo piloto propuesto, y la definición de umbrales para la detección de los coeficientes de la CIR. Por un lado, se obtuvo un umbral $\alpha_{1}$ que garantiza una determinada probabilidad de detección errónea de las muestras de la zona de correlación nula, previas a la correspondiente al primer camino del canal, a partir del análisis de la PDF de la correlación cruzada normalizada en las muestras correspondientes. Este umbral resulta una alternativa práctica si no se tiene información estadística de los coeficientes del canal. Luego, se propuso obtener un umbral $\alpha_{\text {ópt }}$ que maximice la probabilidad de sincronismo perfecto (PSP), incluyendo además la PDF de la CCN en el instante correspondiente al arribo del primer camino del canal.

En esta sección se estudia el efecto de la aplicación de la máscara espectral de

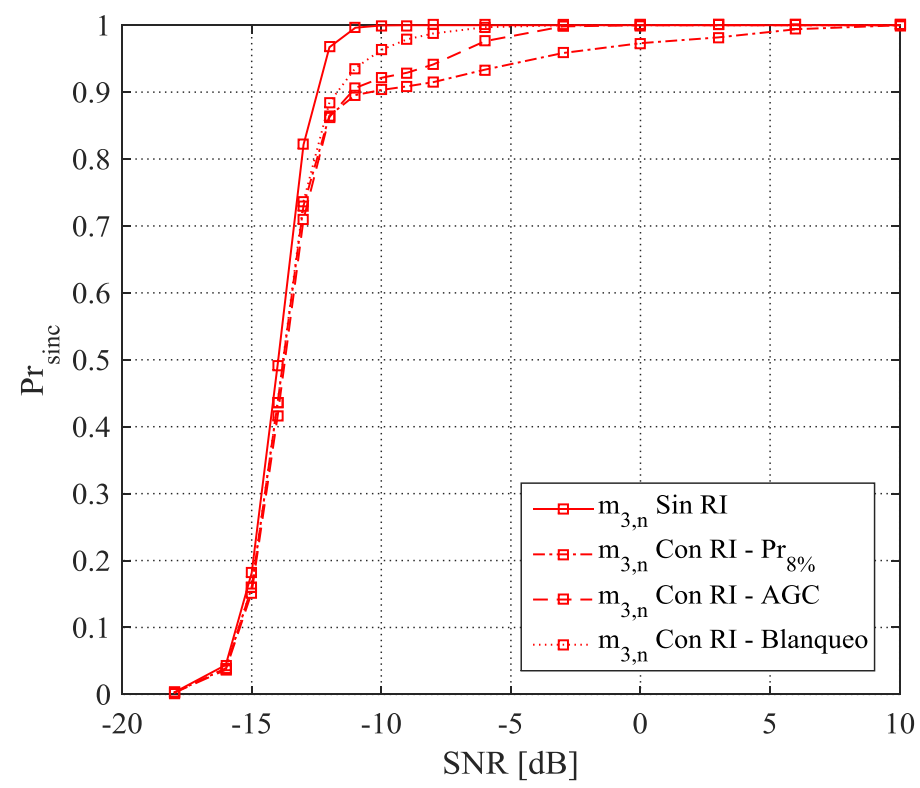

Fig. 6.19 Probabilidad de detección del símbolo piloto con y sin mitigación de eventos de ruido impulsivo con probabilidad de ocurrencia de $8 \%$. 
Capítulo 6: Sincronización y estimación de canal en sistemas DMT-PLC bajo una máscara espectral de transmisión

transmisión en la etapa de sincronización fina, y a partir de ello se estudia la definición de nuevos umbrales que permitan un desempeño adecuado ante las distorsiones introducidas por la máscara de transmisión. Teniendo en cuenta que los resultados obtenidos para la etapa de sincronización gruesa indican que la aplicación de un filtro notch en recepción mejora considerablemente el desempeño, la etapa de sincronización fina se enfoca en el escenario en que se incluye el filtro en el receptor además de la máscara espectral en el transmisor. En la etapa de sincronización gruesa, se demostró que incluyendo el filtro se obtiene una condición de $S N R$ equivalente en las bandas activas dada por $S N R_{a c t} \approx S N R+9 \mathrm{~dB}$, donde $S N R$ es la relación señal a ruido cuando se transmite en todo el ancho de banda teóricamente disponible. Como a partir de esta sección se considera únicamente al caso en que se incluye el filtro notch en recepción, se denomina indistintamente la relación señal a ruido $S N R_{\text {act }}$ como $S N R$.

\subsubsection{Efecto en la sincronización fina}

Las subportadoras anuladas por la máscara provocan una distorsión en las propiedades de correlación cruzada del símbolo piloto. En la Fig. 6.20 se presenta la salida del filtro acoplado en condiciones ideales sin el agregado de ruido, para una realización del canal PLC tipo A con y sin la aplicación de la máscara espectral, en la que puede apreciarse la dispersión en el tiempo que degrada la zona de correlación nula en las muestras previas al primer camino del canal.

Recordando el algoritmo de sincronización fina propuesto en el capítulo 5, este resulta:

$$
\begin{aligned}
& n_{f}=n_{f_{1}} \\
& \text { while } \underset{n \in\{0,1, \ldots, D\}}{\arg \max }\left(n \mid c_{n_{f}-n}^{\prime} \geq \alpha\right)>0 \\
& n_{f}=n_{f-n} \\
& \text { end }
\end{aligned}
$$

donde $n_{f_{1}}$ es la posición del máximo de la correlación cruzada normalizada utilizada como condición inicial del algoritmo, y $D$ es el tamaño de la ventana para la búsqueda hacia atrás de muestras que superen un umbral de detección $\alpha$. Por su parte, se definieron dos variantes de correlación cruzada normalizada, de acuerdo a:

$$
c_{1, n}^{\prime}=\frac{1 / N\left|c_{n}^{\mathbf{r}, \mathbf{x}^{\prime}}\right|^{2}}{\sum_{m=0}^{N-1}\left|r_{n+m}\right|^{2}}
$$


Diseño de Estrategias de Sincronización y Estimación de Canal para la Mejora de Comunicaciones en Redes Inteligentes de Energía

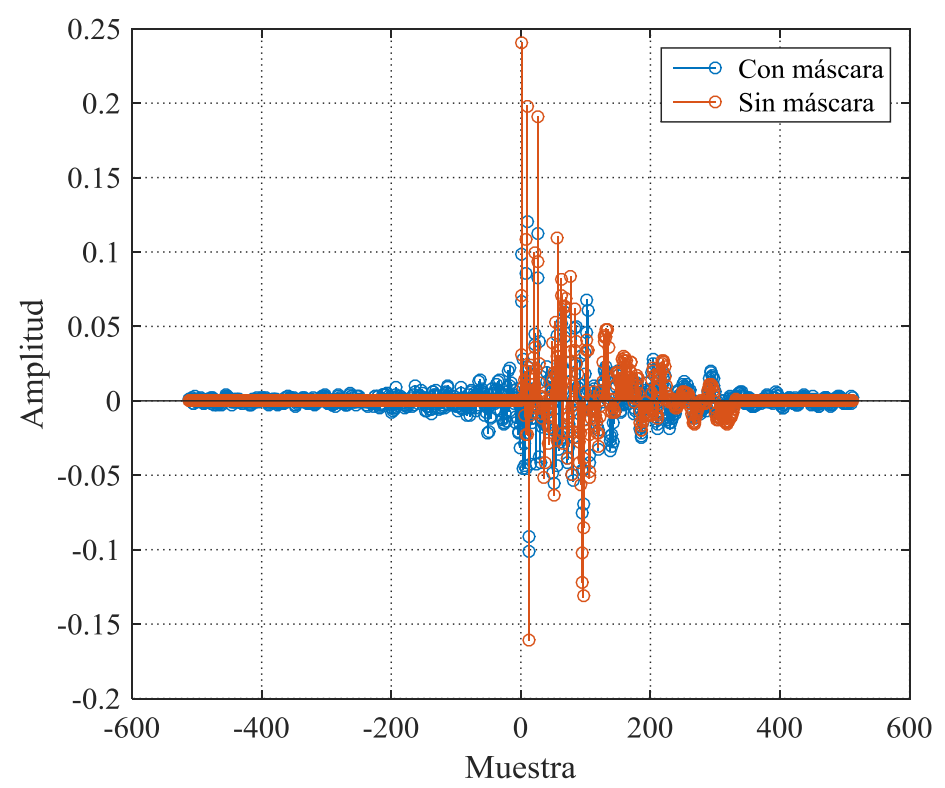

Fig. 6.20 Salida del filtro acoplado con y sin máscara espectral de transmisión, para una realización del canal A sin el agregado de ruido.

$$
c^{\prime}{ }_{2, n}=\frac{c_{1, n}^{\prime}}{1-m_{3, n}}
$$

donde $c_{n}^{\mathbf{r}, \mathbf{x}^{\prime}}$ es la correlación cruzada de la señal recibida $r_{n}$ con el filtro acoplado al símbolo piloto propuesto $\mathbf{x}^{\prime}$, y $m_{3, n}$ es la métrica definida en la etapa de sincronización gruesa. A su vez, los umbrales $\alpha_{1}$ que garantizan una probabilidad de falsa detección de, por ejemplo $10^{-7}$, pueden obtenerse de acuerdo a lo desarrollado en el capítulo 5 como:

$$
\begin{aligned}
& \alpha_{1, c_{1, n}^{\prime}}=\frac{28,374 \sum_{k=-\infty}^{\infty} f_{1_{k}}^{2}}{N^{2}(S N R+1)} \\
& \alpha_{1, c_{2, n}^{\prime}}=\frac{28,374 \sum_{k=-\infty}^{\infty} f_{1_{k}}^{2}}{N^{2}\left(1-\frac{N_{2}}{N}\right)}
\end{aligned}
$$

donde $N$ es la cantidad de puntos de la DFT del sistema DMT, $N_{2}$ la cantidad de muestras acumuladas para el cómputo de la métrica de sincronización gruesa, y $f_{1_{k}}$ son las muestras correspondientes a la secuencia de correlación cruzada entre el filtro que colorea el ruido y el símbolo piloto. Evidentemente, el desempeño de la etapa de sincronización fina a partir de los umbrales derivados en el capítulo 5 se verá sustancialmente degradado, porque las muestras previas al primer camino contienen interferencia debido a la dispersión temporal del canal. En las Fig. 6.21 y Fig. 6.22 se presentan el desempeño obtenido en términos de la probabilidad de sincronización 
Capítulo 6: Sincronización y estimación de canal en sistemas DMT-PLC bajo una máscara espectral de transmisión

perfecta (PSP) y el RMSE de la estimación del instante de sincronismo, respectivamente. En ambos casos, se utiliza $\alpha_{1}$ para la sincronización fina, y el sistema se encuentra modificado por la aplicación de la máscara espectral, y la inclusión del filtro notch en recepción. Adicionalmente, se incluyen los resultados obtenidos a partir de la etapa de sincronización gruesa, así como de la ubicación $n_{f_{1}}$ del máximo de las dos variantes de CCN propuestas, considerando los dos tipos de canal PLC A y B. Por un lado, puede apreciarse que la sincronización fina bajo el umbral $\alpha_{1}$ no presenta un correcto desempeño, debido a que se obtienen mejores resultados a partir del máximo de la $\mathrm{CCN}$ en casi todo el rango de $S N R$, que en la búsqueda hacia atrás del primer camino del canal; esto sucede para las dos variantes de CCN. El problema radica en que $\alpha_{1}$ no considera la interferencia producida por la extensión en el tiempo de la CIR del canal, y por lo tanto los valores de umbral resultan demasiado pequeños, provocando una alta probabilidad de detección de muestras previas al primer camino del canal como instante de sincronismo. A medida que aumenta el nivel de $S N R$, predomina el efecto de interferencia en el valor de las muestras previas por sobre el del ruido, lo que explica la reducción en la PSP a medida que aumenta $S N R$. Por su parte, los valores de umbrales óptimos $\alpha_{\text {ópt }}$ son menores a $\alpha_{1}$ (véase la Tabla 5.3), por lo que, en caso de aplicarlos para la sincronización fina, el desempeño resultante presentaría peores resultados aún.

Por otro lado, puede apreciarse que el sincronismo a partir del máximo de la $\mathrm{CCN} c_{2, n}^{\prime}$ presenta una mayor PSP que $c_{1, n}^{\prime}$, debido a que la modificación aplicada al dividir por $1-m_{3, n}$ en (6.31) implica una reducción en la amplitud de la CCN en las muestras correspondientes a los caminos más alejados al instante óptimo de sincronismo, derivado de la reducción de $m_{3, n}$, como se puede observar en la Fig. 5.3. Sin embargo, el desempeño al aplicar el umbral $\alpha_{1}$ resulta peor para la $\operatorname{CCN} c_{2, n}^{\prime}$ que para $c_{1, n}^{\prime}$, lo que indica que la degradación en las propiedades de correlación es mayor en $c_{2, n}^{\prime}$.

Adicionalmente, se obtiene una mayor PSP para el canal B respecto A, mientras que el RMSE resulta similar en ambos casos. Esto se debe a que el canal B posee en promedio una menor cantidad de caminos que el $\mathrm{A}$, y los caminos resultan más espaciados temporalmente (veáse como ejemplo la Fig. 4.1). Como la ganancia promedio del primer camino es la mayor en los dos casos, la probabilidad de que el primer camino sea el de mayor amplitud se incrementa para el canal B. 
Diseño de Estrategias de Sincronización y Estimación de Canal para la Mejora de Comunicaciones en Redes Inteligentes de Energía

Finalmente, la sincronización gruesa produce una PSP prácticamente nula y un elevado RMSE, y son justamente las razones por las que resulta necesaria la aplicación de la etapa de sincronización fina.
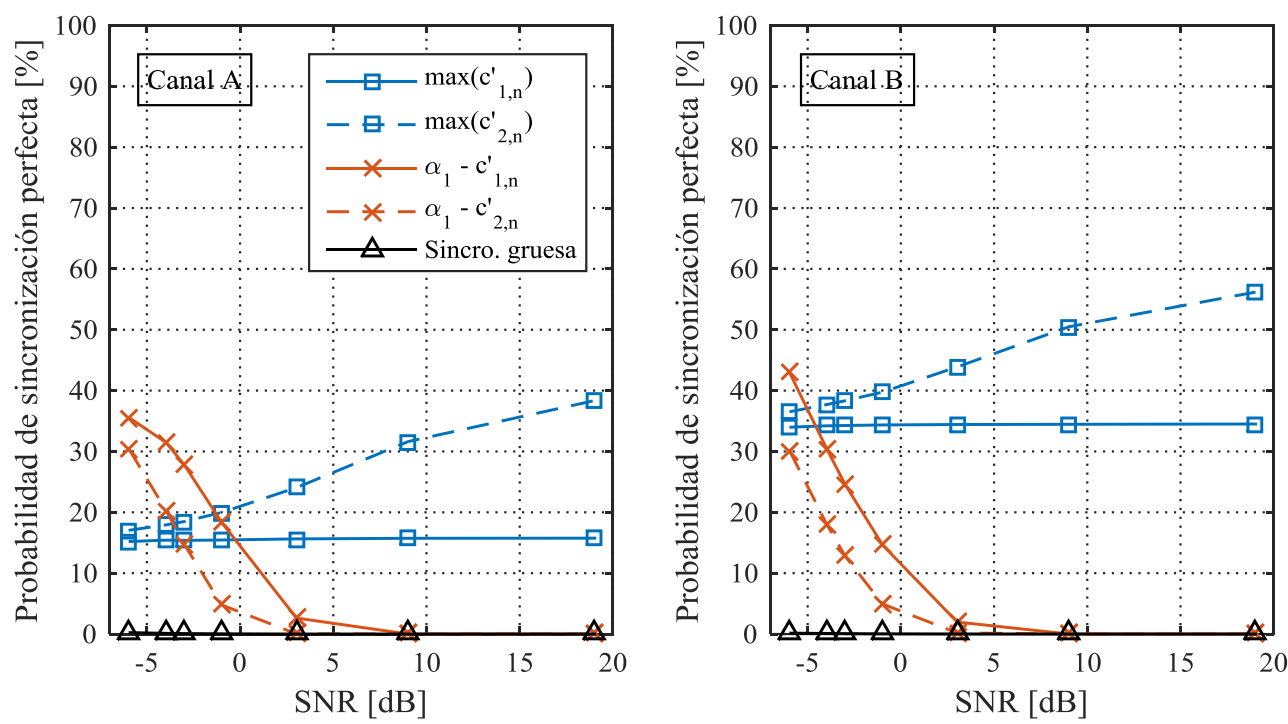

Fig. 6.21 Probabilidad de sincronización fina perfecta considerando los umbrales $\alpha_{1}$ para las dos variantes de CCN, la ubicación del máximo de cada una, y el resultado de la etapa de sincronización gruesa, para los canales tipo A (izquierda y B (derecha).
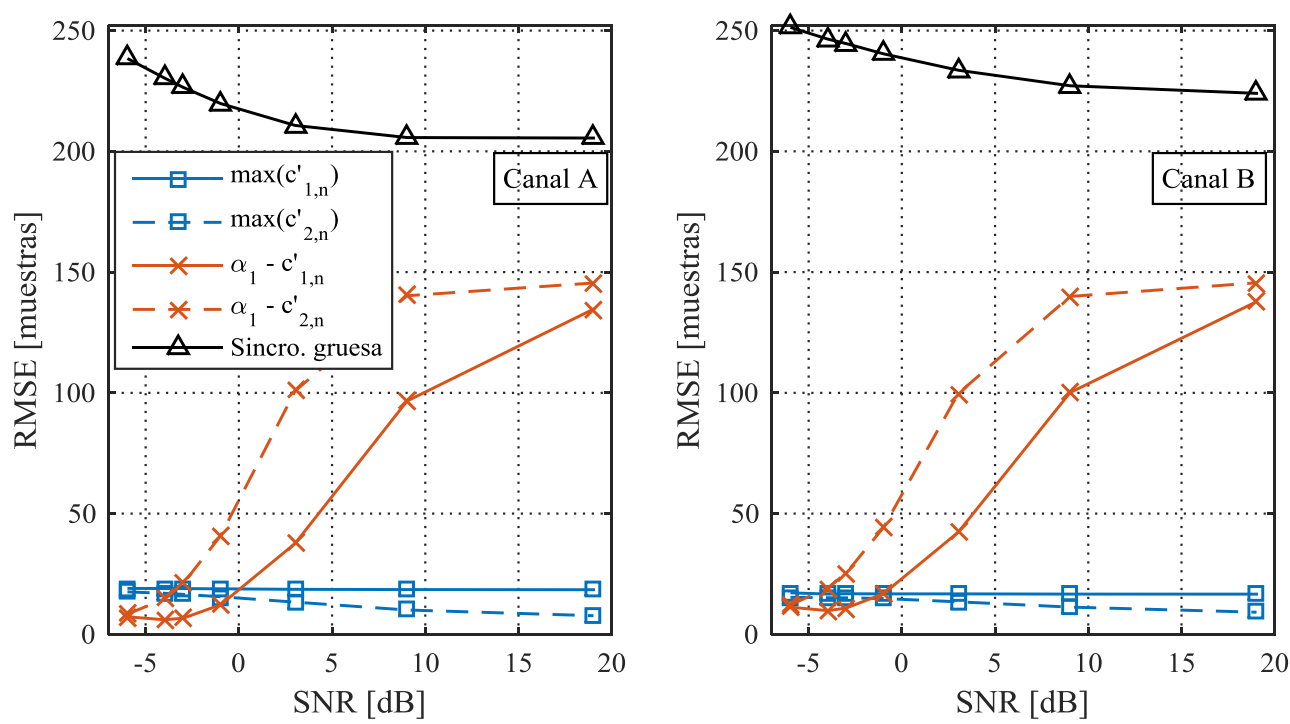

Fig. 6.22 RMSE de la sincronización fina considerando los umbrales $\alpha_{1}$ para las dos variantes de CCN, la ubicación del máximo de cada una, y el resultado de la etapa de sincronización gruesa, para los canales tipo A (izquierda y B (derecha). 


\subsubsection{Propuesta de ajuste de umbral para sincronización fina}

Teniendo en cuenta que la aplicación de la máscara espectral de transmisión produce una importante degradación del desempeño del algoritmo de sincronización fina, resulta necesario desarrollar una nueva técnica de umbralización que implique una mejora en el desempeño. El factor preponderante en el error de estimación es la interferencia debido a la extensión en el tiempo de la CIR, por encima del ruido presente en las muestras de la señal recibida. Así, se incrementa la probabilidad de falsa detección de las muestras previas a la correspondiente al primer camino del canal, disparando el error de estimación. Como el cómputo de las $\mathrm{CCN}$ de acuerdo a (6.30) se basa en la correlación cruzada, en la Fig. 6.23 se presentan los valores de la correlación del símbolo piloto propuesto, normalizada respecto de su valor máximo, al aplicar la máscara espectral de transmisión. Es importante destacar que la interferencia introducida depende no sólo de la función de correlación del símbolo piloto, sino también de la amplitud de los coeficientes del canal, y a la vez de la correlación existente entre coeficientes próximos. Por lo tanto, se propone definir un umbral dinámico que se ajuste a partir del valor máximo de las dos variantes de $\mathrm{CCN}$ de acuerdo a:

$$
\begin{aligned}
& \alpha_{k_{1}}=k_{t h} \cdot c^{\prime}{ }_{1, n_{f_{1}}} \\
& \alpha_{k_{2}}=k_{t h} \cdot c^{\prime}{ }_{2, n_{f_{1}}}
\end{aligned}
$$

donde $k_{t h} \in(0,1)$ es el factor que define el valor del umbral respecto del máximo obtenido a partir de cada CCN. El valor del factor se ve acotado por la relación de compromiso entre obtener una buena capacidad de detección de caminos del canal de menor amplitud que el máximo, y por la probabilidad de detectar erróneamente muestras previas debido a la dispersión temporal de la CC. Bajo estas consideraciones, y de acuerdo a la CC del símbolo piloto de la Fig. 6.23, se propone un valor de factor $k_{t h}=0,15$, que en principio otorga una baja probabilidad de detección de muestras demasiado alejadas al instante de llegada del camino del canal.

En las Fig. 6.24 y Fig. 6.25 se presenta el desempeño de la sincronización fina a partir del umbral propuesto en términos de la PSP y del RMSE, respectivamente. Puede observarse como la técnica de umbralización propuesta se comporta significativamente mejor para la $\mathrm{CCN} c_{1, n}^{\prime}$ que $c^{\prime}{ }_{2, n}$, tanto en términos de la PSP como del RMSE, como sucede también para el umbral $\alpha_{1}$. Por otro lado, la CCN $c_{2, n}^{\prime}$ presenta una complejidad 
Diseño de Estrategias de Sincronización y Estimación de Canal para la Mejora de Comunicaciones en Redes Inteligentes de Energía

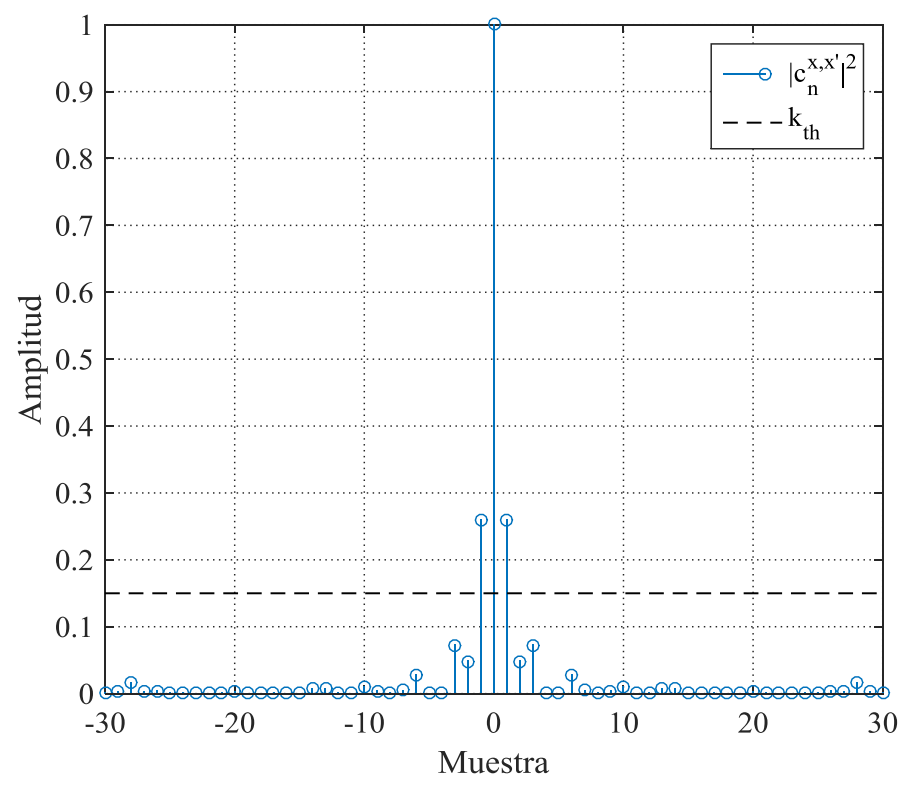

Fig. 6.23 Muestras de la correlación cruzada del símbolo piloto propuesto, luego de aplicar la máscara espectral de transmisión.

mayor debido al cómputo de (6.18) a partir de $c^{\prime}{ }_{1, n}$, por lo que se propone a la CCN $c_{1, n}^{\prime}$ como la opción más atractiva para aplicar el algoritmo de sincronización fina.

Comparando la sincronización fina bajo el umbral $\alpha_{k_{1}}$ respecto a utilizar $\alpha_{1}$, el desempeño es significativamente superior a altas $S N R$, mientras que para bajos valores de $S N R$ el umbral $\alpha_{1}$ obtiene mejores resultados. Esto se debe a que, para muy bajos valores de $S N R$, la interferencia por dispersión temporal del canal resulta despreciable respecto al efecto del ruido coloreado del canal PLC, y mientras que el umbral $\alpha_{1}$ fue derivado considerando únicamente el efecto del ruido, los umbrales $\alpha_{k_{1}}$ y $\alpha_{k_{2}}$ fueron obtenidos considerando como factor preponderante el efecto de la dispersión del canal. Teniendo esto en cuenta, y en caso de conocer (a partir de una estimación) la condición de $S N R$, entonces se propone modificar la técnica de umbralización para la $\mathrm{CCN} c^{\prime}{ }_{1, n}$ para mejorar el desempeño para bajos valores de $S N R$, de acuerdo al siguiente criterio:

$$
\begin{gathered}
\alpha_{k_{1}-\text { mod. }}=k_{t h} \cdot c^{\prime}{ }_{1, \widetilde{\theta}_{f_{1}}{ }^{\prime}} \quad \text { si } k_{t h} \cdot c^{\prime}{ }_{1, \widetilde{\theta}_{f_{1}}}>\alpha_{1, c_{1, n}^{\prime}} \\
\alpha_{k_{1}-\text { mod. }}=\alpha_{1}, \quad \text { si } k_{t h} \cdot c^{\prime}{ }_{1, \widetilde{\theta}_{f_{1}}} \leq \alpha_{1, c_{1, n}^{\prime}}
\end{gathered}
$$

donde $\alpha_{1, c_{1, n}^{\prime}}$ es el umbral de (6.19) que requiere el conocimiento de $S N R$. El criterio aplicado se resume a la restricción que el umbral dinámico no sea inferior al umbral $\alpha_{1, c_{1, n}^{\prime}}$ correspondiente, y se fundamenta en el hecho de que, para altos valores de $S N R$, el umbral $\alpha_{1, c_{1, n}^{\prime}}$ resulta demasiado pequeño como para evitar la interferencia por 
Capítulo 6: Sincronización y estimación de canal en sistemas DMT-PLC bajo una máscara espectral de transmisión
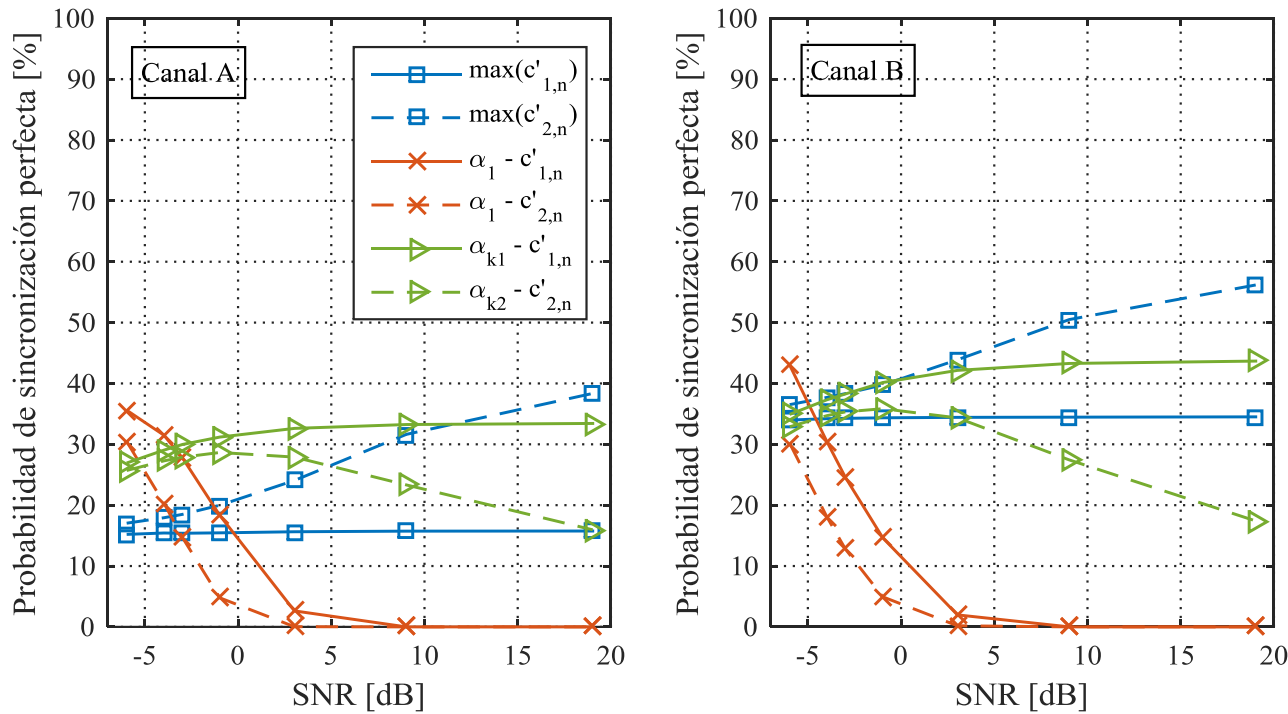

Fig. 6.24 Probabilidad de sincronización fina perfecta considerando los umbrales $\alpha_{k}$ para las dos variantes de CCN, los umbrales $\alpha_{1}$ y la ubicación del máximo, para los canales tipo A (izquierda y $B$ (derecha).
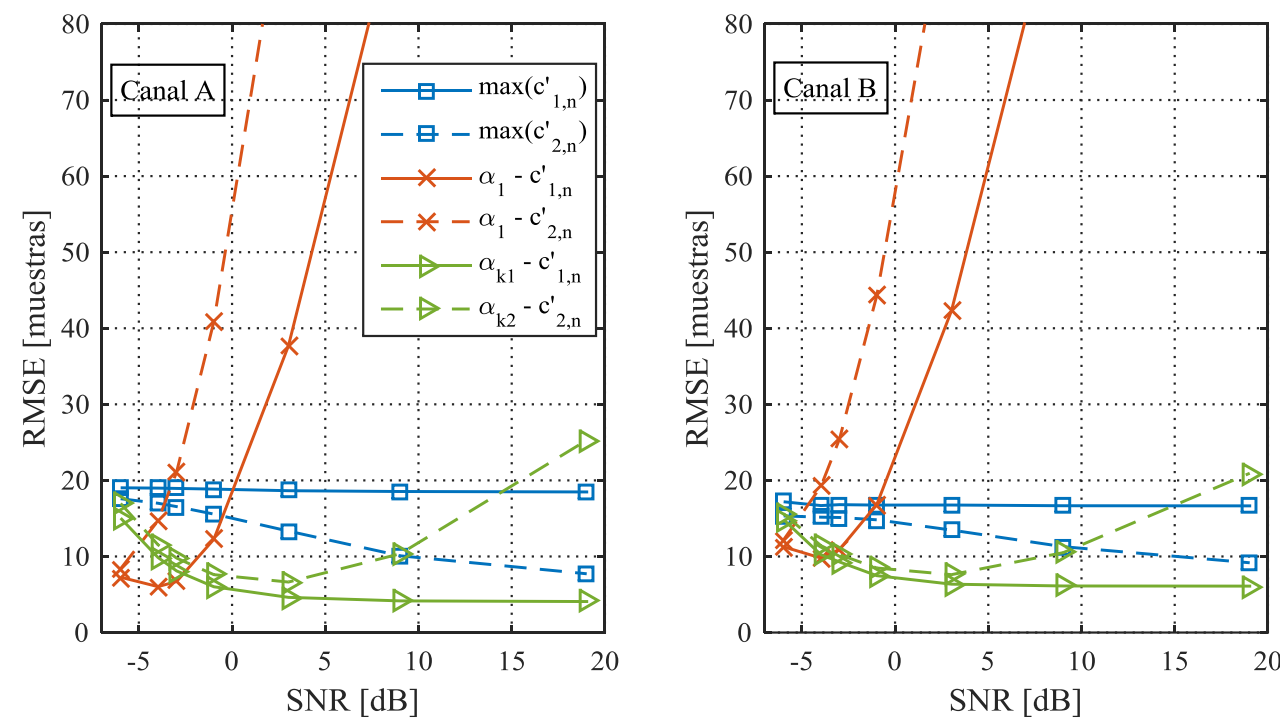

Fig. 6.25 RMSE de la sincronización fina considerando los umbrales $\alpha_{k}$ para las dos variantes de CCN, los umbrales $\alpha_{1}$ y la ubicación del máximo, para los canales tipo A (izquierda y B (derecha).

dispersión de la CIR, mientras que para bajos valores de $S N R$ el umbral $\alpha_{k_{1}}$ resulta en promedio demasiado bajo al no considerar el efecto del ruido que se incrementa a medida que disminuye $S N R$.

La técnica propuesta posee la ventaja de que utiliza el valor inicial del algoritmo de sincronización fina y lo multiplica por un factor constante, independiente de la condición de $S N R$, resultando en una alternativa de baja complejidad que logra un 
Diseño de Estrategias de Sincronización y Estimación de Canal para la Mejora de Comunicaciones en Redes Inteligentes de Energía

aceptable desempeño. Si además se dispone de un estimador de la $S N R$, la técnica de umbralización modificada permite mejorar el desempeño para bajos valores de $S N R$. Sin embargo, pueden desarrollarse estrategias para definir umbrales que se ajusten a la condición de $S N R$ para obtener resultados superiores. En esa línea, se obtuvieron cuatro variantes de umbrales a partir de simulaciones de Monte Carlo cuyos desempeños resultaran óptimos para maximizar la PSP, o bien minimizar el MSE, de acuerdo a:

$$
\begin{gathered}
\alpha_{P S P}(S N R) \\
\alpha_{M S E}(S N R) \\
\alpha_{k-P S P}=k_{t h-P S P}(S N R) \cdot c_{1, \widetilde{\theta}_{f_{1}}} \\
\alpha_{k-M S E}=k_{t h-M S E}(S N R) \cdot c_{1, \widetilde{\theta}_{f_{1}}}^{\prime}
\end{gathered}
$$

donde $\alpha_{P S P}$ y $\alpha_{M S E}$ son umbrales fijos para cada valor de $S N R$ que maximizan la PSP y minimizan el RMSE, respectivamente; $\alpha_{k-P S P}$ y $\alpha_{k-M S E}$ son umbrales dinámicos que, al igual que el umbral propuesto $\alpha_{k_{1}}$, se obtienen como resultado de multiplicar el máximo de la CCN por los factores óptimos $k_{t h-P S P} \mathrm{y} k_{t h-M S E}$. Los factores se fijan en función de la condición de $S N R$, y se obtienen a partir de los mismos criterios de optimización que los umbrales anteriores. En todos los casos, se efectuaron 50000 realizaciones de canal para cada valor de SNR. En la Tabla 6.2 se presentan los valores de los umbrales $\alpha_{P S P}$ y $\alpha_{M S E}$ y de los factores de umbralización $k_{t h-P S P}$ y $k_{t h-M S E}$ para los canales PLC tipo A y B con ruido de fondo coloreado, y distintos valores de SNR. A su vez, en la Fig. 6.26 y Fig. 6.27 se presenta el desempeño de la sincronización fina a de las distintas estrategias de umbralización en términos de la PSP y del RMSE, respectivamente.

En primer lugar, se verifica que la estrategia de umbralización propuesta modificada $\alpha_{k_{1}-\text { mod. }}$ produce una mejora respecto a $\alpha_{k_{1}}$ en el desempeño para bajos valores de $S N R$ tanto en términos de la PSP como del RMSE, sin alterar el desempeño para valores de $S N R$ altos.

Con respecto a los factores de umbralización óptimos $\alpha_{k-P S P}$ y $\alpha_{k-M S E}$, se observa que los valores decrecen a medida que se incrementa la $S N R$, y que los factores $\alpha_{k-P S P}$ son siempre mayores a los respectivos $\alpha_{k-M S E}$ para cualquier valor de $S N R$ en cada uno de los dos tipos de canal. Lógicamente, para maximizar la PSP el valor del factor debe 
Capítulo 6: Sincronización y estimación de canal en sistemas DMT-PLC bajo una máscara espectral de transmisión

Tabla 6.2 Valores de umbrales de sincronización fina para los canales PLC tipo A y B bajo ruido de fondo coloreado.

\begin{tabular}{|c|c|c|c|c|c|c|}
\hline & \multicolumn{6}{|c|}{ SNR } \\
\hline & $-6 \mathrm{~dB}$ & $-4 d B$ & $-1 d B$ & $3 \mathbf{d B}$ & $9 \mathrm{~dB}$ & $19 \mathrm{~dB}$ \\
\hline$k_{t h}$ & 0,15 & 0,15 & 0,15 & 0,15 & 0,15 & 0,15 \\
\hline$k_{t h-P S P / A}$ & 0,333 & 0,309 & 0,302 & 0,292 & 0,291 & 0,284 \\
\hline$k_{t h-M S E / A}$ & 0,293 & 0,233 & 0,195 & 0,168 & 0,151 & 0,144 \\
\hline$k_{t h-P S P / B}$ & 0,317 & 0,278 & 0,271 & 0,269 & 0,258 & 0,257 \\
\hline$k_{t h-M S E / B}$ & 0,282 & 0,248 & 0,182 & 0,149 & 0,141 & 0,125 \\
\hline$\alpha_{P S P / A}\left(\cdot 10^{-3}\right)$ & 6,09 & 8,11 & 12,34 & 18,92 & 25,44 & 28,22 \\
\hline$\alpha_{M S E / A}\left(\cdot 10^{-3}\right)$ & 4,42 & 5,21 & 6,42 & 8,95 & 10,74 & 11,89 \\
\hline$\alpha_{P S P / B}\left(\cdot 10^{-3}\right)$ & 8,94 & 12,17 & 18,26 & 26,25 & 35,23 & 38,65 \\
\hline$\alpha_{M S E / B}\left(\cdot 10^{-3}\right)$ & 4,58 & 5,53 & 6,89 & 8,74 & 10,74 & 11,89 \\
\hline
\end{tabular}

elevarse para evitar la detección de lóbulos laterales correspondientes al primer camino del canal. De hecho, los valores de $\alpha_{k-P S P}$ a altas $S N R$ se aproximan al valor que adopta la correlación del símbolo piloto en su primer lóbulo lateral, que puede observarse en la Fig. 6.23 con un valor aproximado de 0,26 respecto del máximo. También resulta que un factor que maximice la PSP no garantiza un desempeño óptimo en términos del RMSE. Así, mientras que el RMSE obtenido para $\alpha_{k-P S P}$ tiene un piso de aproximadamente 6 muestras para el canal A y 8 para el canal B, el factor de $\alpha_{k-M S E}$ reduce el piso a 4 y 6 muestras, respectivamente. Evidentemente, los factores de $\alpha_{k-M S E}$ disminuyen la PSP respecto de los umbrales $\alpha_{k-P S P}$.

Puede observarse que el desempeño del umbral propuesto $\alpha_{k_{1}}$ presenta una PSP inferior a las obtenidas por $\alpha_{k-P S P}$ en todo el rango de SNR considerado, y un mayor RMSE que el obtenido a partir de $\alpha_{k-M S E}$, como es de esperarse. Para altos valores de $S N R$, el RMSE que se obtiene para $\alpha_{k_{1}}$ se aproxima a los obtenidos para $\alpha_{k-M S E}$ en los dos

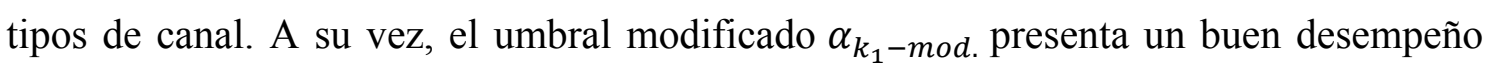
en términos del RMSE en todo el rango de $S N R$ bajo estudio. En ese sentido, resulta en general más provechoso mantener un RMSE reducido que aumentar la PSP, ya que la 
Diseño de Estrategias de Sincronización y Estimación de Canal para la Mejora de Comunicaciones en Redes Inteligentes de Energía
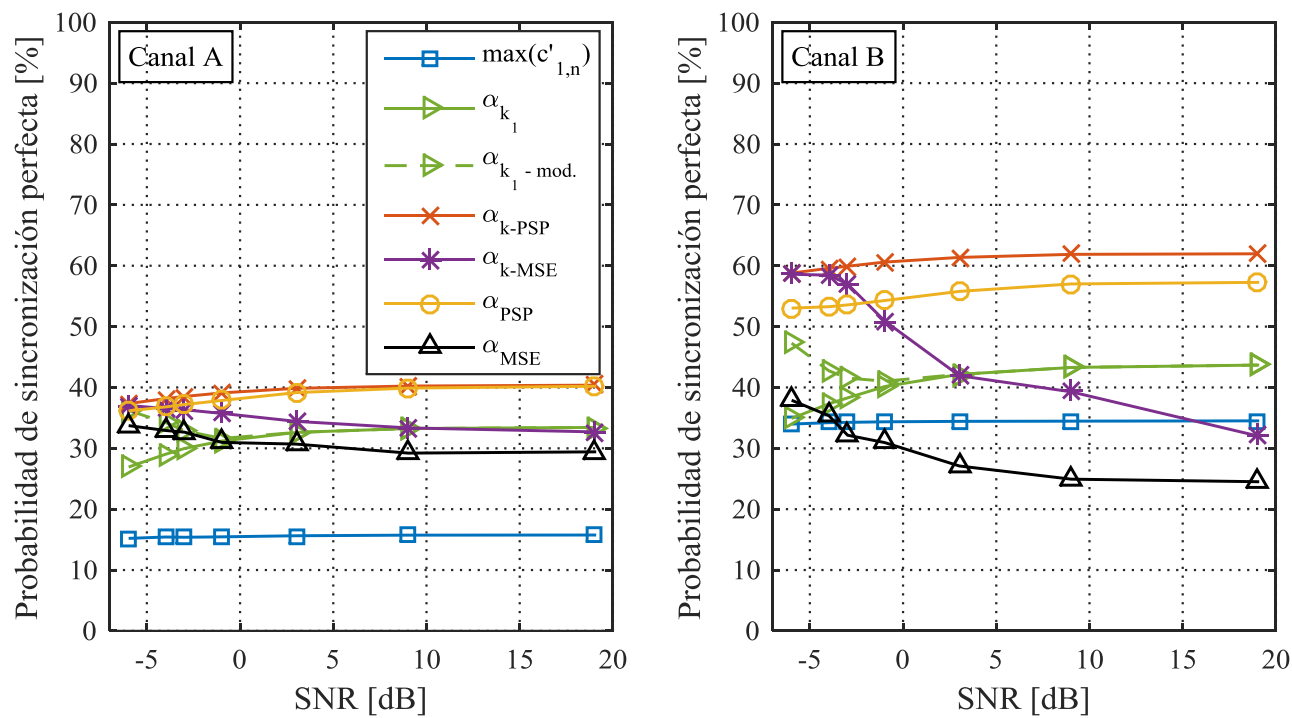

Fig. 6.26 Probabilidad de sincronización fina perfecta bajo distintas estrategias de umbralización para los canales tipo A (izquierda y $B$ (derecha).
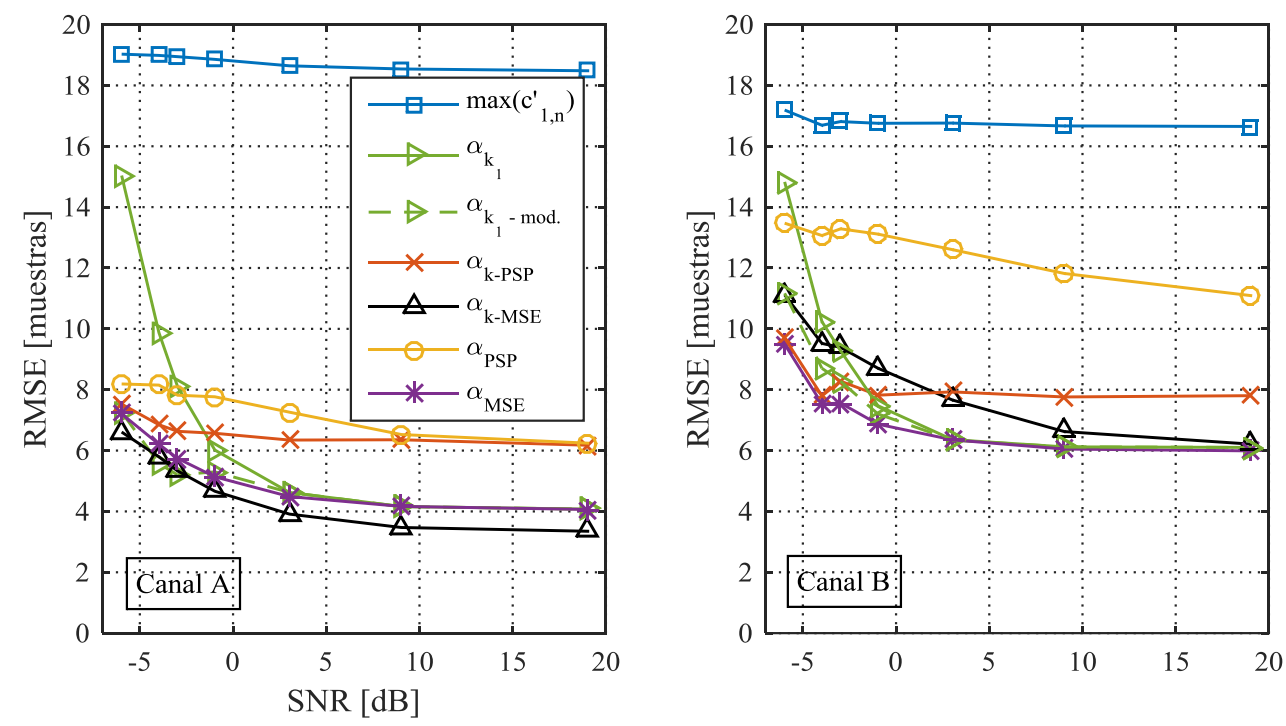

SNR $[\mathrm{dB}]$

Fig. 6.27 RMSE de la sincronización fina bajo distintas estrategias de umbralización para los canales tipo A (izquierda y B (derecha).

reducción en la sincronización perfecta se produce principalmente por la detección de muestras previas pero cercanas a la llegada del primer camino del canal. Estos desvíos, si se mantienen acotados, no producen ISI y se compensan en la etapa de estimación de canal posterior a la sincronización, en la que se estima el canal afectado por el retardo correspondiente al error en la sincronización temporal.

Con respecto al orden de magnitud del RMSE máximo, de 15 muestras para $\alpha_{k_{1}}$ y 7 muestras para $\alpha_{k_{1}-\text { mod. }}$, representan alrededor del 3\% y $1,4 \%$ de las 512 muestras de 
longitud del prefijo cíclico del sistema multiportadora. Si el sistema fue correctamente diseñado, el margen de zona libre de ISI de $N_{c p}-L$ muestras previas al instante de sincronismo óptimo debería ser superior. Los modelos de los canales A y B incluyen una dispersión máxima de 400 muestras para la CIR, lo que implica un margen en simulación de 112 muestras.

Finalmente, se agregaron los resultados obtenidos para cada valor de $S N R$ bajo los umbrales fijos que maximizan la PSP y minimizan el RMSE: $\alpha_{P S P}$ y $\alpha_{M S E}$, para verificar si existe una diferencia importante entre la estrategia de umbralización a partir del valor máximo de la $\mathrm{CCN}$ con respecto a definir umbrales estáticos para cada condición de $S N R$. En términos de la PSP, se obtiene para los dos tipos de canal un valor mayor para el umbral $\alpha_{k-P S P}$ que para $\alpha_{P S P}$, en todo el rango de $S N R$. Con respecto al RMSE, el umbral $\alpha_{M S E}$ presenta un desempeño superior respecto a $\alpha_{k-M S E}$ para el caso del canal A, pero no así para el canal B.

\subsubsection{Efecto del ruido impulsivo en la sincronización fina}

Un evento de ruido impulsivo en el canal PLC afectará la CCN y por lo tanto el proceso de sincronización fina, de manera similar que a la etapa de sincronización gruesa. Por un lado, cuando ocurre un evento de ruido impulsivo, los valores que adopta la CCN en las muestras correspondientes a la CIR del canal disminuyen significativamente, debido al incremento del denominador de (6.17). A su vez, también se espera una atenuación en los valores de la $\mathrm{CCN}$ en las muestras previas al instante de sincronismo óptimo. Nuevamente, como la probabilidad de ocurrencia de un evento de ruido impulsivo es relativamente baja, deben mantenerse los umbrales de detección obtenidos para el sistema cuando no ocurren eventos de ruido impulsivo. Adicionalmente, se puede reducir el efecto nocivo del ruido impulsivo a partir de las técnicas de mitigación por procesamiento no lineal, o bien a partir del AGC. No obstante, aunque no se realiza en este trabajo, podría desarrollarse un algoritmo que permita detectar y cuantificar los eventos de ruido, y reducir el valor del umbral de detección en consecuencia.

Para analizar el desempeño de la etapa de sincronización fina bajo ruido impulsivo con probabilidad de ocurrencia del $8 \%$, se incluye el resultado de simulaciones de dicho algoritmo considerando los umbrales $\alpha_{k_{1}}$ y $\alpha_{k_{1}-\text { mod. }}$. En la Fig. 6.28 se representan los valores de RMSE obtenidos bajo los umbrales de detección $\alpha_{k_{1}}$ y $\alpha_{k_{1}-\text { mod, }}$ y cuatro escenarios distintos respecto de la condición del ruido presente en la señal recibida: con 

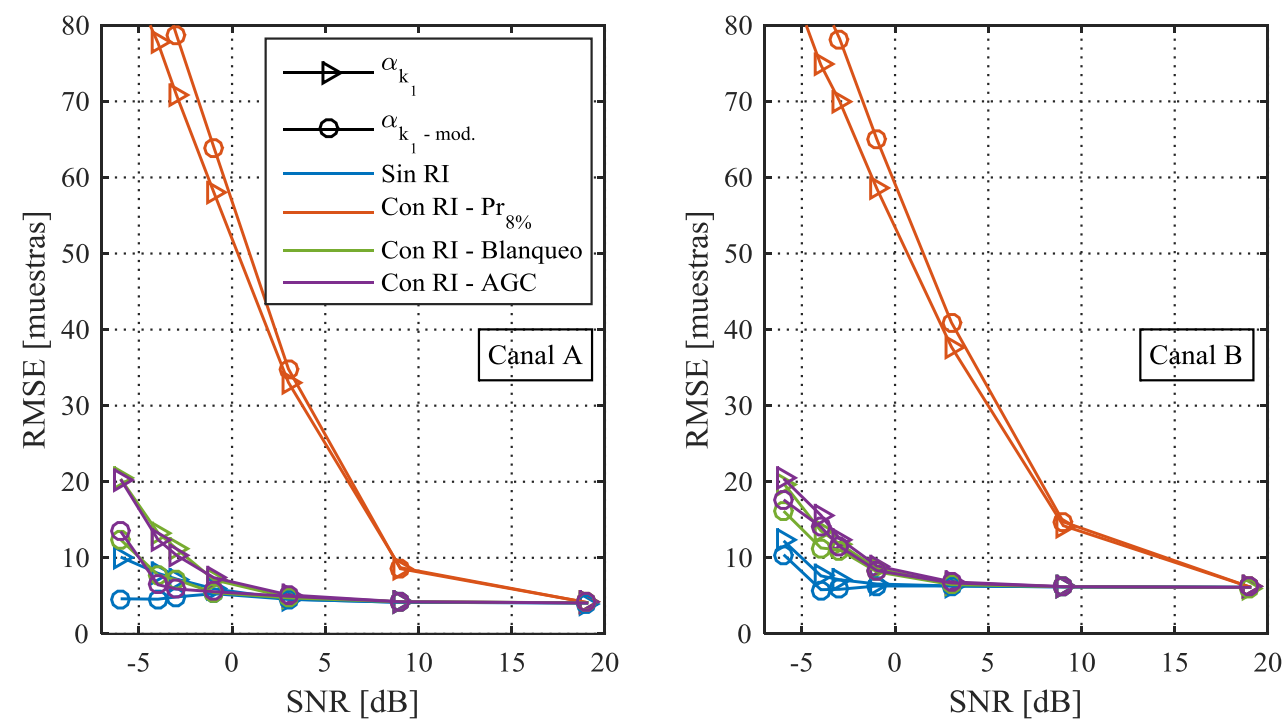

Fig. 6.28 Desempeño de la etapa de sincronización fina en términos del RMSE, para los canales tipo A (izquierda y B (derecha), con ruido de fondo coloreado e impulsivo.

ruido de fondo coloreado sin ruido impulsivo; ruido de fondo coloreado con eventos de ruido impulsivo con probabilidad de ocurrencia típica del $8 \%$; y ruido de fondo coloreado con eventos de ruido impulsivo mitigados a partir de la técnica basada en AGC; o bien por blanqueo no lineal. Puede observarse como el RMSE se dispara a bajas $S N R$ cuando se incluye el ruido impulsivo, y luego las técnicas de mitigación reducen el error acercándolo al RMSE del escenario sin ruido impulsivo. En todos los casos, el RMSE obtenido a partir del método de blanqueo es ligeramente inferior al obtenido por la estrategia basada en AGC, principalmente porque el umbral de blanqueo se define en simulaciones de manera ideal a partir del conocimiento de la amplitud esperada por la señal recibida sin ruido impulsivo, lo que no es factible en la práctica, como se menciona en la sección 4.3.6.2.

El valor de RMSE obtenido por la propuesta de sincronización fina aplicando la máscara espectral de transmisión y el filtro notch en recepción resulta ligeramente superior, pero de orden de magnitud similar al obtenido para el escenario en que se transmite en todo el ancho de banda teóricamente disponible, a partir de un valor de $S N R$ de $3 \mathrm{~dB}$. En esa línea, el lector puede comparar la Fig. 6.28 con la Fig. 5.16 de la sección 5.1.3.3.

Evidentemente, la aplicación de la máscara espectral de transmisión distorsiona las propiedades de correlación del símbolo piloto y, por ende, el error promedio cometido es mayor que en el caso en que se transmite el símbolo completo. Sin embargo, el error 
no se dispara porque la amplitud al cuadrado de la ACS del símbolo piloto, utilizada en el cómputo de la $\mathrm{CCN}$, aún conserva un lóbulo principal importante y se atenúa rápidamente, por lo que, cuando se comete un error de sincronismo adicional por detección de un lóbulo lateral, suele ser de unas pocas muestras y con baja probabilidad de ocurrencia. Adicionalmente, para bajos valores de $S N R$, el principal limitante del sistema está dado por la baja probabilidad de detección en la etapa de sincronización gruesa, como se analizó a partir de la Fig. 6.19. Por su parte, puede concluirse que, si bien un evento de ruido impulsivo degrada considerablemente el desempeño para bajos valores de $S N R$, resulta factible aplicar las técnicas de mitigación bajo estudio.

\subsection{Estimación de canal bajo una máscara espectral de transmisión}

La aplicación de la máscara espectral de emisión afecta, no sólo a la estrategia de sincronización, sino también a la etapa de estimación de canal. El recorte espectral del símbolo piloto transmitido provoca una degradación en las propiedades de correlación del mismo (Fig. 6.3), afectando la zona de correlación nula. Las subportadoras anuladas por la máscara provocan que la estimación de la respuesta al impulso del canal en la salida del filtro acoplado se extienda en el tiempo, debido a las transiciones abruptas en el dominio de la frecuencia. Este fenómeno se presentó en la Fig. 6.20, donde se presenta la salida del filtro acoplado con y sin la aplicación de la máscara espectral, en condiciones ideales sin el agregado de ruido para una realización del canal PLC tipo A. Como la respuesta al impulso del canal no se encuentra contenida en las $N_{c s}$ muestras posteriores al instante de sincronismo óptimo, se comete un gran error si se aplica directamente la técnica de estimación de canal presentada en el capítulo 5 basada en la correlación cruzada del símbolo piloto, aún bajo una situación de sincronismo perfecto, dada por:

$$
\tilde{h}_{n}=\frac{1}{\sqrt{P_{T}} N} c_{n+n_{\text {opt }}}^{\mathbf{r}, \mathbf{x}^{\prime}}, \quad 0 \leq n \leq N_{c s}-1
$$

donde $\tilde{h}_{n}$ representa el valor estimado del $n$-ésimo elemento de la CIR, $P_{T}$ es la potencia transmitida y $N$ el tamaño de FFT del sistema DMT. En la Fig. 6.29 se presentan los resultados de la estimación del canal al aplicar la máscara espectral de transmisión, de la propuesta inicial denominada CPS-CC, y puede apreciarse la degradación de desempeño respecto al caso en que no se aplica la máscara. Para 
Diseño de Estrategias de Sincronización y Estimación de Canal para la Mejora de Comunicaciones en Redes Inteligentes de Energía

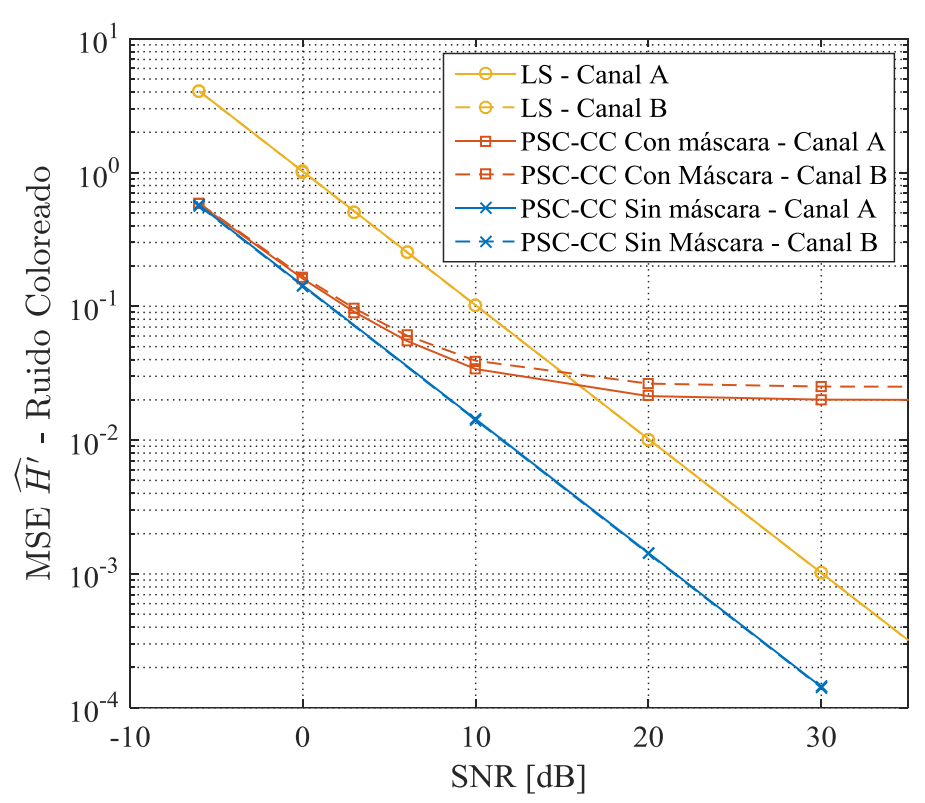

Fig. 6.29 MSE de la estimación de canal en las subportadoras activas, para el sistema perfectamente sincronizado, con y sin máscara espectral de transmisión aplicada.

comparar ambas situaciones, se computa el MSE de la estimación de la respuesta en frecuencia del canal $\mathbf{H}^{\prime}$, que contiene únicamente al subconjunto de subportadoras activas definidas por la máscara espectral. Así, el error se computa de acuerdo a:

$$
M S E_{\widetilde{\mathbf{H}}^{\prime}}=\frac{1}{N_{a}} E\left\{\left\|\mathbf{H}^{\prime}-\widetilde{\mathbf{H}}^{\prime}\right\|^{2}\right\}
$$

Puede observarse cómo el truncamiento de la respuesta al impulso del canal conlleva a un piso de error a altos valores de $S N R^{3}$, ligeramente superior en el caso del canal PLC tipo B. Adicionalmente, se añade el MSE de la estimación de canal aplicando la técnica clásica LS, y se aprecia cómo al aplicar la máscara espectral la técnica propuesta presenta un mayor error para valores de $S N R$ mayores a $15 \mathrm{~dB}$. El diseño del símbolo piloto propuesto no fue optimizado para una estimación en el dominio de la frecuencia, por lo que la estimación se efectúa a partir de un símbolo piloto de amplitud constante en el dominio de la frecuencia basado en una secuencia Zadoff-Chu ${ }^{4}$ de longitud $N$.

La degradación en el desempeño de la técnica de estimación de canal propuesta al

\footnotetext{
3 Recuérdese que $S N R$ es la relación señal a ruido equivalente al aplicar la máscara espectral de transmisión y el filtro notch en recepción.

4 El MSE obtenido utilizando una secuencia Zadoff-Chu es el mismo que para cualquier símbolo piloto de amplitud constante en el dominio de la frecuencia.
} 
aplicar la máscara espectral de transmisión es tal que impide su aplicabilidad. Por lo tanto, debe desarrollarse alguna modificación que permita mejorar el desempeño, intentando a su vez que no se incremente demasiado la complejidad asociada al proceso de estimación.

La problemática de estimación de canal con subportadoras inactivas ha sido ampliamente investigada. Cuando se utilizan estructuras de subportadoras piloto tipo peine, en las que se obtiene la estimación en un determinado número de subportadoras piloto mientras el resto de las subportadoras disponibles se utilizan para transmitir datos, se puede realizar una interpolación a partir de la estimación en las subportadoras piloto para obtener la estimación de la respuesta en frecuencia en las restantes. Las técnicas de interpolación más utilizadas son la interpolación lineal, cúbica, segmentaria (splines) y por filtro pasa bajos [Bogdanović 2014], [Mousa 2010]. Por otro lado, la problemática que generan las subportadoras inactivas en la estimación de canal basada en DFT se analiza en [Xiong 2013], y es el mismo efecto distorsivo que se produce en la estimación de canal en el dominio del tiempo a partir de la salida del filtro acoplado al símbolo piloto del esquema propuesto. Para mitigar la distorsión, pueden completarse las subportadoras inactivas a partir de alguna técnica de interpolación, o bien asignando valores que minimicen algún criterio de error en la estimación, como se propone en [Huang 2006]. A continuación, se presentan alternativas a la estimación de canal propuesta, en las que se estima la CFR en las subportadoras inactivas para reducir el piso de error que se observa en la Fig. 6.29.

\subsubsection{Estimación de canal LS-DFT}

La estrategia denominada LS-DFT es una particular entre las desarrolladas en [Huang 2006], que no demanda el conocimiento de las propiedades estadísticas del canal en términos de la matriz de autocorrelación, ni del nivel de ruido. La técnica es una estimación de canal basada en DFT, es decir, que efectúa una primera estimación en el dominio de la frecuencia $\widetilde{\mathbf{H}}$, para luego aplicar la IDFT de $N$ puntos y obtener un vector $\tilde{\mathbf{h}}$ que contiene la estimación de la CIR. Esto permite descartar muestras ruidosas del vector que no contienen coeficientes de la CIR, cuya máxima dispersión temporal se asume menor que la longitud del prefijo cíclico. Para que la técnica tenga éxito, se debe evitar que la CIR del canal presente un fenómeno de extensión en el tiempo debido a las $N_{\text {vir }}$ subportadoras inactivas (virtuales). Para ello, propone primero estimar la CFR en 
Diseño de Estrategias de Sincronización y Estimación de Canal para la Mejora de Comunicaciones en Redes Inteligentes de Energía

las $N_{\text {act }}=N-N_{\text {vir }}$ subportadoras activas a partir de la técnica LS, y luego completar las subportadoras virtuales de manera tal que se minimicen los valores del vector $\tilde{\mathbf{h}}$ en las muestras que no contienen a la verdadera CIR, es decir, las muestras desde $N_{h}$ a $N$, donde $N_{h}$ es la longitud máxima de la CIR. Esto se traduce en resolver:

$$
\mathbf{F}_{a c t} \widetilde{\mathbf{H}}_{a c t}+\mathbf{F}_{v i r} \widetilde{\mathbf{H}}_{v i r}=\mathbf{0}
$$

donde $\widetilde{\mathbf{H}}_{\text {act }}$ es el vector columna que contiene la estimación LS en las subportadoras activas, $\mathbf{F}_{\text {act }}$ contiene las últimas $N-N_{h}$ filas de la matriz de IDFT de $N$ puntos, y las columnas correspondientes a la ubicación de las subportadoras activas. Por su parte, $\mathbf{F}_{\text {vir }}$ contiene las últimas $N-N_{h}$ filas de la matriz de IDFT, y las columnas correspondientes a la ubicación de las subportadoras inactivas. Por último, $\widetilde{\mathbf{H}}_{\text {vir }}$ es el vector de estimación de la CFR en las subportadoras inactivas que se desea obtener, y $\mathbf{0}$ es un vector columna de $N-N_{h}$ ceros. La solución por LS se obtiene entonces de acuerdo a:

$$
\widetilde{\mathbf{H}}_{v i r}=-\mathbf{F}_{v i r}^{+} \mathbf{F}_{a c t} \widetilde{\mathbf{H}}_{a c t}
$$

donde $\mathbf{F}_{v i r}^{+}$es la pseudoinversa de Moore-Penrose de $\mathbf{F}_{v i r}$. La combinación de la estimación en las subportadoras activas $\widetilde{\mathbf{H}}_{a c t}$ e inactivas $\widetilde{\mathbf{H}}_{v i r}$ proporcionan la estimación de la CFR completa $\widetilde{\mathbf{H}}_{t o t}$, a partir de la cual se aplica la técnica basada en DFT para obtener la estimación final en las subportadoras activas:

$$
\widetilde{\mathbf{H}}_{L S-D F T}^{\prime}=\mathbf{F}_{1}^{H} \mathbf{F}_{2} \widetilde{\mathbf{H}}_{t o t}
$$

donde $\mathbf{F}_{1}$ contiene las primeras $N_{h}$ filas de la matriz de IDFT, y las columnas correspondientes a la ubicación de las subportadoras activas; y $\mathbf{F}_{2}$ las primeras $N_{h}$ filas y las $N$ columas de la matriz de IDFT. En general, la longitud $N_{h}$ es una pequeña fracción de $N$, por lo que $N-N_{h}$ resulta mayor que la cantidad de subportadoras inactivas $N_{i}$, y se debe garantizar que el rango de $\mathbf{F}_{v i r}$ sea $N_{i}$. La estabilidad numérica del sistema de ecuaciones se garantiza para bajos valores de $N$, y de la relación de subportadoras inactivas $N_{i} / N$. Como referencia, en [Huang 2006] se realizan simulaciones con $N=64, N_{i}=12$, mientras que en la alternativa de estimación de [Xiong 2013] $N=256, N_{i}=148$.

En el caso del sistema PLC bajo estudio con $N=4096$, aplicando la máscara espectral de transmisión, de acuerdo a la Tabla 6.1, la cantidad de subportadoras inactivas resulta $N_{i}=2262$. Por su parte, la dispersión máxima del canal se asume desconocida y, por lo 


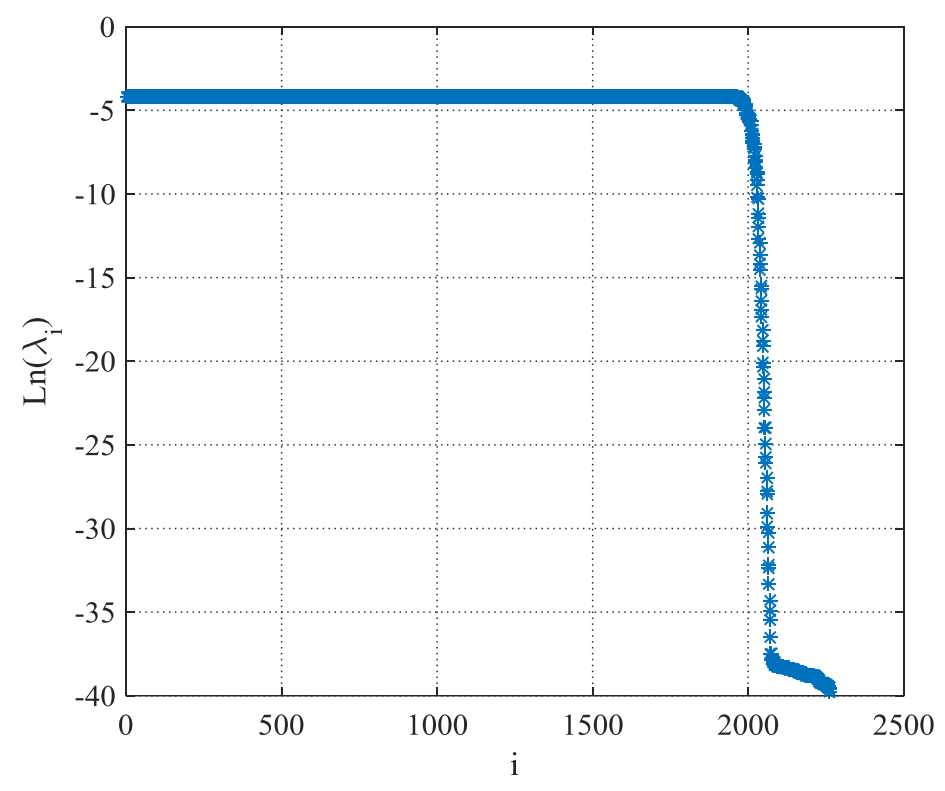

Fig. 6.30 Autovalores de la matriz del sistema lineal $\mathbf{F}_{\text {vir. }}$

tanto, se considera directamente igual a la longitud del prefijo cíclico $N_{h}=N_{c p}$. Así, $\mathbf{F}_{\text {vir }}$ resulta en una matriz de 3584 filas y 2262 columnas. El elevado número de ecuaciones e incógnitas del sistema provoca que $\mathbf{F}_{v i r}$ se encuentre próxima a la singularidad, provocando inestabilildad numérica en el cálculo de $\mathbf{F}_{v i r}^{+} \mathrm{y}$ resultados de estimación no confiables, aún con operaciones efectuadas en punto flotante. En la Fig. 6.30 se hace una representación de los autovalores $\lambda_{i}$ de $\mathbf{F}_{\text {vir }}$, donde se observa que varios resultan cercanos a cero.

Para poder aplicar el método mencionado, y teniendo en cuenta que la máscara impone una gran cantidad de subportadoras inactivas en frecuencias superiores a la última subportadora activa se propone reducir la cantidad de subportadoras inactivas utilizadas para la estimación, desestimando las de frecuencia superior. A su vez, se desestiman también algunas de las subportadoras inactivas de baja frecuencia hasta alcanzar una suficiente estabilidad numérica que genere resultados numéricos confiables en $\mathbf{F}_{v i r}^{+}$. Se considera resultado confiable cuando el producto $\mathbf{F}_{\text {vir }}^{+} \mathbf{F}_{\text {vir }}$ da como resultado una matriz aproximada a la identidad. Desestimando las primeras 3 subportadoras inactivas de baja frecuencia y las últimas 800 subportadoras de alta frecuencia, se obtiene un sistema reducido numéricamente estable, obteniendo una suma de errores absolutos calculados a partir de $\left|\mathbf{F}_{\text {vir }}^{+} \mathbf{F}_{\text {vir }}-\mathbf{I}\right|$ inferior a $10^{-3}$, donde $\mathbf{I}$ es la matriz identidad. En la Fig. 6.31 se aprecia la diferencia en las subportadoras inactivas utilizadas para el cálculo de la estimación de canal en el sistema completo (inestable) y con la reducción propuesta. 
Diseño de Estrategias de Sincronización y Estimación de Canal para la Mejora de Comunicaciones en Redes Inteligentes de Energía

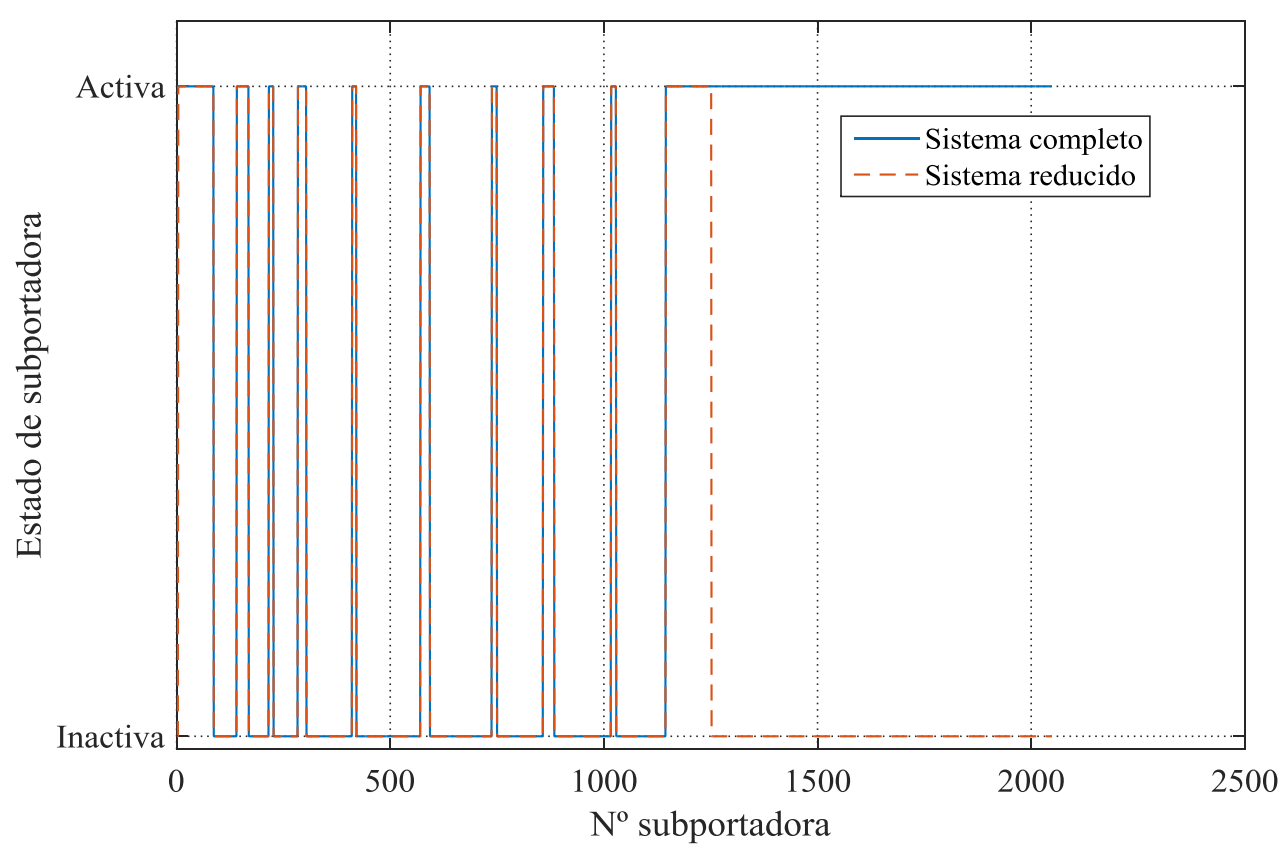

Fig. 6.31 Subportadoras inactivas pertenecientes al sistema de ecuaciones completo y reducción propuesta.

Los resultados obtenidos para esta técnica de estimación de canal LS-DFT se presentan en la Fig. 6.32 en términos del MSE. Puede apreciarse que el problema del elevado piso de error se ve sustancialmente reducido, por lo que para altos valores de $S N R$ implica una mejora respecto a la propuesta inicial de estimación de canal a partir de la CC del símbolo piloto. Sin embargo, para valores de $S N R$ menores a $6 \mathrm{~dB}$, la propuesta supera a la técnica LS-DFT en las condiciones analizadas. Adicionalmente, si bien existe una mejora sustancial respecto a la estimación clásica por LS, debe considerarse la alta complejidad asociada al cálculo de $\widetilde{\mathbf{H}}_{L S-D F T}^{\prime}$, puesto que para cada procesamiento del símbolo piloto, además del calcular la estimación por LS clásica $\widetilde{\mathbf{H}}_{a c t}$ que requiere $N_{\text {act }}$ multiplicaciones complejas, debe además calcularse el producto de (6.32) y luego aplicar las transformadas IDFT y DFT para aplicar la técnica de estimación por DFT de (6.33).

\subsubsection{Estimación de canal DFT con interpolación}

A continuación, se propone evaluar la efectividad de la estimación de canal basada en DFT cuando las subportadoras inactivas se completan por medio de interpolación (y extrapolación) a partir de la estimación en frecuencia de las suportadoras activas, a diferencia de la técnica de estimación LS-DFT en que las subportadoras inactivas se completaron a partir de un criterio LS. 


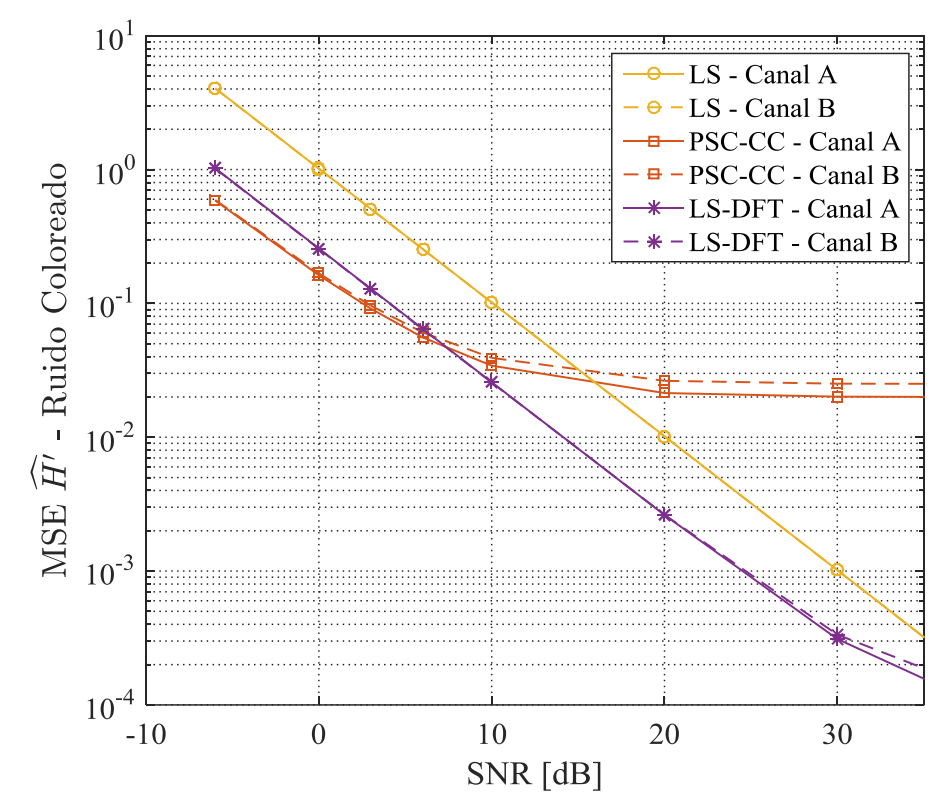

Fig. 6.32 MSE de la estimación de canal al aplicar la técnica LS-DFT.

El proceso de estimación de canal se basa entonces en tres etapas: primero, una estimación en el dominio de la frecuencia por LS en las subportadoras activas; luego, la interpolación en las subportadoras inactivas intermedias entre la primer y última subportadora activa, y extrapolación de las subportadoras inactivas de baja frecuencia hasta DC y de alta frecuencia hasta $N / 2$; y, finalmente, se extiende la estimación al tamaño $N$ aplicando simetría hermítica y se aplica la estimación basada en DFT.

La etapa distintiva del proceso de estimación respecto a la técnica LS-DFT es la interpolación y extrapolación. Las técnicas de interpolación incluidas en este trabajo son lineal, polinomial de Hermite a trozos (cúbica), y segmentaria cúbica (splines cúbicos); computadas en todos los casos a partir de la función interpl disponible en Matlab. La interpolación de las muestras complejas se realiza sobre las partes real e imaginaria de manera independiente. En la Fig. 6.33 se presenta la interpolación de las subportadoras inactivas intermedias por las tres técnicas, para una realización del canal tipo A y una condición de $S N R$ de $30 \mathrm{~dB}$. Puede observarse que en ningún caso la estimación en las subportadoras inactivas se aproxima al canal real, pero en los tres casos se suavizan considerablemente las transiciones abruptas en frecuencia.

Con respecto a las subportadoras inactivas de bajas y altas frecuencias (menores a la frecuencia de la primer subportadora activa y superiores a la frecuencia de la última subportadora activa), en primera instancia no es conveniente considerarlas nulas debido a que se producen transiciones abruptas en los límites de la banda de transmisión, 
Diseño de Estrategias de Sincronización y Estimación de Canal para la Mejora de Comunicaciones en Redes Inteligentes de Energía

generando una importante dispersión en el dominio del tiempo que afecta la estimación de canal basada en DFT. Por lo tanto, se debe extrapolar el resultado obtenido a partir de la etapa de interpolación de alguna manera.

No resulta conveniente utilizar las funciones de interpolación por splines y cúbica directamente para extrapolar, debido a que se produce una divergencia en los valores extrapolados, como se muestra en la Fig. 6.34 en los casos "Cúbica" y "Splines". Una

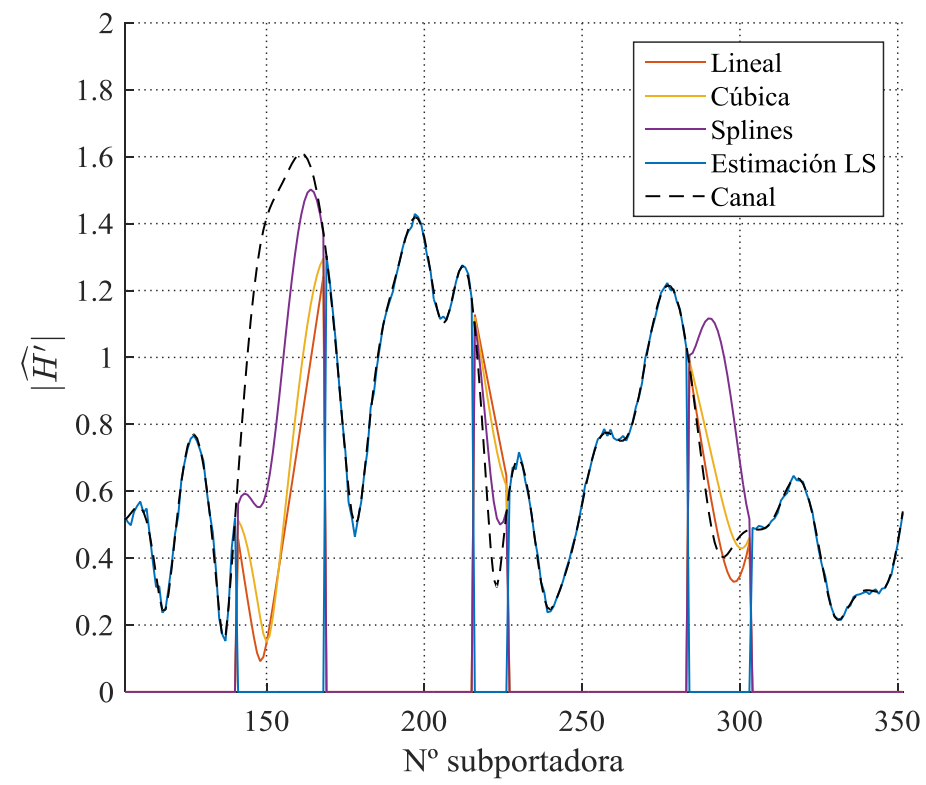

Fig. 6.33 Interpolación en el dominio de la frecuencia en las subportadoras inactivas intermedias para realización del canal tipo $A$ y $S N R=30 d B$.

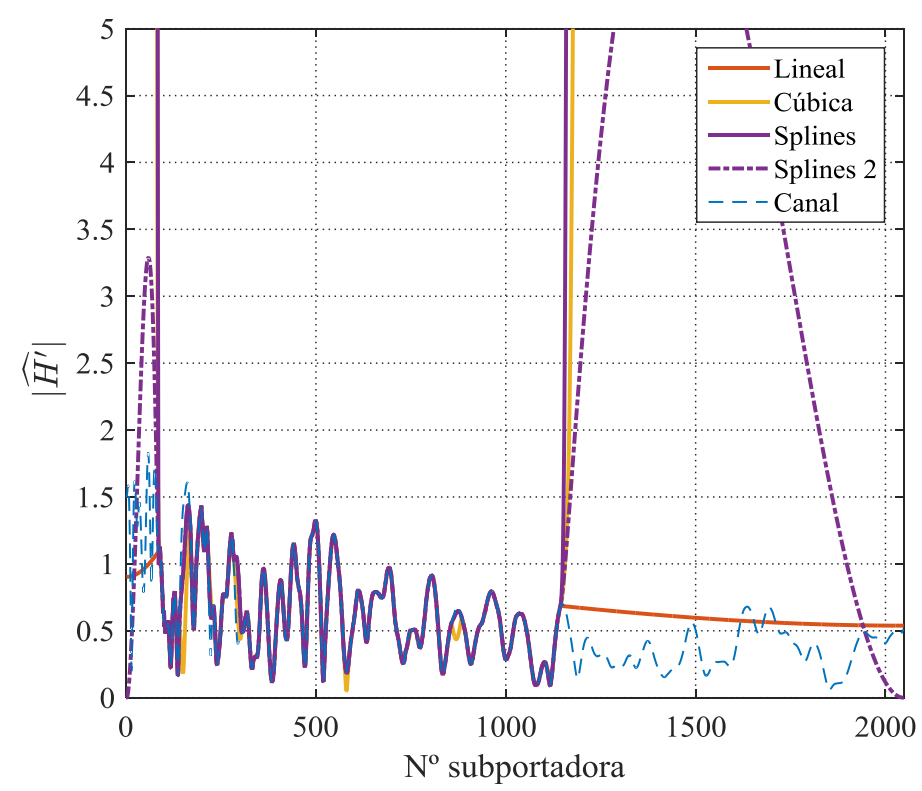

Fig. 6.34 Resultado de extrapolar las muestras obtenidas a partir de los tres métodos de interpolación utilizados para completar las subportadoras inactivas fuera de la banda de transmisión. 
Capítulo 6: Sincronización y estimación de canal en sistemas DMT-PLC bajo una máscara espectral de transmisión

opción es forzar a cero la primera y última subportadora, de manera que la extrapolación se encuentre contenida. Este caso se señala como "Splines 2" en la Fig. 6.34. Por último se agrega una extrapolación lineal en la que se modifica solamente la parte imaginaria. En todo el rango de extrapolación se mantiene constante la parte real de la estimación en la subportadora límite, mientras que la parte imaginaria se reduce linealmente, alcanzando el valor cero en la primera y última subportadora, respectivamente. Por un lado, permite lograr una atenuación suave de la respuesta en frecuencia, y además cumplir el requerimiento de la IDFT $\left\{\widetilde{H}_{0}, \widetilde{H}_{2048}\right\} \in \mathbb{R}$ para obtener una estimación de la CIR $\mathbf{h}$ real, al realizar la extensión con simetría hermítica para completar la IDFT de tamaño $N=4096$.

Luego de la interpolación y extrapolación, se aplica el proceso de IDFT como primer paso de la estimación de canal basada en DFT, obteniendo la estimación de la respuesta al impulso. En la Fig. 6.35 se presenta el resultado obtenido de la estimación de la respuesta al impulso al completar las subportadoras inactivas a partir de interpolación/extrapolación. Puede observarse que las curvas obtenidas aplicando cada uno de los tres tipos de interpolación resultan muy similares en el dominio del tiempo, y que se reduce la dispersión temporal de la CIR en las muestras previas al primer camino del canal, respecto a aplicar la IDFT sin completar las subportadoras inactivas. Sin embargo, como existe una parte de energía del canal en las muestras previas, resulta conveniente incluir una determinada cantidad $N_{\text {pre }}$ de muestras previas como parte del

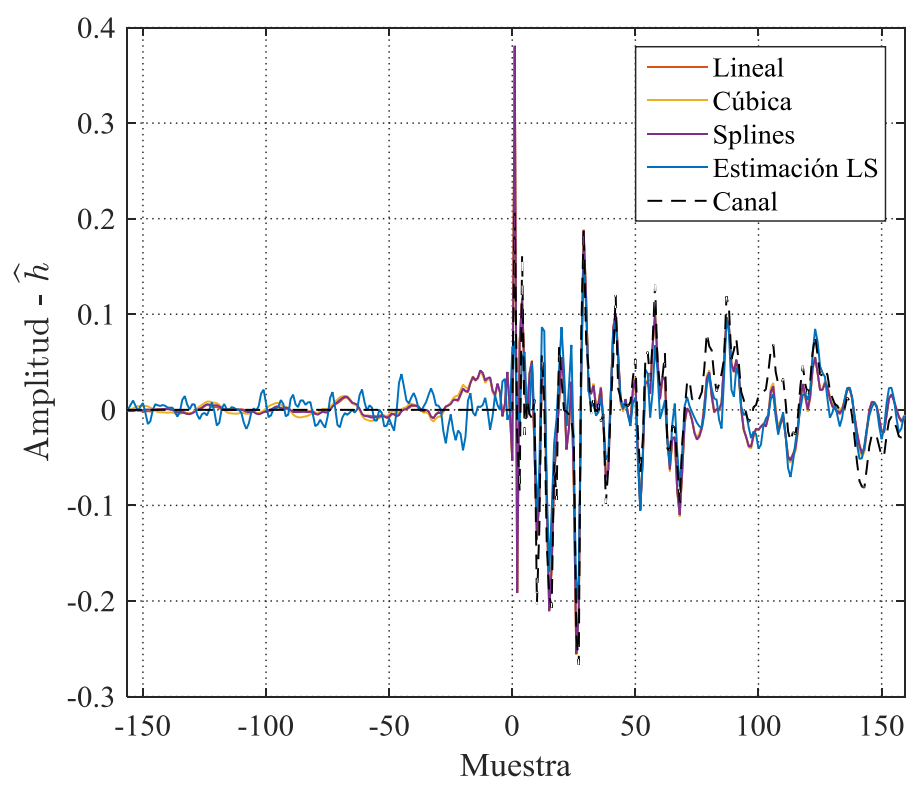

Fig. 6.35 Estimación de la respuesta al impulso luego del proceso de interpolación. 
Diseño de Estrategias de Sincronización y Estimación de Canal para la Mejora de Comunicaciones en Redes Inteligentes de Energía

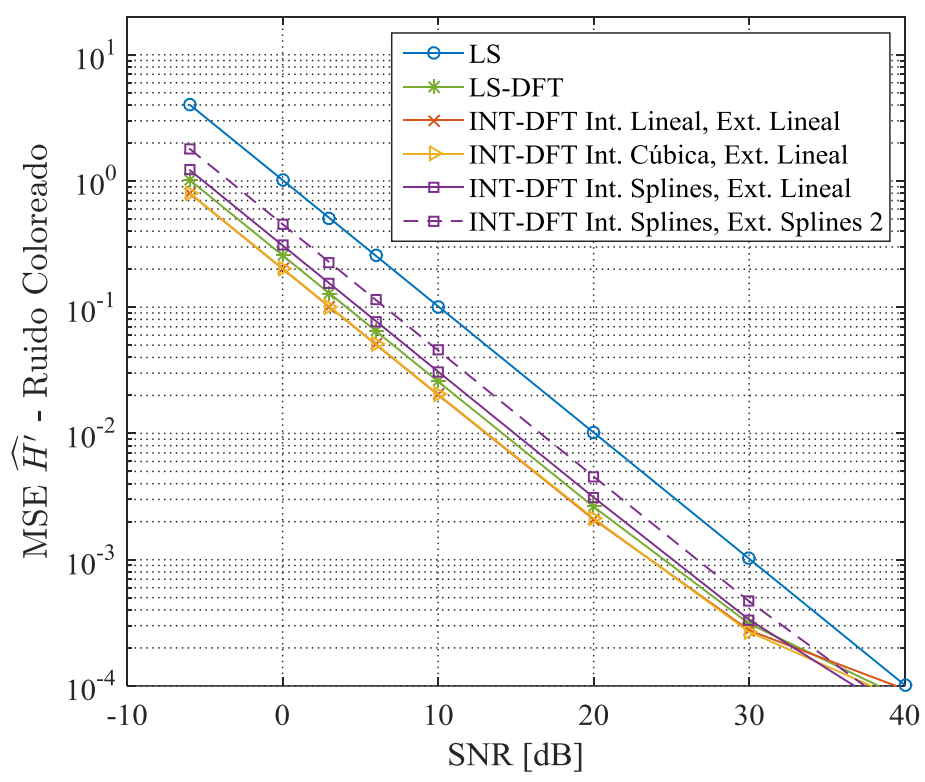

Fig. 6.36 MSE de la estimación de canal al aplicar las distintas técnicas INT-DFT para el canal PLC tipo A.

vector $\tilde{\mathbf{h}}$ a transformar al dominio de la frecuencia. Por otro lado, es evidente que cuantas más muestras se incluyan, menor será la reducción de ruido de la estimación basada en DFT.

Como última etapa de la estimación de canal, se seleccionan las $N_{\text {pre }}+N_{h}$ muestras de la estimación de la CIR que se incluyen en el vector $\tilde{\mathbf{h}} \mathrm{y}$, a partir de la DFT se obtiene la estimación en las subportadoras activas basada en DFT con interpolación, denominada $\widetilde{\mathbf{H}}_{I N T-D F T}^{\prime}$. En la Fig. 6.36 se presenta el desempeño considerando las distintas técnicas de interpolación para obtener $\widetilde{\mathbf{H}}_{I N T-D F T}^{\prime}$. Se fija $N_{\text {pre }}=200$ muestras, y, como los errores son muy similares para los canales PLC tipo A y B, se incluyen únicamente los resultados obtenidos para el canal A. Por un lado, el desempeño de la interpolación por splines resulta inferior a la interpolación lineal y cúbica prácticamente todo el rango de $S N R$, ya que comienza a superarlas a partir de aproximadamente $35 \mathrm{~dB}$. Por otro lado, se observa que la extrapolación lineal propuesta es superior a la extrapolación denominada “Splines 2" de la Fig. 6.34, salvo para valores de SNR muy elevados, mayores a $40 \mathrm{~dB}$. La estimación con interpolación lineal obtiene resultados muy similares a la cúbica, aunque esta última mejora el desempeño para $S N R$ mayores a $30 \mathrm{~dB}$. Adicionalmente, el MSE de éstas es ligeramente inferior a la estimación obtenida por LS-DFT. Por todo esto, como la interpolación lineal es la que menor complejidad computacional demanda y posee un desempeño altamente competitivo en todo el rango de $S N R$ analizado, resulta la opción más atractiva para su potencial implementación. 
Todas las técnicas de estimación presentadas hasta el momento en este capítulo se basan en una primera estimación en el dominio de la frecuencia. El símbolo piloto propuesto diseñado a partir de CPS no posee amplitud constante en el dominio de la frecuencia, lo que reduce el desempeño al aplicar este tipo de técnicas respecto de su aplicación a símbolos piloto con amplitud constante. Por otro lado, no se puede aprovechar la correlación cruzada eficiente utilizada para la etapa de sincronización, ya que, si se utilizan directamente las muestras de la salida del filtro acoplado como estimación de la CIR para obtener la estimación de la CFR en las subportadoras activas, al efectuar la operación de DFT se degrada sustancialmente la estimación mencionada. Esto se produce por la distorsión que generan las subportadoras inactivas, al verse degradadas las propiedades de correlación del símbolo piloto propuesto. Entonces, no resultaría útil la estimación en las subportadoras activas como punto inicial para una posterior aplicación de las técnicas LS-DFT o INT-DFT.

Por todas las razones mencionadas, resulta atractivo desarrollar una técnica que permita aprovechar la CC en el dominio del tiempo del símbolo piloto basado en CPS para la estimación de canal, cuando se aplica una máscara espectral de transmisión.

\subsubsection{Estimación de canal CPS-CC con interpolación}

En este apartado se propone una estrategia de estimación de canal en el dominio del tiempo que permite aprovechar la CC del símbolo piloto basado en CPS de la etapa de sincronización. Se propone agregar la estimación correspondiente a las subportadoras inactivas en el dominio del tiempo para evitar la distorsión producida por las transiciones abruptas en frecuencia, intentando a su vez mantener una complejidad reducida de los algoritmos de sincronización y estimación de canal conjunta. De esta manera, se evita la secuencia de IDFT/DFT requerida por las técnicas de estimación de canal basadas en DFT para obtener la estimación de canal final, ya que a partir de la propuesta se precisaría únicamente la DFT de las muestras compensadas en el dominio del tiempo para obtener la CFR.

Con respecto a la información de la estimación en las subportadoras inactivas se propone modificar la $\mathrm{CC}$ en el dominio del tiempo, de manera tal que el resultado se traduzca en la interpolación (y extrapolación) lineal en el dominio de la frecuencia analizada en el apartado anterior, ya que se concluyó que resulta la opción con mejor relación de compromiso entre desempeño y complejidad. 
Diseño de Estrategias de Sincronización y Estimación de Canal para la Mejora de Comunicaciones en Redes Inteligentes de Energía

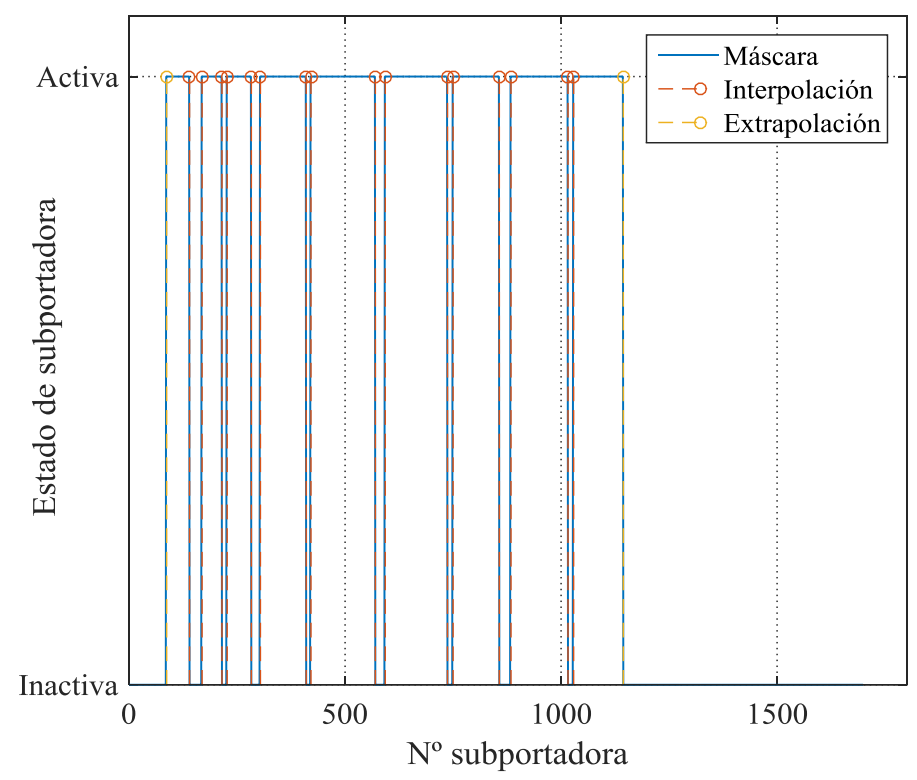

Fig. 6.37 Subportadoras necesarias para efectuar la interpolación y extrapolación lineal propuesta.

\subsubsection{Estimación de canal en subportadoras activas}

Al igual que en las técnicas de estimación LS-DFT e INT-DFT, se precisa partir de una estimación del canal en ciertas subportadoras activas para efectuar una estimación en las subportadoras inactivas. La máscara espectral de transmisión define 9 sub-bandas de transmisión con subportadoras activas, de acuerdo a la Tabla 6.1 y como se aprecia en la Fig. 6.37. Así, para efectuar una interpolación lineal en el dominio de la frecuencia, se precisan estimar las siguientes 16 subportadoras que se encuentran en los límites de las sub-bandas de transmisión:

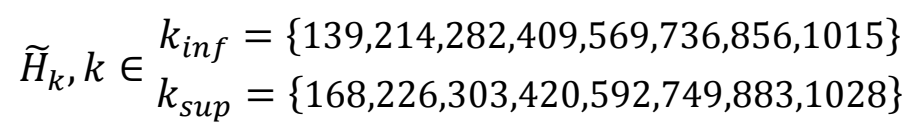

donde $k_{\text {inf }}$ contiene el conjunto de subportadoras activas previas al inicio de cada una de las 8 bandas inactivas, mientras que $k_{\text {sup }}$ contiene a las subportadoras activas posteriores a la última subportadora inactiva de cada banda. Adicionalmente, para la extrapolación, se precisa estimar el canal en la subportadora activa de menor frecuencia $\widetilde{H}_{86}$ para completar las frecuencias inactivas de baja frecuencia $\widetilde{H}_{0-85}$; y la subportadora activa de mayor frecuencia $\widetilde{H}_{1143}$ para extrapolar a las subportadoras de mayor frecuencia $\widetilde{H}_{1144-2048}$.

La estimación propuesta $\widetilde{H}_{k}$ para cada una de las 18 subportadoras es por el método LS, debido a su complejidad reducida. Como la cantidad de subportadoras a estimar es una pequeña fracción del tamaño de DFT $N=4096$, entonces la operación de FFT para 
obtener la estimación no resulta apropiada en términos de complejidad, y resulta una mejor opción evaluar algoritmos de cómputo de la DFT de manera individual para cada subportadora. La ventaja se obtiene a partir de la implementación de filtros IIR recursivos individuales de reducida complejidad, donde la gran mayoría de variantes de este tipo de filtros se basan en el algoritmo de Goerztel [Yang 2002]. El algoritmo recursivo de Goertzel de segundo orden permite obtener la DFT $R_{k}$ de $N$ muestras de la señal de entrada $r_{n}$, en la $k$-ésima subportadora, de acuerdo al cómputo de $N$ recursiones de las ecuaciones:

$$
\begin{gathered}
v_{k, n}=2 \cos \left(\frac{2 \pi k}{N}\right) v_{k, n-1}-v_{k, n-2}+r_{n} \\
R_{k, n}=v_{k, n}-\exp \left(j \frac{2 \pi k}{N}\right) v_{n-1}
\end{gathered}
$$

donde, inicialmente para $n=0, v_{-1}=v_{-2}=0$. Adicionalmente, para computar la DFT de manera continua a partir de una ventana deslizante (Sliding, SDFT), (6.35) se modifica por:

$$
v_{k, n}=2 \cos \left(\frac{2 \pi k}{N}\right) v_{k, n-1}-v_{k, n-2}+r_{n}-r_{n-N}
$$

El filtro equivalente para computar la SDFT en la $k$-ésima subportadora se presenta en la Fig. 6.38, y requiere sólo operaciones reales para computar $v_{n}$ : tres sumadores y un multiplicador por una constante, más los elementos de retardo. Para computar la salida $R_{k, n}$, se requiere un multplicador constante y un sumador, ambos complejos. Para obtener la DFT de las subportadoras necesarias a la vez, se requiere repetir 18 veces en paralelo la estructura del filtro. Como la respuesta al impulso es la respuesta de un oscilador truncada en $N$ muestras, la estabilidad del filtro IIR es una de las cuestiones críticas a resolver al momento de una implementación. Unas posibles soluciones al problema de estabilidad son la inserción de un factor de amortiguamiento como se

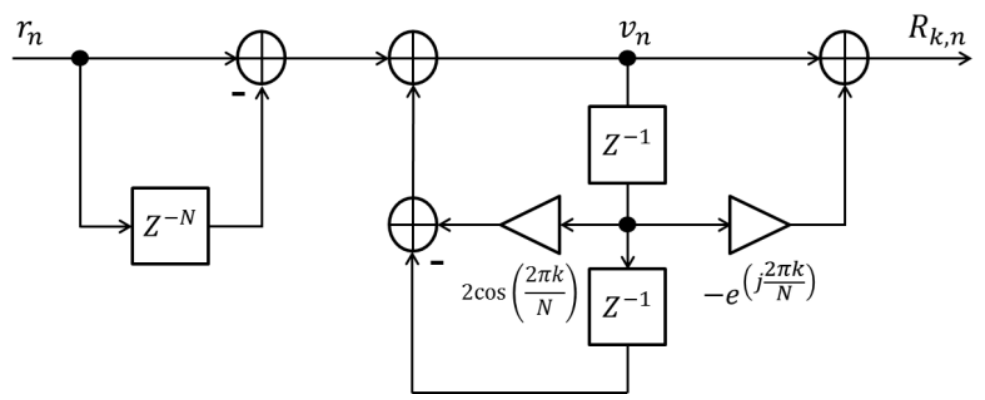

Fig. 6.38 Filtro equivalente para el cómputo de la SDFT. 
Diseño de Estrategias de Sincronización y Estimación de Canal para la Mejora de Comunicaciones en Redes Inteligentes de Energía

plantea en [Orallo 2015], o bien a partir la duplicación del filtro y el reseteo periódico de los registros, como se propone en [Talla 2017]. Por otro lado, es factible definir una muestra determinada para el inicio del procesamiento de la señal, como puede ser el instante obtenido a partir de la detección del símbolo piloto, o bien en la muestra $n_{g}$, al finalizar la etapa de sincronización gruesa. Esto permite mantener acotado el error de la DFT en el instante resultante de la etapa de sincronización fina $n_{f}$.

La señal recibida en el dominio de la frecuencia al aplicar la DFT considerando un sincronismo perfecto puede escribirse como:

$$
R_{k, n_{\mathrm{o} p t}}=\sqrt{P_{T}} H_{k} X^{\prime}{ }_{k}+W_{k}
$$

donde $P_{T}$ es la potencia transmitida; $H_{k}$ el coeficiente complejo del canal; $X^{\prime}{ }_{k}$ el símbolo piloto; $\mathrm{y} W_{k}$ el ruido, en la frecuencia correspondiente a la $k$-ésima subportadora. Por lo tanto, la estimación por LS en cada una de las 18 subportadoras puede obtenerse de acuerdo a

$$
\widetilde{H}_{k}=\frac{R_{k, n_{\mathrm{o} p t}}}{\sqrt{P_{T}} X^{\prime}{ }_{k}}=\frac{R_{k, n_{\mathrm{o} p t}}}{\sqrt{P_{T}}\left|X^{\prime}{ }_{k}\right|} e^{-j \varphi_{k}}, \quad k \in k_{\text {inf }} \cup k_{\text {sup }}
$$

donde $\varphi_{k}$ es el ángulo de $X^{\prime}{ }_{k}$. Si bien puede aplicarse un factor multiplicador complejo en la salida de la estructura de la Fig. 6.38 con valor $e^{-j \varphi_{k}} /\left(\sqrt{P_{T}}\left|X^{\prime}{ }_{k}\right|\right)$, este coeficiente se integrará en la estructura de modificación de la correlación cruzada, que se presenta a continuación.

\subsubsection{Modificación de la correlación cruzada}

Una vez obtenidas las estimaciones del canal en las subportadoras necesarias, se procede a efectuar la interpolación y extrapolación lineal. El objetivo es adicionar esta información en el dominio del tiempo a las muestras de la CC del símbolo piloto disponibles en la salida del filtro acoplado utilizado para la etapa de sincronización. Es importante destacar que la modificación de las muestras debe ser tal que incorpore una estimación de la correlación cruzada, y no la estimación de canal propiamente dicha. Para clarificar esto, la correlación cruzada en el dominio de la frecuencia en una subportadora activa puede escribirse como:

$$
C_{k, n_{\text {ópt }}}^{\mathbf{r}, \mathbf{x}^{\prime}}=R_{k, n_{\mathrm{o} p t}} X^{\prime *}=\sqrt{P_{T}} H_{k}\left|X^{\prime}{ }_{k}\right|^{2}+W_{k} X_{k}^{\prime *}
$$


Capítulo 6: Sincronización y estimación de canal en sistemas DMT-PLC bajo una máscara espectral de transmisión

La estimación de canal $\widetilde{H}_{k^{\prime}, q}$ correspondiente a la interpolación lineal en la $k^{\prime}$-ésima subportadora inactiva de la $q$-ésima banda, a partir de las dos subportadoras límites de la banda $k_{\text {inf,q }} \mathrm{y} k_{\text {sup }, q}$, se puede escribir como (6.41). Nótese que $k$ ' representa cada una de las portadoras inactivas incluidas en las bandas definidas por los $q=\{1,2, \ldots, 8\}$ pares límites indicados en (6.34), denotados aquí genéricamente por $k_{\text {inf,q }} \mathrm{y} k_{s u p, q}$. Una representación gráfica de la interpolación lineal a partir de la cual se obtiene (6.41) se encuentra en la Fig. 6.39, a partir de un ejemplo de interpolación de la parte real.

$$
\begin{aligned}
& \widetilde{H}_{k^{\prime}, q}=\operatorname{Re}\left\{\widetilde{H}_{k_{\text {inf }, q}}\right\} \frac{k_{\text {sup }, q}-k^{\prime}}{k_{\text {sup }, q}-k_{\text {inf }, q}}+j \operatorname{Im}\left\{\widetilde{H}_{k_{\text {inf }, q}}\right\} \frac{k_{\text {sup }, q}-k^{\prime}}{k_{\text {sup }, q}-k_{\text {inf }, q}} \\
& +\operatorname{Re}\left\{\widetilde{H}_{k_{\text {sup }, q}}\right\} \frac{k^{\prime}-k_{\text {inf }, q}}{k_{\text {sup }, q}-k_{\text {inf }, q}}+j \operatorname{Im}\left\{\widetilde{H}_{k_{\text {sup }, q}}\right\} \frac{k^{\prime}-k_{\text {inf }, q}}{k_{\text {sup }, q}-k_{\text {inf }, q}}, \quad k_{\text {inf }, q}<k^{\prime}<k_{\text {sup }, q}(6.41)
\end{aligned}
$$

Finalmente, de acuerdo a (6.40) la estimación de la correlación cruzada en cada subportadora inactiva se puede escribir como:

$$
\begin{gathered}
\tilde{C}_{k^{\prime}, q}^{\mathbf{r}, \mathbf{x}^{\prime}}=\sqrt{P_{T}} \widetilde{H}_{k^{\prime}, q}\left|X_{k^{\prime}}^{\prime}\right|^{2} \\
\tilde{C}_{k^{\prime}, q, m}=\frac{1}{N} \widetilde{C}_{k^{\prime}, q}^{\mathbf{r}, \mathbf{x}} \mathrm{e}^{\left(j \frac{2 \pi k^{\prime} m}{N}\right)}
\end{gathered}
$$

A partir de aplicar la técnica de interpolación para la estimación de canal y obtener la estimación de la correlación cruzada, deben completarse las ocho bandas de frecuencias inactivas. Para esto, como el sistema DMT procesa muestras reales (a partir de la simetría hermítica en el domino de la frecuencia), pueden añadirse las funciones

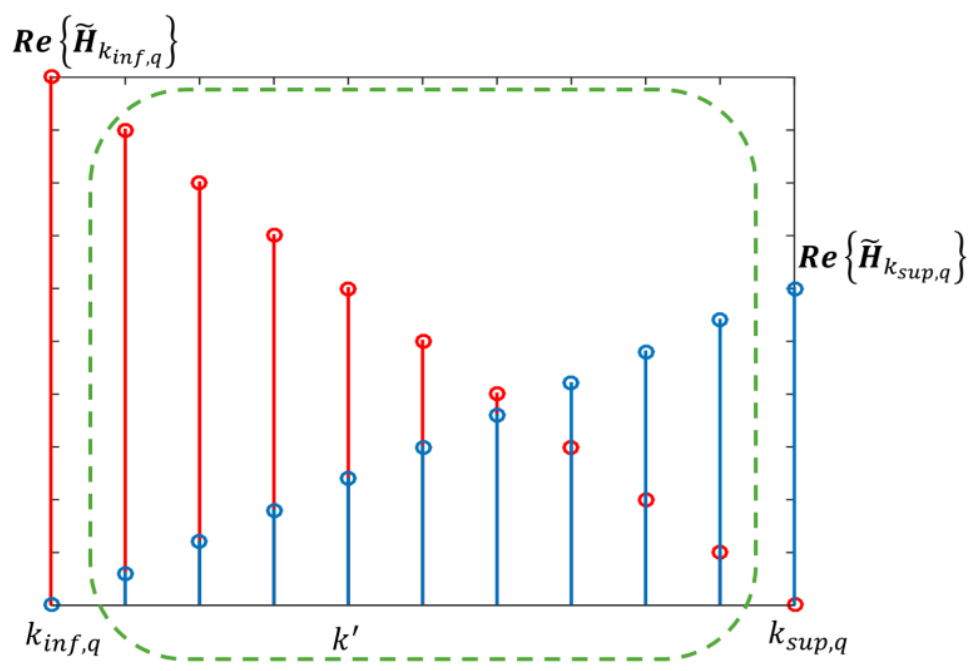

Fig. 6.39 Representación gráfica de la interpolación lineal propuesta para completar la parte real de cada banda intermedia inactiva. 
Diseño de Estrategias de Sincronización y Estimación de Canal para la Mejora de Comunicaciones en Redes Inteligentes de Energía

senoidales en el dominio del tiempo de cada una de las subortadoras pertenecientes a la banda inactiva. Las muestras a adicionar $\tilde{c}_{k^{\prime}, q, m}$ correspondientes a la $k^{\prime}$-ésima subportadora de la $q$-ésima banda inactiva, a partir de las dos subportadoras límites de la banda $k_{\text {inf,q }} \mathrm{y} k_{\text {sup }, q}$, se pueden escribir como:

$$
\begin{aligned}
& \tilde{c}_{k^{\prime}, q, m}=\operatorname{Re}\left\{R_{k_{\text {inf }, q}, n_{\text {ópt }}}\right\} 2 \frac{k_{\text {sup }, q}-k^{\prime}}{k_{\text {sup }, q}-k_{\text {inf }, q}} \cos \left(\frac{2 \pi m}{N} k^{\prime}-\varphi_{k_{\text {inf }, q}}\right) \frac{\left|X_{k^{\prime}}^{\prime}\right|^{2}}{N\left|X_{k_{\text {inf }, q} \mid}\right|} \\
& +\operatorname{Im}\left\{R_{k_{\text {inf }, q}, n_{\mathrm{o} p t}}\right\} 2 \frac{k_{\text {sup }, q}-k^{\prime}}{k_{\text {sup }, q}-k_{\text {inf }, q}} \sin \left(\frac{2 \pi m}{N} k^{\prime}-\varphi_{k_{\text {inf }, q}}\right) \frac{\left|X_{{ }^{\prime}{ }^{\prime}}\right|^{2}}{N\left|X^{\prime}{ }_{k_{\text {inf }, q}}\right|} \\
& +\operatorname{Re}\left\{R_{k_{\text {sup }, q}, n_{\mathrm{opt}}}\right\} 2 \frac{k^{\prime}-k_{\text {inf }, q}}{k_{\text {sup }, q}-k_{\text {inf }, q}} \cos \left(\frac{2 \pi m}{N} k^{\prime}-\varphi_{k_{\text {sup }, q}}\right) \frac{\left|X^{\prime}{ }_{k^{\prime}}\right|^{2}}{N\left|X^{\prime}{ }_{{ }_{\text {sup }, q}}\right|}
\end{aligned}
$$

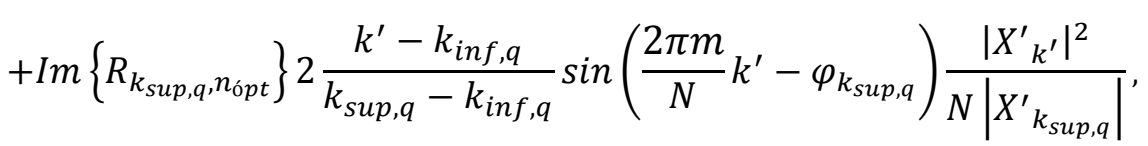

$$
\begin{aligned}
& k_{\text {inf }, q}<k^{\prime}<k_{\text {sup }, q}
\end{aligned}
$$

Por otro lado, para efectuar la extrapolación propuesta para la banda inactiva de subportadoras de baja frecuencia $(q=0)$ se precisa la estimación en la primera subportadora activa $\widetilde{H}_{86}$, a partir de (6.39). Del mismo modo, para las subportadoras inactivas de alta frecuencia $(q=9)$ se precisa la estimación del canal en la última subportadora activa $\widetilde{H}_{1143}$. Luego, la estimación en las subportadoras inactivas en cada caso se puede expresar como:

$$
\begin{gathered}
\widetilde{H}_{k^{\prime}, 0}=\operatorname{Re}\left\{\widetilde{H}_{86}\right\}+j \operatorname{Im}\left\{\widetilde{H}_{86}\right\} \frac{k^{\prime}}{86} \quad 0 \leq k^{\prime} \leq 85 \\
\widetilde{H}_{k^{\prime}, 9}=\operatorname{Re}\left\{\widetilde{H}_{1143}\right\}+j \operatorname{Im}\left\{\widetilde{H}_{1143}\right\} \frac{2048-k^{\prime}}{2048-1143} \quad 1144 \leq k^{\prime} \leq 2048
\end{gathered}
$$

Al igual que para el caso analizado de las ocho bandas inactivas intermedias, a partir de (6.42), se obtienen las muestras que completan la correlación cruzada en el dominio del tiempo:

$$
\begin{aligned}
& \tilde{c}_{k^{\prime}, 0, m}=\operatorname{Re}\left\{R_{86, n_{\text {opt }}}\right\} 2 \cos \left(\frac{2 \pi m}{N} k^{\prime}-\varphi_{86}\right) \frac{\left|X_{k^{\prime}}^{\prime}\right|^{2}}{N\left|X_{86}^{\prime}\right|} \\
& +\operatorname{Im}\left\{R_{86, n_{\mathrm{o} p t}}\right\} 2 \frac{k^{\prime}}{86} \sin \left(\frac{2 \pi m}{N} k^{\prime}-\varphi_{86}\right) \frac{\left|X_{k^{\prime}}^{\prime}\right|^{2}}{N\left|X^{\prime}{ }_{86}\right|}, \quad 0 \leq k^{\prime} \leq 85
\end{aligned}
$$




$$
\begin{aligned}
& \tilde{c}_{k^{\prime}, 9, m}=\operatorname{Re}\left\{R_{1143, n_{\text {opt }}}\right\} 2 \cos \left(\frac{2 \pi m}{N} k^{\prime}-\varphi_{1143}\right) \frac{\left|X_{k^{\prime}}^{\prime}\right|^{2}}{N\left|X^{\prime}{ }_{1143}\right|} \\
& +\operatorname{Im}\left\{R_{1143, n_{\text {op }}}\right\} 2 \frac{2048-k^{\prime}}{2048-1143} \sin \left(\frac{2 \pi m}{N} k^{\prime}-\varphi_{1143}\right) \frac{\left|X^{\prime}{ }_{k^{\prime}}\right|^{2}}{N\left|X^{\prime}{ }_{1143}\right|},
\end{aligned}
$$

$$
1144 \leq k^{\prime} \leq 2048
$$

Es importante destacar que, para los casos particulares en que $k^{\prime}=0$ y $k^{\prime}=2048$, el factor 2 presente en (6.46) y (6.47) no se aplica, por propiedades de la simetría hermítica de la IDFT. Finalmente, las muestras a agregar para completar la correlación cruzada correspondiente a todas las subportadoras inactivas se computan a partir de (6.48), y éstas se adicionan a la salida del filtro acoplado para obtener la CIR de acuerdo a (6.49).

$$
\begin{aligned}
& \widetilde{c}_{I_{m}}=\sum_{k^{\prime}=0}^{85} \tilde{c}_{k^{\prime}, 0, m}+\sum_{k^{\prime}=1144}^{2048} \tilde{c}_{k^{\prime}, 9, m}+\sum_{q=1}^{8} \sum_{k^{\prime}=k_{\text {inf }, q}+1}^{k_{\text {sup }, q}-1} \tilde{c}_{k^{\prime}, q, m} \\
& \tilde{h}_{m}=\frac{1}{\sqrt{P_{T}} N}\left(c_{n_{\text {ópt }}+\mathbf{r}, \mathbf{x}^{\prime}}+{\widetilde{c_{I m}}}\right), \quad-N_{\text {pre }} \leq m \leq N_{h}-1
\end{aligned}
$$

Donde $N_{h}$ es la dispersión máxima del canal que se estima en muestras, y $N_{\text {pre }}$ la cantidad de muestras previas requeridas para evitar un elevado piso de ruido, al igual que lo analizado para la técnica INT-DFT. El proceso de modificación de la correlación cruzada para la estimación de canal se esquematiza en la Fig. 6.40, para una realización del canal PLC tipo A y $S N R=20 \mathrm{~dB}$. Estas muestras definen el vector de estimación de la CIR $\tilde{\mathbf{h}}, \mathrm{y}$, luego, para obtener la estimación de la CFR, las primeras $N_{h}$ entradas de la DFT se completan con las muestras $\tilde{h}_{m}$ con $0 \leq m \leq N_{h}-1$. Las últimas $N_{\text {pre }}$ entradas de la DFT se completan a su vez con las muestras $\tilde{h}_{m}$ con $-N_{\text {pre }} \leq m<0$, y el resto se completa con ceros. Luego, se efectúa la DFT correspondiente para obtener la estimación de la CFR. Este proceso se esquematiza en la Fig. 6.41.

\subsubsection{Desempeño de la estimación de canal}

En esta sección se evalúa el desempeño de la estimación de canal propuesta a partir de simulaciones, en las que se fija $N_{h}=512$ y $N_{\text {pre }}=200$ muestras. Es importante tener en cuenta que la inclusión del filtro notch en recepción, que permitió una mejora 
Diseño de Estrategias de Sincronización y Estimación de Canal para la Mejora de Comunicaciones en Redes Inteligentes de Energía
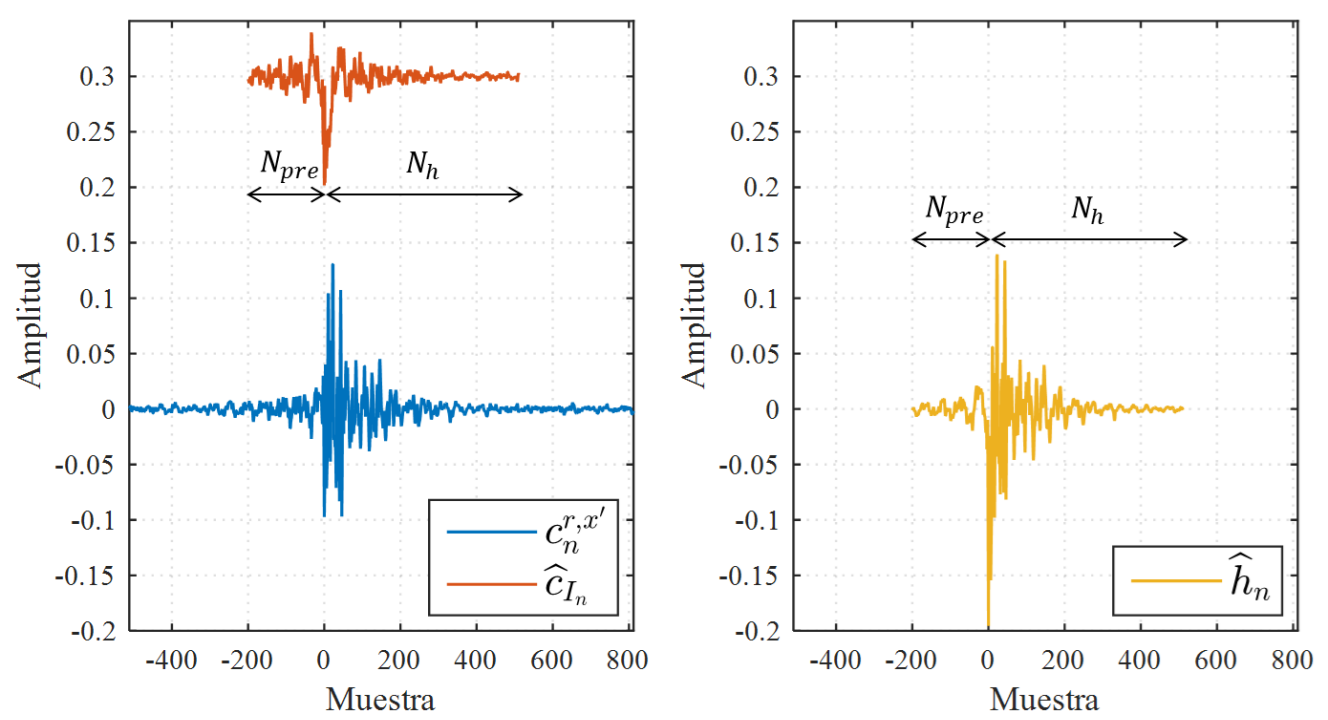

Fig. 6.40 Proceso de modificación de la correlación cruzada a partir de la interpolación y extrapolación en las subportadoras inactivas, para una realización del canal PLC tipo A y $S N R=20 d B$.

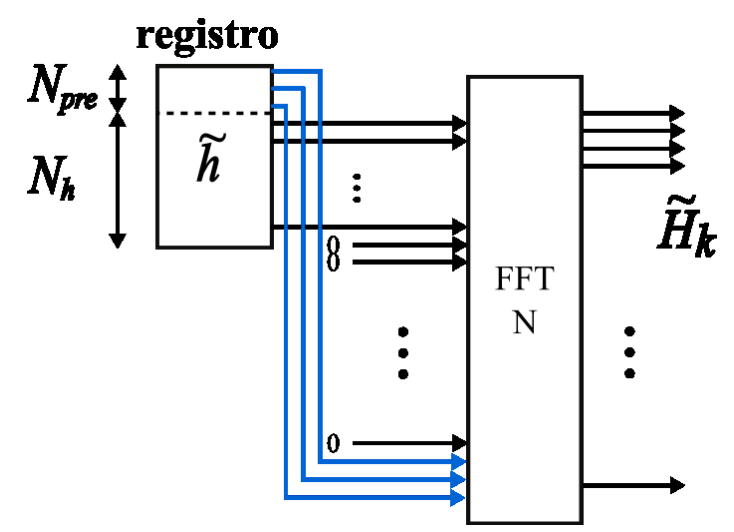

Fig. 6.41 Diagrama del proceso de estimación de la CFR a partir del vector de estimación de la CIR.

sustancial en la etapa de sincronización, tiene un alto impacto en la estimación de canal, puesto que la distorsión en la señal recibida producida por el mismo debe ser estimada y compensada.

Puede pensarse entonces en un canal equivalente entre el canal PLC con vector de CIR $\mathbf{h}$ y el filtro notch $\mathbf{c}$, a partir de $\mathbf{h}_{\boldsymbol{e q}}=\mathbf{h} * \mathbf{c}$ que afecta al símbolo piloto transmitido. Al haber utilizado un filtro de alto orden $\left(N_{c}=2048\right)$, la dispersión temporal del canal equivalente aumenta, por lo que se deben incrementar la longitud del prefijo y sufijo cíclico del símbolo piloto para evitar un elevado error en la estimación de la CFR. Así, se adicionaron 512 muestras tanto al prefijo como sufijo cíclico, resultando en un total de $N_{c p}=1536$ y $N_{c s}=1024$ muestras. El criterio para seleccionar la cantidad de 
Capítulo 6: Sincronización y estimación de canal en sistemas DMT-PLC bajo una máscara espectral de transmisión

muestras se basó en incrementar el número de muestras hasta que el desempeño de la estimación de canal por LS incluyendo el filtro notch resulte prácticamente igual a la estimación LS cuando no se incluye el filtro notch.

\subsubsection{Desempeño de la estimación de canal con sincronización perfecta}

En la Fig. 6.42 se presenta el desempeño de la estimación del canal PLC tipo A en términos del MSE, considerando un sistema perfectamente sincronizado, y ruido de fondo coloreado únicamente. Se incluyen la técnica propuesta CPS-CC y las técnicas LS, LS-DFT e INT-DFT, con y sin la inclusión del filtro notch. Por un lado, de las curvas de estimación LS puede observarse que la extensión del prefijo y sufijo cíclico cuando se incluye el filtro notch permite una estimación de canal adecuada del canal equivalente, ya que las curvas son prácticamente idénticas. Sin embargo, puede observarse que las técnicas LS, LS-DFT y CPS-CC presentan un incremento del error para valores de $S N R$ por encima de $20 \mathrm{~dB}$ cuando se incluye el filtro notch. Esto se debe a que el filtro diseñado es de un alto orden y posee un ripple considerable en las frecuencias cercanas al límite de cada banda inactiva.

Como las técnicas de estimación mencionadas truncan la estimación de la CIR considerando $N_{c p}+N_{\text {Pre }}$ muestras, y luego se efectúa la transformación por DFT con relleno con ceros para obtener la CFR, entonces las transiciones abruptas en frecuencia se suavizan en el proceso de estimación de canal, generando un piso de error a muy

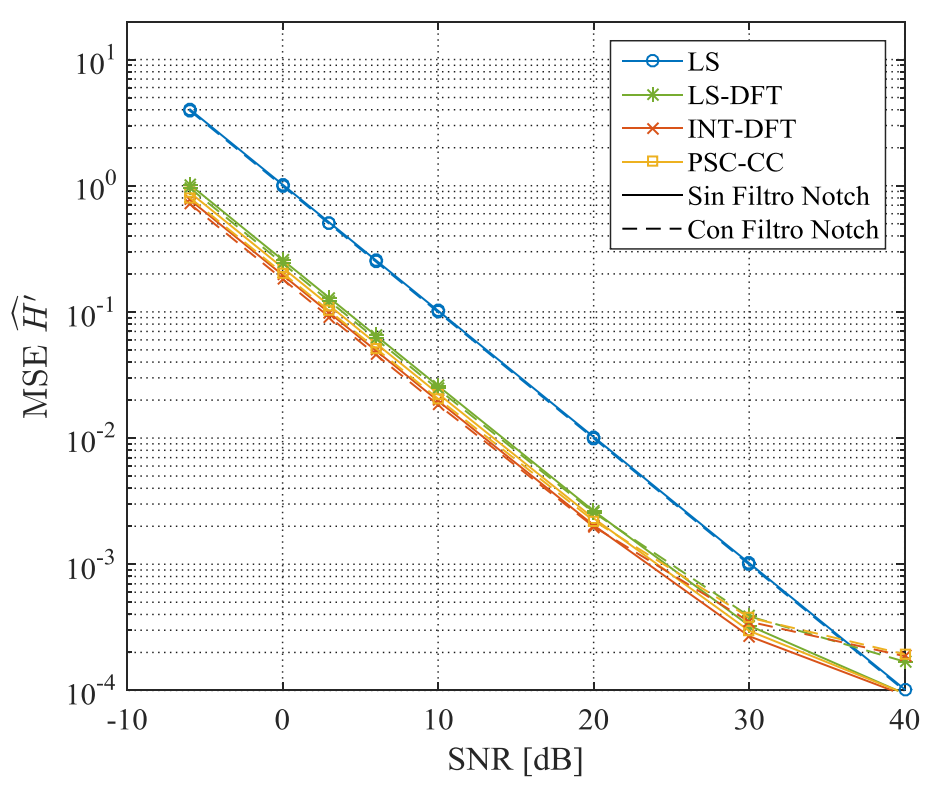

Fig. 6.42 MSE de la estimación del canal PLC tipo A bajo sincronización perfecta para distintas técnicas de estimación de canal y la propuesta CPS-CC con interpolación. 
Diseño de Estrategias de Sincronización y Estimación de Canal para la Mejora de Comunicaciones en Redes Inteligentes de Energía

altos valores de $S N R$. Este fenómeno se puede observar en la Fig. 6.43, donde se presenta la amplitud de la respuesta en frecuencia de una realización del canal PLC tipo A, del canal equivalente y el resultado de la estimación CPS-CC del canal equivalente, sin el agregado de ruido. En adelante, se evalúa únicamente el desempeño de la estimación de canal con el filtro notch incluido en el sistema, y se considera la CFR del canal equivalente en las subportadoras activas directamente como $\mathbf{H}^{\prime}$.

Además, puede apreciarse que el desempeño de la estimación de canal CPS-CC supera a LS y LS-DFT, y es ligeramente inferior a la técnica INT-DFT. Resulta importante recordar que tanto la técnica INT-DFT como CPS-CC basan su estimación en obtener un número reducido de muestras en el dominio del tiempo que contengan a la CIR del canal, lo que permite una reducción del error respecto a la técnica LS. En esa línea, para una estimación de canal $\widetilde{\mathbf{H}}^{\prime}$ se define la ganancia $\rho$ de reducción de error respecto a la estimación por LS como:

$$
\rho=\frac{M S E_{\widetilde{\mathbf{H}}_{L S}^{\prime}}}{M S E_{\widetilde{\mathbf{H}}^{\prime}}}
$$

Donde un valor $\rho>1$ implica un menor MSE de la técnica de estimación respecto a LS, y $0<\rho<1$ un mayor error. En estas técnicas puede esperarse como máximo un factor de error dado por $\rho=N /\left(L_{h}+L_{\text {pre }}\right)$, que para los parámetros del sistema evaluado tiene un valor aproximado de 5,75. El lector puede referirse al análisis del

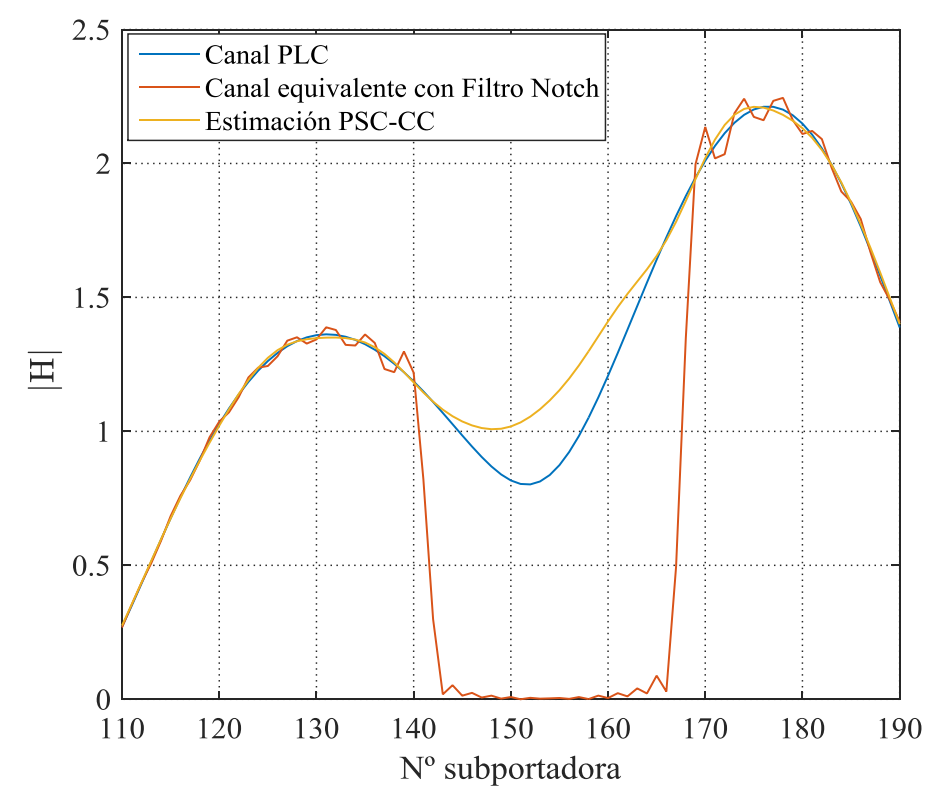

Fig. 6.43 Respuesta en frecuencia del canal PLC sin el agregado de ruido, canal equivalente al incluir el filtro notch y la estimación de canal propuesta. 
error de estimación del canal para el sistema sincronizado de la sección 5.2, donde se verificó el factor de reducción con un símbolo piloto completo y propiedades de correlación ideales. En la Tabla 6.3 se incluye el resultado de evaluar la ganancia de error de las técnicas LS-DFT, INT-DFT y CPS-CC a distintos valores de $S N R$ para los canales tipo A y B. Se observa que, en el rango $-6 \mathrm{~dB} \leq S N R \leq 10 \mathrm{~dB}$, el factor de error se mantiene prácticamente constante en todos los casos, y la propuesta CPS-CC presenta una reducción del orden de cinco veces. Obviamente, en línea con la Fig. 6.42, el mejor desempeño se obtiene con la técnica INT-DFT, obteniendo una reducción extra respecto a CPS-CC del 10\%. En resumen, se puede concluir que la propuesta CPS-CC provee una estimación de canal competitiva a partir de las muestras de la salida del filtro acoplado utilizado en la etapa de sincronización, sin la necesidad de efectuar el proceso de estimación por DFT.

Una vez evaluado el sistema considerando una sincronización perfecta y ruido de fondo coloreado, se procede a adicionar eventos de ruido impulsivo a la señal para estudiar su efecto en la estimación de canal. En la Fig. 6.44 se incluye el MSE de la estimación de canal PLC tipo A (izquierda) y B (derecha) CPS-CC en tres escenarios: sin ruido impulsivo, incluyendo eventos de ruido impulsivo (RI) con probabilidad de ocurrencia del $8 \%$, y finalmente el resultado al aplicar la técnica de mitigación no lineal de blanqueo. También se evalúa la técnica de estimación LS bajo los distintos escenarios para comparación. Puede observarse en primer lugar que los resultados obtenidos en los

Tabla 6.3 Ganancia de error de las técnicas de estimación de canal respecto a LS, para distintos valores de SNR.

\begin{tabular}{|c|c|c|c|c|c|c|c|c|c|}
\hline & \multicolumn{8}{|c|}{ SNR } \\
\hline & & $-6 d B$ & $0 \mathrm{~dB}$ & $3 \mathrm{~dB}$ & $6 \mathrm{~dB}$ & $10 \mathrm{~dB}$ & $20 \mathrm{~dB}$ & $30 \mathrm{~dB}$ & $40 \mathrm{~dB}$ \\
\hline \multirow{3}{*}{$\frac{\mathbb{Z}}{\mathbb{Z}}$} & LS - DFT & 4,21 & 4,21 & 4,20 & 4,20 & 4,18 & 3,97 & 2,64 & 0,60 \\
\hline & INT - DFT & 5,62 & 5,61 & 5,61 & 5,60 & 5,57 & 5,14 & 2,91 & 0,55 \\
\hline & PSC - CC & 5,03 & 5,02 & 5,02 & 5,01 & 4,98 & 4,61 & 2,70 & 0,53 \\
\hline \multirow{3}{*}{ 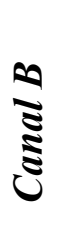 } & LS - DFT & 4,28 & 4,27 & 4,27 & 4,27 & 4,25 & 3,99 & 2,49 & 0,52 \\
\hline & INT - DFT & 5,53 & 5,52 & 5,52 & 5,51 & 5,47 & 5,03 & 2,78 & 0,51 \\
\hline & PSC - CC & 5,10 & 5,09 & 5,09 & 5,08 & 5,05 & 4,66 & 2,64 & 0,50 \\
\hline
\end{tabular}


Diseño de Estrategias de Sincronización y Estimación de Canal para la Mejora de Comunicaciones en Redes Inteligentes de Energía

dos tipos de canales son prácticamente idénticos para el caso de estimación LS, y muy similares para CPS-CC, en el que el MSE al incluir el RI resulta ligeramente superior para el canal.

Los eventos de RI degradan la estimación de canal, y para la técnica LS representa un incremento de aproximadamente 3,5 veces del error de estimación en todo el rango de $S N R$ evaluado. Esto se produce debido a que al agregar el ruido impulsivo la potencia promedio del ruido que afecta al símbolo piloto incrementa en ese orden, y fue verificado por simulaciones. Con respecto a la propuesta CPS-CC, en el rango $-6 \mathrm{~dB} \leq$ $S N R \leq 10 \mathrm{~dB}$ también se presenta el incremento del orden de 3,5 veces de error al incluir el RI, pero a medida que el valor de $S N R$ se incrementa por encima de $30 \mathrm{~dB}$ las curvas con y $\sin$ RI se aproximan, debido que ambas tienden al piso de error de la estimación de canal que comienza a ser más preponderante que el efecto del RI. A su vez, la técnica de blanqueo mejora en general el desempeño bajo RI, dependiendo del nivel de $S N R$. Cuando la $S N R$ es muy baja, la señal recibida está compuesta principalmente por el ruido de fondo coloreado más el ruido impulsivo, y blanquear las muestras afectadas por RI reduce considerablemente el error de estimación. Este error se aproxima asintóticamente al caso sin RI debido a que la señal recibida incluye cada vez una menor proporción de señal útil para la estimación cuando la $S N R$ decrece, y como la amplitud del RI es mucho mayor que del ruido de fondo coloreado, el blanqueo representa prácticamente la remoción del RI. A medida que el valor de $S N R$ aumenta, la
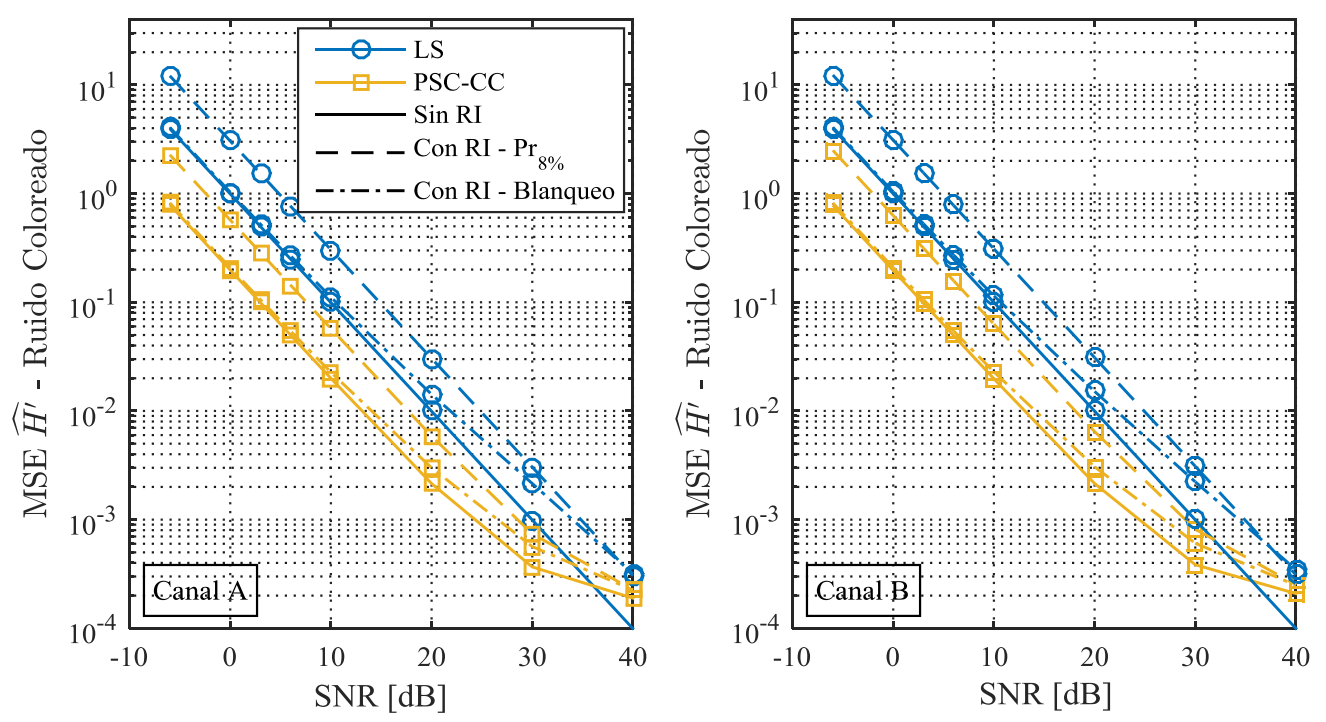

Fig. 6.44 MSE de la estimación del canal bajo sincronización perfecta al incorporar eventos de ruido impulsivo y la técnica de mitigación de blanqueo, para los canales tipo A (izquierda y B (derecha). 
técnica de blanqueo proporciona un desempeño intermedio entre los casos con y sin RI. Sin embargo, para muy altos valores de $S N R$, el MSE obtenido a partir de la técnica de blanqueo supera al escenario con RI en el que no se aplica la técnica de mitigación. Esto se debe a que, ante un valor de $S N R$ lo suficientemente elevado, los eventos de RI que distorsionan la señal dejan de ser significativos en la amplitud de las muestras afectadas, por lo que al blanquearlas se anula información del canal en mayor proporción que lo que se reduce de ruido. Es posible obtener mejores resultados para altos valores de $S N R$ si se utiliza una técnica no lineal de recorte (clipping), o bien si se obtiene un umbral de blanqueo dinámico dependiente de la condición de $S N R$.

6.4.4.1 Desempeño de la estimación de canal con sincronización conjunta.

Los resultados obtenidos para la estimación de canal en el escenario de sincronismo perfecto son útiles como punto de partida para evaluar el esquema propuesto de sincronización y estimación de canal conjunta. A partir de los resultados de la etapa de sincronización gruesa de la sección 6.2 y de la etapa de sincronización fina de la sección 6.3, se evalúa a continuación el desempeño de la estimación de canal CPS-CC y LS. Así, el proceso contempla la capacidad de detección del arribo del símbolo piloto y la sincronización a partir de la ubicación del máximo de la métrica de sincronización gruesa, la ejecución del algoritmo de sincronización fina para obtener el punto final de sincronismo, y finalmente la aplicación de la técnica de estimación de canal correspondiente. Por lo tanto, si durante el proceso de simulaciones en una realización no se logra la detección primaria del símbolo piloto, entonces se descarta y no se aplica el algoritmo de sincronización fina ni la estimación del canal. En todos los casos, se utiliza la métrica de sincronización gruesa $m_{3, n}$ de la sección 6.2 , y la ejecución del algoritmo de sincronización fina se realiza con el umbral de basado en la amplitud del máximo de la CC, $\alpha_{k_{1}}$, de la sección 6.3.

Para evaluar el error de la estimación de canal luego de la etapa de sincronización deben tenerse en cuenta algunas consideraciones. Puede inicialmente computarse el MSE de la estimación a partir del error entre la CFR del canal PLC en las subportadoras activas $\mathbf{H}^{\prime}$ y la estimación obtenida por cualquier técnica de estimación $\widetilde{\mathbf{H}}^{\prime}$, como:

$$
M S E_{\widetilde{\mathbf{H}}^{\prime}}=\frac{1}{N_{a}} E\left\{\left\|\mathbf{H}^{\prime}-\widetilde{\mathbf{H}}^{\prime}\right\|^{2}\right\}
$$


Diseño de Estrategias de Sincronización y Estimación de Canal para la Mejora de Comunicaciones en Redes Inteligentes de Energía

En ese caso, el error que se obtiene cuando la sincronización no es perfecta es elevado porque en el canal estimado aparece el efecto del desplazamiento temporal del instante de sincronismo, que se traduce en un cambio de fase lineal en la CFR estimada $\widetilde{\mathbf{H}}^{\prime}$. El error de sincronización afecta el instante de inicio de la ventana de $N$ muestras de DFT, no sólo para el símbolo piloto sino también para los símbolos de datos de la trama, por lo que finalmente debe ecualizarse el canal desplazado $\mathbf{H}_{\mathbf{d}}^{\prime}$, del que puede escribirse cada uno de sus puntos frecuenciales como:

$$
\mathrm{H}_{\mathrm{d}_{k}}^{\prime}=\mathrm{H}_{k}^{\prime} e^{j \frac{2 \pi k\left(n_{f}-n_{\mathrm{o} p t}\right)}{N}}
$$

donde $n_{f}$ es el instante obtenido a partir de la etapa de sincronización fina y $n_{\text {ópt }}$ es el instante de sincronismo óptimo. Finalmente, puede computarse el error de estimación respecto al canal desplazado de acuerdo a:

$$
M S E_{\widetilde{\mathbf{H}}_{d}^{\prime}}=\frac{1}{N_{a}} E\left\{\left\|\mathbf{H}_{\mathbf{d}}^{\prime}-\widetilde{\mathbf{H}}^{\prime}\right\|^{2}\right\}
$$

Con respecto a la sensibilidad a los errores de sincronismo de la modificación de la correlación cruzada a partir de (6.49), es importante destacar que no producirá un mayor valor de distorsión siempre que el instante de sincronización fina $n_{f}$ se encuentre dentro de la zona libre de interferencia otorgada por el prefijo y sufijo cíclicos. Bajo esa consideración, reemplazando $n_{\text {ópt }}$ por $n_{f}$ en (6.39), se obtiene la estimación LS en las subportadoras activas como:

$$
\widetilde{H}_{k}=\frac{R_{k, n_{f}}}{\sqrt{P_{T}}\left|X^{\prime}{ }_{k}\right|} e^{-j \varphi_{k}}, \quad k \in k_{\text {inf }} \cup k_{\text {sup }}
$$

donde la relación entre el cómputo de la DFT de la señal recibida $R_{k, n_{f}}$ y $R_{k, n_{\text {ópt }}}$ está dada por:

$$
R_{k, n_{f}}=R_{k, n_{\mathrm{o} p t}} e^{j \frac{2 \pi k\left(n_{f}-n_{\mathrm{o} p t}\right)}{N}}
$$

Es decir, el proceso de interpolación y extrapolación se realiza sobre una estimación del canal desplazado. Además, resulta obvio que, tomando las muestras de la CC a partir del instante $n_{f}$, también se está realizando una estimación parcial de la CIR del canal desplazado. Por lo tanto, la suma de las muestras compone la estimación final de la CIR del canal con el desplazamiento incluido. Sin embargo, la estimación de la CIR $\mathbf{h}$ no será numéricamente idéntica (con un desplazamiento), a la obtenida en el escenario de 
Capítulo 6: Sincronización y estimación de canal en sistemas DMT-PLC bajo una máscara espectral de transmisión

sincronización perfecta porque para que eso suceda primero debería efectuarse la interpolación y extrapolación, y luego aplicar el desplazamiento por error de sincronismo, lo que en la realidad sucede en orden contrario. Por otro lado, la cantidad de muestras $N_{\text {pre }}$ y $N_{h}$ tiene un rol importante en la robustez a los errores de sincronismo, ya que el desempeño se mantiene sólo si la CIR del canal desplazado se encuentra contenida dentro de la ventana de $N_{\text {pre }}+N_{h}$ muestras. El proceso de modificación de la CC y la estimación de la CIR bajo un error de sincronismo se representa en la Fig. 6.45, para una realización del canal PLC tipo A y un valor de $S N R$ de $30 \mathrm{~dB}$. El error de sincronismo es forzado a un valor $n_{f}-n_{\mathrm{o} p t}=80$ muestras, lo suficientemente elevado como para que los gráficos resulten con la suficiente claridad. Puede observarse que, a pesar del elevado error, la estimación final de la CIR se atenúa considerablemente en los extremos, lo que indica que se contiene prácticamente toda la energía del canal en el vector de estimación.

En la Fig. 6.46 se presentan los resultados de simulación de MSE de las estimaciones de canal LS y CPS-CC para los canales tipo A y B con ruido de fondo coloreado, sin RI, computando el error respecto al canal PLC $\mathbf{H}^{\prime}$ y el desplazado $\mathbf{H}_{\mathbf{d}}^{\prime}$. Se considera para las dos técnicas de estimación de canal exactamente el mismo resultado del proceso de sincronización. En principio, se observa el gran piso de error al computar (6.51) tanto para LS como para CPS-CC. El piso de error resulta inferior para el caso del canal B,
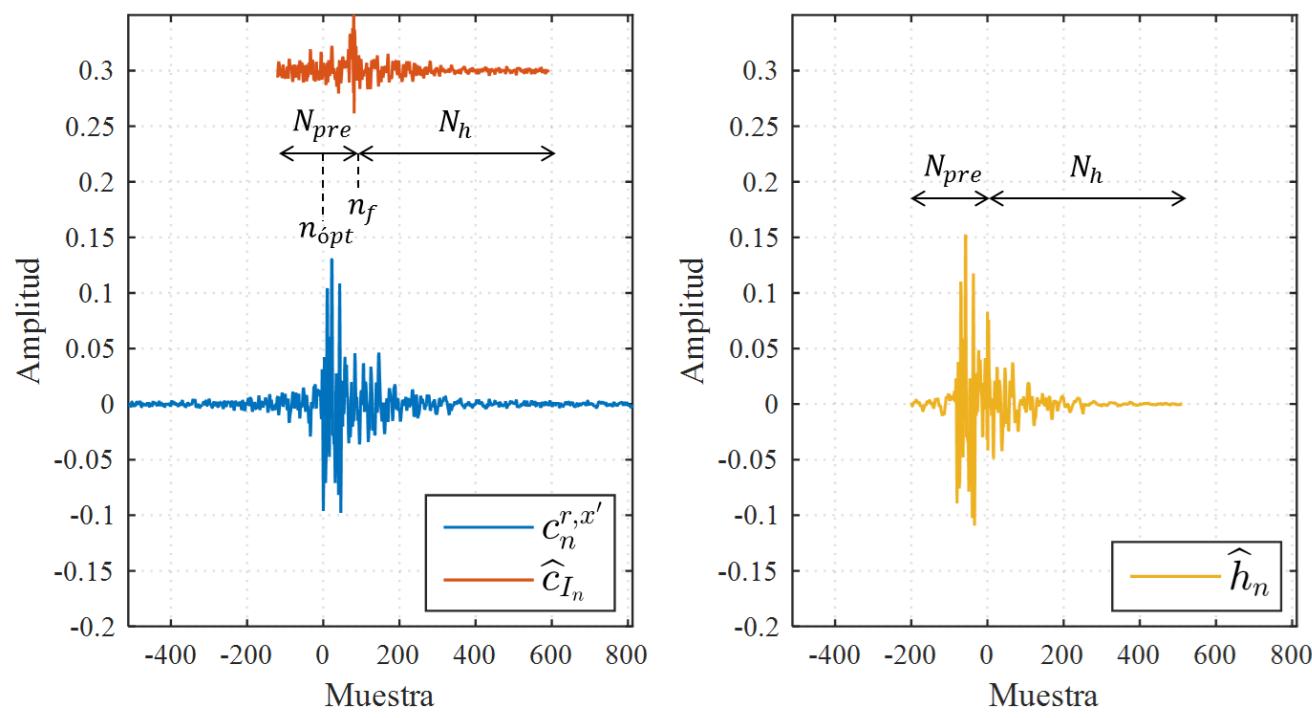

Fig. 6.45 Proceso de modificación de la correlación cruzada, para una realización del canal PLC tipo A, un error de sincronismo de +80 muestras, $y \mathrm{SNR}=30 \mathrm{~dB}$. 
Diseño de Estrategias de Sincronización y Estimación de Canal para la Mejora de Comunicaciones en Redes Inteligentes de Energía

debido a que la probabilidad de sincronización perfecta es mayor que para el canal A.

Por otro lado, se aprecia una estimación adecuada del canal desplazado $\mathbf{H}_{\mathbf{d}}^{\prime}$ en ambos casos. Se agregó en dicha Fig. 6.46 el MSE de la técnica CPS-CC cuando el sistema se encuentra perfectamente sincronizado, y se verifica que la estimación de canal incluyendo los errores asociados al proceso de sincronización es prácticamente la misma (sólo para el valor de $S N R$ de $-6 \mathrm{~dB}$ se observa un ligero incremento en el error). Este resultado tiene una relevancia importante en el desempeño de la sincronización y estimación de canal conjunta, ya que significa que, al considerar las $N_{\text {pre }}$ muestras anteriores y las $N_{h}$ posteriores a la muestra resultante de la etapa de sincronismo $n_{f}$ para la estimación, se garantiza la inclusión de prácticamente la CIR completa del canal en todas las realizaciones, otorgándole robustez a la técnica de estimación ante los errores de sincronismo. Sin embargo, debe tenerse en cuenta que los errores de sincronismo en los que $n_{f}-n_{\text {ópt }}>0$ o $n_{f}-n_{\text {ópt }}<N_{c p}-L+1$ provocarán ISI e ICI al aplicar la DFT de los símbolos de datos, y se traducen en una pérdida de $S N R$. Es decir que en esos casos existirá una reducción en el desempeño del sistema al momento de efectuar la ecualización del canal por los errores de sincronismo respecto a un sistema perfectamente sincronizado.

Si bien el estudio de la posterior ecualización del canal en los símbolos de datos y sus métricas de desempeño exceden el alcance de esta tesis, resulta razonable asumir que la
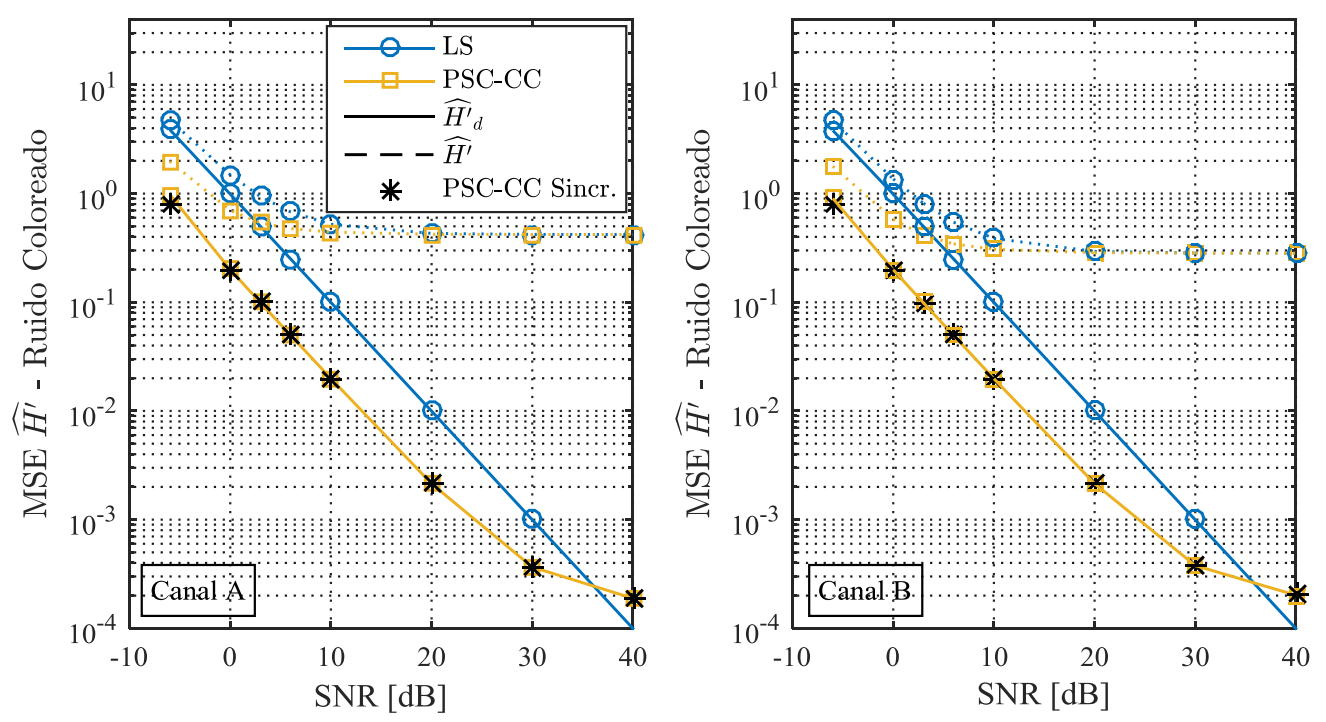

Fig. 6.46 MSE de la estimación del canal con sincronización conjunta, respecto al canal PLC y su versión desplazada por el error de sincronismo, para los canales tipo A (izquierda y B (derecha). 
pérdida de $S N R$ por error en la sincronización se encuentra limitada. De acuerdo a lo expuesto en [Morelli 2007], el error de sincronización debe mantenerse a unas pocas unidades porcentuales de la longitud del símbolo $N$ para obtener una pérdida de $S N R$ tolerable. Si se considera un $1 \%$ de error porcentual para el sistema de $N=4096$, significa un error de sincronización de aproximadamente 40 muestras. De acuerdo a los resultados obtenidos en la etapa de sincronización fina, para valores de SNR por encima de 0dB, el RMSE se mantiene por debajo de las 10 muestras (veáse por ejemplo la Fig. 6.28 de la sección 6.3), lo que indica a priori que la pérdida de $S N R$ se encontrará dentro de niveles tolerables.

Finalmente, se evalúa por simulaciones el impacto del ruido impulsivo en el proceso de sincronización y estimación de canal conjunta. En la Fig. 6.47 se muestra el MSE de la estimación del canal desplazado $\widetilde{\mathbf{H}}_{d}^{\prime}$ a partir de LS y CPS-CC en tres escenarios: sin ruido impulsivo (RI), incluyendo eventos de ruido impulsivo con probabilidad de ocurrencia del 8\%, y aplicando la técnica de mitigación no lineal de blanqueo. En cada caso, se ejecuta el algoritmo de sincronización de manera independiente y luego se realiza la estimación de canal. Se observa claramente que, a medida que el valor de SNR disminuye, la curva correspondiente al caso con RI se aproxima al caso sin RI, y en consecuencia también la curva al aplicar la técnica de blanqueo. Esto se produce porque, a medida que $S N R$ decrece, también lo hace la probabilidad de detección del símbolo piloto en la etapa de sincronización gruesa, y a su vez la métrica se torna más vulnerable a los eventos de RI. Entonces, a muy bajas $S N R$, la detección del símbolo se logra únicamente en las realizaciones en las que no ocurre un evento de RI. A medida que aumenta el valor de $S N R$ el sistema es capaz de sincronizarse aún bajo eventos de RI, y en esos casos el error de estimación del canal es superior respecto al caso sin RI. A su vez, también en esos casos la técnica de blanqueo proporciona una reducción en el error, dando por resultado una curva de menor MSE respecto al caso con RI. En las curvas de estimación LS puede observarse que a partir de $S N R$ de $30 \mathrm{~dB}$ la diferencia entre las curvas sin y con RI alcanza la relación de 3,5 veces analizada en la Fig. 6.44, del sistema bajo sincronización perfecta. 
Diseño de Estrategias de Sincronización y Estimación de Canal para la Mejora de Comunicaciones en Redes Inteligentes de Energía
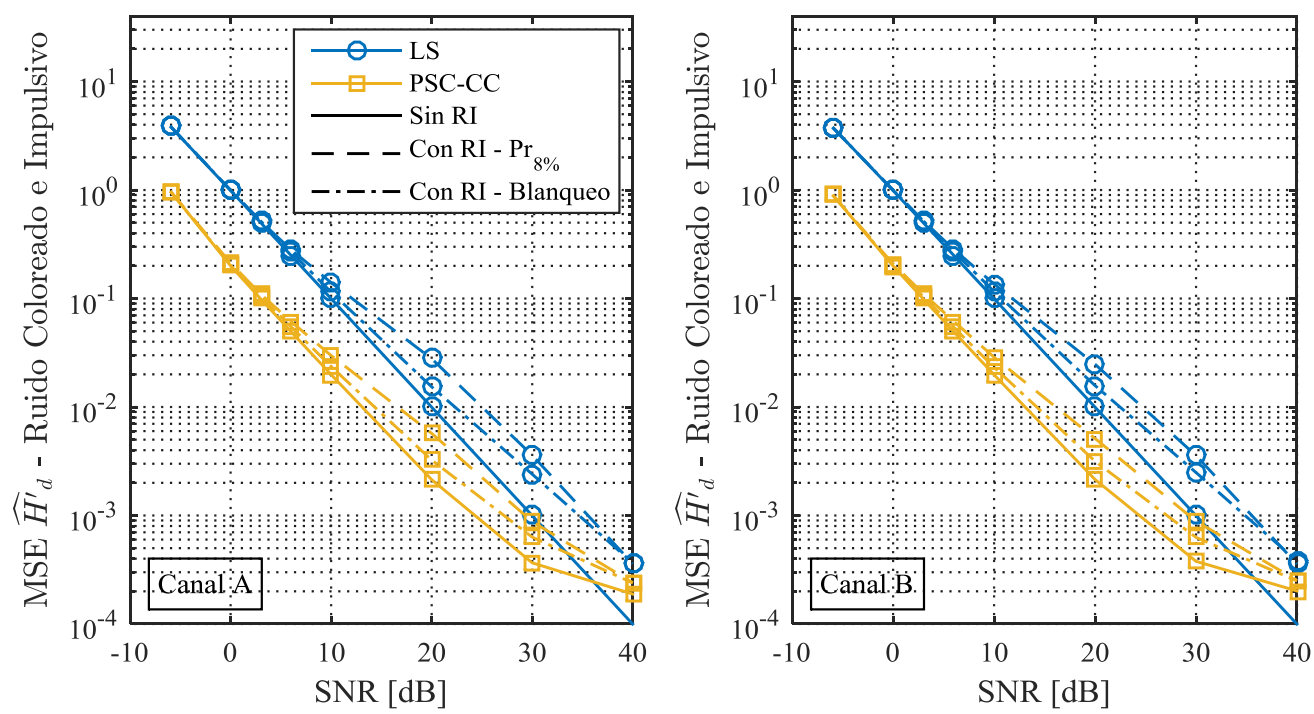

Fig. 6.47 MSE de la estimación del canal con sincronización conjunta, al incorporar eventos de ruido impulsivo y la técnica de mitigación de blanqueo, para los canales tipo A (izquierda y B (derecha)..

\subsection{Complejidad de la propuesta}

En esta sección se efectúa un análisis simplificado de la complejidad asociada a la propuesta de sincronización y estimación de canal conjunta para el sistema DMT bajo una máscara espectral de transmisión. Con respecto al análisis realizado para el sistema sin máscara espectral en la sección 5.3, resumido en la Fig. 5.22, las etapas de sincronización gruesa y fina son muy similares; mientras que las modificaciones más importantes se encuentran en la estimación de canal.

Como al aplicar la máscara espectral de transmisión se incluyó un filtro notch en recepción para reducir el ruido correspondiente a las bandas inactivas, la etapa de filtrado previo al procesamiento de la señal es necesario independientemente de la estrategia de sincronización y estimación de canal, por lo que no se considera en la evaluación de la complejidad asociada. Además, se definió un filtro digital de alto orden para el contexto de simulaciones, pero evidentemente en un sistema de implementación práctica se realiza un filtrado analógico en la estructura AFE, previo a la etapa de ADC.

Con respecto a la etapa de sincronización gruesa, la métrica utilizada $m_{3, n}$ es la misma que en la sección 4.3, y se calcula a partir de:

$$
m_{3, n}=\frac{N_{2} \sum_{k=0}^{N_{2}-1}\left|c_{n+k}^{\mathbf{r}, \mathbf{x}^{\prime}}\right|^{2}}{N \sum_{k=0}^{N_{2}-1} \sum_{m=0}^{N-1}\left|r_{n+k+m}\right|^{2}}=\frac{P_{3, n}}{E_{3, n}}
$$


El numerador $P_{3, n}$ contiene muestras de la correlación de la señal recibida con el símbolo piloto elevadas al cuadrado, se computan de acuerdo a (5.51), y la estructura equivalente para su cómputo es la de la Fig. 6.48. Resulta oportuno recordar que el primer bloque corresponde al correlador eficiente de CPS de longitud $M=N / 8$, de acuerdo a la estructura del símbolo piloto propuesto en el capítulo 4 (4.6). Por su parte, el denominador resulta de la suma de muestras de la señal recibida elevadas al cuadrado, computada a partir de una ecuación recursiva, con la arquitectura equivalente de la Fig. 6.49, donde el primer bloque de la izquierda corresponde a un multiplicador de dos entradas. Para la detección inicial del símbolo piloto se realiza la comparación $P_{3, n}>\gamma \cdot E_{3, n}$, y luego se identifica la ubicación del máximo de la métrica, calculada a partir de $P_{3, n} / E_{3, n}$.

A partir del resultado de la sincronización gruesa, se activa la etapa de sincronización fina. Contrario al resultado obtenido en la sección 5.1, al incluir la máscara espectral de transmisión se obtuvo como resultado en la sección 6.3 que la CCN modificada $c_{1, n}^{\prime}$ presenta un desempeño superior (y una complejidad inferior) para la sincronización fina respecto a $c^{\prime}{ }_{2, n}$. Así, es necesario en este caso computar únicamente $c^{\prime}{ }_{1, n}$ como

$$
c_{1, n}^{\prime}=\frac{\left|c_{n}^{\mathbf{r}, \mathbf{x}^{\prime}}\right|^{2}}{N \sum_{m=0}^{N-1} r_{n+m} \cdot r_{n+m}}=\frac{P_{1, n}^{\prime}}{E_{1, n}^{\prime}}
$$

donde tanto el numerador como el denominador de $c_{1, n}^{\prime}$, pueden obtenerse de resultados intermedios del cálculo del numerador y denominador de la métrica de sincronización gruesa $m_{3, n}$. Luego de obtener la ubicación del máximo $n_{f_{1}}$, se ejecuta el algoritmo de sincronización fina, identificando como coeficientes del canal a las muestras de $c_{1, n}^{\prime}$

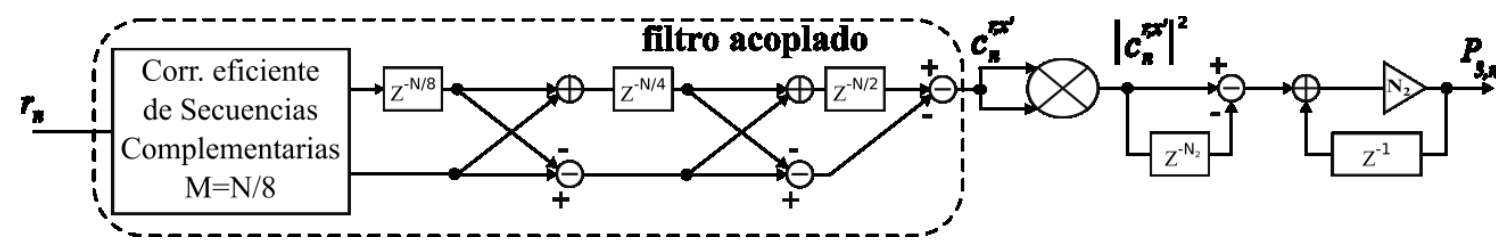

Fig. 6.48 Estructura equivalente para el cómputo del numerador de la métrica de sincronización gruesa.

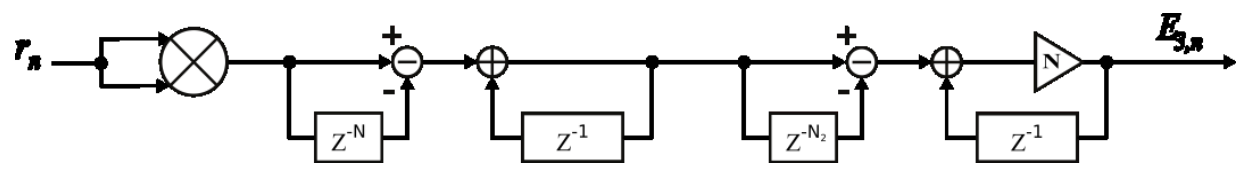

Fig. 6.49 Estructura equivalente para el cómputo del denominador de la métrica de sincronización gruesa. 
Diseño de Estrategias de Sincronización y Estimación de Canal para la Mejora de Comunicaciones en Redes Inteligentes de Energía

que superan el umbral de detección. Para esto, se considera el umbral propuesto $\alpha_{k_{1}}=k_{t h} \cdot c_{1, n_{f_{1}}}^{\prime}$, con $k_{t h}=0.15$. La comparación de la CCN con el umbral puede realizarse a partir de:

$$
c_{1, n}^{\prime}>\alpha_{k_{1}} \rightarrow P_{1, n}^{\prime}>\alpha_{k_{1}} \cdot E_{1, n}^{\prime}
$$

El diagrama en bloques que resume el procesamiento digital de señal requerido por la las etapas de sincronización, por lo comentado previamente, resulta muy similar a los bloques involucrados en la sincronización de la Fig. 5.22.

Durante el proceso de sincronización, las muestras correspondientes a la salida del filtro acoplado multiplicadas por $1 / \sqrt{P_{T}} N$ se almacenan secuencialmente en un registro de desplazamiento. Una vez que se obtiene el resultado de la etapa de sincronización fina, se deben modificar las muestras de la CC de acuerdo a (6.48) y (6.49) para el proceso de estimación de canal. Para ello, debe primero computarse la SDFT de la señal recibida $R_{k, n_{f}}$ en las 18 subportadoras activas necesarias para la interpolación y extrapolación de la correlación cruzada (16 para la interpolación en bandas inactivas intermedias y 2 para la extrapolación de baja y alta frecuencia). Como se debe computar (6.37) para cada subportadora, se requieren 18 bloques con la arquitectura equivalente de la Fig. 6.50, para obtener la SDFT simultáneamente, e ir almacenando las muestras hasta extraer la correspondiente al instante de sincronismo $n_{f}$. Nótese que la Fig. 6.50 es una réplica conveniente de la Fig. 6.38, para una mayor claridad acerca de la arquitectura y su complejidad. Si bien cada bloque requiere de 4 sumadores y 2 multiplicadores constantes reales (y elementos de memoria), la señal resultante del primer sumador es la misma en todos los casos, por lo que cada bloque adicional insume 3 sumadores y 2 multiplicadores. Así, se requieren en total 55 sumadores y 36 multiplicadores para obtener la SDFT en las 18 subportadoras.

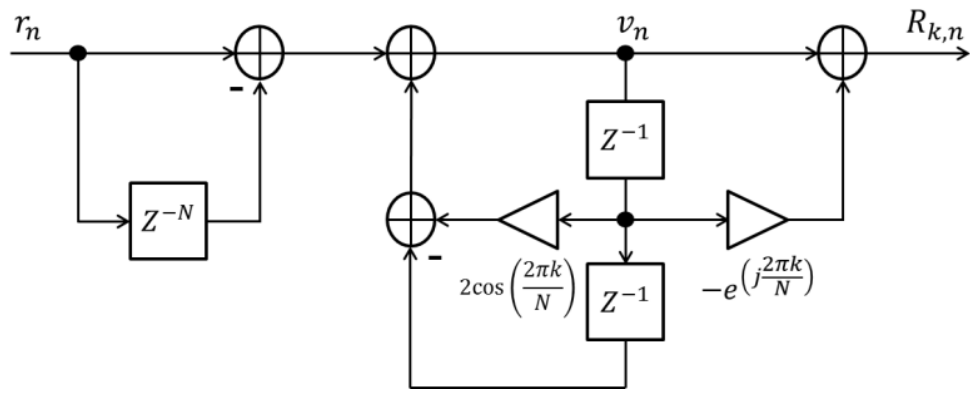

Fig. 6.50 Filtro equivalente para el cómputo de la SDFT. 
Capítulo 6: Sincronización y estimación de canal en sistemas DMT-PLC bajo una máscara espectral de transmisión

Por cada banda inactiva, se deben completar varias subportadoras inactivas. Tomando como ejemplo una banda inactiva $q$ que requiere interpolación, las muestras para completar la CC están dadas por la sumatoria:

$$
\sum_{k^{\prime}=k_{i n f, q^{+1}}}^{k_{\text {sup }, q^{-1}}} \tilde{c}_{k^{\prime}, q, m}
$$

Reemplazando (6.43) en (6.59) se obtiene:

$$
\begin{aligned}
& \sum_{k^{\prime}=k_{\text {inf }, q}+1}^{k_{\text {sup }, q}-1} \tilde{c}_{k^{\prime}, q, m}=\operatorname{Re}\left\{R_{k_{\text {inf }, q}, n_{f}}\right\} \sum_{k^{\prime}=k_{\text {inf }, q}+1}^{k_{\text {sup }, q^{-1}}}\left[2 \frac{k_{\text {sup }, q}-k^{\prime}}{k_{\text {sup }, q}-k_{\text {inf }, q}} \cos \left(\frac{2 \pi m}{N} k^{\prime}-\varphi_{k_{\text {inf }, q}}\right) \frac{\left|X^{\prime}{ }_{k^{\prime}}\right|^{2}}{N\left|X^{\prime}{ }_{k_{\text {inf }, q}}\right|}\right] \\
& +\operatorname{Im}\left\{R_{k_{\text {inf }, q}, n_{f}}\right\} \sum_{k^{\prime}=k_{\text {inf }, q}+1}^{k_{\text {sup }, q}-1}\left[2 \frac{k_{\text {sup }, q}-k^{\prime}}{k_{\text {sup }, q}-k_{\text {inf }, q}} \sin \left(\frac{2 \pi m}{N} k^{\prime}-\varphi_{k_{\text {inf }, q}}\right) \frac{\left|X^{\prime}{ }^{\prime}\right|^{2}}{N\left|X^{\prime}{ }_{k_{\text {inf }, q}}\right|}\right] \\
& +\operatorname{Re}\left\{R_{k_{\text {sup },}, n_{f}}\right\} \sum_{k^{\prime}=k_{\text {inf }, q}+1}^{k_{\text {sup }, q}-1}\left[2 \frac{k^{\prime}-k_{\text {inf }, q}}{k_{\text {sup }, q}-k_{\text {inf }, q}} \cos \left(\frac{2 \pi m}{N} k^{\prime}-\varphi_{k_{\text {sup }, q}}\right) \frac{\left|{X^{\prime}}_{k^{\prime}}\right|^{2}}{N\left|X^{\prime}{ }_{k_{\text {sup }, q}}\right|}\right] \\
& +\operatorname{Im}\left\{R_{k_{\text {sup }, q}, n_{f}}\right\} \sum_{k^{\prime}=k_{\text {inf }, q}+1}^{k_{\text {sup }, q}-1}\left[2 \frac{k^{\prime}-k_{\text {inf }, q}}{k_{\text {sup }, q}-k_{\text {inf }, q}} \sin \left(\frac{2 \pi m}{N} k^{\prime}-\varphi_{k_{\text {sup }, q}}\right) \frac{\left|X^{\prime}{ }_{k^{\prime}}\right|^{2}}{N\left|X^{\prime}{ }_{{ }_{s u p}, q}\right|}\right]
\end{aligned}
$$

Como cada parámetro dentro de las sumatorias es conocido, es posible precomputar las muestras correspondientes de cada sumatoria y almacenar en memoria las $N_{\text {pre }}+N_{h}$ muestras necesarias para completar la estimación de la CIR, con $-N_{\text {pre }} \leq m \leq N_{h}-1$. El factor $\sqrt{P_{T}} N$ de (6.49) se aplica a cada sumatoria en el mismo precómputo. Definiendo la notación $S C_{k_{i n f, q}, m}$ para la primera sumatoria de (6.60), entendida como la $m$-ésima muestra de la sumatoria de cosenos para la subportadora estimada $k_{\text {inf }}$ de la $q$-ésima banda inactiva, y $S S_{k_{i n f, q}, m}$ como la sumatoria de senos, repitiendo el criterio para el resto se puede reescribir (6.60) como:

$$
\begin{aligned}
& \sum_{k^{\prime}=k_{\text {inf }, q}+1}^{k_{\text {sup }, q}-1} \tilde{c}_{k^{\prime}, q, m}=\operatorname{Re}\left\{R_{k_{\text {inf }, q}, n_{f}}\right\} S C_{k_{\text {inf }, q}, m}+\operatorname{Im}\left\{R_{k_{\text {inf }, q}, n_{f}}\right\} S S_{k_{\text {inf }, q}, m} \\
& +\operatorname{Re}\left\{R_{k_{\text {sup }, q}, n_{f}}\right\} S C_{k_{\text {sup }, q}, m}+\operatorname{Im}\left\{R_{k_{\text {sup }, q}, n_{f}}\right\} S S_{k_{\text {sup }, q}, m}
\end{aligned}
$$

Por lo tanto, para generar las muestras que completan una banda inactiva a partir de la interpolación se precisan $4\left(N_{\text {pre }}+N_{h}\right)$ multiplicaciones y $4\left(N_{\text {pre }}+N_{h}\right)$ sumas reales. 
Del mismo modo, a partir de (6.46) y (6.47), las muestras que completan la banda inferior y superior a partir de la extrapolación resultan:

$$
\begin{gathered}
\sum_{k^{\prime}=0}^{85} \tilde{c}_{k^{\prime}, 0, m}=\operatorname{Re}\left\{R_{86, n_{f}}\right\} S C_{86, m}+\operatorname{Im}\left\{R_{86, n_{f}}\right\} S S_{86, m} \\
\sum_{k^{\prime}=1144}^{2048} \tilde{c}_{k^{\prime}, 9, m}=\operatorname{Re}\left\{R_{1143, n_{f}}\right\} S C_{1143, m}+\operatorname{Im}\left\{R_{1143, n_{f}}\right\} S S_{1143, m}
\end{gathered}
$$

Con esto, se completa la estimación de la CIR de acuerdo a:

$$
\tilde{h}_{m}=\frac{c_{n_{\mathrm{o} p t}+m}^{\mathbf{r}, \mathbf{x}^{\prime}}}{\sqrt{P_{T}} N}+\frac{\widetilde{c_{I m}}}{\sqrt{P_{T}} N}, \quad-N_{p r e} \leq m \leq N_{h}-1
$$

Entonces, para completar el cómputo de (6.64) se requieren en total $36\left(N_{\text {pre }}+N_{h}\right)$ multiplicaciones y sumas reales. Sin embargo, se puede obtener una arquitectura que demande para su implementación un número reducido de bloques multiplicadores y sumadores si se diseña una máquina de estados que obtenga secuencialmente cada una de las $N_{\text {pre }}+N_{h}$ muestras de la estimación de la CIR. El diagrama en bloques de esta variante se encuentra en la Fig. 6.51. El fin de la etapa de sincronización fina con la identificación del instante de sincronismo $n_{f}$ da inicio al proceso. Se compone de una máquina de estados en las que por cada pulso de reloj $c l k$ se completa la $m$-ésima muestra de la estimación de la CIR a partir de la modificación de la CC. Por cada ciclo se extrae una muestra de las sumas de senos y cosenos almacenadas en memoria, y se multiplica con la correspondiente parte real o compleja de la SDFT de la señal recibida en el instante de sincronismo (que se mantienen constantes durante todo el proceso). El resultado de los productos correspondientes a las 10 bandas inactivas se suma, completando la muestra de la estimación de la CIR.

En la parte inferior de la Fig. 6.51 puede observarse que cada una de las 8 bandas inactivas que se completan a partir de interpolación requiere 4 multiplicadores de dos entradas y 4 sumadores reales. En su conjunto, esta variante requiere de 36 multiplicadores de dos entradas y 36 sumadores para computar, luego de $N_{\text {pre }}+N_{h}$ ciclos, las muestras necesarias de la estimación de la CIR. Evidentemente, todo el proceso agregará una latencia adicional al requerido por las etapas de sincronización gruesa y fina, y debe evaluarse si la latencia total se encuentra dentro de la aceptable por el sistema. Una opción en caso de que la latencia introducida sea demasiado elevada es 
Capítulo 6: Sincronización y estimación de canal en sistemas DMT-PLC bajo una máscara espectral de transmisión

comenzar la modificación de la CC a partir del instante de sincronización gruesa $n_{g}$. De esta manera, mientras se ejecuta el algoritmo de sincronización fina (búsqueda de máximo, umbralización, etc), se realiza en paralelo la modificación de las muestras de las CC secuencialmente desde el instante $n_{g}$, disminuyendo la latencia. Esto por otro lado implica la necesidad de almacenar las muestras modificadas en un registro lo

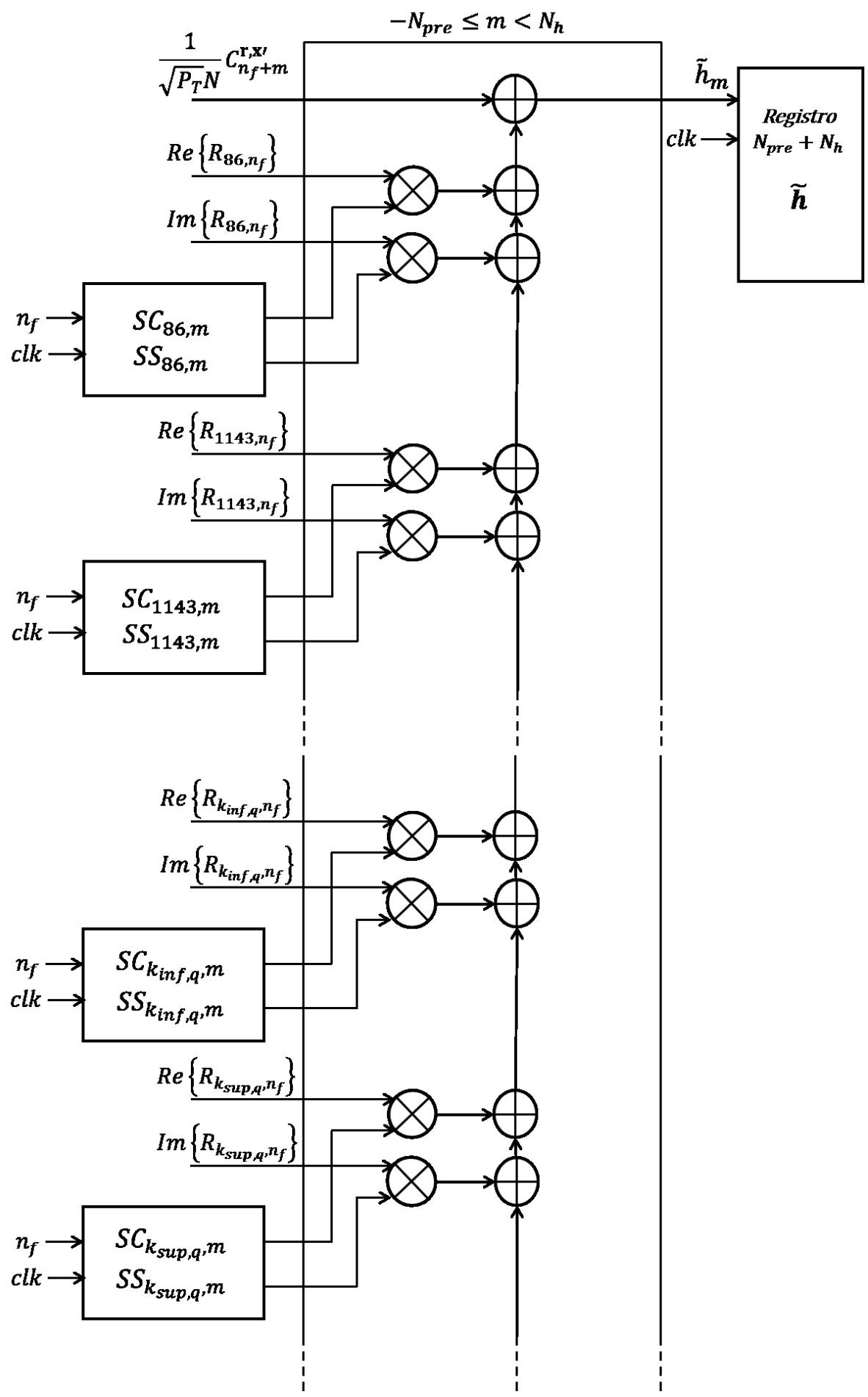

Fig. 6.51 Diagrama en bloques para computar la estimación de la respuesta al impulso del canal. 
Diseño de Estrategias de Sincronización y Estimación de Canal para la Mejora de Comunicaciones en Redes Inteligentes de Energía

suficientemente amplio como para poder seleccionar las muestras que corresponden, una vez obtenido el instante de sincronismo fino $n_{f}$. Luego, se efectúa la DFT de la CIR para obtener la estimación de la CFR, de acuerdo a la Fig. 6.41.

El diagrama en bloques que resume el procesamiento digital de señal requerido por la propuesta de sincronización y estimación de canal conjunta se presenta en la Fig. 6.52. Se considera a la señal $r_{n}$ de entrada al sistema como la resultante luego de que la señal recibida pase por el filtro notch y se aplique una técnica de mitigación del ruido impulsivo. Ésta atraviesa el filtro acoplado eficiente para obtener la CC, a partir de la cual se basan las etapas de sincronización gruesa, fina y la estimación de canal. Con respecto a la transmisión del símbolo piloto sin máscara de transmisión, la principal diferencia se encuentra en la inclusión del banco de SDFTs y la modificación de la correlación cruzada para obtener la estimación de la CIR, que representa un incremento en la complejidad de la estrategia de sincronización y estimación de canal. En conjunto demandan 36 multiplicadores de dos entradas, 36 multiplicadores constantes y 91 sumadores. Esto se evidencia en la Tabla 6.4, en la que se encuentra la cantidad de bloques aritméticos que la propuesta de sincronización y estimación de canal demanda en los escenarios de transmisión con y sin máscara espectral aplicada. Resulta importante destacar que el análisis es simplificado, dado que no se ha detallado en la Fig. 6.52 ni en el análisis de la complejidad: las búsquedas de valores máximos de la métrica de sincronización gruesa y de la $\mathrm{CCN}$, la ejecución del algoritmo de sincronización fina, las señales de control de inicio y fin de las distintas etapas, y elementos de memoria que deben registrar señales intermedias a medida que se avanza con el proceso. Sin embargo, resulta una primera medida para evaluar si la propuesta mantiene una complejidad asociada reducida.

Para tener una referencia de comparación, si se considerara el símbolo piloto de longitud $N=4096$ creado a partir de secuencias ZC utilizado para la estimación de canal por LS también para efectuar la sincronización a partir de la correlación cruzada, la demanda de recursos sería considerablemente mayor. Si bien teóricamente los 4096 elementos del símbolo son diferentes, si se consideran como iguales los coeficientes que difieren en el orden de $0,1 \%$, se puede implementar un filtro acoplado que demandaría alrededor de 1000 multiplicadores constantes y 4095 sumadores. Es decir que el incremento en los recursos demandados para aplicar la estrategia propuesta de sincronización y estimación de canal basada en CPS, incluyendo la máscara espectral de 


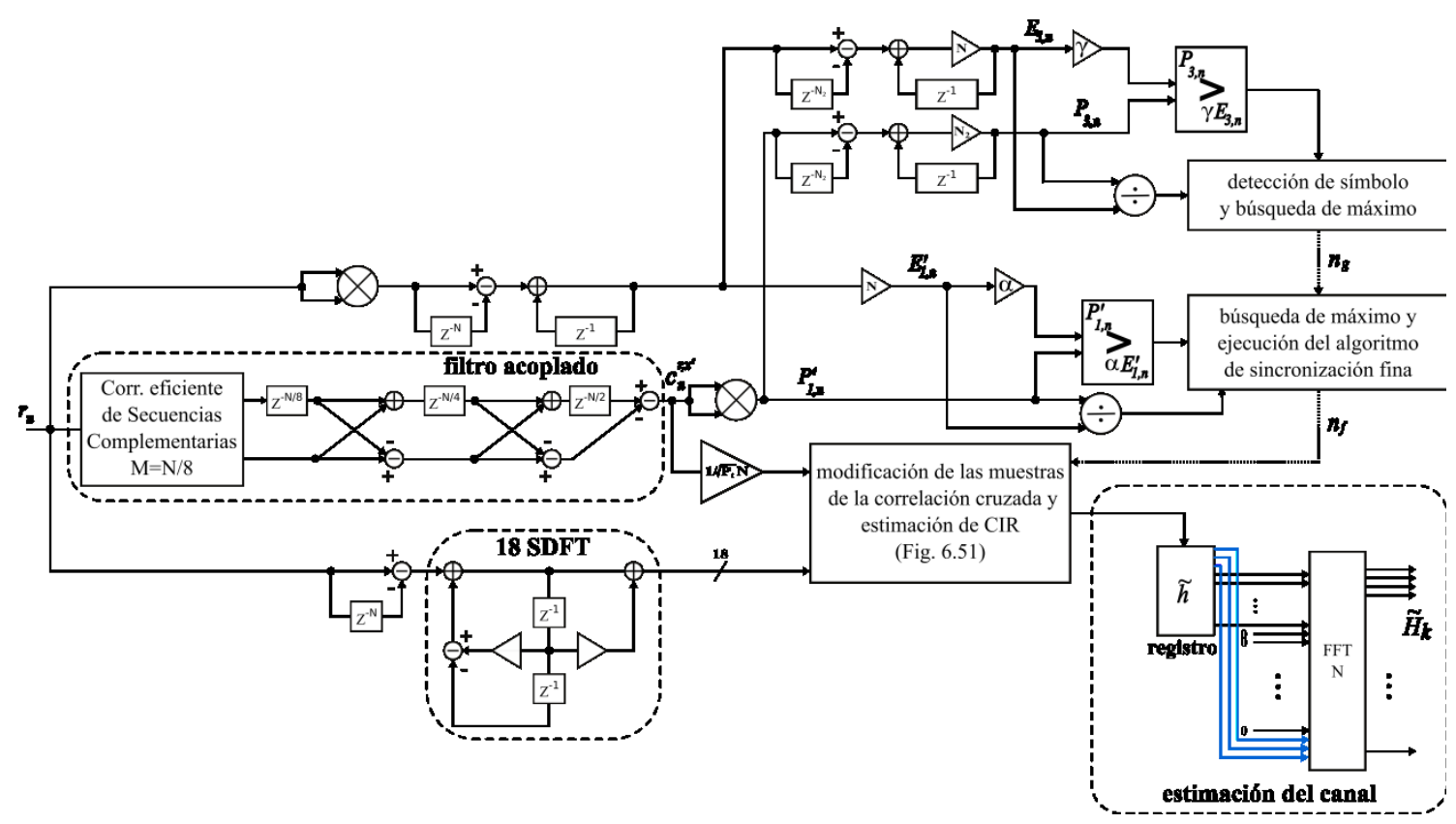

Fig. 6.52 Diagrama en bloques del procesamiento de señal requerido para la propuesta de sincronización y estimación de canal conjunta.

Tabla 6.4 Resumen preliminar de recursos demandados por la propuesta de sincronización y estimación de canal conjunta bajo una transmisión con y sin máscara espectral.

\begin{tabular}{|c|c|c|}
\hline Bloques aritméticos & Sin máscara & Con máscara \\
\hline Sumadores & 30 & 120 \\
\hline Mult. constantes & 6 & 42 \\
\hline Mult./Div. 2 de entradas & 6 & 40 \\
\hline
\end{tabular}

transmisión, mantiene una complejidad reducida respecto a una construcción del símbolo piloto basado en otro tipo de secuencias.

\subsection{Conclusiones}

En este último capítulo se presentó un escenario más realista de transmisión del sistema DMT-PLC respecto a los analizados en capítulos anteriores, en el que se limitó el número de subportadoras activas a partir de una máscara espectral de compatibilidad electromagnética, además de incluir el modelo estadístico del canal PLC y de ruido de fondo coloreado e impulsivo.

Se verificó que la aplicación de la máscara degrada las propiedades de correlación del símbolo piloto propuesto, en detrimento del desempeño de las etapas de sincronización 
Diseño de Estrategias de Sincronización y Estimación de Canal para la Mejora de Comunicaciones en Redes Inteligentes de Energía

y estimación de canal planteadas anteriormente. Por otro lado, como el ruido de fondo coloreado presenta una PSD decreciente con la frecuencia, se evaluó la viabilidad de filtrar una gran parte del ruido presente en las frecuencias correspondientes a las subportadoras inactivas de baja frecuencia, a partir del diseño preliminar de un filtro digital tipo notch.

En ese contexto, se extendió el análisis de las métricas de sincronización gruesa basadas en AC y CC del capítulo 4, caracterizando las PDFs en muestras anteriores a la llegada del símbolo piloto a partir de los parámetros del filtro equivalente que colorea el ruido, el filtro notch incluido, y las propiedades de correlación del símbolo piloto. A partir del análisis, se redefinieron los umbrales de detección para cada métrica, y a partir de simulaciones se contrastaron las PDFs analíticas y posteriormente se evaluó la capacidad de detección primaria de cada métrica. Nuevamente, ante el nuevo contexto de estudio, la métrica basada en CC presentó un mejor desempeño que la que utiliza AC.

Con respecto a la etapa de sincronización fina, se verificó que los umbrales derivados en el capítulo 5 no resultan útiles en el contexto de transmisión bajo la máscara espectral, debido a la distorsión de la zona de correlación ideal del símbolo piloto propuesto. Por lo tanto, se propuso un umbral práctico que mejoró el desempeño, y debido a que derivar umbrales óptimos de manera analítica se encontró fuera del alcance de este trabajo, se comparó el desempeño del algoritmo de sincronización fina utilizando umbrales óptimos obtenidos heurísticamente a partir de simulaciones. Se comprobó que el desempeño del algoritmo de sincronización fina, con el umbral propuesto, presenta a priori un desempeño adecuado, manteniendo un error promedio tolerable por el sistema. Posteriormente, se realizaron simulaciones que dan cuenta que la etapa de estimación de canal es la más comprometida debido a que la distorsión introducida por la máscara espectral de transmisión degrada sustancialmente el desempeño a medias y altas $S N R \mathrm{~s}$, aún bajo un escenario de sincronismo perfecto. Para contrarrestar la pérdida de rendimiento, se propuso la modificación de la correlación cruzada en el receptor a partir de la interpolación lineal y extrapolación en el dominio de la frecuencia para completar las subportadoras inactivas. Se verificó mediante simulaciones que la propuesta presenta un desempeño competitivo respecto a otras estrategias de estimación de canal en el contexto de subportadoras inactivas. 
Capítulo 6: Sincronización y estimación de canal en sistemas DMT-PLC bajo una máscara espectral de transmisión

Además, se planteó la modificación de la correlación cruzada a partir de las muestras en el dominio del tiempo, para aprovechar los cómputos realizados en las etapas de sincronización gruesa y fina, y así mantener en conjunto una complejidad reducida. A partir de un análisis de los bloques asociados a la implementación de la estrategia de sincronización y estimación de canal conjunta propuesta, se comprueba que mantiene una relativa baja complejidad. 
Diseño de Estrategias de Sincronización y Estimación de Canal para la Mejora de Comunicaciones en Redes Inteligentes de Energía

\section{CONCLUSIONES Y TRABAJOS FUTUROS}

En este capítulo se presentan las conclusiones más relevantes que se obtienen de la presente tesis. Posteriormente, se presentan como trabajos futuros distintas líneas de investigación que pueden desarrollarse a partir de las contribuciones de esta tesis. Finalmente, se detallan los artículos publicados (o en proceso de publicación) en revistas internacionales, y en congresos nacionales e internacionales.

\subsection{Conclusiones}

Debido al creciente interés en el desarrollo de las redes eléctricas inteligentes (SGs), la tecnología PLC con modulación multiportadora ha sido motivo de investigación reciente en virtud de ser candidata para cumplimentar los requerimientos de comunicaciones de las SGs. La modulación OFDM es la más divulgada en sistemas PLC actuales, y a pesar del gran avance en OFDM orientado a sistemas inalámbricos en el pasado, recientemente se han realizado esfuerzos en el desarrollo de técnicas de sincronización y estimación de canal que hagan frente a las condiciones particularmente desfavorables del canal: marcada selectividad en frecuencia, ruido de fondo coloreado y diversas fuentes de ruido impulsivo. La presente tesis contribuye al desarrollo de estrategias eficientes de sincronización y estimación de canal para sistemas OFDMPLC, que representan una reducida complejidad conservando un desempeño competitivo. Las contribuciones principales de la presente tesis son las siguientes: 
1. Se realizó el diseño de un símbolo piloto novedoso construido a partir de pares de secuencias complementarias, bajo el criterio de presentar adecuadas propiedades de correlación, un procesamiento de reducida complejidad, y la flexibilidad necesaria para poder aplicar técnicas de sincronización basadas en AC y CC, y obtener una estimación de canal a partir de esta última.

2. Ante la falta en la literatura de una comparación exhaustiva entre las técnicas de sincronización gruesa basadas en $\mathrm{AC}$ y $\mathrm{CC}$ en las condiciones particulares del canal PLC, se demostró analíticamente y por simulaciones para un sistema DMT-PLC que la métrica basada en CC presenta un comportamiento más favorable para la detección primaria del símbolo piloto, en diversos escenarios planteados: con AWGN, ruido de fondo coloreado, ruido impulsivo, con técnicas de mitigación de ruido impulsivo, y error en la frecuencia de muestreo. Para ello, se realizó un análisis detallado de las métricas de sincronización, y se derivaron umbrales de detección adecuados para cada caso.

3. Se desarrolló un algoritmo de sincronización fina a partir de la CC eficiente del símbolo piloto propuesto, en el que los cómputos realizados pueden reutilizarse posteriormente para obtener una estimación de la respuesta al impulso del canal, reduciendo la complejidad del conjunto respecto a un enfoque individual de cada etapa. Se planteó una $\mathrm{CC}$ normalizada y se derivaron umbrales prácticos en un contexto de transmisión OFDM paso banda bajo un canal Rayleigh multicamino. Los umbrales obtenidos dependen de los parámetros del sistema, del modelo del canal y se ajustan en función de la condición de relación señal a ruido. El desempeño de la propuesta se verificó satisfactoriamente mediante simulaciones en un canal multicamino bajo diferentes tipos de ruidos de PLC, y se realizó una comparación de desempeño respecto a la utilización de símbolos piloto construidos a partir de otras secuencias, verificándose su competitividad.

Adicionalmente, se adaptó la propuesta de sincronización fina y estimación de canal para un esquema de transmisión DMT, inlcuyendo un modelo estadístico del canal PLC. A partir del análisis del modelo del canal, se caracterizaron las PDFs de la $\mathrm{CCN}$ en el instante de sincronismo óptimo y en las muestras anteriores, para evaluar de manera analítica la probabilidad de sincronización perfecta. Luego, se propuso una CCN modificada que reduce el impacto negativo de la variación de la ganancia del canal PLC en la CCN. Para las dos variantes de $\mathrm{CCN}$, se derivaron los umbrales: uno que presenta una baja 
Diseño de Estrategias de Sincronización y Estimación de Canal para la Mejora de Comunicaciones en Redes Inteligentes de Energía

probabilidad de falsa detección, y un umbral que maximiza la probabilidad de sincronización perfecta. A partir de simulaciones se contrastó el resultado obtenido en cada caso con el esperado a partir del análisis realizado, y se evaluó el desempeño del algoritmo de sincronización fina, obteniendo un valor de RMSE muy inferior respecto al que se obtiene en la sincronización gruesa, aún bajo la presencia de ruido impulsivo.

4. Se evaluó la propuesta de sincronización y estimación de canal conjunta en un escenario de transmisión DMT-PLC en el que se limita el número de subportadoras activas a partir de una máscara espectral de compatibilidad electromagnética, además de incluir el modelo estadístico del canal PLC y de ruido de fondo coloreado e impulsivo. Se verificó la degradación de las propiedades del símbolo piloto propuesto, y se evaluó la viabilidad de filtrar una gran parte del ruido presente en las frecuencias correspondientes a las subportadoras inactivas de baja frecuencia, a partir del diseño preliminar de un filtro digital tipo notch.

En ese contexto, se extendió el análisis de las métricas de sincronización gruesa basadas en $\mathrm{AC}$ y $\mathrm{CC}$, caracterizando las PDFs en muestras anteriores a la llegada del símbolo piloto a partir de los parámetros del filtro equivalente que colorea el ruido, el filtro notch incluido, y las propiedades de correlación del símbolo piloto. A partir del análisis, se redefinieron los umbrales de detección para cada métrica, y a partir de simulaciones se contrastaron las PDFs analíticas y posteriormente se evaluó la capacidad de detección primaria de cada métrica. Con respecto a la etapa de sincronización fina, se propuso un umbral práctico, y se verificó un adecuado desempeño del algoritmo de sincronización fina a partir de la comparación respecto a utilizar umbrales óptimos obtenidos heurísticamente a partir de simulaciones.

Posteriormente, debido a la degradación de la estrategia de estimación de canal por la inclusión de la máscara espectral, se planteó la modificación de la correlación cruzada en el receptor a partir de la interpolación lineal y extrapolación en el dominio de la frecuencia para completar las subportadoras inactivas. Se verificó mediante simulaciones que la propuesta presenta un desempeño competitivo respecto a otras estrategias de estimación de canal en el contexto de subportadoras inactivas. 
5. Se llevó a cabo un análisis de la complejidad asociada al cómputo de los algoritmos propuestos para la sincronización y estimación de canal conjunta en los distintos escenarios planteados. Por un lado, en un contexto de transmisión con todas las subportadoras disponibles, se verifica que la propuesta representa una opción de reducida complejidad gracias al diseño del símbolo piloto propuesto y su arquitectura de correlación eficiente. Esto se debe principalmente a que las tres etapas (sincronización gruesa, sincronización fina y estimación de canal) utilizan fundamentalmente las muestras correspondientes a la correlación cruzada, y ésta requiere únicamente algunos bloques sumadores y elementos de memoria. En el contexto que incluye una máscara espectral de transmisión, se propuso la modificación de la correlación cruzada a partir de las muestras en el dominio del tiempo, para aprovechar los cómputos realizados en las etapas de sincronización gruesa y fina, y así mantener en conjunto una complejidad reducida. A partir de un análisis de los bloques asociados a la implementación de la estrategia de sincronización y estimación de canal conjunta propuesta, se comprueba que mantiene una relativa baja complejidad.

\subsection{Trabajos Futuros}

En la presente tesis se desarrollaron y analizaron estrategias de baja complejidad para la sincronización y estimación de canal en sistemas PLC. A lo largo del desarrollo de la misma, se han identificado cuestiones que no fueron tratadas intensivamente, pero que pueden ser profundizadas o mejoradas a partir de trabajos futuros:

1. Efectuar la implementación de los algoritmos de sincronización y estimación de canal a partir de las arquitecturas propuestas en dispositivos de lógica programable tipo FPGA (Field-Programmable Gate Array), y realizar pruebas experimentales en entornos PLC reales.

2. Los algoritmos propuestos se han desarrollado considerando sistemas PLC SISO (Single-Input Single-Output), es decir, utilizando dos conductores para la transmisión. En realidad, las instalaciones domiciliarias de baja tensión utilizan tres conductores, y las de media y alta tensión suelen tener cuatro o más conductores disponibles. Esto ha posibilitado el desarrollo de técnicas MIMOPLC, que logran un desempeño superador aprovechando la diversidad espacial que las caracteriza [Berger 2014]. Los algoritmos de sincronización y estimación de canal propuestos pueden extenderse a este tipo de sistemas, utilizando 
Diseño de Estrategias de Sincronización y Estimación de Canal para la Mejora de Comunicaciones en Redes Inteligentes de Energía

códigos derivados de los PSC con correlación eficiente, como pueden ser secuencias LS o ZCZ [Wang 2016].

3. En la presente tesis se consideró un esquema de modulación multiportadora tipo OFDM, que forma parte de la capa física de diversos estándares PLC. Sin embargo, ante las restricciones en el espectro utilizado para BB-PLC, OFDM presenta una seria desventaja debido a su reducida selectividad en frecuencia. Por ello, se han propuesto nuevos sistemas de modulación multiportadora basados en bancos de filtros para PLC, como puede ser: Wavelet OFDM [Nombela 2015b], FMT (Filtered Multitone) [Tonello 2009] y CB-FMT (Cyclic Block Filtered Multitone) [Tonello 2013]. Las particularidades de cada esquema de modulación requiere de un estudio detallado para el diseño de símbolos piloto y algoritmos de sincronización y estimación de canal adecuados.

4. En la estrategia propuesta de sincronización fina basada en CC y umbralización se realizó la suposición de que todos los coeficientes de la CIR del canal son independientes. En realidad, existe cierta correlación entre muestras contiguas de la CIR, que depende de las características del modelo estadístico del canal PLC, como puede osbervarse en la Fig. 4.1. Esto puede aprovecharse para mejorar la capacidad de detección del primer camino del canal, a partir de una adecuada combinación de muestras de la CC con similitud.

5. Los algoritmos propuestos hacen frente a la detección primaria, sincronización y estimación de canal a partir del procesamiento del símbolo piloto transmitido. Sin embargo, los resultados obtenidos pueden refinarse de manera recursiva a partir de los símbolos de datos. Para ello, pueden aplicarse las técnicas en las que se realimenta la información luego de la decisión tomada al demodular los datos en las subportadoras [Akhtman 2007] [Shi 2005], o en los que se explotan los códigos de control de errores utilizados en la codificación de la información [Al-Rawi 2003].

\subsection{Publicaciones derivadas de la tesis}

A continuación, se exponen las publicaciones en revistas indexadas y en congresos nacionales e internacionales derivadas del trabajo de la presente tesis: 
M. Colombo, Á. Hernández, and J. Ureña, "Low-Complexity Joint Time Synchronization and Channel Estimation for OFDM-Based PLC Systems." IEEE Access, vol. 7, pp. 121446-121456, 2019.

M. Colombo, A. Hernández, J. Ureña, and C. De Marziani, "Sincronización y Estimación de Canal mediante Secuencias Complementarias para Sistemas DMT." SAAEI 2018 XXV Seminario Anual de Automárica, Electrónica Industrial e Instrumentación, 2018. Universidad Politécnica de Cataluña.

M. Colombo, C. De Marziani, Á. Hernández, J. Ureña, and M. Mayosky, "Lowcomplexity timing synchronization for OFDM based on CAZAC and Golay sequences." 2017 IEEE 17th International Conference on Ubiquitous Wireless Broadband (ICUWB), 2017, pp. 1-5.

M. Colombo, S. Murano, E. García, C. De Marziani, D. Roldos, "Improved Correlation Algorithm of Multilevel Complementary Set of Sequences. " Congreso Bienal de IEEE Argentina ARGENCON 2016.

M. Colombo, S. Murano, D. Roldos, C. D. Marziani, R. Alcoleas, M. C. Pérez, and E. García, "FPGA hardware resources optimization on efficient correlation algorithm for multilevel CSS." 2015 XVI Workshop on Information Processing and Control (RPIC), 2015, pp. 1-6.

J. Pires, M. Colombo, C. De Marziani, J. Gallardo, R. Alcoleas, S. Murano, and D. Roldos, "Ecualizador Basado en el Algoritmo BCJR para Canales Acústicos Subacuáticos en Sistemas OFDM." XVII Reunión de trabajo en Procesamiento de la Información y Control, 2018. Universidad Nacional de Mar del Plata.

S. Murano, M. Colombo, J. Aparicio, and A. Jiménez, "Using Multilevel Complementary Sets of Sequences for Acoustic Signals Emission in Underwater Links." 3er Simposio Internacional de la Sociedad de Ingeniería Oceánica en América del Sur, IEEE Oceanic Engineering Society, 2016. Buenos Aires.

C. De Marziani, M. Colombo, R. Alcoleas, S. Murano, J. Gallardo, A. Colombo, and J. Pires, "An optimization algortihm for relative positioning systems based on Harmony Search.” 2015 IEEE International Conference on Industrial Technology (ICIT), 2015, pp. 3392-3397.

S. Murano, M.C. Pérez, E. García, J. Ureña, C. De Marziani, R. Alcoleas, and M. Colombo, "Implementación Hardware de un Algoritmo de Correlación Eficiente de 
Diseño de Estrategias de Sincronización y Estimación de Canal para la Mejora de Comunicaciones en Redes Inteligentes de Energía

Códigos CSS Multinivel." SAAEI 2015 XII Seminario Anual de Automática, Electrónica Industrial e Instrumentación, 2015. Universidad de Zaragoza.

Adicionalmente, los siguientes artículos se encuentran en proceso de redacción/revisión: M.Colombo, A. Hernández, J. Ureña, M. Mayosky, and C. De Marziani, “A comparison between autocorrelation- and crosscorrelation-based coarse frame synchronization schemes for OFDM-PLC systems." Signal Processing, Elsevier, 2021.

M.Colombo, J. Ureña, A. Hernández, M. Mayosky, and C. De Marziani, "Fine timing synchronization and channel estimation in OFDM-based PLC systems with null subcarriers." IEEE Access, 2021. 


\section{ANEXO A}

Para facilitar cierta reproducibilidad de los resultados obtenidos por simulaciones en la presente tesis, se incluyen en el presente anexo los coeficientes más significativos del filtro FIR equivalente $g_{n}$ de (4.32), que se utiliza para obtener las muestras del ruido de fondo coloreado a partir del filtrado de un proceso de ruido blanco gaussiano. En la Tabla A.1 se presentan 256 coeficientes seleccionados de la respuesta al impulso del filtro de acuerdo a la Fig. A.1. Los coeficientes del filtro reducido $g_{n}^{\prime}$ se obtienen, considerando a la Tabla A.1 como una matriz T de 32 filas y 8 columnas, de acuerdo a:

$$
g_{n}^{\prime}=T_{i, j}, \quad \text { con } \quad \begin{aligned}
& n=8(i-1)+j \\
& j=1,2, \ldots, 8 \\
& i=1,2, \ldots, 32
\end{aligned}
$$

donde $T_{i, j}$ representa el elemento de la $i$-ésima fila y $j$-ésima columna de la Tabla A.1. Luego, debido a la simetría el filtro FIR, se obtiene el filtro reducido de 511 coeficientes como:

$$
g_{512-n}^{\prime}=g_{n}^{\prime}, \quad \text { con } n=1,2, \ldots, 256
$$

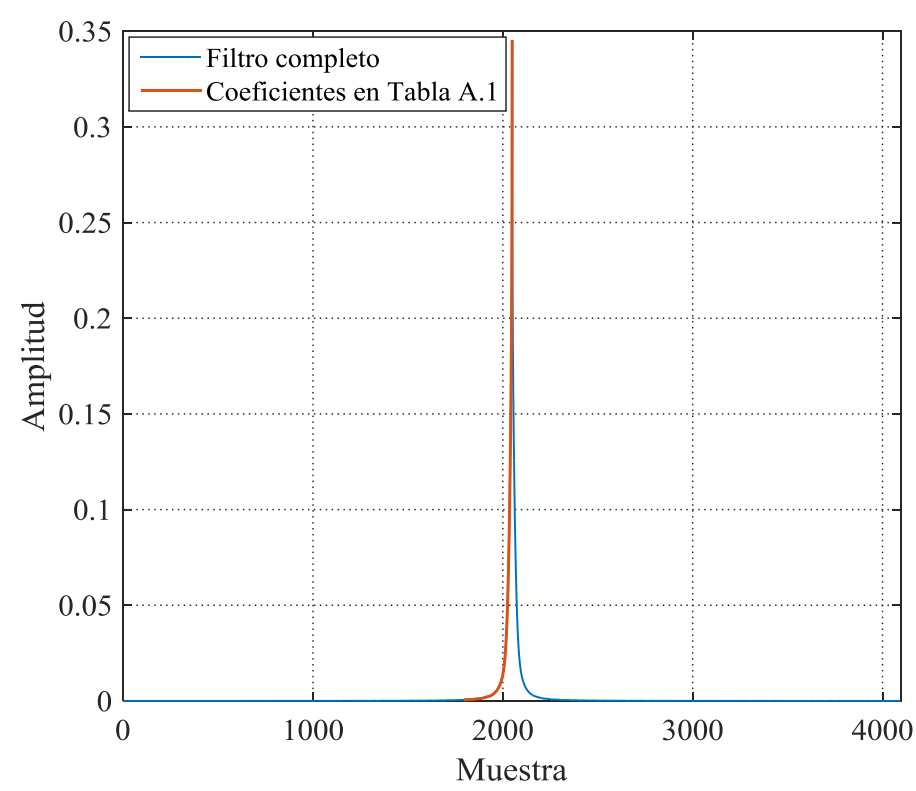

Fig. A.1 Respuesta al impulso del filtro equivalente que colorea el ruido de fondo y coeficientes más significativos incluidos en la Tabla A.1. 
Diseño de Estrategias de Sincronización y Estimación de Canal para la Mejora de Comunicaciones en Redes Inteligentes de Energía

Tabla A.1 Coeficientes más significativos del filtro equivalente utilizado para colorear el proceso de ruido blanco gaussiano.

\begin{tabular}{|c|c|c|c|c|c|c|c|c|}
\hline$j$ & 1 & 2 & 3 & 4 & 5 & 6 & 7 & 8 \\
\hline 1 & $5.370 \mathrm{e}-4$ & $5.448 \mathrm{e}-4$ & $5.535 \mathrm{e}-4$ & $5.622 \mathrm{e}-4$ & $5.716 \mathrm{e}-4$ & $5.808 \mathrm{e}-4$ & $5.907 \mathrm{e}-4$ & $6.002 \mathrm{e}-4$ \\
\hline 2 & $6.102 \mathrm{e}-4$ & $6.196 \mathrm{e}-4$ & $6.294 \mathrm{e}-4$ & $6.385 \mathrm{e}-4$ & $6.478 \mathrm{e}-4$ & $6.562 \mathrm{e}-4$ & $6.648 \mathrm{e}-4$ & $6.723 \mathrm{e}-4$ \\
\hline 3 & $6.799 \mathrm{e}-4$ & $6.864 \mathrm{e}-4$ & $6.929 \mathrm{e}-4$ & $6.982 \mathrm{e}-4$ & $7.035 \mathrm{e}-4$ & $7.077 \mathrm{e}-4$ & $7.118 \mathrm{e}-4$ & $7.149 \mathrm{e}-4$ \\
\hline 4 & $7.180 \mathrm{e}-4$ & $7.201 \mathrm{e}-4$ & $7.223 \mathrm{e}-4$ & $7.236 \mathrm{e}-4$ & $7.252 \mathrm{e}-4$ & $7.261 \mathrm{e}-4$ & $7.274 \mathrm{e}-4$ & $7.281 \mathrm{e}-4$ \\
\hline 5 & $7.294 \mathrm{e}-4$ & $7.304 \mathrm{e}-4$ & $7.321 \mathrm{e}-4$ & $7.335 \mathrm{e}-4$ & $7.360 \mathrm{e}-4$ & $7.383 e-4$ & $7.418 \mathrm{e}-4$ & $7.454 \mathrm{e}-4$ \\
\hline 6 & $7.501 \mathrm{e}-4$ & $7.551 \mathrm{e}-4$ & $7.614 \mathrm{e}-4$ & $7.678 \mathrm{e}-4$ & $7.757 \mathrm{e}-4$ & $7.838 \mathrm{e}-4$ & $7.934 \mathrm{e}-4$ & $8.031 \mathrm{e}-4$ \\
\hline 7 & $8.142 \mathrm{e}-4$ & $8.254 \mathrm{e}-4$ & $8.380 \mathrm{e}-4$ & $8.505 \mathrm{e}-4$ & $8.642 \mathrm{e}-4$ & $8.778 \mathrm{e}-4$ & $8.924 \mathrm{e}-4$ & $9.067 \mathrm{e}-4$ \\
\hline 8 & $9.220 \mathrm{e}-4$ & $9.366 \mathrm{e}-4$ & $9.521 \mathrm{e}-4$ & $9.668 \mathrm{e}-4$ & $9.820 \mathrm{e}-4$ & $9.964 \mathrm{e}-4$ & $1.011 \mathrm{e}-3$ & $1.025 \mathrm{e}-3$ \\
\hline 9 & $1.039 \mathrm{e}-3$ & $1.052 \mathrm{e}-3$ & $1.065 \mathrm{e}-3$ & $1.077 \mathrm{e}-3$ & $1.089 \mathrm{e}-3$ & $1.099 \mathrm{e}-3$ & $1.110 \mathrm{e}-3$ & $1.119 \mathrm{e}-3$ \\
\hline 10 & $1.129 \mathrm{e}-3$ & $1.137 \mathrm{e}-3$ & $1.146 \mathrm{e}-3$ & $1.154 \mathrm{e}-3$ & $1.162 \mathrm{e}-3$ & $1.169 \mathrm{e}-3$ & $1.176 \mathrm{e}-3$ & $1.183 \mathrm{e}-3$ \\
\hline 11 & $1.190 \mathrm{e}-3$ & $1.197 \mathrm{e}-3$ & $1.205 \mathrm{e}-3$ & $1.213 \mathrm{e}-3$ & $1.222 \mathrm{e}-3$ & $1.230 \mathrm{e}-3$ & $1.240 \mathrm{e}-3$ & $1.250 \mathrm{e}-3$ \\
\hline 12 & $1.261 \mathrm{e}-3$ & $1.273 \mathrm{e}-3$ & $1.286 \mathrm{e}-3$ & $1.300 \mathrm{e}-3$ & $1.316 \mathrm{e}-3$ & $1.331 \mathrm{e}-3$ & $1.349 \mathrm{e}-3$ & $1.367 \mathrm{e}-3$ \\
\hline 13 & $1.387 \mathrm{e}-3$ & $1.407 \mathrm{e}-3$ & $1.429 \mathrm{e}-3$ & $1.451 \mathrm{e}-3$ & $1.476 \mathrm{e}-3$ & $1.500 \mathrm{e}-3$ & $1.526 \mathrm{e}-3$ & $1.551 \mathrm{e}-3$ \\
\hline 14 & $1.579 \mathrm{e}-3$ & $1.605 \mathrm{e}-3$ & $1.634 \mathrm{e}-3$ & $1.662 \mathrm{e}-3$ & $1.691 \mathrm{e}-3$ & $1.719 \mathrm{e}-3$ & $1.749 \mathrm{e}-3$ & $1.777 \mathrm{e}-3$ \\
\hline 15 & $1.807 \mathrm{e}-3$ & $1.835 \mathrm{e}-3$ & $1.865 \mathrm{e}-3$ & $1.893 \mathrm{e}-3$ & $1.922 \mathrm{e}-3$ & $1.949 \mathrm{e}-3$ & $1.978 \mathrm{e}-3$ & $2.005 e-3$ \\
\hline 16 & $2.033 \mathrm{e}-3$ & $2.060 \mathrm{e}-3$ & $2.088 \mathrm{e}-3$ & $2.114 \mathrm{e}-3$ & $2.143 \mathrm{e}-3$ & $2.169 \mathrm{e}-3$ & $2.198 \mathrm{e}-3$ & $2.225 \mathrm{e}-3$ \\
\hline 17 & $2.254 \mathrm{e}-3$ & $2.282 \mathrm{e}-3$ & $2.313 \mathrm{e}-3$ & $2.342 \mathrm{e}-3$ & $2.375 \mathrm{e}-3$ & $2.406 \mathrm{e}-3$ & $2.441 \mathrm{e}-3$ & $2.475 \mathrm{e}-3$ \\
\hline 18 & $2.512 \mathrm{e}-3$ & $2.549 \mathrm{e}-3$ & $2.590 \mathrm{e}-3$ & $2.630 \mathrm{e}-3$ & $2.674 \mathrm{e}-3$ & $2.718 \mathrm{e}-3$ & $2.766 \mathrm{e}-3$ & $2.814 \mathrm{e}-3$ \\
\hline 19 & $2.866 \mathrm{e}-3$ & $2.918 \mathrm{e}-3$ & $2.974 \mathrm{e}-3$ & $3.030 \mathrm{e}-3$ & $3.091 \mathrm{e}-3$ & $3.151 \mathrm{e}-3$ & $3.216 \mathrm{e}-3$ & $3.280 \mathrm{e}-3$ \\
\hline 20 & $3.349 \mathrm{e}-3$ & $3.417 \mathrm{e}-3$ & $3.491 \mathrm{e}-3$ & $3.563 \mathrm{e}-3$ & $3.641 \mathrm{e}-3$ & $3.718 \mathrm{e}-3$ & $3.799 \mathrm{e}-3$ & $3.880 \mathrm{e}-3$ \\
\hline 21 & $3.967 \mathrm{e}-3$ & $4.052 \mathrm{e}-3$ & $4.143 \mathrm{e}-3$ & $4.234 \mathrm{e}-3$ & $4.330 \mathrm{e}-3$ & $4.425 \mathrm{e}-3$ & $4.527 \mathrm{e}-3$ & $4.628 \mathrm{e}-3$ \\
\hline 22 & $4.736 \mathrm{e}-3$ & $4.844 \mathrm{e}-3$ & $4.959 \mathrm{e}-3$ & $5.074 \mathrm{e}-3$ & $5.197 \mathrm{e}-3$ & $5.320 \mathrm{e}-3$ & $5.452 \mathrm{e}-3$ & $5.584 \mathrm{e}-3$ \\
\hline 23 & $5.726 \mathrm{e}-3$ & $5.868 \mathrm{e}-3$ & $6.020 \mathrm{e}-3$ & $6.173 \mathrm{e}-3$ & $6.337 \mathrm{e}-3$ & $6.502 \mathrm{e}-3$ & $6.678 \mathrm{e}-3$ & $6.856 \mathrm{e}-3$ \\
\hline 24 & $7.046 \mathrm{e}-3$ & $7.237 \mathrm{e}-3$ & $7.442 \mathrm{e}-3$ & $7.648 \mathrm{e}-3$ & $7.868 \mathrm{e}-3$ & $8.091 \mathrm{e}-3$ & $8.328 \mathrm{e}-3$ & $8.567 \mathrm{e}-3$ \\
\hline 25 & $8.823 \mathrm{e}-3$ & $9.082 \mathrm{e}-3$ & $9.358 \mathrm{e}-3$ & $9.638 \mathrm{e}-3$ & $9.938 \mathrm{e}-3$ & $1.024 \mathrm{e}-2$ & $1.057 \mathrm{e}-2$ & $1.090 \mathrm{e}-2$ \\
\hline 26 & $1.126 \mathrm{e}-2$ & $1.163 \mathrm{e}-2$ & $1.203 \mathrm{e}-2$ & $1.244 \mathrm{e}-2$ & $1.288 \mathrm{e}-2$ & $1.334 \mathrm{e}-2$ & $1.384 \mathrm{e}-2$ & $1.436 \mathrm{e}-2$ \\
\hline 27 & $1.493 \mathrm{e}-2$ & $1.553 \mathrm{e}-2$ & $1.618 \mathrm{e}-2$ & $1.687 \mathrm{e}-2$ & $1.762 \mathrm{e}-2$ & $1.842 \mathrm{e}-2$ & $1.930 \mathrm{e}-2$ & $2.023 \mathrm{e}-2$ \\
\hline 28 & $2.126 \mathrm{e}-2$ & $2.235 \mathrm{e}-2$ & $2.355 \mathrm{e}-2$ & $2.482 \mathrm{e}-2$ & $2.623 \mathrm{e}-2$ & $2.772 \mathrm{e}-2$ & $2.937 \mathrm{e}-2$ & $3.112 \mathrm{e}-2$ \\
\hline 29 & $3.303 \mathrm{e}-2$ & $3.507 \mathrm{e}-2$ & $3.730 \mathrm{e}-2$ & $3.967 \mathrm{e}-2$ & $4.226 \mathrm{e}-2$ & $4.500 \mathrm{e}-2$ & $4.799 \mathrm{e}-2$ & $5.115 \mathrm{e}-2$ \\
\hline 30 & $5.458 \mathrm{e}-2$ & $5.820 \mathrm{e}-2$ & $6.213 \mathrm{e}-2$ & $6.626 \mathrm{e}-2$ & $7.073 \mathrm{e}-2$ & $7.542 \mathrm{e}-2$ & $8.049 \mathrm{e}-2$ & $8.580 \mathrm{e}-2$ \\
\hline 31 & $9.154 \mathrm{e}-2$ & $9.752 \mathrm{e}-2$ & $1.040 \mathrm{e}-1$ & $1.107 \mathrm{e}-1$ & $1.181 \mathrm{e}-1$ & $1.257 \mathrm{e}-1$ & $1.341 \mathrm{e}-1$ & $1.427 \mathrm{e}-1$ \\
\hline 32 & $1.524 \mathrm{e}-1$ & $1.624 \mathrm{e}-1$ & $1.741 \mathrm{e}-1$ & $1.860 \mathrm{e}-1$ & $2.023 \mathrm{e}-1$ & $2.181 \mathrm{e}-1$ & $2.523 \mathrm{e}-1$ & $3.453 \mathrm{e}-1$ \\
\hline
\end{tabular}




\section{ANEXO B}

El presente anexo resume brevemente el efecto de transformaciones lineales y no lineales en procesos WSS y procesos WSS gaussianos, en particular en el valor medio del proceso resultante, y su ACS. Para un estudio más detallado, el lector puede referirse a los capítulos 17, 18 y 20 de [Kay 2006], de donde se extraen las siguientes propiedades.

- Si $X_{n}$ es un proceso WSS, entonces la media $\mu_{X}$ y la secuencia de autocorrelación (ACS) no dependen del instante de tiempo $n$, y se definen como:

$$
\begin{gathered}
\mu_{X}=E\left[X_{n}\right] \\
\Upsilon_{X_{k}}=E\left[X_{n} \cdot X_{n+k}\right]
\end{gathered}
$$

- A partir de la media y la ACS de un proceso WSS, la varianza del proceso $\sigma_{X}^{2}$ y la covarianza entre dos muestras $C_{X_{n, n+k}}$ pueden expresarse como:

$$
\begin{gathered}
\sigma_{X}^{2}=\operatorname{var}\left(X_{n}\right)=\Upsilon_{X_{0}}-\mu_{X}^{2} \\
C_{X_{n, n+k}}=\Upsilon_{X_{k}}-\mu_{X}^{2}
\end{gathered}
$$

- Si un proceso WSS $U_{n}$ de media $\mu_{U}$, ACS $\Upsilon_{U_{k}}$ y PSD $S_{U_{f}}$ es la entrada a un filtro LSI (Linear Shift-Invariant) con respuesta al impulso $g_{k}$ y respuesta en frecuencia $G_{f}$, entonces la salida es también un proceso WSS con:

$$
\begin{gathered}
\mu_{X}=\sum_{k=-\infty}^{\infty} g_{k} \mu_{U}=G_{0} \mu_{U} \\
\Upsilon_{X_{k}}=g_{-k} * g_{k} * \Upsilon_{U_{k}} \\
S_{X_{f}}=\left|G_{f}\right|^{2} S_{U_{f}}
\end{gathered}
$$

donde $*$ denota la operación de convolución y $g_{-k} * g_{k}=\sum_{n=-\infty}^{\infty} g_{n} \cdot g_{n-k}^{*}$ es la ACS del filtro LSI. Por lo tanto, el valor medio, la ACS y la PSD del proceso de salida $X_{n}$ se ven caracterizados por el filtro LSI $\boldsymbol{g}$, y las propiedades del proceso de entrada $U_{n}$. Adicionalmente, en el caso particular en que $U_{n}$ es un proceso WSS gaussiano, entonces el proceso de salida $X_{n}$ también es un proceso WSS gaussiano. 
Diseño de Estrategias de Sincronización y Estimación de Canal para la Mejora de Comunicaciones en Redes Inteligentes de Energía

- Si $X_{n}$ es un proceso WSS gaussiano de media cero, el proceso $Y_{n}$ resultante de elevar al cuadrado las muestras $Y_{n}=X_{n}^{2}$ ya no será un proceso gaussiano dado que toma sólo valores positivos, pero será un proceso WSS con media y ACS:

$$
\begin{gathered}
\mu_{Y}=E\left[Y_{n}\right]=E\left[X_{n}^{2}\right]=\Upsilon_{X_{0}} \\
\Upsilon_{Y_{k}}=\Upsilon_{X_{0}}^{2}+2 \Upsilon_{X_{k}}^{2}
\end{gathered}
$$

- Si $X_{n}$ es un proceso WSS gaussiano de media cero, el proceso $Y_{n}$ resultante de multiplicar cada muestra con la $D$-ésima muestra posterior $Y_{n}=X_{n} \cdot X_{n+D}$, no será un proceso gaussiano, pero será un proceso WSS con media y ACS:

$$
\begin{gathered}
\mu_{Y}=E\left[Y_{n}\right]=E\left[X_{n} \cdot X_{n-D}\right]=\Upsilon_{X_{D}} \\
\Upsilon_{Y_{k}}=\Upsilon_{X_{D}}^{2}+\Upsilon_{X_{k+D}} \cdot \Upsilon_{X_{k-D}}+\Upsilon_{X_{k}}^{2}
\end{gathered}
$$




\section{ANEXO C}

En el presente anexo se incluyen los coeficientes más significativos del filtro notch tipo FIR $c_{n}$, utilizado para filtrar el ruido en las bandas inactivas de acuerdo a la máscara espectral de transmisión aplicada en el Capítulo 6. En la Tabla C.1 se presentan 512 coeficientes seleccionados de la respuesta al impulso del filtro de acuerdo a la Fig. C.1. Los coeficientes del filtro reducido $c_{n}^{\prime}$ se obtienen, considerando a la Tabla C.1 como una matriz $\mathbf{T}$ de 64 filas y 8 columnas, de acuerdo a:

$$
c_{n}^{\prime}=T_{i, j}, \quad \text { con } \begin{aligned}
& n=8(i-1)+j \\
& j=1,2, \ldots, 8 \\
& i=1,2, \ldots, 64
\end{aligned}
$$

donde $T_{i, j}$ representa el elemento de la $i$-ésima fila y $j$-ésima columna de la Tabla C.1. Luego, debido a la simetría el filtro FIR, se obtiene el filtro reducido de 1023 coeficientes como:

$$
c_{1024-n}^{\prime}=c_{n}^{\prime}, \quad \text { con } n=1,2, \ldots, 512
$$

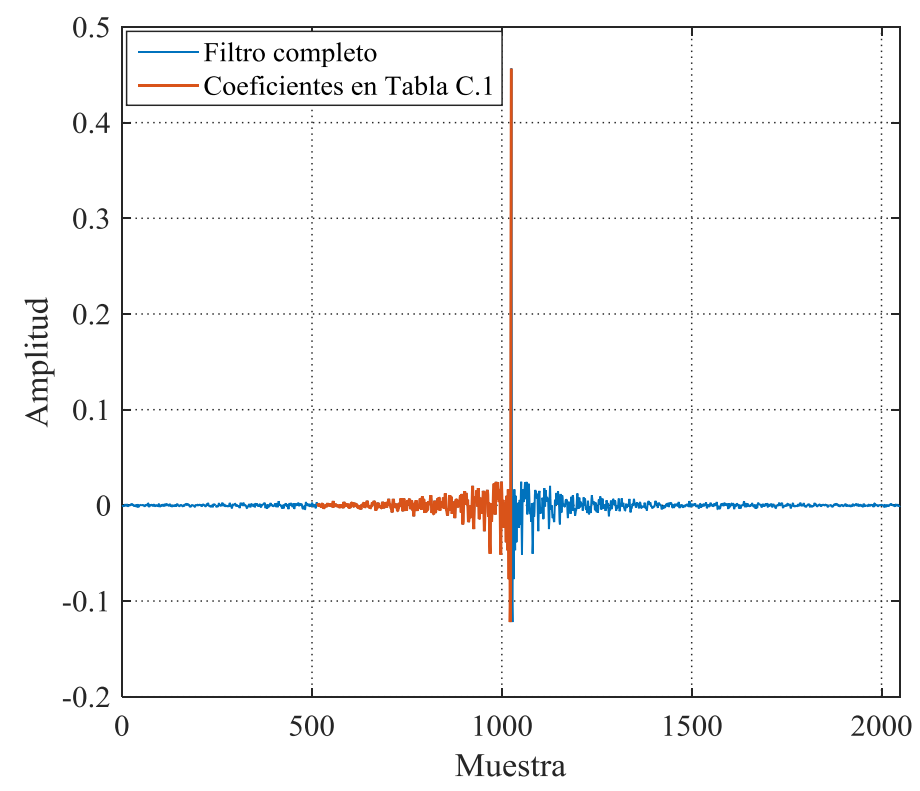

Fig. C.1 Respuesta al impulso del filtro notch y coeficientes más significativos incluidos en la Tabla C.1. 
Diseño de Estrategias de Sincronización y Estimación de Canal para la Mejora de Comunicaciones en Redes Inteligentes de Energía

Tabla C.1 Coeficientes más significativos del filtro notch utilizado para filtrar el ruido en las bandas inactivas en función de las bandas definidas por la máscara espectral de transmisión.

\begin{tabular}{|c|c|c|c|c|c|c|c|c|}
\hline$i$ & 1 & 2 & 3 & 4 & 5 & 6 & 7 & 8 \\
\hline 1 & $1.960 \mathrm{e}-4$ & $8.039 \mathrm{e}-4$ & $1.381 \mathrm{e}-3$ & $-2.958 \mathrm{e}-4$ & $-7.853 e-4$ & $1.100 \mathrm{e}-3$ & $1.273 \mathrm{e}-3$ & $-6.987 e-4$ \\
\hline 2 & $-6.715 e-4$ & $8.404 \mathrm{e}-4$ & $-2.245 e-4$ & $-2.399 \mathrm{e}-3$ & $-1.338 \mathrm{e}-3$ & $1.538 \mathrm{e}-3$ & $1.892 \mathrm{e}-3$ & $-2.816 e-4$ \\
\hline 3 & $-2.355 e-3$ & $-3.209 e-3$ & $-2.593 e-3$ & $-9.121 e-4$ & $5.837 \mathrm{e}-5$ & $-3.815 e-4$ & $-8.802 e-4$ & $-1.033 e-3$ \\
\hline 4 & $-1.332 \mathrm{e}-3$ & $-7.036 e-4$ & $1.214 \mathrm{e}-3$ & $2.075 \mathrm{e}-3$ & $1.166 \mathrm{e}-3$ & $8.191 \mathrm{e}-4$ & $1.128 \mathrm{e}-3$ & $1.404 \mathrm{e}-4$ \\
\hline 5 & $-6.845 e-4$ & $7.509 e-4$ & $1.691 \mathrm{e}-3$ & $-2.614 e-5$ & $-8.055 \mathrm{e}-4$ & $1.019 \mathrm{e}-3$ & $1.034 \mathrm{e}-3$ & $-1.615 e-3$ \\
\hline 6 & $-1.323 e-3$ & $2.775 e-3$ & $4.459 \mathrm{e}-3$ & $1.681 \mathrm{e}-3$ & $-9.742 e-4$ & $-1.088 \mathrm{e}-3$ & $-2.197 e-4$ & $7.788 \mathrm{e}-4$ \\
\hline 7 & $1.416 \mathrm{e}-3$ & $5.873 \mathrm{e}-4$ & $-5.485 e-4$ & $-2.643 e-5$ & $6.028 \mathrm{e}-4$ & $-6.429 e-4$ & $-1.708 e-3$ & $-7.317 \mathrm{e}-4$ \\
\hline 8 & $1.355 \mathrm{e}-4$ & $-7.179 e-4$ & $-1.763 e-3$ & $-2.017 \mathrm{e}-3$ & $-1.701 \mathrm{e}-3$ & $-4.105 e-4$ & $8.360 \mathrm{e}-4$ & $-9.346 e-5$ \\
\hline 9 & $-1.611 e-3$ & $-6.596 e-4$ & $4.890 \mathrm{e}-4$ & $-1.467 e-3$ & $-2.926 e-3$ & $1.717 \mathrm{e}-4$ & $3.556 \mathrm{e}-3$ & $2.190 \mathrm{e}-3$ \\
\hline 10 & $-7.262 \mathrm{e}-4$ & $-6.479 e-4$ & $8.585 \mathrm{e}-4$ & $1.249 \mathrm{e}-3$ & $1.029 \mathrm{e}-3$ & $3.629 \mathrm{e}-4$ & $-6.876 e-4$ & $-1.596 \mathrm{e}-4$ \\
\hline 11 & $1.537 \mathrm{e}-3$ & $7.277 \mathrm{e}-4$ & $-2.085 e-3$ & $-2.171 e-3$ & $1.512 \mathrm{e}-4$ & $6.073 e-5$ & $-2.250 e-3$ & $-2.466 e-3$ \\
\hline 12 & $-2.520 \mathrm{e}-4$ & $1.679 \mathrm{e}-3$ & $2.589 \mathrm{e}-3$ & $2.583 e-3$ & $1.705 \mathrm{e}-3$ & $1.661 \mathrm{e}-3$ & $2.777 \mathrm{e}-3$ & $1.896 \mathrm{e}-3$ \\
\hline 13 & $-1.161 \mathrm{e}-3$ & $-1.539 \mathrm{e}-3$ & $1.494 \mathrm{e}-3$ & $2.497 \mathrm{e}-3$ & $1.979 \mathrm{e}-4$ & $-6.776 e-4$ & $9.508 \mathrm{e}-4$ & $1.489 \mathrm{e}-3$ \\
\hline 14 & $1.462 \mathrm{e}-4$ & $-1.203 e-3$ & $-2.149 e-3$ & $-2.172 \mathrm{e}-3$ & $-6.200 e-4$ & $-2.860 e-5$ & $-2.264 \mathrm{e}-3$ & $-3.629 e-3$ \\
\hline 15 & $-1.656 e-3$ & $-6.805 e-4$ & $-3.126 e-3$ & $-4.208 \mathrm{e}-3$ & $-1.522 \mathrm{e}-3$ & $6.312 \mathrm{e}-4$ & $2.551 \mathrm{e}-4$ & $2.105 \mathrm{e}-4$ \\
\hline 16 & $1.177 \mathrm{e}-3$ & $1.687 \mathrm{e}-3$ & $2.337 \mathrm{e}-3$ & $2.715 e-3$ & $4.196 \mathrm{e}-4$ & $-2.561 e-3$ & $-1.521 e-3$ & $1.800 \mathrm{e}-3$ \\
\hline 17 & $2.210 \mathrm{e}-3$ & $1.069 \mathrm{e}-3$ & $2.566 \mathrm{e}-3$ & $4.708 e-3$ & $3.650 \mathrm{e}-3$ & $8.480 \mathrm{e}-4$ & $-8.883 e-4$ & $-1.625 e-3$ \\
\hline 18 & $-1.616 e-3$ & $-1.056 \mathrm{e}-3$ & $-1.651 e-3$ & $-3.136 e-3$ & $-2.746 e-3$ & $-1.075 e-3$ & $-1.032 \mathrm{e}-3$ & $-1.078 \mathrm{e}-3$ \\
\hline 19 & $1.345 \mathrm{e}-3$ & $3.089 \mathrm{e}-3$ & $1.466 \mathrm{e}-3$ & $4.736 \mathrm{e}-4$ & $2.727 \mathrm{e}-3$ & $4.448 \mathrm{e}-3$ & $3.871 \mathrm{e}-3$ & $3.457 \mathrm{e}-3$ \\
\hline 20 & $2.175 \mathrm{e}-3$ & $-2.168 \mathrm{e}-3$ & $-5.422 e-3$ & $-3.862 e-3$ & $-1.950 \mathrm{e}-3$ & $-3.037 e-3$ & $-2.567 e-3$ & $1.283 \mathrm{e}-3$ \\
\hline 21 & $2.790 \mathrm{e}-3$ & $1.294 \mathrm{e}-5$ & $-2.009 e-3$ & $-1.460 \mathrm{e}-3$ & $-1.200 \mathrm{e}-3$ & $-1.490 \mathrm{e}-3$ & $-1.082 \mathrm{e}-3$ & $-1.391 \mathrm{e}-3$ \\
\hline 22 & $-2.808 e-3$ & $-2.743 e-3$ & $-4.562 \mathrm{e}-4$ & $2.087 \mathrm{e}-3$ & $3.501 \mathrm{e}-3$ & $2.595 e-3$ & $-8.819 e-4$ & $-3.099 e-3$ \\
\hline 23 & $-8.652 \mathrm{e}-4$ & $2.015 \mathrm{e}-3$ & $1.605 e-3$ & $1.199 \mathrm{e}-3$ & $3.008 \mathrm{e}-3$ & $2.463 \mathrm{e}-3$ & $-1.311 e-3$ & $-2.256 e-3$ \\
\hline 24 & $2.758 \mathrm{e}-4$ & $8.877 \mathrm{e}-4$ & $6.435 \mathrm{e}-4$ & $3.992 \mathrm{e}-3$ & $6.813 e-3$ & $3.738 \mathrm{e}-3$ & $-1.743 e-4$ & $6.211 \mathrm{e}-4$ \\
\hline 25 & $1.640 \mathrm{e}-3$ & $-9.941 e-4$ & $-3.101 e-3$ & $-3.063 e-3$ & $-4.926 e-3$ & $-7.345 e-3$ & $-4.237 e-3$ & $3.027 \mathrm{e}-3$ \\
\hline 26 & $6.722 \mathrm{e}-3$ & $4.313 e-3$ & $1.159 \mathrm{e}-4$ & $-1.456 e-3$ & $8.819 \mathrm{e}-5$ & $1.283 \mathrm{e}-3$ & $-7.265 e-4$ & $-2.764 e-3$ \\
\hline 27 & $-6.831 e-4$ & $1.698 \mathrm{e}-3$ & $-1.198 \mathrm{e}-3$ & $-5.654 \mathrm{e}-3$ & $-5.648 e-3$ & $-3.551 e-3$ & $-2.682 \mathrm{e}-3$ & $-4.009 e-4$ \\
\hline 28 & $2.634 \mathrm{e}-3$ & $1.215 \mathrm{e}-3$ & $-1.610 \mathrm{e}-3$ & $1.790 \mathrm{e}-3$ & $7.315 e-3$ & $5.935 e-3$ & $1.280 \mathrm{e}-3$ & $8.063 \mathrm{e}-4$ \\
\hline 29 & $1.614 \mathrm{e}-4$ & $-4.932 e-3$ & $-6.143 e-3$ & $1.335 \mathrm{e}-3$ & $7.093 e-3$ & $3.386 \mathrm{e}-3$ & $-2.364 e-3$ & $-2.328 e-3$ \\
\hline 30 & $6.218 e-5$ & $-9.236 e-4$ & $-4.375 e-3$ & $-5.537 e-3$ & $-1.768 \mathrm{e}-3$ & $3.866 \mathrm{e}-3$ & $5.190 \mathrm{e}-3$ & $1.822 \mathrm{e}-3$ \\
\hline 31 & $3.044 \mathrm{e}-4$ & $3.276 \mathrm{e}-3$ & $6.266 \mathrm{e}-3$ & $6.560 \mathrm{e}-3$ & $4.779 \mathrm{e}-3$ & $3.622 \mathrm{e}-4$ & $-3.964 e-3$ & $-1.578 \mathrm{e}-3$ \\
\hline 32 & $4.876 \mathrm{e}-3$ & $4.192 \mathrm{e}-3$ & $-3.042 \mathrm{e}-3$ & $-5.116 e-3$ & $-2.683 e-3$ & $-6.162 e-3$ & $-1.143 e-2$ & $-6.629 e-3$ \\
\hline 33 & $2.344 \mathrm{e}-3$ & $1.977 \mathrm{e}-3$ & $-2.917 e-3$ & $-2.472 \mathrm{e}-4$ & $5.314 \mathrm{e}-3$ & $2.915 \mathrm{e}-3$ & $-3.949 e-3$ & $-5.773 e-3$ \\
\hline 34 & $-2.571 e-3$ & $3.456 \mathrm{e}-4$ & $6.471 \mathrm{e}-4$ & $-1.463 e-3$ & $-3.328 \mathrm{e}-3$ & $-9.324 \mathrm{e}-4$ & $4.626 \mathrm{e}-3$ & $7.079 \mathrm{e}-3$ \\
\hline 35 & $3.629 \mathrm{e}-3$ & $-2.092 \mathrm{e}-3$ & $-4.395 e-3$ & $1.967 \mathrm{e}-4$ & $7.964 \mathrm{e}-3$ & $9.307 \mathrm{e}-3$ & $3.151 \mathrm{e}-3$ & $9.723 e-4$ \\
\hline 36 & $6.022 \mathrm{e}-3$ & $5.639 \mathrm{e}-3$ & $-3.741 e-3$ & $-7.884 \mathrm{e}-3$ & $-2.704 e-3$ & $-1.777 e-3$ & $-7.139 e-3$ & $-5.332 e-3$ \\
\hline 37 & $2.779 \mathrm{e}-3$ & $2.492 \mathrm{e}-3$ & $-4.707 e-3$ & $-4.523 e-3$ & $2.159 \mathrm{e}-3$ & $3.884 \mathrm{e}-3$ & $1.216 \mathrm{e}-3$ & $1.271 \mathrm{e}-3$ \\
\hline 38 & $3.015 \mathrm{e}-3$ & $4.237 \mathrm{e}-3$ & $7.549 \mathrm{e}-3$ & $1.049 \mathrm{e}-2$ & $6.081 e-3$ & $-3.419 e-3$ & $-7.961 e-3$ & $-4.727 e-3$ \\
\hline 39 & $-9.329 e-4$ & $-3.183 e-3$ & $-8.997 e-3$ & $-9.127 e-3$ & $-8.331 e-4$ & $4.803 \mathrm{e}-3$ & $-7.872 \mathrm{e}-4$ & $-7.960 e-3$ \\
\hline 40 & $-5.916 e-3$ & $-2.035 e-3$ & $-4.195 e-3$ & $-3.478 \mathrm{e}-3$ & $4.577 \mathrm{e}-3$ & $7.073 \mathrm{e}-3$ & $6.873 \mathrm{e}-4$ & $5.067 \mathrm{e}-4$ \\
\hline 41 & $8.345 e-3$ & $7.625 e-3$ & $-2.542 \mathrm{e}-3$ & $-5.462 e-3$ & $2.028 \mathrm{e}-4$ & $1.622 \mathrm{e}-3$ & $-5.608 \mathrm{e}-4$ & $1.807 \mathrm{e}-3$ \\
\hline
\end{tabular}


Capítulo 7: Conclusiones y trabajos futuros

\begin{tabular}{|c|c|c|c|c|c|c|c|c|}
\hline 42 & $3.779 \mathrm{e}-3$ & $-3.892 \mathrm{e}-4$ & $-2.078 \mathrm{e}-3$ & $4.172 \mathrm{e}-3$ & $8.085 \mathrm{e}-3$ & $2.595 \mathrm{e}-3$ & $-3.680 e-3$ & $-1.248 \mathrm{e}-3$ \\
\hline 43 & $7.300 \mathrm{e}-3$ & $1.244 \mathrm{e}-2$ & $8.571 \mathrm{e}-3$ & $-3.066 \mathrm{e}-6$ & $-4.810 \mathrm{e}-3$ & $-5.745 e-3$ & $-8.572 \mathrm{e}-3$ & $-1.076 \mathrm{e}-2$ \\
\hline 44 & $-6.868 \mathrm{e}-3$ & $-3.246 e-3$ & $-5.497 e-3$ & $-3.344 \mathrm{e}-3$ & $7.643 e-3$ & $1.070 \mathrm{e}-2$ & $-4.934 \mathrm{e}-4$ & $-5.391 e-3$ \\
\hline 45 & $4.651 \mathrm{e}-3$ & $8.968 \mathrm{e}-3$ & $-1.598 \mathrm{e}-3$ & $-8.864 \mathrm{e}-3$ & $-4.992 \mathrm{e}-3$ & $-2.623 e-3$ & $-3.575 e-3$ & $8.371 \mathrm{e}-4$ \\
\hline 46 & $3.840 \mathrm{e}-3$ & $-4.638 \mathrm{e}-3$ & $-1.334 \mathrm{e}-2$ & $-8.420 e-3$ & $2.237 \mathrm{e}-3$ & $6.146 \mathrm{e}-3$ & $5.711 \mathrm{e}-3$ & $6.153 e-3$ \\
\hline 47 & $5.120 \mathrm{e}-3$ & $1.465 \mathrm{e}-3$ & $-1.379 \mathrm{e}-3$ & $-1.412 \mathrm{e}-3$ & $4.475 \mathrm{e}-4$ & $1.249 \mathrm{e}-3$ & $-6.509 \mathrm{e}-4$ & $1.800 \mathrm{e}-4$ \\
\hline 48 & $7.562 \mathrm{e}-3$ & $1.036 \mathrm{e}-2$ & $-1.241 \mathrm{e}-4$ & $-7.514 \mathrm{e}-3$ & $3.004 \mathrm{e}-3$ & $1.192 \mathrm{e}-2$ & $-1.863 \mathrm{e}-3$ & $-1.988 \mathrm{e}-2$ \\
\hline 49 & $-1.674 \mathrm{e}-2$ & $-2.422 \mathrm{e}-3$ & $4.823 \mathrm{e}-3$ & $9.572 \mathrm{e}-3$ & $1.486 \mathrm{e}-2$ & $1.019 \mathrm{e}-2$ & $9.001 \mathrm{e}-4$ & 4.192e-3 \\
\hline 50 & $1.299 \mathrm{e}-2$ & $9.061 \mathrm{e}-3$ & $5.893 \mathrm{e}-4$ & $3.188 \mathrm{e}-3$ & $5.889 \mathrm{e}-3$ & $-5.201 e-3$ & $-1.746 e-2$ & $-1.638 \mathrm{e}-2$ \\
\hline 51 & $1.028 \mathrm{e}-2$ & $-1.058 \mathrm{e}-2$ & $-1.338 \mathrm{e}-2$ & $-1.104 \mathrm{e}-2$ & $-4.163 e-3$ & $1.637 \mathrm{e}-4$ & $-2.748 \mathrm{e}-3$ & $-4.441 e-3$ \\
\hline 52 & $7.183 e-3$ & $2.037 \mathrm{e}-2$ & $9.762 \mathrm{e}-3$ & $-1.707 \mathrm{e}-2$ & $-2.479 \mathrm{e}-2$ & $-7.956 \mathrm{e}-3$ & $5.518 \mathrm{e}-3$ & $6.518 \mathrm{e}-3$ \\
\hline 53 & $7.907 \mathrm{e}-3$ & 8.682 & $4.172 \mathrm{e}-3$ & $6.450 \mathrm{e}-3$ & $.567 \mathrm{e}-2$ & $1.166 \mathrm{e}-2$ & $-1.144 \mathrm{e}-3$ & $3.880 \mathrm{e}-3$ \\
\hline 54 & $1.795 \mathrm{e}-2$ & $8.586 \mathrm{e}-3$ & $-1.414 \mathrm{e}-2$ & $-1.381 \mathrm{e}-2$ & $4.256 \mathrm{e}-3$ & $7.992 \mathrm{e}-3$ & $-2.210 \mathrm{e}-3$ & $-5.522 \mathrm{e}-3$ \\
\hline 55 & $-2.667 \mathrm{e}-3$ & $-4.704 e-3$ & $-8.630 \mathrm{e}-3$ & $-6.413 e-3$ & $3.624 \mathrm{e}-3$ & $1.513 \mathrm{e}-2$ & $1.218 \mathrm{e}-2$ & $-1.052 \mathrm{e}-2$ \\
\hline 56 & $-2.699 e-2$ & $-1.418 \mathrm{e}-2$ & $8.074 \mathrm{e}-3$ & $1.212 \mathrm{e}-2$ & $7.381 \mathrm{e}-3$ & $1.062 \mathrm{e}-2$ & $1.435 \mathrm{e}-2$ & $1.407 \mathrm{e}-2$ \\
\hline 57 & $1.613 \mathrm{e}-2$ & $1.084 \mathrm{e}-2$ & $-7.102 \mathrm{e}-3$ & $-1.186 \mathrm{e}-2$ & $2.710 \mathrm{e}-3$ & $-3.928 \mathrm{e}-3$ & $-3.971 \mathrm{e}-2$ & $-5.058 \mathrm{e}-2$ \\
\hline 58 & $-1.900 \mathrm{e}-2$ & $2.373 e-3$ & $-9.518 \mathrm{e}-3$ & $-1.699 \mathrm{e}-2$ & $-6.538 \mathrm{e}-3$ & $-4.107 e-3$ & $-9.173 e-3$ & $-6.118 \mathrm{e}-4$ \\
\hline 59 & $1.484 \mathrm{e}-2$ & $2.183 e-2$ & $2.260 \mathrm{e}-2$ & $1.422 \mathrm{e}-2$ & $-4.892 \mathrm{e}-3$ & $-9.450 \mathrm{e}-3$ & $1.176 \mathrm{e}-2$ & $2.456 \mathrm{e}-2$ \\
\hline 60 & $1.218 \mathrm{e}-2$ & $6.442 \mathrm{e}-3$ & $1.934 \mathrm{e}-2$ & $2.424 \mathrm{e}-2$ & $1.766 \mathrm{e}-2$ & $1.608 \mathrm{e}-2$ & $1.170 \mathrm{e}-2$ & $3.426 \mathrm{e}-3$ \\
\hline 61 & $1.183 \mathrm{e}-2$ & $1.698 \mathrm{e}-2$ & $-1.829 \mathrm{e}-2$ & $-5.138 \mathrm{e}-2$ & $-2.071 \mathrm{e}-2$ & $2.468 \mathrm{e}-2$ & $1.064 \mathrm{e}-2$ & $-2.009 \mathrm{e}-2$ \\
\hline 62 & $-6.911 \mathrm{e}-3$ & $5.691 \mathrm{e}-3$ & $-1.788 \mathrm{e}-2$ & $-2.322 \mathrm{e}-2$ & $2.721 \mathrm{e}-3$ & $1.521 \mathrm{e}-3$ & $-1.817 \mathrm{e}-2$ & $-7.592 \mathrm{e}-3$ \\
\hline 63 & $-4.127 e-3$ & $-3.849 e-2$ & $-3.806 e-2$ & $9.976 \mathrm{e}-3$ & $4.130 \mathrm{e}-3$ & $-4.692 \mathrm{e}-2$ & $-2.786 e-2$ & $1.670 \mathrm{e}-2$ \\
\hline 64 & $-3.175 \mathrm{e}-2$ & $-7.712 \mathrm{e}-2$ & $-7.541 \mathrm{e}-3$ & $8.901 \mathrm{e}-3$ & $-1.220 \mathrm{e}-1$ & $-9.917 \mathrm{e}-2$ & $2.332 \mathrm{e}-1$ & $4.565 \mathrm{e}-1$ \\
\hline
\end{tabular}


Diseño de Estrategias de Sincronización y Estimación de Canal para la Mejora de Comunicaciones en Redes Inteligentes de Energía

\section{REFERENCIAS}

[Akbari 2007] M. H. Akbari, M. Ahmadian, and S. Salari, "Joint frame and frequency synchronization for MIMO OFDM systems with ZCZ sequences," in 2007 3rd IEEE/IFIP International Conference in Central Asia on Internet, 2007, pp. 1-5.

[Akhtman 2007] J. Akhtman and L. Hanzo, "Decision directed channel estimation aided OFDM employing sample-spaced and fractionally-spaced CIR estimators," IEEE Transactions on Wireless Communications, vol. 6, pp. 1171-1175, 2007.

[Al-Rawi 2003] G. A. Al-Rawi, T. Y. Al-Naffouri, A. Bahai, and J. Cioffi, "Exploiting error-control coding and cyclic-prefix in channel estimation for coded OFDM systems," IEEE Communications Letters, vol. 7, pp. 388-390, 2003.

[Ali 1982] M. M. Ali and M. Obaidullah, "Distribution of linear combination of exponential variates," Communications in Statistics - Theory and Methods, vol. 11, pp. 1453-1463, 1982/01/01 1982.

[Alsusa 2013] E. Alsusa and K. M. Rabie, "Dynamic Peak-Based Threshold Estimation Method for Mitigating Impulsive Noise in Power-Line Communication Systems," IEEE Transactions on Power Delivery, vol. 28, pp. 2201-2208, 2013.

[Awoseyila 2008] A. B. Awoseyila, C. Kasparis, and B. G. Evans, "Improved preamble-aided timing estimation for OFDM systems," IEEE Communications Letters, vol. 12, pp. 825-827, 2008.

[Awoseyila 2009] A. B. Awoseyila, C. Kasparis, and B. G. Evans, "Robust time-domain timing and frequency synchronization for OFDM systems," IEEE Transactions on Consumer Electronics, vol. 55, pp. 391-399, 2009.

[Bamberger 2006] Y. Bamberger, J. Baptista, R. Belmans, B. M. Buchholz, M. Chebbo, J. L. D. V. Doblado, V. Efthymiou, L. Gallo, E. Handschin, and N. Hatziargyriou, "Vision and strategy for europe's electricity networks of the future: European technology PlatformSmartGrids," 2006.

[Bellanger 2010] M. Bellanger, D. Le Ruyet, D. Roviras, M. Terré, J. Nossek, L. Baltar, Q. Bai, D. Waldhauser, M. Renfors, and T. Ihalainen, "FBMC physical layer: a primer," Phydyas, vol. 25, pp. 7-10, 2010.

[Benyoucef 2003] D. Benyoucef, "A new statistical model of the noise power density spectrum for powerline communication," in International symposium on power-line communications and its applications, 2003, pp. 136-141.

[Berger 2014] L. T. Berger, A. Schwager, P. Pagani, and D. M. Schneider, "MIMO power line communications," IEEE Communications Surveys \& Tutorials, vol. 17, pp. 106-124, 2014. 
[Biglieri 2003]E. Biglieri, "Coding and modulation for a horrible channel," IEEE Communications Magazine, vol. 41, pp. 92-98, 2003.

[Bogdanović 2014] M. Bogdanović, "Frequency domain based LS channel estimation in OFDM based Power line communications," automatika, vol. 55, pp. 487-494, 2014.

[Budisin 1990] S. Z. Budisin, "New multilevel complementary pairs of sequences," ELECTRONICS LETTERS, vol. 26, pp. 1861-1863, 1990.

[Budisin 1991] S. Z. Budisin, "Efficient pulse compressor for Golay complementary sequences," ELECTRONICS LETTERS, vol. 27, pp. 219$220,1991$.

[Bumiller 2007] G. Bumiller and L. Lampe, "Fast Burst Synchronization for PLC Systems," in 2007 IEEE International Symposium on Power Line Communications and Its Applications, 2007, pp. 65-70.

[Cano 2016] C. Cano, A. Pittolo, D. Malone, L. Lampe, A. M. Tonello, and A. G. Dabak, "State of the art in power line communications: From the applications to the medium," IEEE Journal on Selected Areas in Communications, vol. 34, pp. 1935-1952, 2016.

[Cañete-Corripio 2005] F. J. Cañete-Corripio, "Caracterización y modelado de redes eléctricas interiores como medio de transmisión de banda ancha," 2005.

[Cortés 2010] J. A. Cortés, L. Diez, F. J. Canete, and J. J. Sánchez-Martínez, "Analysis of the indoor broadband power-line noise scenario," IEEE Transactions on Electromagnetic Compatibility, vol. 52, pp. 849-858, 2010.

[Chapra 2010] S. C. Chapra and R. P. Canale, Numerical methods for engineers: Boston: McGraw-Hill Higher Education, 2010.

[Chen 2013] C. Chen, Y. Chen, N. Ding, Y. Wang, J. Lin, X. Zeng, and D. D. Huang, "Accurate Sampling Timing Acquisition for Baseband OFDM Power-Line Communication in Non-Gaussian Noise," IEEE Transactions on Communications, vol. 61, pp. 1608-1620, 2013.

[Chen 2005] H.-H. Chen, Y.-C. Yeh, X. Zhang, A. Huang, Y. Yang, J. Li, Y. Xiao, H. R. Sharif, and A. H. Vinck, "Generalized pairwise complementary codes with set-wise uniform interference-free windows," IEEE Journal on Selected Areas in Communications, vol. 24, pp. 65-74, 2005.

[Chen 2020] Y. Chen and W. Nie, "A Low Complexity joint synchronization algorithm based on CAZAC sequence for OFDM system," in Journal of Physics: Conference Series, 2020, p. 032140.

[Chien 2015] Y.-R. Chien, "Iterative channel estimation and impulsive noise mitigation algorithm for OFDM-based receivers with application to 
Diseño de Estrategias de Sincronización y Estimación de Canal para la Mejora de Comunicaciones en Redes Inteligentes de Energía

power-line communications," IEEE Transactions on Power Delivery, vol. 30, pp. 2435-2442, 2015.

[Chu 1972] D. Chu, "Polyphase codes with good periodic correlation properties (corresp.)," IEEE Transactions on Information Theory, vol. 18, pp. 531532, 1972.

[Das 2019] A. Das, B. Mohanty, and B. Sahu, "Modified CAZAC Sequence Based Timing Synchronization Scheme for OFDM System," Wireless Personal Communications, vol. 108, pp. 37-49, 2019/09/01 2019.

[Davis 1999] J. A. Davis and J. Jedwab, "Peak-to-mean power control in OFDM, Golay complementary sequences, and Reed-Muller codes," Information Theory, IEEE Transactions on, vol. 45, pp. 2397-2417, 1999.

[De Marziani 2007] C. De Marziani, J. Ureña, A. Hernández, M. Mazo, F. J. Alvarez, J. J. García, and P. Donato, "Modular Architecture for Efficient Generation and Correlation of Complementary Set of Sequences," Signal Processing, IEEE Transactions on, vol. 55, pp. 2323-2337, 2007.

[Donato 2009] P. G. Donato, M. A. Funes, M. N. Hadad, and D. O. Carrica, "Optimised Golay correlator," ELECTRONICS LETTERS, vol. 45, pp. 380-381, 2009.

[Du 2019] X. Du, P.-Y. Kam, and C. Yu, "Joint timing and frequency synchronization in coherent optical OFDM systems," Frontiers of Optoelectronics, vol. 12, pp. 4-14, 2019.

[Dubey 2015] A. Dubey and R. K. Mallik, "PLC System Performance With AF Relaying," IEEE Transactions on Communications, vol. 63, pp. 2337$2345,2015$.

[Edfors 1998] O. Edfors, M. Sandell, J.-J. Van de Beek, S. K. Wilson, and P. O. Borjesson, "OFDM channel estimation by singular value decomposition," IEEE Transactions on Communications, vol. 46, pp. 931-939, 1998.

[Ekanayake 2012] J. Ekanayake, N. Jenkins, K. Liyanage, J. Wu, and A. Yokoyama, Smart Grid: Technology and Applications: Wiley Publishing, 2012.

[Esmailian 2003] T. Esmailian, F. R. Kschischang, and P. Glenn Gulak, "Inbuilding power lines as high-speed communication channels: channel characterization and a test channel ensemble," International Journal of Communication Systems, vol. 16, pp. 381-400, 2003.

[Fang 2012] Y. Fang, Z. Zhang, and G. Liu, "A Novel Synchronization Algorithm Based on CAZAC Sequence for OFDM Systems," in 2012 8th International Conference on Wireless Communications, Networking and Mobile Computing, 2012, pp. 1-4.

[Farouk 2017] E. Farouk, M. Z. Saleh, M. Ibrahim, and S. Elramly, "Joint channel estimation for TDS-OFDM based on Superimposed Training," in 2017 
14th International Conference on Telecommunications (ConTEL), 2017, pp. 55-62.

[Ferrari 2019] A. Ferrari, "A note on sum and difference of correlated chi-squared variables," arXiv preprint arXiv:1906.09982, 2019.

[Frank 1962] R. Frank, S. Zadoff, and R. Heimiller, "Phase shift pulse codes with good periodic correlation properties (Corresp.)," IRE Transactions on Information Theory, vol. 8, pp. 381-382, 1962.

[Galli 2010] S. Galli, M. Koch, H. Latchman, S. Lee, and V. Oksman, "Industrial and International Standards on PLC - Based Networking Technologies," Power Line Communications: Theory and Applications for Narrowband and Broadband Communications Over Power Lines, pp. 363412, 2010.

[Galli 2011] S. Galli, A. Scaglione, and Z. Wang, "For the Grid and Through the Grid: The Role of Power Line Communications in the Smart Grid," Proceedings of the IEEE, vol. 99, pp. 998-1027, 2011.

[Gao 2012] J. Gao, Y. Xiao, J. Liu, W. Liang, and C. L. P. Chen, "A survey of communication/networking in Smart Grids," Future Generation Computer Systems, vol. 28, pp. 391-404, 2012.

[García 2012] E. García, J. Ureña, J. J. García, M. C. Pérez, and D. Ruiz, "Efficient Generator/Correlator of GPC Sequences for QS-CDMA," Communications Letters, IEEE, vol. 16, pp. 1676-1679, 2012.

[García 2013] E. García, J. Ureña, and J. J. García, "Generation and Correlation Architectures of Multilevel Complementary Sets of Sequences," Signal Processing, IEEE Transactions on, vol. 61, pp. 6333-6343, 2013.

[García 2015] E. García, J. A. Paredes, F. J. Álvarez, M. C. Pérez, and J. J. García, "Spreading sequences in active sensing: A review," Signal Processing, vol. 106, pp. 88-105, 2015.

[Girotto 2017] M. Girotto and A. M. Tonello, "EMC regulations and spectral constraints for multicarrier modulation in PLC," IEEE Access, vol. 5, pp. 4954-4966, 2017.

[Godoy 2012] J. Godoy, F. J. Canete, J. A. Cortés, and L. Díez, "A study on symbol timing recovery schemes for broadband in-home PLC," in 2012 IEEE International Symposium on Power Line Communications and Its Applications, 2012, pp. 188-193.

[Golay 1961] M. J. E. Golay, "Complementary series," Information Theory, IRE Transactions on, vol. 7, pp. 82-87, 1961.

[Gul 2012] M. M. U. Gul, S. Lee, and X. Ma, "Robust synchronization for OFDM employing Zadoff-Chu sequence," in 2012 46th Annual Conference on Information Sciences and Systems (CISS), 2012, pp. 1-6. 
Diseño de Estrategias de Sincronización y Estimación de Canal para la Mejora de Comunicaciones en Redes Inteligentes de Energía

[Gul 2015] M. M. U. Gul, X. Ma, and S. Lee, "Timing and Frequency Synchronization for OFDM Downlink Transmissions Using Zadoff-Chu Sequences," IEEE Transactions on Wireless Communications, vol. 14, pp. 1716-1729, 2015.

[Guzel 2011] T. Guzel, E. Ustunel, H. B. Celebi, H. Delic, and K. Mihcak, "Noise Modeling and OFDM Receiver Design in Power-Line Communication," IEEE Transactions on Power Delivery, vol. 26, pp. 2735-2742, 2011.

[Haniph 2013] A. L. Haniph, K. Srinivas, Y. Larry, and G. Sherman, in Homeplug AV and IEEE 1901: A Handbook for PLC Designers and Users, ed: IEEE, 2013, p. 1.

[He 2010] L. He, F. Yang, C. Zhang, and Z. Wang, "Synchronization for TDS-OFDM over multipath fading channels," IEEE Transactions on Consumer Electronics, vol. 56, pp. 2141-2147, 2010.

[Hlaing 2003] M. Hlaing, V. K. Bhargava, and K. B. Letaief, "A robust timing and frequency synchronization for OFDM systems," IEEE Transactions on Wireless Communications, vol. 2, pp. 822-839, 2003.

[Hoch 2011] M. Hoch, "Comparison of PLC G3 and PRIME," in 2011 IEEE International Symposium on Power Line Communications and Its Applications, 2011, pp. 165-169.

[Huang 2006] L. Huang, G. Mathew, and J. W. Bergmans, "Pilot-aided channel estimation for systems with virtual carriers," in 2006 IEEE International Conference on Communications, 2006, pp. 3070-3075.

[IEEE 2010] IEEE, "IEEE Standard for Broadband over Power Line Networks: Medium Access Control and Physical Layer Specifications," IEEE Std 1901-2010, pp. 1-1586, 2010.

[IEEE 2013] IEEE, "IEEE Standard for Low-Frequency (less than $500 \mathrm{kHz}$ ) Narrowband Power Line Communications for Smart Grid Applications," IEEE Std 1901.2-2013, pp. 1-269, 2013.

[Jingfang 2012] H. Jingfang, W. Honggang, and Q. Yi, "Smart grid communications in challenging environments," in Smart Grid Communications (SmartGridComm), 2012 IEEE Third International Conference on, 2012, pp. 552-557.

[Juwono 2014] F. H. Juwono, Q. Guo, D. Huang, and K. P. Wong, "Deep Clipping for Impulsive Noise Mitigation in OFDM-Based Power-Line Communications," IEEE Transactions on Power Delivery, vol. 29, pp. 1335-1343, 2014.

[Juwono 2016] F. H. Juwono, Q. Guo, Y. Chen, L. Xu, D. D. Huang, and K. P. Wong, "Linear Combining of Nonlinear Preprocessors for OFDMBased Power-Line Communications," IEEE Transactions on Smart Grid, vol. 7, pp. 253-260, 2016. 
[Kang 2007] Y. Kang, K. Kim, and H. Park, "Efficient DFT-based channel estimation for OFDM systems on multipath channels," IET Communications, vol. 1, pp. 197-202, 2007.

[Kay 2006] S. Kay, Intuitive probability and random processes using $M A T L A B \circledR$ : Springer Science \& Business Media, 2006.

[Kemp 1989] A. H. Kemp and M. Darnell, "Synthesis of uncorrelated and nonsquare sets of multilevel complementary sequences," ELECTRONICS LETTERS, vol. 25, pp. 791-792, 1989.

[Kim 2006] H. W. Kim, B. Gu, K. Kang, and D.-S. Ahn, "Efficient time and frequency offset estimation for OFDM based MSS systems," in IEEE Vehicular Technology Conference, 2006, pp. 1-5.

[Kim 2011] Y. Kim and J. Lee, "Comparison of Passband and Baseband Transmission Schemes for Power-Line Communication OFDM Systems," IEEE Transactions on Power Delivery, vol. 26, pp. 2466-2475, 2011.

[Kung 2012] T.-L. Kung and K. K. Parhi, "Optimized joint timing synchronization and channel estimation for OFDM systems," IEEE Wireless Communications Letters, vol. 1, pp. 149-152, 2012.

[Lalos 2016] A. S. Lalos, A. Vgenis, F. Gioulekas, and M. Birbas, "Pilot-less time synchronization for OFDM systems: Application to power line receivers," International Journal of Distributed Sensor Networks, vol. 12, p. $8532941,2016$.

[Lee 2018] H. Lee, H. Kim, S. Lim, and D. Hong, "A Novel Preamble Design for Time Synchronization in OFDM-Based Multiple Numerologies," in 2018 IEEE 88th Vehicular Technology Conference (VTC-Fall), 2018, pp. $1-5$.

[Liu 2008] L. Liu, T. Cheng, and LuoYanan, "Analysis and Modeling of Multipath for Indoor Power Line Channel," in 2008 10th International Conference on Advanced Communication Technology, 2008, pp. 19661969.

[Liu 2013] S. Liu, F. Yang, J. Song, F. Ren, and J. Li, "OFDM preamble design for synchronization under narrowband interference," in 2013 IEEE 17th International Symposium on Power Line Communications and Its Applications, 2013, pp. 252-257.

[Liu 2017] X. Liu, J. Liu, H. Sun, H. Liu, and X. Gu, "A timing synchronization method for OFDM based power line communication," in 2017 IEEE 9th International Conference on Communication Software and Networks (ICCSN), 2017, pp. 543-547.

[Liu 2012] Y. Liu, Z. Tan, S. Xu, and K. S. Kwak, "Timing synchronisation for orthogonal frequency division multiplexing using improved crosscorrelation," IET Communications, vol. 6, pp. 1137-1143, 2012. 
Diseño de Estrategias de Sincronización y Estimación de Canal para la Mejora de Comunicaciones en Redes Inteligentes de Energía

[Luo 2012] Z.-n. Luo, J.-j. Zheng, J. Zhu, and E.-y. Zhang, "Golay complementary pair aided time synchronization method for OFDM systems," in 2012 IEEE 14th International Conference on Communication Technology, 2012, pp. 166-170.

[Mak 1982] S. T. Mak and D. L. Reed, "TWACS, a new viable two-way automatic communication system for distribution networks. Part I: Outbound communication," IEEE Transactions on Power Apparatus and systems, pp. 2941-2949, 1982.

[Mak 1984] S. T. Mak and T. G. Moore, "TWACSTM, A New Viable Two-Way Automatic Communication System for Distribution Networks. Part II: Inbound Communication," IEEE Transactions on Power Apparatus and systems, pp. 2141-2147, 1984.

[Maneerung 2011] A. Maneerung, S. Sittichivapak, and K. Hongesombut, "Application of power line communication with OFDM to smart grid system," in Fuzzy Systems and Knowledge Discovery (FSKD), 2011 Eighth International Conference on, 2011, pp. 2239-2244.

[Marumo 1920] N. Marumo, "Simultaneous transmission and reception in radio telephony," Proceedings of the Institute of Radio Engineers, vol. 8, pp. 199-219, 1920.

[Meng 2005] H. Meng, Y. L. Guan, and S. Chen, "Modeling and analysis of noise effects on broadband power-line communications," IEEE Transactions on Power Delivery, vol. 20, pp. 630-637, 2005.

[Minn 2000] H. Minn, M. Zeng, and V. K. Bhargava, "On timing offset estimation for OFDM systems," IEEE Communications Letters, vol. 4, pp. 242-244, 2000.

[Minn 2003] H. Minn, V. K. Bhargava, and K. B. Letaief, "A robust timing and frequency synchronization for OFDM systems," IEEE Transactions on Wireless Communications, vol. 2, pp. 822-839, 2003.

[Minn 2004] H. Minn, V. K. Bhargava, and K. B. Letaief, "A combined timing and frequency synchronization and channel estimation for OFDM," in 2004 IEEE International Conference on Communications (IEEE Cat. No.04CH37577), 2004, pp. 872-876 Vol.2.

[Morelli 2007]M. Morelli, C. C. J. Kuo, and M. O. Pun, "Synchronization Techniques for Orthogonal Frequency Division Multiple Access (OFDMA): A Tutorial Review," Proceedings of the IEEE, vol. 95, pp. 1394-1427, 2007.

[Mostofi 2006] Y. Mostofi and D. C. Cox, "Mathematical analysis of the impact of timing synchronization errors on the performance of an OFDM system," IEEE Transactions on Communications, vol. 54, pp. 226-230, 2006. 
[Mousa 2010] A. Mousa and H. Mahmoud, "Channels estimation in OFDM system over Rician fading channel based on comb-type pilots arrangement," IET signal processing, vol. 4, pp. 598-602, 2010.

[Moya 2015] S. Moya, M. Hadad, P. Donato, M. Funes, and D. Carrica, "PLC equalization method for block-pilot OFDM systems based on complementary sequences," in 2015 XVI Workshop on Information Processing and Control (RPIC), 2015, pp. 1-6.

[Moya 2016a] S. Moya, M. Hadad, P. Donato, M. Funes, and D. Carrica, "Channel estimation and equalization of broadband PLC systems-Part 1: Complementary Sequences based channel estimation in OFDM," in 2016 IEEE Biennial Congress of Argentina (ARGENCON), 2016a, pp. 1-6.

[Moya 2016b] S. Moya, M. Hadad, M. Funes, P. Donato, and D. Carrica, "Broadband PLC-channel equalisation in the frequency domain based on complementary sequences," IET Communications, vol. 10, pp. 1605-1613, $2016 b$.

[Na 2018] Z. Na, M. Zhang, M. Xiong, J. Xia, X. Liu, and W. Lu, "Pseudonoise sequence based synchronization for generalized frequency division multiplexing in 5G communication system," IEEE Access, vol. 6, pp. 14812-14819, 2018.

[Nasraoui 2012] L. Nasraoui, L. N. Atallah, and M. Siala, "An efficient reduced-complexity two-stage differential sliding correlation approach for OFDM synchronization in the multipath channel," in 2012 IEEE Wireless Communications and Networking Conference (WCNC), 2012, pp. 20592063.

[Nasraoui 2014] L. Nasraoui, L. N. Atallah, and M. Siala, "Performance evaluation of an efficient reduced-complexity time synchronization approach for OFDM systems," annals of telecommunications-annales des télécommunications, vol. 69, pp. 321-330, 2014.

[Nikfar 2017] B. Nikfar and G. Bumiller, "Real-time synchronization and multiband detection for narrowband power line communication," in 2017 IEEE International Symposium on Power Line Communications and its Applications (ISPLC), 2017, pp. 1-5.

[Nombela 2015a] F. Nombela, E. García, R. Mateos, and Á. Hernández, "Efficient implementation of a symbol timing estimator for broadband PLC," Sensors, vol. 15, pp. 20825-20844, 2015a.

[Nombela 2015b] F. Nombela, E. García, J. Ureña, H. Á, and P. Poudereux, "Robust synchronization algorithm for broadband PLC based on WaveletOFDM," in 2015 IEEE 20th Conference on Emerging Technologies \& Factory Automation (ETFA), 2015b, pp. 1-7.

[Nombela 2017] F. Nombela, E. García, and Á. Hernández, "Preliminary study between frequency-domain equalizer and 0-ASCET for PLC," in 2017 IEEE 17th International Conference on Ubiquitous Wireless Broadband (ICUWB), 2017, pp. 1-5. 
Diseño de Estrategias de Sincronización y Estimación de Canal para la Mejora de Comunicaciones en Redes Inteligentes de Energía

[Nombela 2019] F. Nombela, E. García, R. Mateos, and Á. Hernández, "Real-time architecture for channel estimation and equalization in broadband PLC," Microprocessors and Microsystems, vol. 65, pp. 121$135,2019$.

[Offiong 2020] F. B. Offiong, S. Sinanović, and W. O. Popoola, "PilotAided Frame Synchronization in Optical OFDM Systems," Applied Sciences, vol. 10, p. 4034, 2020.

[Oksman 2009] V. Oksman and S. Galli, "G. hn: The new ITU-T home networking standard," IEEE Communications Magazine, vol. 47, pp. 138$145,2009$.

[OMEGA 2008] OMEGA, "PLC Channel Characterization and Modelling," Dec. 20182008.

[Orallo 2015] C. M. Orallo, I. Carugati, P. G. Donato, and S. Maestri, "Study on Single-bin Sliding DFT algorithms: Comparison, stability issues and frequency adaptivity," Measurement, vol. 69, pp. 9-19, 2015.

[Park 2003] B. Park, H. Cheon, C. Kang, and D. Hong, "A novel timing estimation method for OFDM systems," IEEE Communications Letters, vol. 7, pp. 239-241, 2003.

[Pérez 2008] M. C. Pérez, J. Ureña, A. Hernández, F. Álvarez, A. Jimenez, and C. De Marziani, "Efficient Correlator for LS Codes Generated from Orthogonal CSS," Communications Letters, IEEE, vol. 12, pp. 764-766, 2008.

[Pérez 2009] M. C. Pérez, J. Ureña, A. Hernández, A. Jiménez, and C. De Marziani, "Efficient Generation and Correlation of Sequence Pairs With Three Zero-Correlation Zones," Signal Processing, IEEE Transactions on, vol. 57, pp. 3450-3465, 2009.

[Pollet 1999] T. Pollet and M. Peeters, "Synchronization with DMT modulation," IEEE Communications Magazine, vol. 37, pp. 80-86, 1999.

[Popovic 1999] B. M. Popovic, "Efficient Golay correlator," ELECTRONICS LETTERS, vol. 35, pp. 1427-1428, 1999.

[Prasad 2016] G. Prasad, L. Lampe, and S. Shekhar, "In-band full duplex broadband power line communications," IEEE Transactions on Communications, vol. 64, pp. 3915-3931, 2016.

[Prasad 2019] G. Prasad, H. Ma, M. Rahman, F. Aalamifar, and L. Lampe. (2019, accesed on Feb. 15). A Cumulative Power Line Noise Generator. Available: [Online].

http://www.ece.ubc.ca/ gauthamp/PLCnoise/

[Qian 2017a] X. Qian, H. Deng, and H. He, "Pilot-based parametric channel estimation algorithm for DCO-OFDM-based visual light communications," Optics Communications, vol. 400, pp. 150-155, 2017a. 
[Qian 2017b] X. Qian, Y. Deng, H. Deng, C. Zhang, and J. Du, "Synchronisation algorithm for OFDM/OQAM systems based on zero autocorrelation code," IET Communications, vol. 12, pp. 283-289, $2017 \mathrm{~b}$.

[Qian 2018] X. Qian, H. Deng, and H. He, "Joint synchronization and channel estimation of ACO-OFDM systems with simplified transceiver," IEEE Photonics Technology Letters, vol. 30, pp. 383-386, 2018.

[Rabie 2015] K. M. Rabie and E. Alsusae, "On Improving Communication Robustness in PLC Systems for More Reliable Smart Grid Applications," IEEE Transactions on Smart Grid, vol. 6, pp. 2746-2756, 2015.

[Ren 2005] G. Ren, Y. Chang, H. Zhang, and H. Zhang, "Synchronization method based on a new constant envelop preamble for OFDM systems," IEEE Transactions on Broadcasting, vol. 51, pp. 139-143, 2005.

[Rezgui 2012] C. Rezgui, S. Jarboui, and K. Grayaa, "A PAPR reduction technique based on Golay sequences and Fractional Fourier Transform for OFDM systems," in 2012 Computing, Communications and Applications Conference, 2012, pp. 383-386.

[Rosati 2012] S. Rosati, G. E. Corazza, and A. Vanelli-Coralli, "OFDM Channel Estimation Based on Impulse Response Decimation: Analysis and Novel Algorithms," IEEE Transactions on Communications, vol. 60, pp. 19962008, 2012.

[Schmidl 1997] T. M. Schmidl and D. C. Cox, "Robust frequency and timing synchronization for OFDM," IEEE Transactions on Communications, vol. 45, pp. 1613-1621, 1997.

[Shao 2014] H. Shao, Y. Li, J. Tan, Y. Xu, and G. Liu, "Robust timing and frequency synchronization based on constant amplitude zero autocorrelation sequence for OFDM systems," in 2014 IEEE International Conference on Communiction Problem-solving, 2014, pp. 14-17.

[Shi 2005] K. Shi, E. Serpedin, and P. Ciblat, "Decision-directed fine synchronization in OFDM systems," IEEE Transactions on Communications, vol. 53, pp. 408-412, 2005.

[Shrestha 2018] D. Shrestha, X. Mestre, and M. Payaró, "On channel estimation for power line communication systems in the presence of impulsive noise," Computers \& Electrical Engineering, vol. 72, pp. 406419, 2018.

[Slaughter 1924] N. Slaughter and W. Wolfe, "Carrier telephony on power lines," Journal of the AIEE, vol. 43, pp. 377-381, 1924.

[Stanczak 2001] S. Stanczak, H. Boche, and M. Haardt, "Are LAS-codes a miracle?," in GLOBECOM'01. IEEE Global Telecommunications Conference (Cat. No. 01CH37270), 2001, pp. 589-593.

[Stantchev 1998] B. Stantchev and G. Fettweis, "Burst synchronization for OFDM-based cellular systems with separate signaling channel," in 
Diseño de Estrategias de Sincronización y Estimación de Canal para la Mejora de Comunicaciones en Redes Inteligentes de Energía

VTC'98. 48th IEEE Vehicular Technology Conference. Pathway to Global Wireless Revolution (Cat. No. 98CH36151), 1998, pp. 758-762.

[Suehiro 1988] N. Suehiro and M. Hatori, "Modulatable orthogonal sequences and their application to SSMA systems," IEEE Transactions on Information Theory, vol. 34, pp. 93-100, 1988.

[Talla 2017] J. Talla and V. Blahnik, "Sliding DFT based grid to ground admittance estimation with guaranteed numerical stability," in IECON 2017-43rd Annual Conference of the IEEE Industrial Electronics Society, 2017, pp. 4344-4349.

[Tan 2012] B. Tan and J. Thompson. (2012, Powerline Communications Channel Modelling Methodology Based on Statistical Features. Available: [Online]. Available: http://arxiv.org/abs/1203.3879

[Tonello 2007] A. M. Tonello, "Wideband Impulse Modulation and Receiver Algorithms for Multiuser Power Line Communications," EURASIP Journal on Advances in Signal Processing, vol. 2007, p. 096747, 2007/12/01 2007.

[Tonello 2009] A. M. Tonello and F. Pecile, "Efficient Architectures for Multiuser FMT Systems and Application to Power Line Communications," IEEE Transactions on Communications, vol. 57, pp. 1275-1279, 2009.

[Tonello 2010] A. M. Tonello and F. Versolatto, "Bottom-Up Statistical PLC Channel Modeling\&\#x2014;Part II: Inferring the Statistics," IEEE Transactions on Power Delivery, vol. 25, pp. 2356-2363, 2010.

[Tonello 2012] A. M. Tonello, F. Versolatto, B. Bejar, and S. Zazo, "A Fitting Algorithm for Random Modeling the PLC Channel," IEEE Transactions on Power Delivery, vol. 27, pp. 1477-1484, 2012.

[Tonello 2013] A. M. Tonello and M. Girotto, "Cyclic block FMT modulation for broadband power line communications," in 2013 IEEE 17th International Symposium on Power Line Communications and Its Applications, 2013, pp. 247-251.

[Tonello 2014] A. M. Tonello, F. Versolatto, and A. Pittolo, "In-Home Power Line Communication Channel: Statistical Characterization," IEEE Transactions on Communications, vol. 62, pp. 2096-2106, 2014.

[Top 2012] P. Top, M. R. Bell, E. Coyle, and O. Wasynczuk, "Observing the power grid: Working toward a more intelligent, efficient, and reliable smart grid with increasing user visibility," IEEE Signal Processing Magazine, vol. 29, pp. 24-32, 2012.

[Torres-Alvarado 2012] D. Torres-Alvarado, G. A. Laguna-Sanchez, and A. Prieto-Guerrero, "Real time synchronization for an OFDM/PLC system implemented with a DSP," in 2012 IEEE Latin-America Conference on Communications, 2012, pp. 1-6. 
[Tseng 1972] C.-C. Tseng and C. L. Liu, "Complementary Set of Sequences," IEEE Transactions on Information Theory, vol. IT-18, pp. 644-652, September 1972.

[Val Beitia 2011] I. Val Beitia, "Long polyphase sequences for adaptive mmse detector in asynchronous cdma plc network with impulsive noise," PhD., Universidad Politécnica de Madrid, 2011.

[Van De Beek 1995] J.-J. Van De Beek, O. Edfors, M. Sandell, S. K. Wilson, and P. O. Borjesson, "On channel estimation in OFDM systems," in 1995 IEEE 45th Vehicular Technology Conference. Countdown to the Wireless Twenty-First Century, 1995, pp. 815-819.

[Walpole 2007] R. E. Walpole, R. H. Myers, S. L. Myers, and K. Ye, Probability and statistics for engineers and scientists, 8th ed., 2007.

[Wang 2009] C. Wang and H. Wang, "On joint fine time adjustment and channel estimation for OFDM systems," IEEE Transactions on Wireless Communications, vol. 8, pp. 4940-4944, 2009.

[Wang 2006] C. 1. Wang and H. c. Wang, "A Low-Complexity Joint Time Synchronization and Channel Estimation Scheme for Orthogonal Frequency Division Multiplexing Systems," in 2006 IEEE International Conference on Communications, 2006, pp. 5670-5675.

[Wang 2008] C. L. Wang and H. C. Wang, "An Optimized Joint Time Synchronization and Channel Estimation Scheme for OFDM Systems," in VTC Spring 2008 - IEEE Vehicular Technology Conference, 2008, pp. 908-912.

[Wang 2011] C. L. Wang and H. C. Wang, "Low-Complexity Joint Timing Synchronization and Channel Estimation for MIMO OFDM Systems," in 2011 IEEE 73rd Vehicular Technology Conference (VTC Spring), 2011, pp. 1-5.

[Wang 2007] J. Wang, J. Lin, and D. Li, "Synchronization for MIMO OFDM systems with loosely synchronous (LS) codes," in 2007 International Conference on Wireless Communications, Networking and Mobile Computing, 2007, pp. 254-258.

[Wang 2016] Y. Wang, S. He, Y. Sun, Q. Xu, H. Wang, and L. Yang, "Joint timing synchronization and channel estimation based on ZCZ sequence set in SC-MIMO-FDE system," EURASIP Journal on Wireless Communications and Networking, vol. 2016, pp. 1-17, 2016.

[Wu 2014] W. Wu, H. Bie, and X. Zhang, "An improved timing synchronization method for broadband OFDM based power line communication," in 9th International Conference on Communications and Networking in China, 2014, pp. 214-219.

[Xie 2013] Z.-y. Xie, L. Luo, F.-f. Dong, S.-x. Deng, and W.-b. Cao, "An improved synchronization algorithm for OFDM based powerline 
Diseño de Estrategias de Sincronización y Estimación de Canal para la Mejora de Comunicaciones en Redes Inteligentes de Energía

communication," in 4th International Conference on Power Engineering, Energy and Electrical Drives, 2013, pp. 1613-1618.

[Xiong 2013] X. Xiong, B. Jiang, X. Gao, and X. You, "DFT-based channel estimator for OFDM systems with leakage estimation," IEEE Communications Letters, vol. 17, pp. 1592-1595, 2013.

[Yan 2003] C. Yan, J. Fang, Y. Tang, S. Li, and Y. Li, "OFDM synchronization using PN sequence and performance," in 14th IEEE Proceedings on Personal, Indoor and Mobile Radio Communications, 2003. PIMRC 2003., 2003, pp. 936-939.

[Yan 2017] K. Yan, H. Zhang, and H.-C. Wu, "Robust multipath channel estimation in the presence of impulsive noise," IET Communications, vol. 12, pp. 228-235, 2017.

[Yang 2002] J.-F. Yang and F.-K. Chen, "Recursive discrete Fourier transform with unified IIR filter structures," Signal Processing, vol. 82, pp. 31-41, 2002.

[Ye 2013] Y. Ye, Q. Yi, H. Sharif, and D. Tipper, "A Survey on Smart Grid Communication Infrastructures: Motivations, Requirements and Challenges," Communications Surveys \& Tutorials, IEEE, vol. 15, pp. 520, 2013.

[Yong Soo 2010a] C. Yong Soo, K. Jaekwon, Y. Won Young, and G. K. Chung, "Channel Estimation," in MIMO-OFDM Wireless Communications with MATLAB $<$ sup $>\& \# 174 ;</$ sup $>$, ed: Wiley-IEEE Press, 2010a, p. 544.

[Yong Soo 2010b] C. Yong Soo, K. Jaekwon, Y. Won Young, and G. K. Chung, "Synchronization for OFDM," in MIMO-OFDM Wireless Communications with MATLABß, ed: IEEE, 2010b, p. 1.

[Zhang 2005] C. Zhang, X. Lin, and M. Hatori, "Novel sequence pair and set with three zero correlation windows," IEICE transactions on communications, vol. 88, pp. 1517-1522, 2005.

[Zhang 2004] Z. Zhang, M. Zhao, H. Zhou, Y. Liu, and J. Gao, "Frequency offset estimation with fast acquisition in OFDM system," IEEE Communications Letters, vol. 8, pp. 171-173, 2004.

[Zimmermann 2002] M. Zimmermann and K. Dostert, "A multipath model for the powerline channel," IEEE Transactions on Communications, vol. 50, pp. 553-559, 2002. 